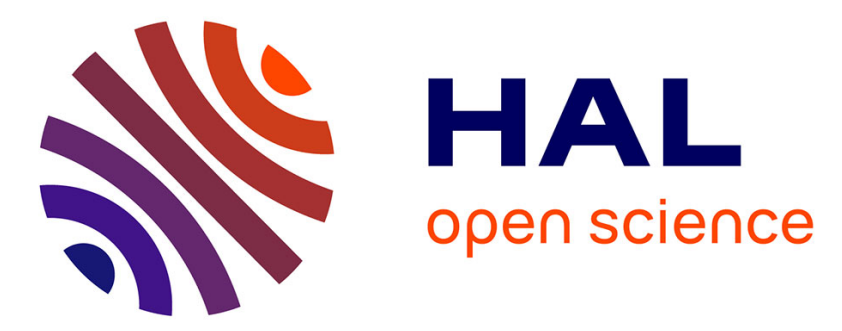

\title{
Seismic imaging of thrust fault structures in Zagros iranian oil fields, from surface and well data \\ Kazem Kazemi
}

\section{To cite this version:}

Kazem Kazemi. Seismic imaging of thrust fault structures in Zagros iranian oil fields, from surface and well data. Geophysics [physics.geo-ph]. Université de Cergy Pontoise, 2009. English. NNT: . tel-00403617v2

\section{HAL Id: tel-00403617 \\ https://theses.hal.science/tel-00403617v2}

Submitted on 9 Sep 2009

HAL is a multi-disciplinary open access archive for the deposit and dissemination of scientific research documents, whether they are published or not. The documents may come from teaching and research institutions in France or abroad, or from public or private research centers.
L'archive ouverte pluridisciplinaire HAL, est destinée au dépôt et à la diffusion de documents scientifiques de niveau recherche, publiés ou non, émanant des établissements d'enseignement et de recherche français ou étrangers, des laboratoires publics ou privés. 
ECOLE DOCTORALE SCIENCES ET INGENIERIE

de l'Université de Cergy-Pontoise

\title{
THESE
}

Présentée pour obtenir le grade de

Docteur de l'Université de Cergy-Pontoise

Discipline : Sciences de la Terre et de l'Univers

Spécialité : Géophysique

\section{SEISMIC IMAGING OF THRUST FAULT STRUCTURES IN ZAGROS IRANIAN OIL FIELDS, FROM SURFACE AND WELL DATA}

\author{
Imagerie sismique de structures pétrolières \\ plissées et faillées du Zagros (Iran), \\ à partir de données de surface et de puits
}

\section{Kazem KAZEMI}

Institut Français du Pétrole

Laboratoire Géosciences \& Environnement Cergy

Soutenue le 2 juillet 2009

Devant le jury composé de :

Pr. Raul Madariaga (Laboratoire de Géologie,ENS)

Président du jury

Pr. Jean-Luc Mari ( Université Paris VI, IFP School)

Rapporteur

Pr. Jean-Michel Marthelot (Institut de Physique du Globe de Strasbourg)

Rapporteur

Pr. Christian David (Université de Cergy-Pontoise)

Co-directeur de thèse

Dr. Laurence Nicoletis (IFP)

Co-directeur de thèse

M. Charles Naville (IFP)

Co-directeur de thèse

Dr. Jean Letouzey (IFP)

Examinateur

Dr. Ramin Nawab (Total)

M. Mahmoud Mohaddess (NIOC)

Examinateur

M. Eric Suaudeau (CGGV)

Invité

Invité 

ECOLE DOCTORALE SCIENCES ET INGENIERIE

de l’Université de Cergy-Pontoise

\section{THESE}

Présentée pour obtenir le grade de

Docteur de l’Université de Cergy-Pontoise

Discipline : Sciences de la Terre et de l'Univers

Spécialité : Géophysique

\section{SEISMIC IMAGING OF THRUST FAULT STRUCTURES IN ZAGROS IRANIAN OIL FIELDS, FROM SURFACE AND WELL}

Imagerie sismique de structures pétrolières

plissées et faillées du Zagros (Iran),

à partir de données de surface et de puits

Par

Kazem KAZEMI

National Iranian Oil Company

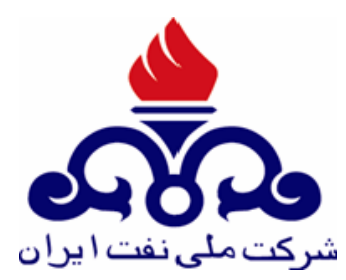





$$
\text { تان بدان جا رسيد دانش من }
$$

"My knowledge has reached the point where I realized how little it still is" 



\section{Contents}

Acknowledgements................................................................... V V

Résumé

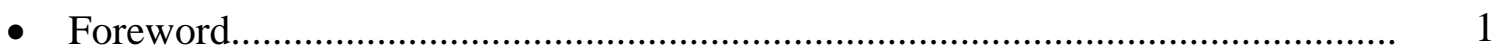

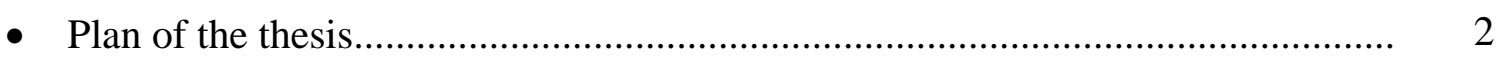

Chapter 1 Regional Geology ……………………………………………... $\quad 7$

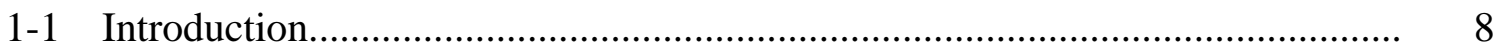

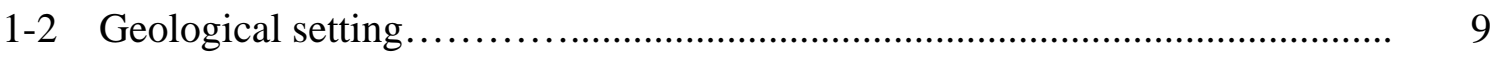

$1-3$ Aghajari field.................................................................. 13

1-4 Structural of Aghajari field................................................... 15

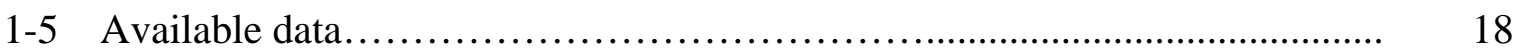

Chapter 2 VSP processing and interpretation on well A...................... 21

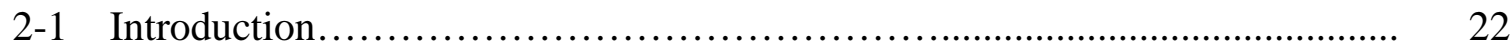

$2-2$ Vertical component processing.................................................. 24

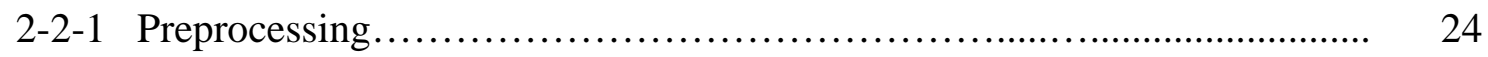

2-2-2 Processing flow chart (single axial component)........................ 24

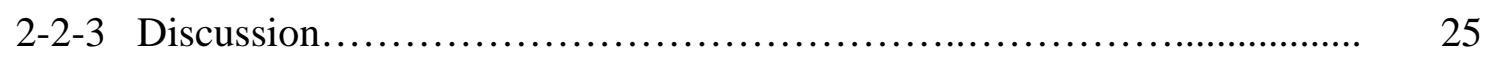

2-3 Three components processing................................................ 33

2-3-1 Orientation of three components...................................... 33

2-3-1-1 Three components at the low to medium deviated angle (Deviation angle $>12^{\circ}$ )...... 33

2-3-1-2 Three components orientation for weak deviated angle (Deviation angle $<12^{\circ}$ )...... $\quad 35$

2-3-2 Isotropic processing flow chart (On oriented three components).......... 40

2-4 Polarization analysis of P-P reflection and Dip/Azimuth computation............. 47

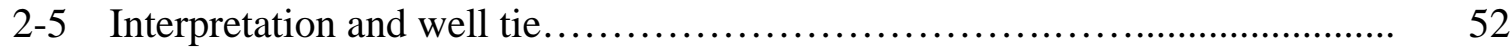

2-6 Conclusions................................................................. 54 
Chapter 3 Provisional interpretation and QC on the first PSTM

cube.

3-1 Introduction........................................................................................

3-2 Structural interpretation............................................................................... 59

3-3 Well Velocity data .....................................................................................

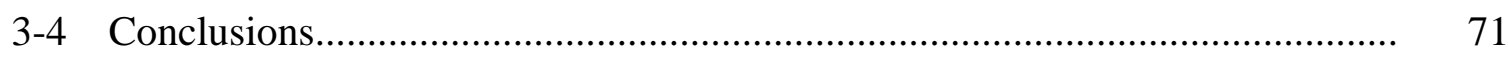

Chapter 4 Application of Beyond Dix workflow.................................... 73

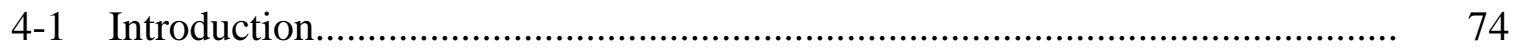

4-2 PSTM velocity analysis with TIKIM tool........................................................ 75

4-2-1 Principle of PSTM..............................................................................

4-2-2 Noise attenuation and mute........................................................................ 76

4-2-3 Velocity preparation................................................................................

4-2-4 Velocity perturbation................................................................................ $\quad 78$

4-2-5 Post migration processing and RMO picking............................................. 82

4-3 Depth velocity model building and PSDM imaging ……………………......... 85

4-3-1 Beyond Dix inversion ......................................................................... 85

4-4 Analysis of depth results .......................................................................... 95

4-5 Possible prospect on the south side of the Aghajari field .................................. 104

4-6 Conclusions................................................................................................ 109

\section{Chapter 5 Improvement of PSTM results by aperture optimization}

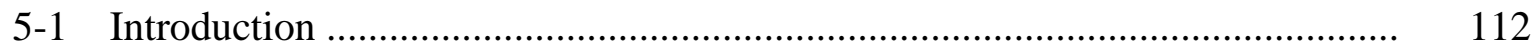

5-2 IFP PSTM migration description.................................................................... 114

5-2-1 Aperture solution and "Migration offset" gathers.......................................... 116

5-3 Test of parameters......................................................................................... 120

5-3-1 azimuth sector selection.......................................................................... 120

5-3-2 Offset range................................................................................... 127

5-3-3 Dip max parameters............................................................................... 127

5-4 Comparison with previous results............................................................. 127 
5-4-1 Main result

5-4-2 Tentative explication of differences.

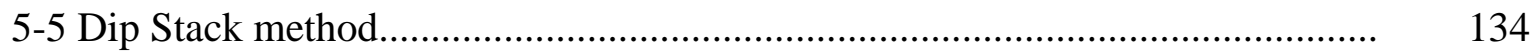

5-5-1 Expected move-out shape in "aperture gathers" for planar reflector............. 134

5-5-2 Short description of algorithm for controlling aperture.............................. 137

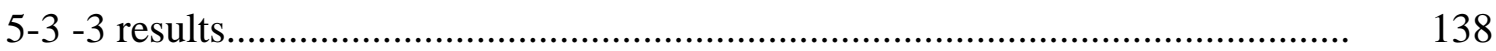

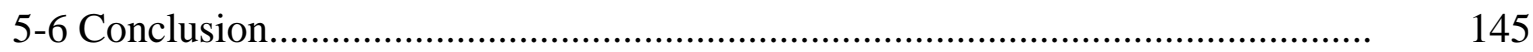

\section{Chapter 6 Method of 3 component VSP data orientation in vertical} to low deviated well.

6-1 Introduction................................................................ 148

6-2 Description of the current VSP orientation practice............................ 149

6-3 Basic summary of propagation of seismic body waves.......................... 155

6-4 Orientation method of recorded horizontal components into a coherent system using down going shear wave residuals....

6-6 Discussions

\section{Chapter 7 Look ahead VSP using oriented 3 Components in a deep} exploration well in mountainous Zagros, Iran, and post drilling integrated interpretation with surface seismic and well data (study on well B).....

Abstract

Introduction.

VSP survey and Pre-processing.... 174

VSP Processing 175

Synthetic seismogram.

Confrontation of synthetic seismogram and VSP corridor stack. ...

Electrical resistivity results and interpretation.... 180

Confrontation of results from 2D surface seismic section, VSP, Synthetic

Seismogram from calibrated sonic log, edited Density and resistivity imager. 


\section{Chapter 8 Conclusion and recommendations}

8-1 What information could be deduced from VSP................................................. 208

8-2 3D imaging from surface seismic.................................................................. 209

8-3 Conclusion concerning The Aghajari structure................................................ 210

8-4 Suggestion for future work....................................................................... 210

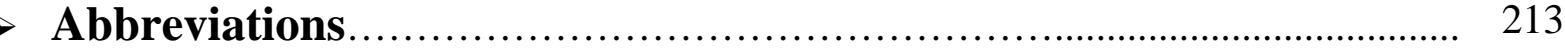

References..................................................................... 215

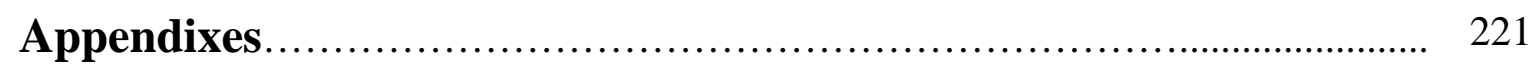

- Appendix 1

Two patents were recently filed in France as follow:

I) Méthode de pointe temps et d'orientation de signaux de puits

à trois composantes; application number: Fr 09/00.643., 12 Feb. 2009

II) Méthode d’orientation de signaux sismiques de puits à trois composantes; application number: Fr 09/00.916. 26

Feb.2009

\section{- Appendix 2}

I) An expanded abstract accepted in EAGE has been orally presented with following title:

Seismic imaging on the Aghajari field: pitfalls and solutions

II) Another article have been presented in SAID and submitted in IFP website as follow:

Look ahead VSP using oriented 3 components in a deep exploration well in mountainous Zagros, Iran 


\section{Acknowledgments}

First I would like to thank Jean Letouzey from Institut Français de Pétrole (IFP), Patrick Canal and Michel Manin from CGGVeritas and Mahmoud Mohhaddess head of NIOC exploration who initiated this $\mathrm{PhD}$ project.

I am grateful to the University Cergy-Pontoise, Département des Sciences de la Terre et Environnement and particularly to Christian David who agreed to host this PhD.

I thank my directors, Laurence Nicoletis and Charles Naville who helped me during the three years and gave me the opportunity to choose the direction of my work. The beginning of the thesis was quite difficult and they helped me to find and reorient the topic that would make me work during these three years. Laurence and Charles made my life easier in accommodating me and my family and in solving diverse administrative issues. I am grateful of their kindness and generous support.

I most thank CGGVeritas for their financial and technical support. I have benefited from the facilities provided at the CGGVeritas research centre in Massy, near Paris. I cannot ever forget their excellent cooperation. I particularly appreciate Serge Zimine for his patient explanations and detailed discussions during the whole project. Special thank to Pierre Mituard, Pierre Charrier and Raffik Oukhedouma for their great technical help during the PSDM and PSTM imaging process. Gilles Lambaré and Eric Suaudeau gave me opportunity to truly understand the PSDM and PSTM jobs and flows. I also thank Julien Meunier for useful exchanges about acquisition and processing practical techniques. Abdelkader Djeddou is highly appreciated for his patient and support in solving the administrative issues. Besides, it has not been easy everyday to deal with long hours for commuting from IFP/Rueil Malmaison to CGGV/Massy using the Paris Metro system especially during the few memorable days of typical French Metro strikes, fortunately not to often.

National Iranian Oil company (NIOC), exploration directorate has provided the whole dataset for this thesis including 3D surface seismic, well data, reservoir and geological information. I am indebted to Mr. Mohaddess and Mr. Zad Mohammadi, for their support in the long-term task of improving the imaging and understanding of the complex Zagros structures and for permission of publishing the results. I appreciate the friendly cooperation from Geophysics department colleagues, particularly Mojtaba Khorasani, Mostafa Naini, Iraj Abdollahifard, 
Mahmoud Reza Torabi and Ghasem Heidary. I also thank Shahram Sherkati from geology department for his constructive comments.

I am very appreciative of all the help and support from Remi Echard, Bernard Colleta and Michel Dietrich. Great thanks to Jean Letouzey and Jean Claude Lecomte from IFP geology department for faithful discussions in seismic interpretation and evaluation of the information content of seismic images.

I always had a lot of support from all the people from IFP geophysics department, during this $\mathrm{PhD}$ thesis. The general atmosphere was very friendly and favourable to a good work, using diverse processing computers/hardware's and various software packages: Geocluster and Teamview (CGGV), Interwell and Easytrace(IFP), Charisma workstation (GeoQuest), GOCad structural modeling (Paradigm) Tesseral seismic modeling software (although I barely touched it): I had the pleasure in cooperating with Louis Cherel, Patrick Rasolofosaon, Josette Bruneau, Martine Ancel, Gisele Etienne, Christian Le Bras, Jean Marc Richard, Jean Luc Rudkiewicz, Nathalie Lucet and karine Labat in IFP Rueil. I would like to thank Christine Le Cleach, Secretary of geophysics department (IFP) for all her help.

I am deeply appreciative of Pascal Froidevaux's contribution for his PSTM migration expertise during the course of my thesis, which was quite decisive in improving the imaging of the steep dipping reflectors. I have been positively surprised by the unexpected results obtained by Pascal's prototype routines, using the same Kirchhoff principals of seismic imaging.

During these three years I have met many researchers in IFP, CGGVeritas, and Schlumberger who work in the domains of geophysics and geology. I had very interesting discussions with some of these researchers, discussions which helped me to take a step backwards and see how my work could fit in the general picture.

The remaining difficulties of seismic imaging in the Zagros area make me realize and appreciate the talent of all explorationists who discovered the giant Iranian oil fields using rudimentary techniques.

As field geophysicists who acquired the 3D seismic survey on the Aghajari Field, this PhD gave me a wide overview about the long path between the design of the field seismic operations and the actual results and evaluation of the ultimate benefits of the 3D seismic 
surveying for understanding a given reservoir and structural geology, and to prepare eventual drilling decisions.

I thank my parents, from whom I learned the values of hard work. They always show me the honor of truly life.

The most important acknowledgment, to me, is to thank my family for support and helping me find the time to work during the thesis. My wife, Shohreh for her patience, love and support gave me strength to complete this work. She always supports me to move forward. There are no words to express how happy and grateful I am to have her by my side. I also dedicate this thesis to my dear daughter Melika for her understanding during my $\mathrm{PhD}$. 



\begin{abstract}
The Aghajari field is located in the Dezful Embayment, one of major structural zone in Zagros fold-thrust belt containing most of Iranian oil fields. The late Oligocene-early Miocene Asmari formation is one of the main reservoir rocks of SW Iran with several decades of production history from different oil fields in the region.

The objective of the present $\mathrm{PhD}$ thesis is to improve the structural understanding of the Aghajari field using 3D seismic imaging for the first time, and well seismic. the lack of seismic response of the dipping horizons in the reservoir oil zone constitutes a major identified difficulty.

Given the dip values of $30^{\circ}$ to $45^{\circ}$ of the reflectors in the pay zone, the P-P reflections are expected to appear mainly on the horizontal components of the VSP data, justifying the processing of the 3 components (3C). An innovative method of 3C VSP orientation was developed. The 3C VSP results confirm the presence of dipping reflectors, with dip values in agreement with surface seismic, on the eastern flank of the structure.

Several new approaches of seismic imaging have been applied in order to investigate and improve the reservoir illumination. The "Beyond Dix" workflow of CGGVERITAS was applied to land seismic data for the first time; this process enables to build a depth/velocity model from the dips read on the PSTM image (pre-stack time migration), and the residual move out corrections. The depth migrated image is improved in the overburden, unfortunately not at reservoir level, due to low signal to noise, interference with multiples, thus poor dip and velocity determination in the reservoir interval.

A new Kirchhoff PSTM prototype technique was developed in IFP, allowing a selection of azimuth sector, offset range and geological dip, with automatic optimization of the local dip. The results from this new technique show clear improvements in dip determination and signal to noise ratio of the migrated image.

The additional study of another exploration anticline in Zagros, using surface seismic, well data, VSP and geological information illustrates how an integrated data analysis can improve the understanding of deep structures, and therefore help making more reliable drilling decisions, if achieved timely.

In Zagros, Surface seismic is definitely a good tool for the structural exploration, but its application to fine reservoir studies has not been demonstrated, in spite of the efforts achieved in the present study.
\end{abstract}

Keywords: Zagros, Aghajari field, 3-Component VSP, Beyond Dix, PSTM, PSDM, Azimuth sector, Borehole data integration;

\title{
Résumé
}

Le Champ d'Aghajari est situé dans le Dezful, une des zones du Zagros structurée par d'importantes failles de charriage, et contenant la majeure partie des champs d'huile iraniens. La formation calcaire de l'Asmari, datant de la fin Oligocène à début Miocène, constitue l'une des principales couches réservoir du Sud-Ouest de l'Iran, avec un historique de production de plus d'un demi siècle pour plusieurs champs de cette région. L'objectif de cette thèse est de mieux comprendre la structure de ce champ, connu principalement par les puits de production, grâce à la première sismique 3D récemment acquise et en intégrant les informations fournies par la sismique de puits. Le problème principal est que le toit et la base du réservoir n’apparaît pas dans la zone à huile du réservoir, où les interfaces sont très pentés.

Étant donné l'importance des pendages (environ $40^{\circ}$ ), les réflexions en sismique de puits zéro offset sont attendues principalement sur les composantes horizontales, ce qui rend impératif le traitement des 3 composantes. Une méthode originale d'orientation des 3 composantes est employée et les résultats confirment la présence de réflecteurs pentés, en accord avec la sismique de surface sur le côté Est de la structure.

Plusieurs nouvelles approches d'imagerie ont été appliquées afin de comprendre et résoudre les problèmes d'illumination de la zone réservoir. Nous avons appliqué pour la première fois en terrestre l'approche « Beyond Dix » de CGGVERITAS qui permet de construire un modèle profondeur à partir de pendages et courbure résiduelles move-out mesurée sur les images PSTM (pré-stack time migration). Les résultats migrés en profondeur sont plus précis dans le recouvrement mais la zone réservoir n’est pas améliorée. La raison en est que les images temps ne permettent pas de mesurer les pendages et courbures résiduelles dans la zone d'intérêt en raison d'une part de fort multiples et d'autre part d'u niveau de bruit important.

Une nouvelle technique prototype de migration PSTM a été développée à l' IFP, permettant une sélection de l' azimut, du pendage géologique et la recherche automatique du meilleur pendage locale. L'application de ces techniques est très encourageante et montre qu'il est possible d'extraire les réflexions pentées en améliorant le rapport signal sur bruit de la migration elle-même.

L'étude additionnelle d'exploration d'une autre structure anticlinale du Zagros, par utilisation combinée de sismique de surface, des données de puits, du PSV et de l'information géologique, illustre comment l'analyse intégrée des données permet d'améliorer la compréhension des structures profondes, et de contribuer à prendre de meilleures décisions de forage, dans la mesure où cette intégration est suffisamment rapide.

En final, dans le Zagros, la sismique de surface est définitivement une bonne méthode d'exploration structurale, mais son application aux études fines de réservoir n'a pas été démontrée, malgré le efforts effectués.

Mots-clés: Zagros, Aghajari, PSV 3-Composantes, Beyond Dix, PSTM, PSDM, Secteur azimutal, études intégrées. 



\section{Foreword}

The Aghajari field is located in the Dezful Embayment, one of major structural zone in Zagros fold-thrust belt containing most of Iranian oil fields. This field is one of the largest oil producing fields in southwest Iran. The Aghajari reservoir consists in a large buried anticline structure, discovered by drilling in 1936. The main reservoir formation is the carbonates of the late Oligocene-early Miocene Asmari Formation. It is covered by salt and evaporates of the Gasharan Formation which form a major structural disharmonic level between deep carbonates structures, the evaporates and clastics upper levels. The previous studies and maps were based on well data, logs and few 2D seismic lines which were acquired in the 1970's. Those 2D seismic lines only allowed imaging the crest of the structure, but not the flanks. Based on reservoir and well reports there is a gas cap on the top of the Aghajari field where the present gas oil contact (G.O.C) and water oil contact (W.O.C) are located around 1930m and $2460 \mathrm{~m}$ depths respectively. So, most of the production wells are located at the periphery of the structure while gas injection wells are on the top. The Asmari reservoir unit consists of dolomite and limestone, which are highly fractured in the more producing area, and small faults and fractures with different amount of displacements were suggested to explain fluctuation in gas-oil level or reservoir parameters in different part of the field. The reservoir producing zones are from different faulted/fractured compartments, which motivated the initial title of the present thesis" Using 3D seismic \&VSP data for structural interpretation and analysis of fractures on the Asmari reservoir, Aghajari field, Zagros"; the aim was to refine the evaluation of the reservoir using the $3 \mathrm{D}$ seismic surface survey recently acquired over the field.

The current 3D seismic dataset is new recent data allows us to test, in a selected subset area of the Aghajari field, the most recent methods, processing and interpretation techniques. The objective is to image the structure and the fault and fracture pattern, study the field and draw new information for further reservoir characterization/monitoring. The illumination of deep horizons is an additional aim, for further exploration of deeply buried reservoirs.

The numerous structures of the Dezful Embayment, and the present or further 3D seismic datasets, should benefit from the methods, processing and interpretation developed in this study. 


\section{Plan of the thesis}

The initial aim of this project is to improve the image of the reservoir over the whole Aghajari structure, and to characterize the detectable faults and fractures in the Asmari reservoir using new available data including:

- 3D seismic data, which has large offsets and a fair azimuth distributions,

- 3-Component VSP acquired in a deviated well on the North-Western flank (well A)

- Conventional well logs such as mud loss, DT, GR etc. and well completion reports,

- Recent borehole imaging logs: formation micro scanner, FMI.

During the case of study a question was raised concerning the poor or lack of reflection on the flank of the Aghajari anticline structure, and especially in south western flank: the old 2D seismic lines and provisional 3D processing results (obtained by BGP company) were not able to image correctly these area where most of the oil producing wells are located. This seismic imaging problem could therefore be crucial for field optimization and recovery. Additionally, with advanced seismic processing methods, we also expect to map the anticline of deeper reservoirs.

Based on available data and advanced processing systems we utilized different methods to approach the objective of the project.

Chapter I introduces the geological setting and the background of the Aghajari field, and summarizes the available data.

Chapter II focuses on the processing and interpretation of VSP on the producing well (well A) located on the North-Eastern flank of Aghajari field in order to establish a correct velocity field along well trajectory, study P- and S-wave reflection and tie the reflectivity observed on the VSP with the 3D seismic time image. First, the VSP data were processed using the CGGV Geocluster seismic software to obtain dip and azimuth of the main reflectors and evaluate any fine structural feature related to fault and fracturation in the well vicinity especially at reservoir level. The common well tie operations and the determination of the velocity profile around the well have been performed in order to check the velocity used for producing the 3D surface seismic block and verify the efficiency and fidelity of the deconvolution process.

3-Component VSP processing results show P-wave velocity vertical variations in overburden and reservoir level. We also measured the S-wave velocity profile which singles out a depth 
interval in the overburden exhibiting a weak S-wave anisotropy related to layering. No sign of S-Wave anisotropy could be detected at reservoir level due to the irreversible degradation of the VSP data quality at acquisition stage.

Chapter III presents the provisional interpretation and QC on the first PSTM cube processed by BGP(Chinese seismic service company) using the Omega software from Western-Geco. The provisional PSTM cube was evaluated and five main horizons were interpreted for the sake of imaging the main structural limbs and find out the location of oil and gas zone in the Asmari reservoir. Attributes such as "Dip" and "Variance" cube were computed in order to investigate the trend and extension of faults and fracture system above the Asmari reservoir. It could be seen right away that the flanks of the reservoir structure right over the oil producing zones, are badly imaged.

Chapter IV is devoted to the optimization of the pre-stack seismic imaging workflow over a reduced subset of the 3D dataset $\left(\sim 280 \mathrm{Km}^{2}\right)$, about 300 Giga byte of raw data, extracted from the pre-stack data preprocessed by BGP. The performed operations include pre-stack time migration (PSTM), tomography for depth velocity building and pre-stack depth migration (PSDM) to enhance the illumination of the Aghajari structure. The PSTM step was performed to prepare the initial velocity field and kinematic attributes used as input for the "Beyond Dix" workflow. "Beyond Dix" tool is a new method developed by CGGV that combines demigration of local slopes to produce kinematic invariants and slope tomography. Although we devoted a lot of our time to optimize this workflow, with the help of CGGV experts, we did not succeed to image the dipping flank in the reservoir area. At this stage, we wanted to understand if any reflection existed from the outmost dipping flanks of the reservoir, and the possible cause of this imaging difficulty.

Chapter V presents an attempt to answer to the question raised in chapter IV, using a PSTM tool developed independently in house by IFP, which allows various selections in migration apertures. This Kirchhoff summation prototype algorithm is not optimized but very flexible as we can select any azimuth sectors and offset range in the image dip space. Surprisingly, this prototype tool enables for the first time to image the dipping flanks, opening a new hope for processing/imaging the Aghajari 3D data and similar prospects in the area. 
Chapter VI exposes a new method for orienting the three component (3C) of zero offset VSPs. This method was applied to the well A VSP described in chapter II and to a very deep exploration well (well B) in mountainous part of Zagros.

Chapter VII presents the well B VSP which was processed and integrated with Electrical Micro Scanner dip meter results and surface seismic data. Seismic reflection were identified on the VSP from thrust fault surfaces with dip indications very different from micro scanner dip meter results in an area where surface seismic image was very poor.

Chapter VIII summarized the conclusions: The main point is that the seismic imaging in Zagros is still challenging even in the areas which are not structurally so complex and dips are not very high at the top of Asmari reservoir.

Deriving appropriate velocity field from velocity analysis of surface seismic reflection is not an easy task although respecting well velocity data is simple.

The Prototype Kirchhoff tool developed by Pascal Froidevaux at IFP opens new perspectives to improve the images in the structural limbs of Aghajari structure where previous industrial tools have failed.

Well tie with VSP, logs and surface seismic have been successfully achieved resulting in a fair interpretation in areas of poor surface seismic images.

Finally, this project can be considered as a pilot study which could be extended to neighboring fields with similar difficulties.

Appendices: Publications underway

- Two patents were filed on the orientation of 3 component of the VSP.

I) Méthode de pointé temps et d'orientation de signaux de puits a trois composantes; application number Fr 09/00.643., 12 Feb. 2009; by Charles Naville, Sylvain Serbutoviez, Jean-Claude Lecomte

II) Méthode D’orientation de signaux sismiques de puits à trois composantes; application number Fr 09/00.916. 26 Feb. 2009; by Charles Naville, Sylvain Serbutoviez, Jean-Claude Lecomte, Pascal Froidevaux et Kazem Kazemi

An oral presentation/paper of the imaging work has been presented at EAGE 2009 in Amsterdam, convention entitled:

\footnotetext{
"Seismic imaging on the Aghajari field: pitfalls and solutions" by Kazem Kazemi* , Pascal Froidevaux¹, Laurence Nicoletis',
} 
- A paper has been presented in SAID (June 2009) and published in IFP review about well B VSP and well tie (ref. chapter VI).

by Kazem Kazemi*, Charles Naville (IFP), 

Chapter 1

Regional geology 


\section{1-1 Introduction}

The Aghajari field is located in the Khuzestan province in the south-west Iran more precisely in the Dezful Embayment, geological province of the Zagros Fold and Thrust Belt (ZFTB) (Fig. 1-1 and 1-2). The Khuzestan is the most prolific oil province of Iran.

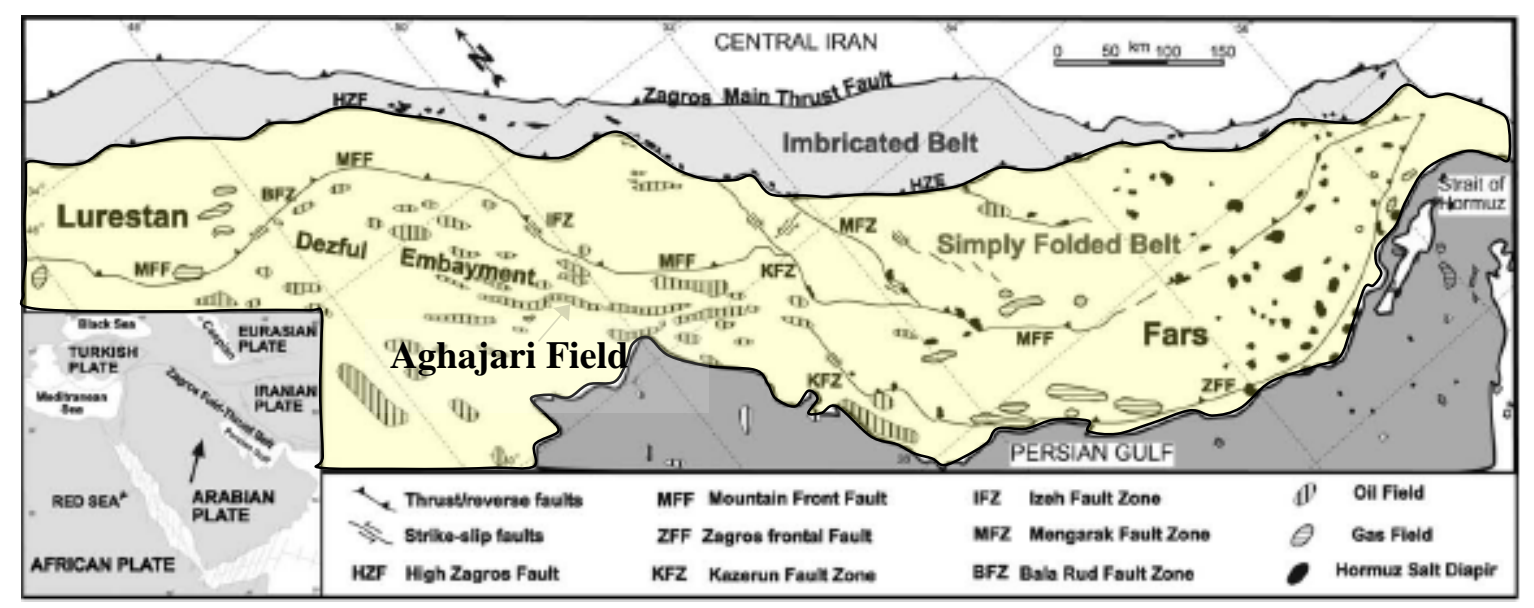

Fig.1-1 structural setting of the Zagros Fold and Thrust Belt showing the major fault zones, distribution of oil and gas fields and the Hormuz salt diapirs (From Sepehr and Cosgrove, 2004).

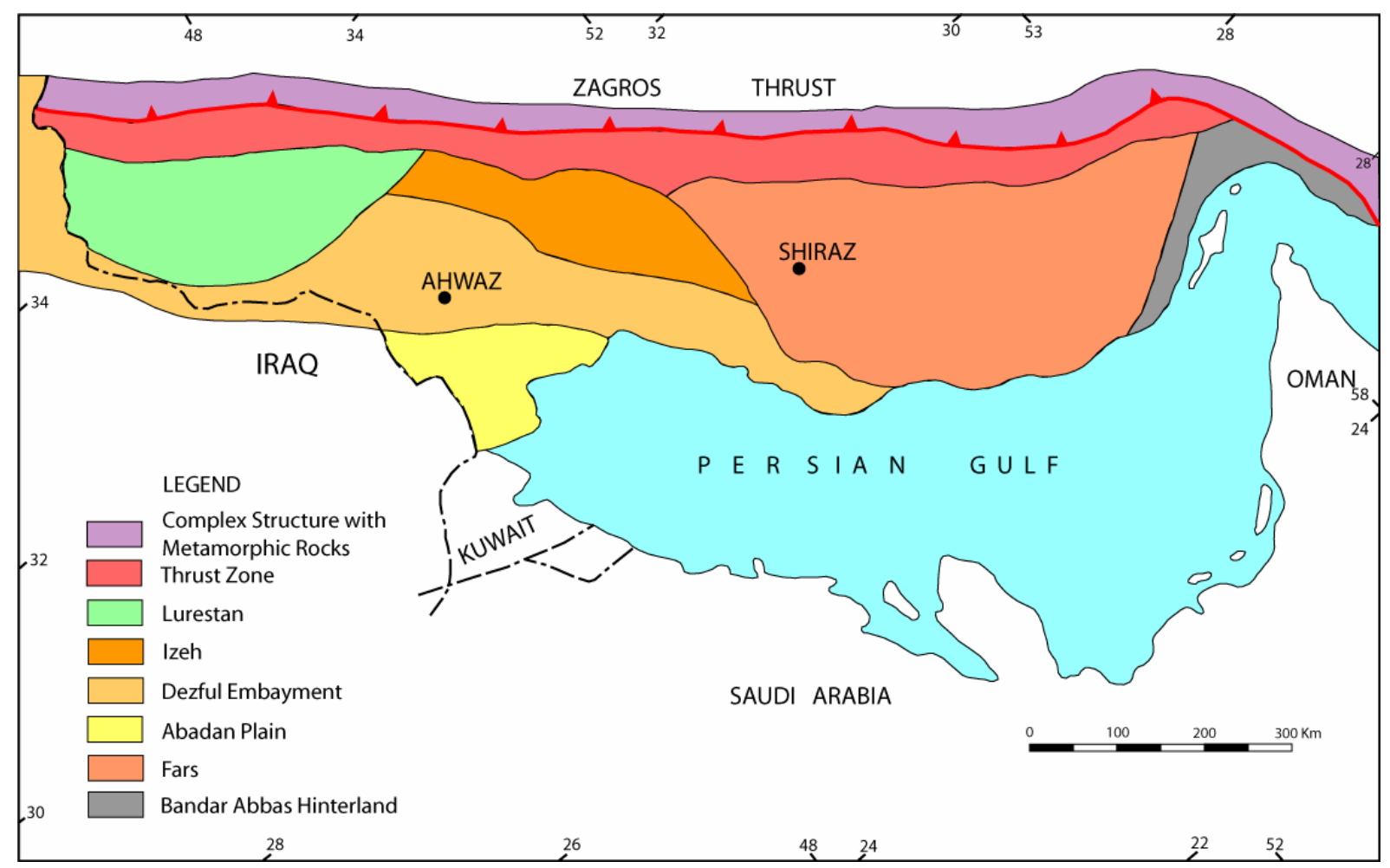

Fig.1-2 Zagros units' classification based on structural and Petroleum characteristics (From H. Motiei, 1995). 


\section{1-2 Geological setting}

The Zagros mountain belt of Iran, a part of the Alpine-Himalayan system, extends from the NW Iranian border through to SW Iran; up to the Strait of Hormuz. This orogenic belt is the result of the collision between the continental Arabian Plate and the so-called Iranian Block belonging to Eurasia (Takin, 1972; Berberian and King, 1981). It has been divided laterally to the Lurestan, Dezful Embayment and Fars regions from northwest to the southeast (Fig.1-2). Dezful Embayment is a structural depression developed mainly after the early Miocene in front of the Izeh folded zone (Fig. 1-2, 1-3 and 1-4). Subsidence is related to the developing foredeep of the Zagros Fold and Thrust Belt (ZFTB) . The Dezfull Embayment is surrounded by Balarud fault in north-north-western limit, the Mountain Front Fault (MFF) in the east north-eastern limit, and its south-south-eastern limit is the Kazerun fault.(Fig.1-1 and 1-3). These N-S and E-W trending structures played an important role in controlling sedimentation of the Basin (Sepher and Cosgrove, 2003).

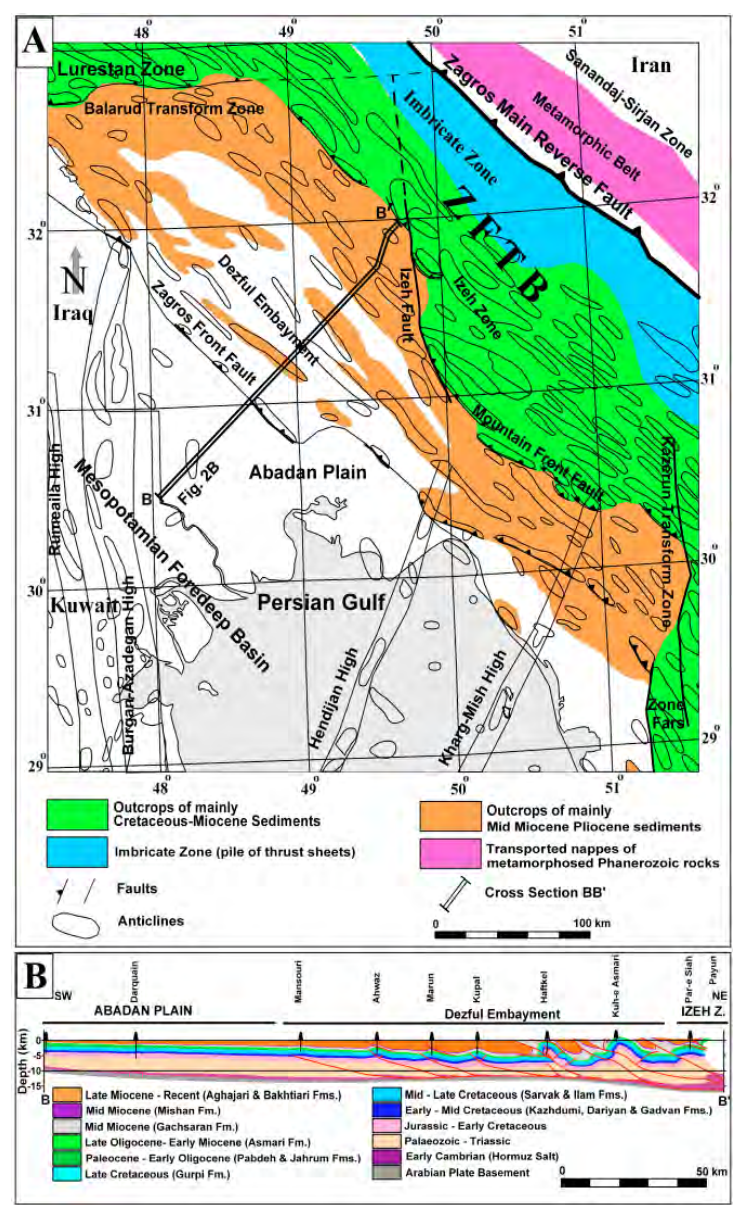

Fig.1-3 Simplified geological map of the western ZFTB, and Cross-section through the Abadan Plain and the Dezful Embayment along line B-B, (From Abdollahi et al. 2006) 


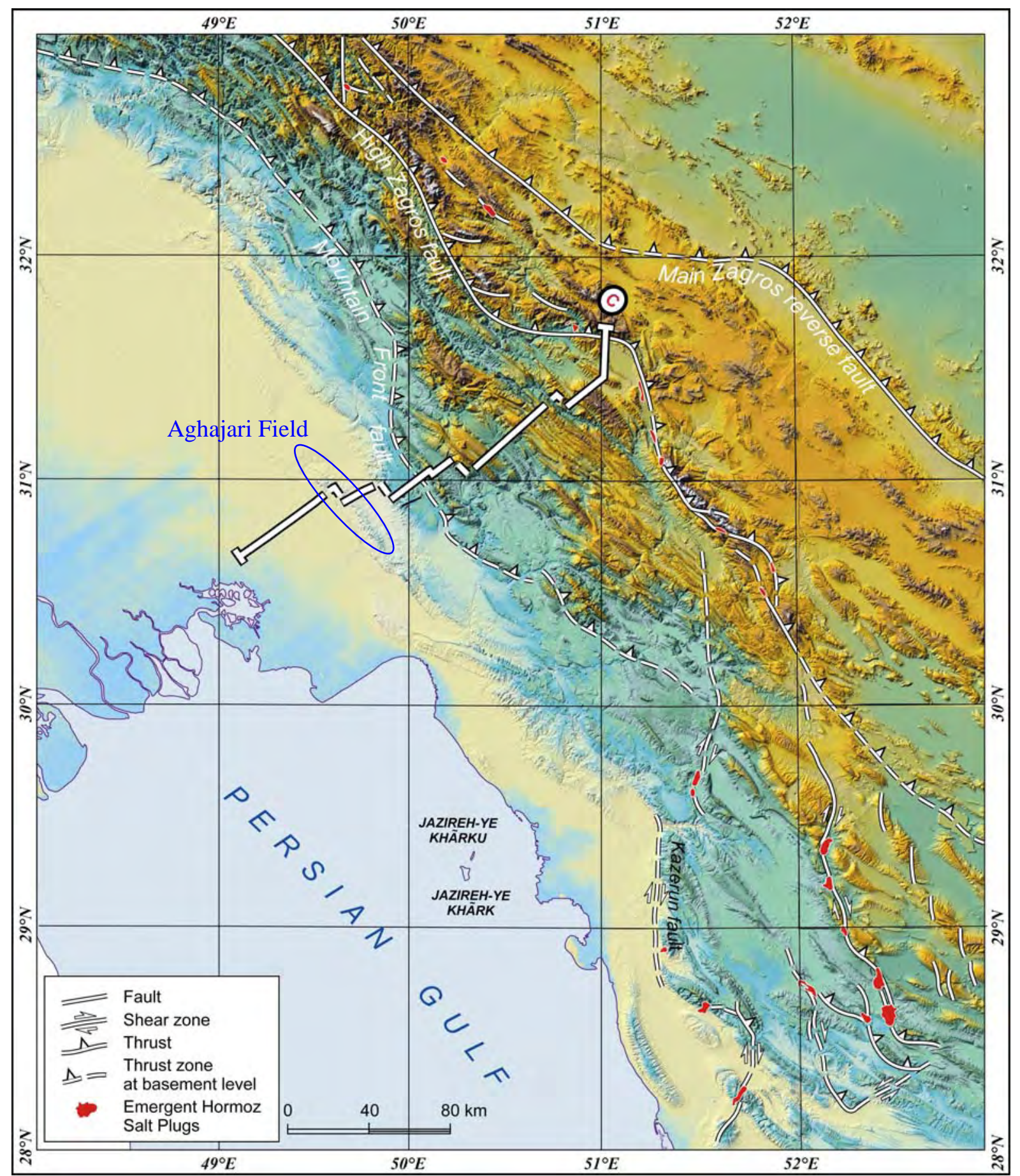

Fig.1-4 Topographic map of Central Zagros with location of five regional structural transects. Transect $C$ is shown in Fig.1-5

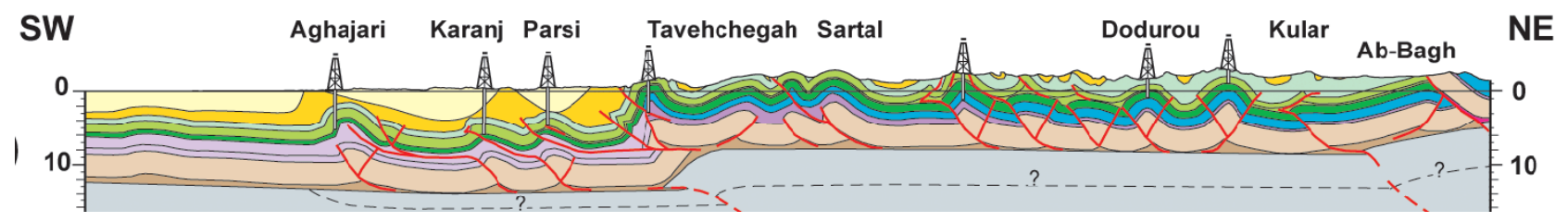

Fig.1- 5 Transect C crossing the Agahjari field (Sherkati et al. 2006) 
The stratigraphic section of the Khuzestan province (Dezful Embayment) is summarize on figure 1-5, but it must be noticed that the stratigraphic control by wells in the Dezful is limited to the Jurassic succession .

The sedimentary cover is supported by a Panafrican basement, which is exposed in the western part of the Arabian shield. There is no exposure of the basement in Iranian Zagros. Information about its depth and composition are gained from seismic, gravity and aeromagnetic surveys. It is generally considered that the evaporates of the Hormuz Formation (latest Proterozoic-Early Cambrian) were deposited directly on the basement. However seismic evidence from northern Fars suggests the existence of pre-Hormuz sediments in some parts of the Iranian Zagros (Letouzey and Sherkati, 2004). The Hormuz Formation is the basal decollement level in the Fars, Lurestan and High Zagros area. In the Izeh zone and Dezful Embayment, there is no surface occurrence of Hormuz salt plugs suggesting the absence of thick salt at depth. Eo-Cambrian evaporite horizons or Cambrian shale may be, in this case, the best alternative for basal decollement (Sherkati and Letouzey, 2004).

Epicontinental deposits containing mostly clastic sediments were deposited during the lower Palaeozoic. These rocks are overlain unconformably by the platform succession of Permian to Triassic age, containing clastic, carbonate and a thick evaporitic succession (Szabo and Kheradpir, 1978). Facies changes in Jurassic from limestone in the Izeh zone north of the Agahjari field to alternations of shale and anhydrite to the south .The carbonate Cretaceous sequence was accumulated on a shallow continental shelf. Northeast of the Arabian plate, a flexural basin has been formed due to the overloading of continental crust by ophiolite obduction in the late Cretaceous.

A rapid shift in sedimentation towards more detritic facies marks the passage from passive margin to foreland basin conditions in the region (Molinaro et al., 2004), and by the accumulation of late Cretaceous-Paleocene marl and flysch (Pabdeh-Gourpi formation). This subsidence was followed by a relatively calm period during the late Eocene (deposition of the Jahrom carbonate). 
During the late Oligocene-early Miocene, the limestones of the Asmari Formation were deposited, whereas basinal facies (turbidite) developed in the northeast of the High Zagros, in response to the onset of continental collision between Arabian Plate and Eurrasia (Sherkati et al., 2006).

\section{Dezful embayment}

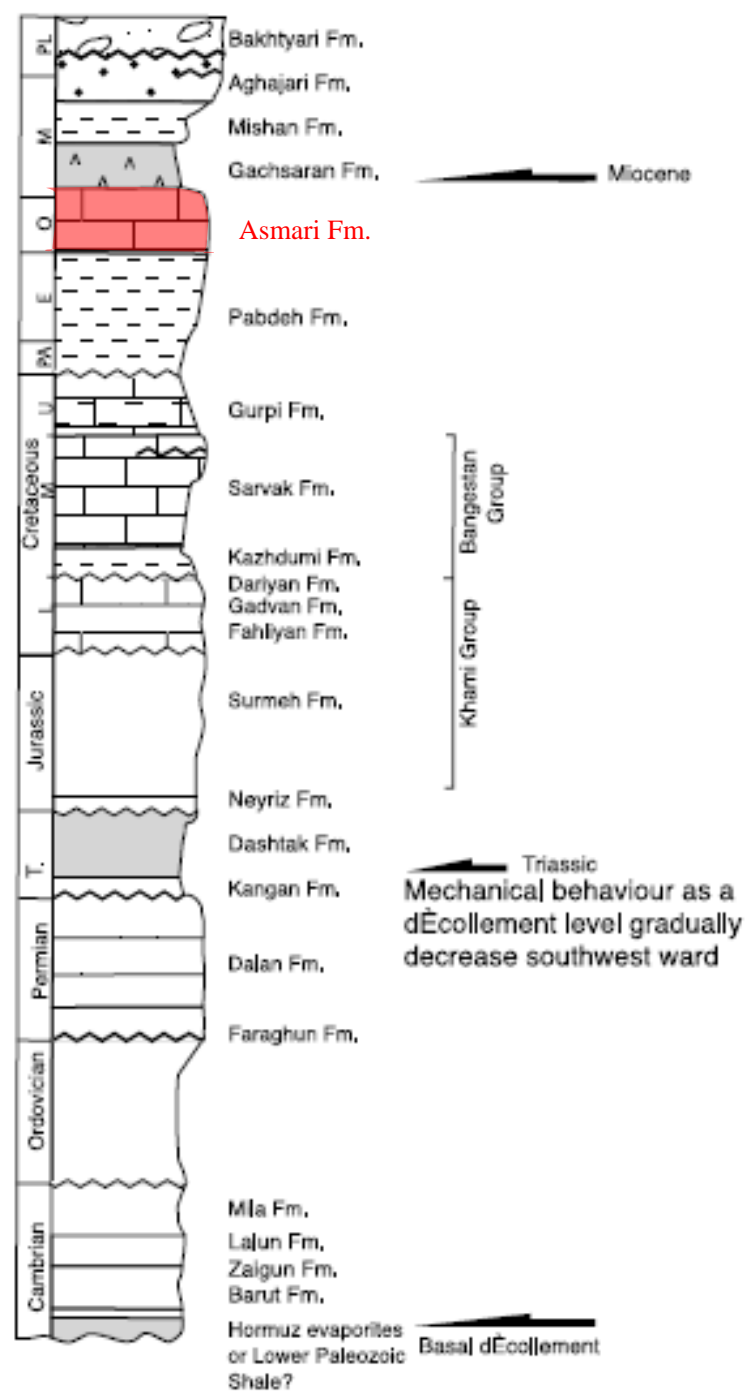

Fig. 1-6 Stratigraphy and main decollement levels in the the Dezful Embayment (From Sherkati and Letouzey, 2004). 
The Neogene sedimentary sequence began with the deposition of the evaporitic Gachsaran Formation above the Asmari limestone reservoir. It is usually subdivided into cap rock, salt and upper evaporitic units. The basal unit include salt intercalated with anhydrite some marls and some bituminous shales developed specially in the S-W Dezful zone. Upper Gachsaran is mostly composed of anhydrite, marls and limestone. The extent of this facies is restricted to the Dezful zone, marking the evolution of the Central Zagros area toward a fold belt and its associated flexural basin. The Gachsaran salt level is a major decollement and disharmony level in the northeast Dezful Embayment zone, south of the Mountain Front Fault (Fig 1-4). Large thickness variations of the Gachsaran Formation are observed on seismic lines and in the wells. This evaporatic sequence plays an important role in the sealing the Oligocene-Miocene Asmari reservoirs in the Dezful Embayment and offshore region.

The Gachsaran Formation is covered by middle Miocene marines marls limestones and shales; Upper Miocene Aghajari continental silstones and sandstones, and Pliocene Baktiari conglomerates. Folding in Dezful Embayment began or greatly accelerated as recently as the Late Miocene- Early Pliocene (Sherkati et al., 2006).

Recently the Zagros Fold and Thrust Belt is the site of intense geological studies, which led to the publication of generalized balanced cross sections across the whole domain. National Iranian Oil Company (NIOC) has launched different vintages of 2D/3D seismic surveys since 1970 's. These valuable seismic data were subject to new geological and geophysical studies (Letouzey et al. 2002; Sherkati and Letouzey 2004; Sherkati et al., 2005; Sherkati et al., 2006; Sepehr and Cosgrove 2004; Abdollahi et al., 2006).

\section{1-3 Aghajari field}

The Aghajari field is one of the big oil producing field located in southwest of Iran, in Dezful Embayment (Fig.1-1). It produces mostly from the late Oligocene-early Miocene Asmari limestone reservoir, and additionally from the Cretaceous Ilam-Sarvak, Fahliyan and Dariyan formations (Fig 1-6). The deepest well from this field touched top Fahliyan (third reservoir) at 4150m and drilled till $5592 \mathrm{~m}$ from GL. 


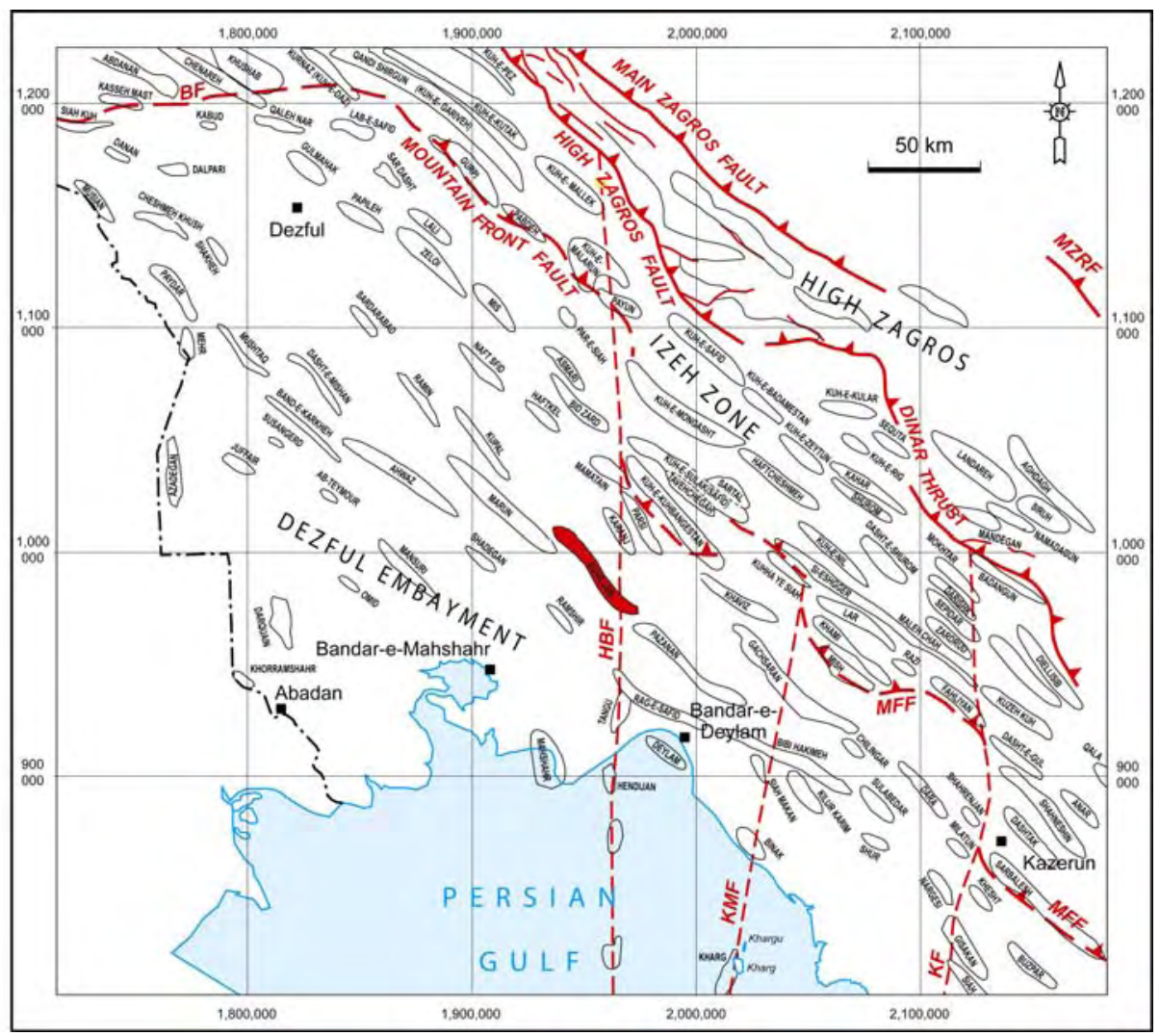

Fig.1-7 Location map of oil and Gas discoveries in Central Zagros (Sherkati et Letouzey, 2004), Aghajari field marked with red color.

The Aghajari Asmari oil reservoir discovered by drilling well AJ-2 in 1936. The Asmari Formation is the main reservoir rocks of SW Iran with several decades of production history from different oil fields in the region. Despite a generally poor porosity and matrix permeability in this reservoir, production rates are high because of the fractures. This reservoir rocks consist largely of carbonate; dolomites in upper part and limestone in the lower part. Sandstone beds occur at a number of horizons within of the field especially in the lower parts of the formation. Asmari reservoir unit consists of dolomite and limestone which are highly fractured in the more producing area. The original primary reserves are calculated to have been 8.6 billion barrels. The high well productivities obtained from the reservoir indicate the presence of a well developed fracture system (Barker et al., 1977). A number of 
studies of the geology of the Asmari reservoir at Aghajari have been done since discovering the field: stratigraphy and lithofacies study (Watson, 1962a); fracture study based on cores, cutting, mud losses, wire-line logs and flow meter data (Watson, 1962b) were performed. The fractures were interpreted as being oriented parallel to the axis of the structure (Barker, et al., 1977).

A detailed description of the origin of the porosity in the Asmari reservoir of Aghajari was prepared by Lacassagne and Shirmohammadi (1967). A review of the geology of the reservoir was carried out by core laboratories (1975a,b and c). A geological study was undertaken by Barker for planning of gas injection in the reservoir (Barker,1977). And a number of studies have been undertaken at NIOC in the past twenty years.

\section{1-4 Structure of Aghajari field}

The Aghajari oil field consists of an anticline that is generally slightly asymmetrical with the axis oriented approximately northwest to southeast. Southern flank is usually the steeper with dips of up to $70^{\circ}$ but averaging about $40^{\circ}$, while the average dip on the northeast flank is about $30^{\circ}$,values of up to $50^{\circ}$ are locally developed. The oil accumulation extends over an area which is about $56 \mathrm{Kms}$ in length and has a maximum width of $5.9 \mathrm{Kms}$. Based on reservoir and well reports there is a gas cap on the top of the Aghajari field where G.O.L and W.O.L are located around 1933m and 2462m measure depth respectively.

There is a thrust fault in the southwest flank of Aghajari field, which assume to Aghajari field up thrust against the Marun anticline. The separation between the Asmari reservoir in Aghajari and Pazanan fields was and is a big doubt. Primary geological study suggested a 
thrust fault between two fields, which Aghajari up thrust against Pazanan field. But based on gas-oil level in the Aghajari (1406 meters Sub Sea) and Pazanan (2195 meters Sub Sea) a saddle was suggested in which a vertical permeability barrier in the saddle region caused separation these reservoirs. In the previous studies based on wire-line logs, cores and other information's small faults and fractures with different amount of displacements suggested to explaining fluctuation in gas-oil level or reservoir parameters in different part of the field. Because of lack of seismic data no attempt was made to describe the distribution of fractures and faults between the wells over the entire field. 


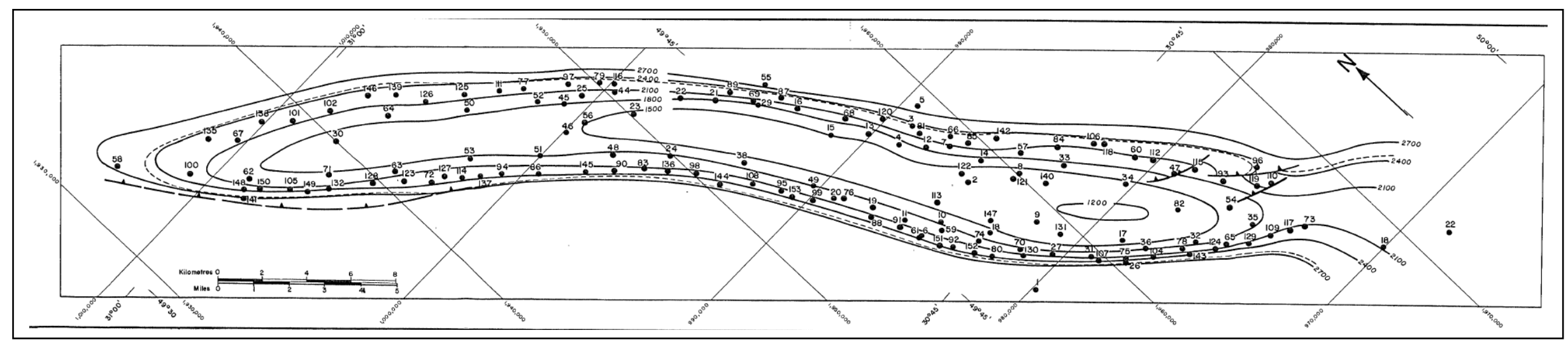

Fig 1-8 Asmari contour map of Aghajari field before the 3D seismic acquisition, with well locations (structure dimensions are about 56km / 6km), dash line shows water oil level in 1977. 


\section{1-5 Available data}

In 2005 NIOC exploration launched a 3D seismic survey over three giant fields (Aghajari,

Marun and Kupal) with the parameters summarized in Table 1.

Table1: The summary of the acquisition parameters of the 3D dataset used in the study.

\begin{tabular}{|l|l|}
\hline Group interval & $50 \mathrm{~m}$ \\
\hline Source interval & $50 \mathrm{~m}$ \\
\hline Receiver line interval & $300 \mathrm{~m}$ \\
\hline Source line interval & $300 \mathrm{~m}$ \\
\hline Number of active channel per line & 192 \\
\hline Number of line per patch & 10 \\
\hline Bin size & $25 * 25$ \\
\hline Fold & $16^{*} 5=80$ \\
\hline Maximum offset in line & $6000 \mathrm{~m}$ \\
\hline Maximum offset in cross line & $1800 \mathrm{~m}$ \\
\hline Number of channel & 1920 \\
\hline Patch & 10 X 192 \\
\hline Inline roll & Rolling 6 station for one salvo, roll \\
& on, roll off \\
\hline X-line roll & Rolling one line \\
\hline X max & 6400 \\
\hline Xmin & 475 \\
\hline Aspect ratio & 10 X 192 \\
\hline
\end{tabular}

The instrument parameters of the 3D dataset

\begin{tabular}{|l|l|}
\hline Sample rate & $2 \mathrm{~ms}$ \\
\hline Recording length & $7 \mathrm{sec}$ \\
\hline Preamplifier gain & $24 \mathrm{~dB}$ \\
\hline notch & out \\
\hline Low frequency cut & Out \\
\hline High frequency cut & $128 \mathrm{~Hz}$ \\
\hline
\end{tabular}

Figure 1-9 shows the patch, offset and azimuth distribution of this survey. We have chosen around $280 \mathrm{Km}^{2}$ of this survey over middle of the Aghajari field as study project (NIOC technical report). 

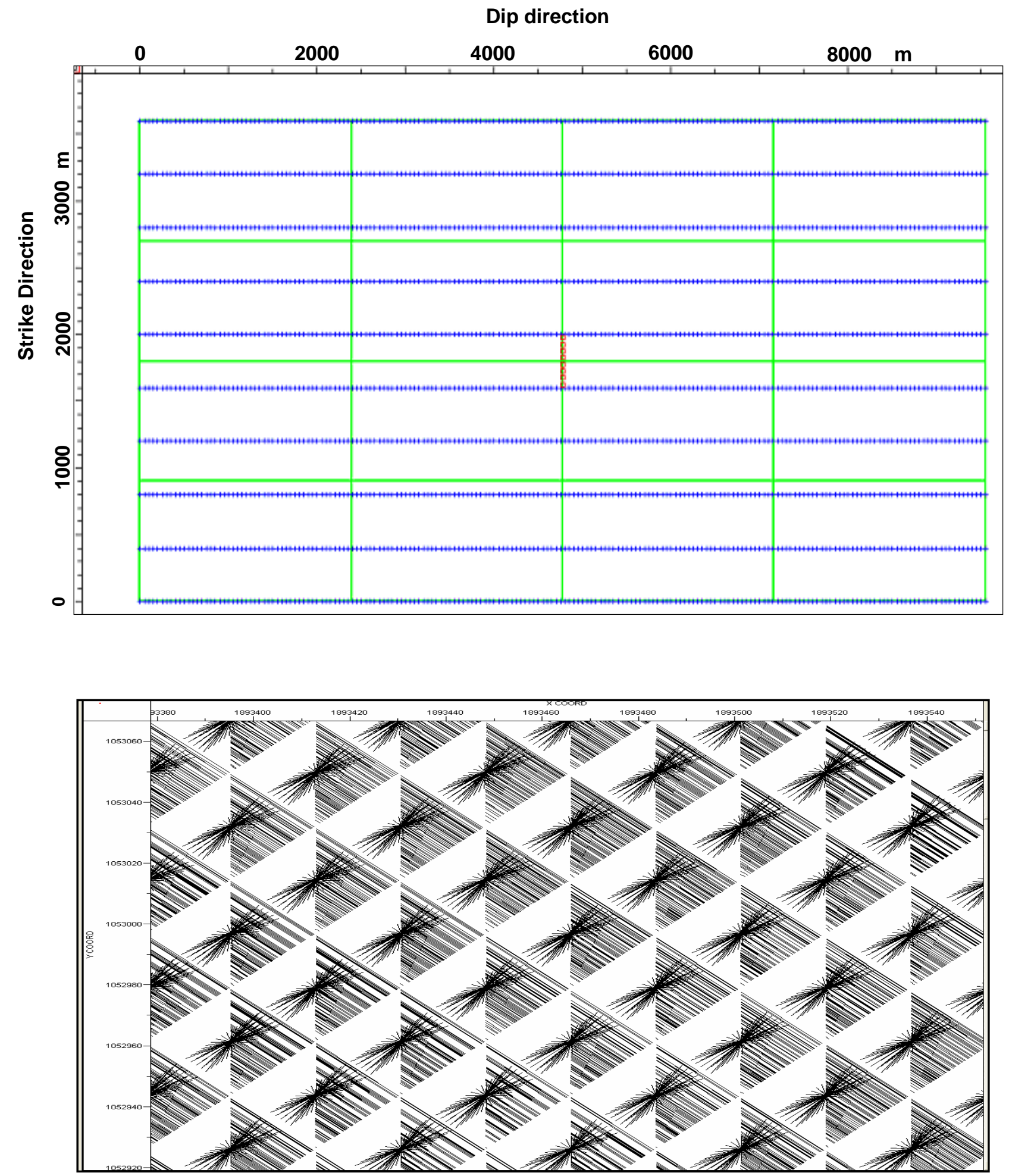

Fig 1-9 Above: acquisition template. Below: Distribution of azimuth and offset in each bin. Each box is $25 \times 25 \mathrm{~m}$, spiders show the azimuths distribution and lines correspond to offset distribution in each box. 
Three check shots and well logs such as DT, GR etc. with convenient distribution on the study area were gathered. The well completion reports are available as well. Because all delineation wells were drilled before 1970, all well logs and velocity data are analogue, which recently were digitized. In addition there is a new acquired VSP dataset over the study area. The VSP run during work-over operation in the well A located in the north eastern flank. We reprocessed the VSP 3-components data to tie the VSP with the 3D seismic cube, to measure reflectivity/attenuation and also to investigate if the noise within the reservoir interval is due to microseismicity or induced by any fluid flow. The results of VSP are discussed in the next chapter. We also used the checkshot data to extract velocity variations in the whole field. 


\section{Chapter 2}

\section{Dip/azimuth from 3-Component VSP and improved well tie}




\section{2-1 Introduction}

VSP surveys are commercially recorded with a single zero-offset source position and with a downhole tool including 3 orthogonal seismic sensors. Appropriate three component (3C) VSP processing can yield three dimension reflector dips and azimuths and values of the reflection coefficient series of the seismic reflectors around the well.

Advanced, isotropic processing of the oriented 3 components have been applied, in the aim of measuring the dip and azimuth of a few selected reflectors of good quality. Isotropic processing means that all the processing operations even time variant ones, are identically applied on the 3 components at any seismic time. The 3-components are rotated into true geographic coordinate system/ or at least into a coherent coordinate system prior to processing.

We tested this technique (chapter VI) on a deviated well A (up to $42^{\circ}$ at well bottom) while the dip and azimuth of the main geological layers were already measured by geologic data derived from the well data and the 3D seismic horizon maps over a major oil field. Dip values derived from the VSP in well A are in agreement with geological and geophysical data but the azimuth shows \pm 20 degree difference which is not fully understood. As a matter of fact the absolute accuracy of the Relative Bearing Sensor (RBS) mounted inside the VSP tool is better than $\pm 2^{\circ}\left(\right.$ ?) above $13^{\circ}$ in well deviation as measured at the bottom of the well about $2300 \mathrm{~m}$. It seems that the slow rotation trend versus depth of the oriented coordinate system might be presented over hectometres depth interval, meaning that the orientation method is not very accurate over depth of extrapolation of $20^{\circ}$ difference in $300 \mathrm{~m}$. Further investigation should be conducted in order to evaluate the accuracy of orientation method. We have meaningfully applied this technique which can be used in exploration wells where structural geology is complex, well logs are hard to interpret and surface seismic is 
poor. The present example demonstrates that valuable information can be obtained from a VSP recorded in the cased part of old well, after drilling a deviated leg, or during a work over operation.

Well A is located in the Dezful Embayment of Zagros fold -thrust belt southwest Iran (Fig2-1). The VSP data were acquired in a single logging run using the three-axis, VSP tool, every $15.12 \mathrm{~m}$ apart. The VSP survey covers a measured depth range of 2740.0 to $-100.1 \mathrm{~m}$ (from RT) in low to moderately deviated well. The deviation is maximum $\left(42.5^{\circ}\right)$ at total depth (NIOC, Field survey report, Well A).

The VSP data were processed using the CGGV Geocluster seismic software to obtain dip and azimuth of geological formations and evaluate any fine structural feature related to fault and fracturation in the well vicinity especially in the Asmari reservoir interval. The common well tie operations and the determination of the velocity profile around the well have been performed in order to calibrate the 3D surface seismic block. We reprocessed the VSP data in order to tie the reflectivity observed on VSP with the 3D seismic image and also to investigate if the noise within the reservoir interval is due to microseismicity or induced by any fluid flow (Heloise, B et al. 1999; Liu, Q. et al, 2006). 


\section{2-2 Vertical component processing}

\section{2-2-1 Pre-processing}

We perform pre-processing to quality control the initial VSP data acquired. We displayed all initial traces, edited and QC them at different depths for four shuttles. We tried to keep good traces over different shuttles and rejected the noisy ones at different depths on the different components. Then all traces at the same depth were vertically stacked. Due to different amount of noise on the horizontal and axial components we kept different number of the VSP stations for the vertical component VSP dataset (138 levels) in comparison with the 3-component isotropic VSP dataset (121 levels) of the vertical stack.

\section{2-2-2 Processing flow chart (Single axial component)}

The following processing workflow was applied:

- Reading the raw field data transferred into SEG-Y by the logging contractor, and editing before isotropic 3C vertical stack (not median)

- Well trajectory display (Vertical \& horizontal projections)

- Time picking of $\mathrm{P}$ down wave and associated velocity curves,

- On flattened down going P-wave train position, band pass FK velocity filter to extract the down going P-wave train and define the deconvolution signature (limited to 350ms window after first arrival time)

-Deconvolution of total wave field with down going $\mathrm{P}$ wave signature - Spherical divergence gain expansion $\left(\mathrm{V}^{2} \mathrm{~T}\right)$ 
- Up/down Wave field separation with FK filter in flattened down going P- and S-wave train data position, reject FK-filter of the deconvolved down going wave train in order to keep all other events

- Shift of P-P reflections into Two way time.

- Random noise attenuation in F-X domain $(5-70 \mathrm{~Hz})$.

-Amplitude spectrum balancing $(5-70 \mathrm{~Hz})$ after frequency analysis.

-Polarity after deconvolution SEG normal or Peak= negative reflection coefficient (decrease of impedance with increasing depth)

\section{2-2-3 Discussion}

First, processing was applied on the axial component as explained in above flow chart. Figure 22 illustrates the raw Z-component with the casing and liner completion of the well which exhibits the high noise level in the reservoir part. The noise is continuous with white frequency spectrum over the whole $0-200 \mathrm{~Hz}$ band, characteristic of electrical tool noise possibly increasing with temperature? in the deep part of the well. After applying band pass filter $(2,8,60,80)$, first breaks are clearer but from $2407 \mathrm{~m}$ to the TD (well bottom), we had to select the traces with first breaks sharp enough to be picked. Down going P-waves first arrival were picked and deconvolution signature was extracted. Then down going P-wave were accurately removed by FK filter and down going S-wave, were partially removed. After picking the first breaks and applying signature deconvolution, the P-wave arrivals are compressed into zero phase wavelet and horizontalized (Figure 2- 3). Fig2- 4a and figure 2-4b show the well trajectory in horizontal and vertical planes respectively. Figure 2-4a shows the azimuth distribution of the ray path in projection in the horizontal plane. The incidence angle was computed for each level and the result 
is compatible with the illustration on figure $2-4 \mathrm{~b}$. The interval velocity variations are confronted with lithology. Fig 2-5a illustrates the vertical time from ground level, the interval, RMS and average velocities versus true vertical depth. RMS velocity versus two way time is displayed on Figure2-5b. The RMS velocity profile derived from VSP constitutes a lower limit for the best RMS stacking velocity of surface seismic, as this latter is affected by the layering anisotropy and might be slightly higher( by up to 5\% ) than VSP derived RMS velocity, but not smaller.

After removal of downgoing P-wave, the wave field reflection arrivals was transferred into two way time (figure 2-6). Fig 2-6 is the final result of well axis component associated with corridor stack. Different geological markers are traceable (detail on figure2-6). Although Cap rock has high reflectivity response on the surface seismic, it is absent on the VSP single component data due to high dip of the cap rock reflector and its geometrical disposition. It would be expected to be more visible on the horizontal components. The depth of geological markers, top Cap Rock is $2498 \mathrm{~m}$ corresponds to $1340 \mathrm{~ms}$ and top Asmari reservoir is $2544 \mathrm{~m}$ corresponds to $1350 \mathrm{~ms}$ (Fig26). There is also a deep coherent event (B) around 1520ms which correspond to a reflector located above Cap rock on the eastern flank of the Aghajari anticline. Event A might be an internal Asmari reflection but it needs to be confirmed by $3 \mathrm{C}$ processing. Events $\mathrm{S} 1$ and $\mathrm{S} 2$ are $\mathrm{S}$ $\mathrm{S}$ reflection arriving right after the direct downgoing S-wave. One should not be surprised that S$\mathrm{S}$ reflections on dipping structures exhibit significant energy on the $\mathrm{Z}$ component. Corridor stack domain represents the best P-P reflection data in VSP section are located between $\mathrm{P}$ and $\mathrm{S}$ direct arrivals. 


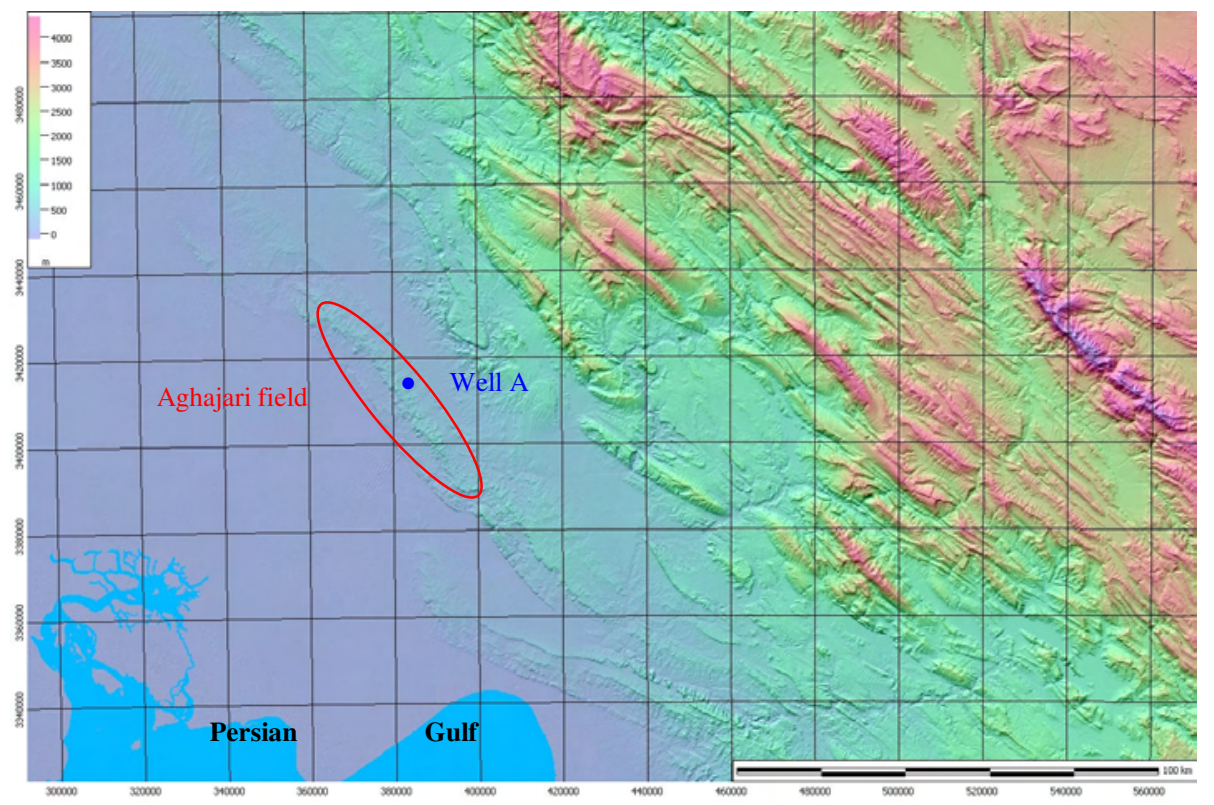

Fig 2-1 Location of the well A on the north flank of the study field

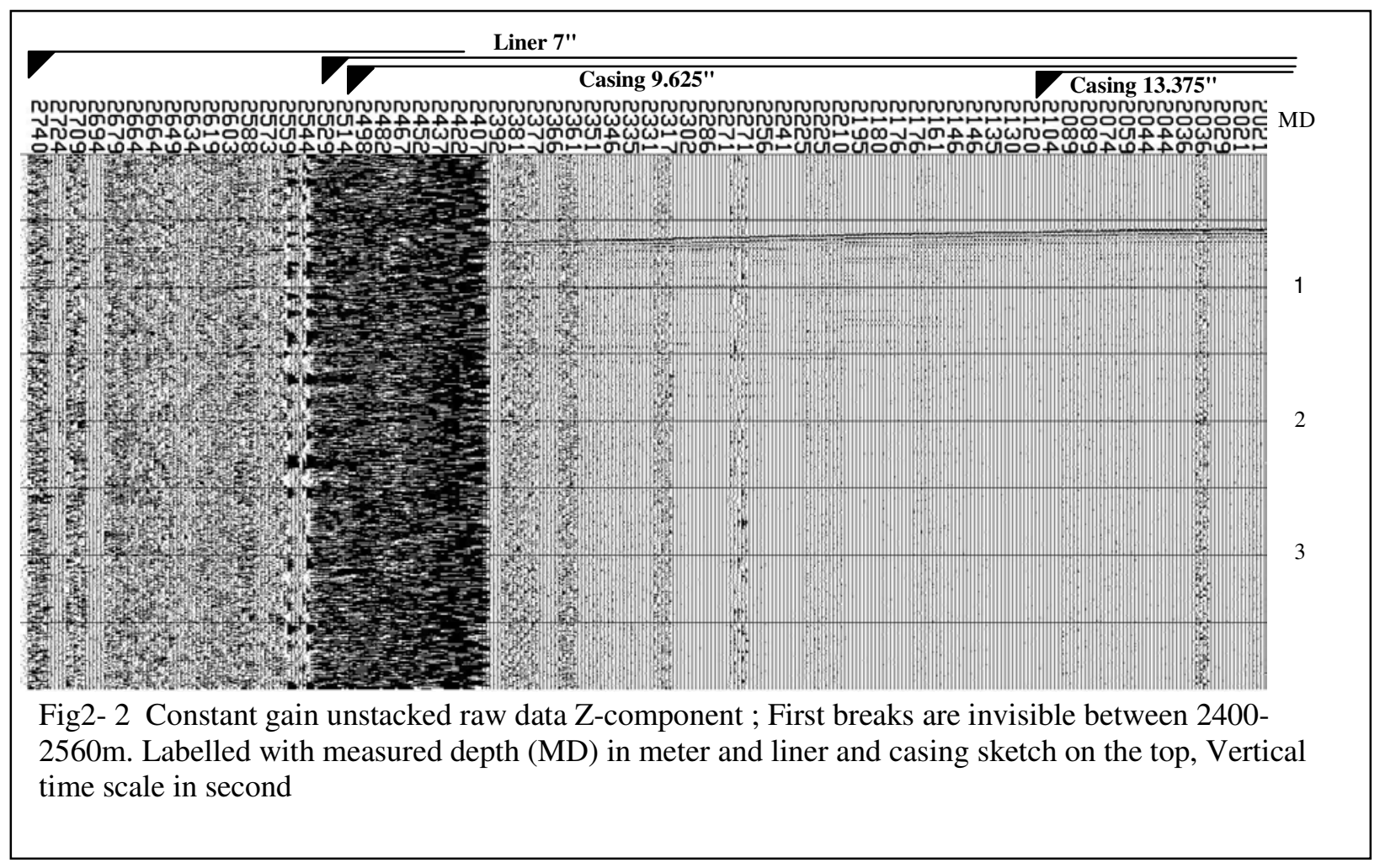




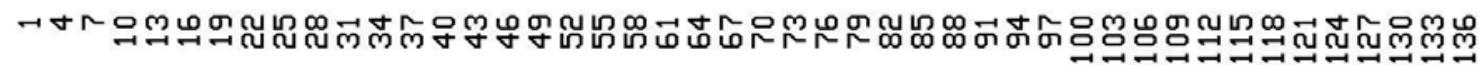

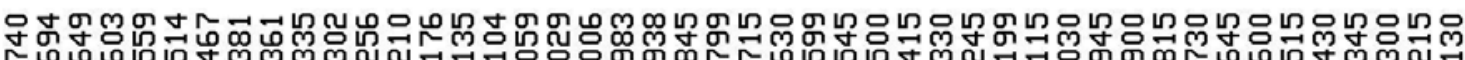

MD ผ

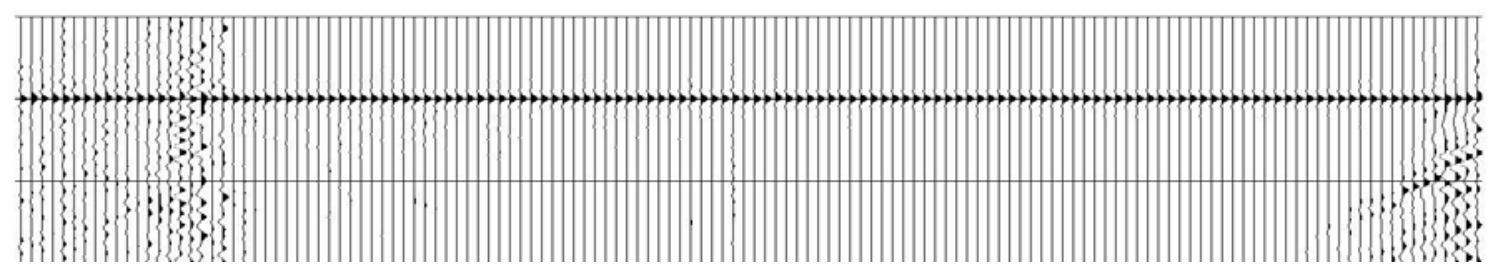

Fig 2-3 Time pick control display of P-measured wave down going horizontalized at $100 \mathrm{~ms}$ $(5-80 \mathrm{~Hz})$, labelled with trace number and measure depth (MD) in meter

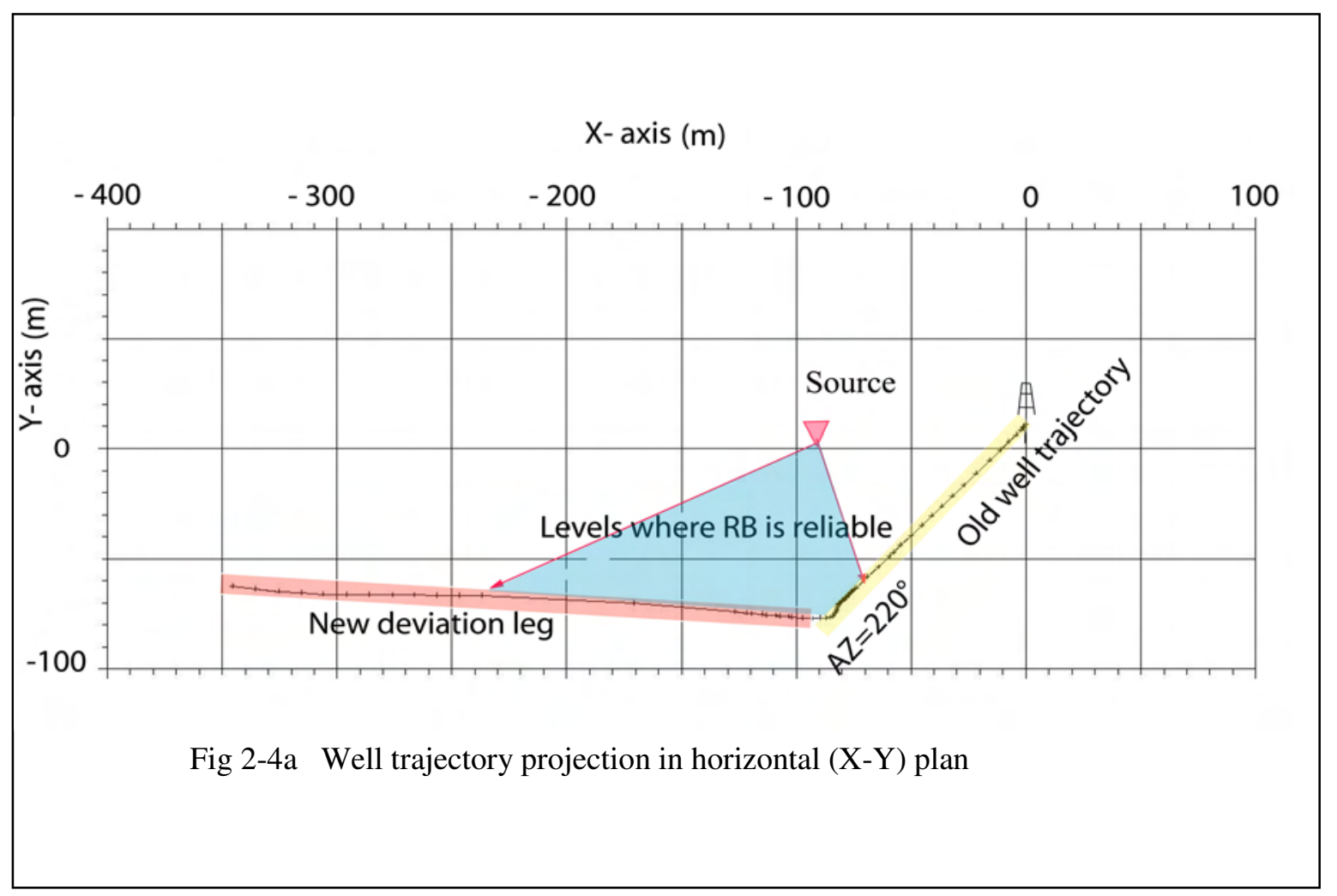




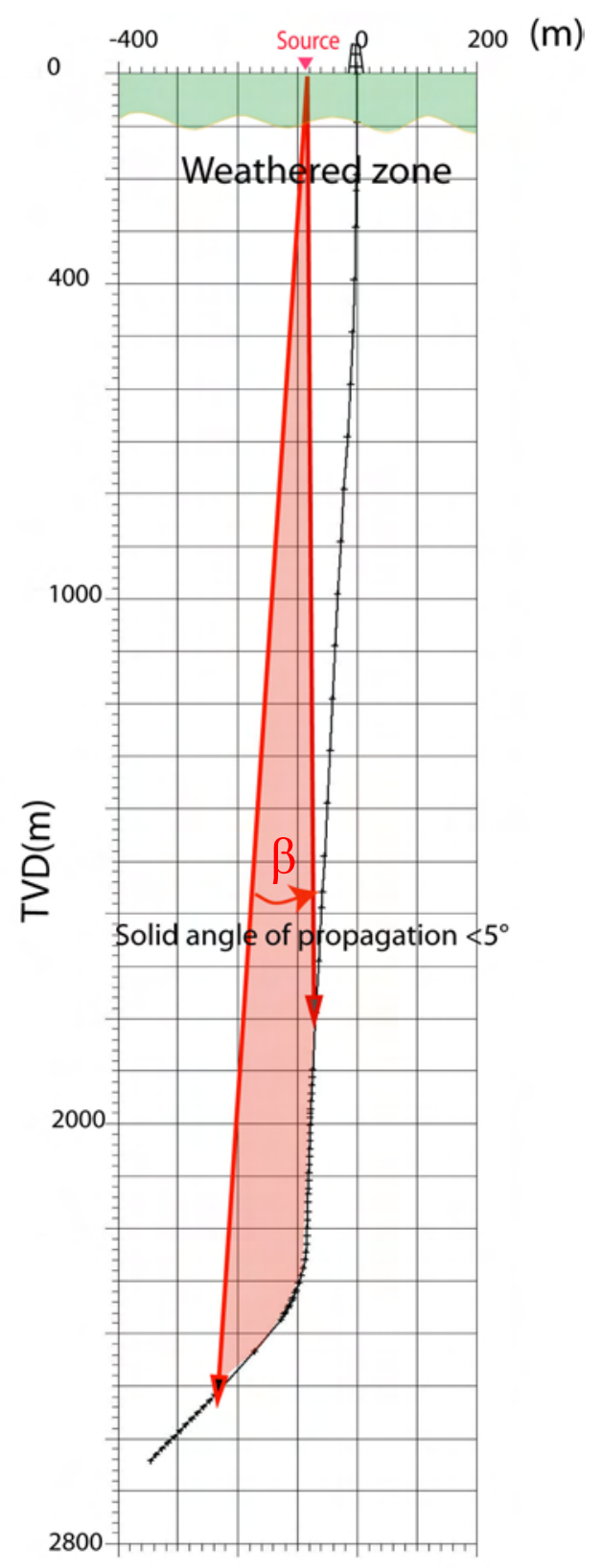

Fig 2-4b Well trajectory projection in vertical plane. $\beta$ shows the incident angle in the solid angle propagation of the wave shape of $\mathrm{P}$ - and $\mathrm{S}$-wave first arrivals Eigen mode versus depth, even if a small S-wave anisotropy occurs. 


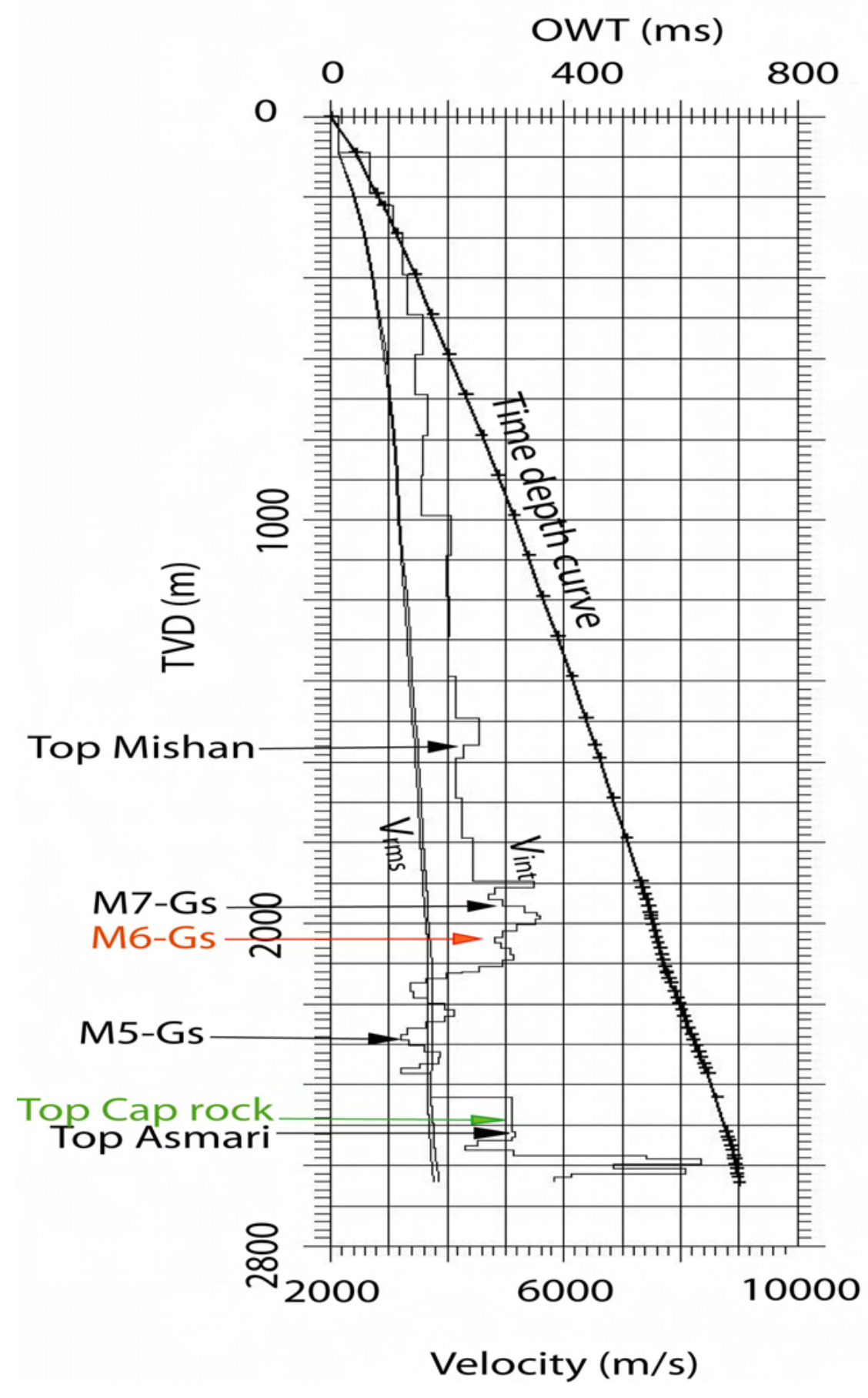

Fig2-5a Time depth curve from surface (GL), Interval and RMS velocities vs. true vertical depth, Main geological markers are shown. 


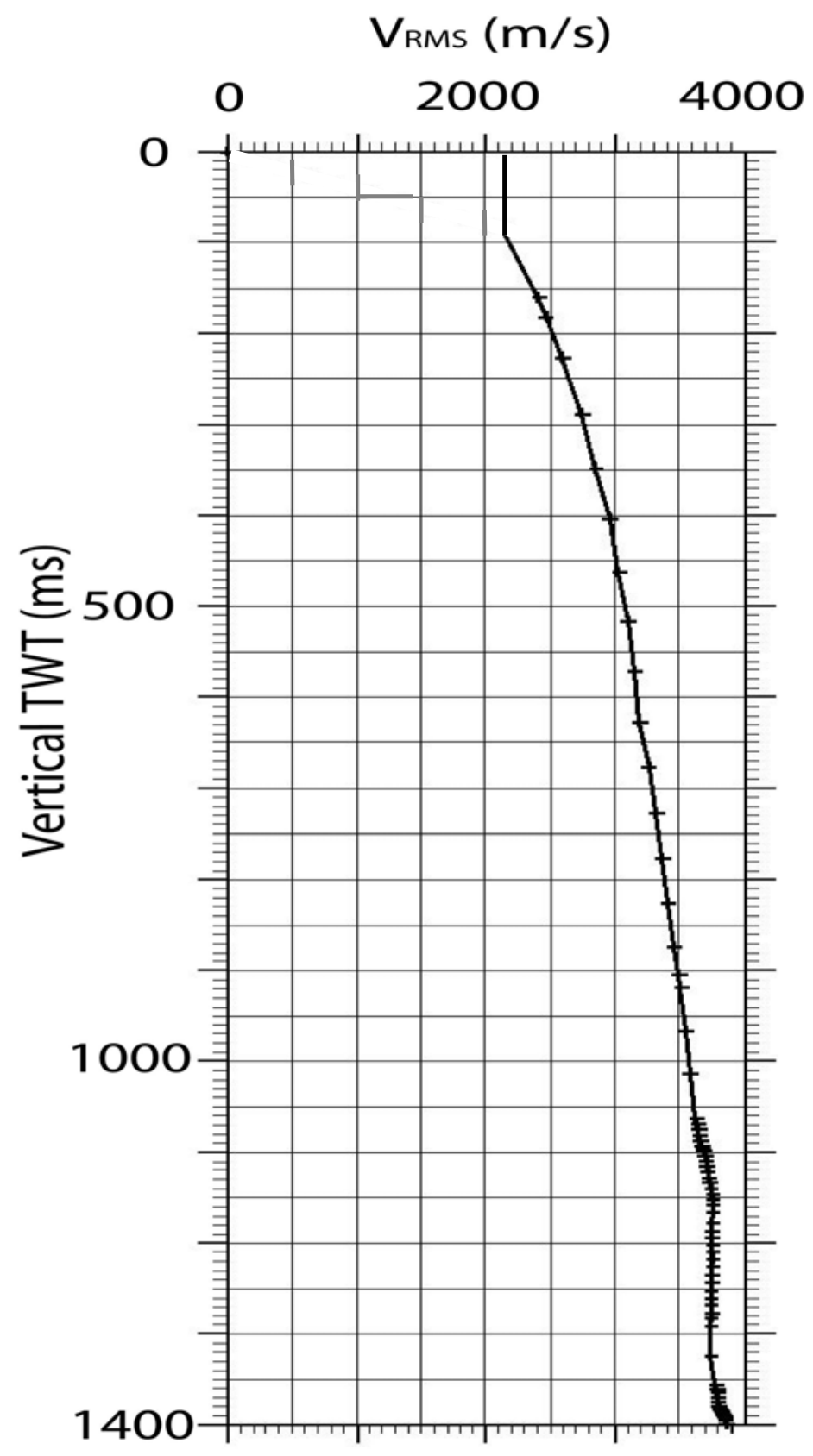

Fig2-5b RMS velocity vs. TWT from surface (GL), to be compared with stacking velocity of surface seismic. 


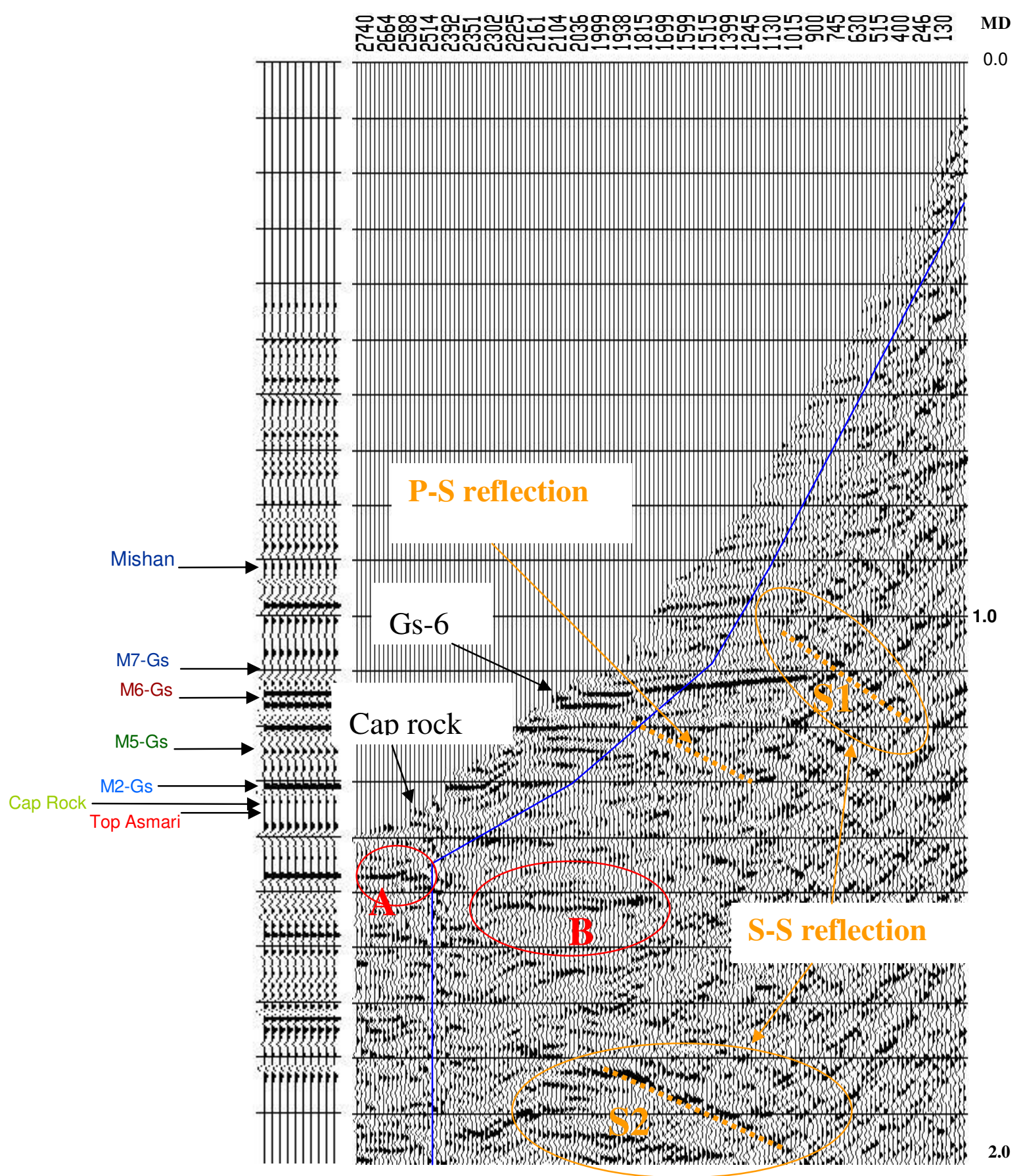

Fig 2-6 Display of deconvolved upgoing wavefield Z-component equalized.

The Corridor stack domain is marked by blue line following the direct arrival.

Top geological formations are mentioned. 


\section{2-3 Three Components processing}

\section{2-3-1 Orientation of three components}

\section{2-3-1-1 Three components at the low to medium deviated angle (Deviation}

angle $>12^{\circ}$ )

Each shuttle of the VSP tool comprises three fixed orthogonal components (XT, YT, ZT), and a Relative Bearing (RB) pendulum device connected to a circular resistor in the plane orthogonal to the well axis. Component ZT is oriented along the tool axis i.e. the borehole axis. Component XT is oriented along the direction of arm opening and correspond to the origin of relative bearing $(\mathrm{RB})$ angle: $\mathrm{RB}(\mathrm{XT})=0$. YT component is oriented $90^{\circ}$ from $\mathrm{XT}$ counter clockwise looking down.

The RB angle is significant when the well deviation is sufficiently high, above $12^{\circ}$ (chapter 6 ).

To obtain the true geographic component orientation, 3 rotations were applied.

1-The first rotation, Rot (RB) occurs in the plane orthogonal to well axis. It means XT and YT components are turned around $\mathrm{Z}$ axis as much as $\mathrm{RB}$ angle. The results will be $\mathrm{XV}, \mathrm{YH}$ and $\mathrm{ZT}$ 2- The second rotation, Rot (SDEV) occurs in the vertical plane locally tangent to the well. It means $\mathrm{ZT}$ and $\mathrm{XV}$ are turned around $\mathrm{YH}$ axis as much as DEV angle. The results are ZV, HA and $\mathrm{YH}$ now. Eventually, $\mathrm{HA}$ and $\mathrm{YH}$ are in horizontal plane and $\mathrm{ZV}$ is vertical component down ward.

3- The third rotation, Rot (HAZI) occurs in the horizontal plane. It means HA and YH turned around the $\mathrm{ZV}$ axis as much as azimuth of the well. The results are $\mathrm{HN}$, $\mathrm{HE}$ and $\mathrm{ZV}$. Based on tool specifications, these rotations are reliable only where well deviation angle SDEV $>12^{\circ}$. After the three rotations, Z-component $(\mathrm{ZV})$ is vertical pointing downward, $\mathrm{X}$-component $(\mathrm{HN})$ is 
horizontal pointing to North and Y-component (HE) is horizontal pointing the East true geographic direction(figure 2-7).

As the direct P-wave amplitudes were too weak in the horizontal plane (Figures 2-8\&10), the direct S-wave particle motion was used and rendered coherent level to level, in order to obtain a reliable orientation on adjacent depth level.

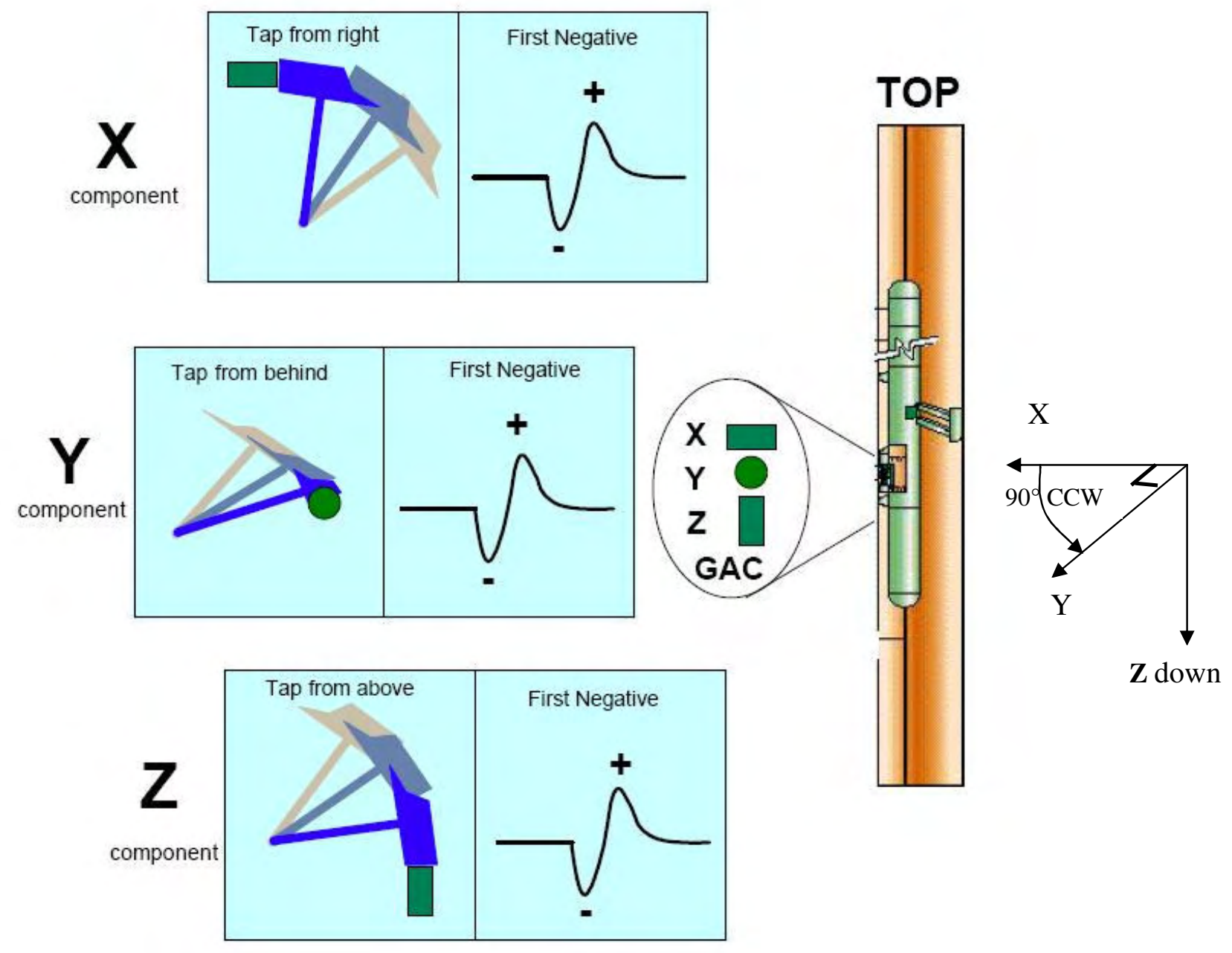

Figure 2-7 3-component polarities in VSP tool 
2-3-1-2 Three components orientation for weak well deviated angle (Deviation angle $<12^{\circ}$ )

The modulus component $\{(\mathrm{X} * 2+\mathrm{Y} * * 2) * * 0.5\}$ was computed and filtered (figure2-8 and 2-10 left). We applied a low cut $5 \mathrm{~Hz}$ filter on the modulus to improve the detection of high frequency variations. The modulus is invariant with respect to rotation thus independent from orientation of input recorded components. Therefore, the time of direct shear wave can be accurately picked on the filtered modulus trace (figure 2-8left). Then a short time window can be accurately defined along the shear wave direct arrival in which the energy of horizontal components of shear wave is maximized versus the azimuth; it occurs that the fast S-wave component (S-fast) is maximized right before 100ms(Figure 2-9 pink strips). The first break of direct fast and slow shear waves can be picked with higher accuracy after maximizing their energy on the horizontal components. The observed wave shape coherency of shear wave after the orientation (Fig. 2-10 middle) and shear wave particle motion in the maximization window (fig. 2-11) give confidence in this process. Calibration of direction of maximum S-wave amplitude was performed on the VSP levels previously oriented into geographic coordination using the RBS sensor (2200-2400m) in the deep deviated part of the well. The final output is fully oriented data in geographic coordinates ( $\mathrm{Z}$ up, H North and H East) .

In the reservoir part the well has a medium deviation of $40^{\circ}$, unfortunately all three components are very noisy and the 3 components can not be processed.

The orientation process above described looks reliable, and can be used even when no hardware orientation device is added on the VSP tool during acquisition. Therefore, a patent is being filed on this orientation method (summarized in chapter6, full patent in Appendix I in French). 


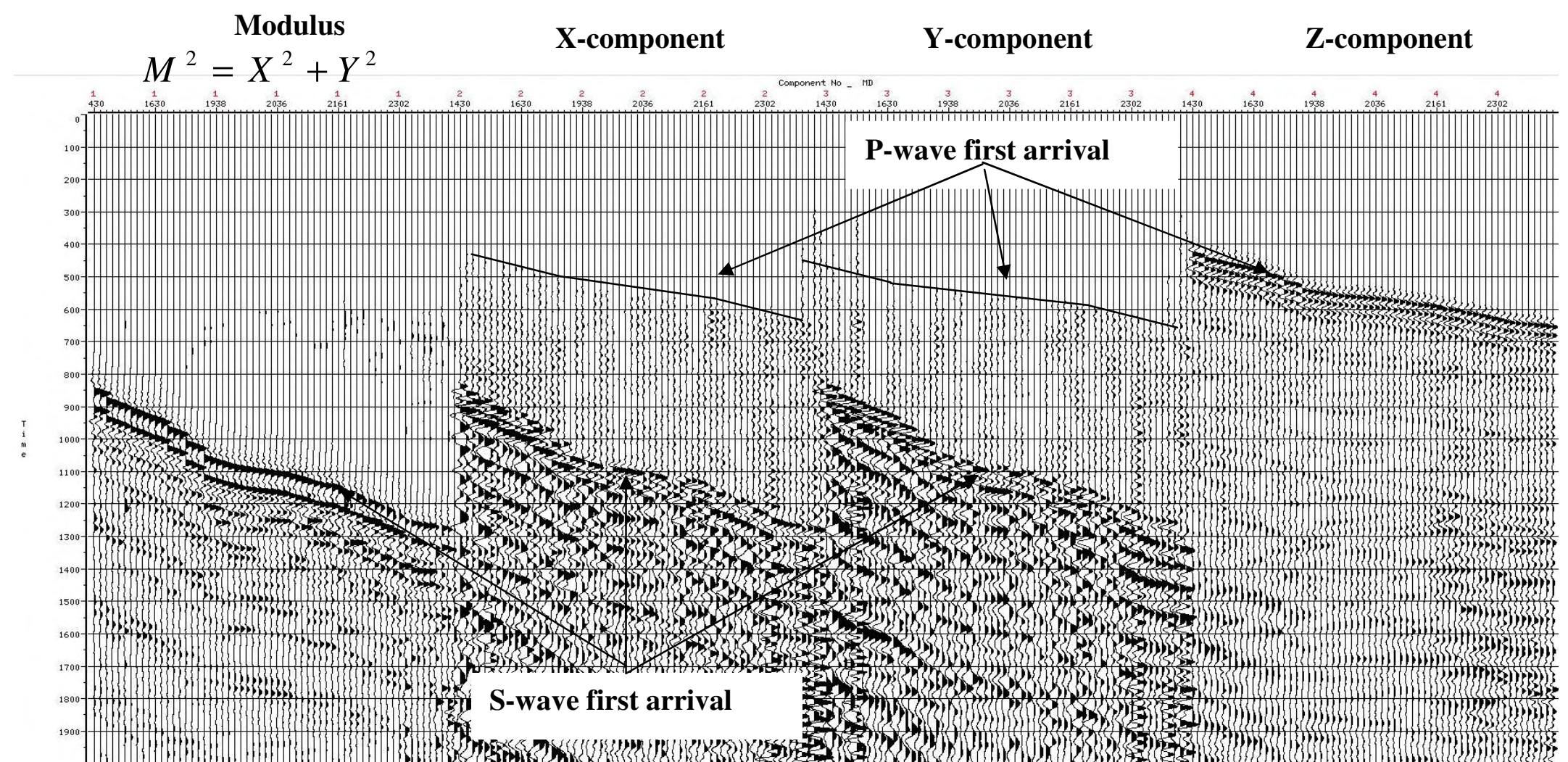

Fig 2-8 From left to right Modulus, $\mathrm{X}, \mathrm{Y}$ and Z-components before orientation. First arrival S-waves are clear on horizontal components while on the Z-component P-wave first arrivals are sharp to pick. First arrival S-waves are not consistent before orientation while modulus first arrivals are identical before and after orientation. X, Y and Z components are displayed with same constant gain while modulus has been normalized. 


\section{Direct Shear wavetrain Horizontalized}

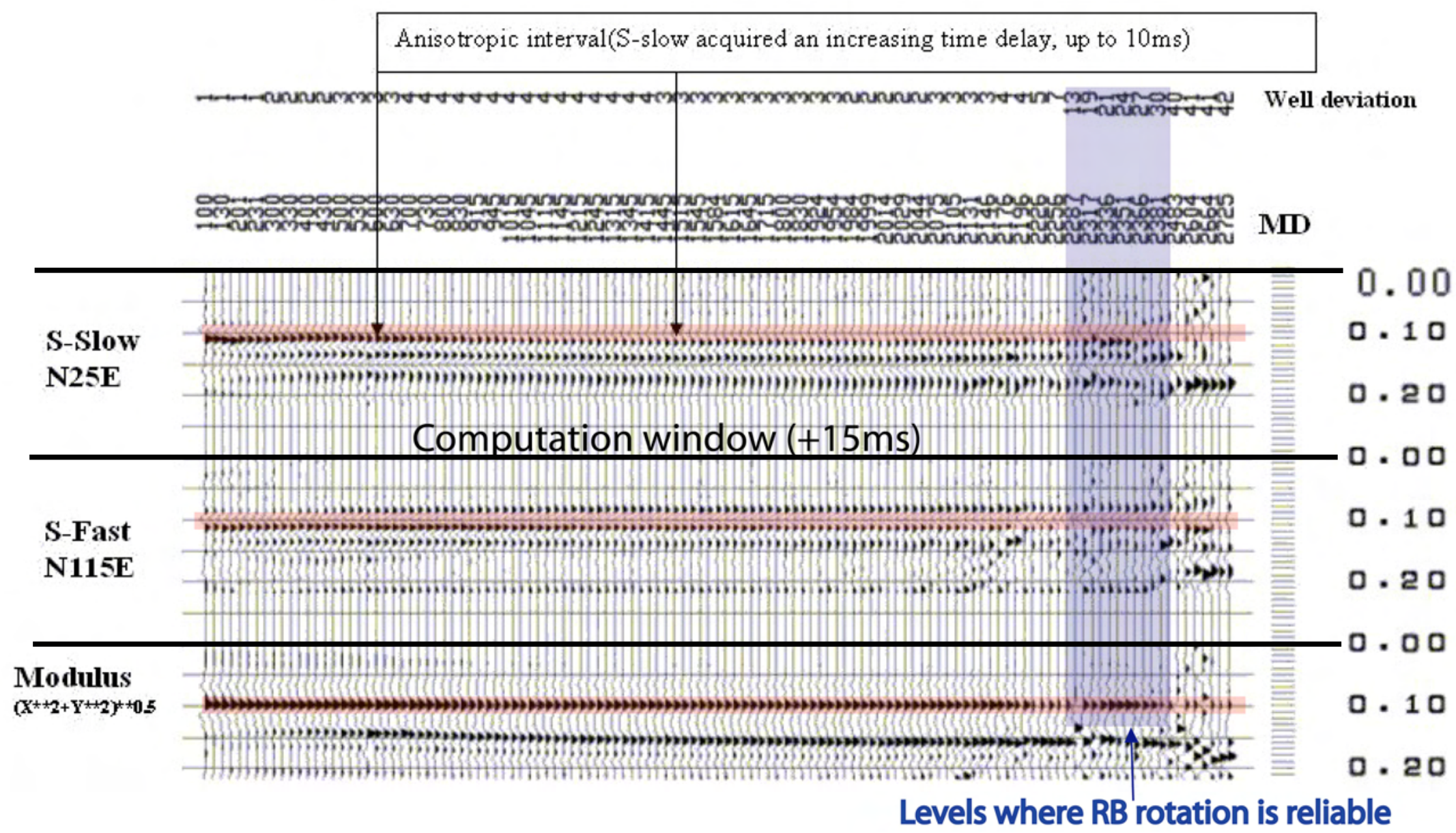

Fig 2-9 Direct shear wave horizontalized, first is S-slow in azimuth N25E and second is S-fast wave orthogonal to first one $90^{\circ}$ clockwise. In the 600-1500m anisotropic depth interval, the slow S-wave is seen to acquire an increasing time delay, up to 10 $\mathrm{ms}$, relatively to the horizontalized fast s-wave. Below $1500 \mathrm{~m}$, both S-wave propagate with same velocity, so formation are isotropic 


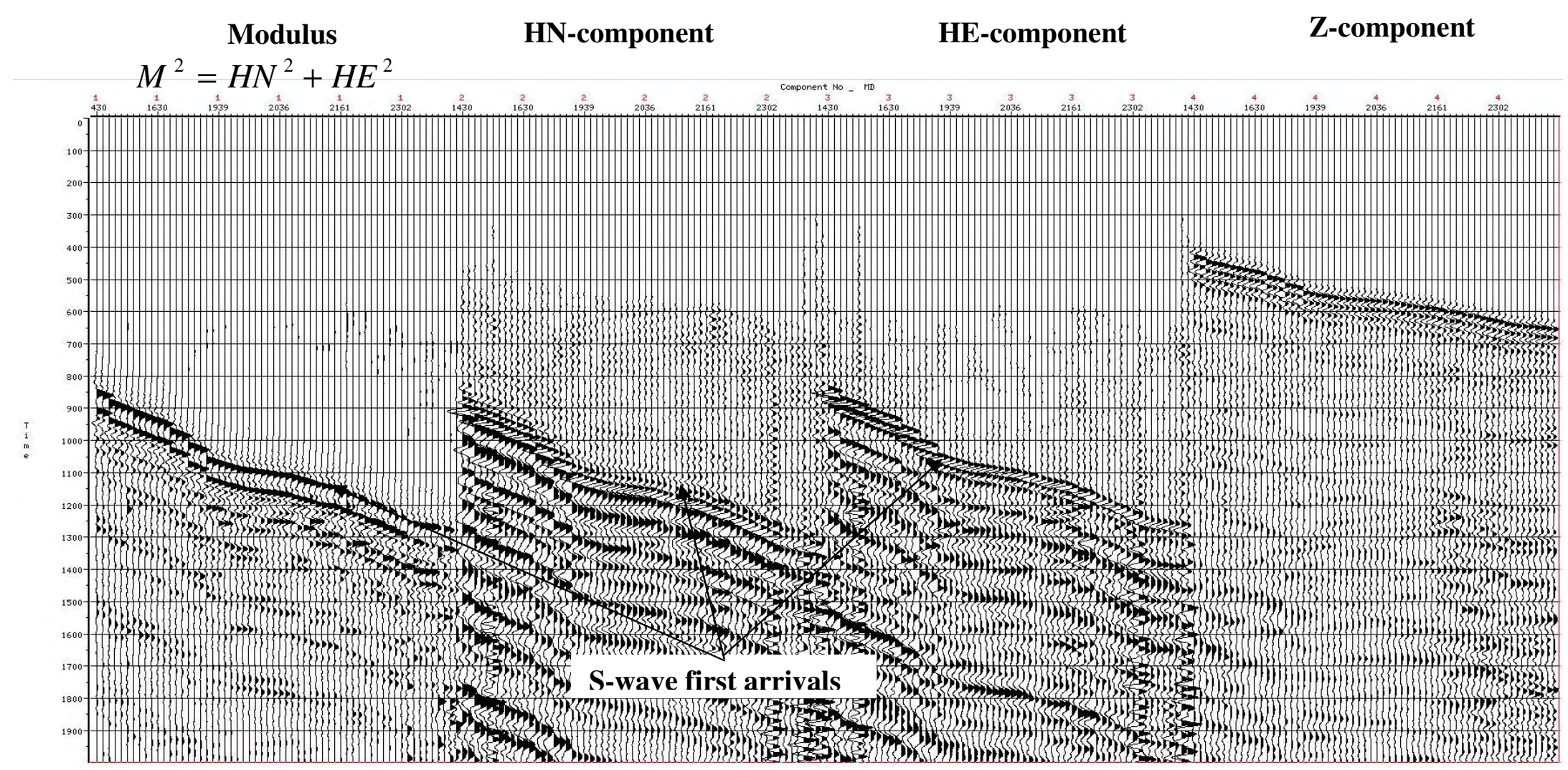

Fig 2-10 From left to right Modulus, HN, HE and Z-components after orientation. On the Z-component P-wave first arrivals are sharp to pick. First arrival S-waves are coherent after orientation. The filtered modulus signal first arrivals is identical before and after orientation. $\mathrm{HN}, \mathrm{HE}$ and $\mathrm{Z}$ components are displayed in constant gain while modulus has been normalized. 


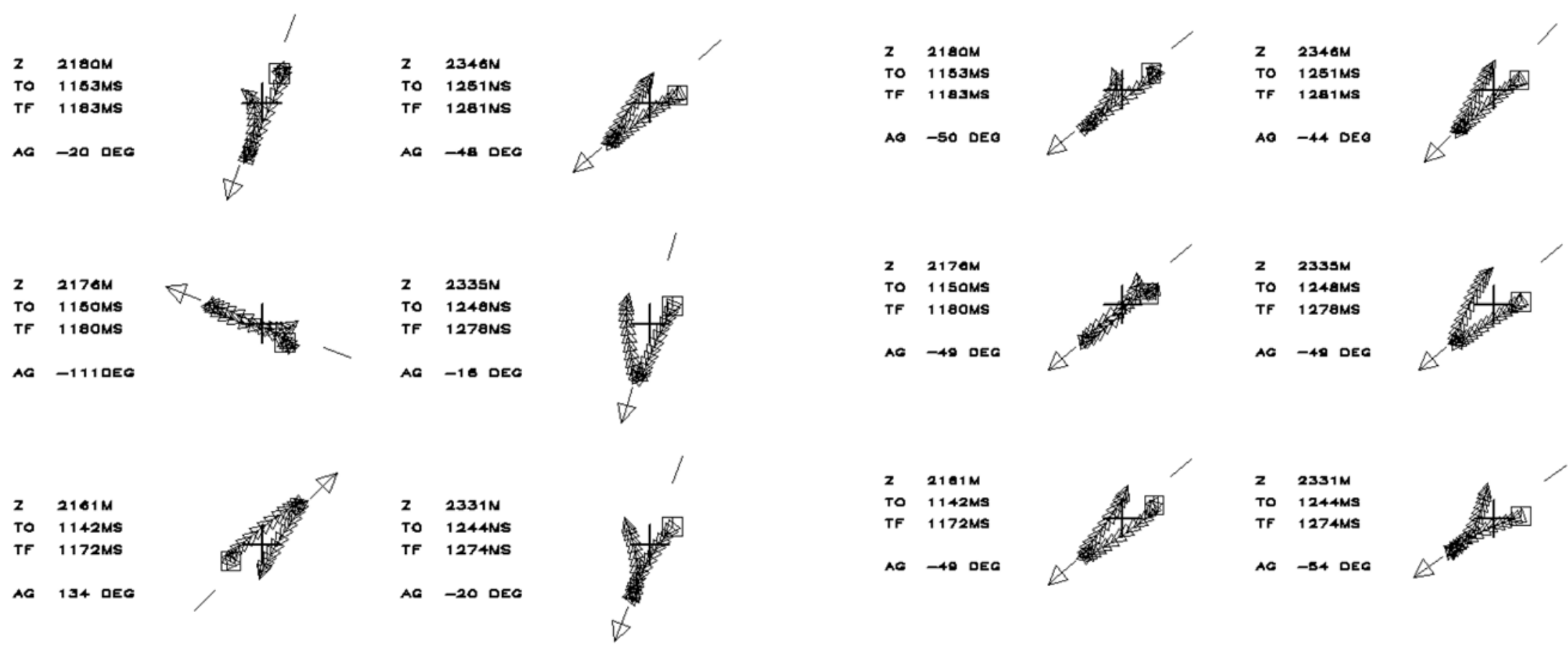

Figure2-11 Shear wave particle motion before (left) and after (right) orientation in true geographic coordinates. Consistency is observed for all traces after orientation. On the left side of any hodogram; $\mathrm{Z}$, TO, TF and AG represents the depth, beginning and ending of time computation window and relative bearing angle respectively. 
On figure 2-9 the SH(Fast, strike direction) and SV(slow, dip direction) waves can clearly be observed between $600 \mathrm{~m}$ and $1500 \mathrm{~m}$ with a $10 \mathrm{~ms}$ propagation delay, corresponds to around $1.5 \%$ velocity anisotropy.

The maximized S-wave arrivals have been extracted from measured polarization of S-wave in the plane perpendicular to wave propagation direction. Multi-component VSP processing is required, because the up going reflection ray path are generally at oblique angle to the borehole, thus all three components must be used to capture the entire reflected wavefield. Horizontal components are used in zero offset processing to measure direct S-wave velocities and to deal with geophone tilting that occurs in deviated boreholes. Additionally all three components are needed to recognize structure in $3 \mathrm{D}$ view.

$\mathrm{S}$-wave velocity derived from $\mathrm{S}$-wave time picks can be confronted with $\mathrm{P}$-wave velocity. $\mathrm{Vs} / \mathrm{Vp}$ ratio was computed (figure 2-12). This ratio $(\mathrm{Vs} / \mathrm{Vp})$ increases from 0.5 near surface (Poisson coefficient $=0.33$ ) to 0.55 in the Asmari reservoir $($ Poisson coefficient $=0.28)$, which corresponds to very compacted formations from top to the T.D, in agreement with tectonic and geological history.

\section{2-3-2 Isotropic Processing flow chart (On oriented three components)}

The processing flow chart of 3 components is characterized by its isotropy, meaning that the same processing operation have been applied identically on the 3 components possibly in a time variant manner. After removing down-going S-wave with FK filter isotropic, spectral balancing (time-variant amplitude deconvolution and equalization) was applied on 3 components. Fig2-13 demonstrates P-P, P-S and S-S reflections and 3C isotropic P-P up going wave field. 
Many operations are identical to the single processing ones but all three component processing operations must be isotropic.

- Time picking of $\mathrm{P}$ and $\mathrm{S}$ down wave and associated velocity curves,

- Isotropic up/down Wave field separation with FK filter on flattened down going P-wave train position, band pass velocity filter to extract the down going P-wave train and define the deconvolution signature

-Isotropic deconvolution of 3 components total wave field with down going $\mathrm{P}$ wave signature

- Spherical divergence gain expansion (same on the 3 components)

- Isotropic up/down Wave field separation with FK filter in flattened down going P- and S-wave train data position, reject FK-filter of the deconvolved down going wave train in order to keep all other events

- Shift of P-P reflections into two way time (same on the 3 components)

-Isotropic random noise attenuation in F-X domain (5-70HZ)

-Isotropic amplitude spectrum balancing $(5-70 \mathrm{~Hz})$ after frequency analysis.

-Polarity after deconvolution: SEG normal or Peak= reflection coefficient $<0$ (decrease of impedance) 


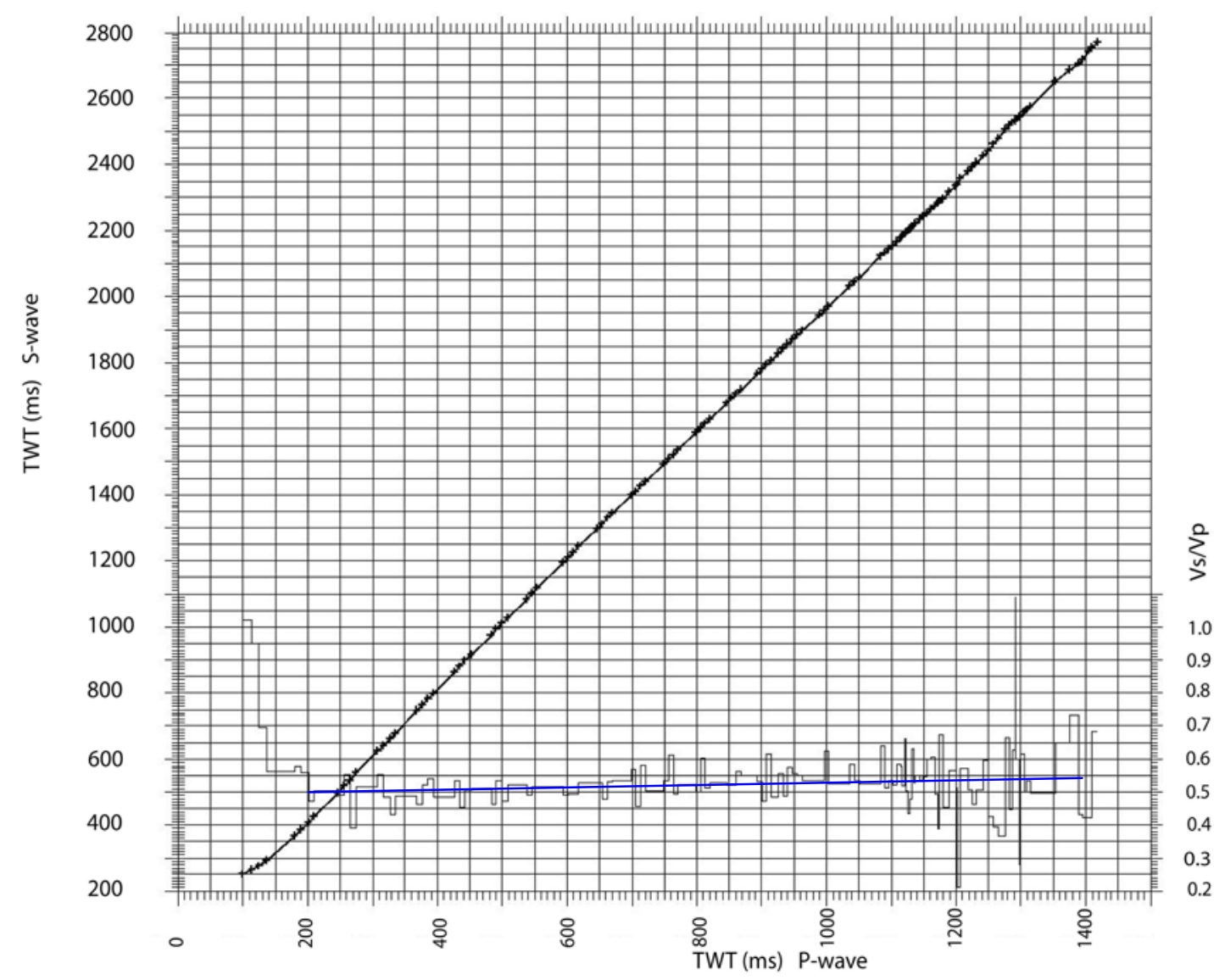

Figure 2-12 Vs/Vp ratio versus $\mathrm{P}$-wave and S-wave time;

After we obtain the isotropic 3 component reflected wavefield, the polarization of specific reflected events can be analyzed and determined, either by direct computation or by polarization scan (see next paragraph on polarization analysis of P-P reflection and dip computation below). P-P and P-S reflections are seen on the isotropic 3 component final wave field. Cap rock reflection is visible on the horizontal components especially on the HN due to its geometry and dip while it can not be seen on the vertical one (figure 2-13 \& 14). 


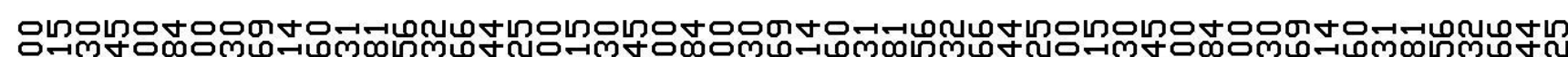

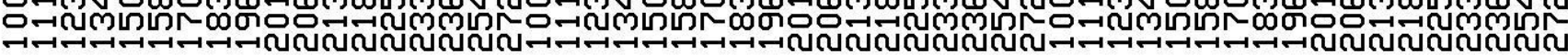

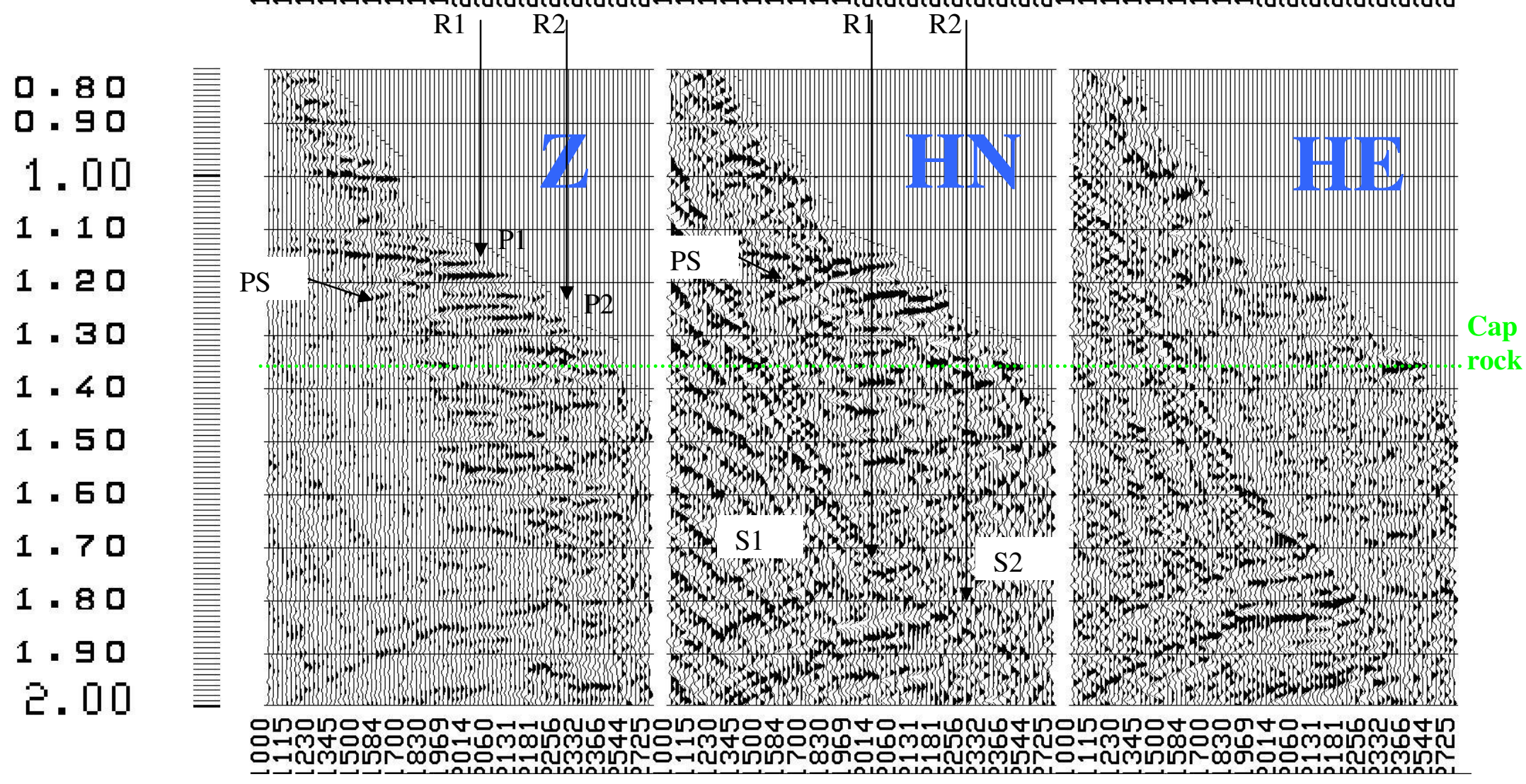

Fig2-13 P-P reflection display TWT Isotropic spectrum balancing equalization for 3 components, time from ground level. 
POLARIZATION ANALYSIS PRINCIPLE

A linearly polarized event shows a null in the plane orthogonal to its polarization.

Z-Time windows of the 3-component data are projected on a cylinder

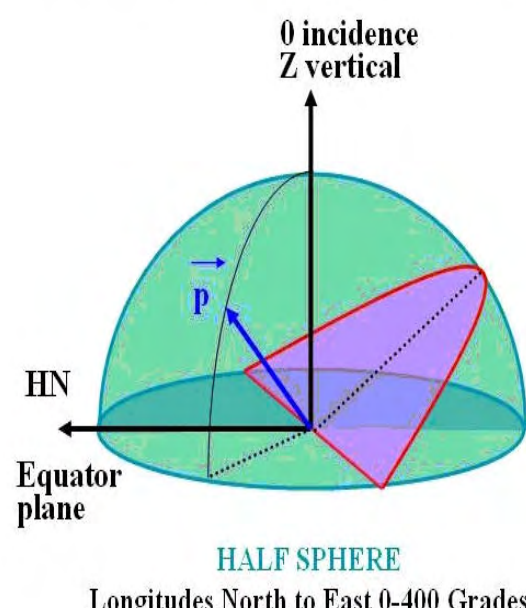

Longitudes North to East $0-400$ Grades

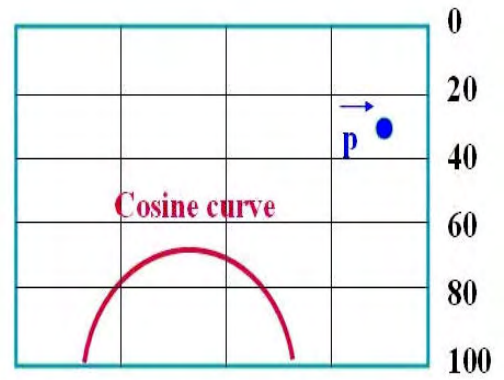

$\begin{array}{lllll}0 & 100 & 200 & 300 & 400\end{array}$

$\mathrm{HN} \quad \mathrm{HE} \quad-\mathrm{HN} \quad-\mathrm{HE} \quad \mathrm{HN}$

CYLINDRICAL PROJECTION

Longitudes and vertical incidence in Grades

\section{POLSCAN ON SYNTHETIC EVENT C Vertical \\ vertical incidence $66 \mathrm{Gd}$ and azimuth $33 \mathrm{Gd}$ \\ incidence \\ in grades}

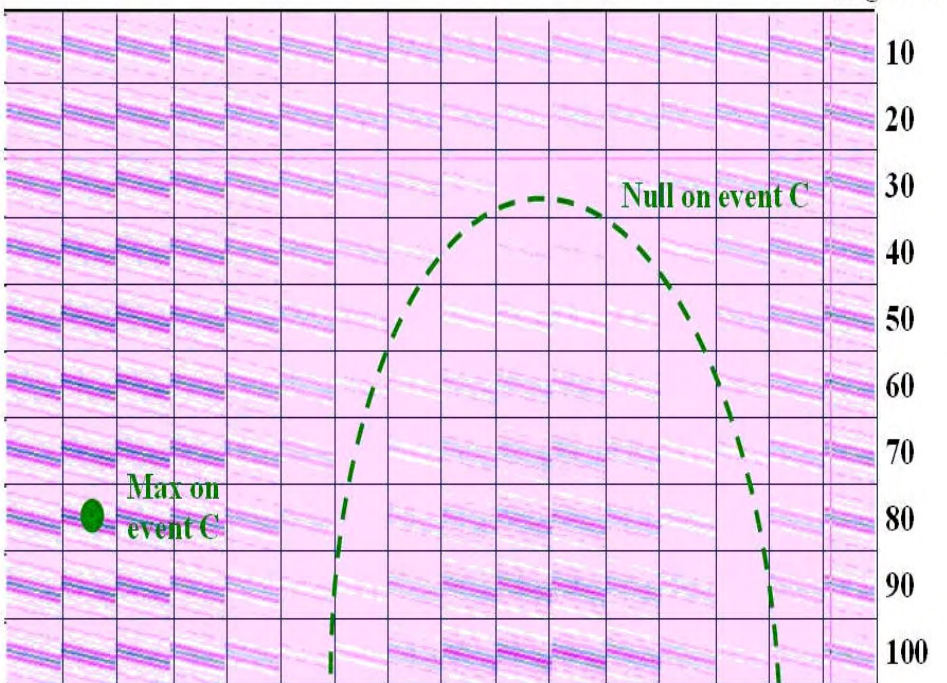

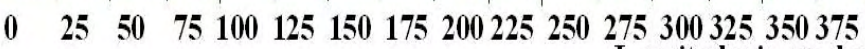

Fig2-14a Schematic figures show the polarization analysis and computation dip and Azimuth of certain event on cylinder projection ( Naville, et al, 2001) 

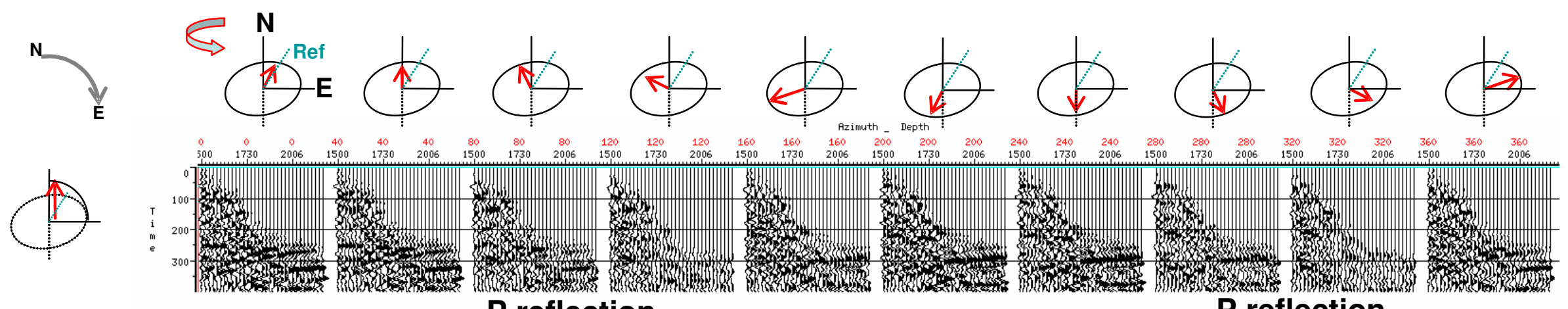

\section{P reflection}

\section{$\mathbf{P}$ reflection}
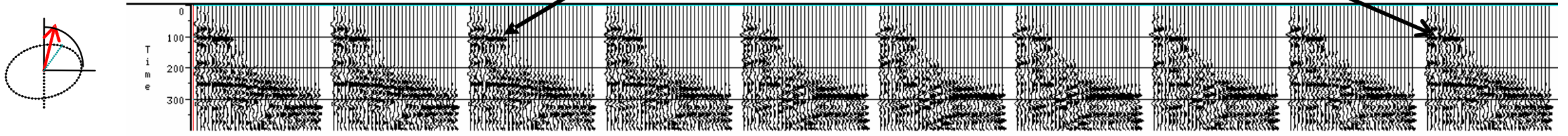

(7) Max. P-P reflection $\because " \cdot$
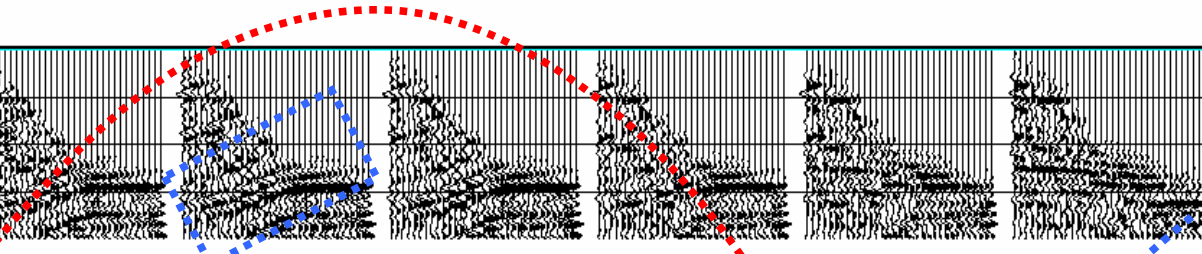

Max. P-S reflection
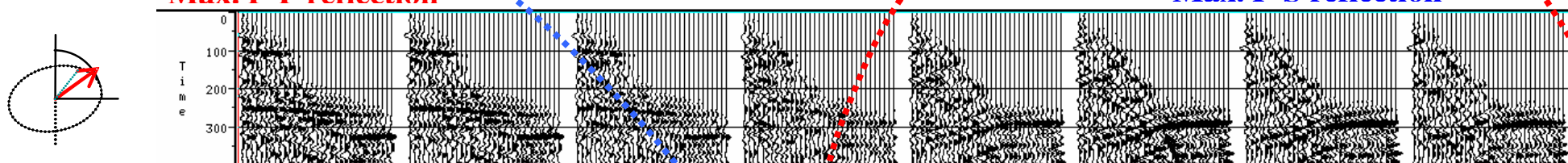

:

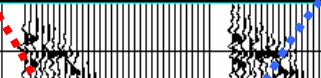

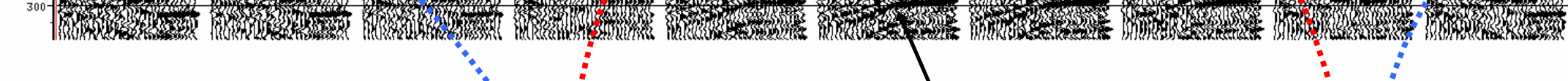
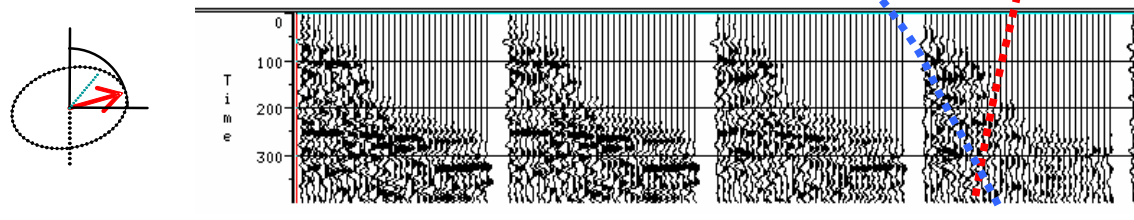

P - S reflection

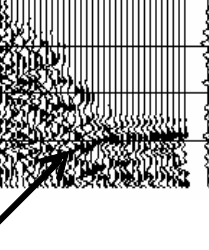

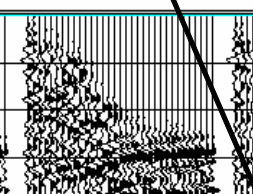

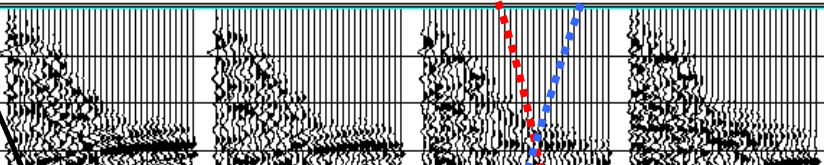

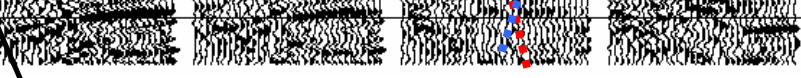

\section{P-S reflection}

Figure 2-14b Scan polarization of reflected wave field as cylindrical projection, all angle are in Grade, vertical step of 20 and horizontal step of 40 


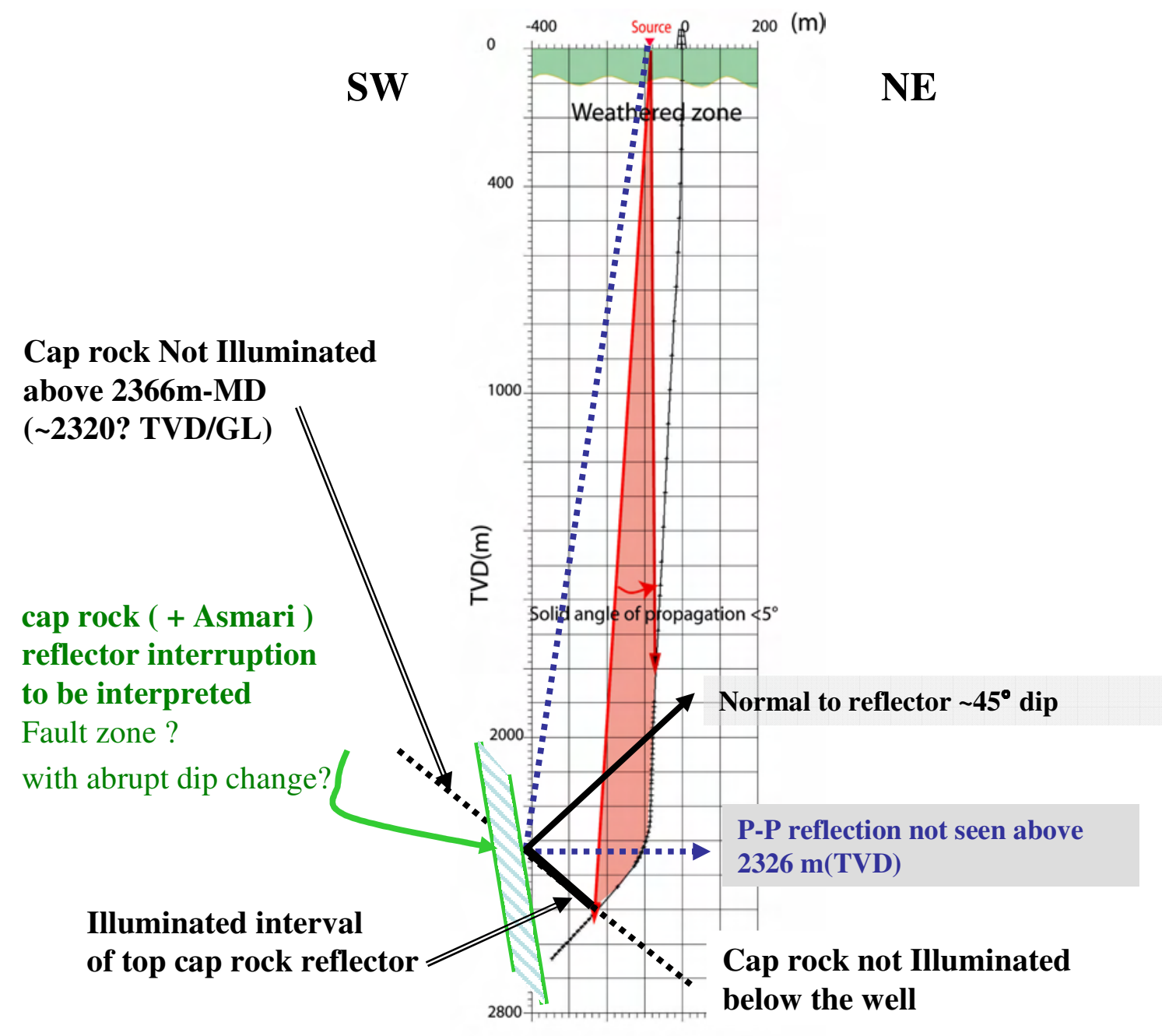

Fig 2-15 Structural sketch of Cap rock reflector seen by VSP 3 component 


\section{2-4 Polarization analysis of P-P reflection and Dip/Azimuth}

\section{computation}

To compute the dip and azimuth of reflectors within an analysis time- depth window and discriminate interfered waves, we computed the amplitude for all azimuths from 0 to 400 grades and all incident angle from 0 to 100 grades simultaneously and plot them (Fig 2-14

). This kind of plot corresponds to a cylindrical projection of the 3 component data at discrete angle values of latitude and azimuth. This document enables to discriminate the P$\mathrm{P}$ reflections from $\mathrm{P}-\mathrm{S}$ converted in the chosen window and to estimate the polarization (Figure2-14a and b). A linearly polarized event shows a null in the plane orthogonal to its polarization. Z -Time window of the 3-components are projected on a cylinder (Naville, patent no. 6076045, Naville et al, 2001).

A refined structural sketch of the dipping Cap rock reflector has been outlined on Figure 215, showing how the Cap rock reflector, dipping about $45^{\circ}$, generate a P-P reflection visible mainly on the horizontal component HE, and only below $2326 \mathrm{~m}$ (True Vertical Depth, TVD). The reason for interrupted reflection has been suggested as an accident affecting only the anhydrite Cap rock reflector, in agreement with the surface seismic image locally to the well (figure2-17 and 2-20).

The incidence angle of the GS- 6 reflection has been estimated to $45^{\circ}$ in the $\mathrm{N} 20^{\circ} \mathrm{E}$ azimuth which resulting of the dip value of $22^{\circ}$ in the same down dip azimuth $\mathrm{N} 20^{\circ} \mathrm{E}$ (Figure 2-16). 


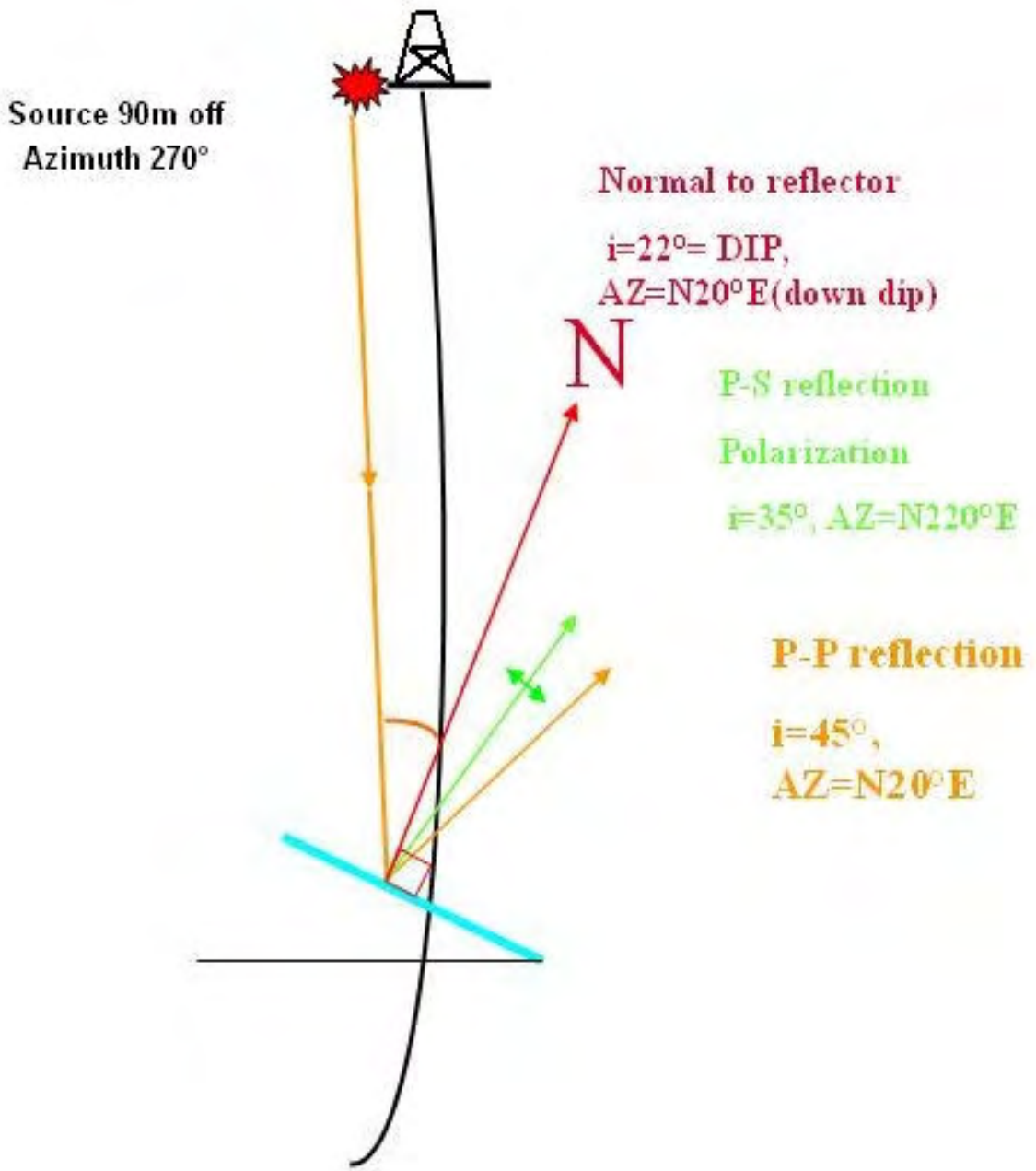

Fig2-16 Sketch of P-P and P-S waves reflected from Gachsaran level. if the dip was $60^{\circ}$, the incidence angle should be $60^{\circ}$ so the lateral VSP coverage could be around $1 \mathrm{Km}$ 

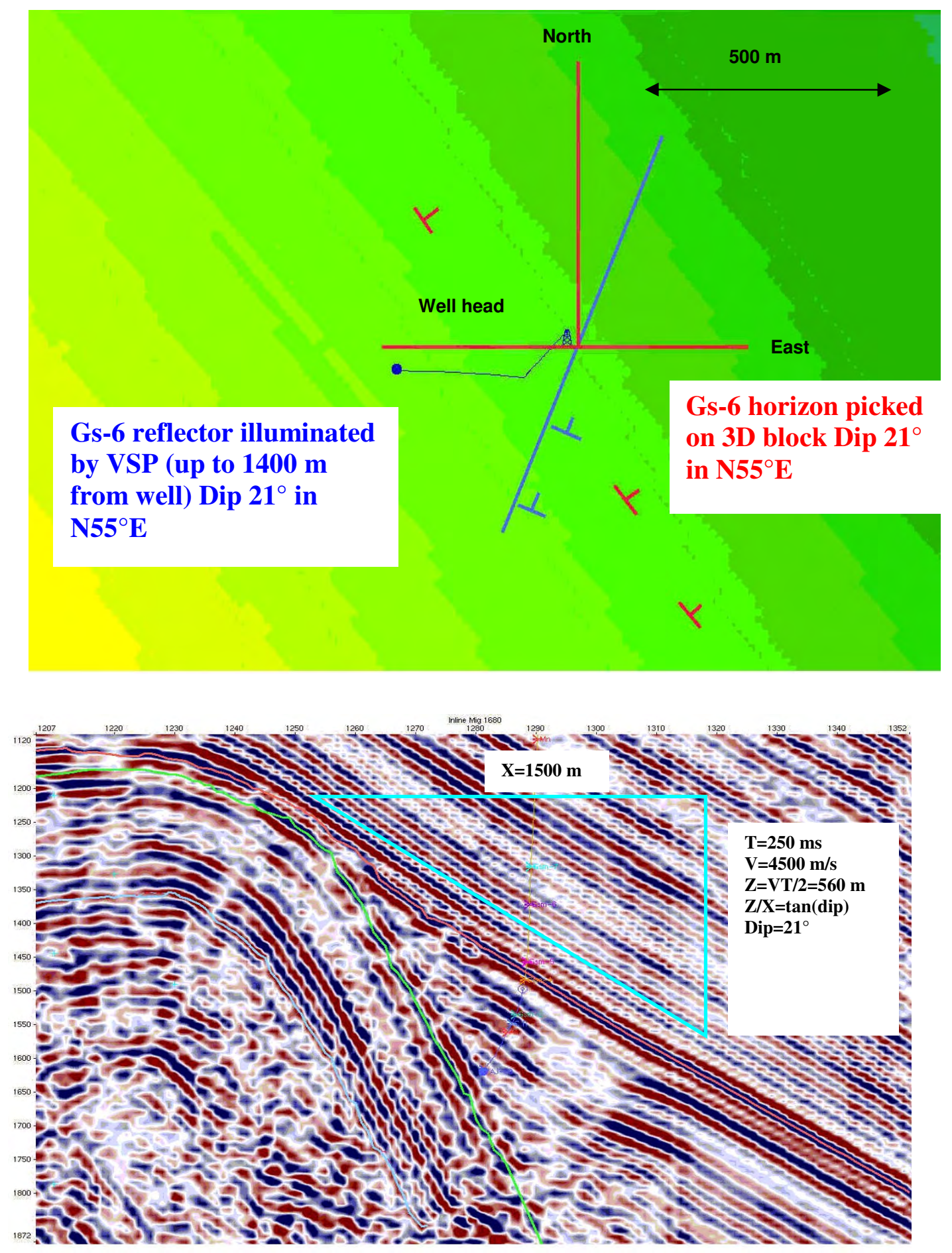

Fig2-17 Dip and Azimuth of Gachsaran member6 from time map (above) and from 3D seismic interpretation (below) compared with VSP results 


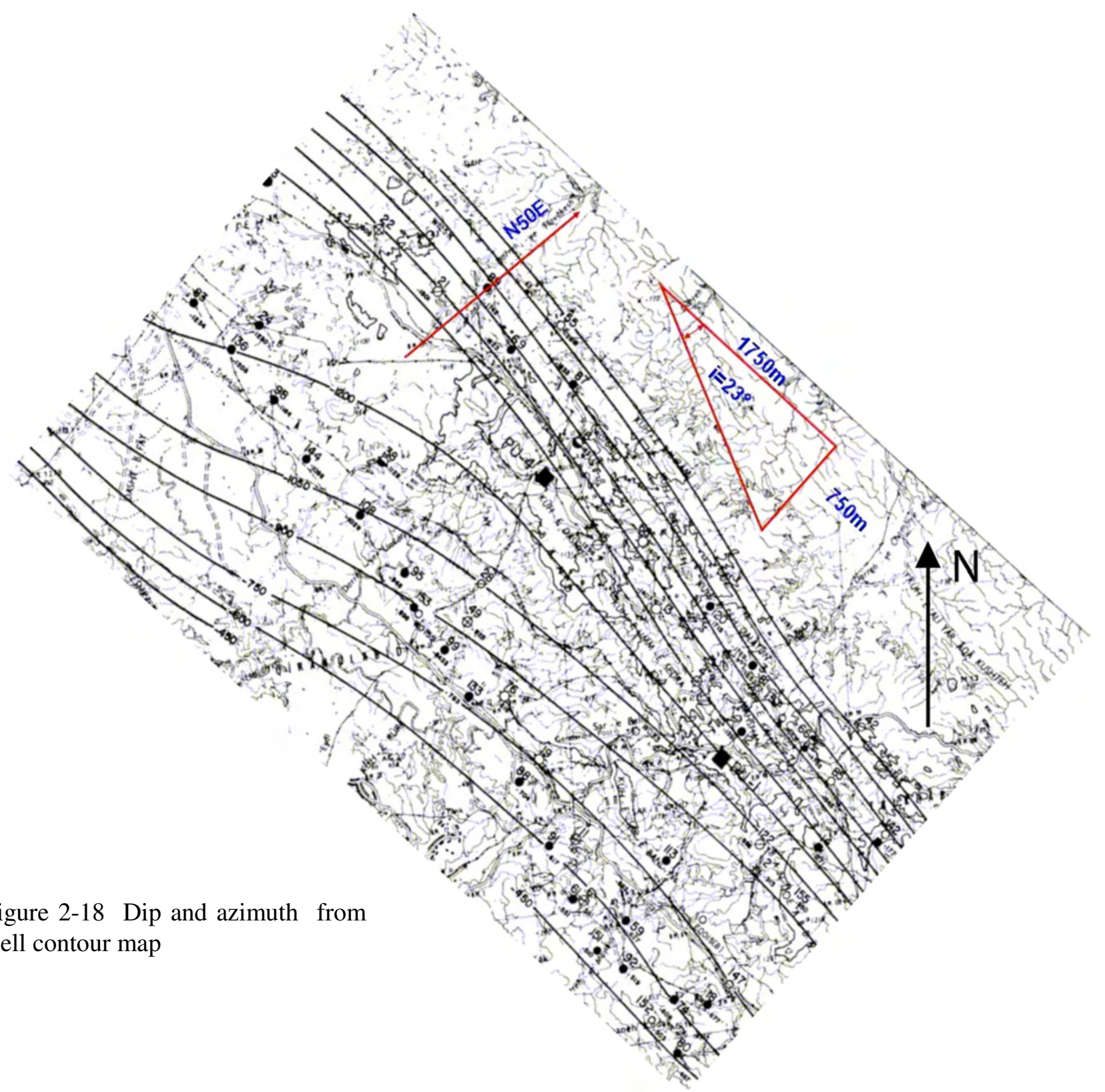

For the GS-6 reflector, P-P and P-S waves were identified and dip of any event is obtained from the polarization analysis (see on $3 \mathrm{C}$ isotropic reflected wave field on figure 2-13). The dip value of $25^{\circ}$ is consistent with geology(Figure 2-18) and surface seismic (Figure2-17) while the azimuth shows around $20^{\circ}$ mismatches, due to the uncertainty of the tool orientation in the deep part of the well. The coherency of Shear wave particle motion looks correct level to level but, uncertainty of dip and azimuth depends partially on the precision of the RB pendulum tool. However, this discrepancy in azimuth measurement also might be due to slight changes of shear wave particle motion level to level. 
Table 1 High confidence bedding dip characteristics from micro scanner measurements in well A at Asmari reservoir interval

$\begin{array}{cccccccc}\text { MD } & \text { QUAF } & \text { DPTR } & \text { DPAZ } & \text { DPAP } & \text { DPAA_NAZ } & \text { DPAA_TOH } & \text { DIP_HEIGHT } \\ \text { m } & & \text { Deg } & \text { Deg } & \text { Deg } & \text { Deg } & \text { Deg } & \text { m } \\ 2750.4 & 20 & 41 & 61 & 24 & 352 & 72 & 0.0787 \\ 2733.3 & 20 & 52 & 59 & 29 & 14 & 95 & 0.1067 \\ 2731.7 & 20 & 49 & 36 & 43 & 356 & 76 & 0.1803 \\ 2696.4 & 20 & 45 & 47 & 32 & 354 & 79 & 0.1245 \\ 2695.9 & 20 & 38 & 59 & 22 & 344 & 68 & 0.0787 \\ 2689.2 & 20 & 44 & 48 & 30 & 352 & 77 & 0.1118 \\ 2688.5 & 20 & 46 & 57 & 25 & 3 & 89 & 0.0965 \\ 2688.2 & 20 & 50 & 50 & 32 & 4 & 90 & 0.1194 \\ 2664.5 & 20 & 42 & 61 & 20 & 350 & 79 & 0.0686 \\ 2661.1 & 20 & 52 & 49 & 32 & 5 & 94 & 0.1168 \\ 2652.9 & 20 & 42 & 52 & 26 & 347 & 76 & 0.0889 \\ 2629.9 & 20 & 43 & 45 & 30 & 345 & 75 & 0.1067 \\ 2612.6 & 20 & 52 & 54 & 28 & 10 & 100 & 0.1118 \\ 2607.2 & 20 & 43 & 52 & 25 & 351 & 80 & 0.0889 \\ 2606.4 & 20 & 51 & 54 & 28 & 9 & 99 & 0.1016 \\ 2605.0 & 20 & 44 & 53 & 25 & 354 & 83 & 0.0889 \\ 2599.8 & 20 & 45 & 50 & 28 & 355 & 84 & 0.1016 \\ 2595.0 & 20 & 51 & 51 & 30 & 8 & 97 & 0.1067 \\ 2590.0 & 20 & 49 & 60 & 23 & 13 & 102 & 0.0787 \\ 2588.0 & 20 & 49 & 60 & 23 & 13 & 102 & 0.0787 \\ 2551.7 & 20 & 52 & 59 & 26 & 21 & 110 & 0.0965 \\ 2545.8 & 20 & 51 & 55 & 27 & 14 & 103 & 0.1016 \\ 2542.8 & 20 & 51 & 54 & 29 & 14 & 103 & 0.1067 \\ 2539.8 & 20 & 50 & 59 & 26 & 18 & 105 & 0.1016 \\ 2538.0 & 20 & 54 & 56 & 30 & 21 & 108 & 0.1118 \\ 2537.1 & 20 & 68 & 81 & 31 & 72 & 158 & 0.1118\end{array}$

QUAF $=$ Dip quality factor $\quad$ DPTR $=$ True dip angle $\quad$ DPAZ $=$ true dip azimuth DPAP= Apparent dip angle-tool DPAA_NAZ= Apparent dip azimuth north DPAA_TOH= Apparent dip azimuth top of the hole DIP_HIGHT= Height of dip plane

Table 1 shows the micro scanner dip measurement results in well A at Asmari reservoir level. The blue columns show true dip angle and true azimuth of the dip respectively. These dip measurements are also in agreement with VSP dip measurement. Although azimuth measurement has $\pm 20^{\circ}$ variations which is explained in chapter6 in detail. 


\section{2-5 Interpretation and well tie}

Well tie with surface seismic (3D seismic block) was undertaken. Figure2-19 shows the VSP corridor stack, time adjusted on the seismic section ( $7 \mathrm{~ms}$, compatible with static correction). The phase matching in the correlation window indicated by a box on figure 2-19 is about $40^{\circ}$. The deviated leg enters the Asmari reservoir. The well trajectory is converted in the time domain by vertical stretch and squeeze operation from depth domain (Fig 2-19), using the correspondence between vertical depth and two way time, therefore, it is not fully accurate for confrontation with a migrated section in the time domain; actually, in presence of dip, the well trajectory should be computed in the migrated time domain of confrontation in order to correct for lateral offsets induced by seismic propagation (Hubral's correction).

Figure 2-19 also illustrates that the anhydrite Cap rock formation is faulted although the faults probably do not penetrate the Asmari reservoir on the Eastern flank. Due to low frequency of the seismic image of the reservoir, the elongated wavelet the Cap rock discrete reflector corresponds to an elongated wavelet (60ms peak-trough-peak series) right above the top of the Asmari reservoir.

Small faults with oblique displacement are visible on the seismic section on Cap rock reflection which is in agreement with the lateral extension of the P- wave reflection on the few traces of VSP horizontal component HE (Figure 2-13), resulting in the structural sketch of Figure2-14. Figure 2-20 illustrates the faulted Cap Rock which is obtained by 3 component VSP wavefield and confirmed by 3D seismic cube. Cap rock has negative polarity (SEG convention) response on the seismic section while top Asmari reservoir cannot be seen. Due to lack of velocity or impedance contrast between the anhydrite Cap rock and the Asmari carbonates. Most probably 
around well A on the Eastern flank of anticline structure, the faults do not penetrate into the Asmari reservoir, in contrast with the top of the anticline as illustrated by figure 2-20.

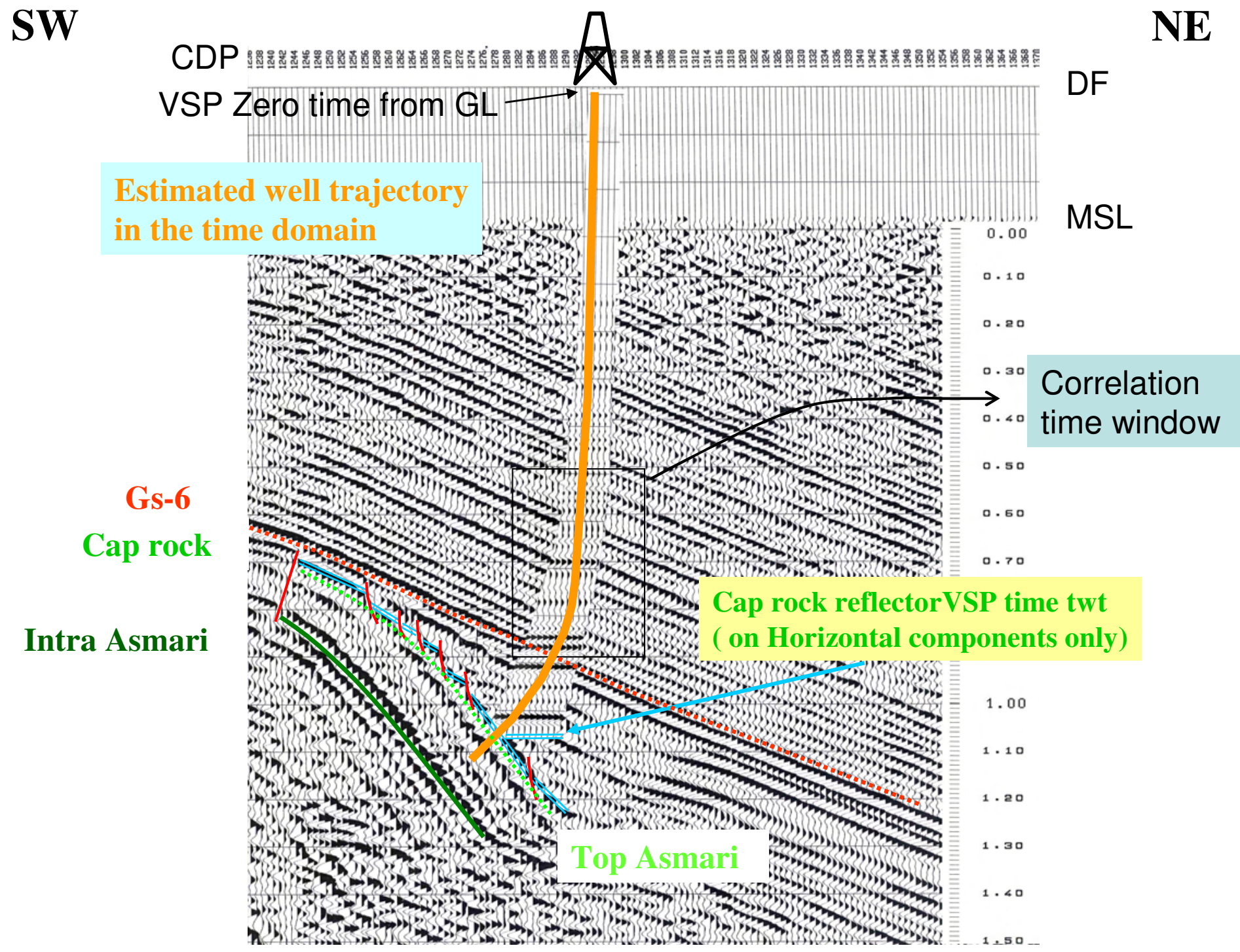

Figure 2-19 well ties with 3D seismic block; the rectangle shows the appropriate window where well tie performed due to low well deviation and high reflectivity. Time scale in the right side shows datum from mean sea level while section is measured from ground level 


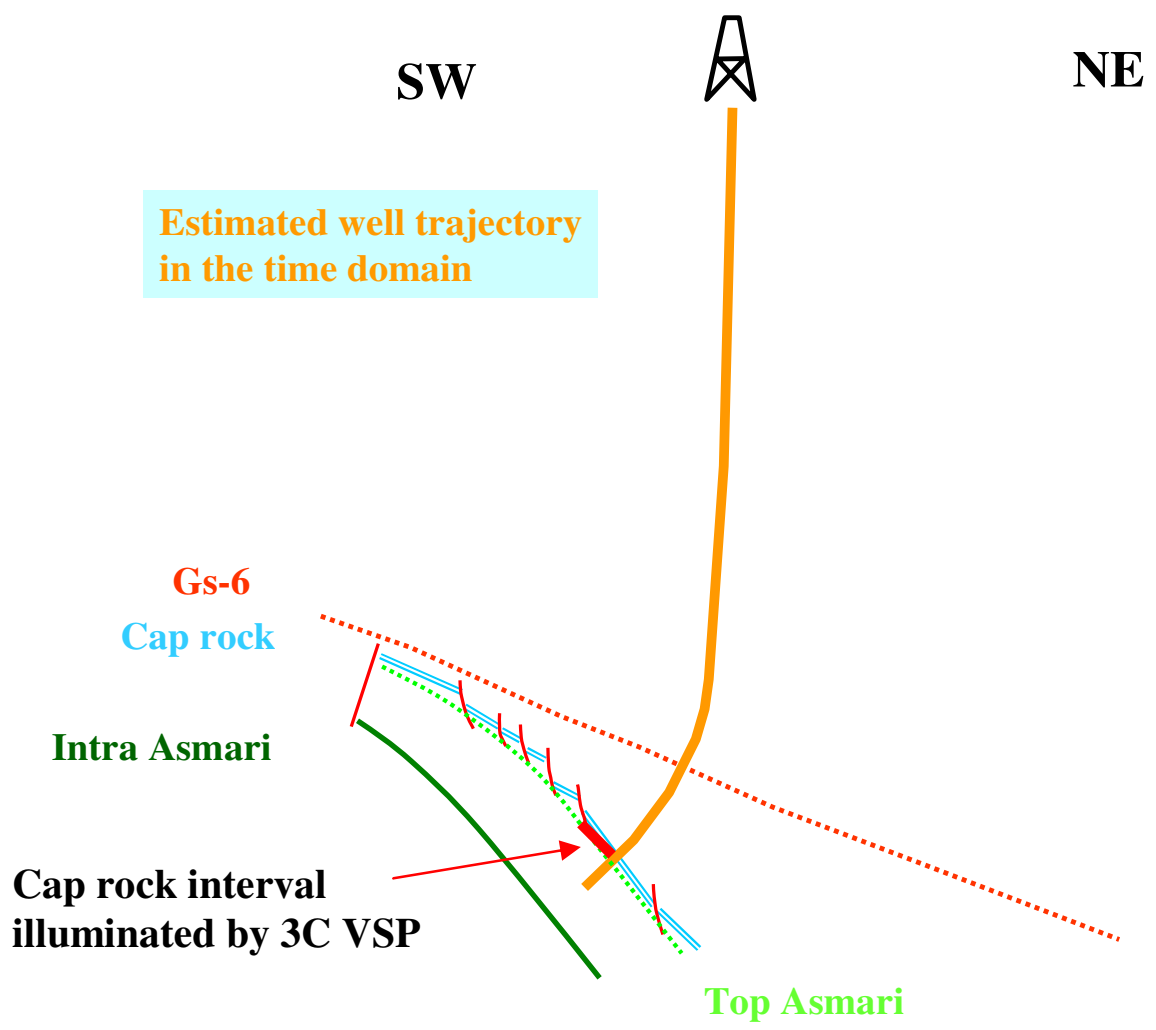

Figure2-20 Structural sketch of eastern flank of Asmari reservoir around well A, the faulting of anhydrite Cap rock layer does not likely affect underlying carbonate Asmari reservoir

\section{2-6 Conclusion}

Because of sensitivity of the 3-component orientation to RB angle accuracy from the VSP tool, it is suggested to combine an inclinometer or Gyroscope to orient the VSP tool in the wells deviated less than $12^{\circ}$ surrounded by structural complexity, which cannot be illuminated by surface seismic. 
The expected precision of the dip measurements is less than $5^{\circ}$ while the precision on the azimuth is about $\pm 10^{\circ}$.

Splitting of direct shear wave arrivals into S-fast and S-slow components confirms that the seismic anisotropy is due to layering, and the S-slow is polarized in the dip direction, the Fast S-wave is parallel to the strike.

Three Component processing of oriented 3C VSP signals is an independent and reliable method to obtain the structural dip and azimuth in the well vicinity from oriented P-P reflections, potentially up to distance of a few hundred meters away from the well and below total depth in both open and cased holes. This technique complements the micro scanner dip results, as both techniques do not necessary read the same dipping geological features. In cased hole only the VSP measurements can be performed.

With 3C VSP, the interpreters can also study with higher confidence shear wave behavior, PS and S-S reflectivity. The VSP technique is a good tool to investigate the P-S reflectivity.

Well tie between VSP corridor stack and surface seismic has been successful on the overburden part of the well A where the well is near vertical. The Cap rock reflector is dipping about $45^{\circ}$ and its reflection is recorded only on the horizontal components.

In the case of well $\mathrm{A}$, the integration of 3 component interpretation results with $3 \mathrm{D}$ surface seismic image gives better understanding of geometry and faults trends in the well vicinity, for the benefit of geologists and reservoir engineers. 



\section{Chapter 3}

\section{Provisional interpretation and QC on first PSTM cube}




\section{3-1 Introduction}

Primary goals of this study are a) improve the structural image and b) detecting the fractures within the Asmari reservoir. Based on reservoir and well reports there is a gas cap on the top of the Aghajari field where G.O.L and W.O.L are located around 1933m and 2462m measured depth respectively( Barkers, S. N, Speers, R. G, 1977, Report No. P-3319, NIOC). On the production side, it is preferable to focus our effort on the producing oil interval where well data (well log, mud loss) show fractures and where stimulation by gas injection is planned. On the seismic side, it is not sure that a good image of the reservoir can be achieved because of large dip and velocity variations. The southwest flank is usually the steeper with dips up to $70^{\circ}$ but Averaging about $40^{\circ}$ while the average dip on the northeast flank is about $30^{\circ}$, values of up to $50^{\circ}$ are locally developed.

The seismic data that we have used for this first interpretation come from the very first 3D acquired in the field in 2005. The data have been processed by a Chinese contractor including pre-stack time migration. The corresponding cube and migration velocity were delivered to us by March 2007. We performed a first interpretation of these data in order to check if PSTM processing could already bring information's on faults and fractures in Asmari reservoirs (section 3.2) and if migration velocities were in agreement with well data available (section 3.3). In addition, structural interpretation of main horizons and well velocities are essential data that will be required in the next step of depth imaging. 


\section{3-2 Structural interpretation}

I interpreted five main horizons to have better knowledge of faults trend and structural style of the field:

-Top Gachsaran member 6, mainly anhydrite and marl (high velocity) is a guide reflector due to good acoustic contrast,

-Top and base of Amari reservoir,

-Top Kazhdomi shale formation (low velocity) which act as decollement level beneath the Bangestan group,

- Top Khami group that is the deepest reservoir in this field.

The figures 3.1 (a) and (b) show 2 vertical sections in dip and strike direction with these 5 horizons. The limit between the gas cap and the oil zone is also shown. Top Asmari time map shown in Figure 3.2 illustrates that we are not able to image south western flank and only partially the north East flank: Not only we are not able to map fault or fractures in the flanks but we cannot even image the reservoir in the southwestern flank where producing oil wells( oil zone) are located.

In the anticline submittal part where the image is good, a variance cube with convenient parameters was also created to visualize faults and fractures within the Asmari reservoir. In this area, the time map of Top Asmari (Figure 3.2) shows a crestal graben faults system over top of the structure. It could come from a big thrust fault in the Southwestern flank with a few back thrusts in the north Eastern one. The crestal graben fault system on the top of Asmari was confirmed with the Variance cube and Dip measurements (Figures 3-7a and b). 


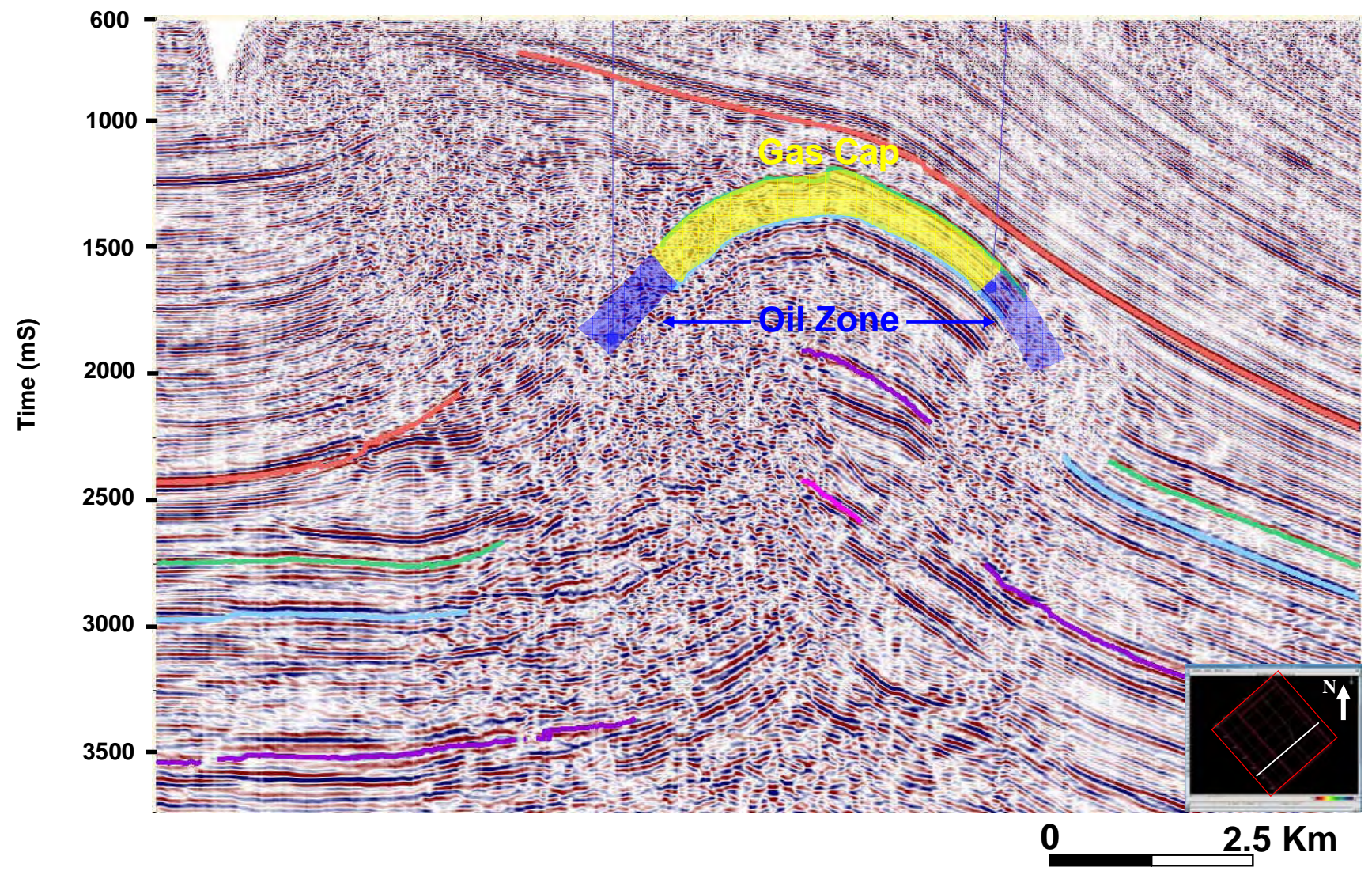

Figure 3-1a: Profile in dip direction, The interpreted horizons display from top to bottom, Gs-7(brown), top Asmari(Green), Base Asmari (blue),top Kazhdomi(Violet), and top Fahliyan (Cyan) 


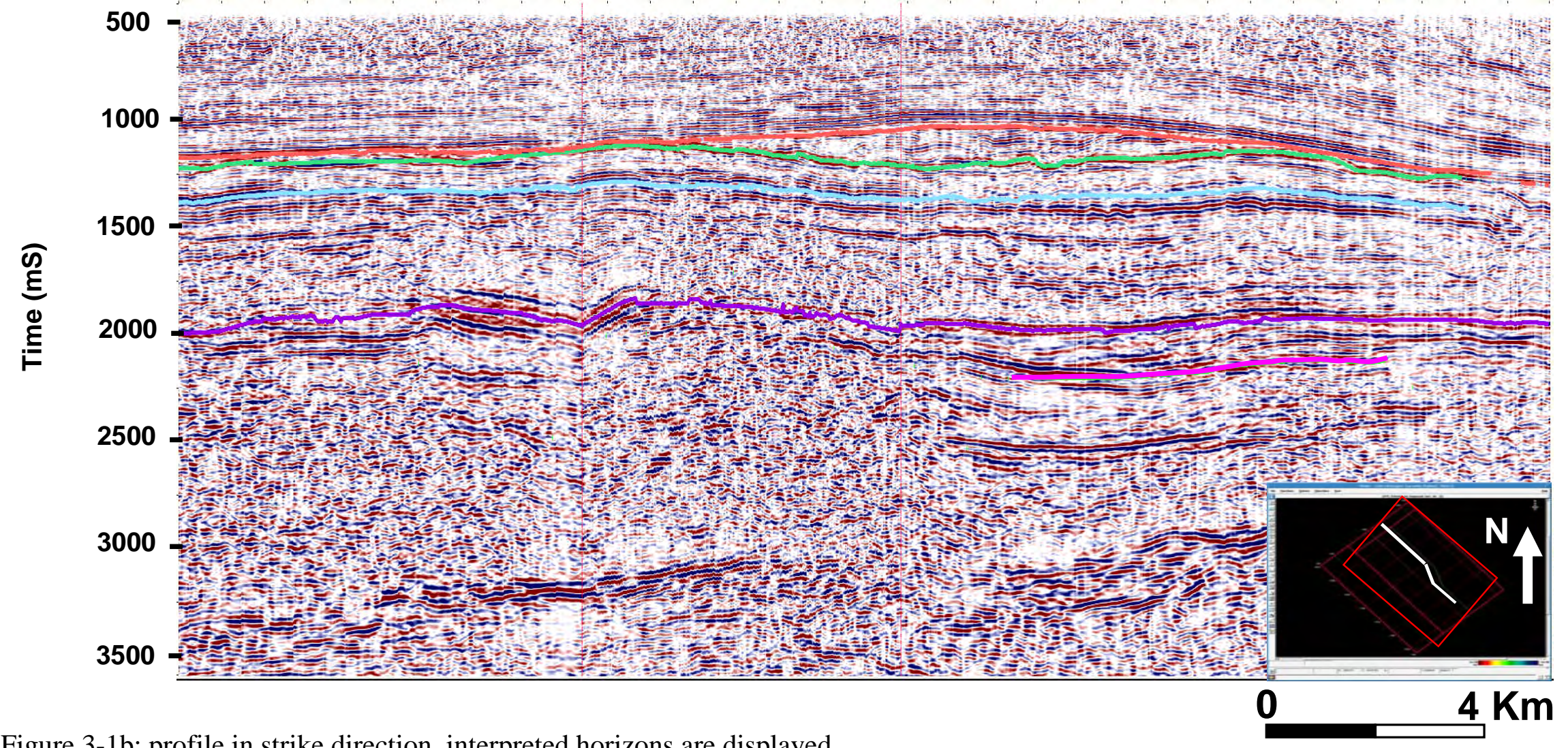

Figure 3-1b: profile in strike direction, interpreted horizons are displayed 
The poor reconstruction in the south flank might be a problem of illumination of steep dips with our acquisition design but is more probably due to the complexity of the velocity that presents large lateral variation in these areas: in the south part, the thickness of evaporates series is much higher and the Asmari is much deeper and steeper, with probably the presence of a major fault (or several faults). The velocities of shales and evaporates are much smaller than the velocity of carbonates in the Asmari layer which results in large velocity difference across the faults. The other problem is that migration algorithm migrates also some strong noise and multiple present in the prestack data.

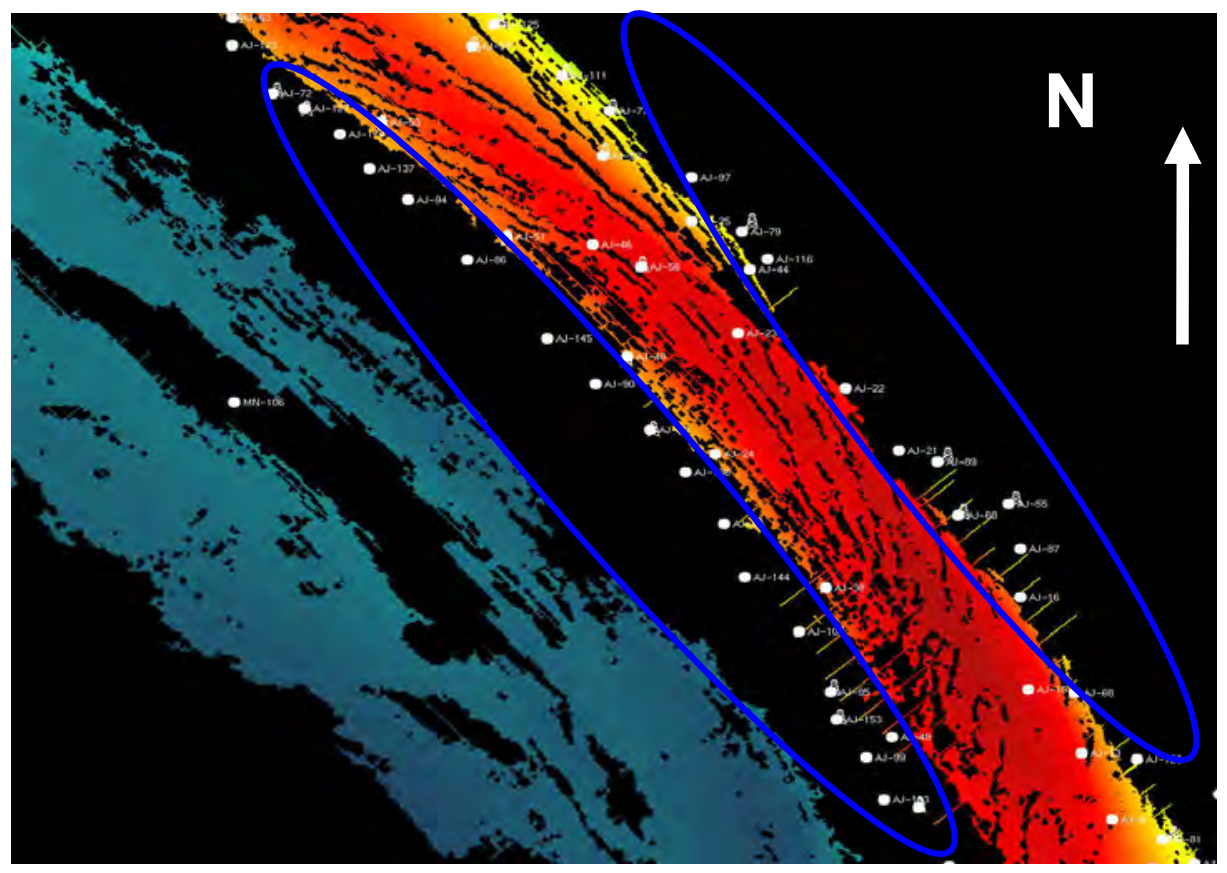

Figure 3-2 Time map of top Asmari reservoir illustrate the crestal graben faults system on the top of structure 


\section{3-3 Well Velocity data}

We have used velocity information of 4 wells to investigate the velocity variation over the Aghajari field that are shown on Figure 3-3. The single zero offset VSP data on the well A in the North-East flank, the check shot and sonic data on deep exploration well on the top, Eastern part of the field, the check shot on the South-Western flank and check shot in production well on the top of the structure in the western part of the study area.

The deep exploration well locates on the top of the Aghajari structure in the eastern part of the field. It is an exploration well that penetrates up to 5592m for investigating the third reservoir in this field (reached top of Fahliyan, third reservoir in $4147 \mathrm{~m}$ from ground level). There are DT, GR, RHOB and check shot data in deep exploration well where sonic log runs from $2400 \mathrm{~m}$ (MD) below the Asmari reservoir to the TD and Check shot from surface to the $4150 \mathrm{~m}$ (MD). Interval velocity was calculated and calibrated with check shot then projected on the seismic cube to investigate the velocity variation along the well trajectory (Figure 3-4). 


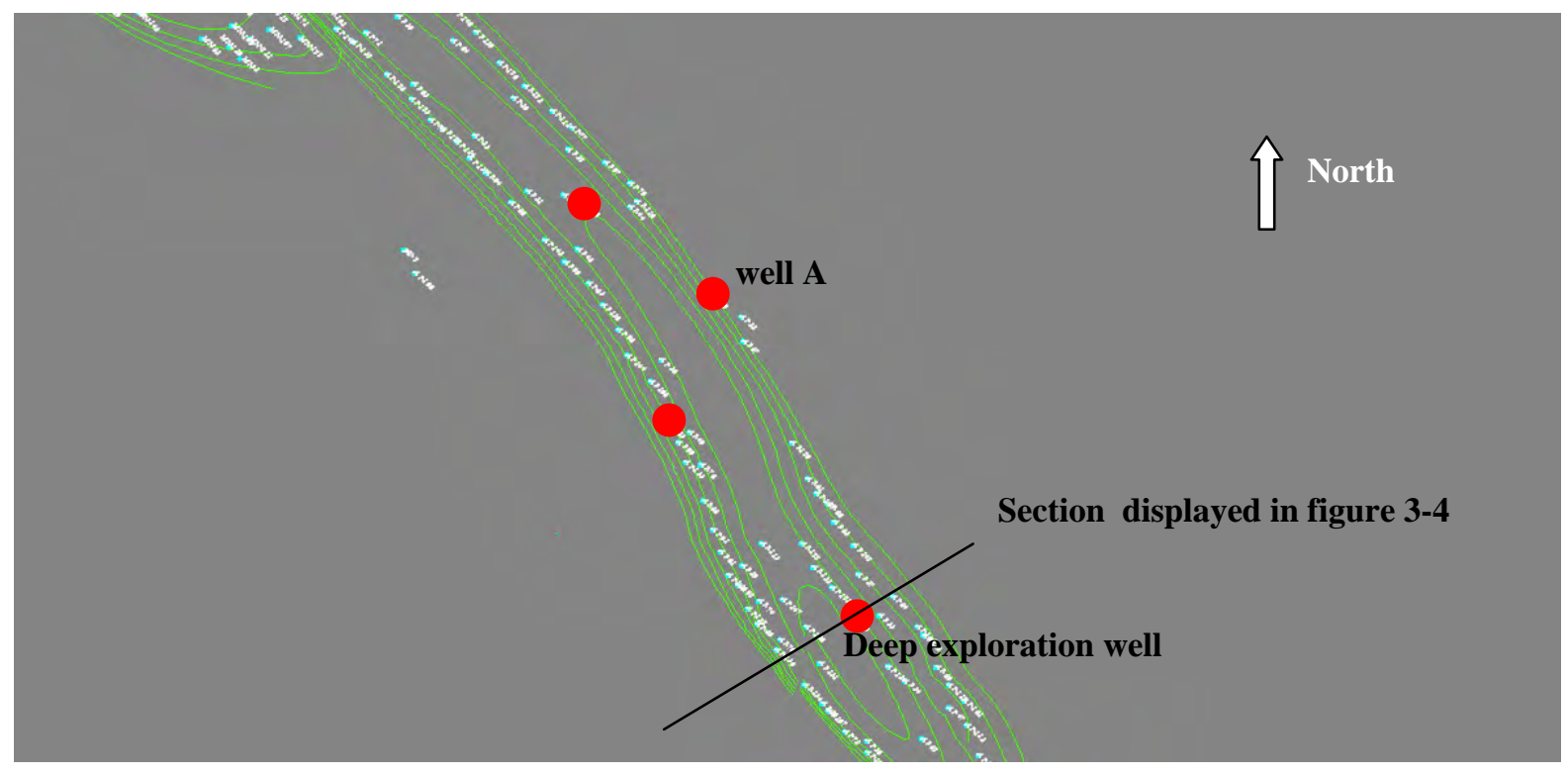

Figure3-3 The wells (red point) that have been used for velocity analysis 


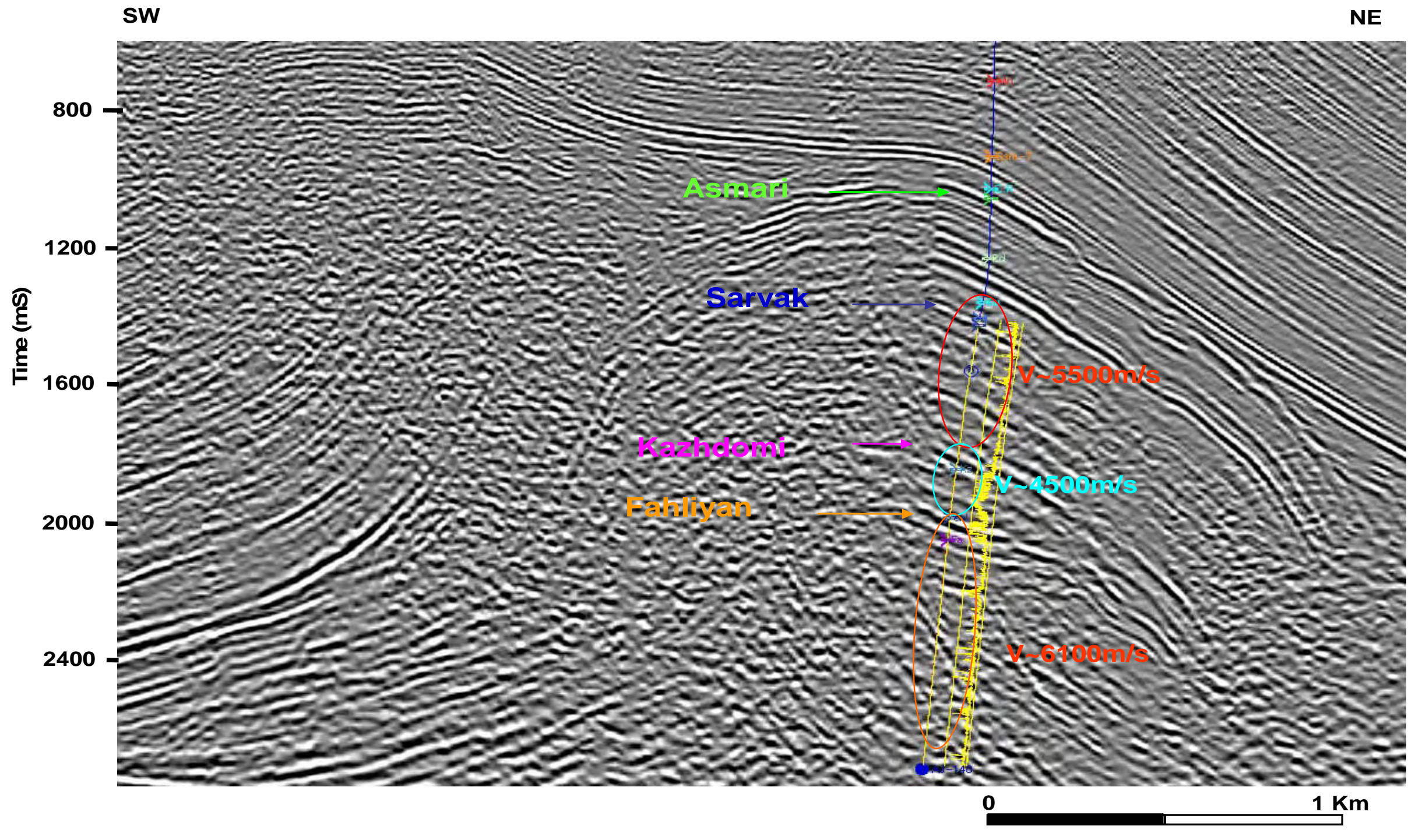

Figure3-4 projection of interval velocity on the seismic section along the well trajectory 
We realized that interval velocity decreases in the Kazhdomi formation (3360m), which should be taking into account during the next PSDM step.

Well with check shot and sonic which located in the South-Western flank in the middle of the field. It penetrated up to 2680 m (MD) and was completed as Asmari oil producing well. Based on well completion report, Gachsaran formation member 4-2 was repeated due to faulting in this well. The fault is around 2053m, which corresponds to about 1350ms in vertical two-way time. This repetition of Gachsaran member 4 to 2 occurs in this well, whilst an important repetition is reported in some other wells in southwestern flank. Moderate to heavy losses was reported in Asmari formation, which leads to fractures in Asmari level (well completion reports).

There are some misfits between well trajectories and PSTM cube on the wells which are located in the flanks with high dip but for the wells on the crest misfit is negligible. It is possible that the PSTM cube is not fully migrated to correct lateral position and therefore some misfits with well projection are normal (Figure 3-5).

From sonic, check shots and VSP velocities and main geological markers (formation) a 2D velocity model was established to use in next PSDM step.Figure3-6 shows this model. The other issue is dipping top reservoir reflector which apparent stacking velocity increases due to dip. Although the geological formations in both flanks are the same but due to different tectonic responses, thickness and formation setting changes, consequently velocities are getting azimuthally variable. 

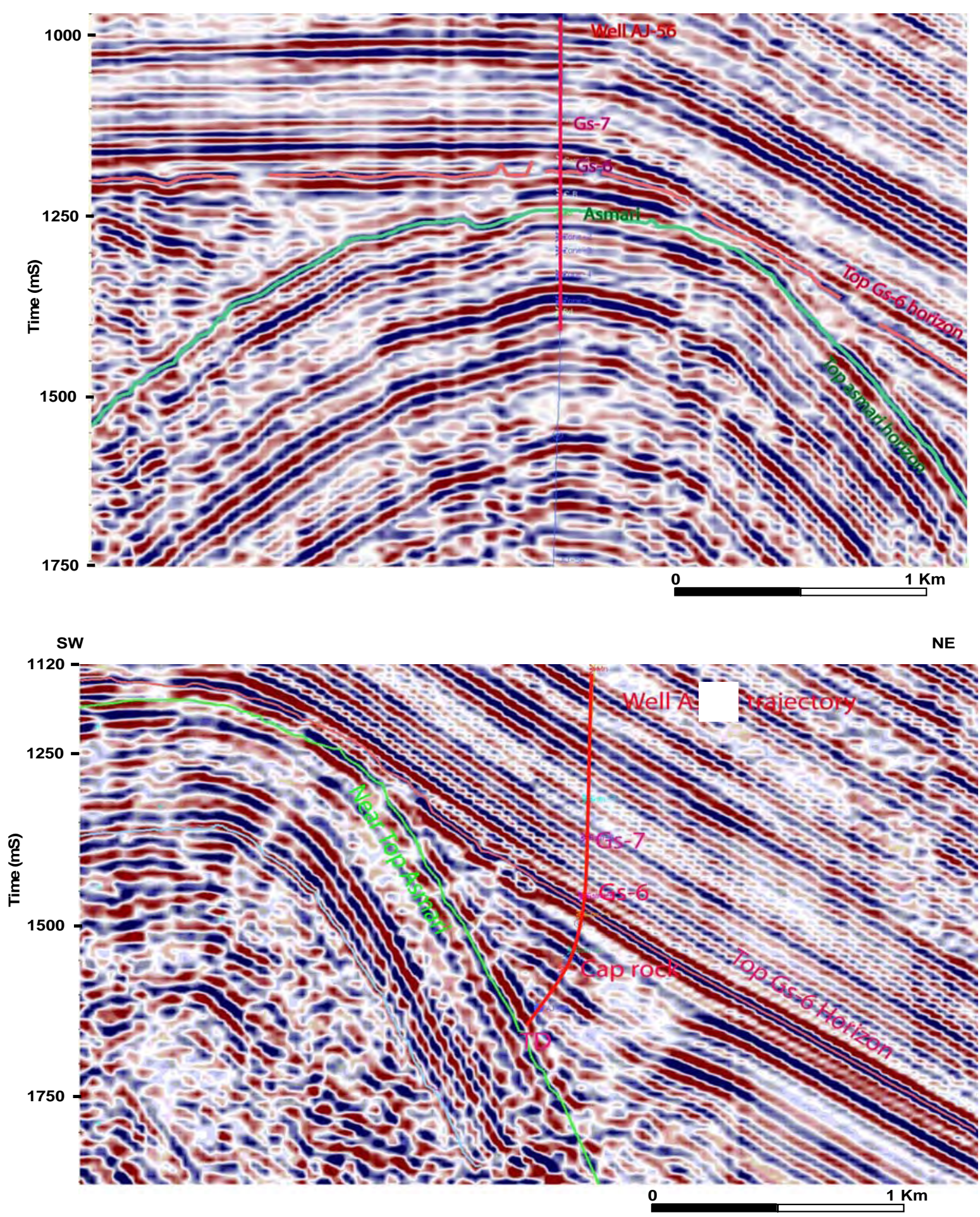

Figure3-5: Above) well is fit with seismic cube due to its location on the crest below)misfit between well trajectory and PSTM cube in the northern flank 


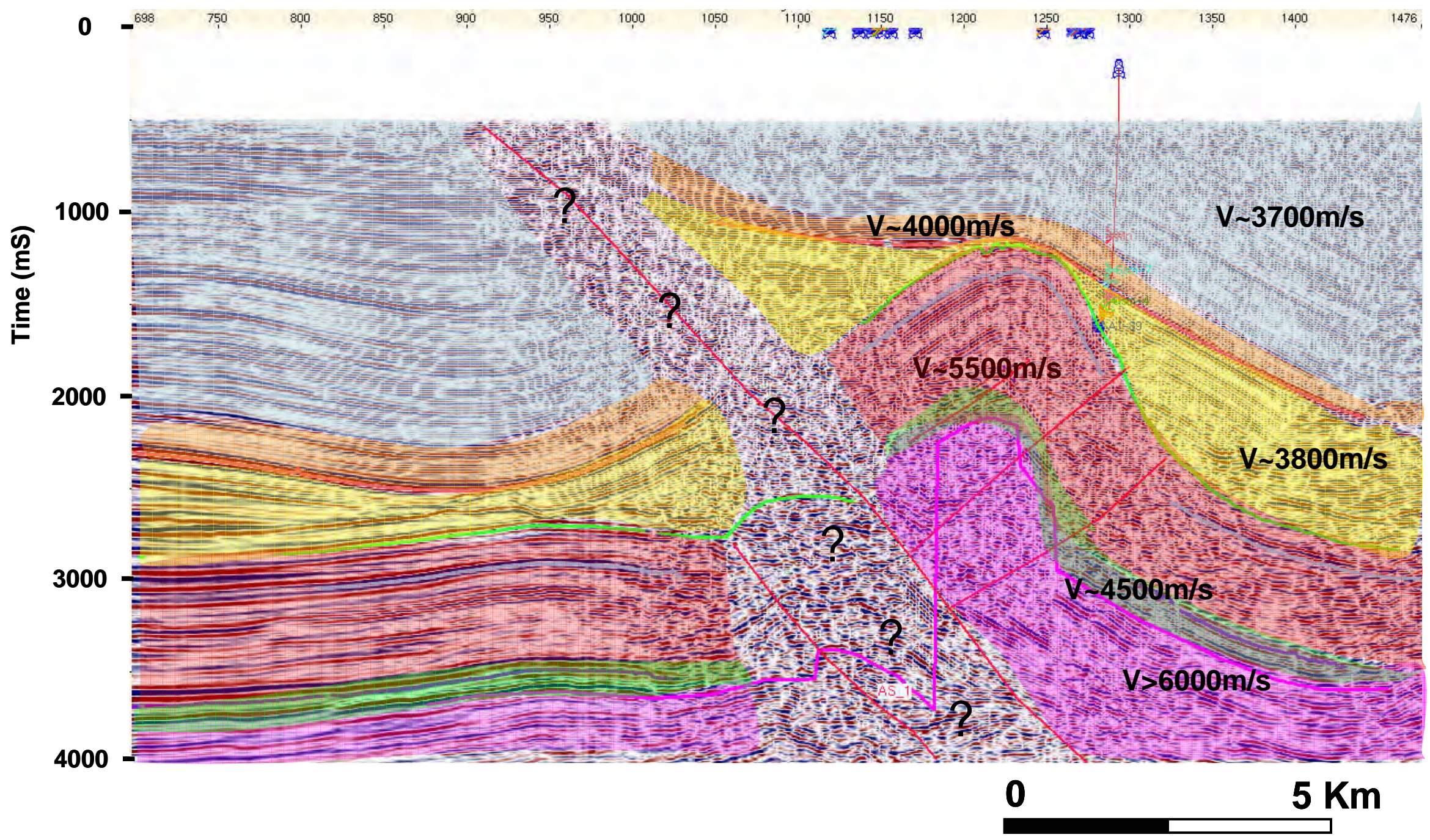

Figure 3-6 2D velocity model based on geological interpretation (colors represent different lithology, velocities derived from well data) 


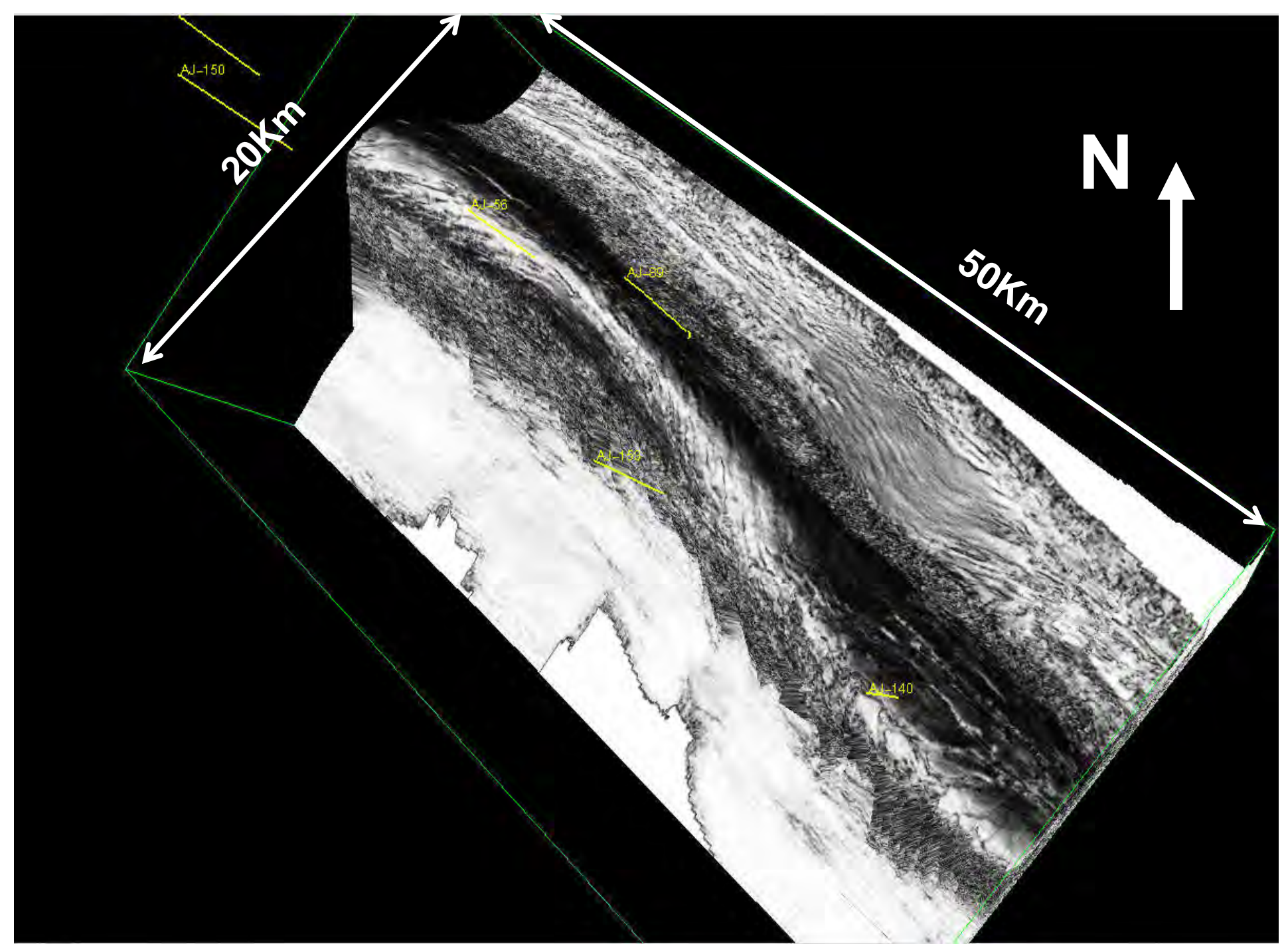

Figure 3-7a Variance Attribute shows fault on the Top of Asmari reservoir 


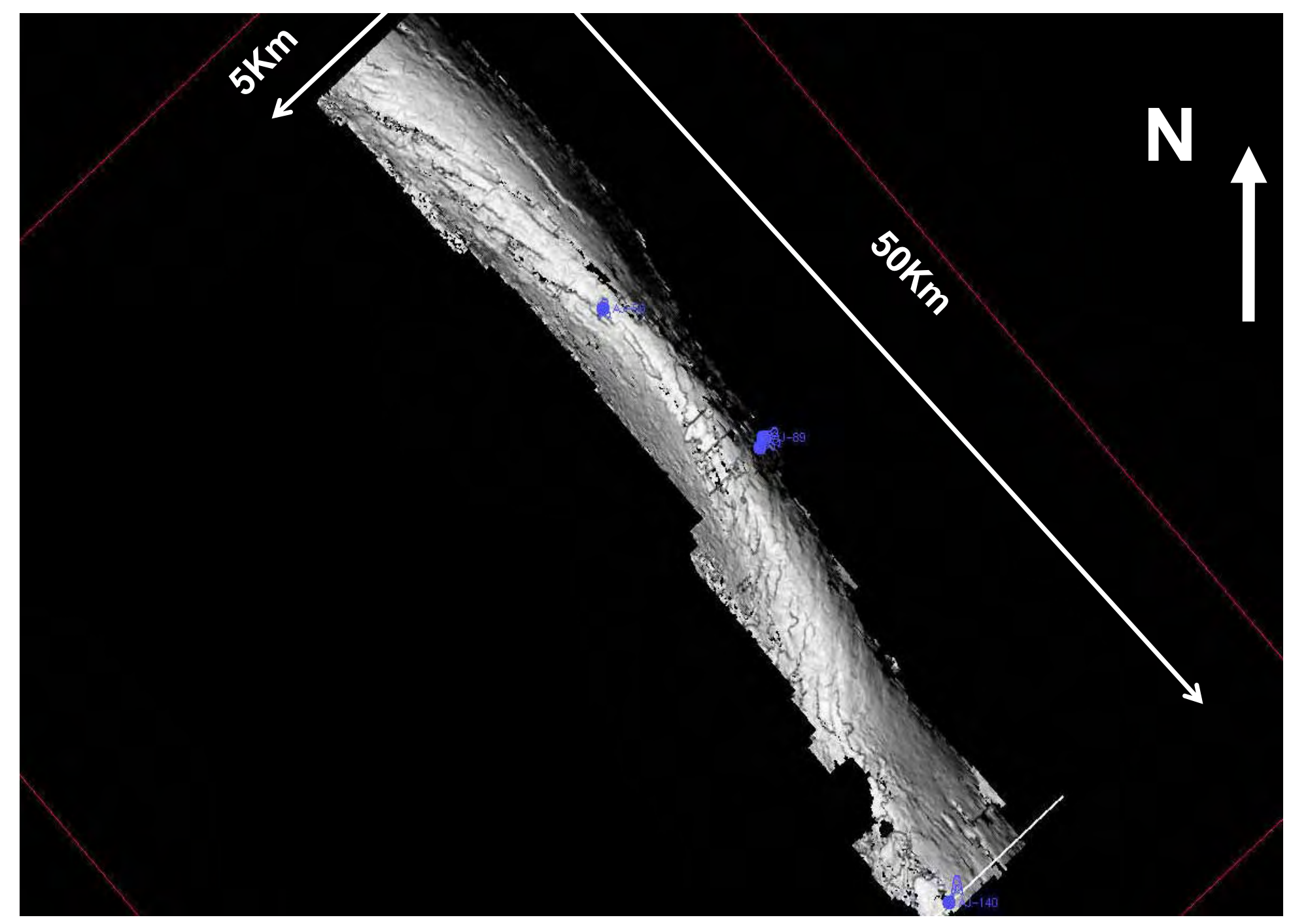

Figure 3-7 b Dip attribute illustrates fault on the Top of Asmari reservoir 


\section{3-4 Conclusion}

The 3D PSTM image on the Aghajarri field did not solve the imaging problem already recognized on pre-existing 2D lines. The oil producing interval where all wells are located is still poorly or not imaged by 3D seismic. A tentative explanation is the strong velocity variation in the overburden in particular in evaporates Gachsaran formation. This formation is affected by strong deformations due to folding and faulting (swelling salt members and faulting), and simultaneously by large thickness variation of salt, anhydrite and marls. It is well known that lateral velocity variation cannot be accounted for by PSTM imaging. We hope that it is possible to build an accurate depth velocity model and improve the reservoir image by use of PSDM method. However, the top of Asmari formation presents medium to steep dips in the reservoir part $\left(40^{\circ}\right.$ to $70^{\circ}$ in southwest flank) and the seismic illumination on these steep flank should be verified by modeling. The field acquisition was planned with large aperture (up to 6km) but we will check if it is sufficient by pre-stack migration analysis. Based on VSP data on well A, check shots and sonic logs in other three wells on the top of structure and in the south flank (including deep an exploration well), a mild interval velocity inversion, or velocity stabilization to $3800 \mathrm{~m} / \mathrm{s}$ is evidenced in the Gs-5 to Gs-2 interval , corresponding to interbedded massive salt and very soft and ductile gray marls, and to the deep shaly Kazhdomi formation. An abrupt increase of velocity occurs on top of anhydrite Cap rock (5800 $\mathrm{m} / \mathrm{s})$, in the Asmari reservoir immediately below it $(5500 \mathrm{~km} / \mathrm{s})$. 
The above observations, justify the need for applying depth migration using a precise depth velocity model based on surface seismic and well data.

Before engaging the depth migration process, we have thoroughly repeated the PSTM velocity analysis. Indeed, we cannot start a depth conversion without careful analysis of all pre-stack seismic data. In addition, we want to use a new workflow to build depth velocity model that makes use of PSTM velocity and residuals picked in the time migrated domain. The next chapter describes our work on time and depth velocity migration analysis. In this chapter I will describe the depth model building starting from the new CGGV product "Beyond DIX". 


\section{Chapter 4}

\section{D surface seismic imaging}

Application of Beyond Dix workflow 


\section{4-1 Introduction}

Pre-Stack time migration still represents the majority of seismic imaging activity in the industry. The reason for this is the speed and robustness of time imaging and its ability to focus seismic reflectors for most geological settings. Limitations appear in the case of strong lateral velocity variations, which require the more rigorous imaging and more accurate velocity models offered by Pre-Stack Depth Migration (PSDM).

In areas of moderate complexity, where Pre-Stack Time Migration (PSTM) begins to suffer, the "Beyond Dix" workflow provides accurate fast-track depth migration from time imaging projects. It takes full advantage of the speed, good focusing and high signal-to-noise ratio available from time imaging to jump-start the depth imaging process. The name comes from the fact that it goes beyond the limitations of the 1D Dix inversion commonly used to derive depth interval velocities from the time imaging velocities. It uses the full kinematic information available in the time migrated domain to directly build an accurate depth velocity model and by-pass the 1D Dix Inversion altogether" (from Lambaré et al., 2007).

Conventional processing of this dataset was done by BGP Company in China. We started with CDP-gathers for which all pre-processing steps except NMO and mute have already been applied. After quality control of geometry, topography and static corrections, the data set was loaded into CGGVeritas proprietary Geocluster system. All gathers were shifted to the floating datum plane and migration velocities were then measured referring to this floating datum (smoothed version of the topography in time).

By experience, pre-stack time Kirchhoff migration is an appropriate method as a first step before depth imaging.

The first PSTM result provided by BGP was not appropriate as input for the Beyond Dix workflow (the kinematic de-migration used in Beyond Dix has to be fully consistent with the PreSTM tool used to generate the migrated gathers); therefore we performed a new PreSTM with the TIKIM algorithm from CGGVeritas.

In this chapter I firstly explain the steps we used to perform the PreSTM velocity analysis used as input for the Beyond Dix workflow. Secondly I describe the "Beyond Dix" workflow which allows obtaining a depth velocity model for PreSDM. We assume that this model will allow improving both focusing and positioning, especially in the south west flank of the Aghajari field. 


\section{4-2 PSTM velocity analysis with TIKIM tool}

\section{4-2-1 Principle of PSTM}

Kirchhoff PSTM aims at relocating reflected dipping events in time at their correct lateral position with the assumptions of hyperbolic events and use of a local velocity. This relocalization requires an effective velocity model. A value of the effective velocity is associated to any point in the migrated image. This value fully determines the travel time curve used in the PSTM to map reflected event from the un-migrated to the migrated domain. In PSTM, for a given image point in $(x, y, t)$ (where $t$ is the vertical coordinate in one-way time), the diffracted travel time curve only depends on its effective velocity parameters and sources and receivers offsets with respect to the image point, by means of the double square root equation:

$T\left(x, y, t, x_{s}, y_{s}, x_{r}, y_{r}\right)=\sqrt{t^{2}+\frac{\left(x-x_{s}\right)^{2}+\left(y-y_{s}\right)^{2}}{V^{2}}}+\sqrt{t^{2}+\frac{\left(x-x_{r}\right)^{2}+\left(y-y_{r}\right)^{2}}{V^{2}}}$

Where $\mathrm{T}$ is two way time, $\mathrm{t}$ is two way time at zero offset and $\mathrm{V}$ is effective velocity

The advantages of this approximation (Robein, E, 2003) are:

- fast computation of travel time,

- there is only one effective and local parameter to optimize (V)

- the focusing of diffractions and optimization of stack is still possible even if the lateral position is not correct (at least for a given offset range, as long as the diffracted wavefront along the normal ray stays spherical).

For Kirchhoff PSTM in practice, we sort data by common offset (without paying attention to the azimuth of this offset), each of which is processed independently. For each offset, the result is a common offset cube in $\mathrm{x}, \mathrm{y}$ and $\mathrm{t}$ ( $\mathrm{x}$ and $\mathrm{y}$ are image coordinate positions and $\mathrm{t}$ is the vertical time from the reference datum to the diffraction point). At each surface point $(\mathrm{x}, \mathrm{y})$, we have a common image gather that represents the image versus $\mathrm{t}$ and $\mathrm{h}$ (offset). If the effective velocity model is good we should have the same image for all offsets and consequently flat common image gathers (CIGs). We can then stack the CIGs over the offset providing the final PSTM stack image. If CIGs are not flat we can scan the migration velocity in order to improve the flatness of the gather (or the semblance or the stack along offset dimension). If the gathers are almost flat, it is sufficient to pick residual move-out curves 
(parametric curves, for instance parabolic) in order to flatten the gathers (by a residual correction) before stack. We worked with the algorithm TIKIM of GEOCLUSTER to perform PSTM and migration velocity analysis.

An important parameter for this algorithm is the aperture along diffraction curve used to perform the diffraction stack. In TIKIM, this aperture is controlled by the aperture width (maximum offset between the midpoint and the image point) and a maximum geological dip (the computation of the local migration dip use approximate ray tracing in a provided 1D model).

Based on acquisition parameters and geological information (in particular steep dips up to $70^{\circ}$ ), aperture width and dips were chosen at maximum values. We sorted the data by offset class (step of $120 \mathrm{~m}$ ) in such a way to preserve the dependency of residual move-out versus offset while insuring that the CMP distribution in $\mathrm{X}$ and $\mathrm{Y}$ is sufficient and homogeneous to produce a correct migration. Others parameters are anti-alias protection (that depends on the acquisition bin step and of the frequency) and the introduction of an a priori anellipticity (due to ray bending in an approximate 1D model, computed by Dix inversion of the migration velocity model, after Al-Khalifa , 1997). A test line was chosen and migration parameters were analyzed and fixed for this line.

\section{4-2-2 Noise attenuation and mute}

Pre-processing for pre-stack time and depth migration can have a dramatic effect on the final results. Since the data will be analyzed before stack, it is very important to have the data as clean and free of noise as possible. There were many spikes in the gathers that could make the migration results noisy. In order to remove spikes and ambient noise from the gathers we used a special module (FDNAT) in Geocluster which attenuates high amplitude noise in decomposed frequency bands. It uses frequency -dependent and time variant threshold values of sample amplitudes, within defined trace neighborhoods to detect and suppress noise specific to different frequency ranges and different times. We removed a lot of spikes in near offset and ambient noise in the whole range of offsets. From figure 4-1 we see a few gathers before and after noise attenuations.

Different mutes were applied to remove the effect of NMO stretch also removing noise from near offset traces. The mutes is important essentially for the stack and restrict the picking of move-out attributes in appropriate range (at large offset, the move out is affected by the NMO stretch and can be more complex than the simple analytic curve used for their picking). 


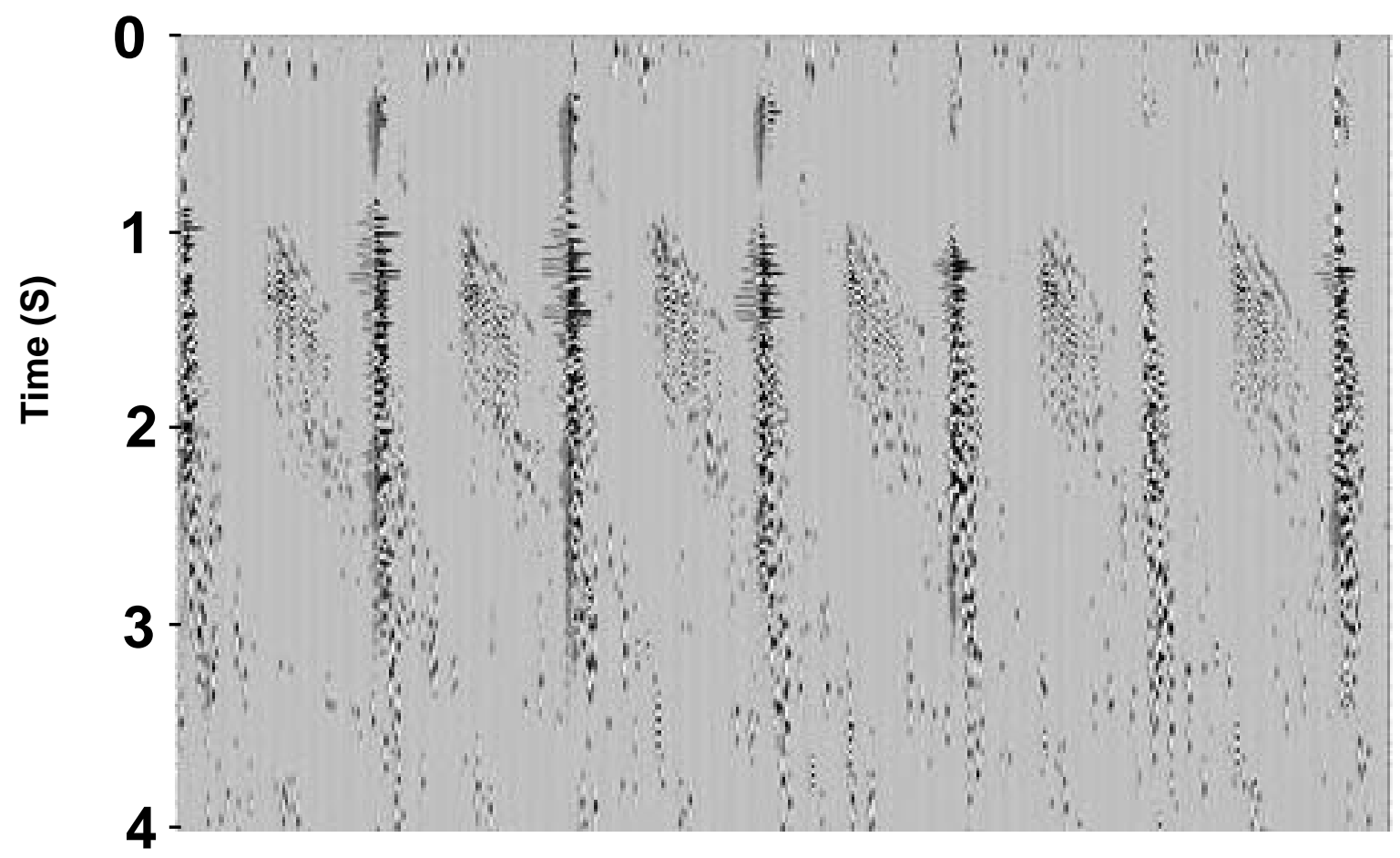

Figure4-1 spikes and noise removed from the gathers

\section{4-2-3 Velocity preparation}

NMO correction was performed with stacking velocity file provided by BGP Company every $1 \mathrm{Km}$ in $\mathrm{x}$ and $\mathrm{y}$. First we smoothed this stacking velocity to avoid negative effect in migration. We used this velocity as a base for PSTM velocity analysis and applied various perturbations (next section) to create a more accurate PSTM velocity model.

For each velocity profile, TIKIM computes the effective anellipticity $\eta$ for non-hyperbolic NMO with the Al-Khalifa formula:

$$
T^{2}=T_{0}^{2}+\frac{X^{2}}{V^{2}}-2 \eta \frac{X^{4}}{V^{4} T^{2}\left(1+\frac{(1+2 \eta) X^{2}}{V^{2} T^{2}}\right)}
$$


The $\eta$ coefficient at this stage only represents the non hyperbolicity induced by the bending of the ray in a 1D layering. An asymptotic development (extension of Dix formula to fourth order) shows that $\eta$ comes from the relative difference between quartic and quadratic mean of interval velocity versus time:

$1+8 \eta=\frac{\left(\sum V_{i}^{4} T_{i}\right) \sum T_{i}}{\left(\sum V_{i}^{2} T_{i}\right)^{2}}$

\section{4-2-4 Velocity perturbation}

As the NMO velocity can be far from the optimum PSTM velocity (in particular the NMO velocity is divided by the cosine of the angle where significant dip is present in the structures (Hale, I. 1983, Levin, K. 1990), we perform velocity perturbations (also called "velocity scans") in the range of $-20 \%$ to $20 \%$ with a $4 \%$ increment . After launching migration job for all offset classes and perturbations over three lines in different part of the study area, we realized that this perturbation set was too large. Therefore, we re-run the migration job for one line in the middle of the study area including all offset classes and velocity perturbations from $-14 \%$ to $8 \%$ every $2 \%$.

With the technique of perturbations scan, we are able to compare the images with different velocity perturbation by overlying them and pick optimum velocities over the migrated section where the image is the best (figure 4-2). We can also pick the optimum velocity by looking at CIGs and select the best flatness (figure 4-3). A new velocity model was then derived from these picked velocities every $500 \mathrm{~m}$ by $500 \mathrm{~m}$. 


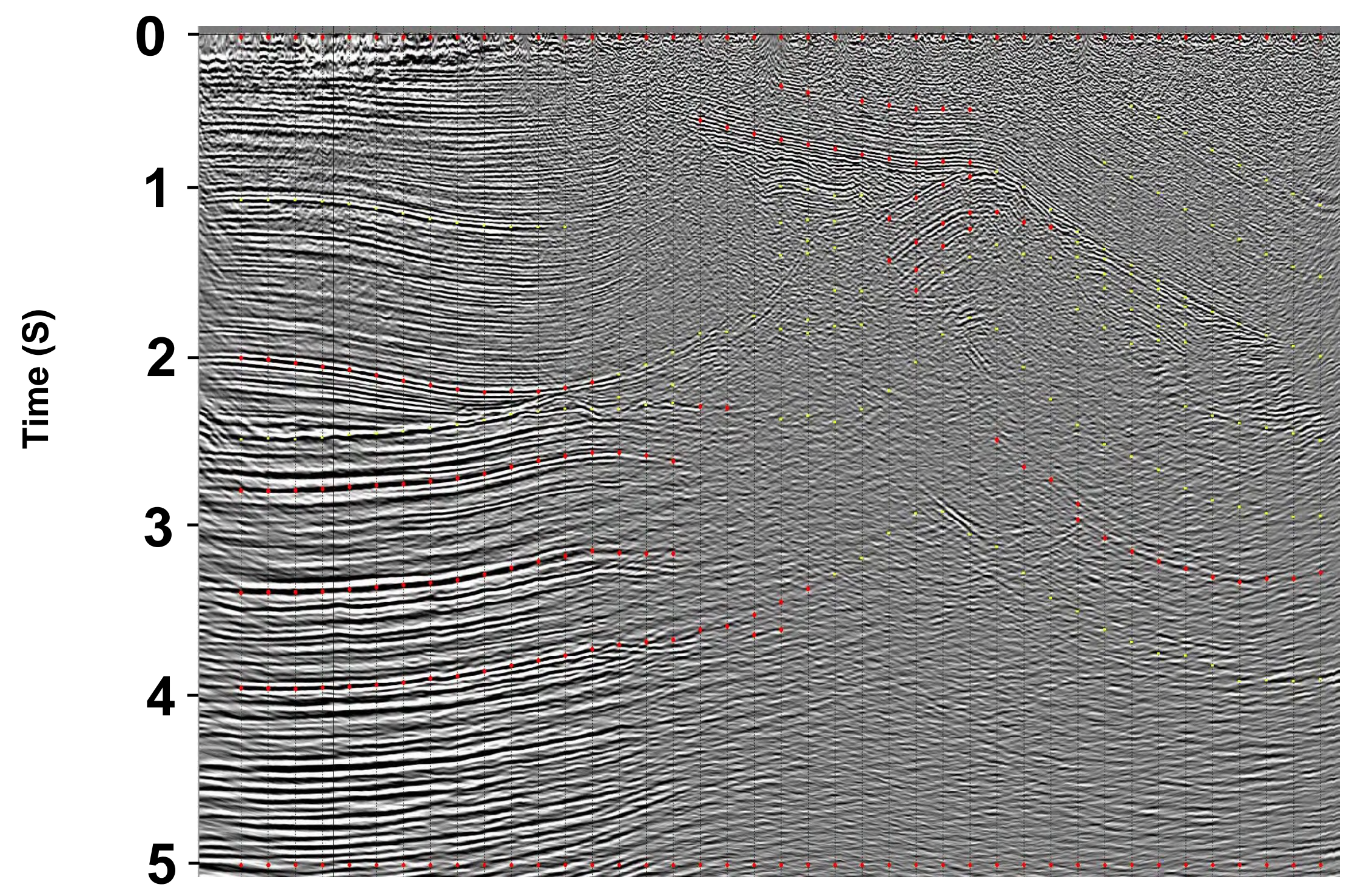

Figure4-2 velocity perturbation scan over the migrated section, red points shows the best image with $100 \%$ velocity picked 


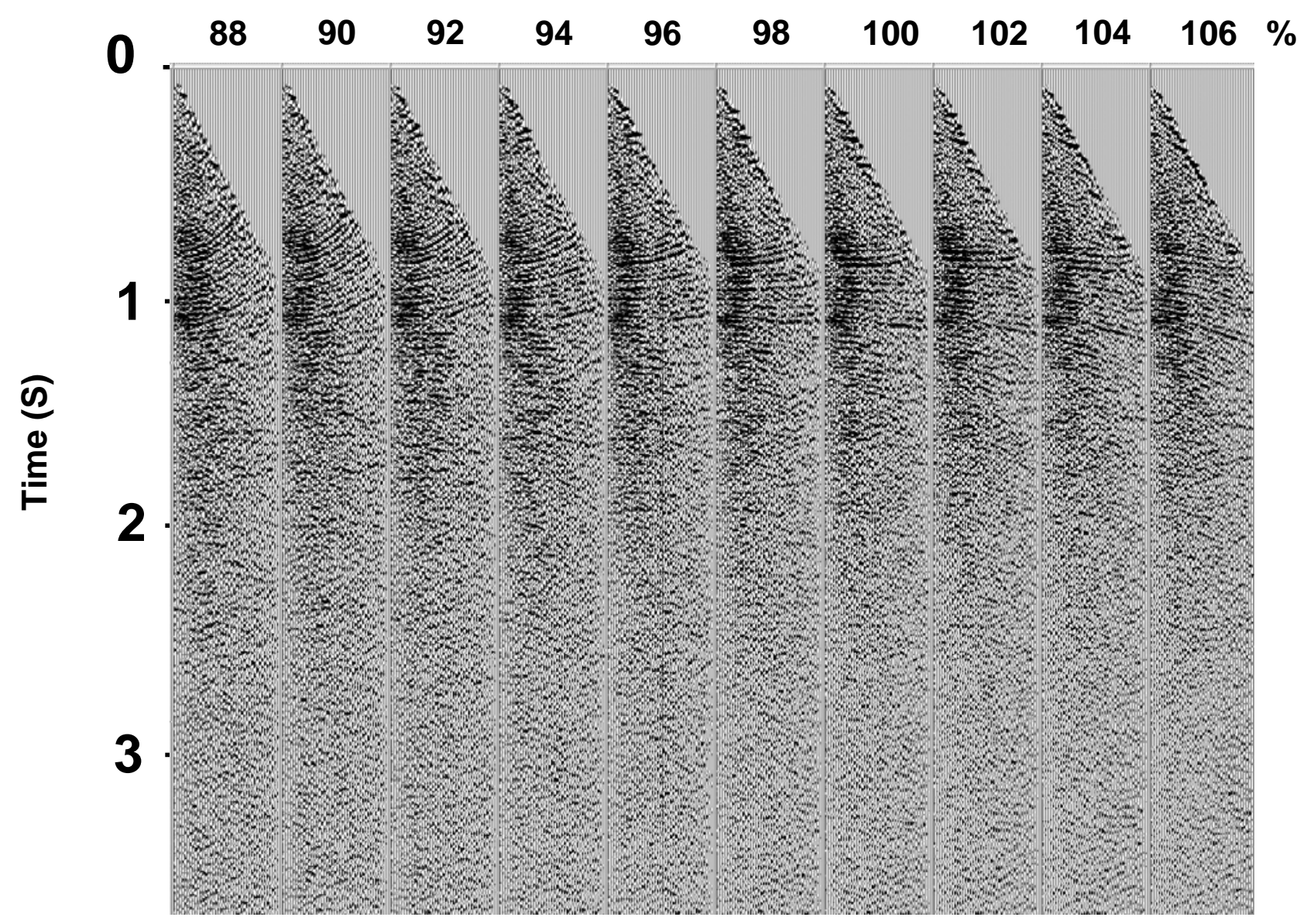

Figure4-3 velocity perturbation scan on the gathers, numbers on the top show the velocity in percentage

The final effective velocity model (Figure 4-4) is consistent in the In-line and X-line directions and shows some variations which can be related to the local geology. Hence it is considered as structurally meaningful and well suited for time migration. 


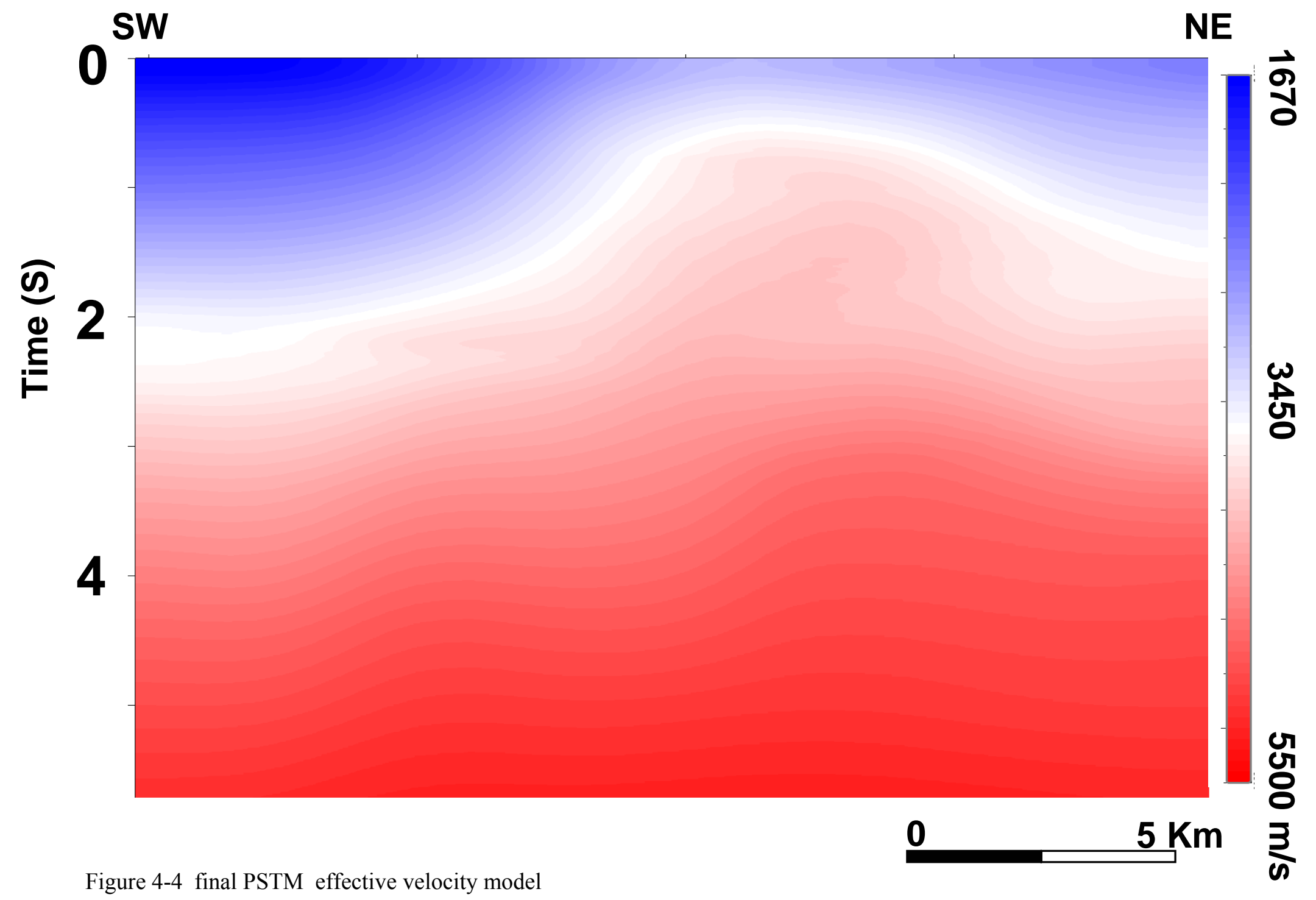




\section{4-2-5 Post Migration processing and RMO picking}

To improve the quality of migrated data and final stack, the following steps were performed:

- Computing multiples and removing them from the migrated gathers (RAMUR module).

- High density automatic RMO picking to compensate for the residual move out of the gathers (Siliqi and al, 2003). The analytic shape can be related to a shifted hyperbola: $\left(t+\tau_{0}\right)^{2}=\left(t_{0}+\tau_{0}\right)^{2}+\frac{x^{2}}{V_{e}{ }^{2}}$

described by 2 parameters $\left(\tau_{0}, d t n\right)$ where $\tau_{0}$ is the shift at zero offset between the true zero offset travel-time and the zero offset travel time corresponding to hyperbola asymptotic to residual move-out at finite reference offset $x_{\operatorname{Re} f}$, and $d t n$ is the time delay at largest offset value:

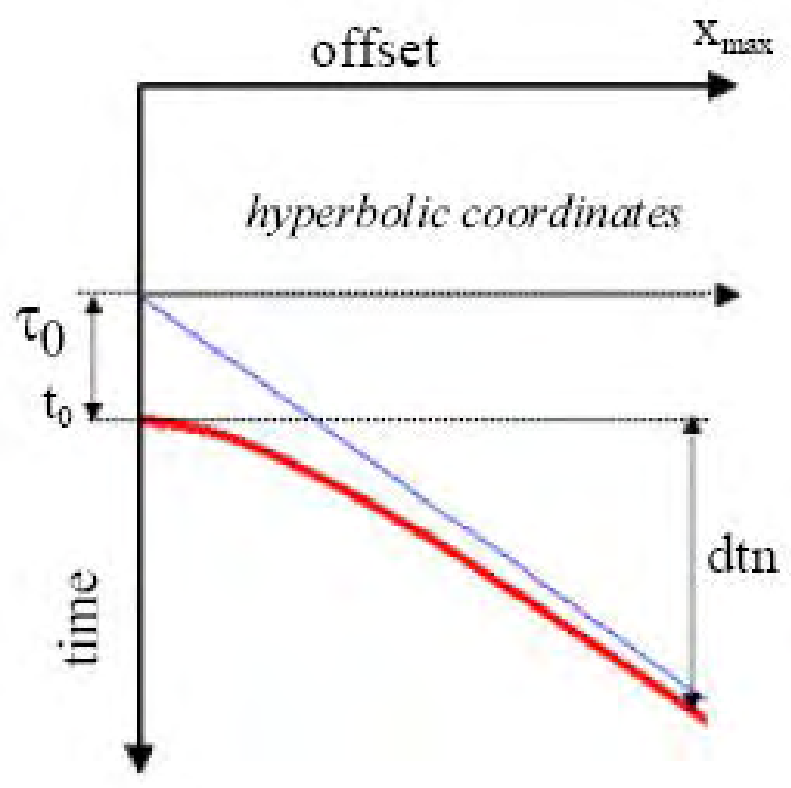

Figure 4-5 relationship between move out parameters $d t n$ and $\tau_{0}$ (from Siliqi et al, 2000)

A picking corridor corresponding to minimum and maximum values of effective velocity and anellipticity is introduced in order to reduce the automatic RMO picking domain and velocity stabilizes the results. The maximum of the semblance is used as the automatic picking criterion. 
- To remove high frequency noise in different time windows on the stack section, we applied a time variant filter.

- At the end we applied a 3D random noise attenuation process to clean the final seismic cube (Soubaras R. 2000). 


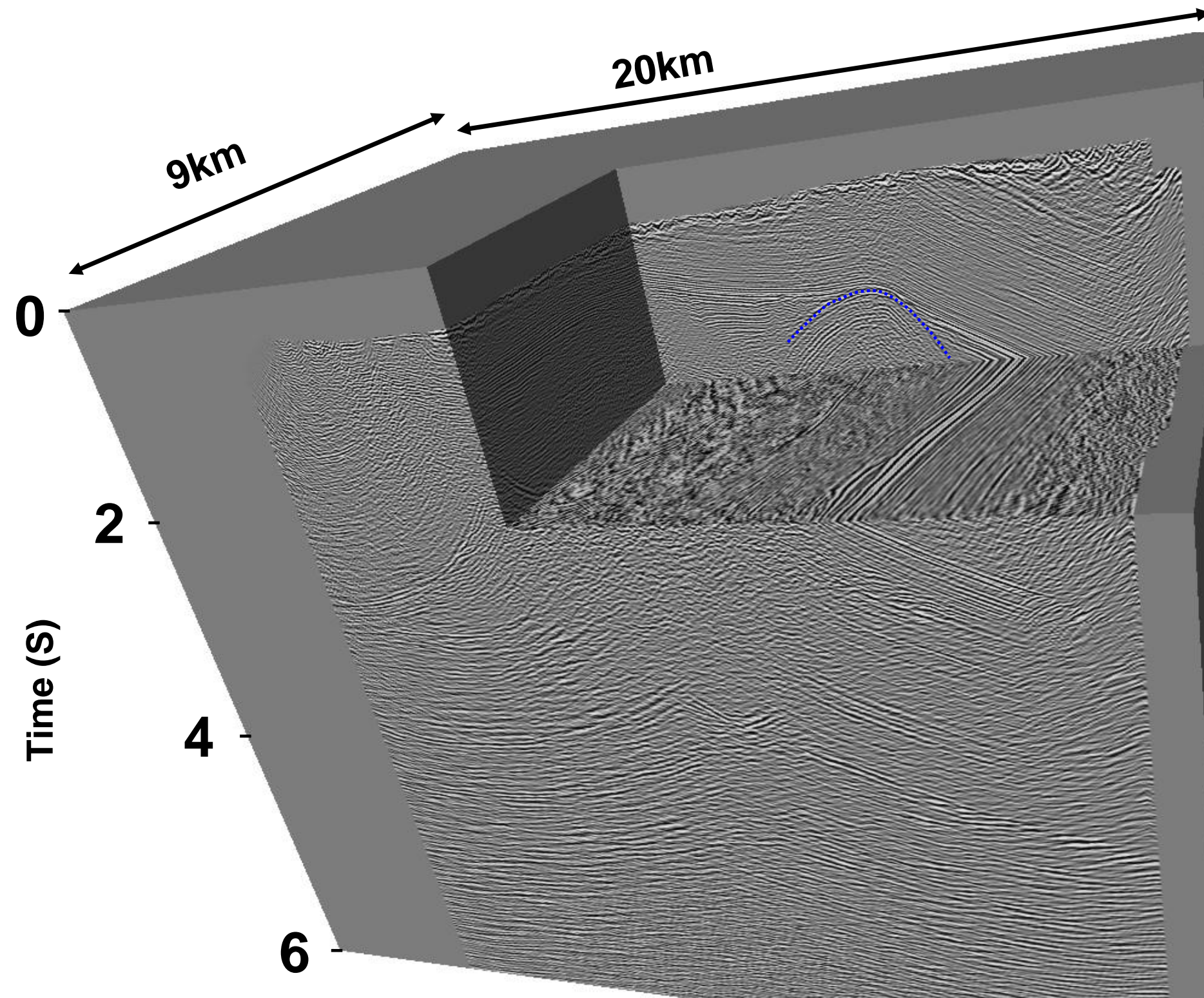

Figure4-6 Final PreSTM seismic cube, Blue dash line shows top Asmari reservoir 


\section{4-3 Depth velocity model building and PSDM Depth Imaging}

\section{4-3-1 Beyond Dix inversion}

The difference of average velocity between wells and surface seismic is about $5 \%$ which may be due to anisotropy effect, in the overlying shale layers.

In Aghajari field we deal with moderate structural complexity and strong lateral velocity variations, which mean that PSDM is required to handle this complexity. PSDM definitely requires an accurate velocity model which can be obtained in a first step by the Beyond Dix approach (Lambaré et al.; 2007b). In this approach a depth velocity model is obtained after a dense automatic picking of dips and RMO (Residual Move Out) attributes performed in the PSTM domain; Kinematic de-migration of the PSTM "migrated facets" provided the kinematic invariants that can be used for the depth velocity model building without any compromise with accuracy (figure 4-7a).

In this approach the quality of the automated RMO picking is crucial. Actually a good focusing improves the performance of automated picking tools (Siliqi et al.; 2007) which explains the success of the Beyond Dix approach where the picking is performed on already well focused PreSTM results. It potentially reduces the number of PreSDM/picking/tomography loops required to obtain the final velocity model.

The following steps were performed to create the final velocity model (Lambaré et al.; 2007):

- Creating initial depth velocity model using PSTM velocity and Dix conversion assuming no dips and no lateral velocity variations.

- Automatic Picking of RMO on the PSTM common image gathers.

- Volumetric dip computation on the time migrated image.

- Kinematic de-migration of the PSTM migrated facets to obtain kinematic invariants.

- Tomographic update of the velocity model using the kinematic invariants.

The last step was repeated several times/iterations to obtain an optimum depth velocity model by parameters fine-tuning. The key point in this approach is the use of the concept of kinematic invariants (Guillaume et al.; 2001). These are computed by de- migration of events picked on the CIGs. Each event on the un-migrated section is characterized by its position, dip and two way time. These parameters are called 'kinematics invariants' due to their non 
dependency with respect to the velocity model. They are used for the tomographic update of the velocity model. Several tomographic inversion loops can be performed to be able to optimize the velocity model but only a single dip and RMO picking is required. The beauty of this tool is because it performs kinematic migrations of invariants to update the velocity model rather than an expensive PSDM of full seismic data. The Beyond Dix workflow had already been applied with success to marine data (Lambaré et al.; 2007). This is the first time it was applied on land dataset with elevation. 


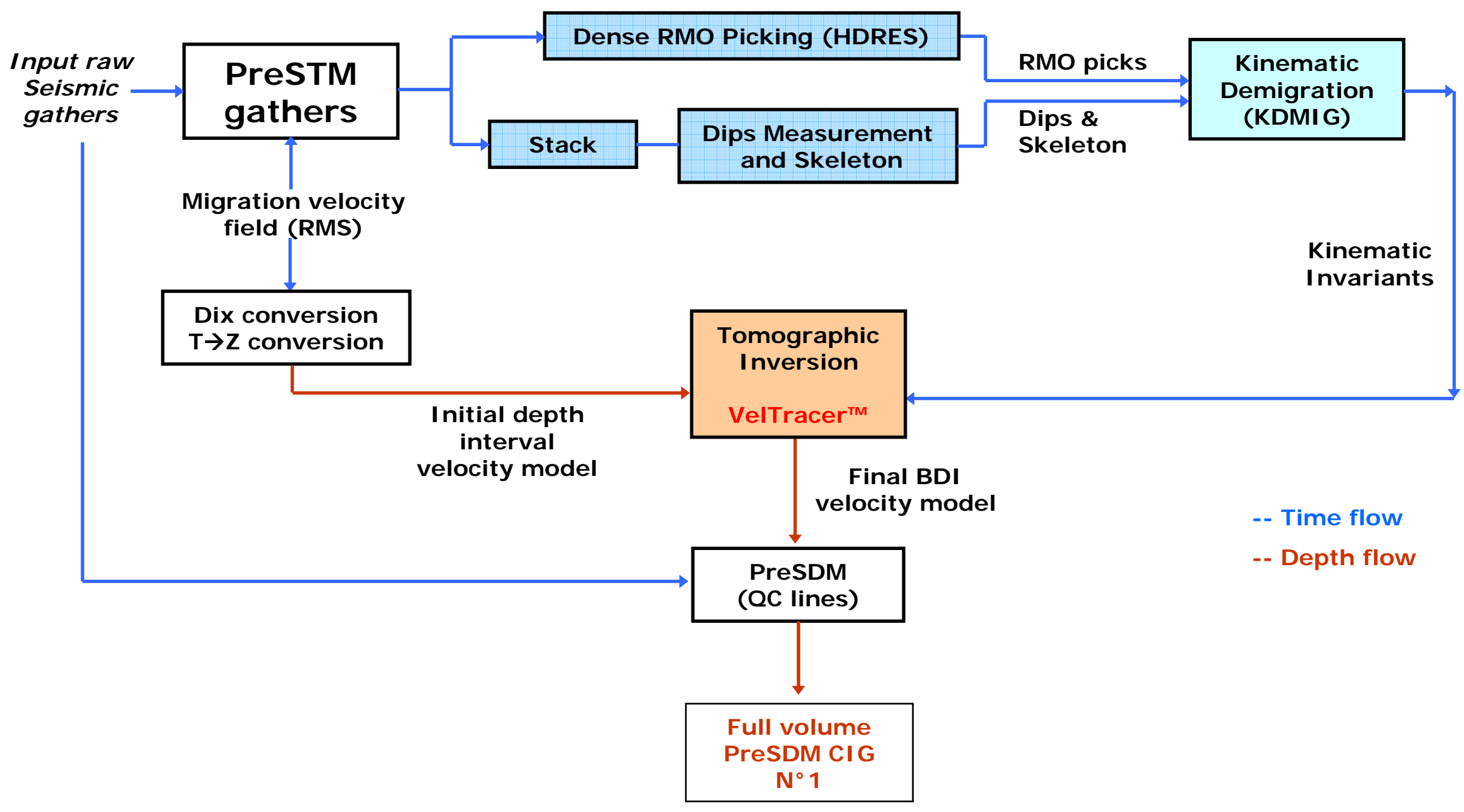

Figure 4-7a flow chart of the Beyond Dix approach (Tomographic inversion, step 1) 


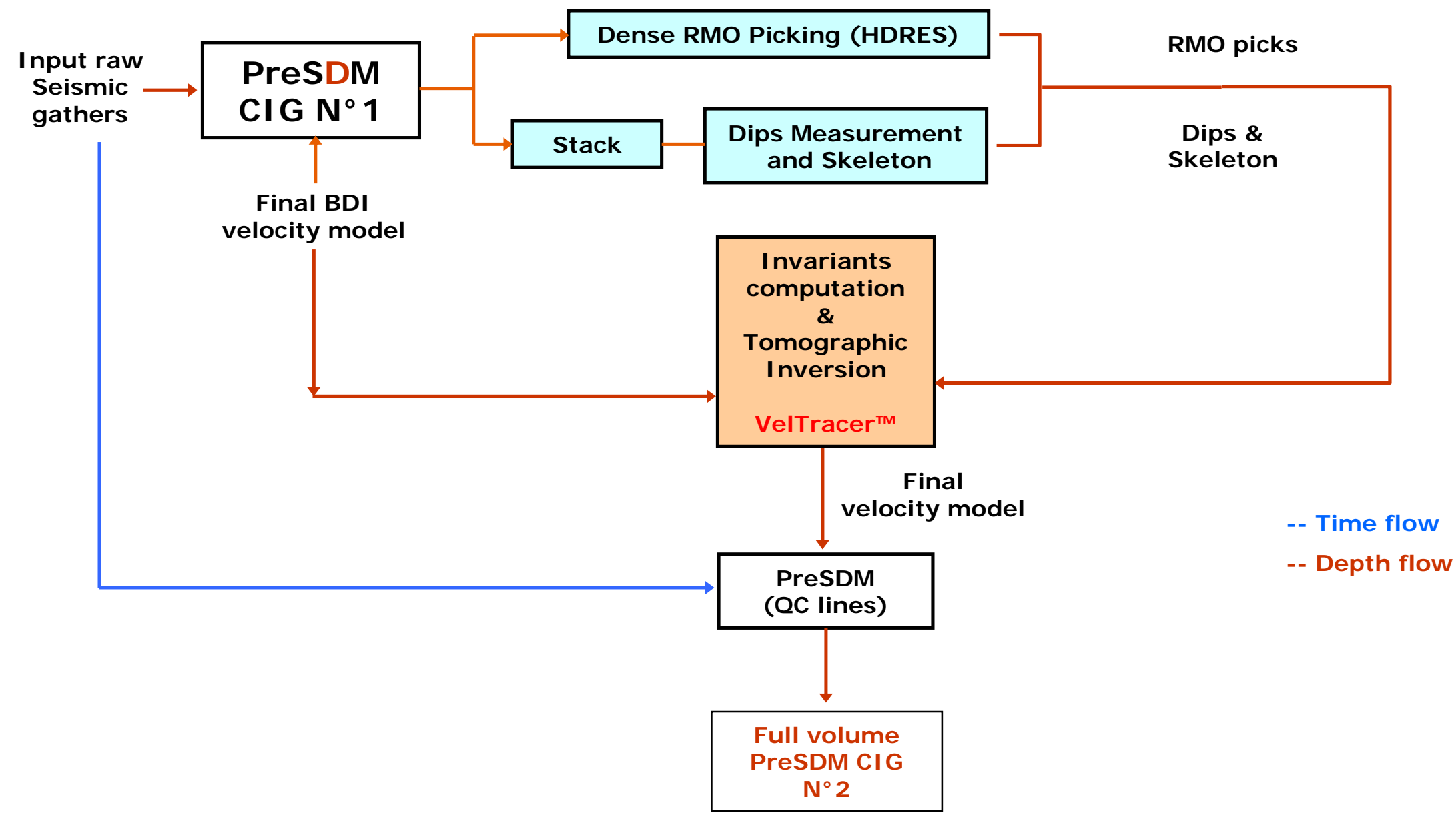

Figure 4-7b flow chart for the 'pure depth' route (Tomographic inversion, step 2) 
Figure 4-8 shows a few PSTM CIG superimposed with RMO picks and figure 4-9a shows the PSTM stack section overlaid with computed dip respectively.

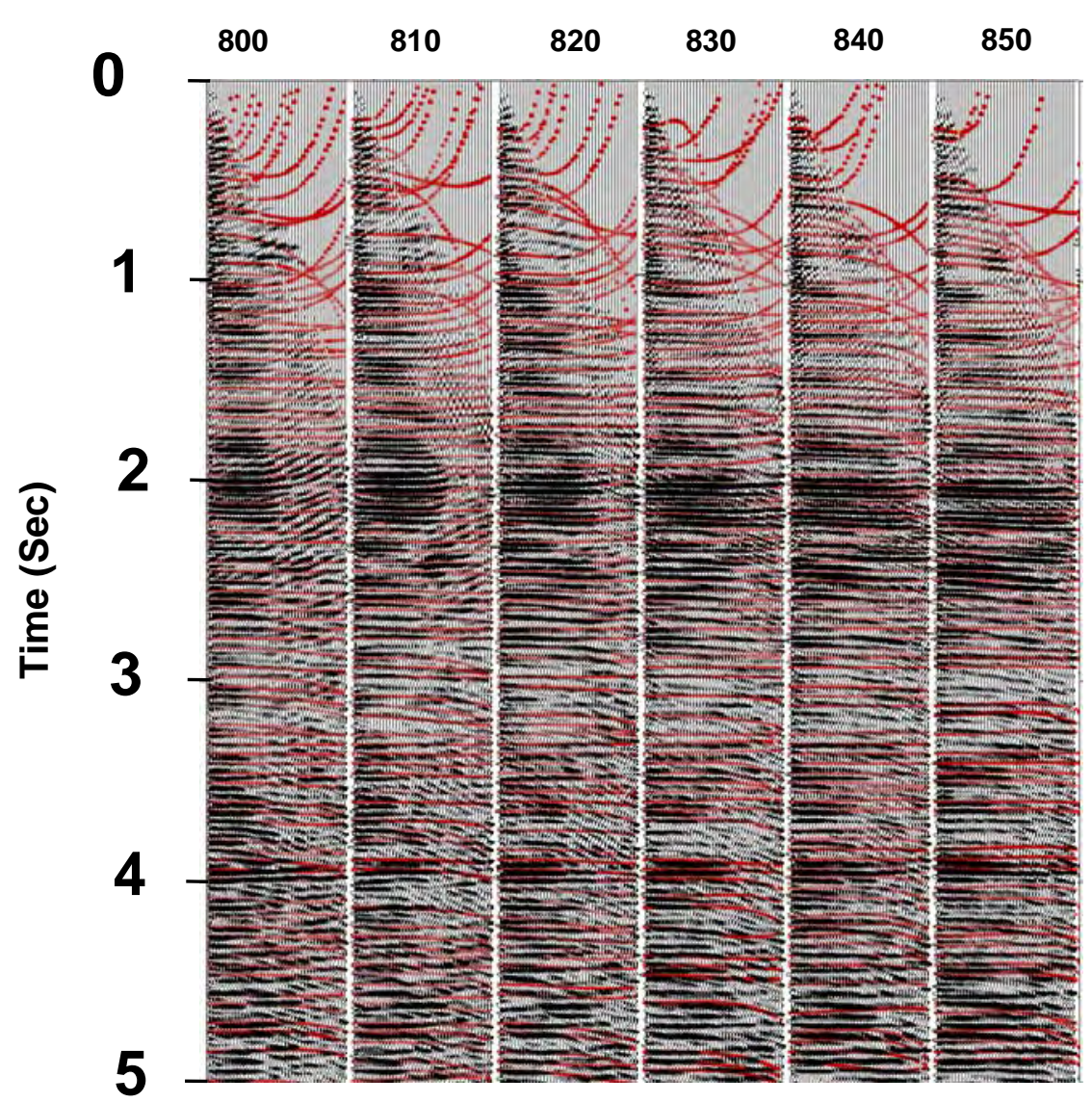

Figure4-8 RMO picks superimposed to the corresponding PSTM CIGs, numbers at top are the CDP numbers. 


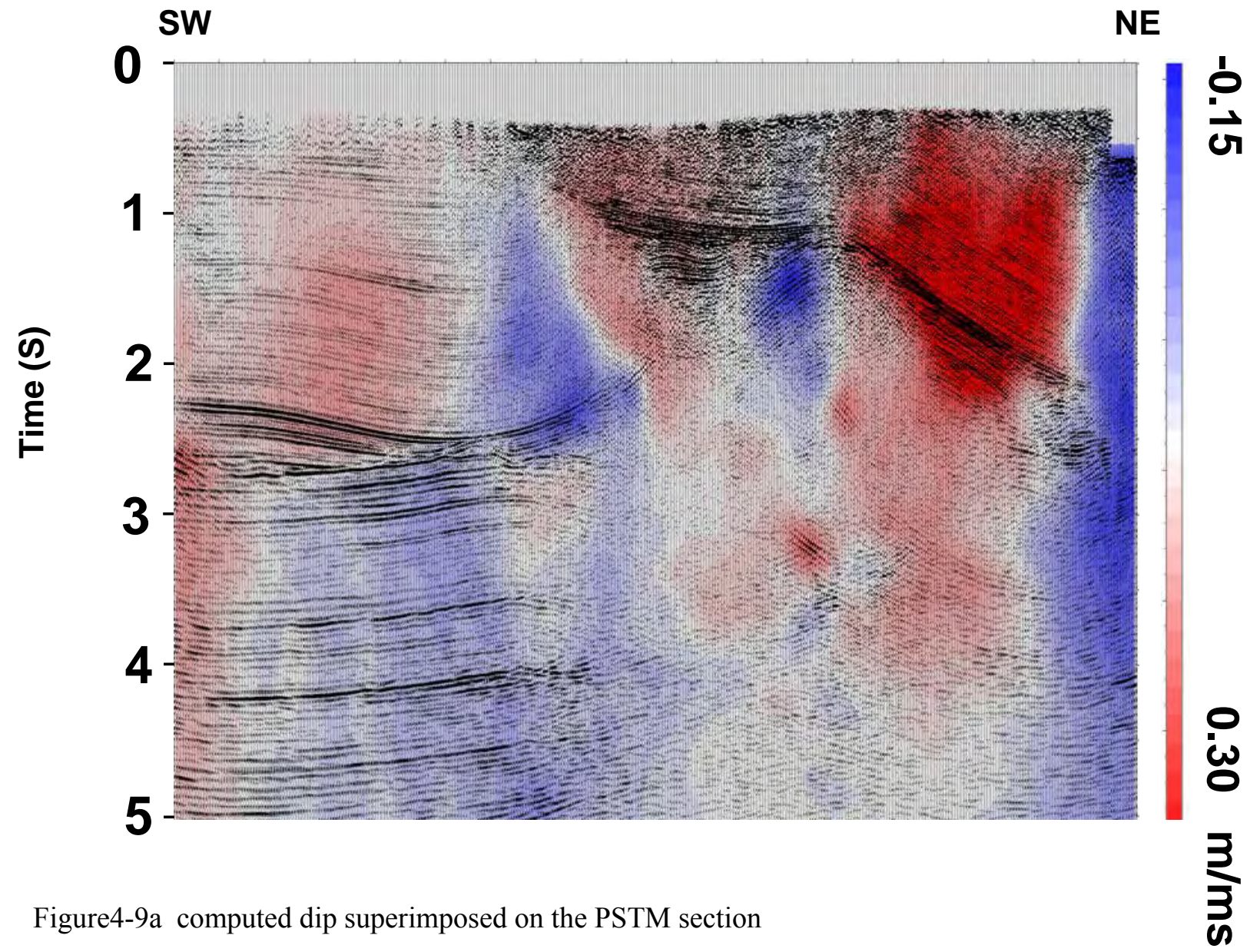

After Beyond Dix and a first run of PSDM, we obtained CIG in depth that should be flat if convergence is achieved. Figure $4-9 \mathrm{~b}$ shows some PSDM CIG with RMOs picks superimposed that are correctly flattened for the selected CIG. These are located in the SW part of the section where the $\mathrm{S} / \mathrm{N}$ ratio is quite good without significant dip. On the contrary, where the dip attribute was difficult to estimate with the automated picking, of course the match is not as good (SW flank of the anticline)!

This is why a second run of tomographic update was launched following a 'pure depth' route as described in Figure 4-7b above. The starting point here are the depth CIG from the Beyond Dix flow as opposed to the time CIG used in the first step. This makes sense as we benefit from better focused data (easier volumetric picking) and already partly updated model.

The final PSDM results are illustrated in Figure 4-9b (CIG and RMO picks) and Figure 4-9c (section superimposed to the final depth velocity model over the Aghajari area). 
We also converted PSDM images back to time to illustrate improved focusing and structural description compared with PSTM images. Figure 4-10a illustrates final pre-stack depth section converted to time to be compared with final pre-stack time result (Figure 4-10b).

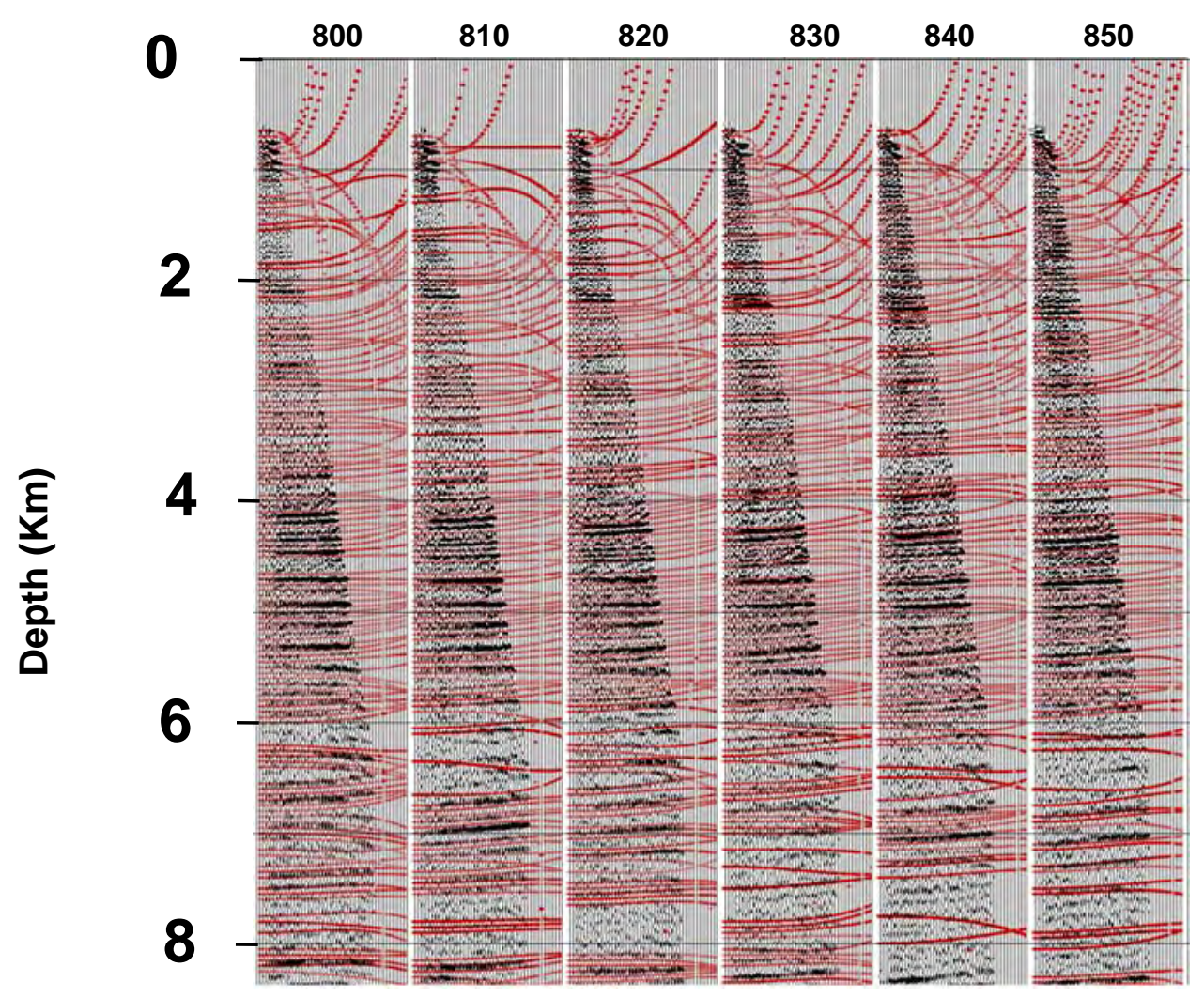

Figure 4-9b RMO picks superimposed to the corresponding PSDM CIGs after the 'pure depth' route 


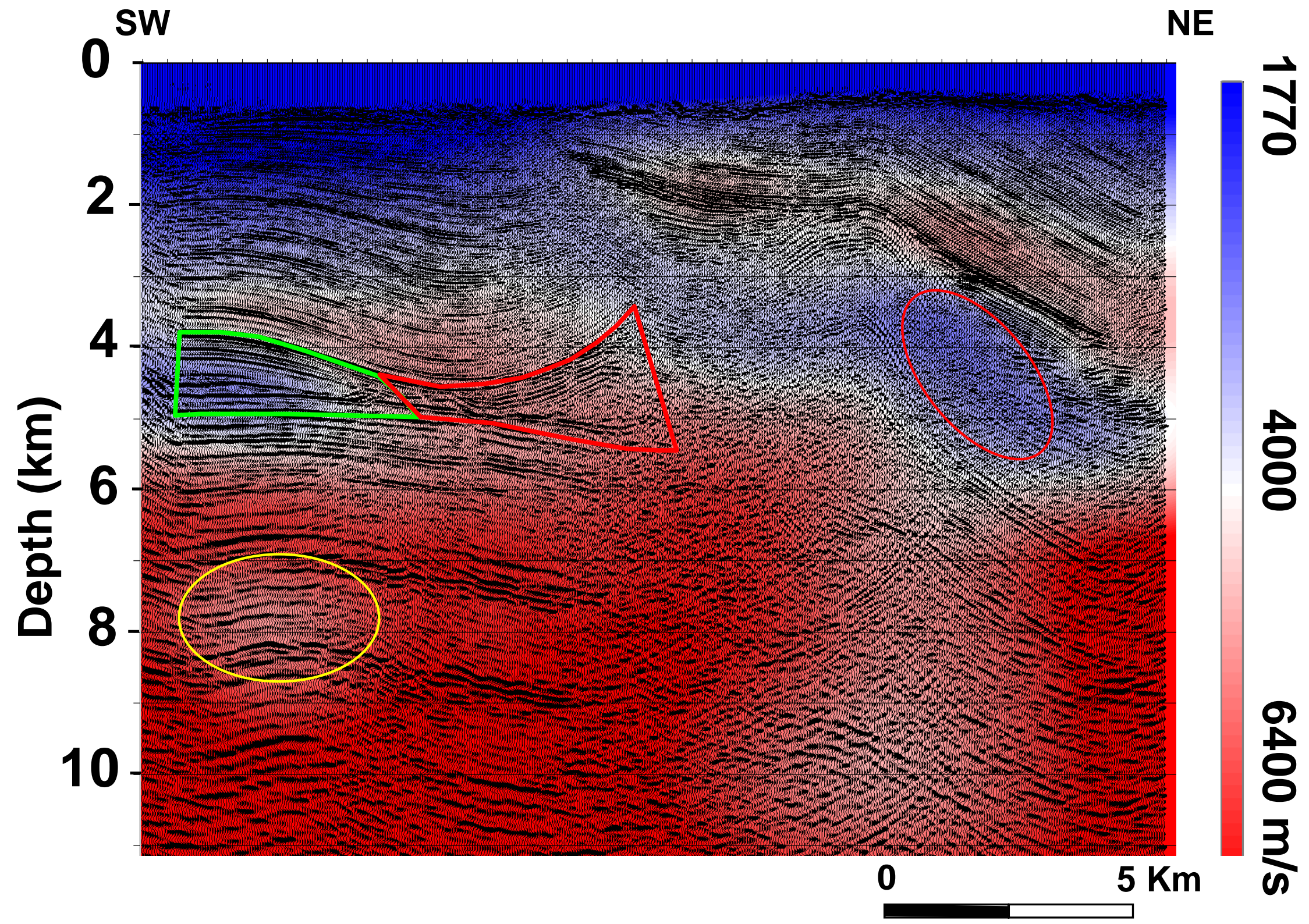

Figure 4-9c Final depth migrated section overlain by final velocity model, red box and ellipse show inaccurate velocity while green one is in better agreement with geology and well data(compare with figure 4-15a), Yellow ellipse shows inaccurate velocity due to edge effect 


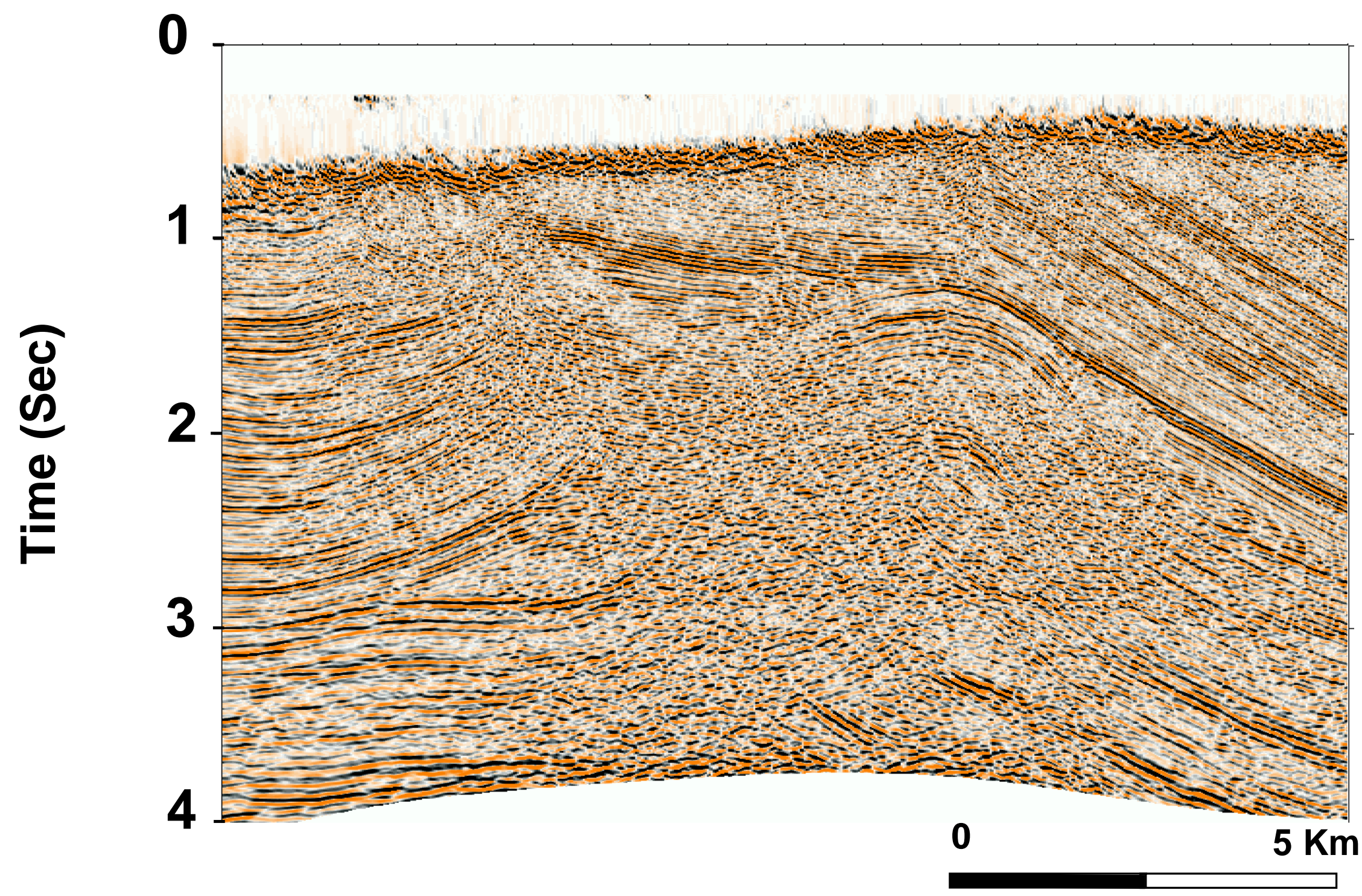

Figure 4-10a Final PSDM section (Inline1525) converted to time to be compared with the final PSTM result in figure 4-10b 


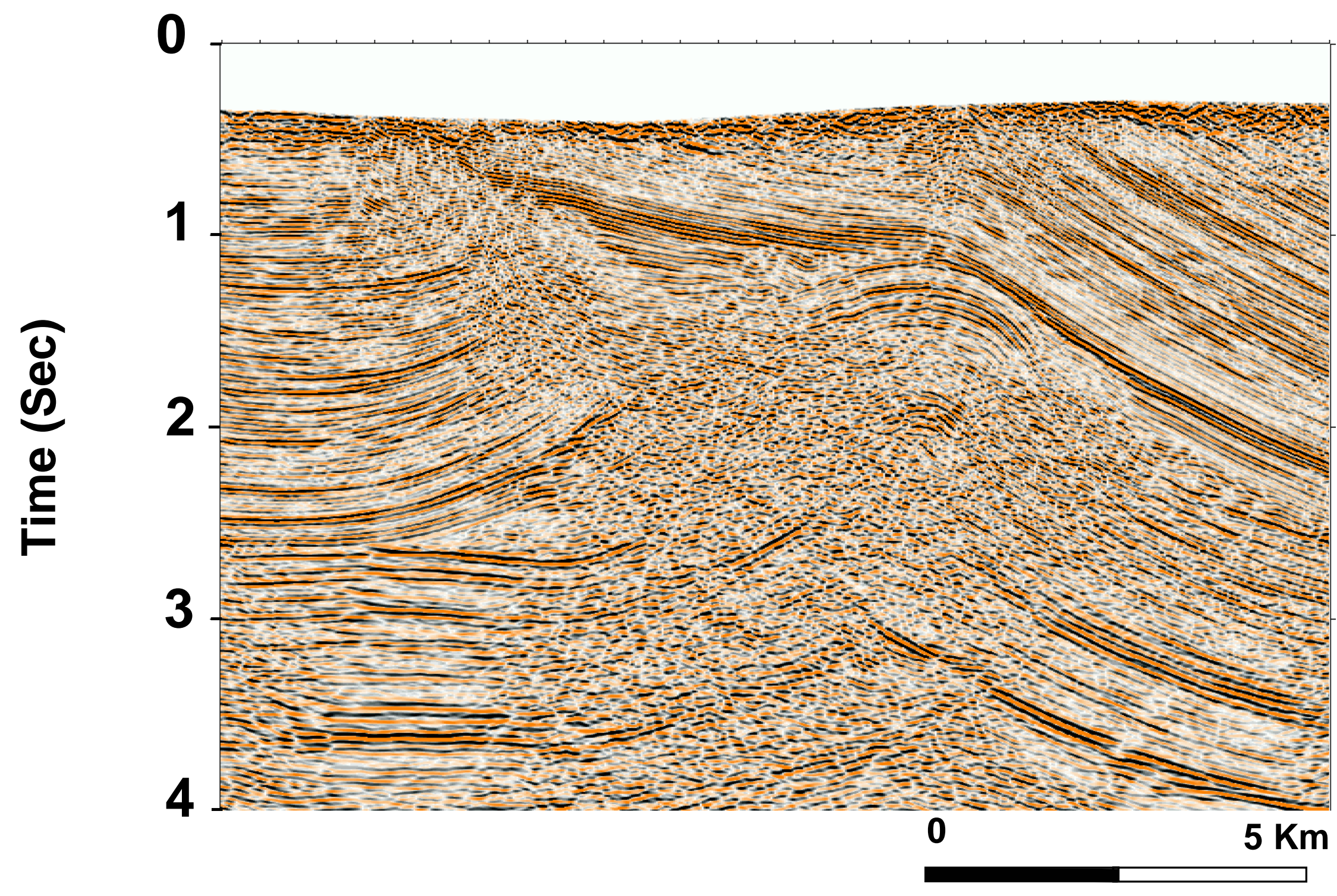

Figure 4-10b Final PSTM result (Inline1525) 


\section{4-4 Analysis of depth results}

The velocity used for PSDM migration was 100 percent of stacking velocity. The top Cap rock reflector and the base of the Asmari reservoir were time picked as well as few more horizons on the final depth cube in order to investigate whether the final cube fits with well data (Figure 4-12a and b). Imaging is better on the top part of the anticline and is in agreement with geological markers derived from well data. The shift between well depths (Gs-6) and PSDM result does not exceed 5\% which indicates that the velocity extracted from seismic velocity analysis is quite accurate above the Asmari. However, this shift on the top part could be due either to the anisotropy or to some uncertainty in the replacement velocity or floating datum (FDP) management. This is possible if the elevation used in the PSDM does not accurately reflect the behavior of the time FDP. Figure 4-11a illustrates the elevation of sources and receivers on the in-line 1760 superimposed by FDP used in the PSTM flow. From CDP 950 to the left, source -receiver elevations and FDP are not consistent. Horizontal scale is CDP number for both while the vertical scale shows the elevations in meter overlain by FDP values in ms. Figure 4-11b illustrates the rms velocity field with FDP in ms at the top. The comparison between two images, illustrates that the inconsistency between FDP and elevations on the left part of the section (from CDP950 to the left) is due to the low velocity at shallow depth which was managed correctly in both PSTM and PSDM routines. A major thrust fault (figure 4-13) outcrops around CDP 1000 which is explains the low velocity zone in the left part of the section where shallow sediments are less compacted and have low velocity.

Static corrections from the pre-processing (BGP) used a replacement velocity of $3000 \mathrm{~m} / \mathrm{s}$ and a seismic reference datum (SRD) of $600 \mathrm{~m}$ above Mean Sea Level (MSL). Nevertheless the data provided as input to both the PreSTM and the PreSDM flows, starts at the FDP time; i.e. the low frequency component of the statics was not applied (the so called 'regional static' between FDP and SRD). In time the migration is carried out from the FDP and the 'regional static' is applied afterwards to bring the migrated result to the actual SRD.

In depth the velocity model used for migration is referenced to the SRD. But travel-times (tt) are computed from the elevation surface; dummy tt is seen between SRD and the time surface elevation, and meaningful tt below. This computation is accounted for by the depth migration and the depth image is output from the SRD. Therefore the static corrections are correctly handled throughout both processing flows. 
However, there is about 5\% difference between the depths of geological markers and the corresponding seismic horizons in the overburden on the flanks. This can be explained by anisotropy in the shaly and layered part of the overburden, above the Gachsaran (Gs-6) horizon since the depth of this level is $5 \%$ deeper at the wells. The medium is expected to be transversely isotropic with an axis of symmetry normal to local bedding. The NMO velocity (which is also the migration velocity) differs from polar velocity by the coefficient $\delta$ (Thomsen, 1986) which is usually positive, which is coherent with the apparent deepening of the PreSDM migration that we also observe in the 3C VSP (chapter 2).

There is abnormal thickening (due to folding) of the Gachsaran layers (below the Gs-6 horizon) with a complex internal repartition of anhydrite and salt, resulting in variable velocities inside the formation ( see the yellow layers on the figure 4-15a). This thickening was already observed in well markers (Figure 4-12a). Steep dips above 40-45 can not be followed on the seismic depth image (Figure 4-12a), and consequently no fault pattern can be observed. Comparison between Figure 4-13 (time image) and figure 4-14 (depth image) illustrates that the PreSDM imaging above Asmari reservoir is better than the PreSTM imaging but there is no significant improvement for deep reflectors including the deep reservoir image.

This mitigated result might be explained as follows:

- Computed dip (facets picking) in the flanks, especially on the north east one, is not sufficiently accurate (figure 4-9a). Dips are computed based on a 'plane wave destructor technique' using a semblance criterion in both in-line and $\mathrm{x}$-line directions. Due to weak reflection energy in the PSTM cube in the north-east flank the computed dip is not in good agreement with geological dip from well information. In addition, noticeable multiple reflections with apparent dips much smaller than primaries may have been picked in the faulted area in the south western flank of the Aghajari anticline.

- The interval Velocity below the Asmari reservoir does not correspond to velocity derived from sonic log, check shot and VSP velocity information (Figure 4-9b); it is significantly too small $(4000$ to $4500 \mathrm{~m} / \mathrm{s}$ instead of 5500 to $6000 \mathrm{~m} / \mathrm{s})$.

In this dataset, using solely surface seismic data to obtain the velocity is not always satisfactory because the reflection energy in/below the south western flank of Aghajari anticline is very weak. Dip computation based on PSTM cube is also questionable due to weak reflection energy in the flanks of the structure. Although PSDM is the best solution for improving imaging in the complex structural zone, due to very weak reflected energy it is difficult to derive the optimal depth velocity model. Consequently, imaging the Aghajari 
structure is still challenging and we need other approaches to tentatively overcome these problems. 


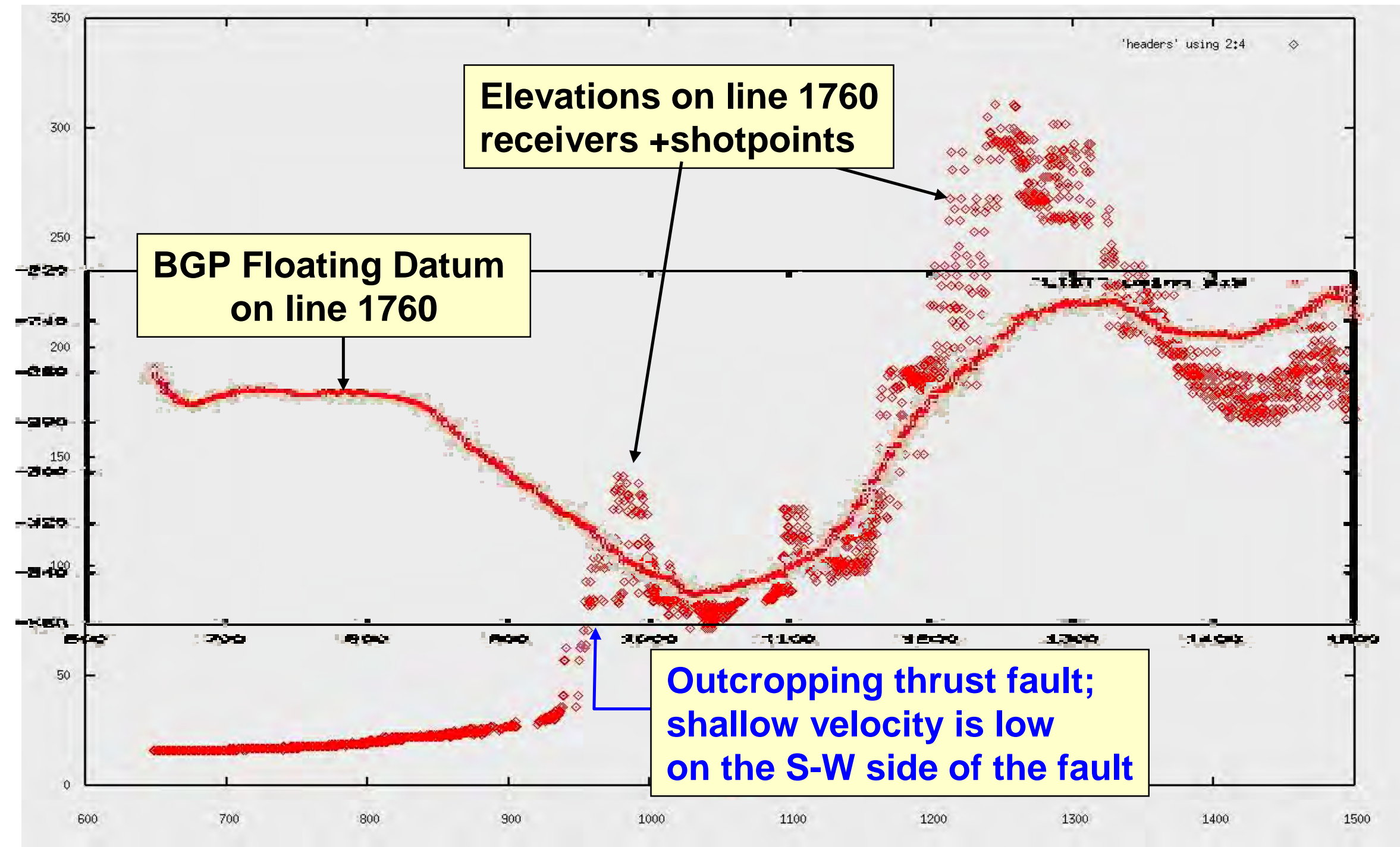

Figure 4-11a Both top and bottom of figure 4-11a are superimposed and adjusted to show the inconsistency in left part of the section 


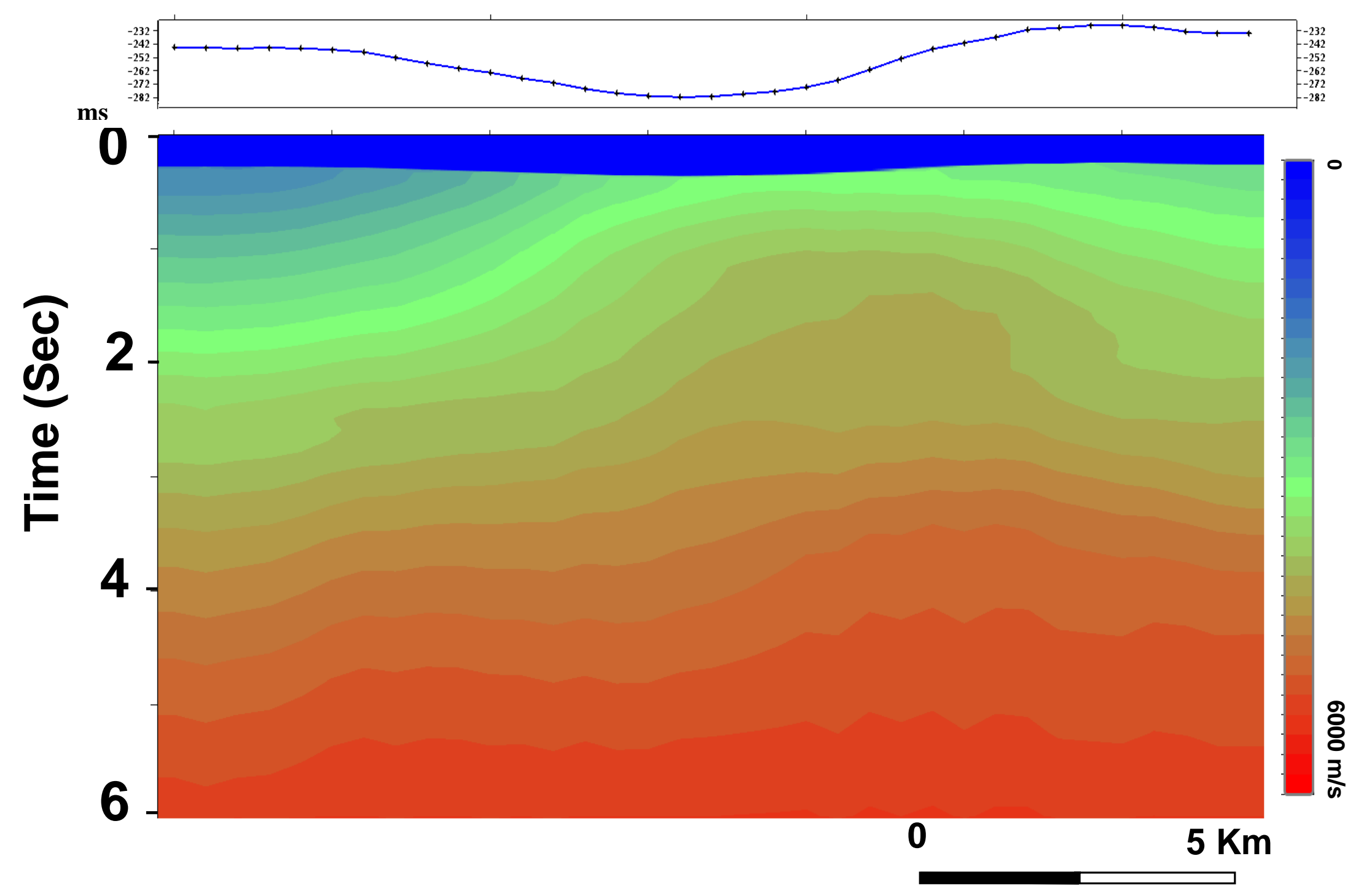

Figure 4-11b source-receiver elevations from in-line1760 (top) and floating datum plane (bottom). Horizontal scales represent cdp numbers. Vertical scale at top represents the elevation in meter and below represents values of FDP in ms. 


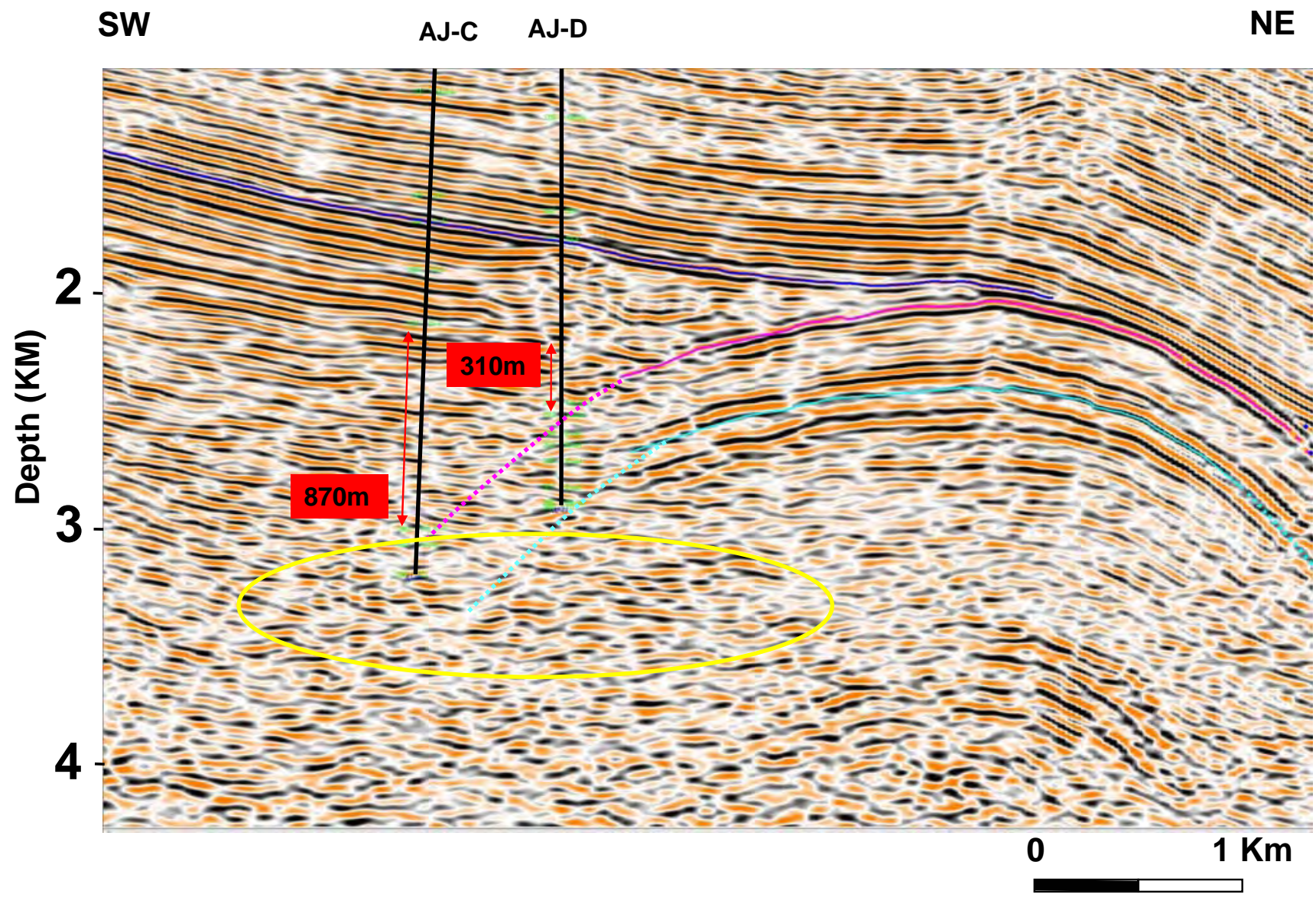

Figure 4-12a Depth section from PSDM cube; geological markers were projected on the final depth cube. Blue horizon is Gachsaran layer (GS-6). Purple and Cyan horizons are top cap rock and base Asmari respectively. Abnormal thickening in Gachsaran layer in well AJ-C is observed respectively to AJ-D. The yellow ellipse shows the residuals of multiple reflections which were probably picked by the automated velocity picking process. 


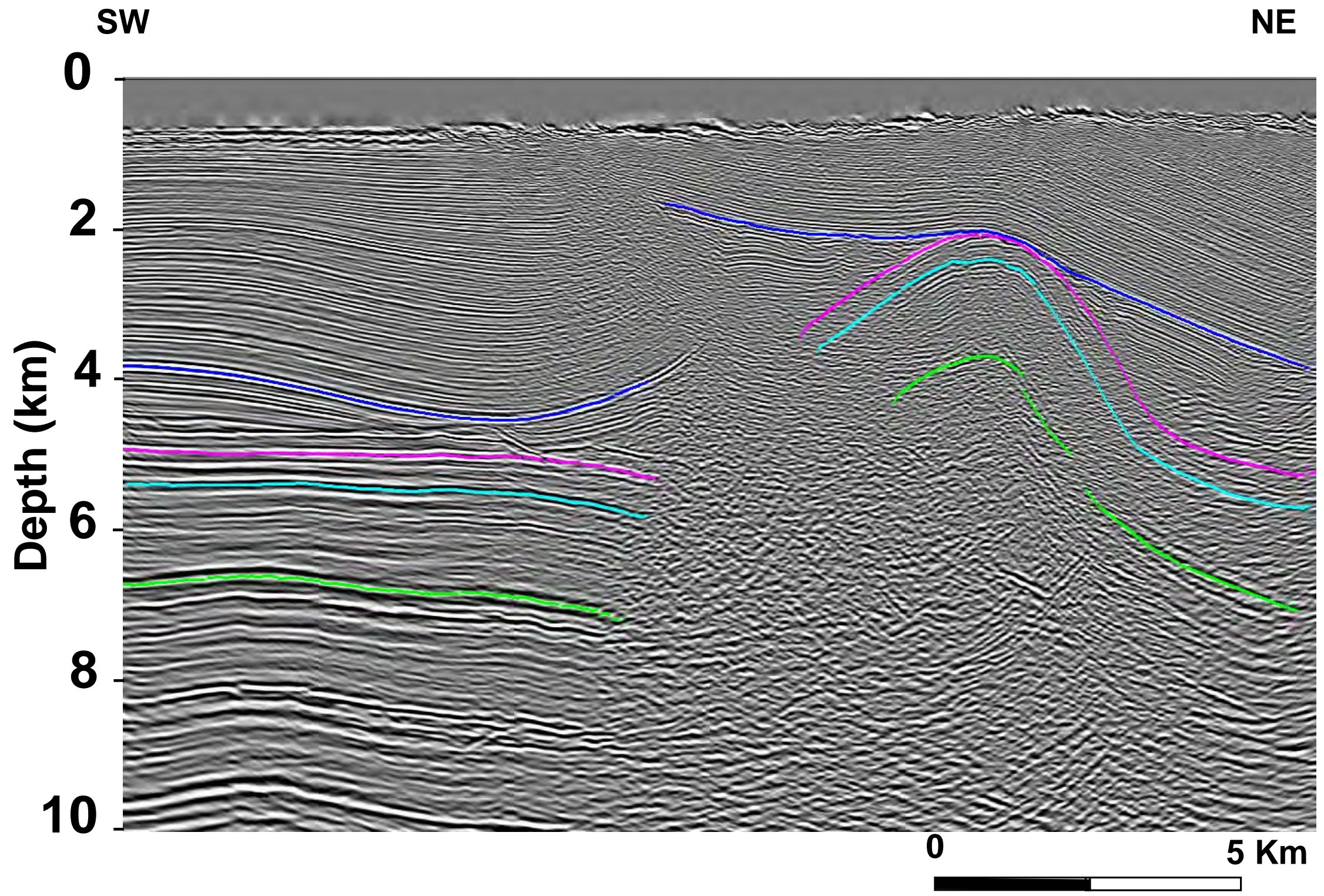

Figure 4-12b main geological horizons. Purple and cyan are top cap rock and base of Asmari reservoir respectively, blue is Gachsaran 6 and green one is a deep shale (Kazhdomi) 


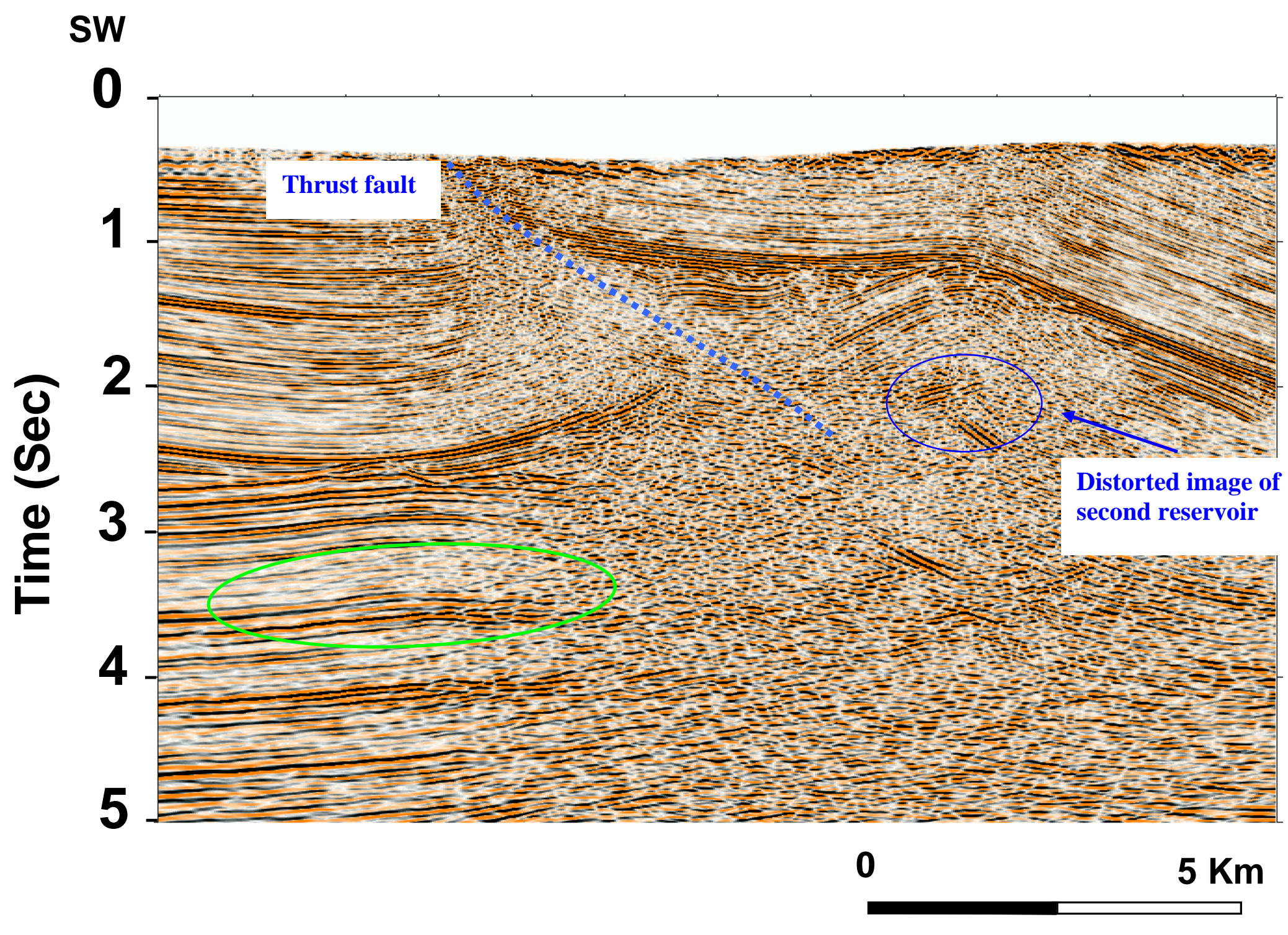

Figure 4-13 PSTM section Inline 1760; an anticline structure is clearly visible at Asmari level around 2.8s twt (green ellipse) 


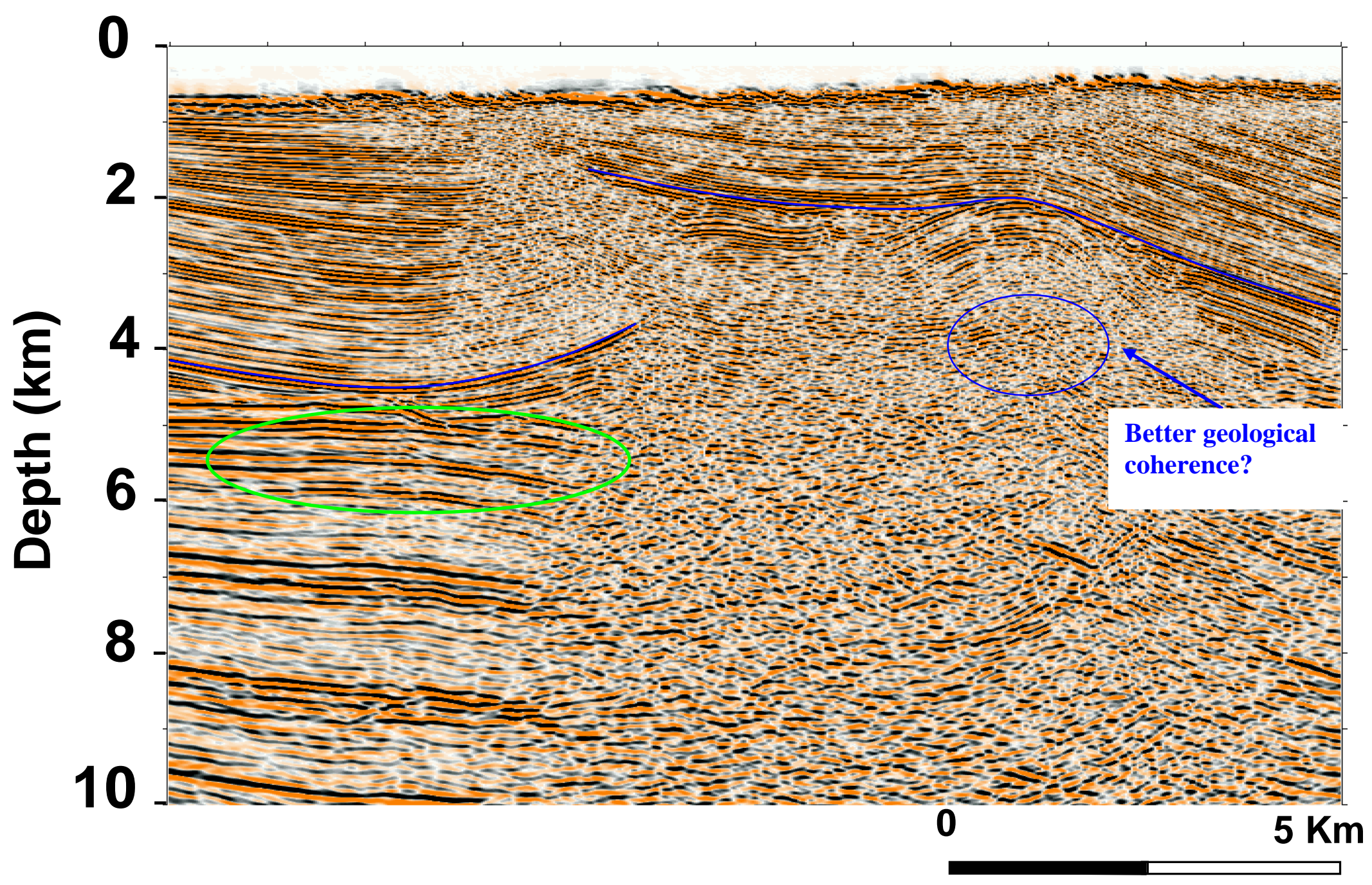

Figure 4-14 PSDM section Inline 1760. Illumination in overburden above Gs-6 (blue line) is better than time section in figure 4-13. However, due to inaccurate velocity variation on the overburdens, the structure on the green ellipse is transformed into a monocline with uncertain closure. 


\section{4-5 Possible prospect on the southwestern side of the Aghajari field}

One of the objectives of this $3 \mathrm{D}$ is to study and to map the Aghajari structures but also to define new prospects. The PSTM results illustrate a gentle anticline in the southwest of the Aghajari structure at Asmari level which is around 4500m depth below surface. In order to confirm the existence of this structure, velocity variation in overburden was examined carefully. Considering that the velocity of pre tectonic sediments (Gasharan and older) is acquired at the time of the maximum burial, the uplift due to the compression does not modify this velocity. In PSTM cube the velocity in the overburden is laterally consistent even if it is slightly different from well velocity, so we can conclude that the PSTM image gives a fidel representation of the structure. In addition, the rms velocity derived from well data ( DT, Check shot and VSP) over the main Aghajari structure were compared with the velocity derived from surface seismic. There is good agreement between rms and well velocities above Asmari level over the south western prospect although Gachsaran velocity (in yellow on figure 4-15a) is slightly higher than true velocity extracted from the wells.

We carefully checked the Static corrections in the western part of Aghajari survey because the velocity field shows strong variation from beginning of the section till CDP 1000 (IL1760) just above the south western prospect.

A simplified 2D velocity model was built from well data and geological interpretation over the main Agahjari field in order to set reliable velocities in the whole overburden down to Gs6 (Fig 4-15a).

Simple 1D depth vertical conversion by stretch and squeeze was done using this velocity model on Inline 1760 resulting in figure 4-15b. It confirms the anticline prospect structure at Asmari level at about $4600 \mathrm{~m}$ with about $150 \mathrm{~m}$ vertical closure on the west of the section. Figure4-16 shows the depth converted model overlain on the PSDM section. It illustrates that the inaccurate overburden velocities used for PSDM conversion (see figure 4-9b red domain area) modified the underlying structures.

The interval velocity in Gachsaran on the right side of the prospect structure is higher than the well velocity but the structure still exists. Green ellipses on the figure 4-13 and 4-14 show this prospect on the final PSTM and PTDM sections respectively, to be compared. Due to inaccurate velocity at Gachsaran level this structure almost disappeared on final PSDM result (figure 4-16). 
Therefore, this gentle western anticline might be considered as a low risk prospect for new exploration at the region as long as the 3D survey indicates an appropriate closure.

In addition, there are a few faults affecting the Asmari reservoir on this prospect which illustrates that the Asmari reservoir at this level is probably fractured, at least in the fault vicinity. 


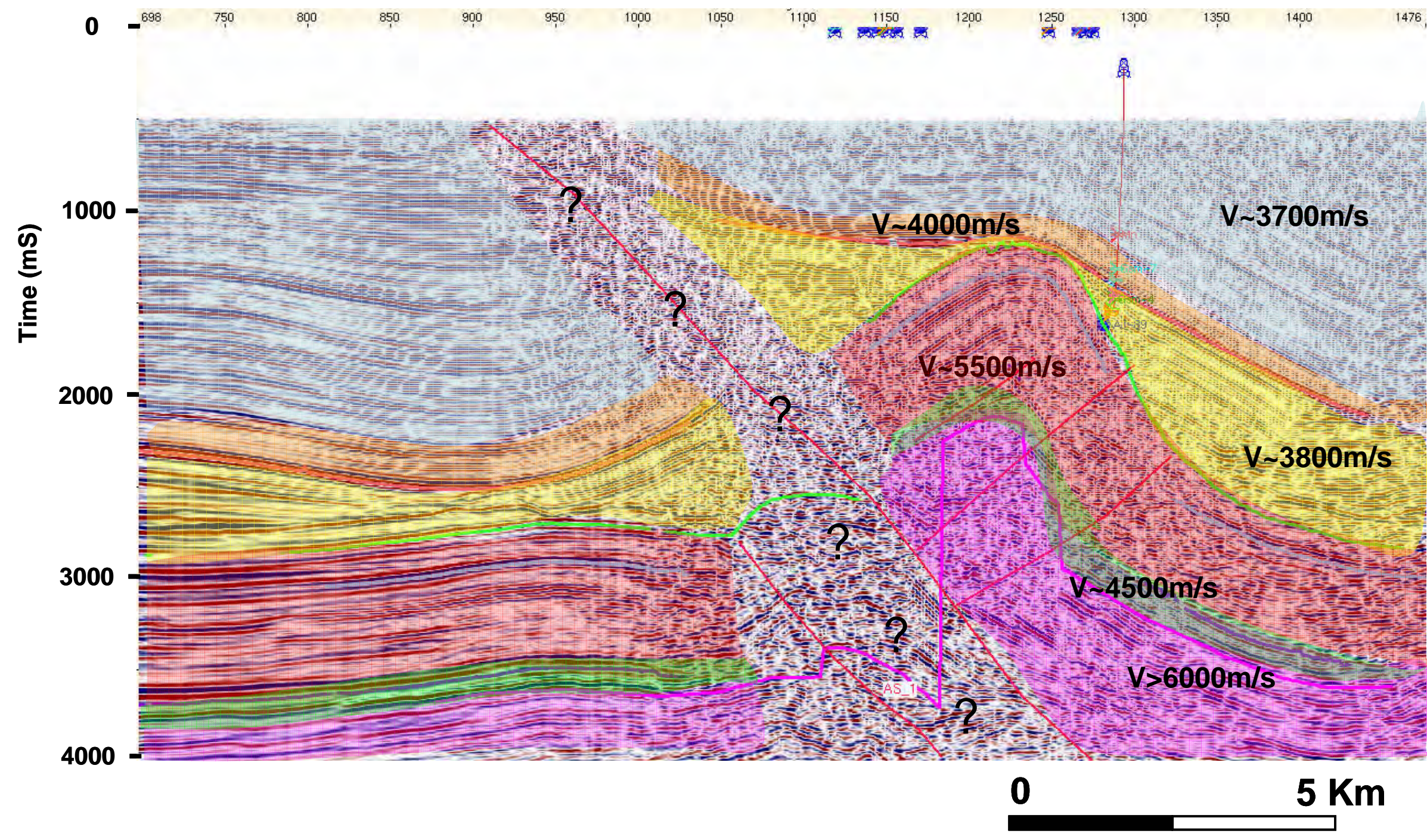

Figure 4-15a 2D velocity model based on geological interpretation (colors represent different lithology, velocities derived from well data) 


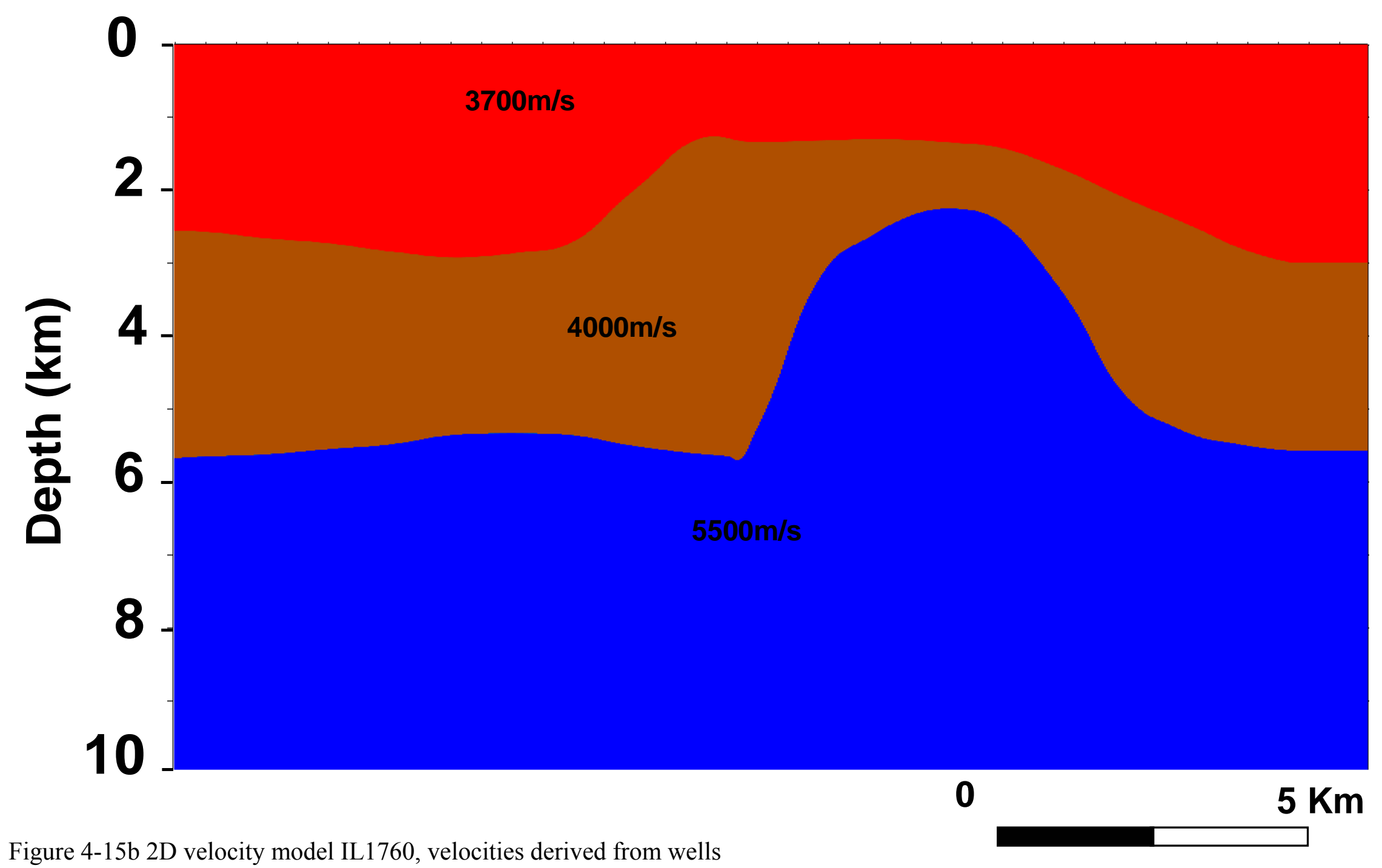




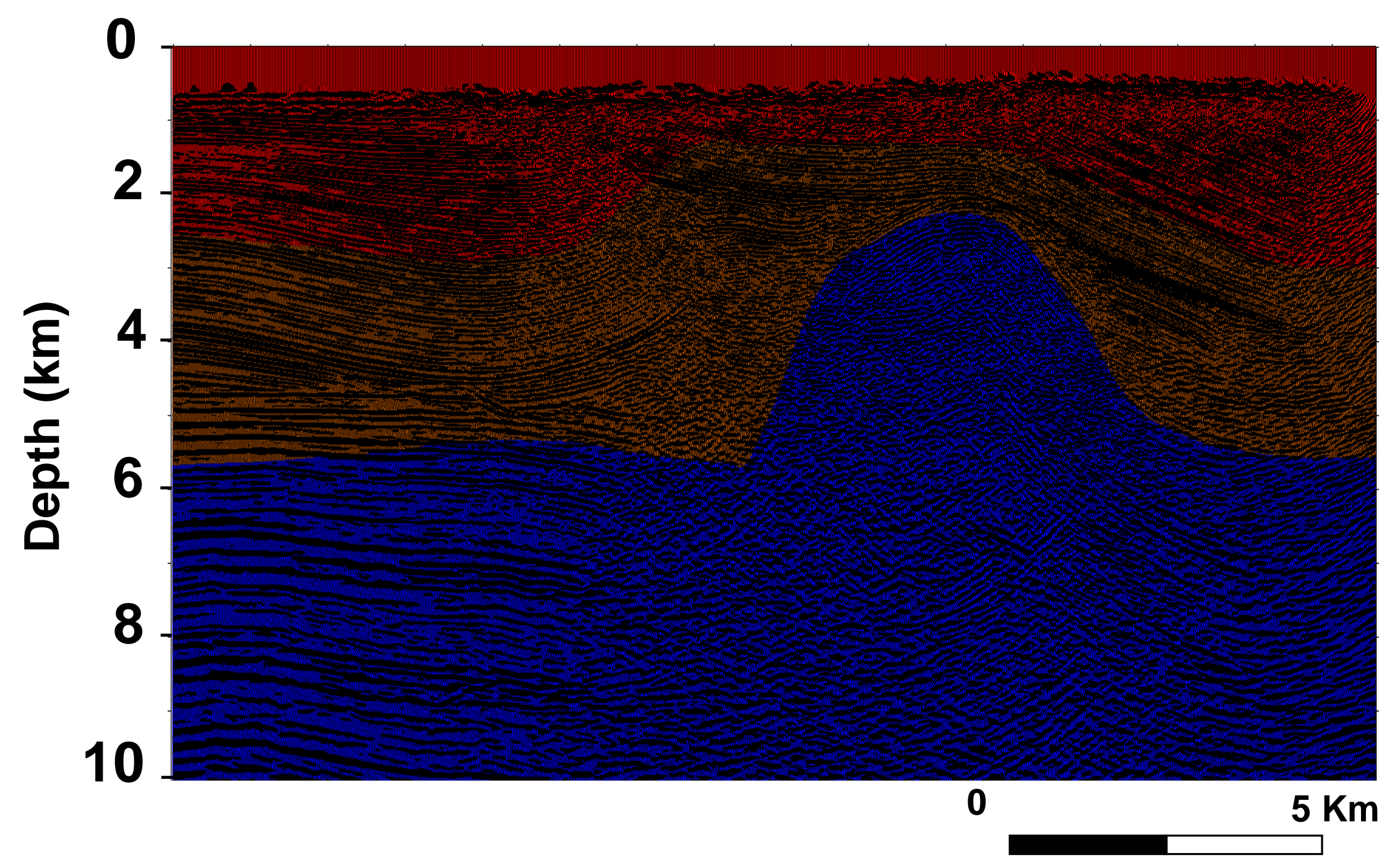

Figure 4-16 2D depth velocity model overlain on final PSDM section colour code same as figure 4-15 


\section{4-6 Conclusion}

The final PSDM volume exhibits a better image on the top of Aghajari structure and in general on the whole field above Asmari reservoir where the velocity field was correctly refined (figure 4-13 and 14). The structure limbs of the Aghajari anticline have no better image in comparison with the PSTM cube which is possibly due to inaccurate picking of kinematic attributes that enters into the tomography; very weak reflection energy is present on the common image gathers $(\mathrm{CIG})$ in the shadow zone faces which limits the tomographic technique in updating the velocity field. Furthermore, some multiples generated by the strong Gs-6 reflector and surface were picked at the reservoir level mainly in the south west flank. Dip computation is also inaccurate at northeastern flank.

Underneath the Aghajari anticline, the reflectors around 4200-4500m (around 2s twt) from surface corresponds to a deep reservoir are not clear, so top reservoir in this level is difficult to be mapped in both PSTM and PSDM final results.

In conclusion, the PSTM and PSDM results do not show drastic improvements of the image of the southern flank in Agahjari. We need a new approach to solve this drawback, which is the object of the next chapter.

Beam migration based algorithms could also be used as another alternative to achieve better imaging in the southern flank where ray paths are possibly distorted due to vertical velocity inversion and lateral velocity variations. 



\section{Chapter 5}

\section{Improvement of PSTM results by aperture optimization}




\section{5-1 Introduction}

We have seen in chapter 4 that "Beyond Dix" workflow did not succeed to solve velocity inside the Asmari reservoir mainly because PSTM failed to image steep flanks of the structure and because of the absence of good reflectors. Therefore, it is important to investigate the reasons of this lack of illumination and look for possible improvement of high dips reconstruction.

In practice, 5 factors contribute to the difficulty to image steep dips:

- The size of the survey that limits the maximum aperture of the operator and therefore the maximum dip that can be reconstructed; in our case the survey design was large enough to include $45^{\circ}$ illumination and beyond, at least in the dip direction.

- The sampling of input trace (for instance the space sampling of the inline and cross lines for constant offset migration): if the sampling is large or irregular, the dipping part of the operator will not interfere destructively (as they should do) during the migration stack, leading to "operator induced aliasing noise". To avoid such artefact, Kirchhoff migration algorithms include 'operator antialias protection” that filters the frequency content and eventually cancels steep dips; The antialias filter is a key point to be investigated in this study.

- The sampling of output image activates another antialias filter (now in the image domain) and eliminate steep events if the image bin is too large; this effect is not considered to be important in our study

- The level of incoherent noise that is rather important in the present dataset, requires also to severely filter steep part of migration operators, and this may attenuate as well the true steep reflections.

- Any coherent noise or events that do not correspond to primary PP arrivals as turning wave, multiples... are migrated as well as primary and can mask weak steep reflectors.

The PSTM results were obtained by TIKIM (CGGVeritas migration tool) using very large values of parameters controlling the apertures and standard antialias filter of the operator. This filter which is a low pass filter ensures that the local linear move-out of 
the operator is not aliased. For instance a planar reflector with $45^{\circ}$ dip, below an overburden of velocity $V=3000 \mathrm{~m} / \mathrm{s}$ will emerge at surface with local slowness $2 \frac{\sin \theta}{V}=0.47$ in $\mathrm{ms} / \mathrm{m}$. With a bin size $=25 \mathrm{~m}$, the time delay is $12 \mathrm{~ms}$ between adjacent bins and the Nyquist of the antialias filter is $42 \mathrm{~Hz}$, which means that frequencies are attenuated by the anti-alias filter between 30 and $40 \mathrm{~Hz}$.

The problems raise by the imaging of steep dips, in particular in noisy environment have been recently studied and some solutions have been proposed (Liu et al. 2002). One approach is based on the exploitation of local kinematic attributes picked on non migrated data. For instance, CRS (Common Reflection Surface) attributes that are already computed for the purpose of 3D interpolation of 4 or 5 dimensional data (Hoecht and Ricarte, 2006) have also been used (Spinner, 2006) to limit the migration aperture in the PSTM domain. In this approach, a local slope (versus the migration variables that are in principles midpoint coordinates), is estimated at each sample and is used during the migration to limit the spread of current contribution along the isochrones. Similarly, multi focusing techniques (Berkovitch et al, 2008) scan simultaneously local dip and curvature of reflectors and effective velocity. Another approach is the beam migration technique (Hill, 2001): the data is decomposed into "beams" by local slant stacks. Each beam component (for all slopes and all beam positions) is individually migrated using Gaussian beam theory, (Gaussian beam ray theory can propagate wave field generated by extended sources while standard rays are for source point). Unfortunately, routines based on these algorithms were not available yet for our study.

The other approach was proposed by Tabti (2005) in the aperture domain, before application of the diffraction stack: this domain corresponds to a given position of the image and a given offset range, in which the horizontal coordinate is the distance between midpoint and migrated location. The method consists in applying a low band pass filter in the horizontal direction that enhances specular locations where the event is locally horizontal. Then an automatic picking selects the maximum values and the stack is applied only in a narrow corridor around this specular point. Kabbej et al (2005) shows an application of such method.

IFP developed a prototype 3D PSTM algorithm that allows creating images for different selections in the migration aperture domain. This prototype tool has no 
antialias protection and the aperture parameters are very easy to understand and control.

In this chapter, we present the particularities of this prototype tool, then the different tests performed to improve the image of the steep flanks using the possibility to select aperture azimuthal sectors. Encouraged by the obtained results, we also present an attempt to further post process “aperture gathers” delivered by the migration program in order to search for the best single planar reflector, as was proposed by Tabti (2005), but with a different approach.

All results shown in this chapter were obtained with a subset data limited to 100 inline mid point gathers (strip of 2500m width) because of our limited computing resources. The velocity field was the same as the velocity field applied in the previous chapter (defined for the CGGVeritas PSTM).

\section{5-2 IFP PSTM migration algorithm description}

This prototype program has been written by Pascal Froidevaux (IFP) and allows to take into account floating datum (surface elevation) and to deliver various types of gathers.

Let us consider the seismic trace $A\left(X_{S}, Y_{S}, X_{R}, Y_{R}, t\right)$ where $\left(X_{S}, Y_{S}, X_{R}, Y_{R}, t\right)$ are the horizontal coordinates of source and receiver and $t$ is two way time of the reflected event on the current trace. This time is zero at surface on floating datum: $Z_{F D}(X, Y)$ referred to an absolute reference horizontal level. The source position $S\left(X_{S}, Y_{S}\right)$ and receiver position $R\left(X_{R}, Y_{R}\right)$ are located on the floating datum with $d Z_{R}=Z_{F D}\left(X_{R}, Y_{R}\right)$ and $d Z_{S}=Z_{F D}\left(X_{S}, Y_{S}\right)$ (Figure 5-1). For each trace, we define the midpoint position $\mathrm{M}$ and the local depth $d Z_{M}$ on the floating datum. 


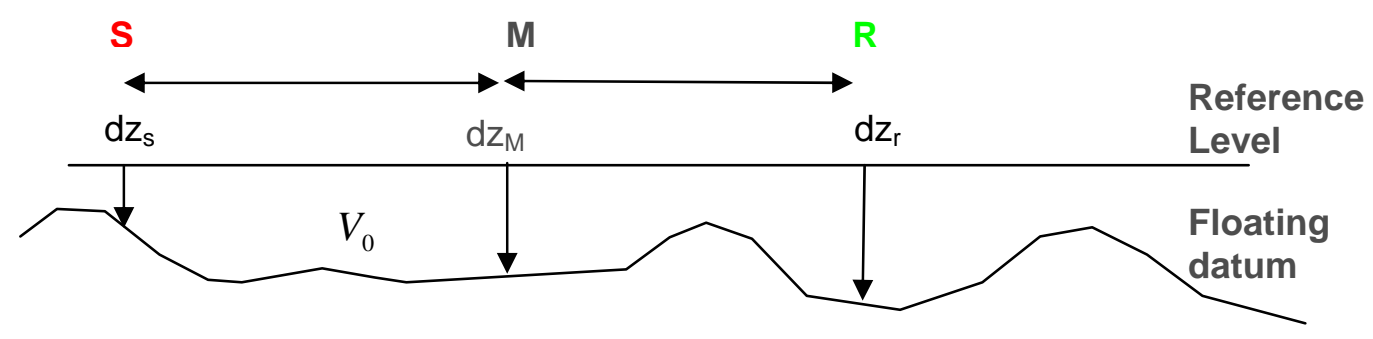

Figure 5-1 Reference and floating datum, preparing a smooth ground surface, in the TWT

In the PSTM migrated domain, the origin of the migrated time must corresponds to the reference level, initially defined in depth domain.

The solution proposed by Pascal Froidevaux is to link the migrated time $\tau$ to a (pseudo) depth $\mathrm{Z}$ by mean of the average migration velocity equal to the correction velocity $V_{0}$. In practice the reference is chosen close to the FDP.

From the figure 5-3, for an image point I with horizontal coordinates $\left(X_{C}, Y_{C}\right)$ the migrated time $\tau$ i corresponds to a pseudo depth Zi such that:

$Z_{i}=\frac{\tau_{i}}{2} V\left(X_{C}, Y_{C}, \tau_{i}\right)$

where $V\left(X_{C}, Y_{C}, \tau_{i}\right)$ is the migration velocity, estimated by migration velocity analysis, that is defined to the reference level. In the interval between reference level and FDP, the velocity is set to a constant value:

$V\left(X_{C}, Y_{C}, \tau_{i}\right)=V_{0}$ for $\tau_{i} \leq \frac{2 * Z_{F D}\left(X_{C}, Y_{C}\right)}{V_{0}}$

The migrated image is built as the sum of traces (eventually restricted to a given offset class) using the diffracted time $T\left(X_{C}, Y_{C}, \tau_{i}, X_{S}, Y_{S}, X_{R}, Y_{R}\right)$ computed for each trace by the double square root: 
$T_{i}^{\text {diff }}=\left(\frac{\sqrt{D_{S}^{2}+\left(Z_{i}-d Z_{S}\right)^{2}}}{V\left(X_{c}, Y_{c}, \tau_{i}\right)}+\frac{\sqrt{D_{R}^{2}+\left(Z_{i}-d Z_{R}\right)^{2}}}{V\left(X_{c}, Y_{c}, \tau_{i}\right)}\right.$

where $D_{S}=\sqrt{\left(X_{C}-X_{S}\right)^{2}+\left(Y_{C}-Y_{S}\right)^{2}} \quad$ is the horizontal distance S to C and $D_{R}=\sqrt{\left(X_{C}-X_{R}\right)^{2}+\left(Y_{C}-Y_{R}\right)^{2}} \quad$ is horizontal distance R to C;

In previous formula, the floating datum is accounted separately for source and receiver. A more commonly used formulation is to use the same datum for source and receiver, defined at midpoint position. This is the option used in this work in order to confirm with previous work done at CGGVERITAS.

When $\mathrm{C}$ is identical to the midpoint $\mathrm{M}$ (case of a flat reflector), equation (1) reduces to the classical Dix formula: $\quad T_{i}^{\text {diff }^{2}}=T_{0}^{2}+\frac{h^{2}}{V\left(X_{c}, Y_{c}, \tau_{i}\right)^{2}}$

where $\quad T_{0}^{2}=\left(\tau_{i}-2 \frac{d Z_{M}}{V\left(X_{c}, Y_{c}, \tau_{i}\right)}\right)^{2}$

$V$ is migration velocity and $h$ is the source to receiver offset.

\section{5-2-1 Aperture selection and "migration offset" gathers}

The aperture of the migration operator is limited by several factors:

\section{I- The maximum dip parameter "dipmax"}

This parameter is in fact the maximum aperture of a single (source or receiver) Green function. (Figures 5-2a and b). 


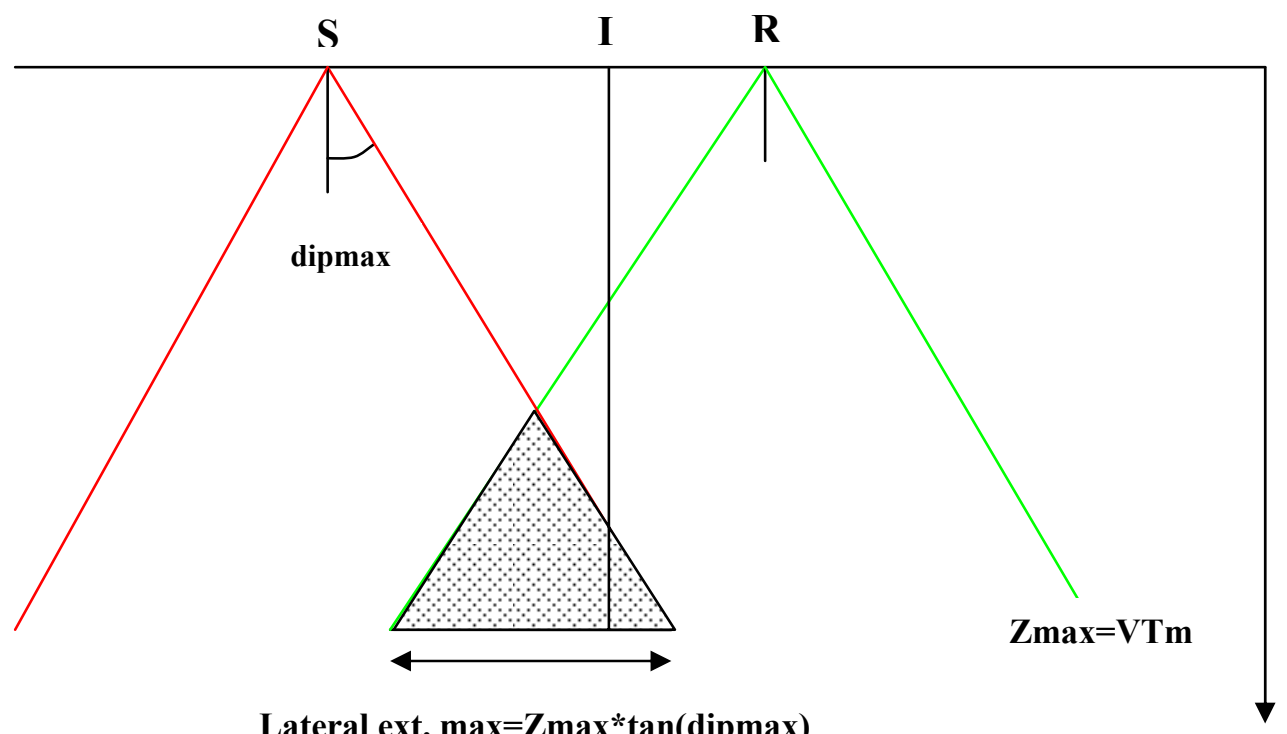

Lateral ext. $\max =\mathbf{Z m a x} * \tan (\operatorname{dipmax})$

Figure 5- 2a Vertical section showing the domain in which the migrated data is computed accordingly to the parameter "Dipmax"

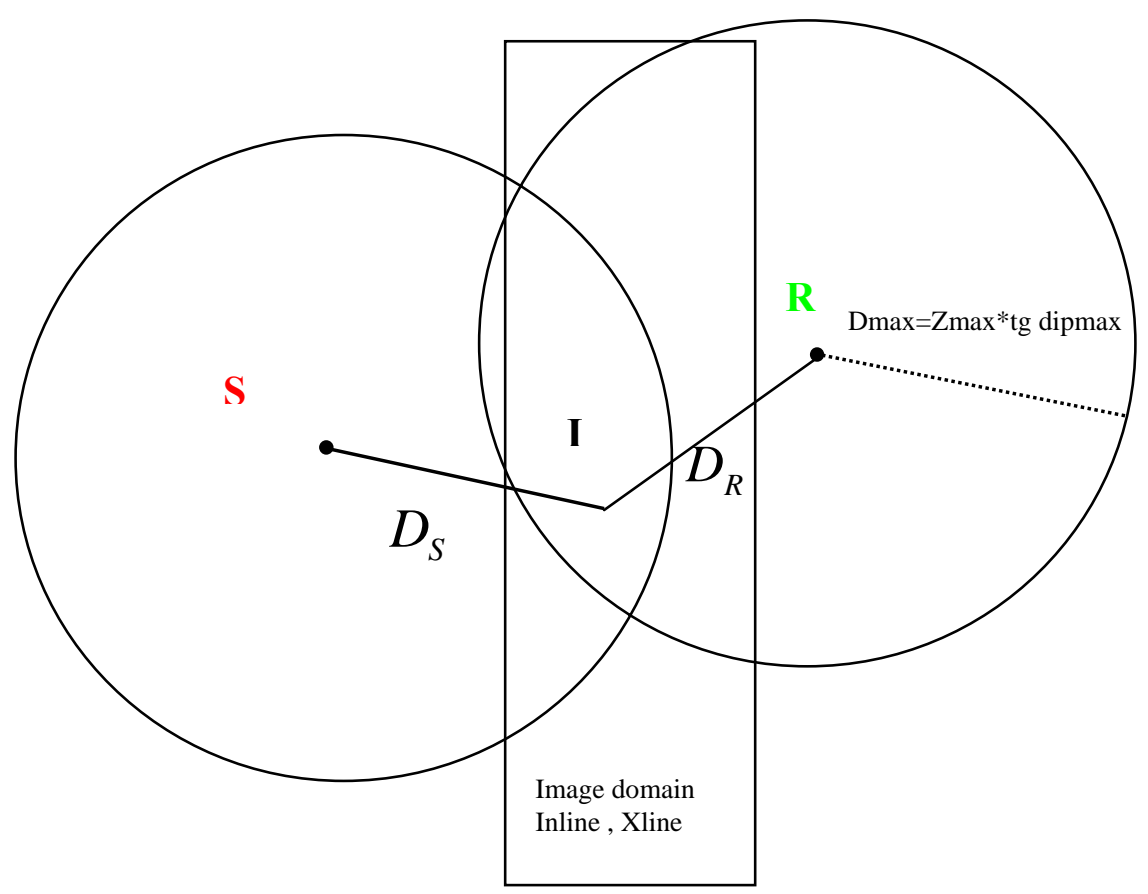

Figure 5-2b Horizontal section 
There is of course a relationship between "DIPMAX" and the maximum geological dip that can be imaged; they are identical for zero-offset. For a finite offset, the size of "DIPMAX” can be checked by looking at impulse response as shown in Figures 5.13. More important are the geological dips so that the bigger dip the longer aperture must be used. Note that large apertures produce noise so that a compromise between dip and migration offset should be made.

\section{II- Azimuth sector}

The image can be output for a selection of azimuths defined for the "apertures vector". It is the azimuth of the horizontal vector that links mid point $M$ to $C$ (the horizontal projection of image point), while the acquisition azimuth is the angle between the source to the receiver line with the North (Figure5-3). The vector M to C is called in this chapter "aperture vector" or "migration offset". Note that the aperture vector azimuth is equal to the azimuth of geological (apparent in PSTM) dip for zero offset and remain close to it for finite offset. 


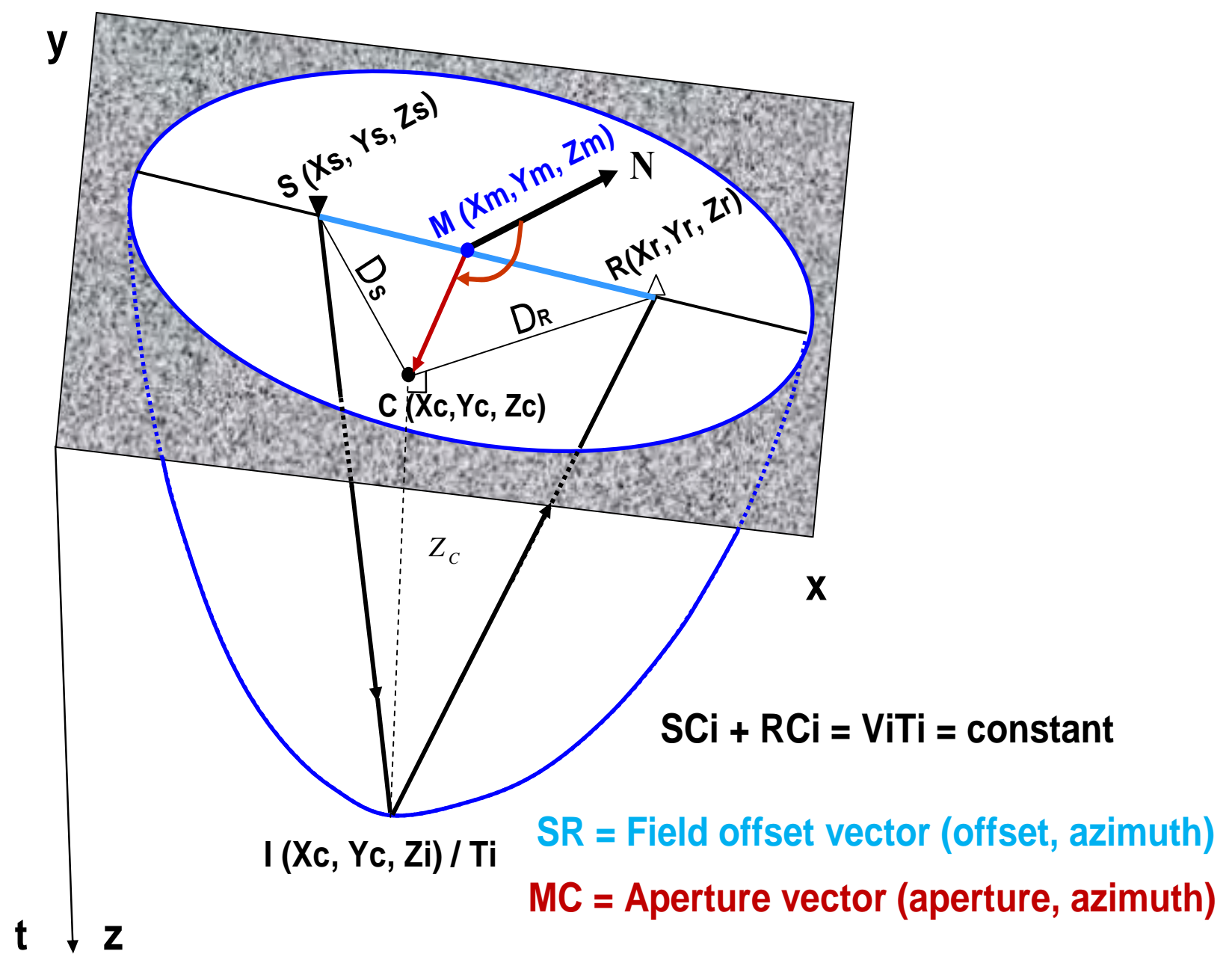

Figure 5-3 isochrone in 3D view and definition of the "migration offset vector MC "

In the IFP program, we can select various type of output gathers:

-Standard gathers versus acquisition offset, useful for velocity analysis; in which the traces have been stacked along a generally large aperture.

-Gathers versus different values of the "aperture vector", all obtained for a single range of acquisition offsets. 
"Aperture vector" can be selected either in polar coordinates (azimuthal sectors and length) or in Cartesian coordinates. The first part of our tests was made using the azimuthal sectors option: In the next section, we show the results of different tests made on the range of acquisition offset, (DIPMAX parameter) and the angle range of azimuthal sectors. Then we show comparisons of best results with previous standard TIKIM (PSTM by CGGV tool) results. In the last section we will show how Cartesian migration offset can be used to enhance the main reflectors.

\section{5-3 Tests of migration parameters}

\section{5-3-1 Azimuth sector selection}

Figure5-4a depicts a swath including 100 CDP lines (IL1750-1850) divided in 6 imaging azimuth sectors every $60^{\circ}$. We can output each sector separately or gather reciprocal sectors together as shown on Figure5-4b for the main dip azimuth direction (here $42^{\circ}$ ). We performed different tests on the width of azimuth sector from $180^{\circ}$, $90^{\circ}, 60^{\circ}$ to $45^{\circ}$ all centered around the main dip direction (Figure5-5). Azimuth sector $180^{\circ}$ means that the whole data contribute in the image while the $45^{\circ}$ represents one fourth azimuth sector of data used in imaging process. We decided to explore the content of $60^{\circ}$ sectors as we found it optimum. Figure5-6 shows the contribution of these different sectors every $60^{\circ}$. It illustrates that each sector illuminates a limited part of the structure and also which one has more signal while the others contain more noise and multiple arrivals. It is clear that the main dip sectors contains the information we are looking for (the steep flank of the anticline), normally masked by multiples and noise that are now visible on the residual sections. The counterpart is that we attenuate the horizontal reflectors (since the azimuthal partition cut by a factor 3 the polar area corresponding to horizontal reflectors). However, these reflectors are strong enough to still subsist . 


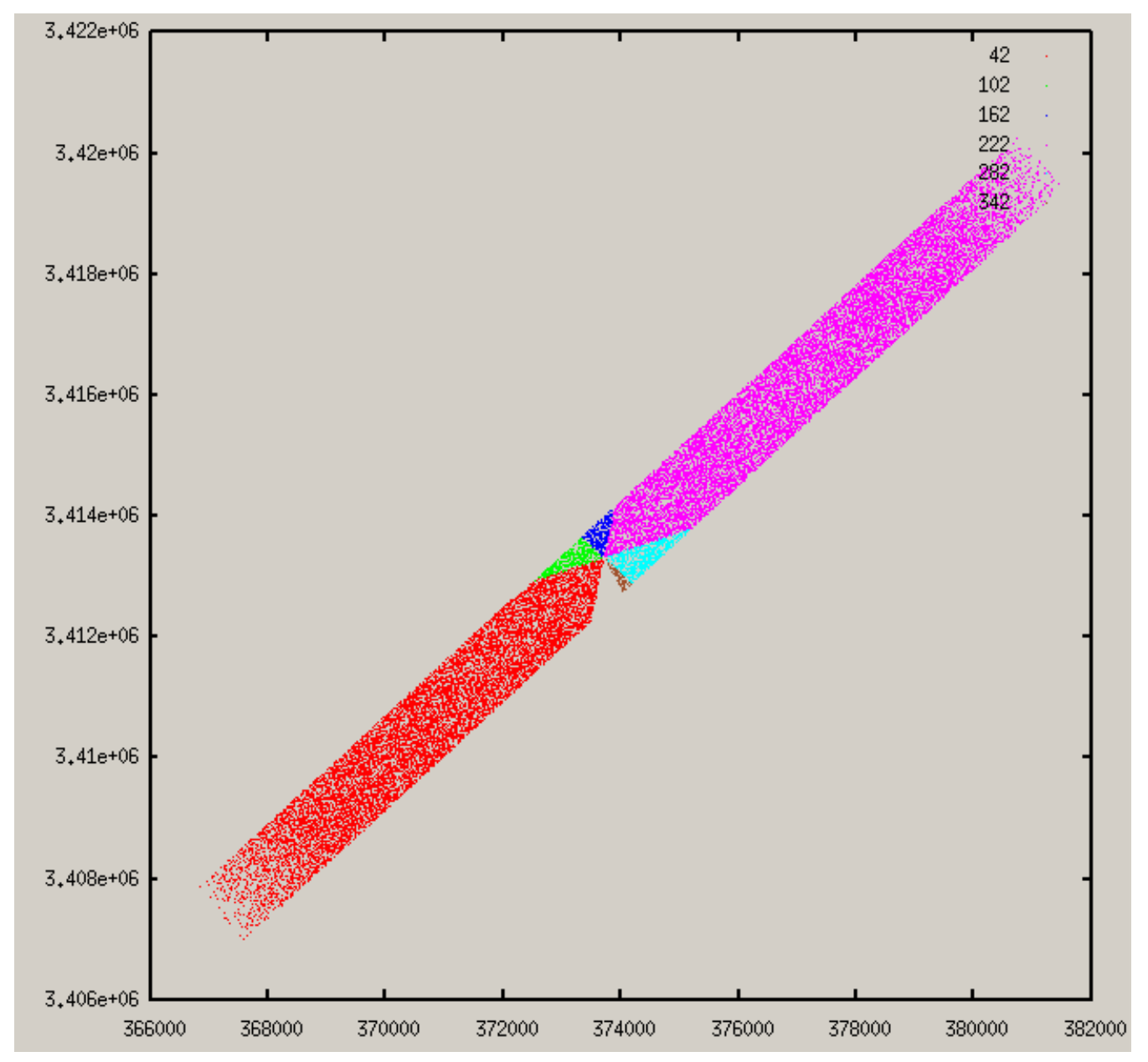

Figure5-4a A swath includes $100 \mathrm{CDP}$ lines used in this test. 6 sectors of $60^{\circ}$ each are identified (red+ purple points are points in two main sectors in dip direction). 


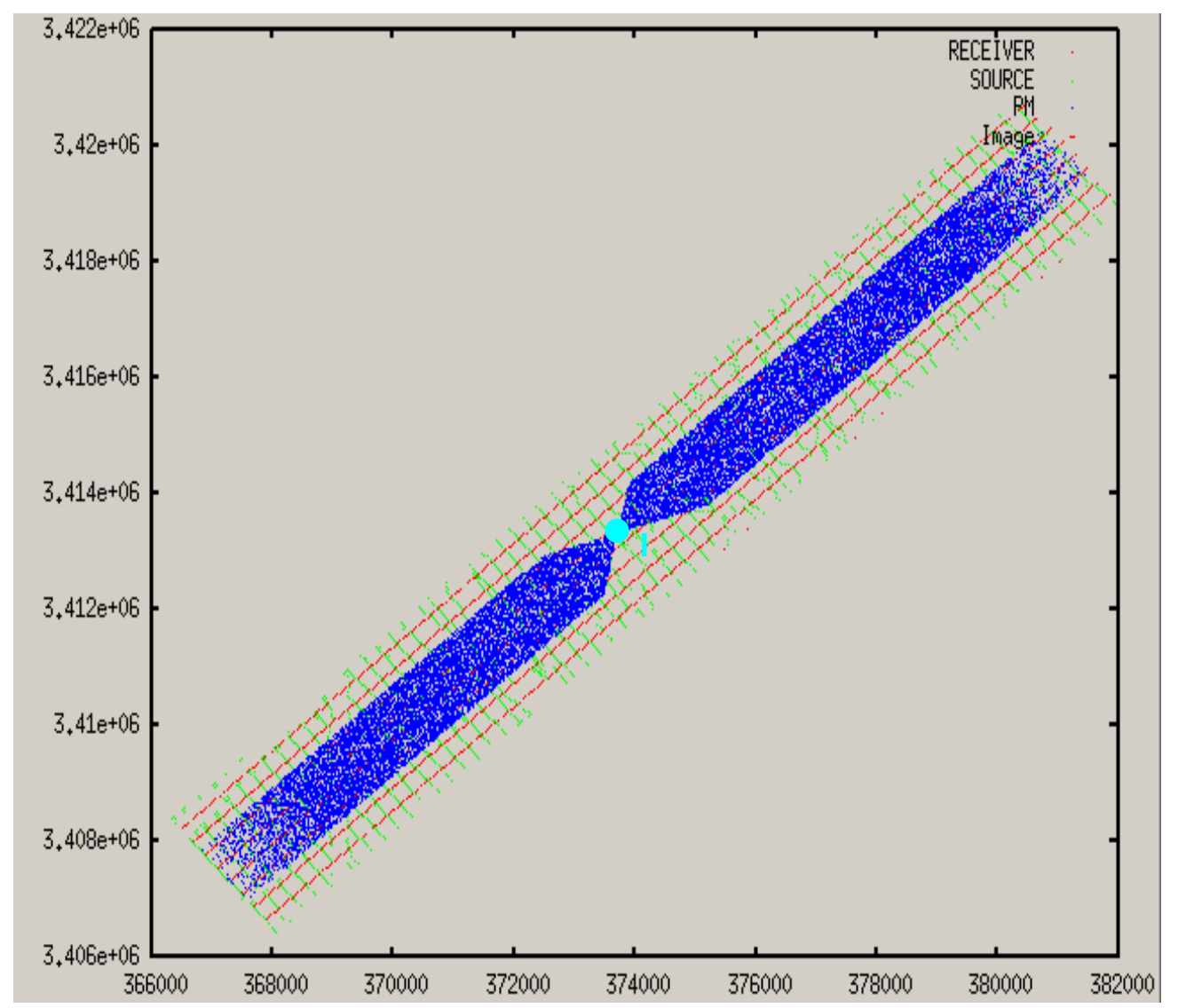

Figure5-4b red and green points represent receiver and source locations respectively. Blue points are mid-points (1\% of whole points) contributed in imaging of point I. 

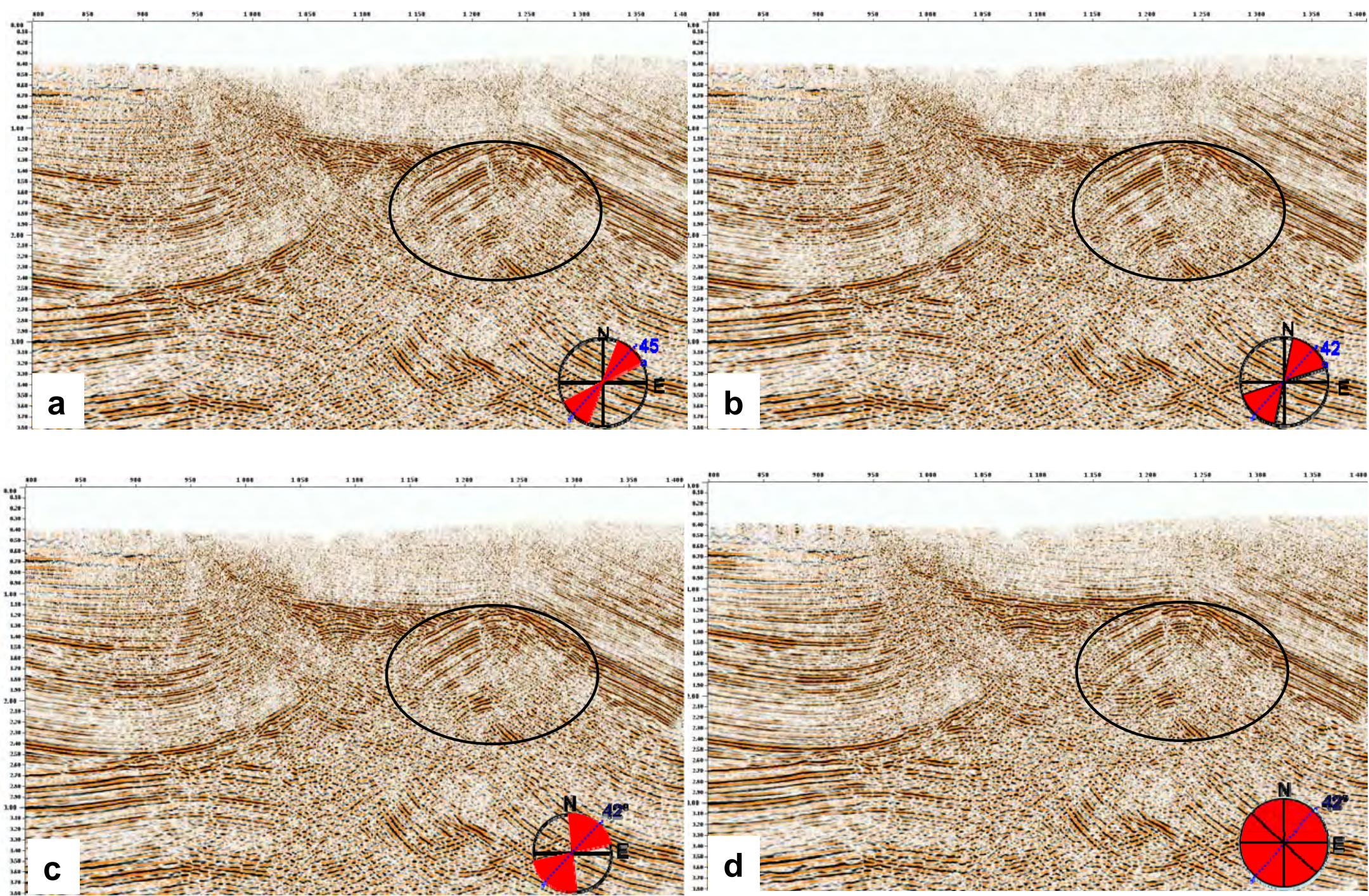

Figur5-5 Azimuth sector selection: a, b, c and d represent azimuth $45^{\circ}, 60^{\circ}, 90^{\circ}$ and $180^{\circ}$ respectively, black ellipses show imaging of the flanks 

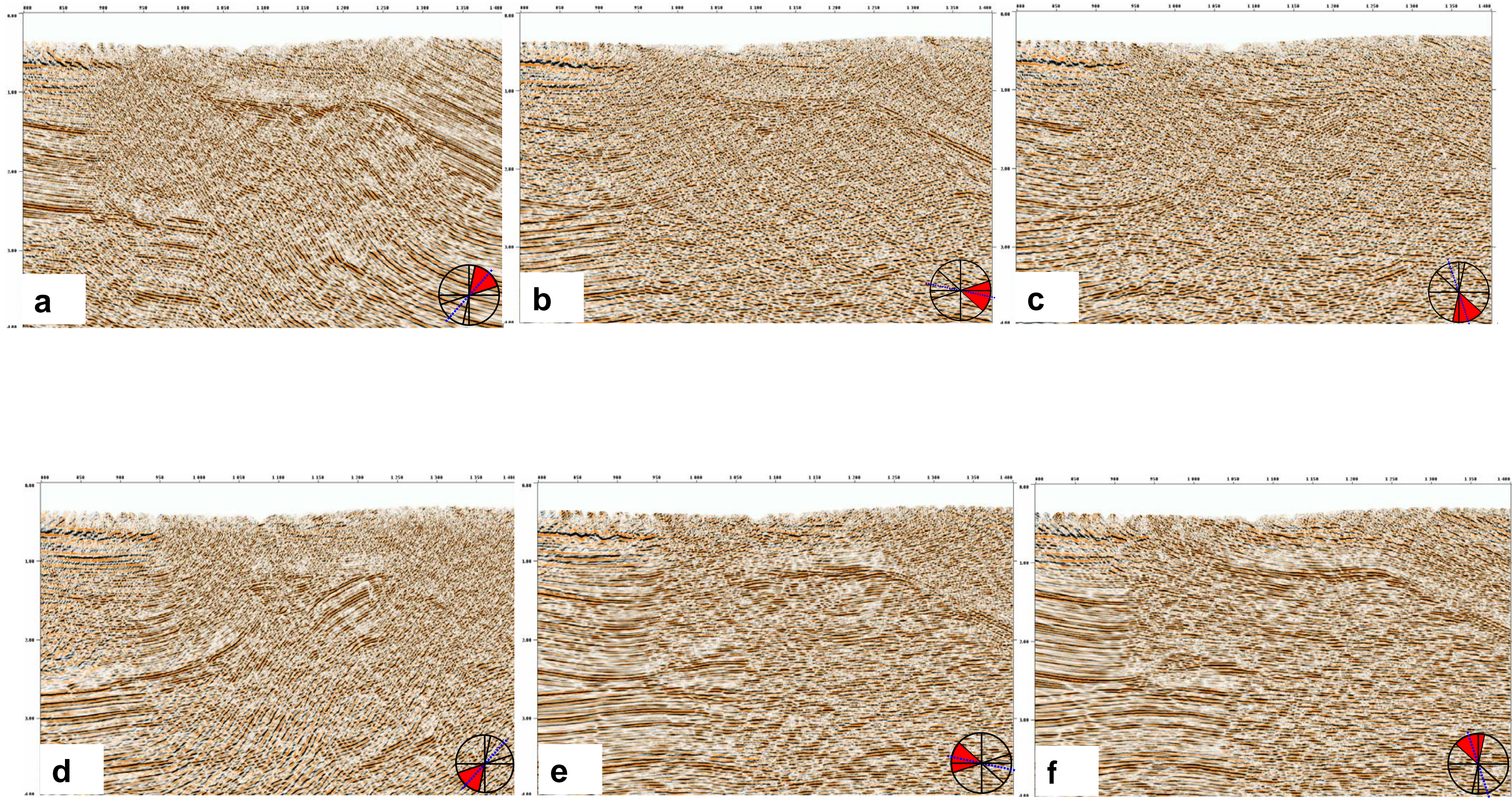

Figure 5-6 migrated results in 6 different sectors of source-geophone azimuths defined at $360^{\circ}$ (red sector in circles at bottom right corner of each image shows the part of mid-points which contribute in imaging,

blue dash lines show azimuth of imaged section). 

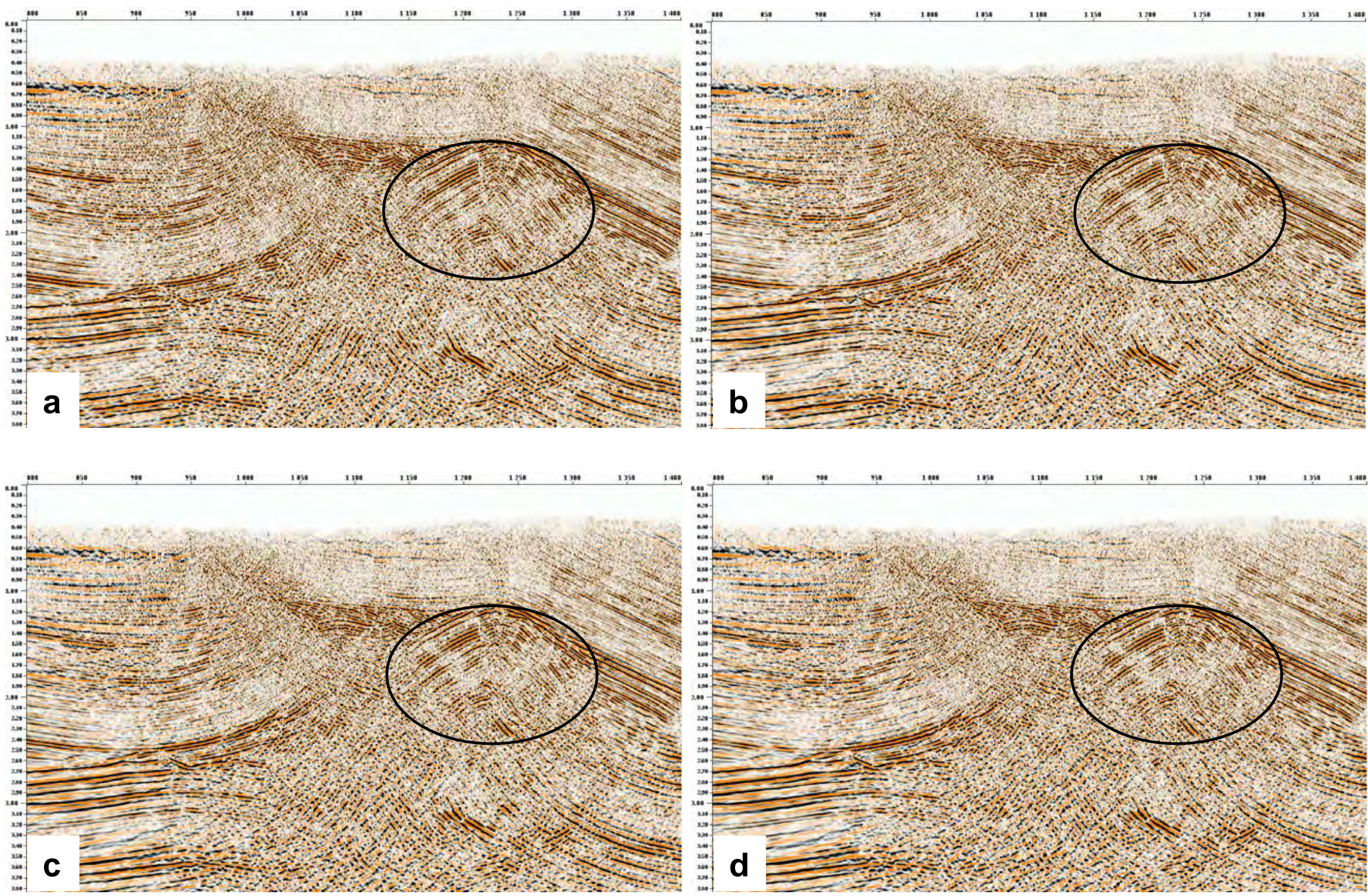

Figure 5-7 Offset scan: a, b, c and d represent the maximum in-line acquisition offset from 0 to 2000, 3000, 4000 and 5000 m respectively 

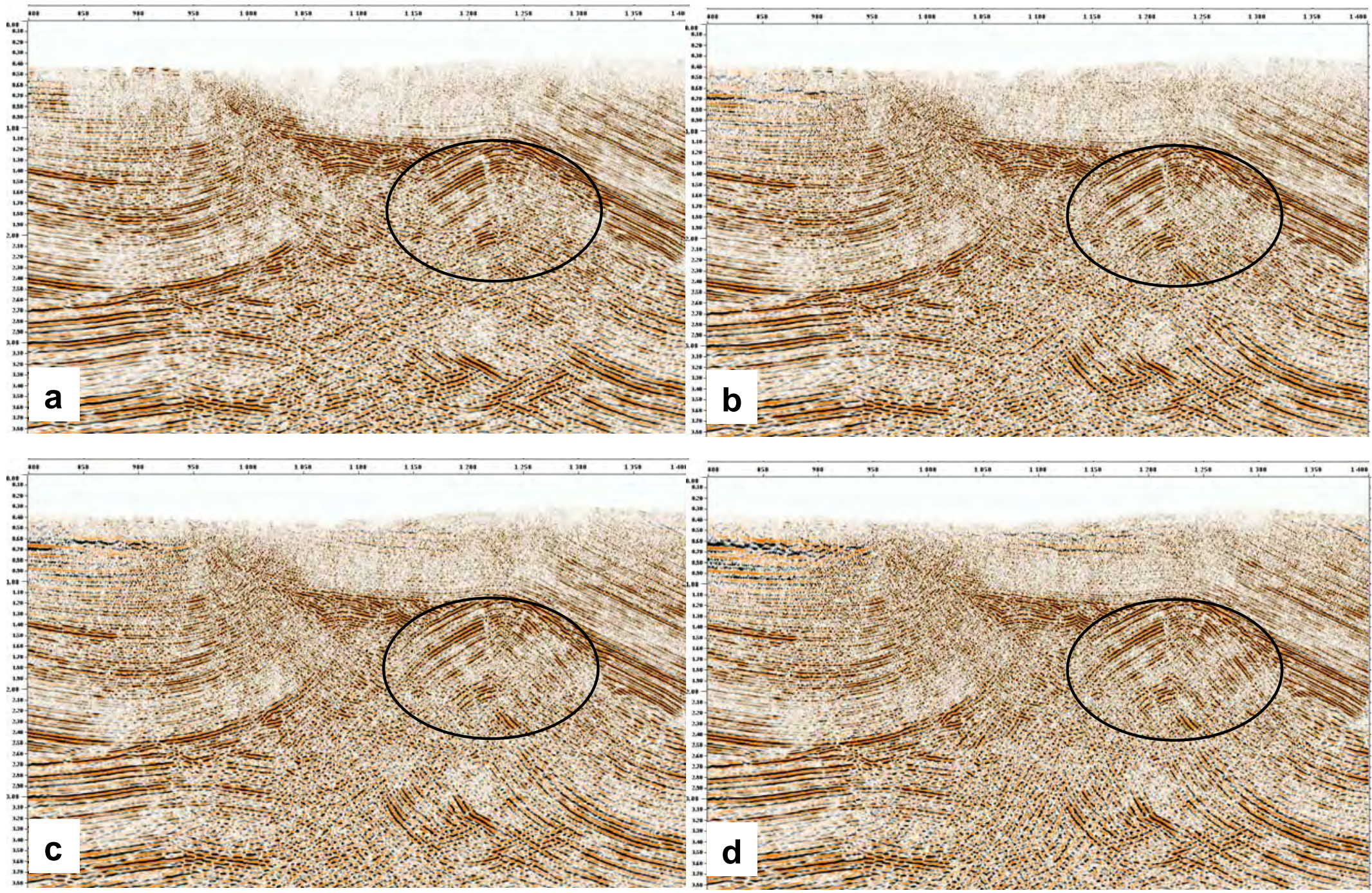

Figure 5-8 Dipmax scan: a, b, c and d represent DIPMAX $=30^{\circ}, 45^{\circ}, 55^{\circ}$ and $70^{\circ}$ respectively (X-Line direction),black ellipses show imaging of the flanks 


\section{5-3-2 Offset range}

Figure 5-7 illustrates a series of tests aimed to check the best acquisition offset range. It is clear that for imaging the deeper horizons long offsets are needed. However, finding the accurate velocity field for long offset seismic data is difficult where the geological setting is complex. We found that maximum acquisition offset to create the best image is $2500 \mathrm{~m}$. Longer offsets generate more noise and migration tails which degrade the quality of final migrated image.

\section{5-3-3 Dipmax parameter}

Figure 5-8 shows the influence of this parameter as it indirectly limits the exploration of geological dip. We decided to keep the maximum value $70^{\circ}$.

\section{5-4 Comparison with previous results}

\section{5-4-1 Main results}

The new results(IFP results) were compared with the two previous results obtained with the Western-Geco software (Omega) used by contractor (BGP) and by our self using TIKIM module of CGGVeritas.

Figures 5-9 to 5-11 show the results to be compared. Figure 5-11 corresponds to azimuth selection of $60^{\circ}$, offset range of $2500 \mathrm{~m}$ and dipmax equal to $70^{\circ}$.

In the ellipse domains corresponding to the oil ring reservoir zones, the new results on figure5-11 exhibit the near $40^{\circ}$ dip flanks of the structure, which are absent on the industrial PSTM images (figures 5-9 and 5-10).

Figure 5-12 shows the multiple arrival residuals which contaminate the data but were removed by the azimuth sector selection (IFP PSTM): this multiple is clearly generated between the strong Gs-6 reflector and the ground surface (floating datum, FDP) 


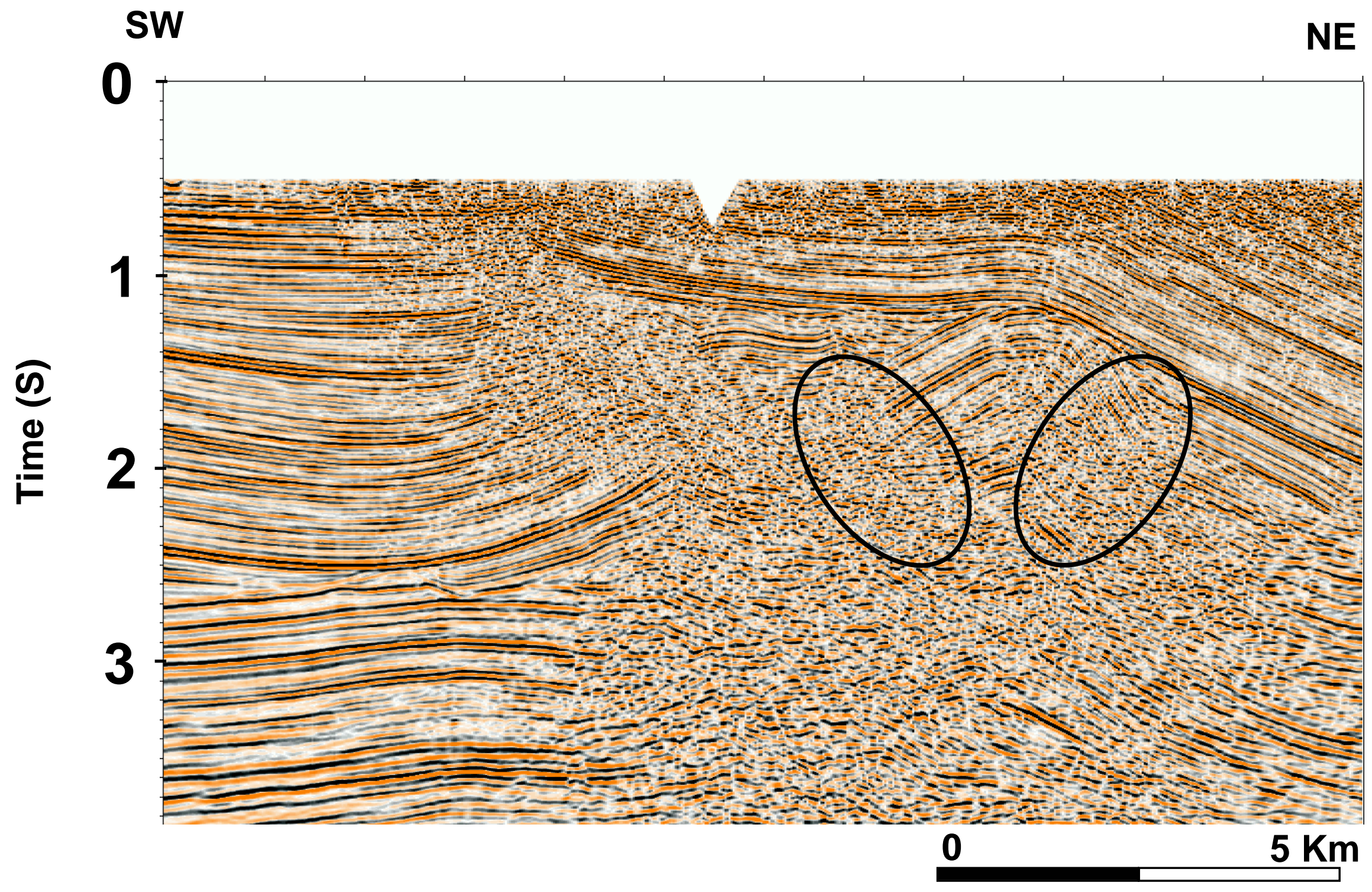

Figure 5-9 Final PSTM result In-Line1760 (Western Geco tool), the black ellipse focuses on the reservoir zone of interest 


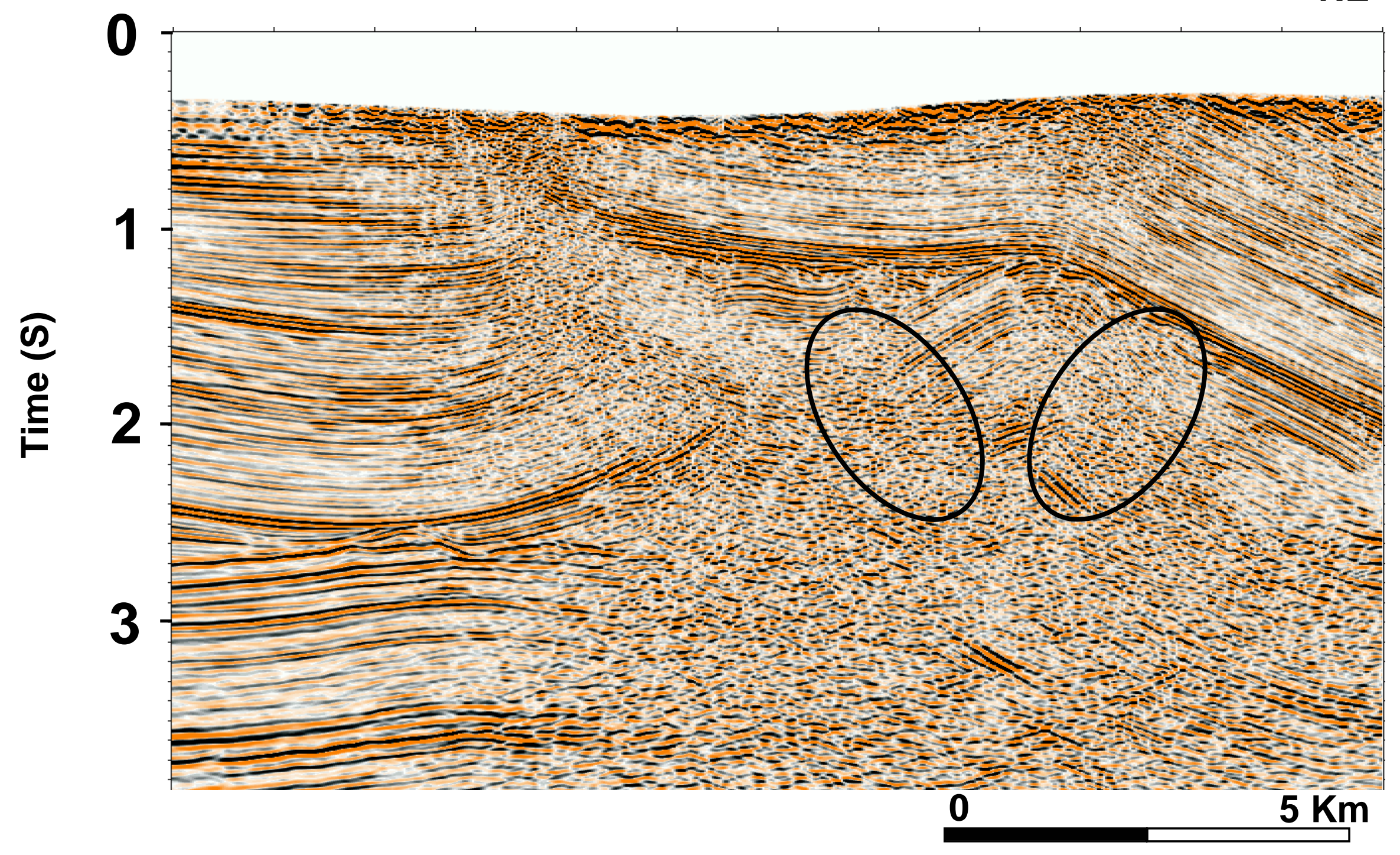

Figure 5-10 Final PSTM result In-Line1760 (TIKIM tool from CGGV), the black ellipse focuses on the reservoir zone of interest 


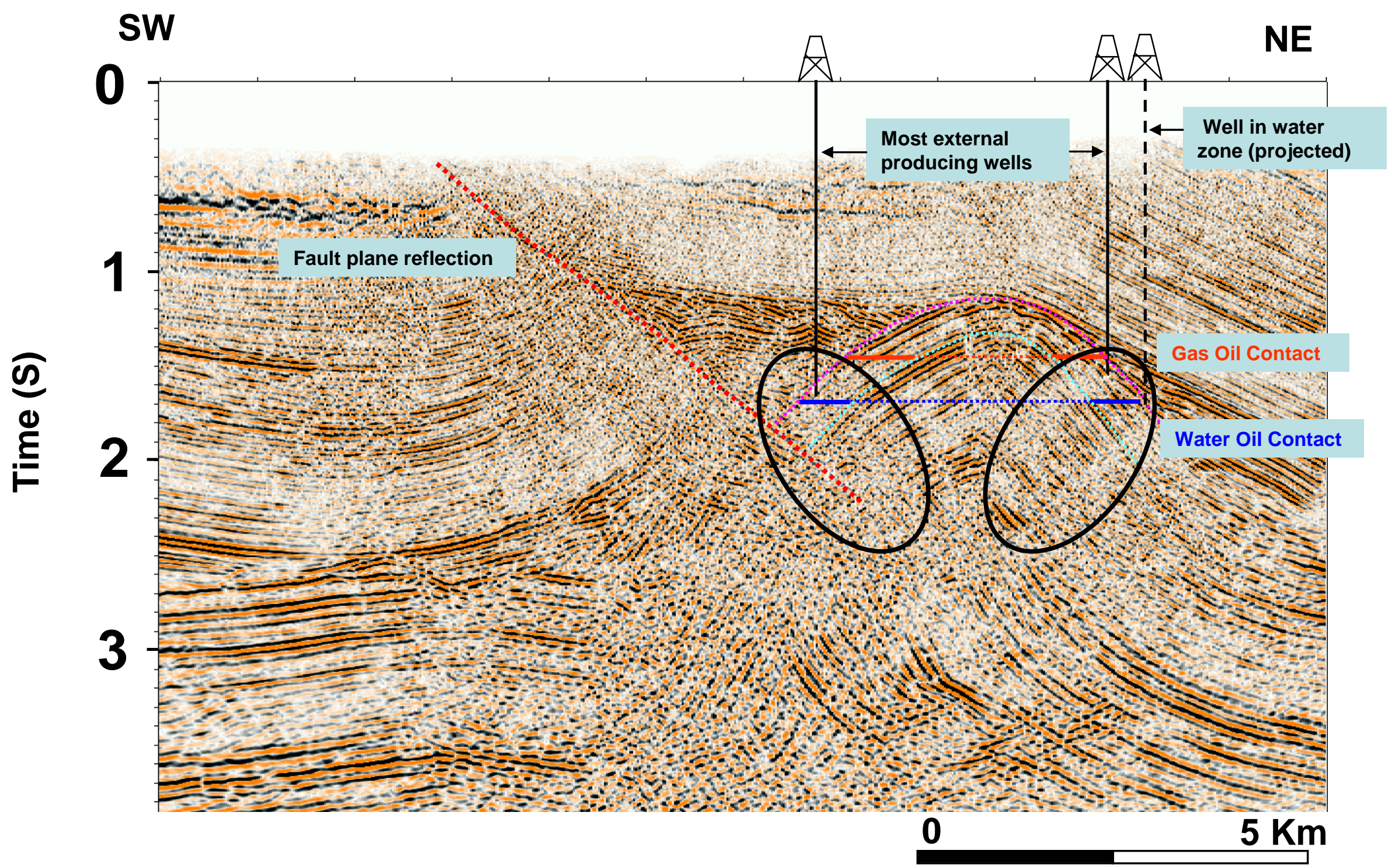

Figure 5-11 Final PSTM result In-Line1760 (IFP tool), the black ellipse focuses on the reservoir zone of interest 


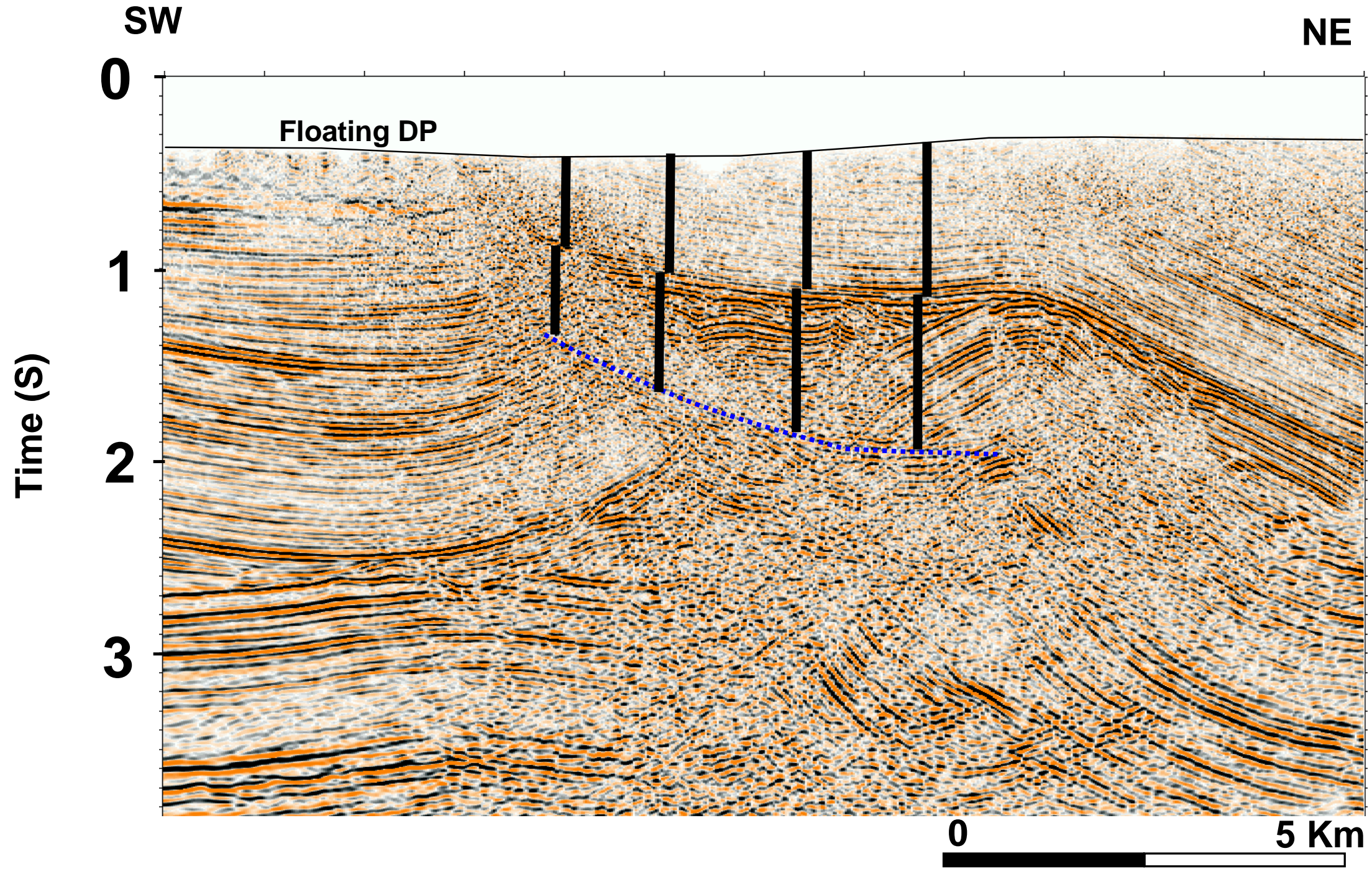

Figure 5-12 IFP result In-Line1760, azimuth sector $180^{\circ}$ in which the multiple between Gs-6 and surface is clearly identified 


\subsubsection{Tentative explication of differences}

To investigate the main reasons of having different PSTM image on CGGV and IFP tools, the following tests were performed. First an impulse test was done to see whether there is any difference in impulse signal for migration in both tools. Comparison between figure5-13a and b shows no major difference in the arrival times of the migration impulse signals.

The differences between the TIKIM and the IFP image come mainly from the following points:

- The antialias filter of TIKIM attenuates so much steep parts,

- The small amplitude steep reflections that could subsist are overlain by strong multiples (on the left part of section) or by noise that we removed partially by making azimutal selection.
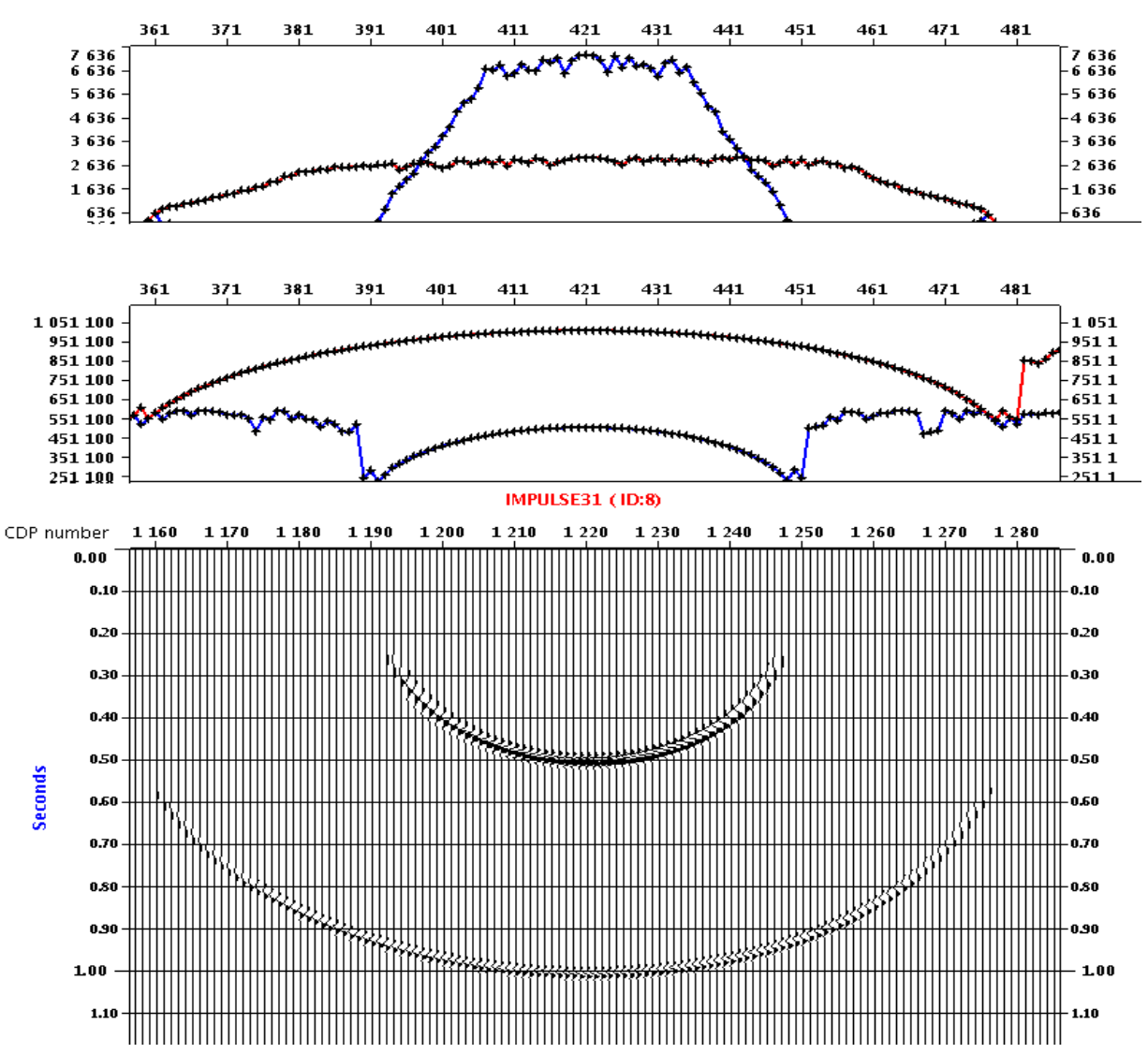

Figure 5-13 a Impulse test by CGGV tool 


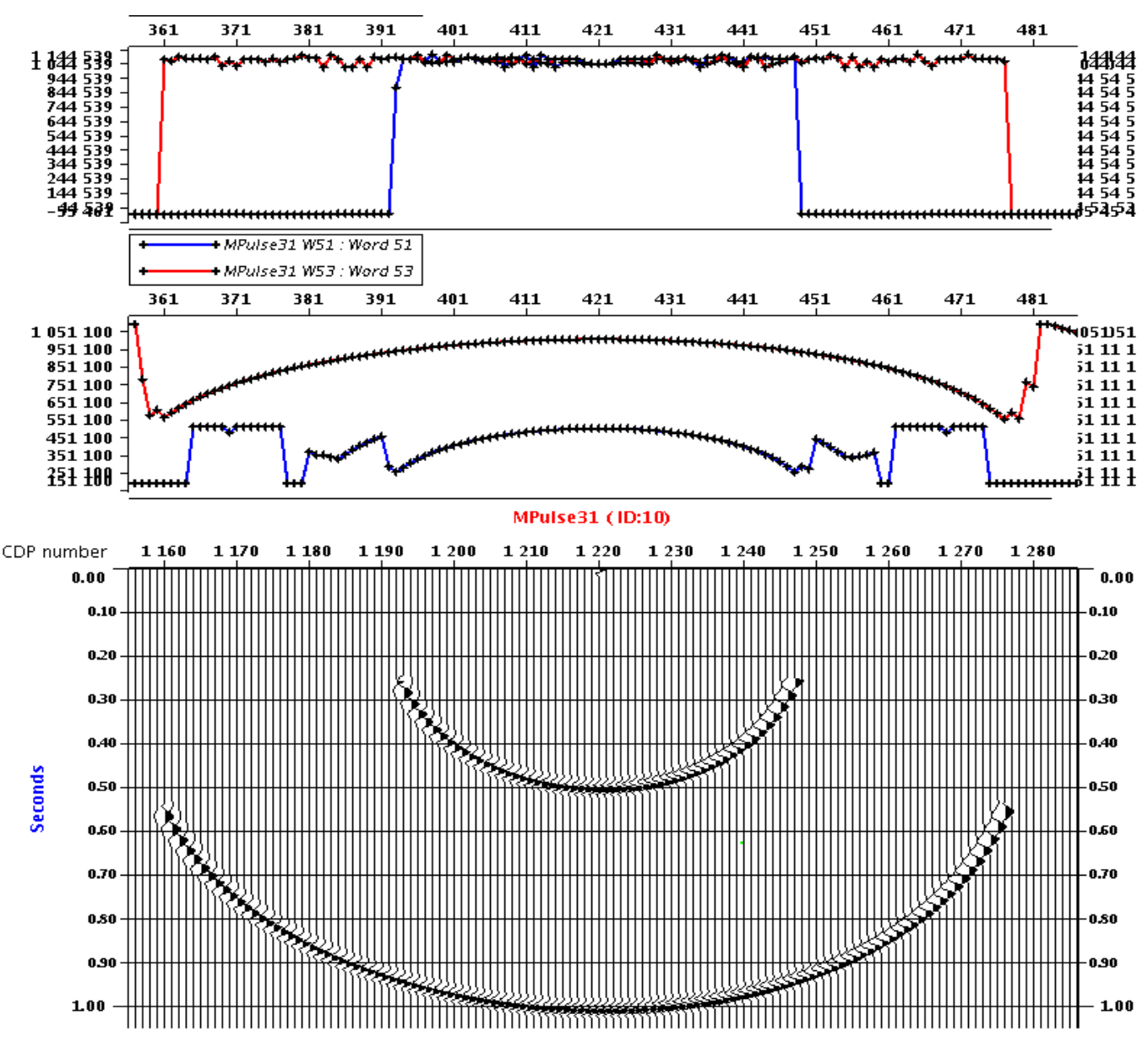

Figure 5-13 b Impulse test by IFP tool.

On figure 5-13a and b two impulses in 500ms and 1000ms are displayed. lower curves at top represents time in ms (blue for 500ms and red for 1000ms). Upper curve at top represents maximum amplitude. Note that in CGGV tool taper was applied while IFP program has no taper. 


\section{5-5 Dip stack method}

\section{5-5-1 Expected move-out shape in "aperture gathers" for planar reflector}

The concept of limited aperture migration is strongly related to a simplified vision of the underground: if the main energy comes from regular, locally planar reflector, we can set the imaging problem as the reconstruction of such events and explicitly search (by inversion or scan) the local dip or equivalently the specular migrated offset $x_{\text {spec }}=z \cdot \tan v_{\text {spec }}$.

On figure5-14a, we see on right a simple model with a planar reflector. We consider an image point $\mathrm{M}$ located on this reflector and represent in red the rays used for computing diffraction time and in green the actual reflected rays that reach the same traces. On the left, we see the travel times associated with the reflection and diffraction that are tangent at specular trace $x_{\text {spec }}$.
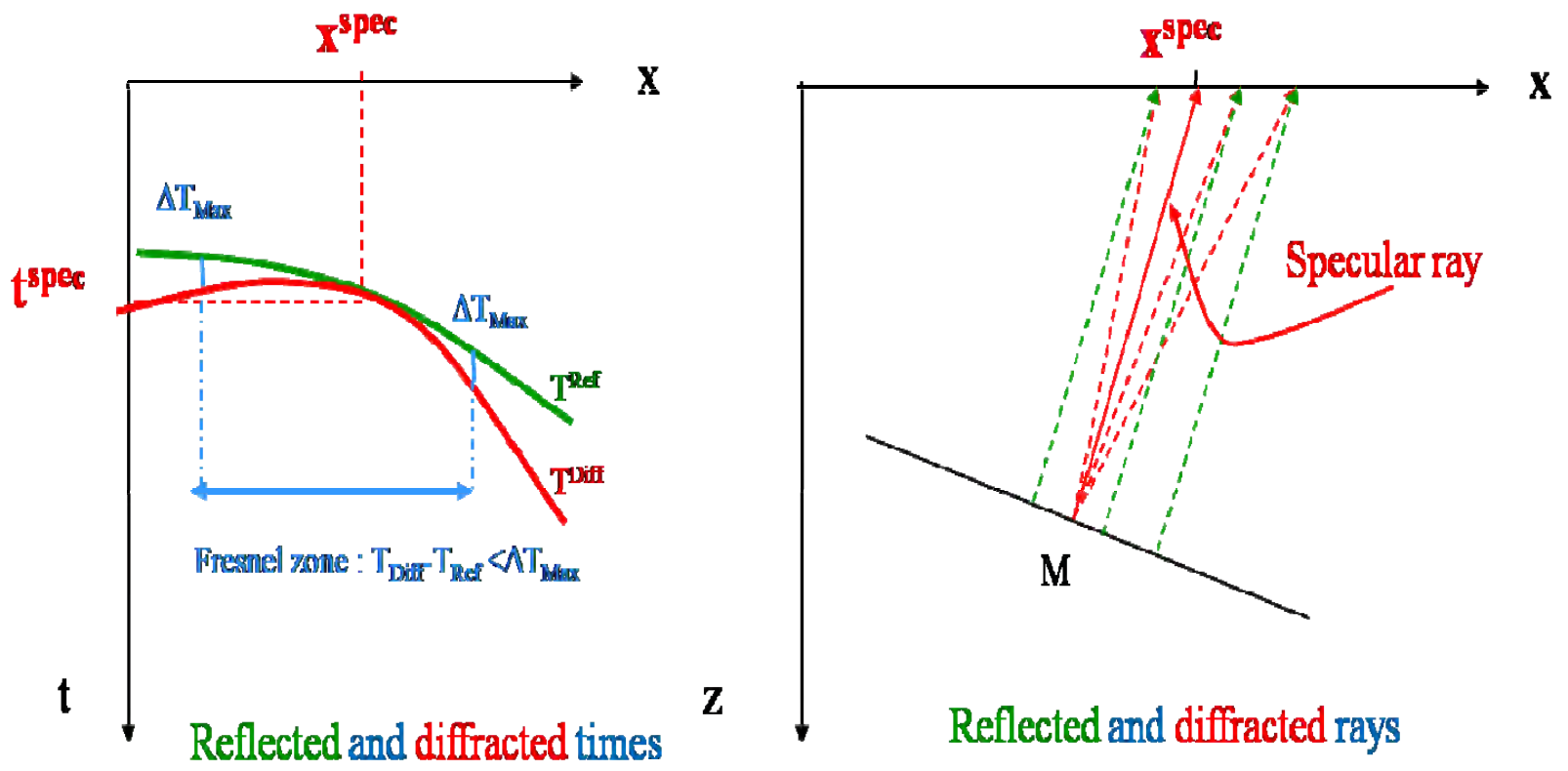

Figure 5-14a Sketch of diffraction and reflection rays (left), and specular ray (right) 


\section{Notation:}

$x$ is the integration variable (CMP coordinate - image coordinate)

$x^{\text {spec }}$ is the values of integration variable at specularity

$\mathrm{M}(0, \mathrm{z})$ is an image point, located along a reflector

$\boldsymbol{v}\left(v_{X}, v_{Z}\right)$ is the migrated dip vector in $\mathrm{M}$, function of $x$, the integration variable $v^{\text {spec }}$ is the specular dip

The image in $\mathrm{M}$ is obtained by shifting reflection time by the value of the diffraction time, then the diffraction stack is performed horizontally along the variable x. Before this stack, the planar reflections appear with tulip shape with apex located at specular trace, as shown on figure5-14b. The migration Fresnel zone is the maximal aperture $\Delta x$ around specular trace ( not yet known) such that

$\Delta T=T^{\text {DIFF }}(x)-T^{\text {SPEC }}(x)$ is smaller than the dominant period of the signal.

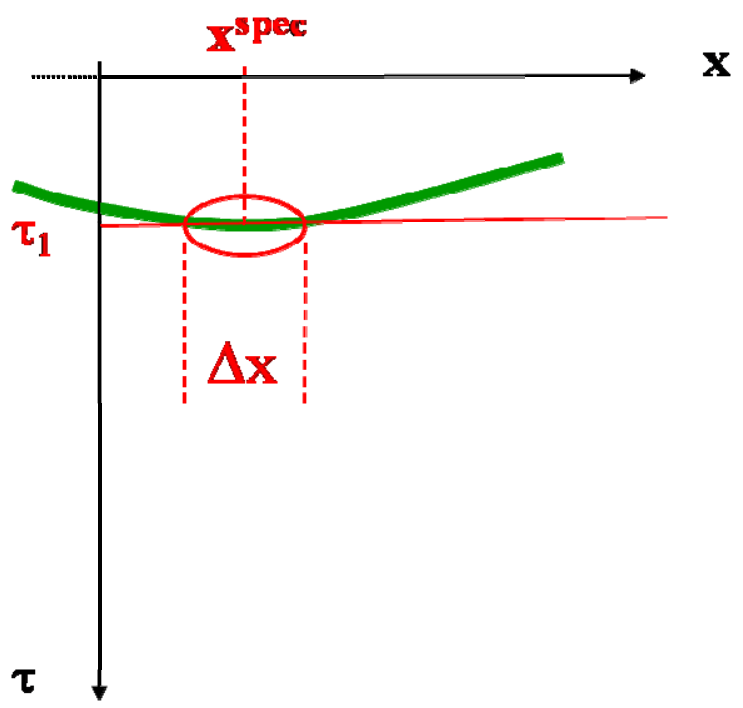

Figure 5-14b smile shape of reflection curve along aperture X, red ellipse shows Fresnel zone

In order to calculate $\Delta T$, it is necessary to expand (Taylor expansion) the diffracted time to second order versus $x$, since the specular time and the first order term between diffracted and reflected times are the same at specularity: 


$$
\begin{aligned}
& T^{D I F F}(x)=T^{D I F F}\left(x^{\mathrm{SPEC}}\right)+\left.\left(x-x^{S P E C}\right) \frac{\partial T^{D I F F}}{\partial x}\right|_{x}+\left.\left(x-x^{S P E E C}\right)^{2} \frac{\partial^{2} T^{D I F F}}{\partial x^{2}}\right|_{x^{\mathrm{SPEC}}} \\
& T^{R E F}(x)=T^{R E F}\left(x^{\mathrm{SPEC}}\right)+\left.\left(x-x^{S P E C}\right) \frac{\partial T^{R E F}}{\partial x}\right|_{x}+\left.\left(x-x^{S P E C C}\right)^{2} \frac{\partial^{2} T^{R E F}}{\partial x^{2}}\right|_{x^{\mathrm{SPEC} C}}
\end{aligned}
$$

with

$$
\begin{aligned}
& T^{\text {DIFF }}\left(x^{\text {SPEC }}\right)=T^{R E F}\left(x^{\text {SPEC }}\right) \\
& \left.\frac{\partial T^{D I F F}}{\partial x}\right|_{x \text { SPEC }}=\left.\frac{\partial T^{R E F}}{\partial x}\right|_{X^{S P E C} \quad \text { (Imaging condition for a reflector). }}
\end{aligned}
$$

Therefore:

$$
\Delta T(x)=\left(x-x^{\operatorname{SPEC}}\right)^{2}\left(\left.\frac{\partial^{2} \mathrm{~T}^{\mathrm{DIFF}}}{\partial x^{2}}\right|_{x^{\text {spec }}}-\left.\frac{\partial^{2} \mathrm{~T}^{\mathrm{REF}}}{\partial x^{2}}\right|_{x^{\mathrm{SPEC}}}\right)
$$

In PSTM, we assume straight rays. Let us consider a planar reflector with dip angle $v_{\text {spec }}$. For a given image point located in $(0, \mathrm{z})$ the specular CMP coordinate is $x_{\text {spec }}=z \cdot \tan v_{\text {spec }}$ and the migration operator will apply on the variable $y=x-x_{\text {spec }}$ where $x$ is the CMP coordinate relative to image point. Variable $y$ is called migrated offset or aperture in the following.

Diffracted time reduces to following expression for zero offset case:

$$
T^{\text {Diff }}=2 \frac{\sqrt{z^{2}+\left(x_{\text {spec }}+y\right)^{2}}}{V}=2 \frac{\sqrt{z^{2}+x_{\text {spec }}^{2}}}{V}\left(1+\frac{x_{\text {spec }}}{z^{2}+x_{\text {spec }}^{2}} y+\frac{y^{2}}{2\left(z^{2}+x_{\text {spec }}^{2}\right)}\left(1-\frac{x_{\text {spec }}^{2}}{\left(z^{2}+x_{\text {spec }}^{2}\right)}\right)+\ldots\right)
$$

The specular time $T_{\text {spec }}=2 \frac{\sqrt{z^{2}+x_{\text {spec }}{ }^{2}}}{V}$ and slope versus $\mathrm{y}$ $p_{\text {spec }}=2 \frac{\sqrt{z^{2}+x_{\text {spec }}^{2}}}{V} \frac{x_{\text {spec }}}{z^{2}+x_{\text {spec }}^{2}}$ are identical for the diffracted and reflected time. The difference is second order term: 


$$
\left.\Delta T(y)=\frac{1}{V} \frac{y^{2}}{\sqrt{z^{2}+x_{\text {spec }}^{2}}}\left(1-\frac{x_{\text {spec }}^{2}}{\left(z^{2}+x_{\text {spec }}^{2}\right.}\right)\right)-y^{2}\left(\left.\frac{\partial^{2} \mathrm{~T}^{\mathrm{REF}}}{\partial x^{2}}\right|_{x^{\mathrm{SEC}}}\right)
$$

The migration Fresnel zone is the maximal aperture $\Delta y$ such that $\Delta T \leq T_{\text {signal }}$ where $T_{\text {signal }}$ is a time length that characterizes the signal (for instance $50 \mathrm{~ms}$ ).

We find for a planar reflector: $\Delta y=\sqrt{\frac{z V \cdot T_{\text {signal }}}{\cos v_{\text {spec }}}} \frac{1}{\cos v_{\text {spec }}}$

For a dip to $45^{\circ}$, a signal of $50 \mathrm{~ms}$ and a velocity of $3000 \mathrm{~m} / \mathrm{s}$, we have: $\Delta y \approx 21 \sqrt{z}$ that defines in turn a maximum contributing migration dip angle:

$\operatorname{tg} v=\operatorname{tg} v_{\text {spec }}+\frac{\Delta y}{Z}$

For instance, for $z=3000 \mathrm{~m}$, the maximum angle for complete reconstruction around $45^{\circ}$ is

$\operatorname{tg} v=1.75$ i.e. about $60^{\circ}$.

Note concerning antialias filter: at this value, the operator antialias filter will have for Nyquist $34 \mathrm{~Hz}$. It means that it is necessary to have a bin sampling of 12.5 meter to fully recover reflector around $45^{\circ}$ with standard (large aperture) migration and standard migration antialias filter.

\section{5-5-2 Short description of algorithm for controlling aperture}

The method proposed by Tabti (2005) was tested but the data were too noisy to have a clear detection of main specular events.

The proposed algorithm to find specular trace and limit the migration diffraction stack to a Fresnel zone is designed for the simple zero offset and 2D case. It can still be applied to our data if :

-we use only a selection of short offset $(<2500 \mathrm{~m})$, and -half migrate the data in strike direction (where apparent dips are negligible). 
The method is applied independently to each gather, corrected for diffraction stack, half migrated for strike direction and where the horizontal dimension of the gather is the inline aperture $x$.

For each time, we scan simultaneously $x^{\text {spec }}$ and the possible curvature of reflector (assumed to be small) $\left(\left.\frac{\partial^{2} \mathrm{~T}^{\mathrm{REF}}}{\partial x}\right|_{x^{\mathrm{SPC}}}\right)$ square added in equation before by computing the semblance on a temporal window of about $50 \mathrm{~ms}$ and an horizontal window tapered between one and two Fresnel zones. The best semblance gives the value of $x^{\text {spec }}$. The diffraction stack is then done on a corridor between $x_{\text {inf }}=x^{\text {spec }}-\Delta x$ and $x_{\text {sup }}=x^{\text {spec }}+\Delta x$, as shown in next figure.

Aperture gather

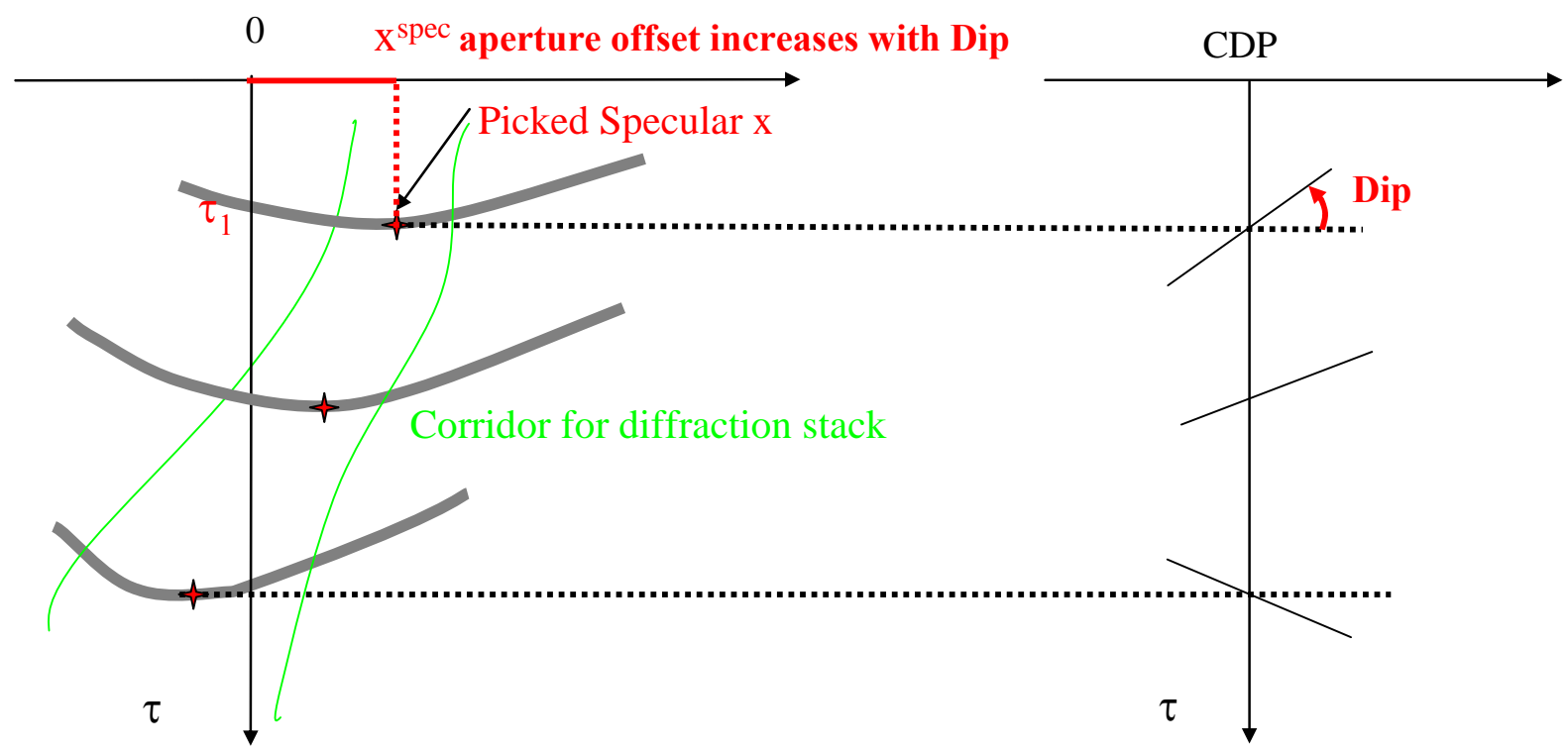

Figure 5-15 corridors in aperture domain which keeps to be stacked

For each time window, the algorithm searches up to 3 quasi planar reflectors in case, we are located at intersection of reflectors. The outputs are the 3 best stacks ranked according to value of semblance. The move-out curve of the aperture gather is simultaneously scanned between half and twice the theoretical curve for planar reflector. 


\section{5-5-3 Results}

In next figures, we show aperture gathers at two locations: one is at CMP 860 in the left part of the survey and the other one is at CMP 1210 in the central part of the anticline.

Figure5-16a shows the aperture gather in location 1 side by side with the migrated section. As expected, the reflectors appear with negative move-out (smile) with apex shifted to right for reflectors dipping toward left and vice versa. The zoom in Figure516b, shows the move-out picked by the algorithm. Next Figure5-17 illustrates the aperture corridor found by the algorithm with events flattened by the picked move-out curves for QC. Figure 5-18 illustrates aperture gather in location2 side by side with migrated section where illumination by other methods are not as well as this routine result. The selection of events in this part depends on the threshold value set on the picked semblance such that empty working windows may happen.

For a given aperture corridor, we can build the final image with 2 options. The first option is to stack horizontally the original aperture gather inside the corridor: only the specular part will contribute mainly coming from a small vicinity of specular trace. This summation will produce half integration of the signal by virtue of stationary phase theorem, property well known in migration (compensated by appropriate derivatives before migration). Another option is to flatten the gather using the move-out resulting from analysis and then to stack horizontally: in this case, the contributions come from a larger area (equivalent to a smoothing along detected reflector, like in CRS stack) and the signal is not integrated and will appear a little higher frequency. These two results are shown in figure 5-19 and figure 5-20. As expected, the image is more noisy without flattening the events (figure 5-19). The stack is improved after flattening as its power is always higher. On the other hand, this stack adds lateral contribution and the information is more mixed laterally. The images appear like a skeleton on the main events. As expected, small details or strong curvatures that are not scan (for instance in the middle of the anticline) cannot be recovered but continuous steep dips appear very clearly; they interrupt sometimes abruptly (because of the limits set on DIPMAX parameter and maximum apertures (here set to $6000 \mathrm{~m}$ ). Unfortunately, the multiples of the top Gs-6 are still remained and the interfering structures are still invisible. However, we can follow the top and bottom of Asmari reservoir better than in standard PSTM results. 


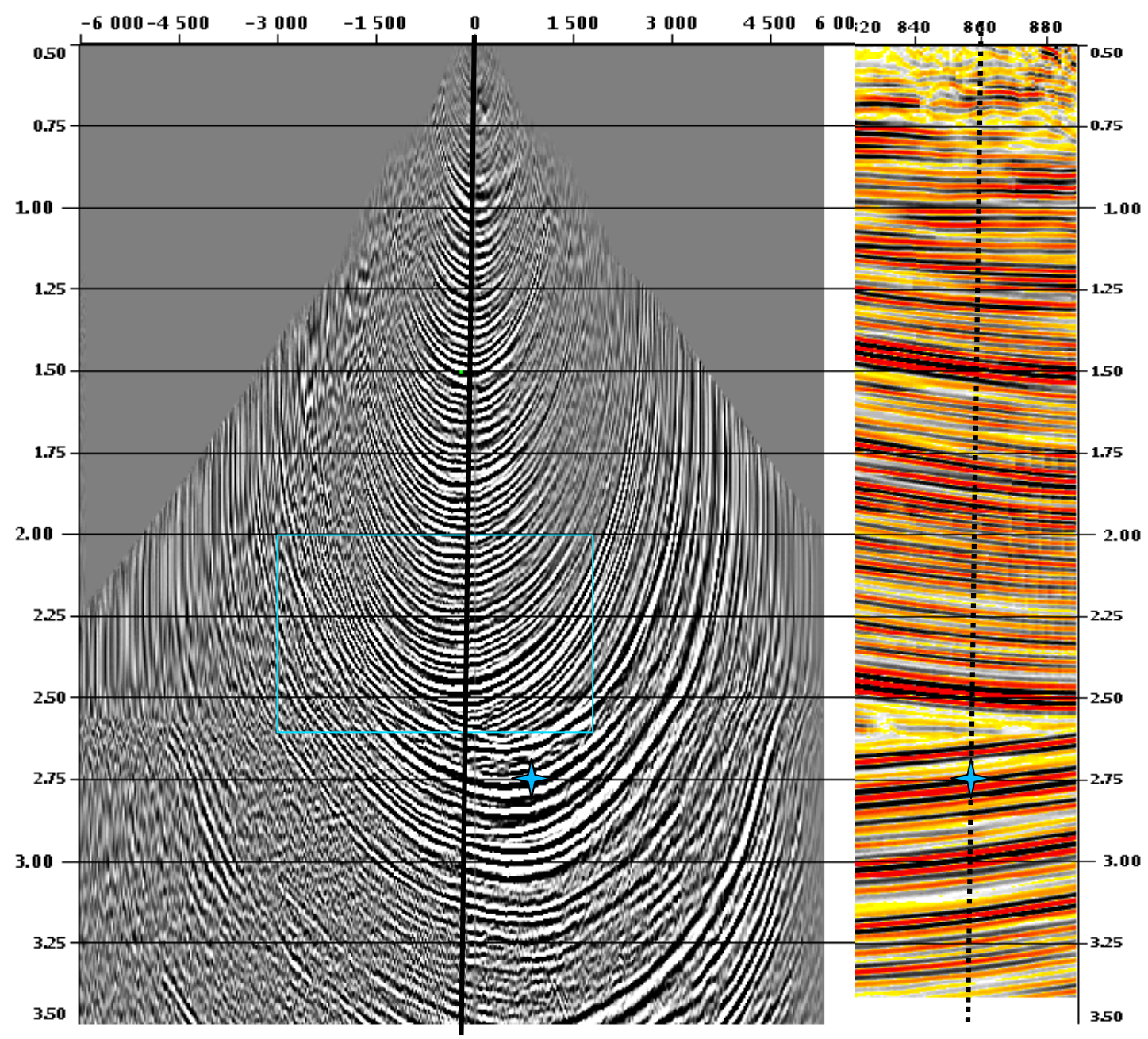

Figure 5-16a Aperture gather with \pm 6000 m offset(right) part of migrated section shows location of this migration gather; blue box area which zoomed in figure 5-16b

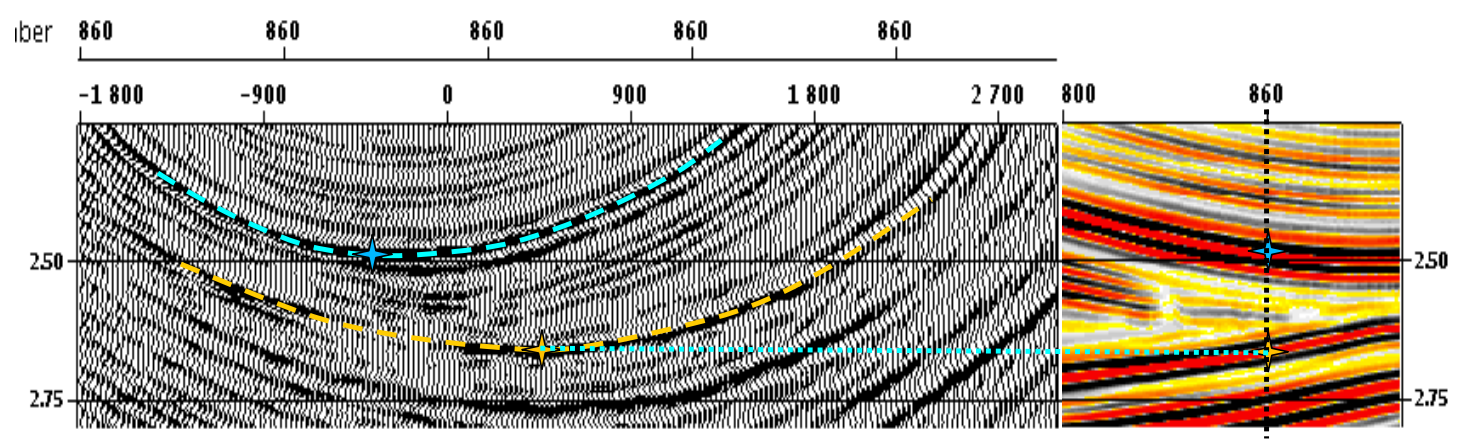

Figure5-16b stars show same location to be compared, apex of smiles on the aperture gathers shift to right or left depending on the dip direction, dash curves were computed by the algorithm 


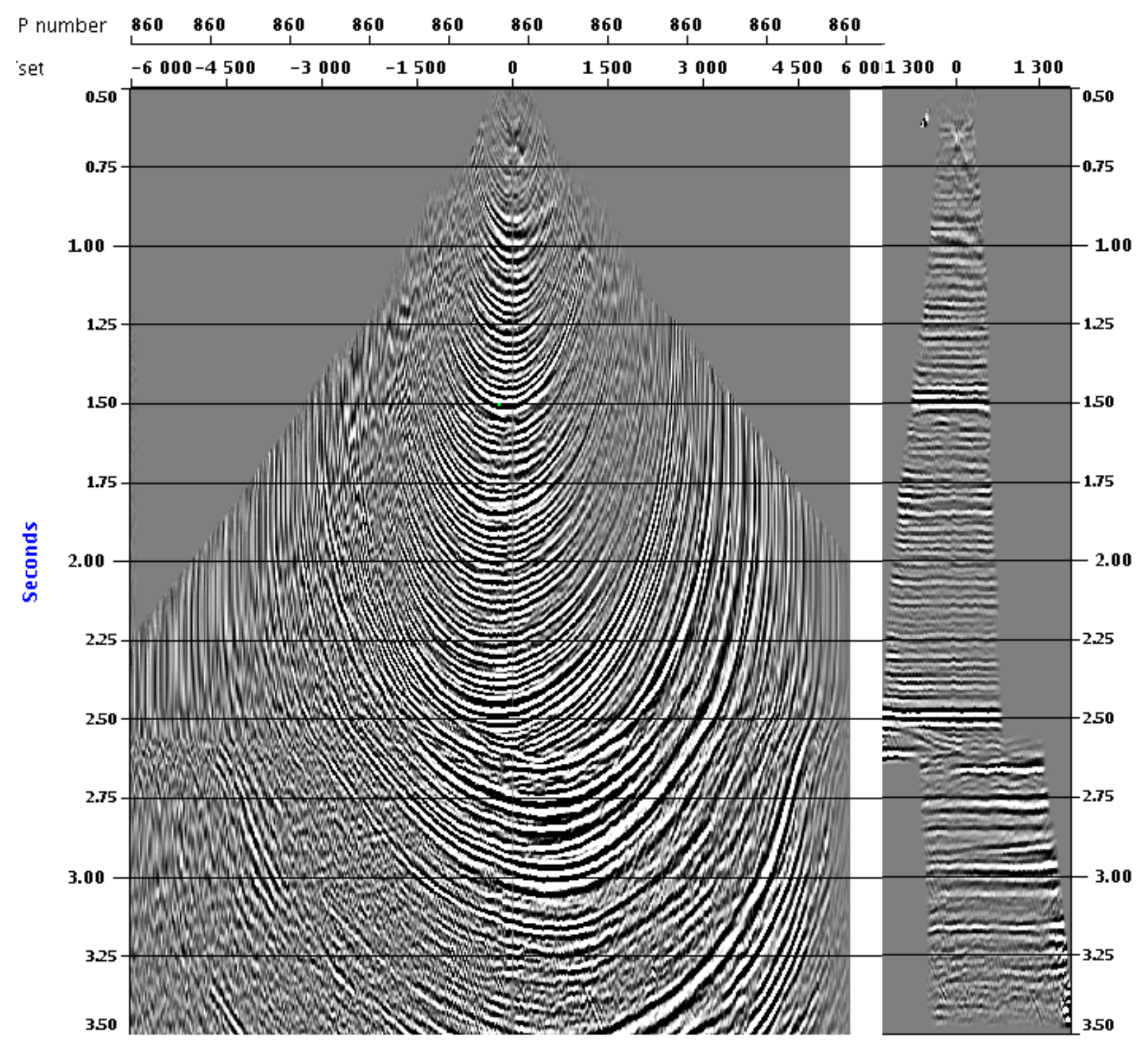

Figure5-17 The aperture gather (left), the corridor which flatten by applying moveout time picked by algorithm (right) 


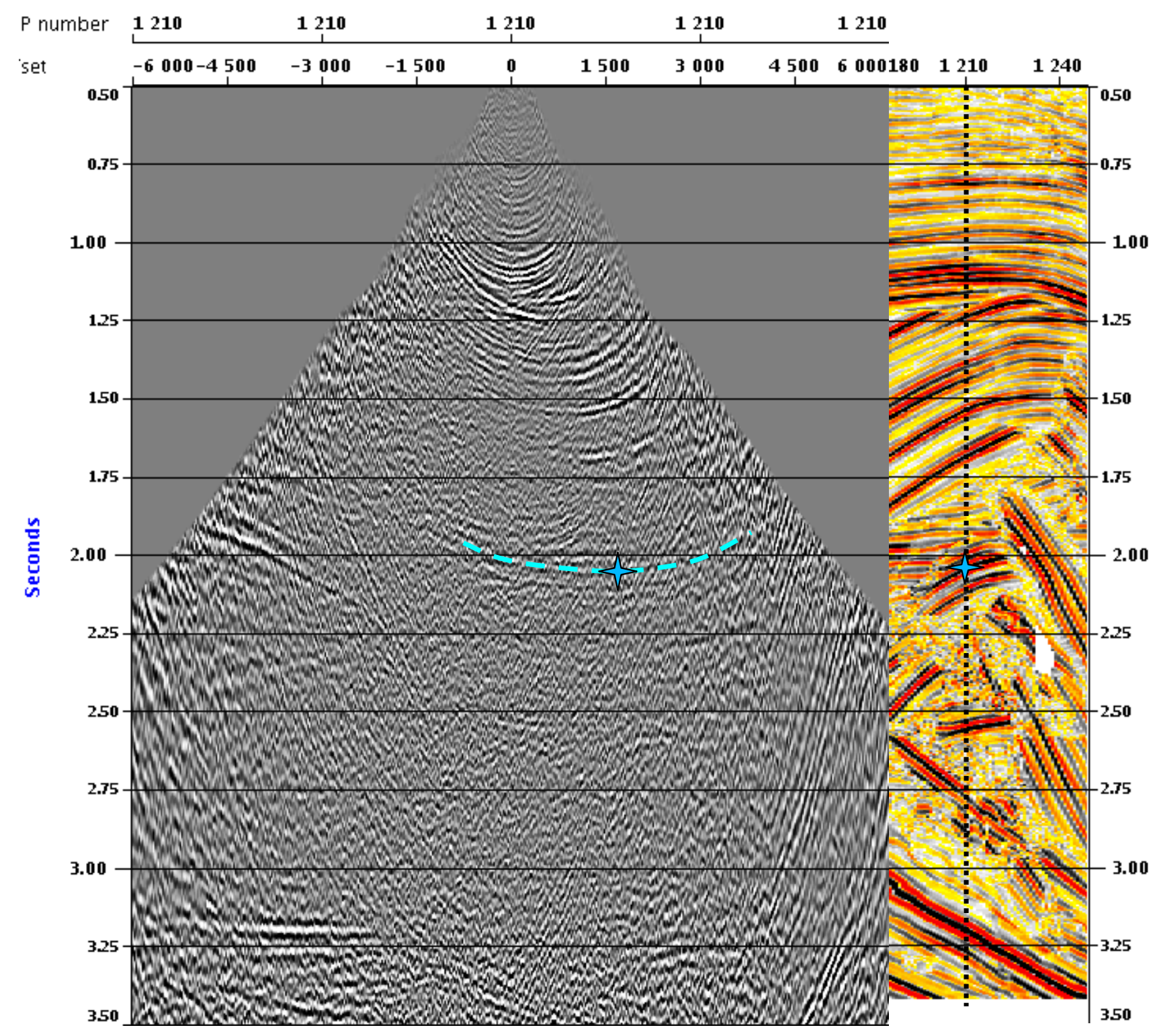

Figure5-18 aperture gather in the location of the reservoir where reflection energy is weak (left) migrated section in corresponding location, the star shows the dipping event which is recovered by this algorithm 


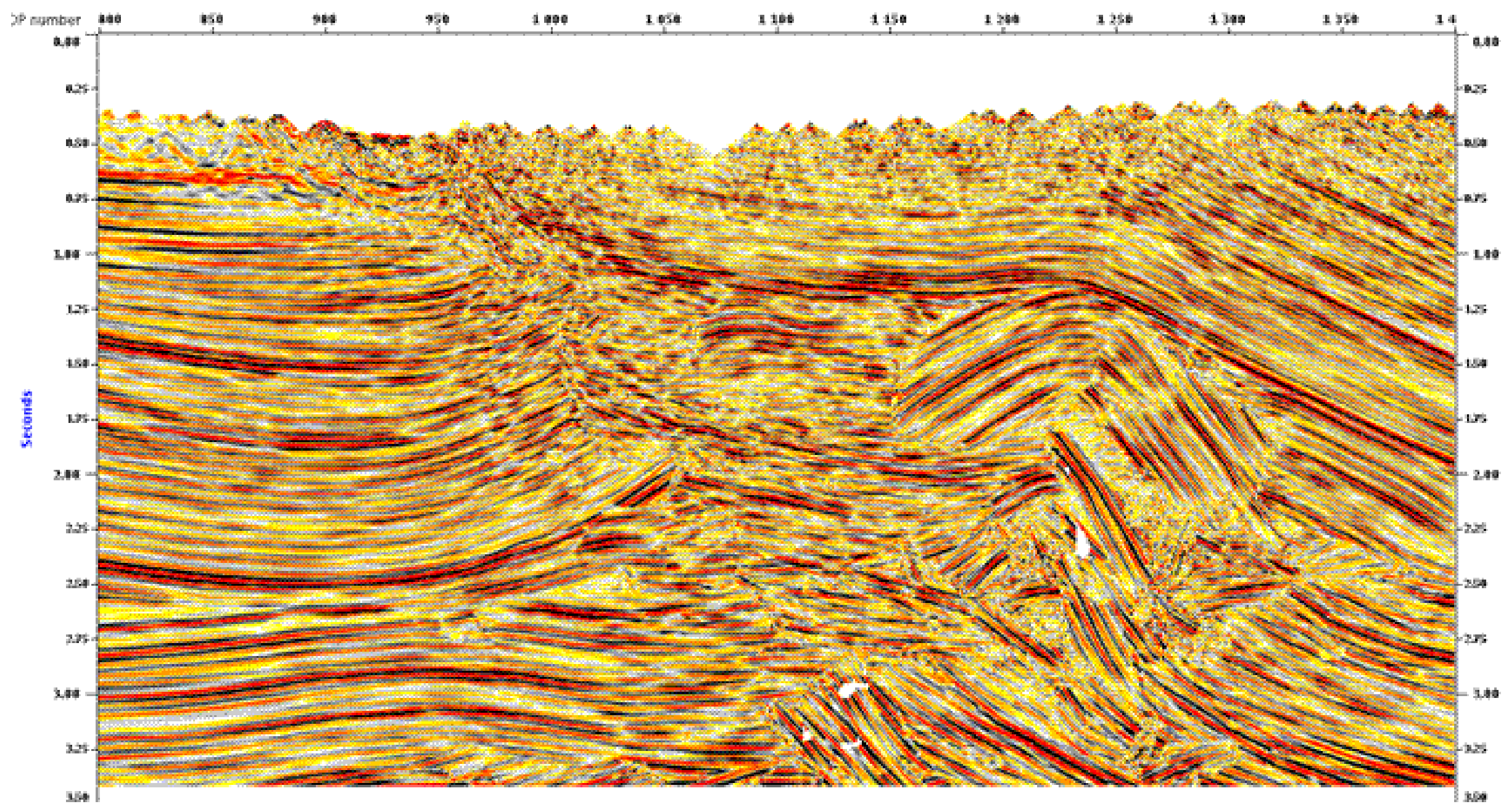

Figure5-19 result of first option, horizontally stack the aperture gathers inside the corridor without applying any computed move out 


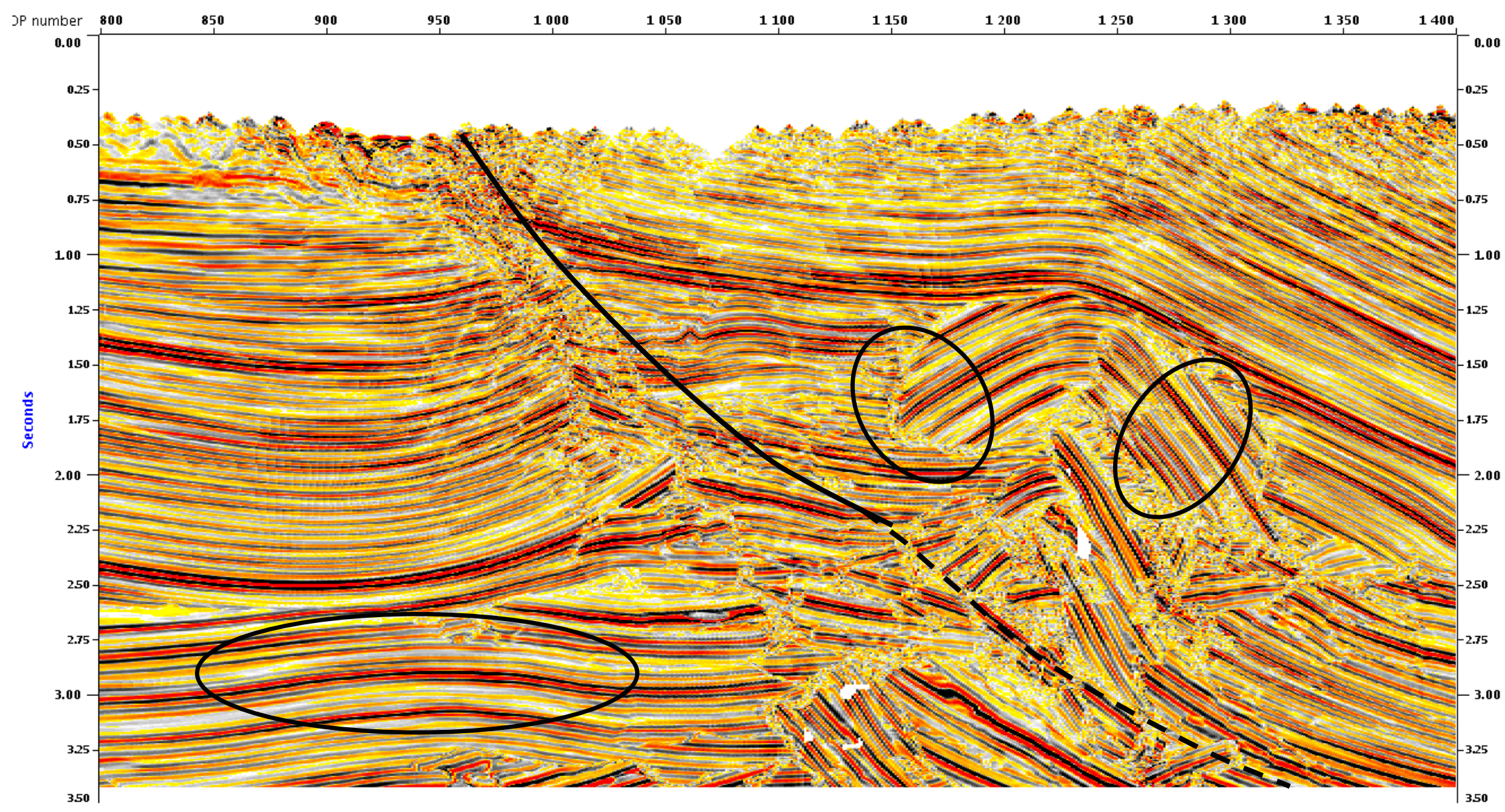

Figure5-20 result of second option, horizontally stack the aperture gathers inside the corridor after applying the computed move out, ellipses show the area of improvement 


\section{5-6 Conclusion}

We have tested two procedures in order to improve the reconstruction of steep dips.

The first method consists in an azimutal filtering in vector aperture domain with no antialias protection. In consequence of the polar partition, the horizontal events associated with multiples were attenuated by a factor 3 ( as the central part of the pie was divided by 3 sectors). The flanks of Asmari were better imaged than in any previous PSTM.

The second method searches explicitly for single (or up to 3) planar events or small curvature events and restricts the diffraction stack to a narrow corridor around the detected specularity. There is no need for antialias filter and steep reflectors can be reconstructed up to the limits set by other parameters (DIPMAX and absolute maximum aperture). Resulting image shows only the main continuous part of the structure. However, the method produces very valuable results: the Cap rock and base of the Asmari can be followed much longer not only on the sides of the anticline but also on the left part under the detachment fault that also seems to be now visible.

These results are very encouraging as they prove that information on the steep dipping parts is present in the data even if it is too noisy to be well imaged by standard methods. Other methods, unfortunately not available at IFP like CRS stack, multi-focusing should work well too and deserve to be tested. Methods like Beam migration that avoid the large spreading of traces along the isochrones should also improve the image. For such methods, the problem may be in the pre-processing step with the picking and decomposition in local coherent events: strong coherent noise present on data may still be picked. The picking done by CRS or multi focusing may be more robust as it searches solutions simultaneously versus dip, reflector curvature and velocity. The presence of free surface multiples of top Gs-6 is still a problem to be resolved. 



\section{Chapter 6}

Method of 3 component VSP data orientation in vertical to low deviated well 


\section{6-1 Introduction}

The present invention concerns a method for estimating the orientation of seismic sensors of zero offset vertical seismic profile (VSP) datasets based on pre-processing of favourable datasets.

The VSP technique is commonly used for a better understanding of the structure around the well in exploration phase or production phase. This technique is also used to define the geological structures in the vicinity of the borehole to guide the drilling or possibly redefine a side track from the well trajectory. Triaxial component VSP tools include usually one component along the well axis and two orthogonal components fix in the borehole tool, of unknown azimuthal direction. The 3 components can be alternatively mounted on gimbals so that one component remains vertical even in deviated or horizontal wells.

The mechanical specification of a VSP tool is important to determine how its components should be rotated in order to orient them in true geographic coordinate system.

To be able to use 3 component VSP wavefield data for computing dip and azimuth of certain reflector in time/depth, it is absolutely necessary to orient the 3 components in true geographic coordinate system/ or at least into a coherent coordinate system prior to processing. Because seismic waves propagate in three dimensions, it is necessary to record, process and interprets them in three components to be able to study the characteristics of the whole seismic propagation wavefield.

The linear character of the polarization of direct P-wave arrival is often used industrially for orientation and processing of offset VSPs when the source is located away from the well head at distance larger than $10 \%$ of vertical depth. In order to do so, the energy of P-wave direct arrival is maximized in the horizontal plane and in a time window around the P-wave first arrival. There are many publications illustrating the use of P-wave direct arrival in order to orient horizontal components in a vertical well, or 3C fix components in a deviated well (M. Becquey, 1990; Bellefleur, Gilles, et al.2001, P. N. Armstong, 2005). Unfortunately, the Pwave polarization method often is not applicable in zero offset VSP due to very low energy of the P-wave first arrivals projection on the horizontal components.

The limitation of conventional processing with one or two components also leads to potential problems in the identification of the mode of certain arrivals received by the $3 \mathrm{C}$ sensor, pressure or shear, and in positioning of the corresponding geological events. The time inversion or the migration of seismic reflected events can lead to multiple solutions, since the azimuthal direction of dip of the reflectors is unknown. Moreover, when two seismic events 
arrive at the same time with similar apparent velocity, on adjacent depth levels (six to twelve, for example), we realize that it is almost impossible to separate them by conventional monocomponent processing and velocity filters only.

It is therefore essential to improve the reliability of the interpretation of seismic VSP data, by processing the three components after orientation. However, the acquisition stage does not really provide the actual orientation of the geophones, while this information is essential to process the $3 \mathrm{C}$ data. Indeed, the orientation of horizontal components from VSP three components is unknown, because the wireline cable which sustains the borehole VSP tool rotates into random direction level to level.

However, although there are accessories which can measure the tool orientation in the well, such as magnetometers/inclinometers or gyroscopes, these sophisticated hardware accessories are seldom used because they induce a substantial extra cost. In addition, they may deteriorate the quality of mechanical anchoring of the VSP tool to which they are combined, depending on the tool design. We note that the step of acquiring VSP allows only rarely delivering the actual orientation of the three axis sensors, while this information is essential to permit processing the three components. In fact, the horizontal components from VSP on vertical wells are most of the time oriented in unknown directions. This is the case particularly for near vertical /deep exploration wells for which the low angle values of vertical inclination are insufficient to actuate the device of partial orientation such as gimbals or of relative bearing sensors.

\section{6-2 Description of the current VSP orientation practice}

Figure 1a illustrates the overall set up and geometry of acquiring a VSP field operation in a well drilled substantially vertical and partially mildly deviated in limited depth intervals. The well depth can typically reach several thousand meters. Figure $1 \mathrm{~b}$ depicts the geometry of propagation of the direct seismic waves emitted by the direct repeatable surface source and received by the down hole seismic sensors. The solid angle $\beta$ (figurelb) covers the whole interval of the well in which the VSP measurements are performed with a regular interval e.g. $15 \mathrm{~m}$. In fact, the narrowness of the emission solid angle $\beta$, guarantees the consistence of the waveforms recorded on adjacent VSP level in a substantially homogeneous or stratified medium. 

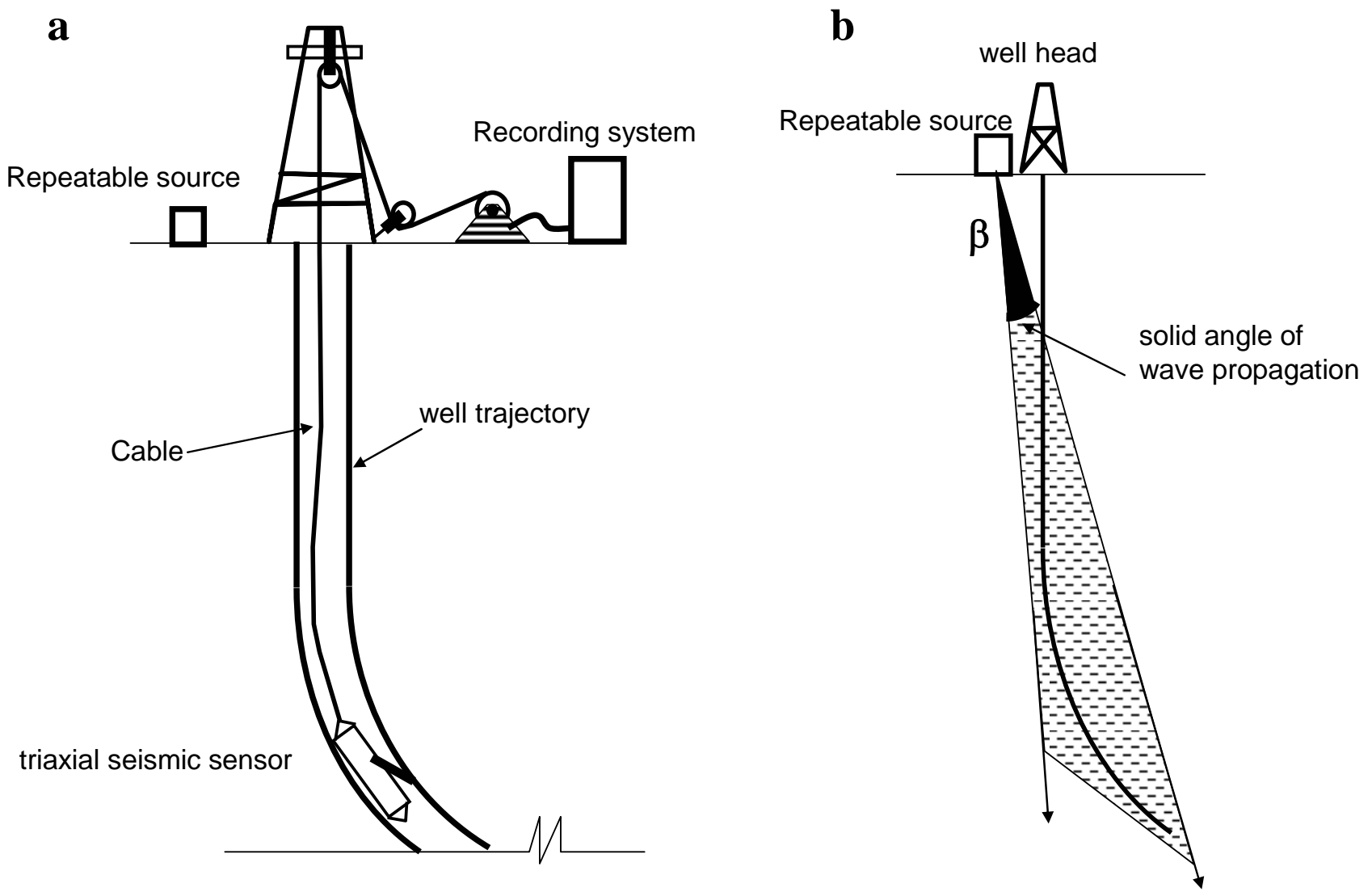

Figure 1 a Well trajectory in vertical to low deviated well b) solid angle of emission of the P- and Swave first arrivals recorded in a vertical seismic profile

In order to determine the spatial direction of a direct P-wave arrival from the three components, one can use the technical analysis of covariance matrix (Benham, at al. 1987). This technique makes the assumption that the direction of polarization is calculated in the vertical plane containing the positions of source and receiver. This is realistic in a stratified sedimentary medium, even with low to medium values of dip (up to approximately $20^{\circ}$ for any azimuth, and for higher dip as long as the source azimuth relatively to the well is close to the azimuth of the general dip, up dip or down dip). 
a

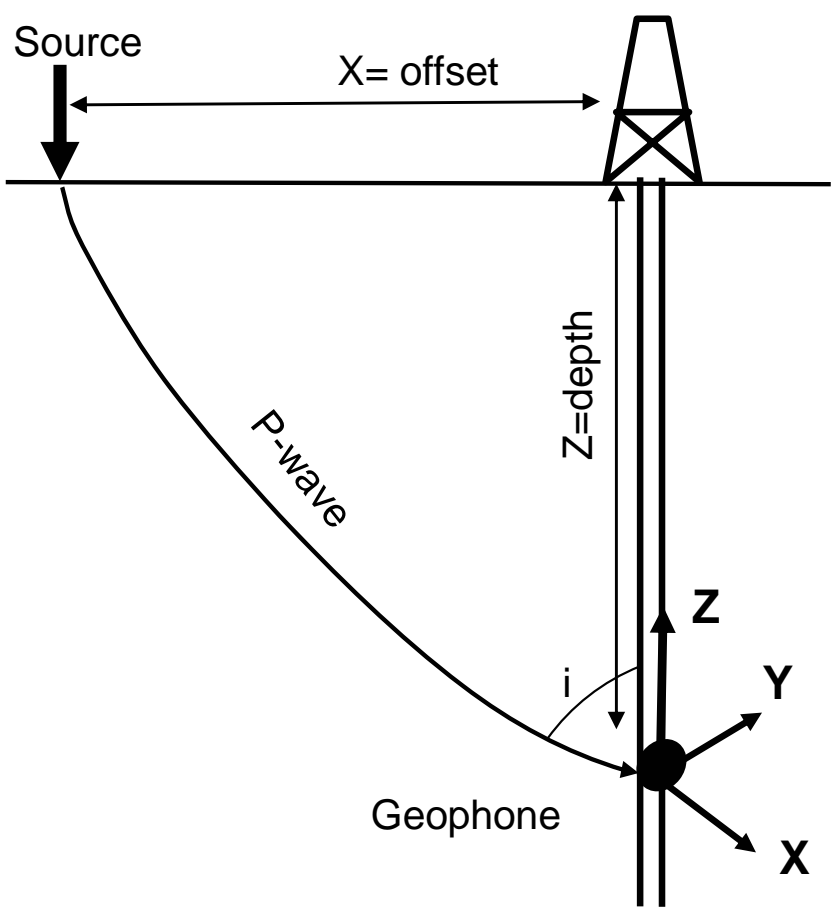

b

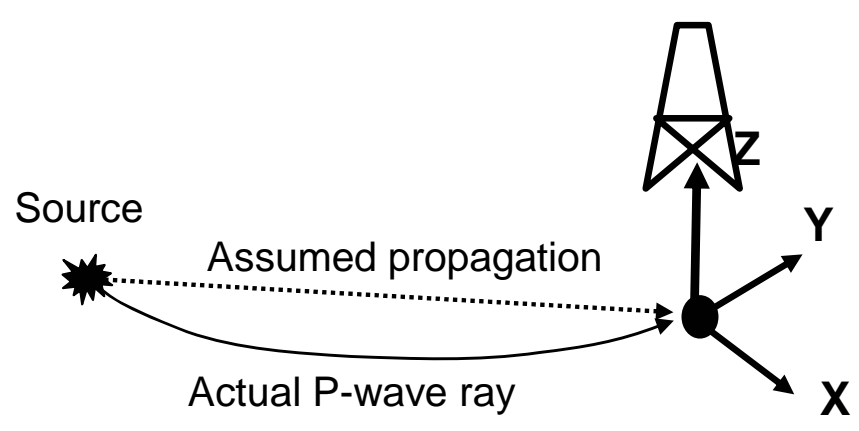

the offset $X$ of the source is large enough so that the incidence angle (i) of direct $\mathrm{P}$-wave is longer than $10-15^{\circ}$ resulting in a sufficient seismic energy of direct arrival on horizontal components

Assumption: the direct P-wave azimuth aligned with the azimuth of the source position relatively to the geophone; this approximation is valid only if the dip does not exceed $20^{\circ}$ laterally to the vertical plane containing source and receiver.

Figure2 VSP orientation from offset P-wave a) vertical plane projection b) Horizontal plane projection

This process uses the time of the direct P-wave made on the vertical axis of the well, whose shape is consistent on adjacent depth levels (figure 3). This process becomes inapplicable if the energy of P-direct arrival is too low on the horizontal components. This method of estimating the orientation of the three sensors by maximizing energy signal of linearly polarized direct P-wave (DiSiena et al., 1984; Becquey and Dubesset, 1990; X. Zeng et al., 2001) in a time window defined by the vertical or axial component at the well, preferably applies to geometries of offset VSP (figure2), which includes at least one seismic 
surface source positioned at a fixed offset distance from the well (typically equal to 0.2 to 1.5 times the total vertical depth of wells).

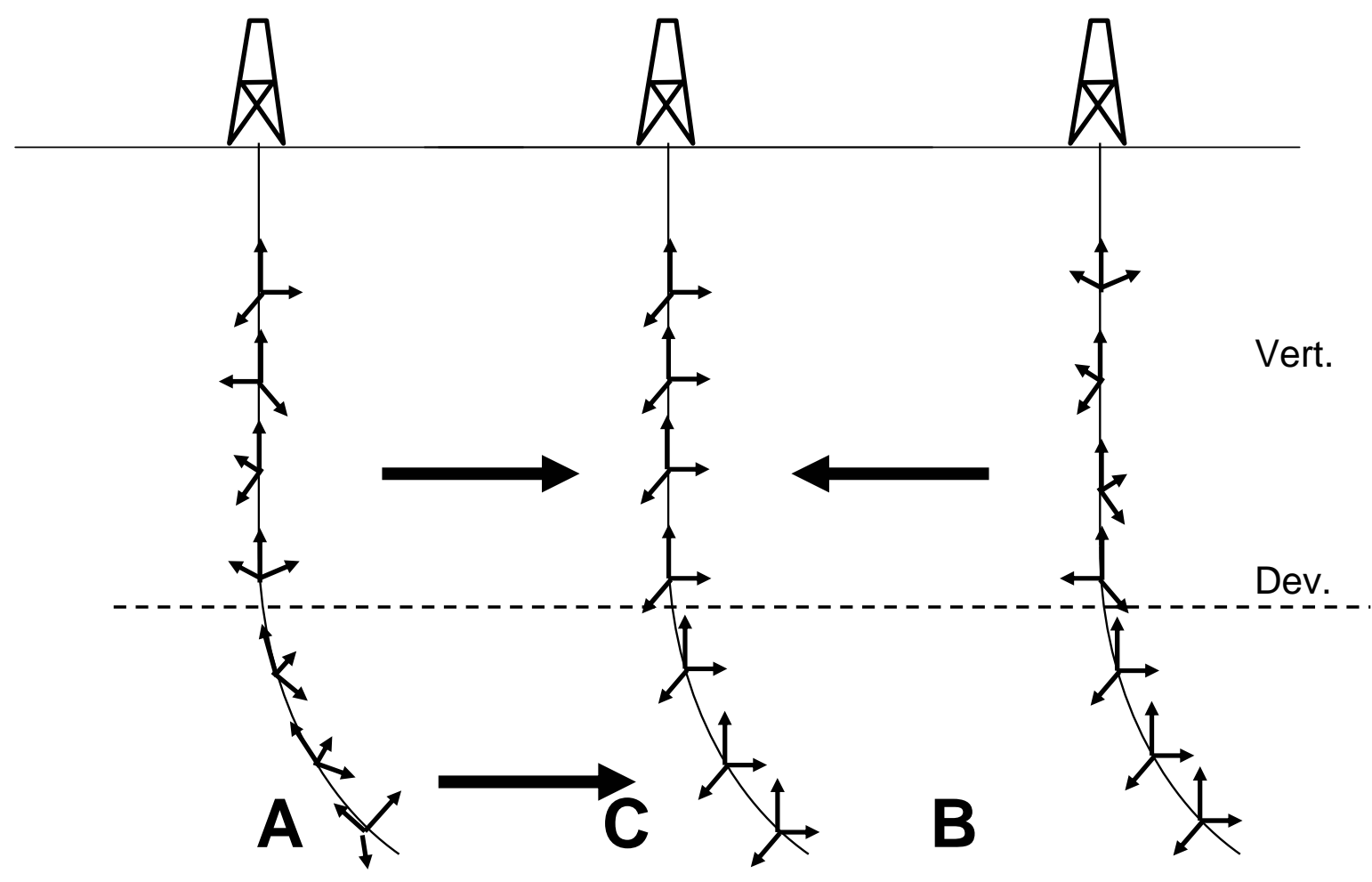

Figure 3 Configurations A or B represent the input 3C data orientation from field measurements. Configuration $\mathrm{C}$ represents the output from the $3 \mathrm{C}$ data orientation pre-processing method

Configuration A of figure 3 corresponds to 3C data recorded with a FIXED 3 component sensor setting in the VSP tool; Z component is aligned with the well axis, $\mathrm{X}, \mathrm{Y}$ are orthogonal to $\mathrm{Z}$, oriented in random direction versus depth station.

Configuration $\mathrm{B}$ corresponds to $3 \mathrm{C}$ data recorded with a GIMBALLED 3 component sensor setting in the VSP tool; $\mathrm{Z}$ component is vertical, $\mathrm{X}, \mathrm{Y}$ are horizontal, oriented in random direction versus depth station. Configuration $\mathrm{C}$ corresponds to $3 \mathrm{C}$ data oriented in a the same coordinate system for all depth stations: Z component is vertical, X, Y are horizontal, oriented in same azimuth, known or unknown, allowing $3 \mathrm{C}$ data processing to be performed; The $\mathrm{X} \&$ $\mathrm{Y}$ azimuth can be calibrated into geographic direction at one or several discrete depths over 
the logged interval, using external information such as tool orientation device, or geological knowledge of down-dip azimuth from 3D surface seismic or other logs.

a

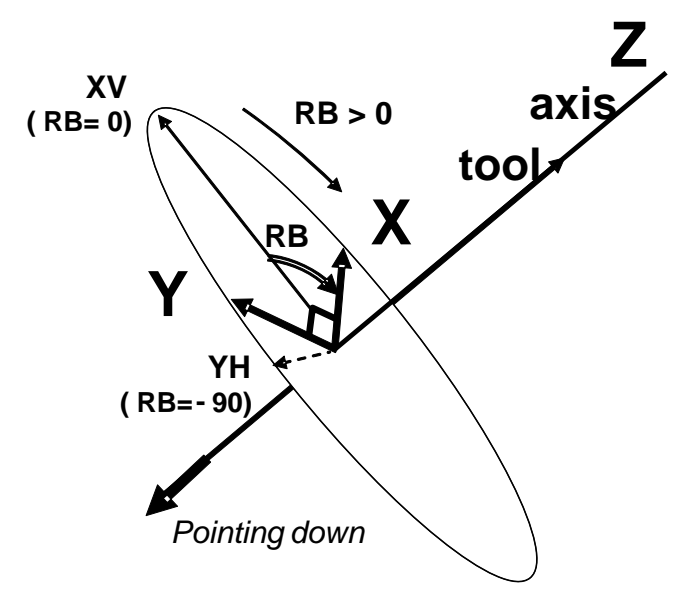

Relative Bearing Angle illustration in plane orthogonal to well/tool axis $[X V, Y H]=\operatorname{Rot}(\mathrm{RB}) .[\mathrm{X}, \mathrm{Y}]$ b

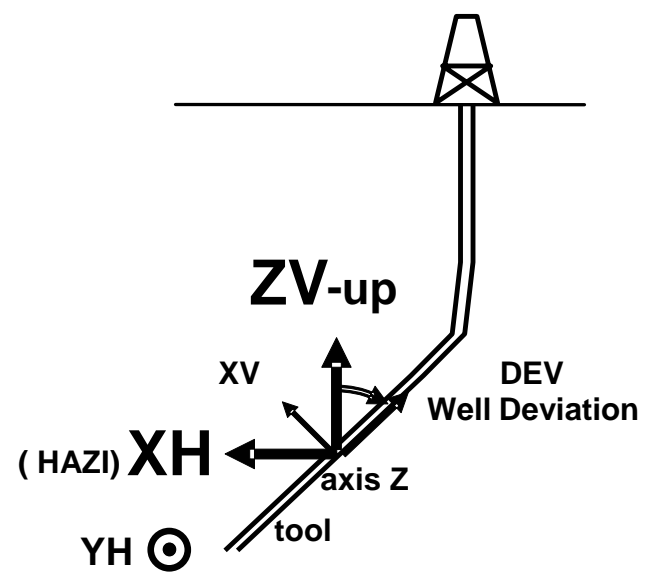

Figure4 Orientation of three fixed components with RB sensor in a deviated well, corresponds to contractor tool notations and conventions; a) relative bearing angle definition in the plane orthogonal to the well axis and rotation into vertical plane of deviation b) sketch of well trajectory and components in the vertical plane of deviation locally tangent to the well 


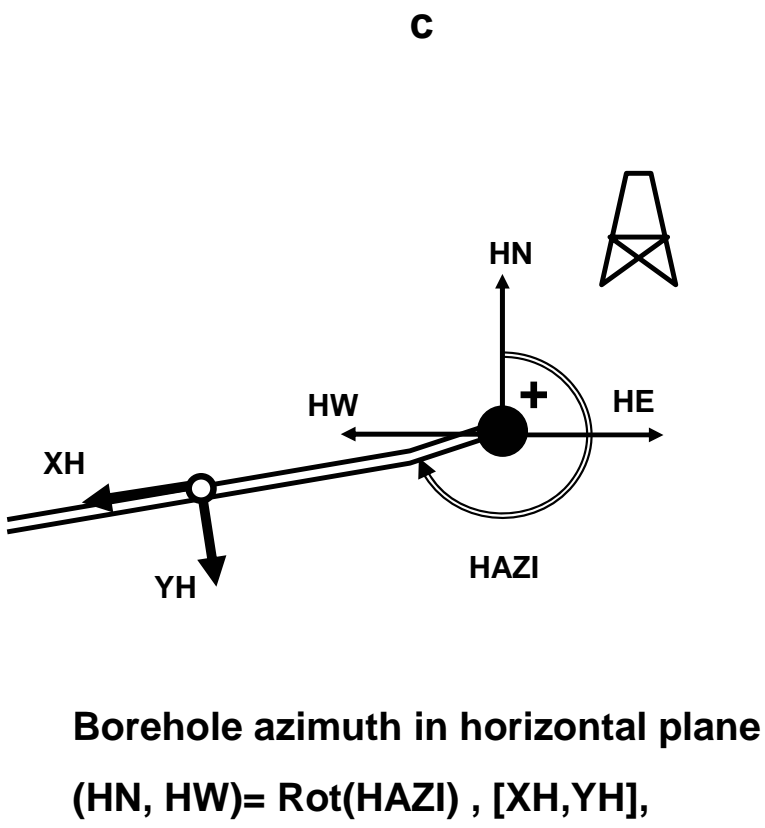

figure 4c) sketch of partially oriented component in the horizontal plane

Figure 4 depicts the orientation steps from three fixed components recorded by a VSP tool in a deviated well, into geographic directions.

Figure 4a shows the relative bearing angle (RB) in a plane orthogonal to the well, and the first rotation with angle $\mathrm{RB}$

Figure $4 \mathrm{~b}$ shows the well trajectory in the vertical plane of deviation locally tangent to the well and the seismic components implied in the second rotation with deviation angle (DEV).

Figure $4 \mathrm{c}$ illustrates the components in the horizontal plane and the seismic components implied in the third rotation with borehole azimuth angle (HAZI).

The recorded fix three components can be oriented into geographic coordinates by applying three successive rotations using the relative bearing angle (RB), the borehole deviation angle (DEV) and the borehole azimuth angles (HAZI) in the following order:

1) $(\mathrm{XV}, \mathrm{YH})=\operatorname{Rot}(\mathrm{RB}) \cdot[\mathrm{X}, \mathrm{Y}] \quad$ in plane orthogonal to the well axis (figure4a)

2) $(\mathrm{ZVup}, \mathrm{XH})=\operatorname{Rot}(\mathrm{DEV}) \cdot[\mathrm{Z}, \mathrm{XV}]$ in horizontal plane of borehole deviation (figure $4 \mathrm{~b})$

3) $(\mathrm{HN}, \mathrm{HW})=\operatorname{Rot}(\mathrm{HAZI}) \cdot[\mathrm{XH}, \mathrm{YH}]$, in horizontal plane (figure $4 \mathrm{c})$

4) $\mathrm{HE}=-\mathrm{HW}$

We applied this method on real data and it successfully oriented the VSP data where the well deviation angle (DEV) is larger than 13 degrees. Below this deviation value the RB sensor is not accurate enough, so that another technique is needed. 


\section{6-3 Basic summary of propagation of seismic body waves}

Figure 5 illustrates the earth motion along the propagation of a plane body wave in P-wave mode (figure 5a) and in S-wave mode (figure 5b). Figure5a shows the alternating compressions (pushes) and dilations (pulls) which are oriented in the same direction as the Pwave propagation along the ray path; and therefore, perpendicular to the wavefront.

In contrast, on figure 5b, shows a shear wave displacement perturbation as an upward motion followed by a downward motion, which are oriented orthogonally to the S-wave ray path.

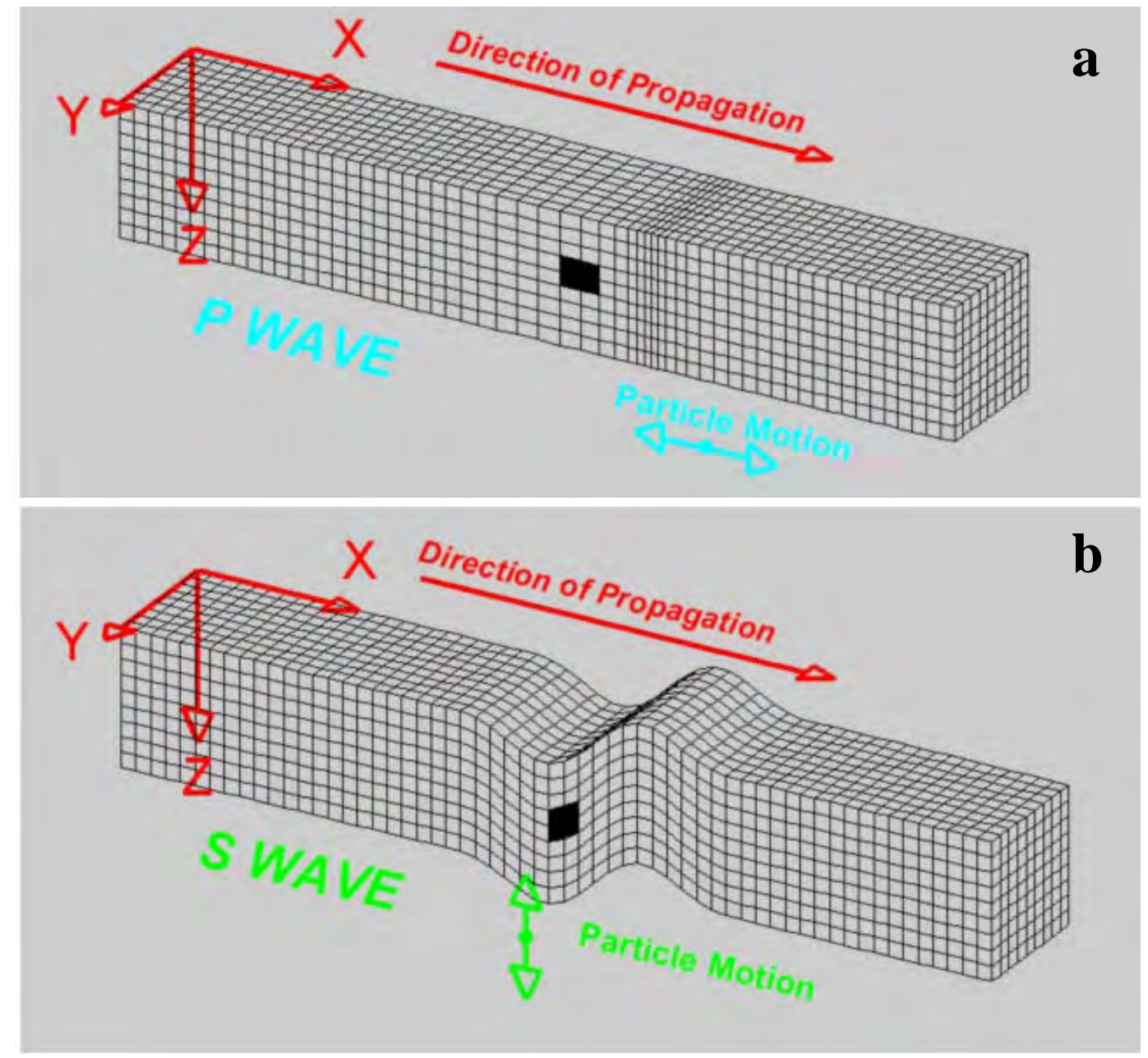

Figure 5

a) Perspective view of elastic $\mathrm{P}$-wave propagation along the $\mathrm{X}$ direction through a grid representing a volume of elastic material.

b) Perspective view of S-wave propagation through a grid representing a volume of elastic material. 
The particle motion of an S-wave in anisotropic medium remains near perpendicular to the direction of propagation but there are two Eigen modes of S-wave, with orthogonal linear polarization in the plane perpendicular to the propagation. In both $\mathrm{P}$ and $\mathrm{S}$ modes, the material returns to its original position after the wave has passed.

a

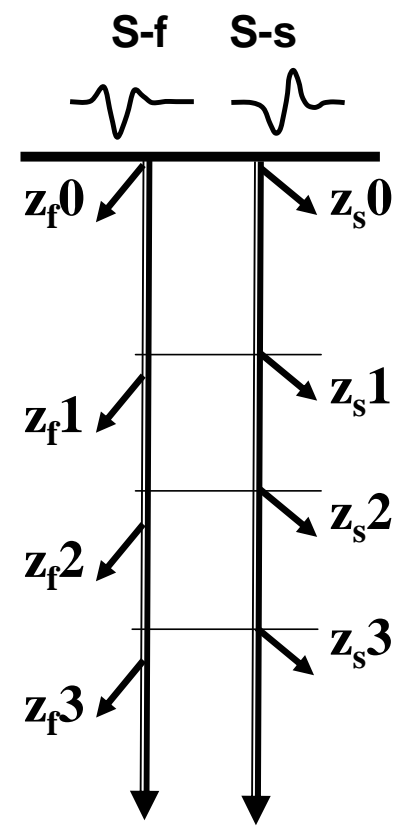

b

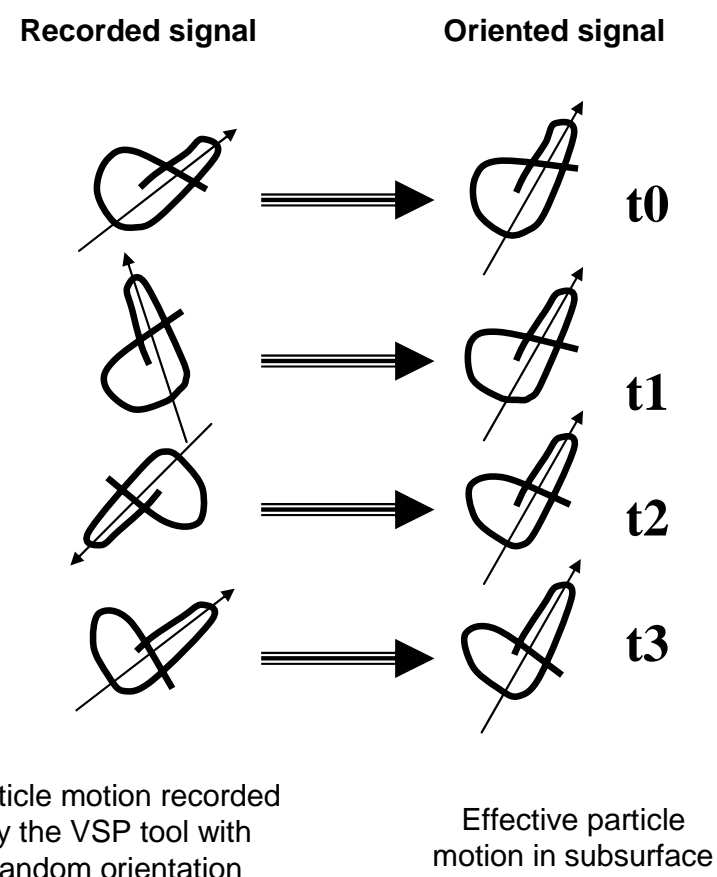

Figure6 Sketch of principle; propagation of eigen modes of shear waves in stratified, low dip and weakly anisotropic medium

Figure 6a illustrates the S-wave splitting phenomenon into two linearly polarized eigen modes S-slow (S-s) and S-fast (S-f) in anisotropic homogenous medium at successive stages of vertical propagation, at times $\mathrm{t} 1, \mathrm{t} 2, . . \mathrm{ti}$, for which the $\mathrm{S}$ wave train reaches the successive depths $\mathrm{z} 1, \mathrm{z} 2, \ldots \mathrm{zi}$.

Figure $6 \mathrm{~b}$ and $\mathrm{c}$ show the particle motion or hodogram of the shear wave train, in the plane orthogonal to the propagation, respectively before (6b) and after (6c) orientation. The general shape of hodograms slightly changes with increasing depth in presence of weak anisotropy. 


\section{6-4 Orientation method of recorded horizontal components into a coherent system using down going shear wave residuals}

The innovative method described hereafter concerns the orientation of geophones of a multicomponents sensors to compensate for the absence of any orientation measurement device combined with the VSP down hole tools for the field acquisition. The orientation of seismic components into a geographic reference system, or at least coherent system of unknown azimuth is necessary prior to processing the three components, for the benefit of the structural and geological interpreters.

The method includes the following steps:

1) Building the "modulus" signal by calculating the square root of the sum of the squares of these two components orthogonal to the known vector $Z$, for each time successive ti $M^{2}\left(t_{i}\right)=X^{2}\left(t_{i}\right)+Y^{2}\left(t_{i}\right) \quad$ Where $\mathrm{M}$ is the modulus, $\mathrm{X}$ and $\mathrm{Y}$ are horizontal components The modulus is shown on the S-wave particle motion of figure7a, and it is independent from the coordinate system orientation, as illustrated on figure $7 \mathrm{~b}$

2) Time picking of down-going shear wave first arrival on a peak or trough amplitude of the filtered modulus signal. As the raw modulus signal is always positive, a low cut filter ( e.g. LC5 Hz ) is applied in order to improve the display of the modulus signal and facilitate the time pick of the main seismic arrivals depth level to depth level(figure 7c).

3) Orientation of the input components $\mathrm{X}$ and $\mathrm{Y}$ into on true geographical coordinate system, using the following steps repeated for each depth level:

3a-Defining a time window along the shear wave time arrivals previously picked

3b-Determining the direction of maximal energy of the $(\mathrm{X}, \mathrm{Y})$ seismic components in the time window.

3c-Rotate the input $(\mathrm{X}, \mathrm{Y})$ seismic components along the direction of maximal energy previously computed, representing a coherent; nearly fix azimuthal geographic direction, generally unknown.

The result of all above steps can be seen on figure 8 (raw data) and figure9, after orientation.

4) Determining the true geographical orientation the coherent system previously defined, at one or several discrete depths or depth intervals using different methods, as explained in the next paragraph (Calibration of the reference system). 


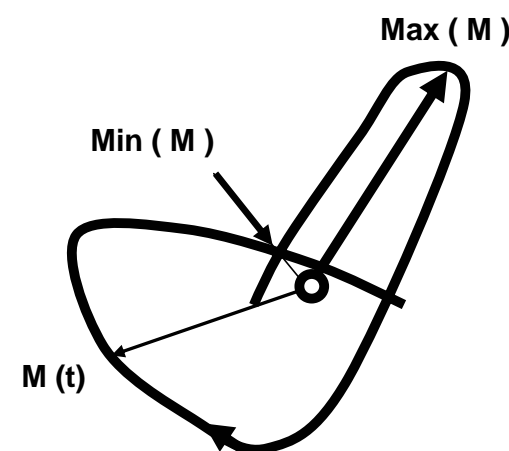

increasing time

Sketch of an eliptical S-wave particle motion

Figure 7a Modulus vector on an S-wave particle motion

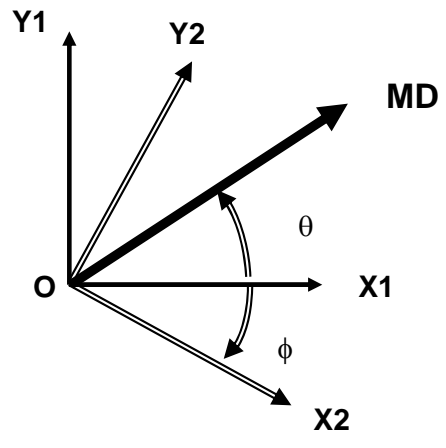

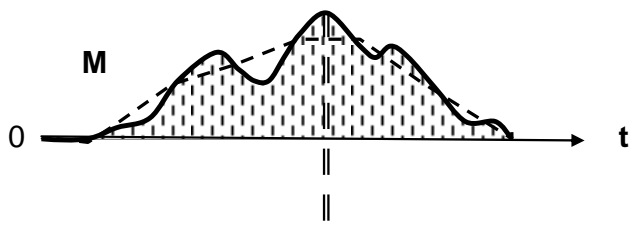

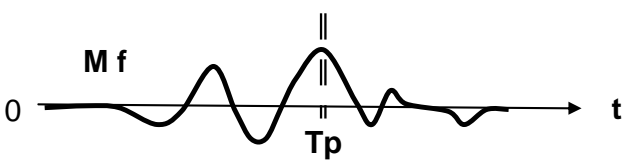

Modulus trace before (above) and after (below) filter

Figure 7c Raw and filtered modulus trace

Modulus Vector MD coordinates in the X1,Y1 system: $\mathrm{MD}=(\mathrm{X} 1, \mathrm{Y} 1)$ in Cartesian coordinates $\mathrm{MD}=[\mathrm{M}, \theta]$ in polar coordinates

Modulus Vector MD coordinates in the X2, Y2 system:

$\mathrm{MD}=(\mathrm{X} 2, \mathrm{Y} 2)$ in Cartesian coordinates

$\mathrm{MD}=[\mathrm{M},(\theta-\Phi)]$ in polar coordinates

By definition, the amplitude of Modulus vector remains invariant versus coordinate system orientation, for any time sample:

therefore, the modulus trace $M(t)$ remains invariant versus coordinate system orientation, which allows to pick times accurately BEFORE orientation

Figure $7 \mathrm{~b}$ Modulus vector in two rotated coordinate systems 


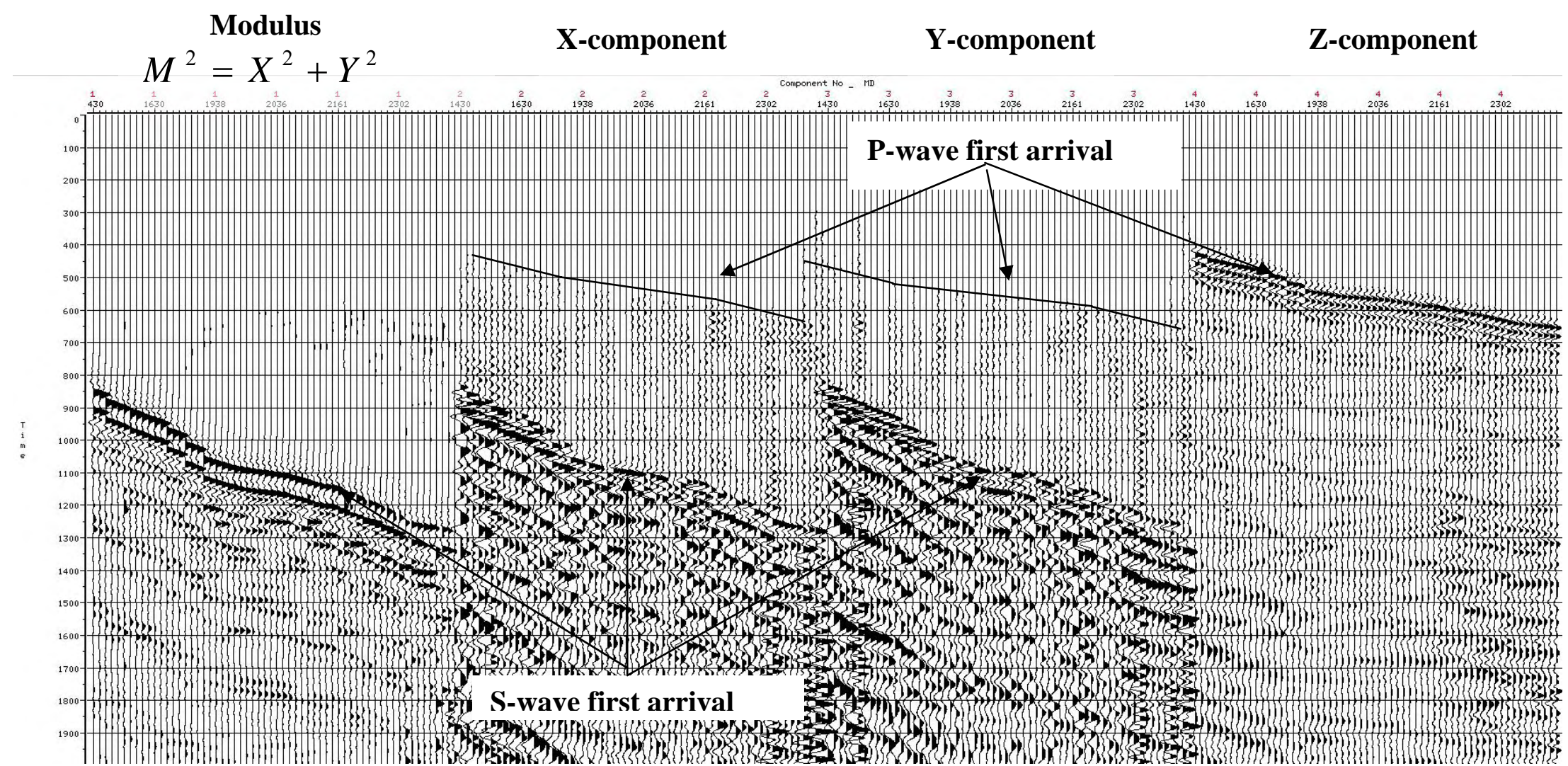

Fig 8 From left to right Modulus, $\mathrm{X}, \mathrm{Y}$ and Z-components before orientation. First arrival S-waves are clear on horizontal components while on the Z-component P-wave first arrivals are sharp to pick. First arrival S-waves are not consistent before orientation while modulus first arrivals are identical before and after orientation. X, Y and Z components are displayed with same constant gain while modulus has been normalized. 


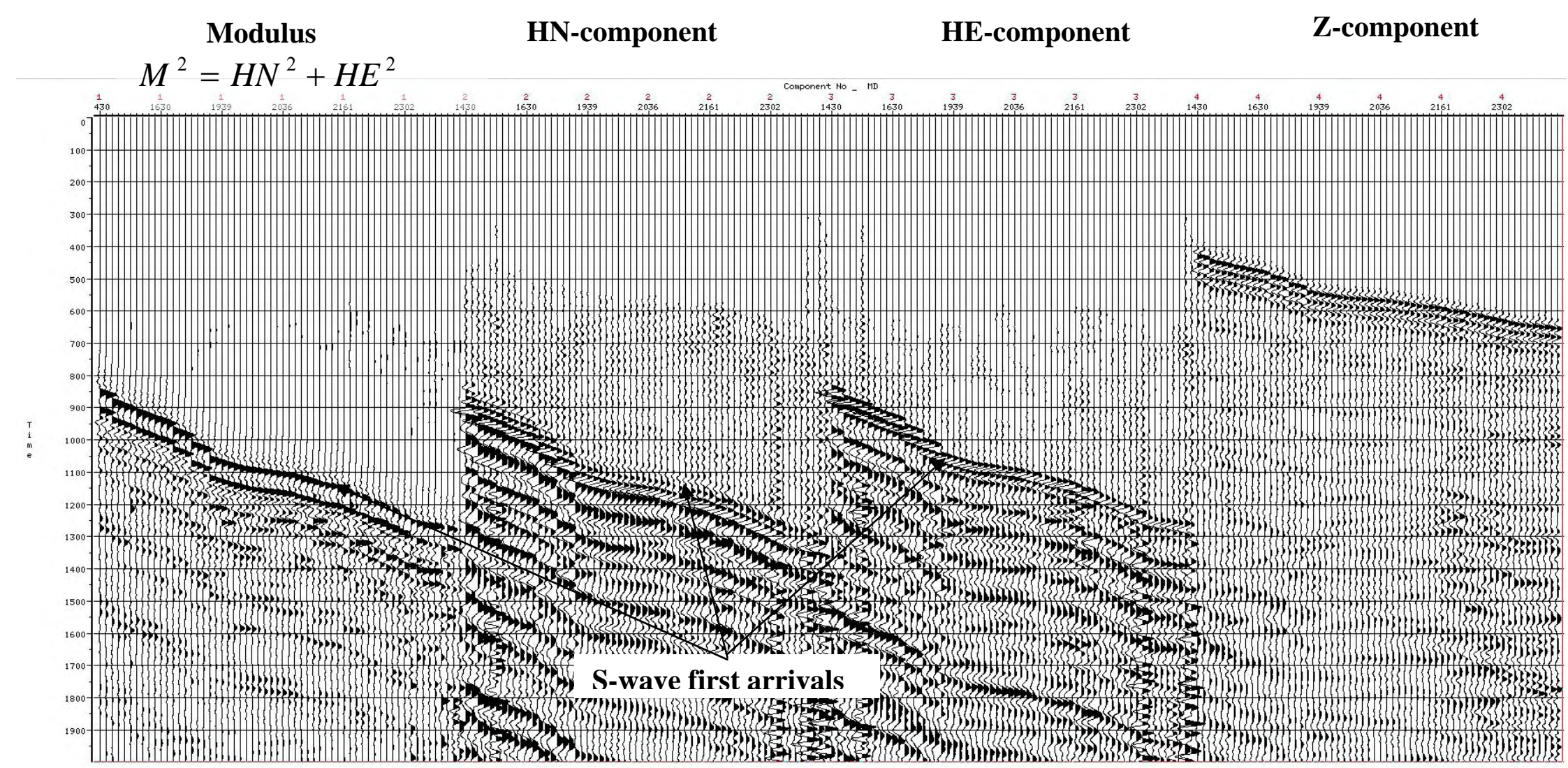

Fig 9 From left to right Modulus, HN, HE and Z-components after orientation. On the Z-component P-wave first arrivals are sharp to pick. First arrival S-waves are coherent after orientation. The filtered modulus signal first arrivals is identical before and after orientation. $\mathrm{HN}, \mathrm{HE}$ and $\mathrm{Z}$ components are displayed in constant gain while modulus has been normalized. 
Figure 8 shows the three recorded components $\mathrm{Z}, \mathrm{X}, \mathrm{Y}$, right to left, in constant gain plus modulus signal: the modulus on far left is computed form $\mathrm{X}$ and $\mathrm{Y}$ only, then filtered bandpass $(5-50 \mathrm{~Hz})$ in order to exhibit more clearly the local troughs and peaks occurring along the high amplitude direct S-wave arrival. One can verify that the filtered modulus signal is very coherent in depth, which enables to pick the S-wave time with high accuracy.

Figure 9 shows the three oriented components ZV, HN, HE, right to left, in constant gain plus modulus signal: the modulus on far left is computed form $\mathrm{HN}$ and $\mathrm{HE}$ only, then filtered bandpass $(5-50 \mathrm{~Hz})$ : it is identical as the modulus displayed on Fig.8 left, which illustrates the invariance of the modulus with respect to the coordinate system.

\section{The depth to depth coherence of the seismic signal on the oriented horizontal components demonstrates the excellent vector fidelity of the VSP tool used for the acquisition, and the efficiency of the orientation method, which are both necessary to render usable the horizontal components of the VSP seismic data.}

Figure10 shows particle motion hodograms of real data before and after orientation which demonstrate coherent trend after successful orientation. Note that shear wave particle motion is stable with depth along a given direction of propagation (in practice within a small solid angle of propagation).

Figure11 illustrates direct shear wavetrain horizontalized. Top display represents S-slow in azimuth $\mathrm{N} 25 \mathrm{E}$ and middle display is $\mathrm{S}$-fast (S115E) wave orthogonal to S-slow by $90^{\circ}$ clockwise. Bottom display represents the filtered modulus traces horizontalized along the first peak, the same time pick has been used to horizontalize S-slow(top) and S-fast(middle) signals. Measured depth and deviation angle are displayed on the top of section. The boxes on the first shear wave arrivals represent the maximization time window $( \pm 15 \mathrm{~ms})$ around $\mathrm{t}=0.10 \mathrm{~s}$. The deep depth interval indicates large deviation angle where RB angle measurement is valid is used for calibration to true geographic coordination system. From 2550 to the total depth was not usable due to noisy shear wave first arrival.

In addition, in the $600-1500 \mathrm{~m}$ depth interval exhibits obvious sign of anisotropy, as the slow S-wave is seen to acquire an increasing time delay, up to $10 \mathrm{~ms}$, relatively to the horizontalized fast S-wave which appears perfectly horizontalized. Below $1500 \mathrm{~m}$, both Swaves propagate with same velocity, meaning that the formations are isotropic. 
a

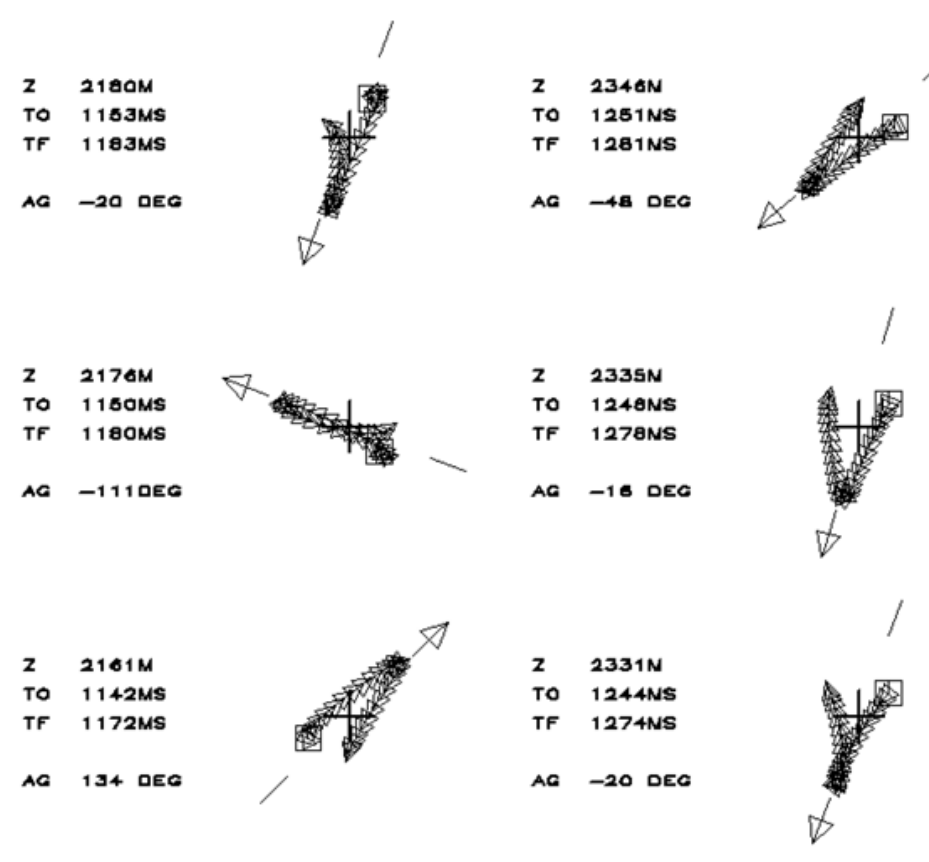

b

$\begin{array}{ll}z & 2180 M \\ \text { TO } & 1183 M S\end{array}$

TO $1183 \mathrm{MS}$

AQ -SO DEO

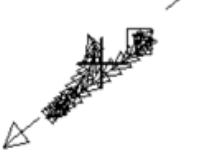

$\begin{array}{ll}Z & 2346 \mathrm{M} \\ \text { TO } & 1251 \mathrm{MS}\end{array}$

To $1281 \mathrm{MS}$
TF $1281 \mathrm{MS}$

AO -44 DEO

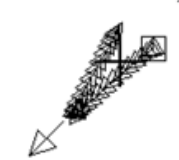

$22170 \mathrm{M}$

TO 11 SOMs

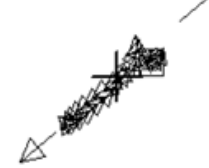

$2 \quad 2335 \mathrm{M}$

TO $124 a \mathrm{Ms}$

TF rans

AC -48 DEC

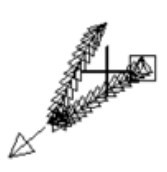
$2 \quad 2101 \mathrm{M}$
$\begin{array}{ll}\text { TO } & 1142 \mathrm{MS} \\ \text { TF } & 1172 \mathrm{MS}\end{array}$

AC to DEC

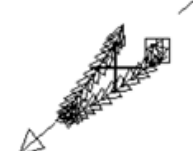
TO $1244 \mathrm{M}$
TF $1274 \mathrm{MS}$

AC -DS DEC

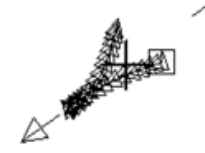

Figure10 Shear wave particle motion before(a) and after (b) orientation in true geographic coordinates. Consistency of orientation is obtained by aligning the direction of maximal energy, along the arrow into the same direction.

On the left side of any hodogram; Z, TO, TF and AG represents the depth, beginning and ending of time maximation window and relative bearing angle respectively. 


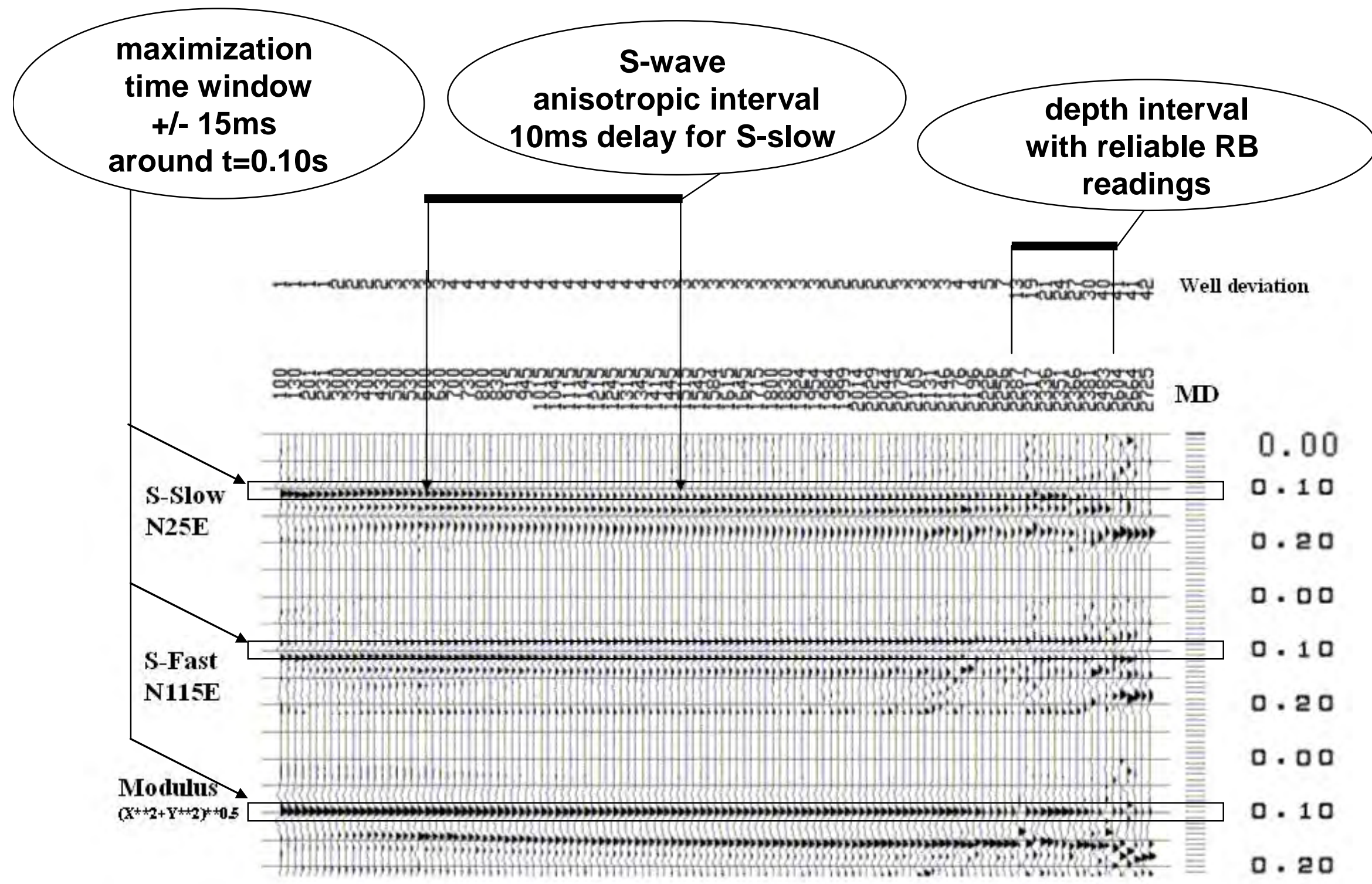

Figure 11 Direct shear wave horizontalized, top display is S-slow in azimuth N25E and middle display represents S-fast wave orthogonal to S-slow $90^{\circ}$ clockwise. Bottom display represents the filtered modulus traces horizontalized along the first peaks; the same time pick has been used to horizontalized Sslow(top) and S-fast(middle)signals. 


\section{6-5 Calibration of the $3 \mathrm{C}$ data into a geographic reference system}

Figure 12a sketches an incident P-wave which travels vertically and encounters a dipping reflector with angle $(\alpha)$. In this configuration, the reflected P-P wave is polarized in the horizontal direction of the down-dip azimuth of the reflector, $\pm 360^{\circ}$, and the vertical incidence angle of the reflected P-P wave equals twice the dip angle $(2 \alpha)$.

The dip and azimuth of a reflector seen by VSP in the corridor stack domain can be defined by the orientation of the Normal $(\mathrm{N})$ to the reflector. The normal $(\mathrm{N})$ to the reflector is the bisector of incident ray (I) and reflected ray (R).

Conversely, the dipping azimuth of a reflector determined from 3C VSP data previously oriented in a coherent coordinate system of unknown azimuth, can be used to calibrate the geographical orientation of the horizontal components of the initial VSP dataset oriented in a coherent system of unknown azimuth.

a

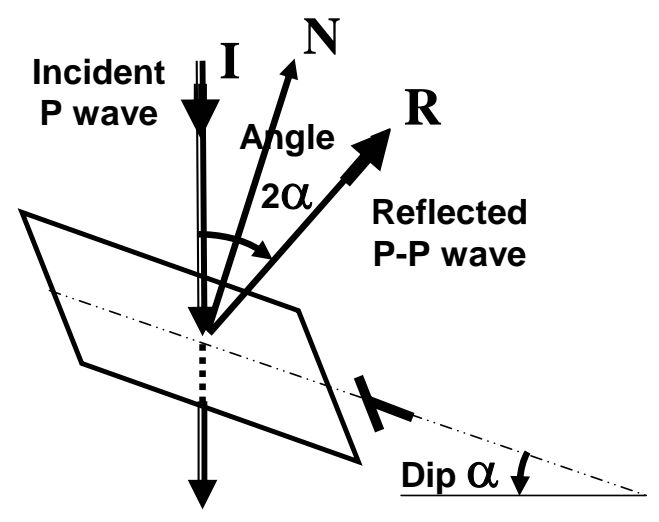

Vertical propagation

for zero-offset VSP b

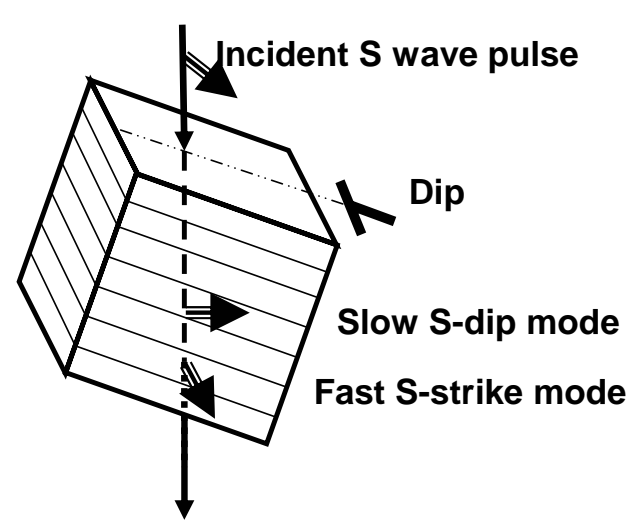

Vertical propagation

for zero-offset VSP

Figure 12 Vertical P-wave propagation and reflection(a) and S-wave propagation(b) in dipping layered medium 
Figure $12 \mathrm{~b}$ illustrates the $\mathrm{S}$-wave propagation in a dipping layered medium.

Layering anisotropy induces the splitting of the incident S-wave into:

a) Fast horizontally polarized S-strike wave, oriented in the strike direction parallel to layering.

b) Slow horizontally polarized S-dip wave, oriented in the azimuth of the dip

Therefore the separation of the direct S-wavetrain split by layering induced birefringence can help to calibrate the azimuth trend of the general dip, when known from external structural information (figure $12 \mathrm{~b}$ )

Actually, the results of figure9 were obtained using the RBS readings in the VSP tool in the deviated part of the well, near the well bottom, in order to "geographically" calibrate the azimuth of the coherent reference system obtained by S-wave maximisation. The three rotation process described in the previous section has been applied. The well deviation angle appears on figure 11 , and is larger than $13^{\circ}$ near well bottom, on the right.

\section{6-6 Discussion}

We applied this technique on two VSPs field data from the Zagros and successfully oriented data. In the first case the orientation result confirmed by 3D seismic section passing through the well, 3D seismic time map and underground well contour map of certain reflector/formation. The simplicity and efficiency of the orientation process prompted the authors to file for a patent, although additional tests would be necessary to assess the actual accuracy of the method. Some progressive rotation of the coordinate system could occur without much control, and it is desirable to obtain a calibration of the orientation at several depth intervals. 


\section{6-7 Summary of patent Claims}

The patented method for orienting the three components of single zero-offset source VSP's recorded in near vertical wells consists in the following steps:

a) compute the modulus signal $\mathrm{M}(\mathrm{t})$ from the $\mathrm{X}(\mathrm{t})$ and $\mathrm{Y}(\mathrm{t})$ signals, the modulus signal $\mathrm{M}(\mathrm{t})$ being independent from the VSP tool orientation; the modulus trace $\mathrm{M}(\mathrm{t})$ can be filtered in order to enhance the visualisation and detection of the main, high energy seismic arrivals (INNOVATIVE procedure)

b) Time pick a coherent downgoing line-up of high energy, corresponding most of the time to the S-wave direct arrival or to a converted-transmitted P-S wave arrival. S-wave time pick and velocity can be derived (INNOVATIVE procedure)

c) Define a time window along above defined time pick for maximisation of the energy of the seismic signal from $\mathrm{X}(\mathrm{t})$ and $\mathrm{Y}(\mathrm{t})$ initial signal, and determination of direction of maximal energy.

d) rotate initial signals $\mathrm{X}(\mathrm{t})$ and $\mathrm{Y}(\mathrm{t})$ into the direction of maximal energy Hmax and orthogonal direction Hmin, which constitute a coherent coordinate system ( Hmax, Hmin) oriented in an unknown fix azimuth angle from geographical directions ( INNOVATIVE procedure).

e) calibrate the above ( Hmax, Hmin) coordinate system relatively to geographical North using one or several of the following INNOVATIVE sub-procedures:

e-1) using the RB angles when measured, or the orientation provided by gimbal systems, whenever the well vertical inclination is larger than $10^{\circ}-15^{\circ}$

e-2) orienting the shallow VSP levels using the P-wave residuals on horizontal components.

e-3) detecting and quantifying the S-wave birefringence anisotropy, which principal direction coincide to the layering dip and strike directions locally to the well.

e-4) extract the reflected wavefield in 3 components by isotropic processing and derive dip and azimuth of main reflectors, to be adjusted on dip and azimuth of horizon maps from external knowledge, such as 3D surface seismic, maps from wells, etc...

f) improve the field QC control of VSP acquired data in real time, and demonstrate that the $3 \mathrm{C}$ VSP data can be oriented, thus processed as a $3 \mathrm{C}$ dataset (INNOVATIVE process) 
g) Simplify the downhole hardware complexity by combining an orientation device on a single shuttle of a multilevel VSP tool, since adjacent levels can be oriented relatively to one another (INNOVATIVE approach) 



\section{Chapter 7}

\section{Look ahead VSP using oriented 3 \\ Components in a deep exploration well in mountainous Zagros, Iran, and post drilling integrated interpretation with surface seismic and well data}


This chapter describes the integrated interpretation of one of the exploration structures in rugged mountainous Zagros. To perform this interpretation, 3 component VSP data, 2D surface seismic line, resistivity image log, well completion report and geological information were used. The image of 2D seismic line which acquired during the drilling stage was very poor. The processing of the VSP vertical component showed signs of P-P reflectors beyond the intermediate total depth $5300 \mathrm{~m}$, however no significant indication of dip could be obtained. VSP 3 component dip results drastically differ from the dip read on the borehole resistivity image.

The reprocessing of the VSP using the oriented 3 components confirmed the existence of a reliable P-P mode seismic reflector located about $500 \mathrm{~m}$ below intermediate T.D, with negligible dip. This information gave confidence to NIOC explorationists for pursuing drilling, down to $6 \mathrm{~km}$ deep, which represented a costly decision.

The following paper demonstrates how the integration of different dataset can enhance the imaging and target geometry and help making more reliable drilling decisions. 


\title{
Look ahead VSP using oriented 3 Components in a deep exploration well in mountainous Zagros, Iran, and post drilling integrated interpretation with surface seismic and well data
}

by K. Kazemi ${ }^{12}$, C. Naville ${ }^{1}$

${ }^{1}$ Institut Français du pétrole, 1-4 avenue de Bois-préau Rueil Malmaison, 92852, France

${ }^{2}$ National Iranian Oil company, NIOC-Exploration, P.O box 19395-6669 Tehran, Iran

\begin{abstract}
Prior to drilling the knowledge of dip and azimuth estimates at target depth is crucial for exploration wells. When surface seismic is very poor the drilling risks are further increased and drilling planning relies mainly on geological considerations.

A deep exploration well was drilled down to $5300 \mathrm{~m}$ on the top of a surface fold in the mountainous Zagros with the aim to encounter a deep anticline at the top of Tertiary reservoir formation known in the area. Structural interpretation was performed using 3 components intermediate VSP, 2D surface seismic, well logs, borehole resistivity imaging and geological data.
\end{abstract}

The processing of the vertical component of VSP executed by the logging contractor showed signs of P-P reflections beyond the total depth 5300m, however no significant indication of dip could be obtained. This reason lead to reprocess the 3 components of the VSP after accurate orientation. The processing of the oriented 3 component VSP evidenced a weak amplitude reflector with negligible dip and substantial lateral extension at about $5840 \mathrm{~m}$ depth, $500 \mathrm{~m}$ below the intermediate drilled depth. Therefore, the interpreters decided to pursue the drilling operation as they hoped to encounter the top 
Tertiary objective not far beyond the $5300 \mathrm{~m}$ temporary drilled depth. The VSP and synthetic seismogram confirmed the absence of good quality reflectors on the surface seismic section which renders exploration more challenging. The present paper exposes a comprehensive structural study using all the data available from surface and borehole in order to understand better the response the different methods in the wildcat area considered. Last, the integration of different dataset enhances the imaging of target geometry and can help making more reliable drilling decisions, as long as this integrated interpretation can be achieved timely.

\section{Introduction}

Well B is a deep exploration well located in the rugged mountainous Zagros region. 2D seismic profiling recorded between drilling periods did not yield any clear structural indication (Figure1). A comprehensive set of logs were recorded down to $5300 \mathrm{~m}$, including an resistivity imager and a zero offset VSP. Reprocessing the VSP in oriented 3 components was undertaken to confirm the identification of P-P reflectors and their dip/azimuth within the drilled interval and below intermediate total depth $(5.3 \mathrm{~km})$.

A fairly good P-P reflector was intersected by the well around $5000 \mathrm{~m}$, but its cinematic and near null dip from VSP drastically differs from the resistivity imager readings, indicating steep dips from $55^{\circ}$ to $70^{\circ}$.

As the well did not reach the targeted Tertiary formation at $5300 \mathrm{~m}$, the reliable assessment of any reflected events below T.D. was crucial to motivate the pursuit of drilling of the present wildcat well. In this respect, it was decided to process the three components of the VSP in order to confirm that the seismic events seen within $600 \mathrm{~ms}$ twt 
below intermediate $5300 \mathrm{~m}$ total depth (T.D), are actually P-P reflections, especially as the drilling operation stopped in late 2006 for the winter season and was postponed to the spring 2007 if it had to be continued.

The reprocessing of the VSP using the oriented 3 components confirmed the existence of a reliable P-P mode seismic reflector located about $500 \mathrm{~m}$ below intermediate T.D, with negligible dip. This information gave confidence to NIOC explorationists for pursuing drilling, down to $6 \mathrm{~km}$ deep, which represented a costly decision. Drilling confirmed that the predicted reflector does not actually correspond to a lithological change but to a thrust fault surface, within the Dashtak overburden formation, therefore, the predicted reflector is unrelated to the targeted reservoir formation.

The absence of reflected events on the 2D seismic section particularly below $5000 \mathrm{~m}$ depth has been investigated using the borehole information.

The sonic log was calibrated with the VSP first arrival times. The density log was confronted with Caliper, GR and sonic in order to evaluate its reliability. The resulting synthetic seismogram shows weak reflection coefficients and clear discrepancies with the VSP corridor stack. Splicing the synthetic seismogram and corridor stack on the 2D section indicates that no reflection appears on the surface seismic section within the logged interval or below it, likely due to the absence of any deep reflecting interface with sufficient lateral coherency. 


\section{VSP survey and Pre-processing}

A rig source VSP survey had been recorded mainly in the open hole of the near vertical deep exploration well, using a 2x 150ci G-gun source in a water pit, using a two depth level VSP tool including fix three components $\mathrm{Z}, \mathrm{X}, \mathrm{Y}$ of seismic sensors, $\mathrm{Z}$ being aligned with the well axis, and a Relative Bearing sensor. The vertical inclination of the well reaches $16^{\circ}$ locally in the logged depth interval, 4800-5000m (Figure2 right). Checkshot stations were recorded from depth $4600 \mathrm{~m}$ up to surface. The quality of the three component VSP data is excellent, with respect to the Signal to Noise ratio $(\mathrm{S} / \mathrm{N})$ and vector fidelity. Soon after reaching the $5300 \mathrm{~m}$ TD, one of the two downhole VSP tool became affected by electrical noise bursts, therefore, the VSP field engineer simply switched it off for the whole acquisition. This incident shows that using a two level VSP tool in the open hole increases the efficiency of the acquisition as it can avoid a time consuming maneuver for pulling a defective tool out of the borehole and resume the whole operation.

Displays of the Z-component appear in raw recording-time on figure 2 left. Figure 2 middle shows the $\mathrm{Z}$ component data after time pick alignment, and confirms that the direct VSP wavelet is stable over the whole borehole depth. Figure 3 shows the timedepth and velocity curves after application of an obliquity correction to obtain vertical times.

As the seismic energy of the direct $\mathrm{P}$-wave arrival is very weak on the $\mathrm{X}, \mathrm{Y}$ components, the VSP data orientation has been achieved by maximization of the S-wave energy in the plane orthogonal to the well axis, followed by a calibration of the orientation into geographic coordinates using the relative bearing angles in the mildly deviated interval of 
the well, around $16^{\circ}$, according to the method described in filed French patents (application numbers: 09/00.643 and 09/00.916). Figure 4 shows the raw components $\mathrm{Z}, \mathrm{X}, \mathrm{Y}$ of the VSP data, left to right, as an isotropically normalized display, the normalization being applied on the whole set of three components at each depth level. Figure 5 shows the three components Z, H-North, H-east of the VSP data oriented into a geographic system, left to right, as an isotropically normalized display. It is remarkable how the orientation rendered the downgoing S-wave arrival totally coherent on adjacent depth levels (Figure5 versus Figure4). The P-wave energy residuals observed on the $\mathrm{X}$ raw component in the $4800-5000 \mathrm{~m}$ deviated interval (Figure4), almost vanished on the oriented horizontal components of Figure5. Conversely, the S-wave residuals observed on the raw $\mathrm{Z}$ component in the $4600-5000 \mathrm{~m}$ deviated interval (Figure4) around $1.7 \mathrm{sec}$, disappeared on the vertical component of Figure5.

\section{VSP Processing}

The result of the VSP processing of the single vertical component appears on Figure6 with the zero phase deconvolved reflected wavefield in two way time, high cut filtered $55 \mathrm{~Hz}$, displayed in constant gain (left), and corridor stack (right), on which a positive reflection coefficient is represented as a white trough; the separation of upgoing from downgoing wavetrains was achieved with a single narrow FK reject filter applied on aligned downgoing events.

Figure6 exhibits a clear reflector intersecting the well around $5000 \mathrm{~m} / 1.86 \mathrm{~s}$ twt, and a possible weak reliable P-P reflector below Total intermediate Depth (T.D.) 5300m, at $2.12 \mathrm{~s}$ two way time, which depth can be estimated at $5800 \mathrm{~m}$, assuming a $6 \mathrm{~km} / \mathrm{s}$ velocity 
beyond T.D. Both reflectors are underlined by a red dash line on Figure 6 and appear as near horizontal.

The isotropic processing of 3 component VSP is summarized by the flow chart of figure7.

The isotropic specification means that the same processing operation has been executed with the same parameters in the same time window on all three components, even in a time-variant manner. By doing so, the polarization of the desired reflected arrivals can be read at the end of the processing operations, in order to derive the dip and azimuth of reflectors (Naville et al. 2001, Serbutoviez, S., 2002).

The result of the VSP processing of the three geographical components is illustrated on Figure8, with the zero phase deconvolved reflected wavefield in two way time, displayed in an isotropic manner .

After isotropic deconvolution and rejection of the P-downgoing wavetrain, an additional isotropic velocity filter has been applied to reject S-wave downgoing residuals, followed by an isotropic spectrum balancing in true amplitude in the $10-38 \mathrm{~Hz}$ bandwidth, as no signal is received in higher frequencies; on the vertical component, a positive reflection coefficient is represented as a white trough. One can observe on Figure8 that most of the coherent upgoing seismic events appear with significant energy on the vertical component only, meaning that the dip of the reflectors at $5000 \mathrm{~m} / 1.86 \mathrm{~s}$ twt, and at $5800 \mathrm{~m} / 2.12 \mathrm{~s}$ twt is null, since the incident and reflected P-wave arrivals propagate vertically ( see the sketch Figure9); locally, some weak reflector facets with very limited lateral extension indicate a significant dip, about $22^{\circ}$ towards South for the blue event, $22^{\circ}$ towards North for the red event in the circled on Figure8. Since the amplitude of 
these reflected events are approximately in phase or out of phase between $\mathrm{Z}$ and $\mathrm{HN}$, with about the same amplitude, their polarization angle is linear, with about $45^{\circ}$ incidence, the dip angle being half of this value $\left(22^{\circ}\right)$.

Figure 10 shows the upgoing VSP wavefield in twt, $\mathrm{Z}$ component, processed by the logging contractor (right side) and reprocessed in this project (left side). The logging contractor result have been obtained after 7 trace median filter applied on horizontalized upgoing P-P reflection and normalized on each trace for display. In contrast, the reprocessed results on figure 10-left have been obtained by removal of the S-down going residual and display in true amplitude. There is good agreement between the two results; the same events are seen at the same time with same polarity. The median filter applied by logging contractor on the reflected wave field in two way time is too strong, therefore it might strongly attenuate possible dipping reflections. Although the reprocessing could have been limited to the vertical component, it was applied to the 3 components for confirmation of the low dip values of P-P reflections.

\section{Synthetic seismogram}

Sonic log calibration: Figure 11 shows the raw sonic log (left track green), and corrected sonic log (left track red), the time depth curve from integrated sonic (second track), the drift curve (third track) and residual drift after correction $\left(4^{\text {th }}\right.$ track, right). The drift corresponds to the difference between the one way vertical time derived from VSP time and the integrated sonic time. The "laminated " aspect of the sonic, density and GR logs in the $3700-5000 \mathrm{~m}$ interval suddenly disappears below 5000m (figure 13), and corresponds to an interval of substantial negative drift of $8 \mathrm{~ms}$ (figure 11), which is not 
expected in such compacted formation. Lithology variations were confirmed by geological information obtained while drilling and Gamma ray. In order to compute the synthetic seismogram (figure 13), the density log was edited according to the caliper log so as to eliminate the invalid measurements at the level of large caves and washouts (figure 12 ).

On figure 12 , three synthetic seismograms were produced using the calibrated sonic log, with raw density ( $3^{\text {rd }}$ track from right), with edited density (second track from right) and without density using just sonic log (first track from right).

The effect of density log on the synthetic seismogram is significant. The synthetic seismogram from raw density shows some strong reflections which are faked due to unreasonably low density values where large caves are present. The comparison between synthetic seismograms obtained from edited density and just using velocity below $5000 \mathrm{~m}$ (two rightmost tracks) shows low reflection values which are due mostly to small contrasts in density only. The constant velocity observed below 5000m depth results on the synthetic seismogram by a total lack of reflectivity on the rightmost track.

The weak reflection coefficients on the synthetic seismogram are coherent with the lack of reflectivity exhibited by the surface seismic section (figure1); the quality of surface seismic images is not likely to be improved drastically using new tools, methods and techniques in acquisition and processing.

Actually the reflectors F1 to F4 correspond to faults detected by the resistivity imager as we will see in the following paragraphs. 


\section{Confrontation of synthetic seismogram and VSP corridor stack}

Figure13: Synthetic seismogram built from calibrated sonic log and edited density log are confronted with the VSP corridor stack at same time scale and with similar frequency bandwidth $(8-40 \mathrm{~Hz})$. On the left the depth scale is displayed in correspondence with linear twt time scale. Both synthetic seismogram and corridor stack are displayed in SEG normal Polarity zero phase (White trough representing an increase contrast of impedance).

The major reflector on VSP corridor stack at about $5000 \mathrm{~m} / 1.86 \mathrm{~s}$ shows a significant mismatch with the synthetic seismogram. This discrepancy is most likely due to a local ruggedness of the reflector surface intersecting the well at this depth very different from a smooth sedimentary interface.

The black peak reflection appearing on the corridor stack at $5840 \mathrm{~m} / 2.13 \mathrm{~s}$, seen in prediction below T.D by the intermediate VSP and noted P.R on figure 13, is not evidenced on the synthetic seismogram, although it corresponds to a horizontal reflector with significant coherency laterally to the well (3C VSP results). This leads us to consider that this reflector does not correspond to any lithological changes but possibly to a fault surface. Unfortunately, there is no borehole resistivity imager measurement in this depth interval to confirm this possible interpretation. Nevertheless, the existence of this horizontal reflector gave confidence to the interpreters to resume drilling the well below $5326 \mathrm{~m}$ in early 2007. 


\section{Eelectrical resistivity results and interpretation}

Figures 14a,b and 15a,b exhibit logs and lithology derived from drilling data (well completion report) and structural cross-sections modeled from dips manually picked on borehole resistivity images. These structural interpretations are obtained assuming similar fold model in reference to the paper by Etchecopar \& Bonnetain 1992.

Figure 14a confront the standard logs (caliper, density, gamma ray and velocity) and the cross-section obtained from tad pole plot on the rightmost track. Sudden changes in dip attitude are clearly visible at depths $4720 \mathrm{~m}$ and 5020m corresponding to faults F1 and F2 respectively. On the expanded illustration on figure $14 \mathrm{~b}$, a zone of caving similar to the one commonly observed when a fault crosses a well, exists where F1 would intersect the well. The second fault F2 seems to be located at $5030 \mathrm{~m}$, i.e. $10 \mathrm{~m}$ below the contrast of velocity, density and gamma ray $(5020 \mathrm{~m})$. The structural section resulting from resistivity imager dips corresponds between F1 and F2 to a fold overturned to the West.

\section{Strikingly, the 3C VSP illuminates a horizontal reflector right at depth $5020 \mathrm{~m}$} (Figure6) corresponding to fault F2, while the resistivity imager results indicate very steep layering dips beyond $70^{\circ}$.

In retrospect, taking into account the very high dips and laminated aspect of the velocity $\log$ above $5020 \mathrm{~m}$, the negative drift observed on figure 11 above $5020 \mathrm{~m}$ can be reasonably interpreted as being generated by a layering anisotropy effect, resulting in VSP velocities higher than sonic velocities as the vertical propagation is parallel to layering in this particular depth interval.

Accordingly the absence of velocity layering below 5020m (Fault F2) corresponds to a null drift (figure 11). 
Figure 15a shows a structural cross-section interpreted from the resistivity imager dip results confronted to lithology and gamma ray(GR) in the 5100 to 5580m interval. Two additional faults (F3 and F4) are detected at depth 5280m and 5550m respectively, without obvious relationship to the lithology.

Figure 15b shows another structural cross-section from interpreted resistivity imager results around the F4 accident at 5543m.

\section{Confrontation of results from 2D surface seismic section, VSP, Synthetic Seismogram from calibrated sonic log, edited Density, and resistivity imager}

A 2D surface seismic line was acquired across the well and processed while drilling the shallow depth interval in order to tentatively image the subsurface structure (figure1), but no continuous events can be seen deeper than $1.3 \mathrm{sec}$. twt around the well location. The processing of this $2 \mathrm{D}$ line was trusted to two additional seismic companies, unfortunately no improvement of the seismic image was obtained (figure1). The single component VSP processing obtained by the logging contractor yielded an uncertain image of the subsurface, altered by excessive coherency enhancement of horizontal events on the upgoing wavefield (figure 10, right).

Figure16 shows the VSP corridor stack and calibrated vertical velocity log superimposed over the 2D seismic section. There is no continuous/coherent reflector matching with the VSP corridor stack. Locally to the well, a coherent reflector with fair local extent appears at $1.3 \mathrm{~s}$ from datum or $1.0 \mathrm{~s} \pm 0.05 \mathrm{~s}$ from ground level/floating datum on the seismic 
section (yellow ellipse); and corresponds probably to top Dashtak 2600-2800m from surface (ref time depth curve from VSP on figure 3); unfortunately this reflector cannot be tied with the VSP corridor stack or to other logs due to the lack of measurement.

No coherent reflection corresponds to the VSP reflector predicted ahead of the bit by VSP at depth 5840m from surface. Moreover, the 3C VSP cannot detect any reflection at all down to $7700 \mathrm{~m}$ below surface (3s twt from datum on the seismic section), while some events appear between $2.6 \mathrm{~s}$ to $2.8 \mathrm{~s}$ on the $2 \mathrm{D}$ section possibly due to reflectors located laterally to the section, or corresponding to processing artefacts(?). Unfortunately, the seismic section display is equalized with a short AGC window and exhibits a lot of coherency enhancement artefacts which do not give much confidence in the existence of any deep reflectors at well location.

Figure 17 shows a comprehensive geological structural sketch based on all the available measurements, processing and interpretation.

Interpretation of the VSP reflector seen at depth 5840m ahead of the intermediate $5326 \mathrm{~m}$ T.D: the predicted reflector P.R on figure 13 is actually detected as horizontal reflecting surface by the 3C VSP. Unfortunately, no borehole resistivity image is available at this depth: However, the steep layering dip indicated by the resistivity imager from $4700 \mathrm{~m}$ to $5580 \mathrm{~m}$ lead us to believe that the P.R reflector at 5840m probably corresponds to another thrust fault noted F5 on figure 17. The expected Tertiary target may or may not correspond to any seismic reflector as no deep reflector can be assessed.

In brief, all the events noted F1 to F5 correspond to thrust faults of different importance: 
- Only F2 and F5 are seen as horizontal reflections by the VSP, implying that a substantial gouge thickness is present along the fault surface along with a rugged fault surface.

- F1,F2,F3 and F4 are clearly detected by the resistivity imager measurements showing typical slip fault patterns

- The sonic and density logs, used to build the synthetic seismogram as a 1D model with horizontal acoustic impedance contrasts, can read only the faults F1 and F2 (although the sudden contrast of dip occurs at 5030m,10m below the contrast on the other logs).

- The faults F3,F4 and F5 are not detected by the caliper, sonic, density, GR or lithology logs.

\section{Discussion and Conclusion}

The 3C VSP detected a horizontal event at 5020m/5030m which disappears a few ten meters laterally from the well axis. A few tens of meters above 5020m, the resistivity imager results indicate a dip of $70^{\circ}$ abruptly changing to horizontal right at $5030 \mathrm{~m}$, then below 5030m, the dip is consistently around $80^{\circ}$ (Figure14a). The strong horizontal reflector at $5020 \mathrm{~m} / 5030 \mathrm{~m}$ seen by the VSP corresponds to the surface of a very low angle

thrust fault. This reflector appears on the synthetic seismogram as well but with a noticeable phase shift relatively to a VSP response, indicating that the reflecting fault surface is probably not smooth (F2 on figures 12 to 17 ). 
The unusual negative drift between VSP times and integrated sonic time, observed in the layered velocity formations above $5020 \mathrm{~m}$, can be plausibly explained by the layering anisotropy effect induced from the steep dip expressed by the resistivity imager readings. At 5840m a weak reflector with negligible dip appears on the VSP and spreads out up to a few hundreds of meters laterally to the well but it cannot be seen on the synthetic seismogram: it probably corresponds to a subhorizontal fault surface (noted F5 on figure 17).

Figure 17 depicts a comprehensive structural sketch based on all information including VSP 3 component result, borehole resistivity image, geological data, log information and drilling result.

Due to weak reflection coefficient and little acoustic impedance contrasts, the surface seismic technique can not exhibit any clear and consistent reflection image at depth; the fact that the top Dashtak reflection can be consistently tracked on surface seismic indicates that the processing of the section is correct, however cannot be improved, probably because of the absence of deep reflectors, or because they are totally blurred.

Resistivity imager and 3 component VSP techniques measure the dip of different geological objects, therefore, they are not necessarily in agreement when confronted to each other. Resistivity imager measures the layering dip along the borehole wall while the 3C-VSP can restitute the dip of seismic reflectors up to a few hundred meters away from the well or below its T.D. Therefore, the, integration of both techniques logically leads to improved geological interpretation. 
In the present case the layering dip from resistivity imager is totally at odds with the dip of seismic reflectors from 3C VSP, implying that resistivity imager, 3C VSP, and all standard logs are complementary tools for the interpreters.

In spite of the very steep dips indicated by the resistivity imager log, we could not observe any P-P reflection, refraction or diffraction on the zero offset VSP data, and it is not surprising to obtain no good concordance between corridor stack and synthetic seismogram.

In complicated cases, it is necessary to use all possible techniques and methods to build a plausible interpretation. Building an integrated interpretation right after drilling further increases the operational difficulty.

\section{Acknowledgements}

The authors thank NIOC exploration for providing data and permission to publish this work. The review and remarks of Jean Letouzey and Jean-Claude Lecomte on the structural geological aspects and the dip readings are highly appreciated. CGGV is acknowledged for financial support and long-term support in the exploitation of oriented $3 \mathrm{C}$ component of VSP's 


\section{References:}

Etchecopar, A., Bonnetain, J.L., 1992 - Cross sections from dipmeter data.

AAPG bull, V. 76, N0.5, Page 621-637

Naville Charles, Sylvain Serbutoviez, Jean-Claude Lecomte, Pascal Froidevaux and Kazem Kazemi, Patent No. 09/00.916 French Patent

Naville, Charles, Sylvain Serbutoviez, Jean-Claude Lecomte,Patent No. 09/00.643 French Patent

Naville C., US patent no. 2003/0086335 A1; May 8, 2003,

Naville Charles., Serbutoviez Sylvain., Bruneau Josette., 2001,SEISDIP: the "VSP dipmeter" from oriented 3 components, AAPG, Expanded Abstracts

NIOC exploration, Graphic well log, well B,

NIOC-Exploration, VSP survey report, Well B ,2006,

Serbutoviez S., Naville C.,Bruneau J. , Huguet F. , Reinier M. ,2002,Pam-3C VSP- Preserved and true amplitude 3 component VSP, EAGE 64th conference \& Exhibition, Italy 


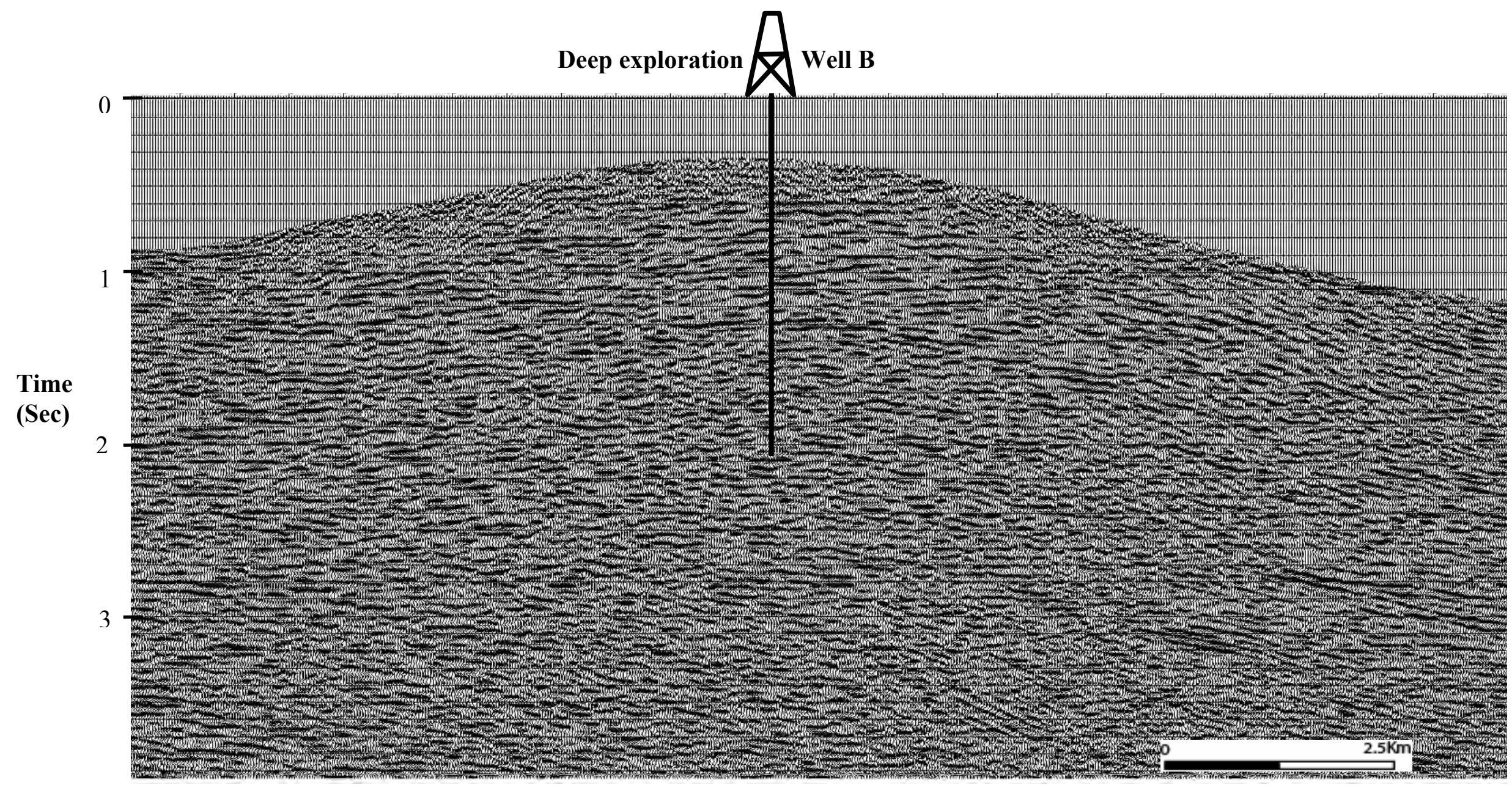

Figure1 2D seismic section over well B: Acquisition 2005, processed three times; The expected target is a deep anticline. The exploration well is located on top of a3000 m mountain, $1200 \mathrm{~m}$ above the lateral valleys and took 3 years to drill. 

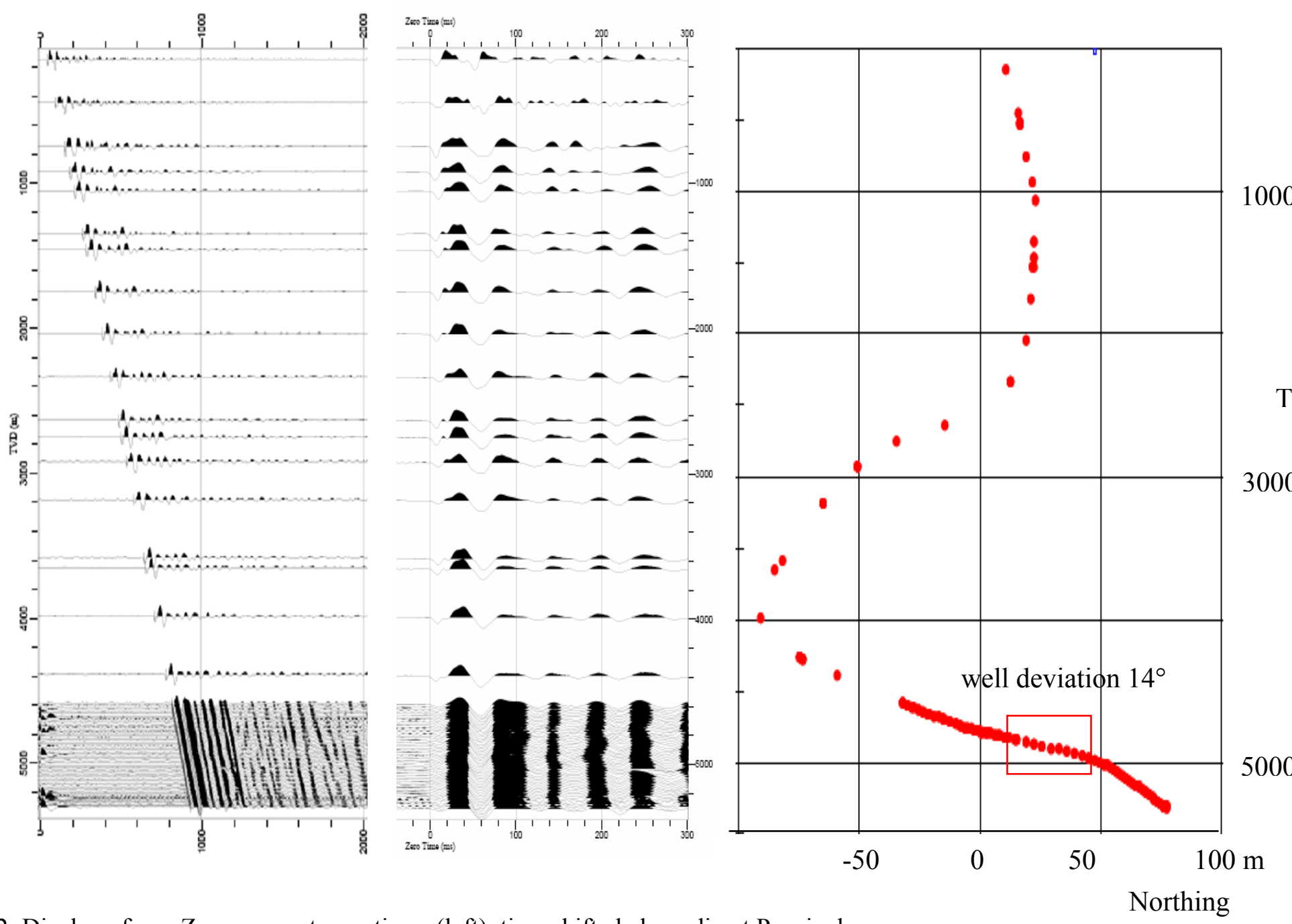

Figure2: Display of raw $\mathrm{Z}$ component, raw times (left), time shifted along direct $\mathrm{P}$ arrival ( centre), and well projection (right) 


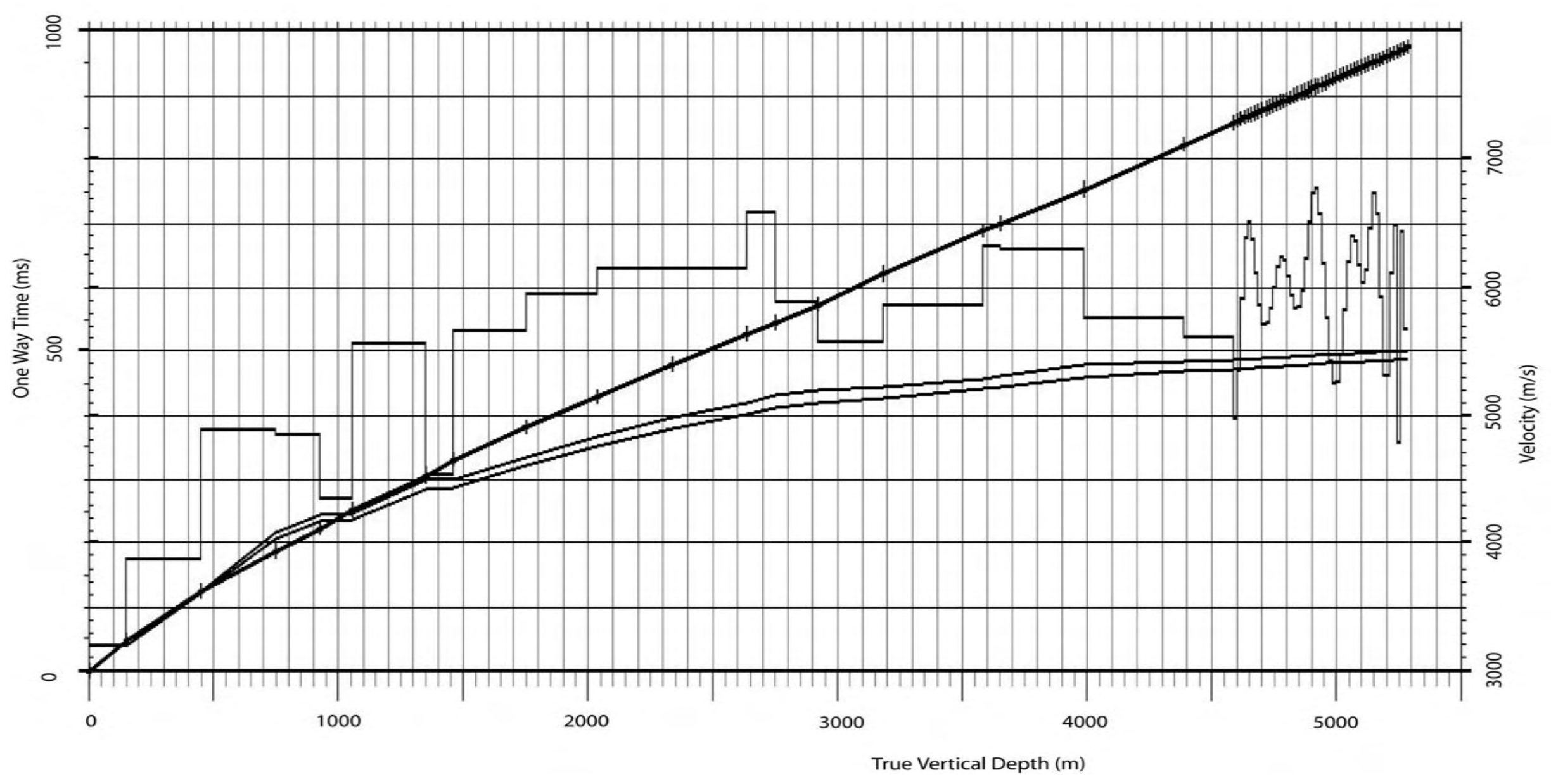

Figure3: Time depth curve after obliquity correction, Interval, Average and RMS velocity versus true vertical depth from Ground level/SRD 


\section{Raw-Z}

Raw-X

Raw-Y

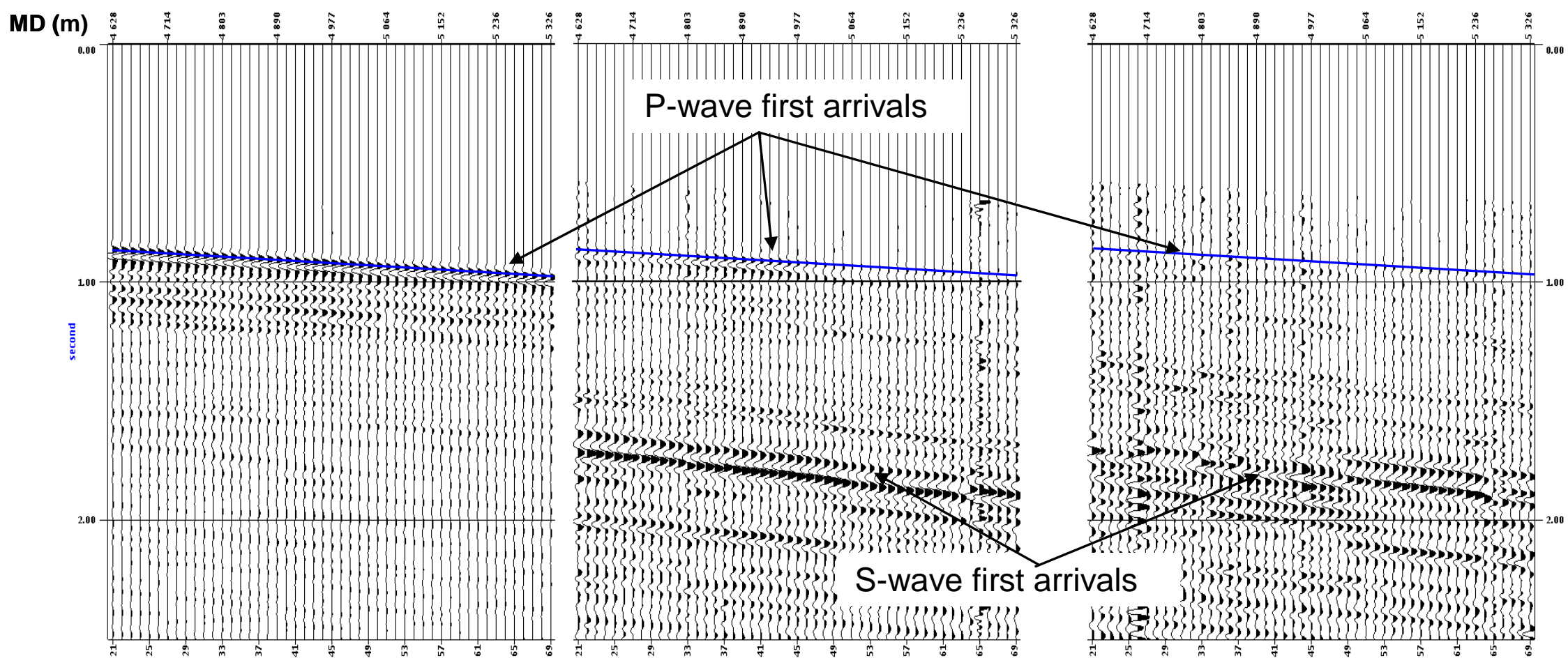

Figure4: Raw VSP data of the recorded three tool components, isotropic vertical stack, isotropic normalized display ( $\mathrm{Z}$ along well axis) 


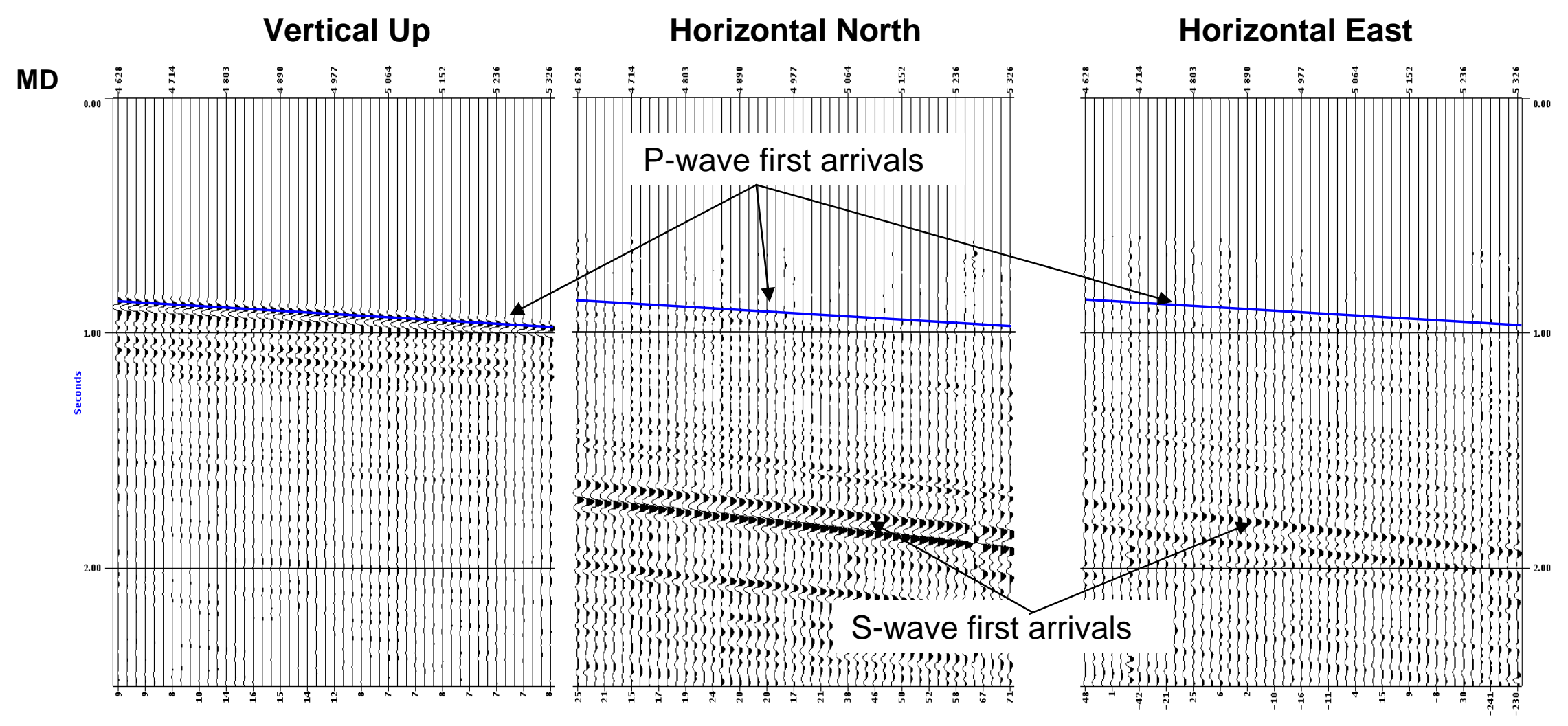

Figure5: Three components of VSP oriented in geographical coordinated, isotropic normalized display. 


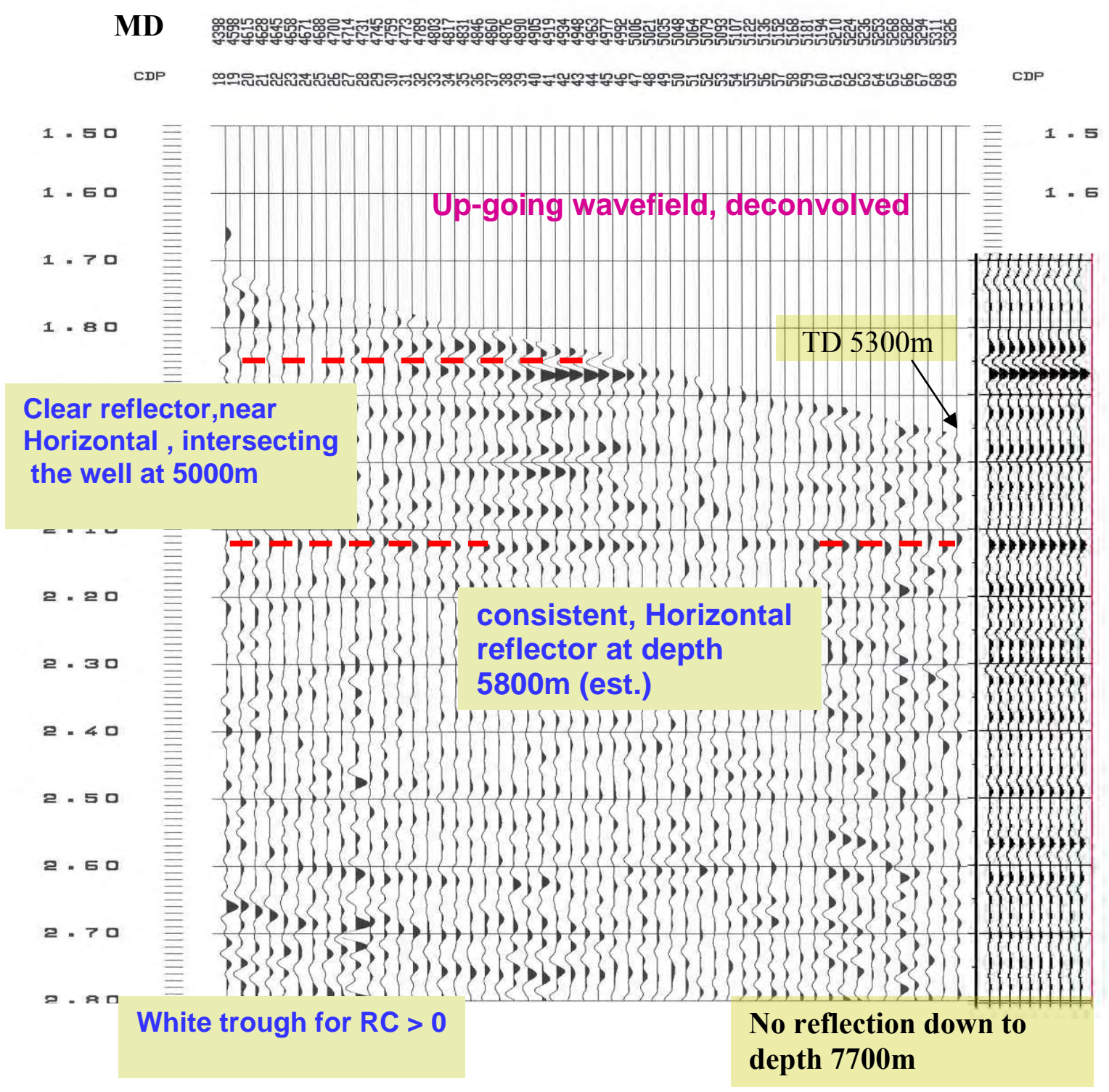

Figure6 Z-component from SINGLE component processing, True amplitude display, TWT 


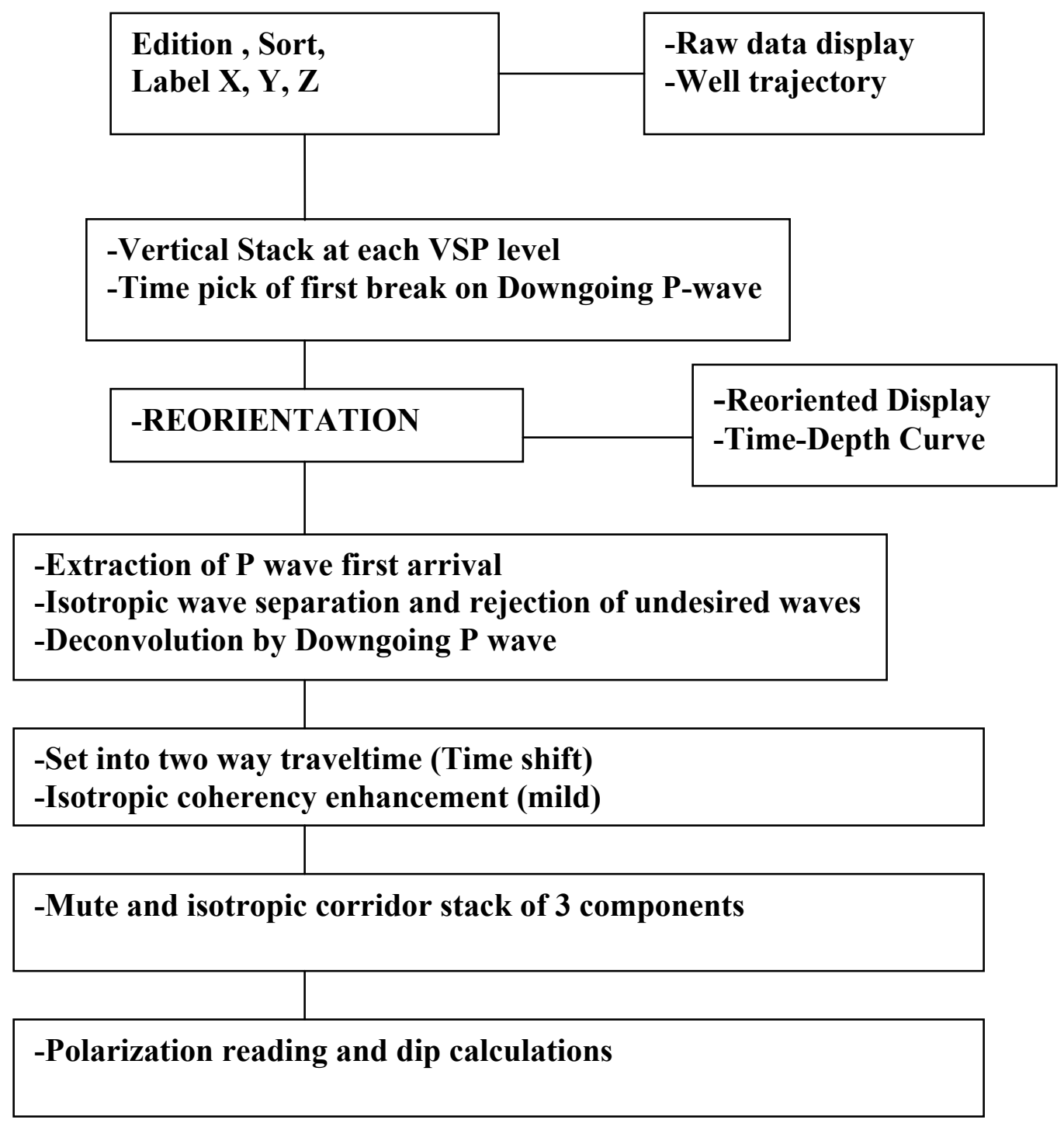

Figure 7 Flowchart of isotropic processing of oriented 3C VSP 


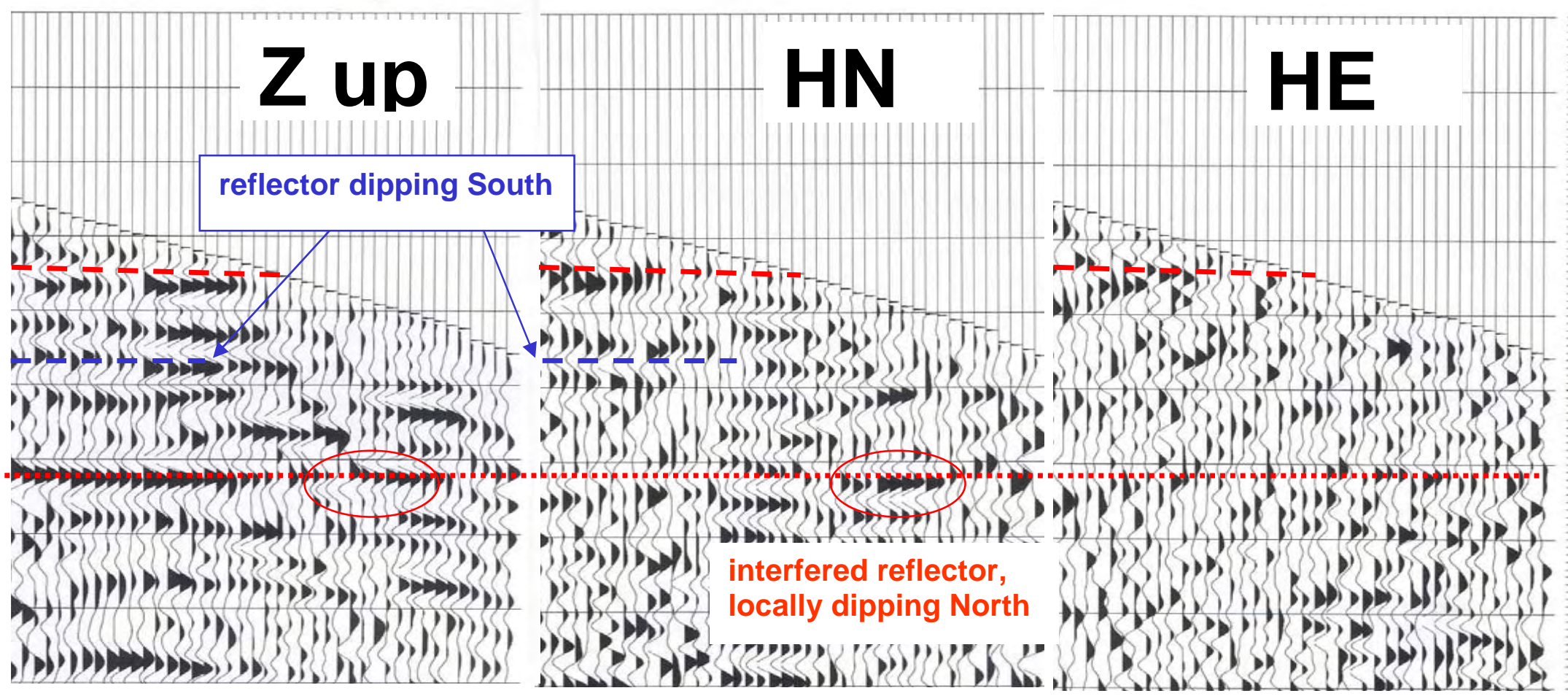

Figure8: Isotropic P-P reflection wave field from 3 component VSP processing results; display in two way time 


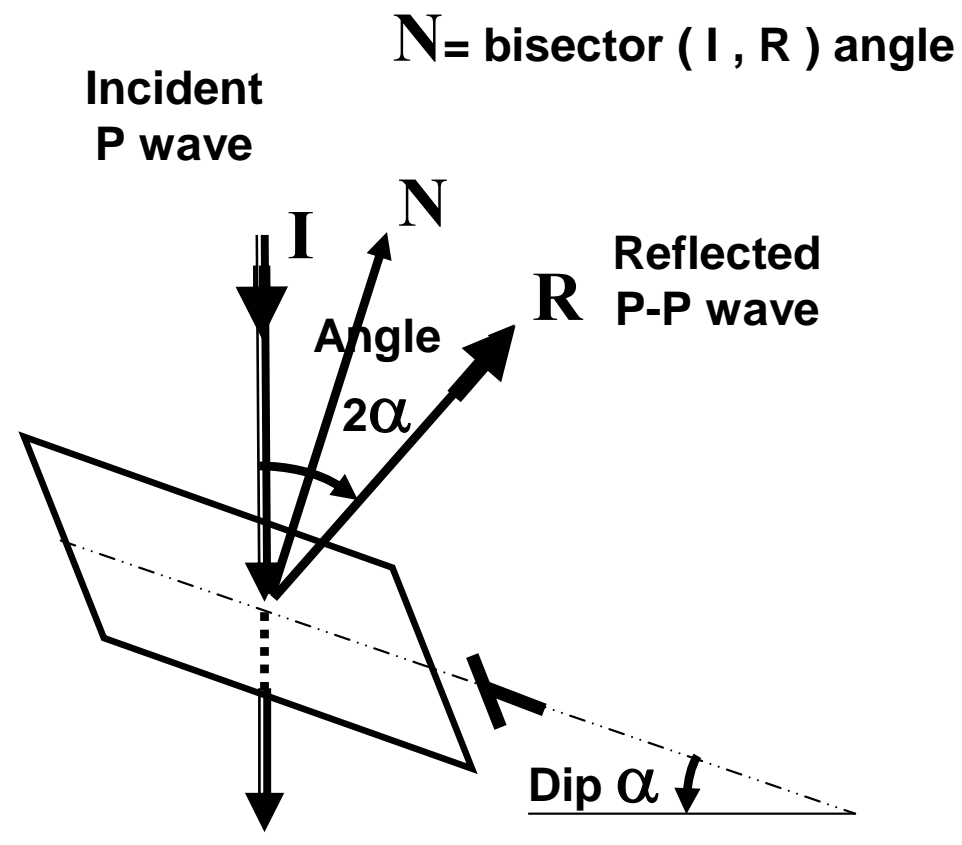

Dip of reflected ray $=2 \alpha$

Azimuth of reflected ray $=$ Azimuth of downdip

Figure9: Sketch of P-P reflection on the dipping reflector with dip angle $=\alpha$ 


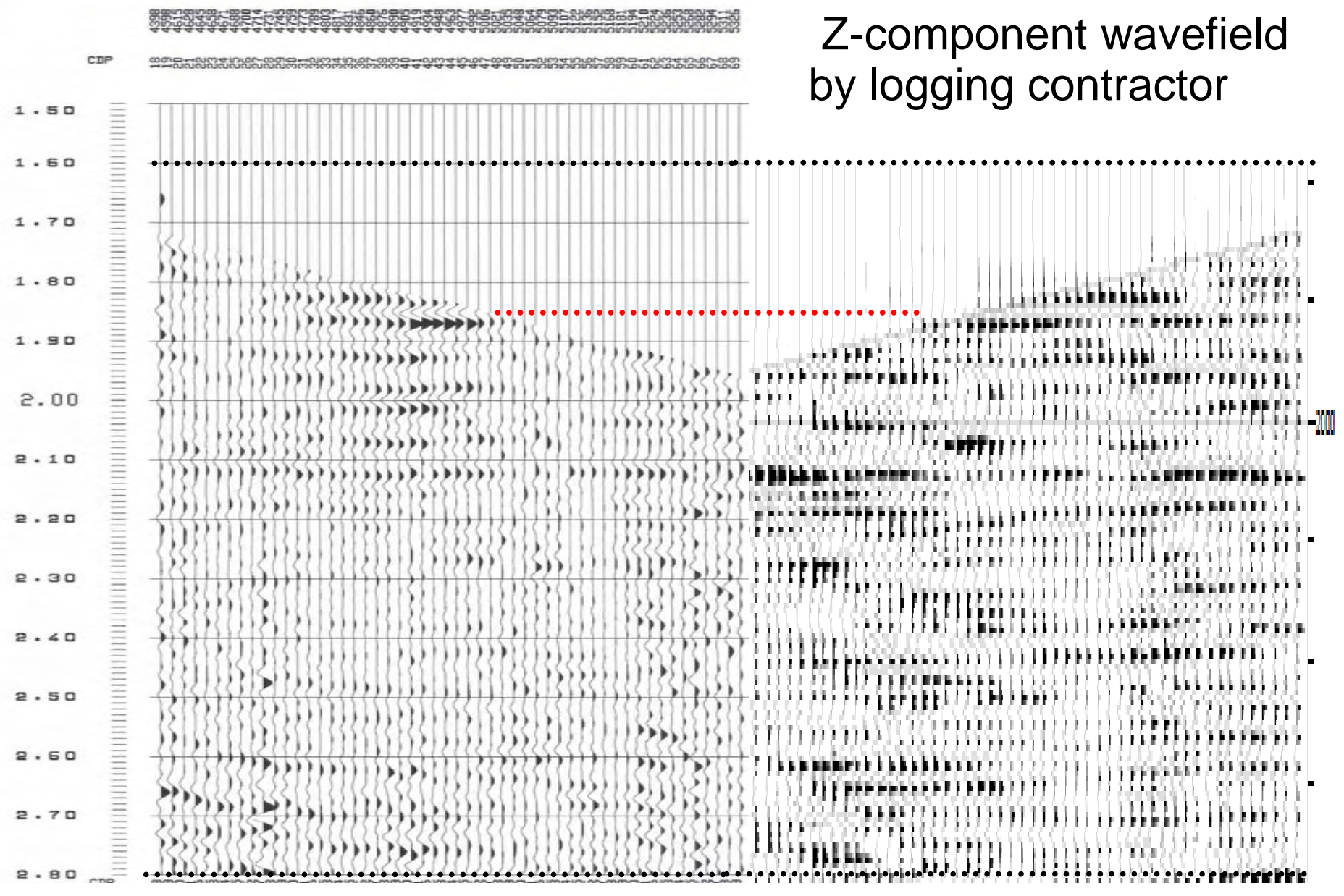

Figure10 Z-component reflected wavefield obtained by logging contractor (right):7 trace median filter applied, display normalized trace to trace; Reprocessed result (left) S-wave removed, no coherency enhancement, true amplitude display, Polarity: SEG normal, increase of impedance $=$ white trough 


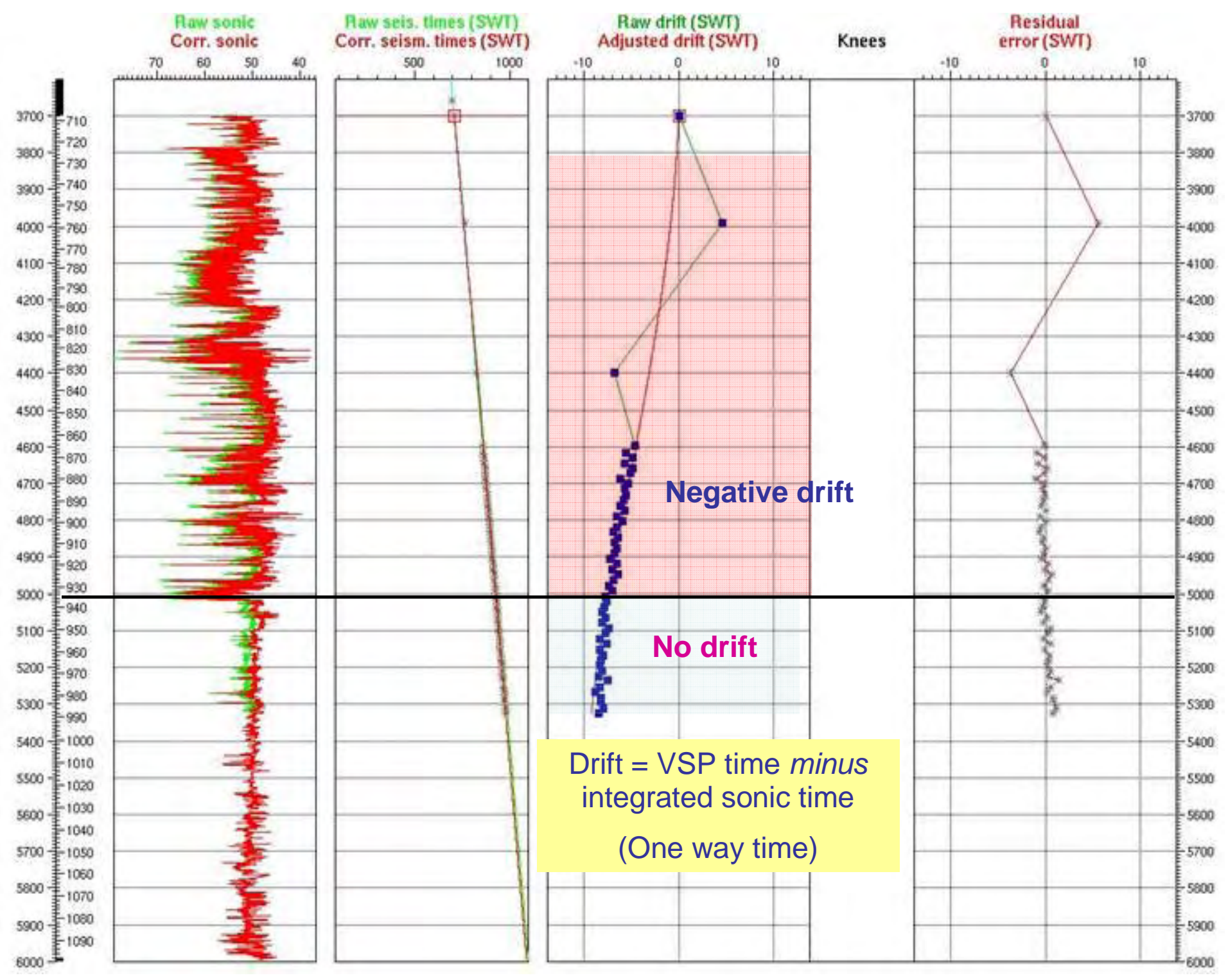

Figure 11 Sonic log calibrated with VSP time 


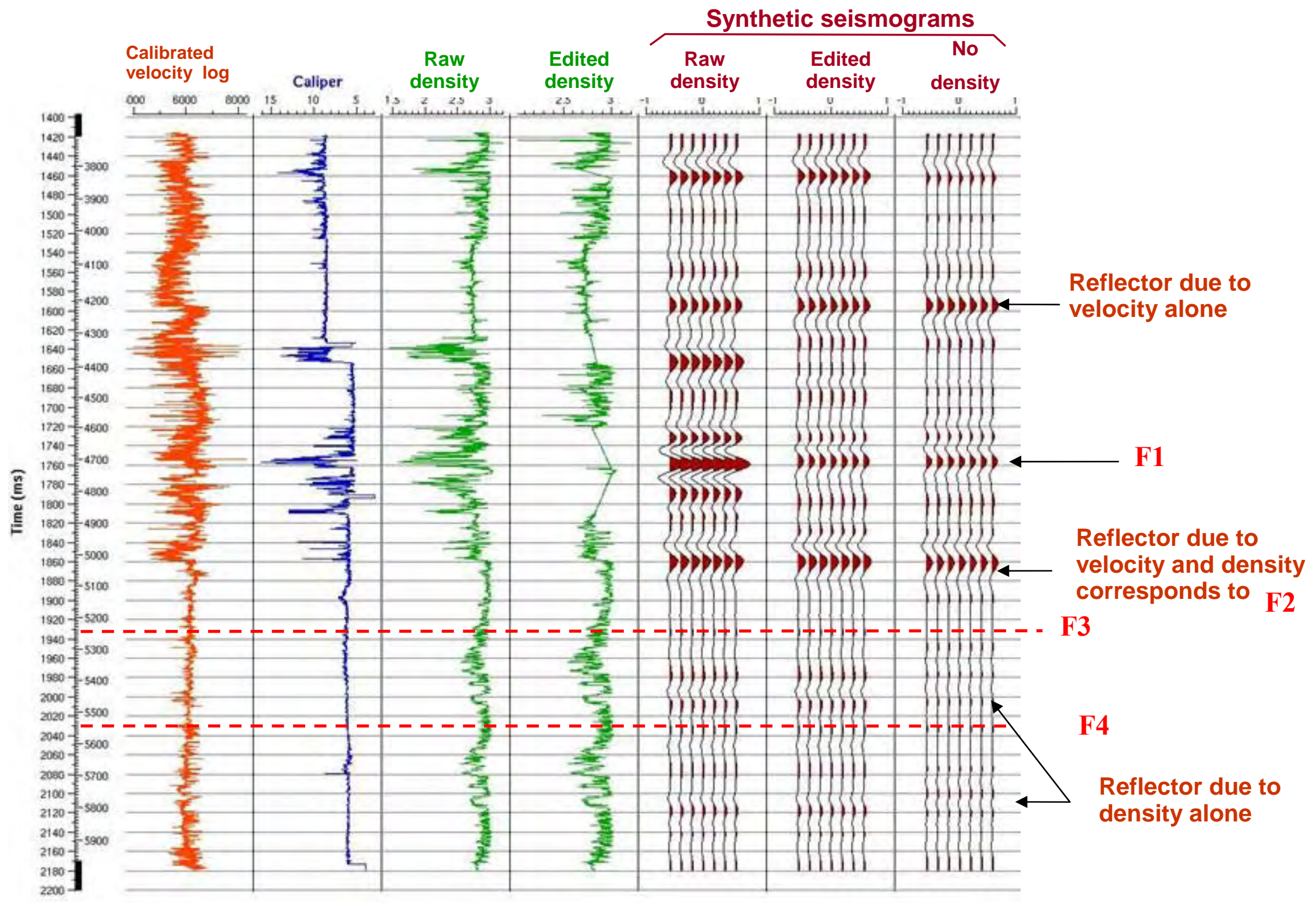

Figure12 Three Synthetic seismograms built with calibrated sonic plus raw density, edited density and with sonic alone all convolved with $30 \mathrm{~Hz}$ zero phase Ricker wavelet; large wash outs Caliper log indicates the wrong readings of the density log 


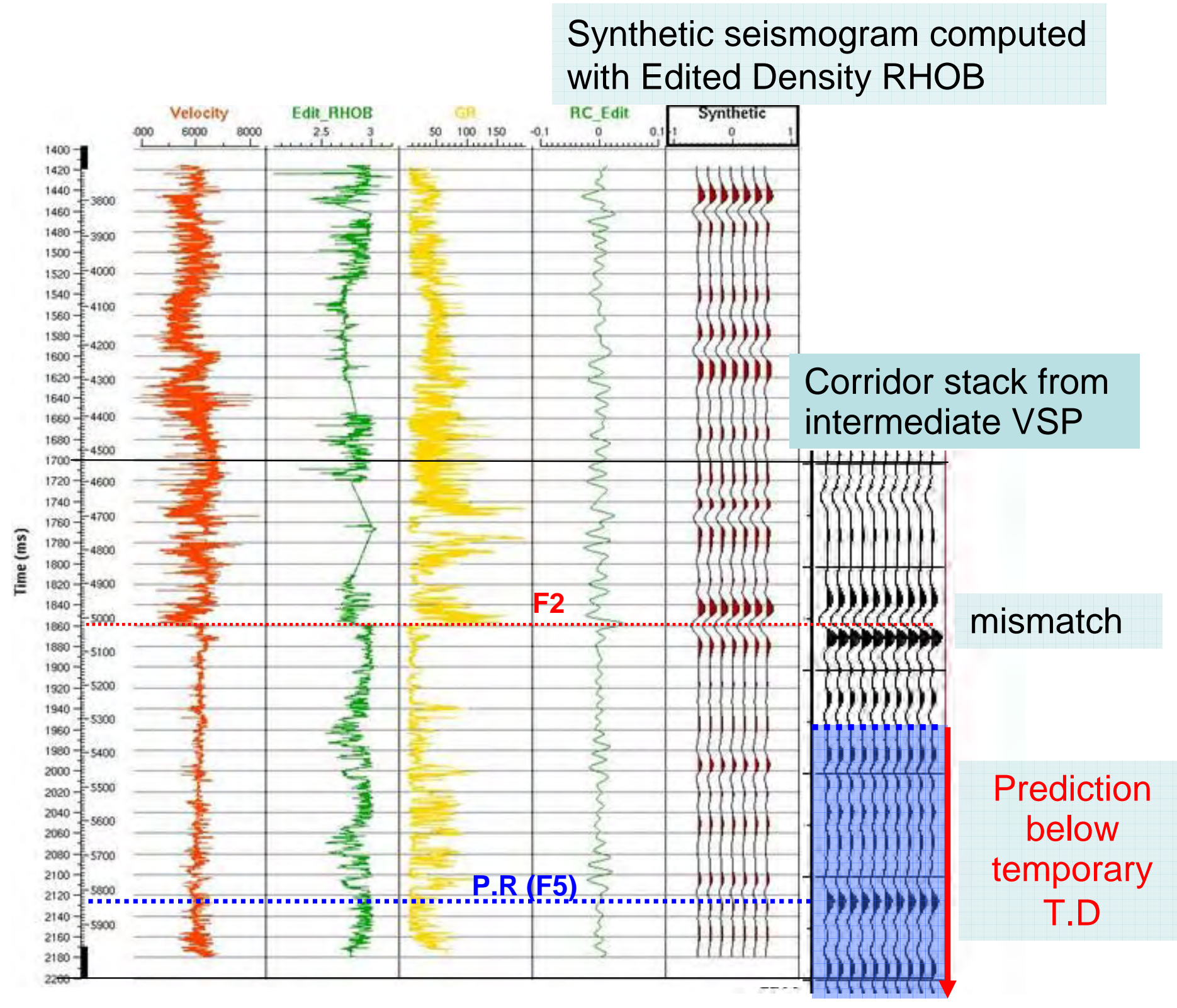

Figure 13 confrontation of VSP corridor stack with synthetic seismogram.

Both results are zero phase, SEG normal polarity (increase of impedance is represented by white trough) 


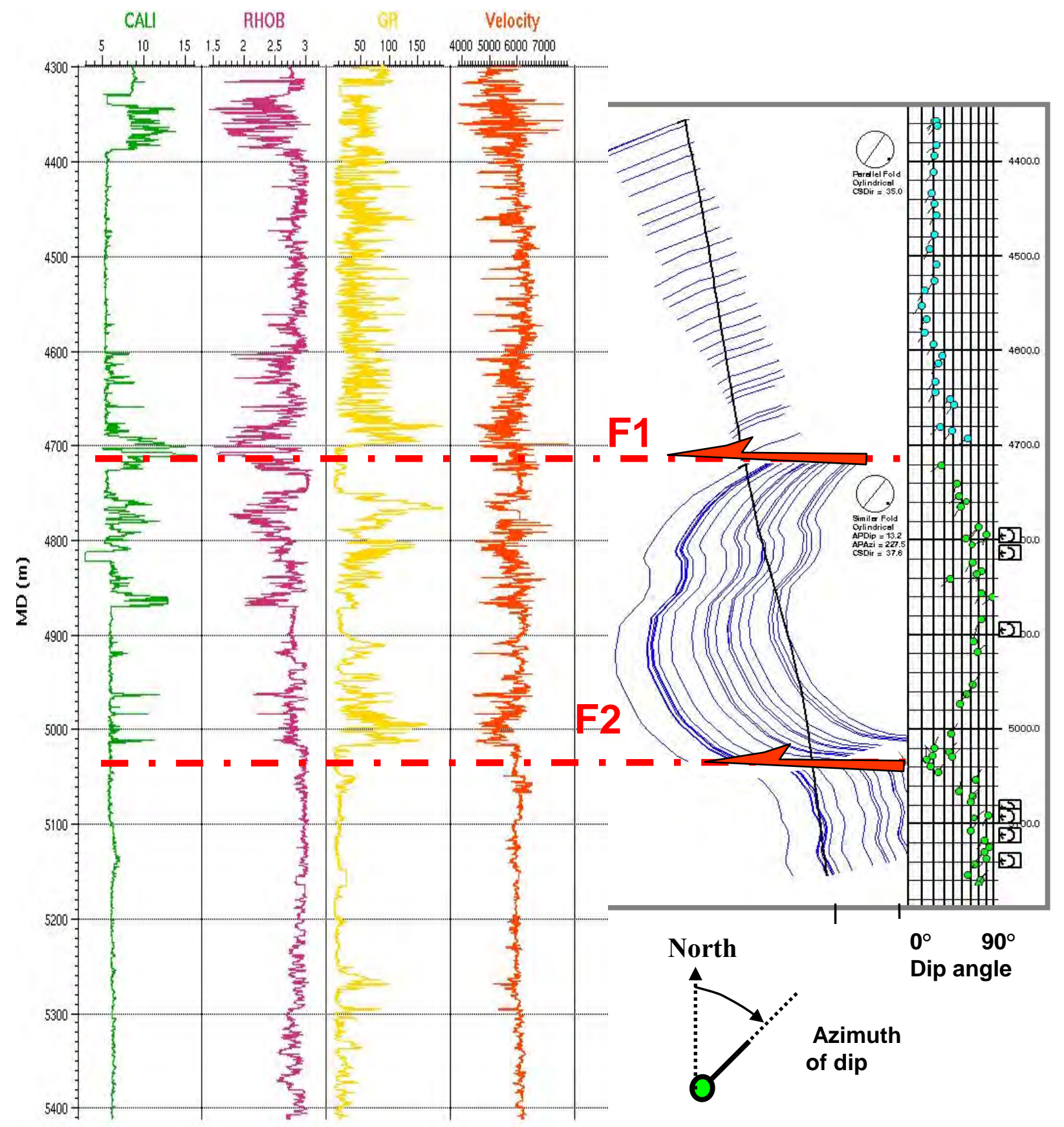

Figure 14a confrontation between logs and resistivity dip-meter results in depth scale, F1 and F2 correspond to thrust faults associated with abrupt change of dip 

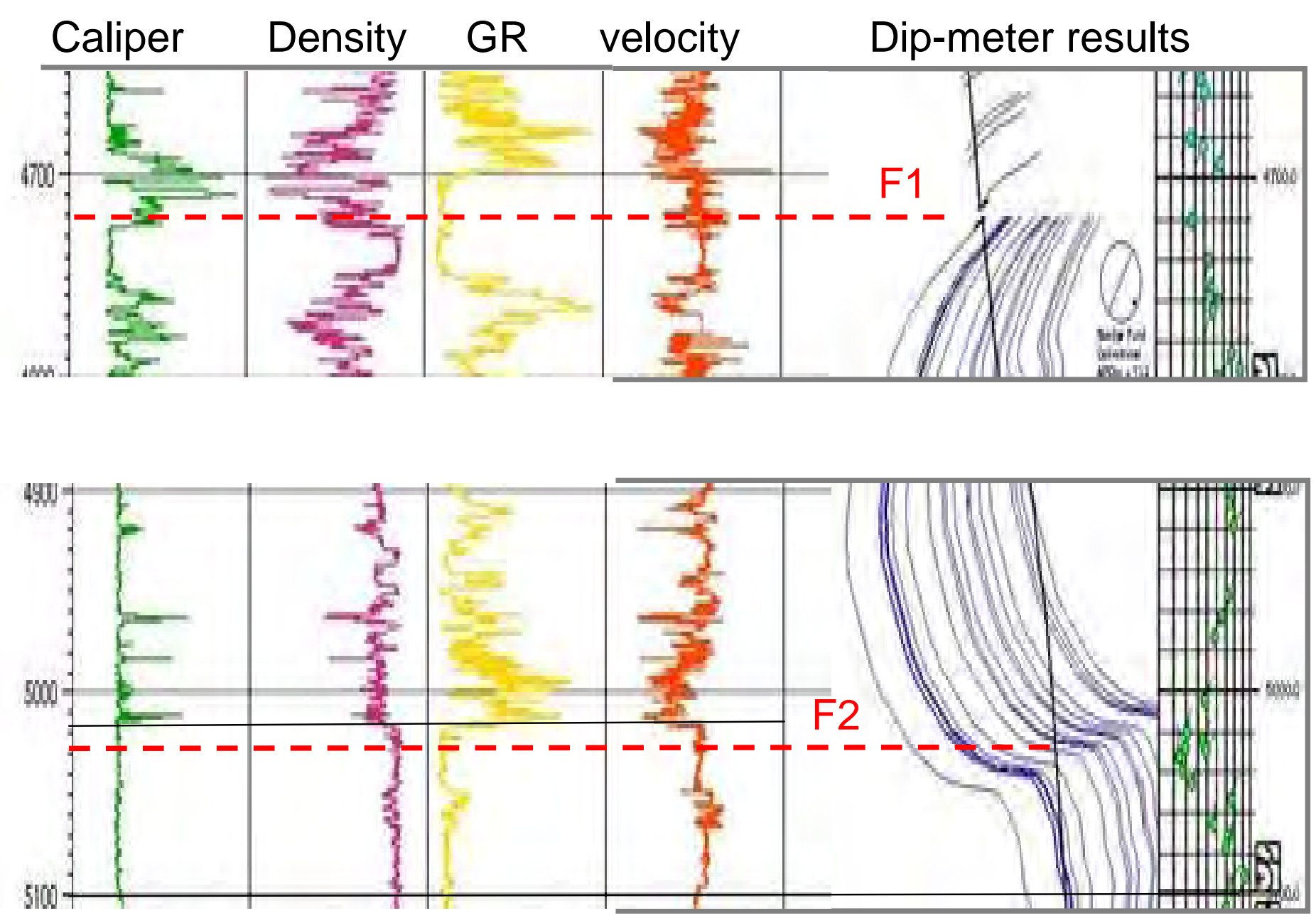

Figure 14b Confrontation between logs and electrical resistivity dip-meter results in depth scale, F1 (above) and F2 (below) are zoomed; there is $10 \mathrm{~m}$ mismatch between velocity abrupt changes and fault position on the resistivity dip-meter results 


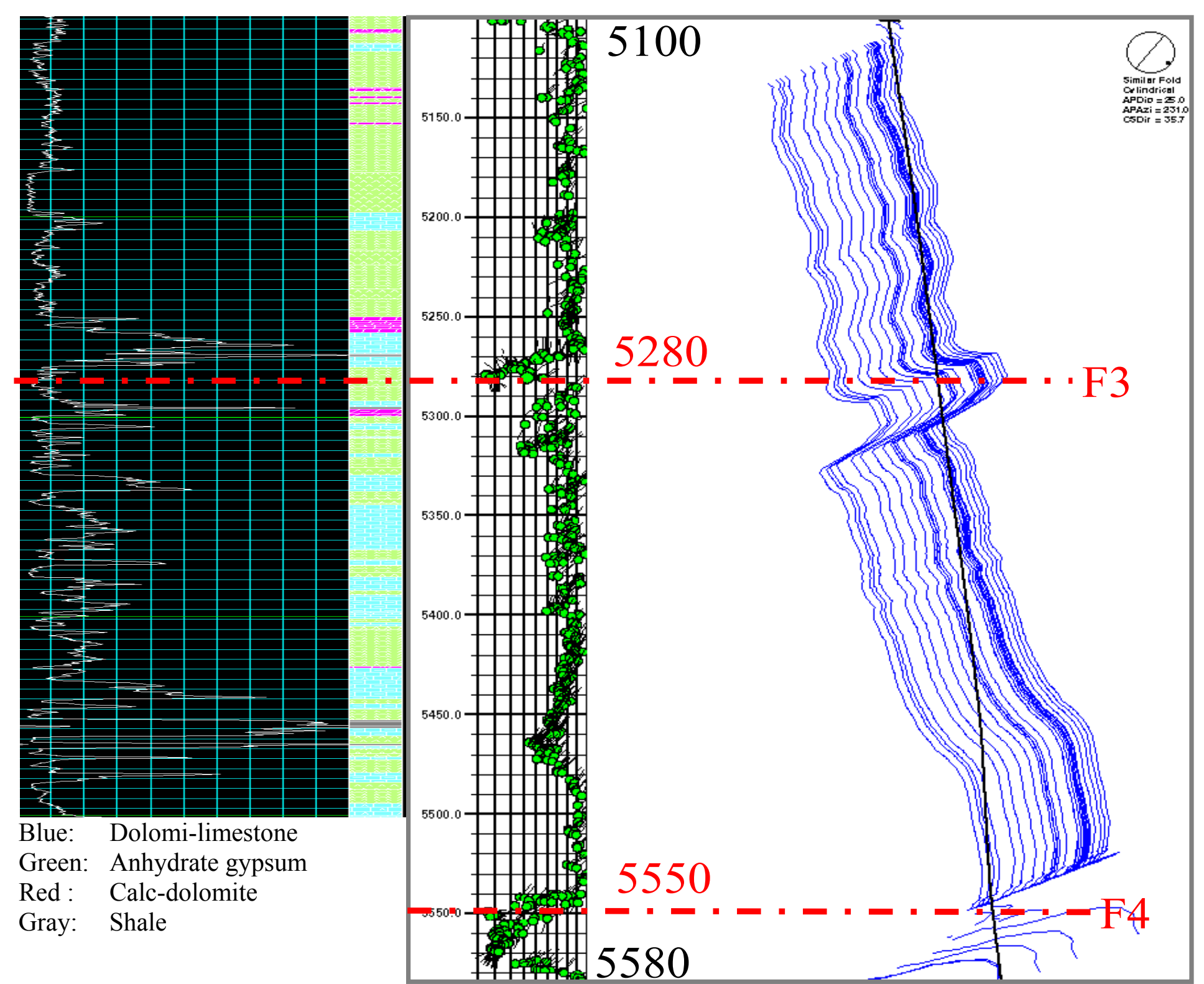

Figure 15a Structural detail of F3 confronted with lithology 


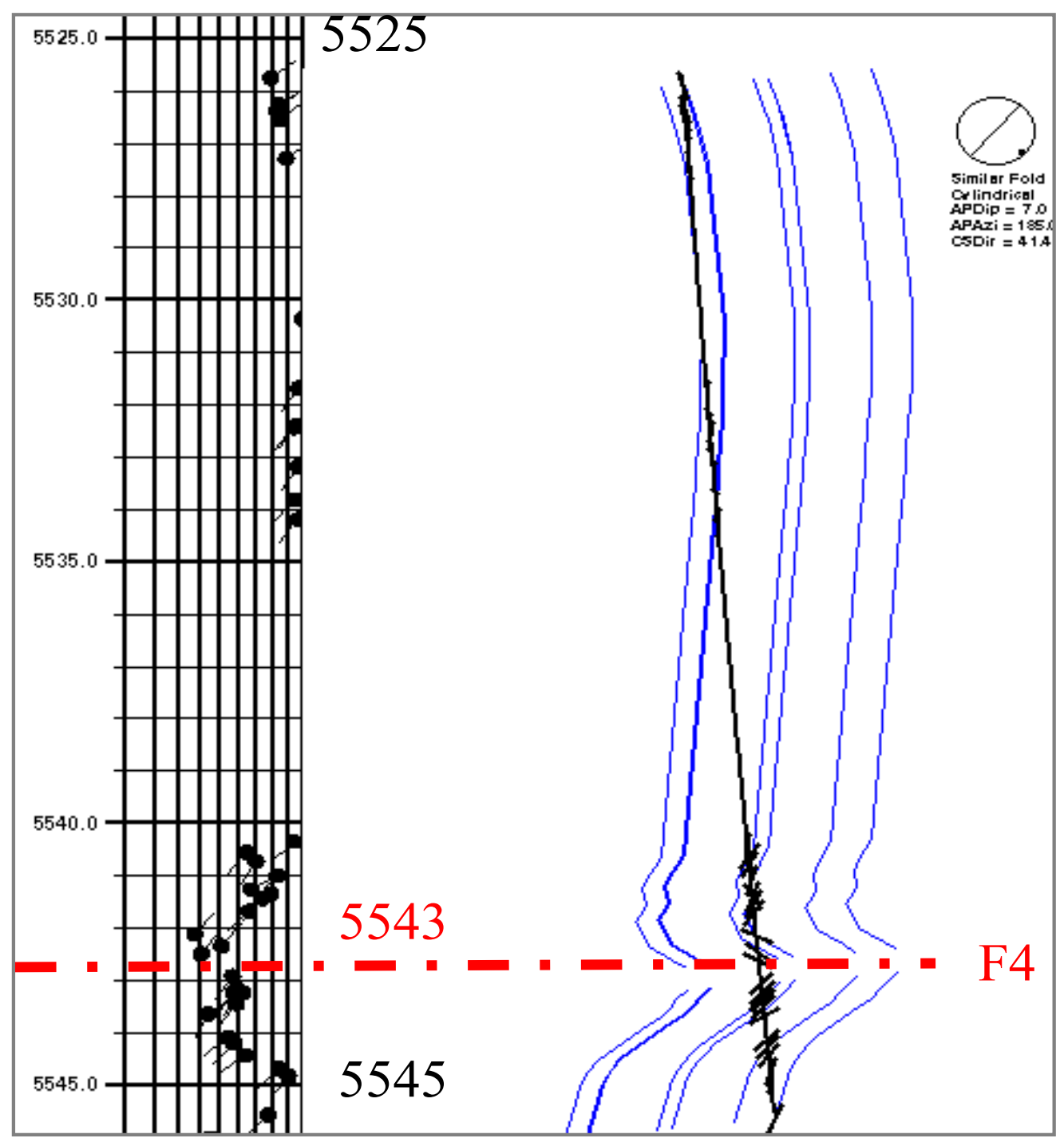

Figure $15 \mathrm{~b} \quad$ Resistivity dip-meter detail of $\mathrm{F} 4$ 


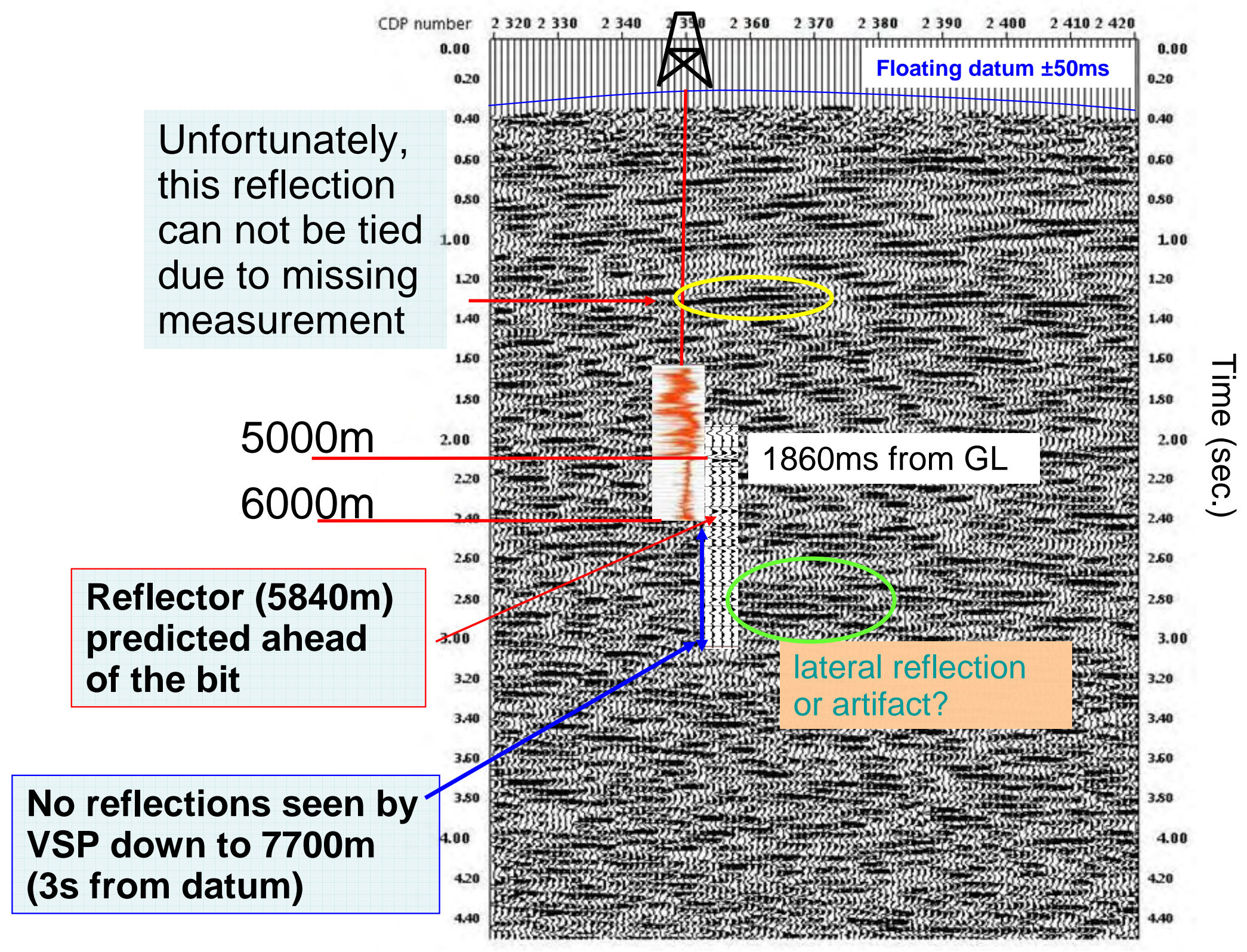

Figure 16 Illustration of well B with sonic log and VSP corridor stack. overlain on the 2D section, 


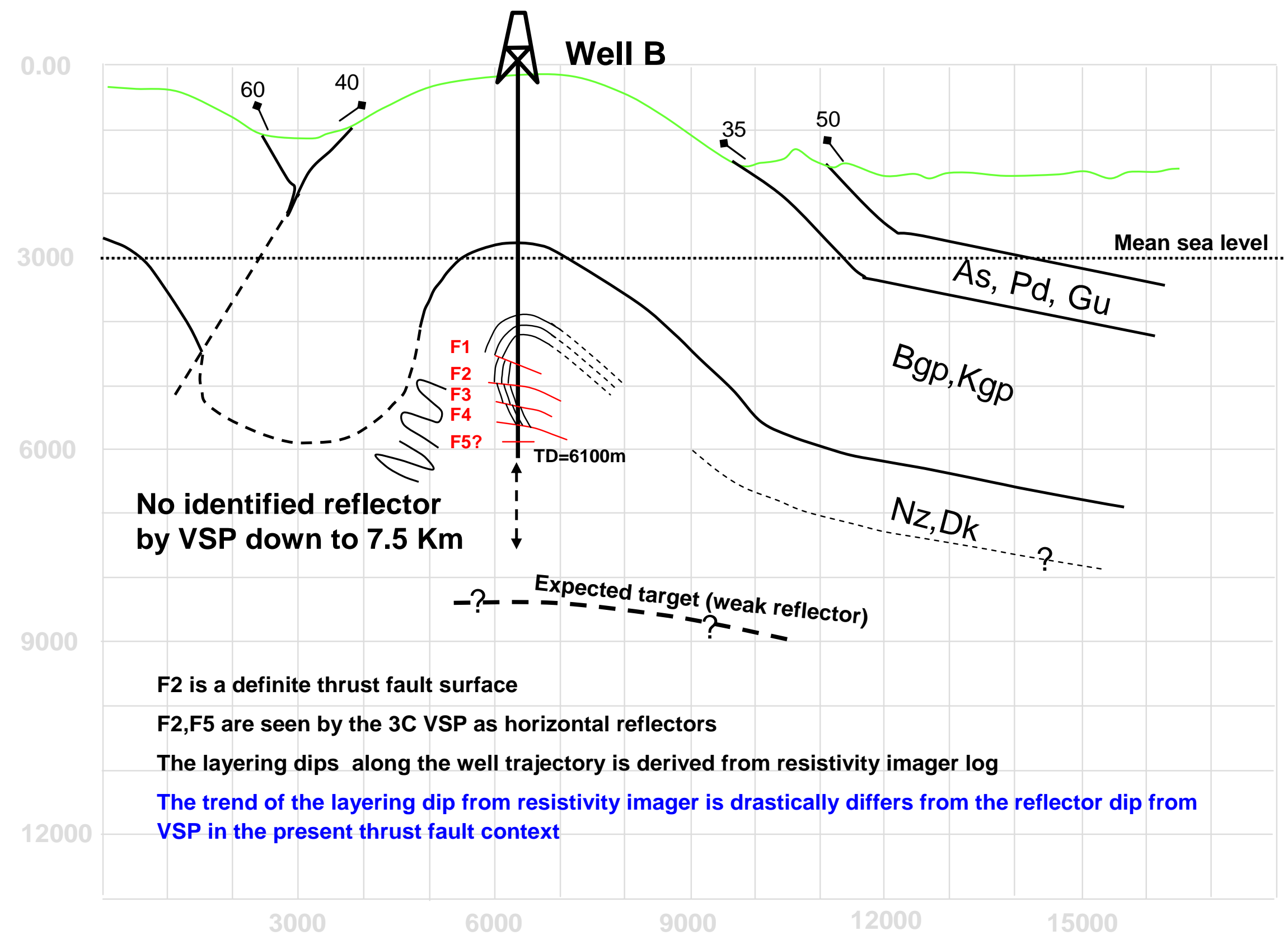

Figure 17 Structural sketch based on surface seismic, 3C VSP, from resistivity imager dip and geological data 



\section{Chapter 8}

\section{Discussion and Conclusion}


The objective of this PHD was initially inspired by the first and recent 3D seismic survey acquired on the Aghajari structure. Although this structure has been drilled by about 150 production wells and the locations of oil zones and gas cap are well known, important issues were in question:

- As Asmari reservoir is tight and only produces by fracturation and faults, it is most important to see if seismic data (both surface and well data) can bring informations on such discontinuities;

- Other reservoirs and plays which exist in deeper part of the Aghajari structure and it is of the most importance to obtain a clear structural image of the overall structure.

The preliminary PSTM cube obtained by the contractor was not able to really answer the above questions. It was possible to map the main faults that intersect the Cap rock (strong reflector) but only on the top and northern flank of the structure in the gas zone. The oil zone, which is the zone of interest, was not imaged well enough to conclude whether faults and fractures presence or not. Indeed, the flanks of structure are quite steep at the level of oil zone and were almost not imaged. This imaging difficulty also seen on the other neighboring fields, reinforcing the need for improvement in usual workflow.

We concentrate of our work in 2 directions:

- What information can be brought by VSP and in particular 3C VSP to the knowledge of the field or/and to an improvement of processing 3D surface seismic data.

- Can we build a reliable depth velocity model and will PSDM solve the poor imaging of the reservoir zone?

The conclusion, focuses on the results obtained concerning these 2 points, as well as the main difficulties encountered and how we dealt with them. By the end, we do some suggestions for future work in order to benefit from the existing VSP and surface seismic data or acquiring new dataset.

\section{8-1 What information could be deduced from VSP}

First well: We spent times to process the 3 components of the only VSP existing in the North East flank. Usually horizontal components are discarded and only the vertical one is 
processed assuming a 1D layered media. We found that no energy from Cap rock was reflected on the vertical component while it was present on the horizontal ones, because the local dip is near $45^{\circ}$. The fractures of Cap rock reflector may not affect the Asmari reservoir immediately underneath cap rock anhydrates.

We used the polarization and an original reorientation method in order to determine the dip and azimuth of dipping reflectors and found good concordance with already known values of these dips (Micro Scanner, structural maps ...). We also identified a clear anisotropy effect on $\mathrm{S}$-waves in the overburden in relation with the stratification ( $\mathrm{S}$-wave birefringence)

We measured true interval velocities that should be used during depth velocity model building.

Second well: We also processed another VSP from a deep exploration well in a more complex area of Zagros. The integration with the resistivity imager data shows a very interesting result: The VSP recorded a reflection that comes from a faulted horizontal surface separating two layers with the same impedance and internal vertical stratification. It is the first time that such evidence is demonstrated and this has important application for understanding VSP in complex thrust area. The radius of investigation of VSP can reach a few hundreds of meters laterally to the well and below the well. The resistivity imager log can be run only in open hole and yields the local dip along the borehole wall. Both techniques are complementary to each other for structural delineation.

The excellent wave propagation is demonstrated by the good signal to noise ratio observed on the VSP /check shot in this well and other deep exploration well on the Aghajari structure acquired in 1976 with low energy seismic air gun sources. This means there is no penetration problems for seismic waves in the this area.

\section{8-2 3D imaging from surface seismic}

Both PSTM and PSDM results allow to clearly image faults on the crest of the structure in the gas zone. Unfortunately, as we could not image the dipping flanks corresponding to the oil zone, and we cannot conclude on the level of faulting inside the oil zone.

We first believed that initial PSTM was sufficient to enter a PSDM workflow with the "Beyond Dix method" and we spend most of our time on the application of this workflow. In spite of our efforts, we did not reach expected results because the PSTM was not able to image properly the steep parts of the structure. In consequence, kinematic attributes are erroneous and the PSDM is not better than the PSTM except in the overburden where PSTM provided correct attributes. 
We improved the PSTM results by trying to optimize the apertures by two methods. The first method consists in a selection of migration azimuths that produces two benefits:

- it decreases the amplitude of the strong free surface multiple of the top Gs-6 that overlaid the south flank of the structure

- it decreases the noise and enhance the contribution of the dipping reflectors.

The second method was an attempt to determine the dip and curvature of main continuous reflectors and produced quite interesting results by reinforcing the coherent reflectors already present, the high dips and a few deep reflectors.

In both PSTM methods, we were able to follow much better the Cap rock inside the oil zone where dips are important and also to follow its structure under the possible detachment fault below the main Aghajari structure. In the second case, the top Asmari over the prospect in the south west of Aghajari field (chapter4) is more continuous while all other previous results do not represent this illumination.

\section{8-3 Conclusion concerning the Aghajari structure}

- $\quad$ The existence of the top Cap rock reflection has been confirmed in the oil zone by the prototype migration program developed in IFP.

One of the positive results of this 3D survey is the image of a previously unknown potential prospect in the south of the Aghajari structure. The shape of this new structure depends on the reliability of FDP and velocity field. We generated a velocity field from stacking velocities, geological interpretation and well data and performed a rough post stack depth conversion, which confirmed the smaller structure at the south west of the Aghajari anticline, potentially with higher reliability than the depth image from PSDM using velocities derived from stacking velocities only.

\section{8-4 Suggestion for future work}

By looking at various 2D/3D surveys in Dezful Embayment, Zagros, it seems that changing acquisition parameters will not bring major improvements on the surface seismic results where the results are poor. Indeed we proved that the reflections on dipping part of the structure are present in the date although not visible in conventional migration and that there is no penetration problem. There are several ways to progress for a better understanding of our structures: 
- first it is important to integrate datasets from different methods: surface seismic, well seismic, well data and geological information in order to approach the objectives and understand the imaging challenge. In particular, a first a priori depth velocity model should be build based on this information and any automatic tool for Depth velocity building should honor these information.

- We found that free surface multiples of top Gasharan limits the reconstruction of south flank and in consequence the picking a needed kinematic attribute in this area: new method based on multi dimensional interpolation and SRME(surface relative multiple elimination) anti multiple algorithm should be tried. The multiple might have been eliminated naturally by using higher stacking velocities if fair primary reflectors would have emerged from data processing, as the interval velocities are too low at reservoir level.

- Full aperture migration are not appropriate but new methods have been developed which focus on the main continuous event and can recover very steep dips and even overhanging part of structure. The PSTM -PSDM workflow should make use of these new techniques as soon as possible in the workflow.

- In order to further investigate the lateral extension of the Asmari reservoir and Cap rock reflector at the level of the oil zone it is desirable to run a few zero-offset or offset VSPs (in new wells or in work over operations) on the south western flank of Aghajari field.

- It would be interesting to have 2D seismic line with very long offset (about $15 \mathrm{~km}$ ) on the Aghajari structure in order to analyze the potential of refraction and wide angle reflection methods for structural imaging especially in areas where the strong Cap rock marker is not visible on the reflection images. Actually, the Cap rock reflector corresponds to an abrupt velocity increase from $3800 \mathrm{~m} / \mathrm{s}$ to $5500 \mathrm{~m} / \mathrm{s}$ in the thick sedimentary section underneath: this confirmation from the sonic logs and Checkshot surveys in many wells is quite encouraging for refraction surveys targeted to mapping the Cap rock interface. 



\section{Abbreviations}

\begin{tabular}{|c|c|}
\hline PSTM & Pre-Stack Time Migration \\
\hline PSDM & Pre-Stack Depth Migration \\
\hline QC & Quality Control \\
\hline GOL & Gas Oil Level \\
\hline WOL & Water Oil Level \\
\hline TD & Total Depth \\
\hline VSP & Vertical Seismic Profiling \\
\hline 3C & Three Components \\
\hline $\mathrm{RB}$ & Relative Bearing \\
\hline TVD & True Vertical Depth \\
\hline OWT & One Way Time \\
\hline twt & two way time \\
\hline GR & Gamma Ray \\
\hline CGGV & $\begin{array}{l}\text { Company name (Company general de geophysics joint } \\
\text { with VERITAS) }\end{array}$ \\
\hline CDP & Common Mid Point \\
\hline NMO & Normal Move Out \\
\hline CIG & Common Image Gathers \\
\hline RMO & Residual Move Out \\
\hline FDP & Floating Datum Plane \\
\hline
\end{tabular}


SRD

MSL

SRME

CRS
Seismic Reference Datum

Mean Sea Level

Surface relative multiple elimination

Common reflection surface 


\section{References}

Abdollahie Fard, I. Braathen, A. Mokhtari M. and Alavi,S. A. "Interaction of the Zagros Fold-Thrust Belt and the Arabian-type, deep-seated folds in the Abadan Plain and the Dezful Embayment, SW Iran", Petroleum Geoscience, Vol. 12, 2006, page 347362

Alaei, B. and Pajichel, J. , "Single Arrival Kirchhoff Prestack Depth Migration of complex faulted folds from Zagros Mountains, Iran" , 2006, CSEG Recorder, page41-48

Alaei, B., "An integrated procedure for migration velocity analysis in complex structures of thrust belts" , 2006, Journal of applied Geophysics 59 , page89-105.

Alex Berkovitch, Igor Belfer, and Evgeny Landa, "Multifocusing as a method of improving subsurface imaging “,The Leading Edge 27, 250, 2008, page 250-256

Alkhalifah, Tariq. "Velocity analysis using nonhyperbolic moveout in transversely isotropic media." Geophysics 62.6, 1997, page1839-54.

Al-Yahya, K. M. 1987, PhD thesis, Standford university

Anquelle, Lorette, Jean Arnaud, Magali Beele, Pierre Esquier, Yves Le Stunff "Anisotropic pre-stack time migration and pre-stack depth migration for reservoir evaluation in offshore West Africa." SEG Technical Program Expanded Abstracts 25.1, 2006, page510-14.

Balch A.H. \& Myung W. Lee; Vertical Seismic Profiling: technique, Applications, and case Histories, 1984, D. Reidel publishing Company;

Barker, S. N and Speers R. G., “The geology of Asmari reservoir, Aghajari Field”, Volume1 \&2, NIOC internal Report No. P-3319, Ahwaz, 1977

Bellefleur, Gilles, Christof Muller, "Reliability of VSP-receiver orientations deduced from direct P-wave polarization." SEG Technical Program Expanded Abstracts 20.1, 2001, page 432-35.

Benhama,A., Cliet, C., and Dubesset, M., 1988, Study and application of spatial directional filtering in three component recordings : geophysical prospecting, 36, page591-613,

Becquey M. and Dubesset, M. " Three component sonde orientation in a deviated well" Geophysics, 1990, vol.55 Nº 10, page1386-1388.

Bush I. and Crampin, S. "Paris Basin VSPs: case history establishing combinations of fine layer (or lithologic) anisotropy and crack anisotropy from modelling shear wavefields near point singulatities" Geophys. Journal Int., 1991, N²107, page 433-437.

Check shot survey reports, well AJ-56,AJ-58,AJ-140, AJ-150, AJ-153, NIOC technical internal reports 
Chuanwen sun and Rubern D. Martinez, 3D Kirchhoff prestack time migration for V(z) and VTI media, PGS Geophysical, EAGE 64th Conference \& Exhibition — Florence, Italy, May 2002

Cliet, C., and Dubesset, M., 1987 : La paramétrisation des trajectoires de particules, Institut Français du pétrole, Report N 35080.

C. Cliet, L. Brodov, A. Tikhonov, D. Marin and D. Michon, "Anisotropy survey for reservoir definition", Geophys. J. Internat., 1991, 107, page417-427.

C. Esmersoy, "Velocity estimation from offset VSP using Direct P and converted SV-waves", POS6.4, SEG abstracts 1987, page538-541

De bazelaire, E. and Viallix, J. R.,1994, "Normal moveout in focus", Geophysical Prospecting, EAGE, 42, page 477-499;

DiSiena, J. P., J. E. Gaiser, and D. Corrigan, 1984, "Horizontal components and shear wave analysis of three-component PSV data”, in M. N. Toksöz and R. R. Stewart, eds., Vertical seismic profiling, Part B : Advanced concepts : Geophysical Press, 177235.(Bellefleur et al.)

Etchecopar, A., Bonnetain, J.L., 1992 - Cross sections from dipmeter data.

AAPG bull, V. 76, N0.5, Page621-637

Ehinger, Andreas, et al. "The depth stack: An economic solution for imaging complex geological structures." SEG Technical Program Expanded Abstracts 15.1, 1996, page332-35.

Guillaume, Patrice, et al. "3D finite-offset tomographic inversion of CRP-scan data, with or without anisotropy." SEG Technical Program Expanded Abstracts 20.1, 2001, page71821.

Gopa S. De, Donald F. Winterstein, Mark A. meadows, 1994, Comparison of P- and S-wave velocities and Q's from VSP and sonic log data, Geophysics, Vol. 59, No. 10, Page $1512-1529$

Hale, Ira David, 1983, PhD thesis, Standford University

Hardage, B., 1983. Vertical seismic profiling: Part A: Principles:

Geophysical Press Prorgam ,CiSL, undun,U K.

Hardage, B.; "Vertical Seismic Profiling" : Principles, Third updated and revised edition; in : Handbook of Geophysical Exploration, seismic exploration,Vol.14, 2000, Pergamon, Elsevier Science;

Helbig K., Thomsen L. 2005, 75-plus years of anisotropy in exploration and reservoir seismic, Geophysics, Vol. 70, No. 6, Page 9-25 
House N. , Fuller ,b., Shemeta J. , 2004, Integration of surface seismic,3D VSP, and Micro seismic Hydraulic fracture Mapping to improve Gas production in a tight complex reservoir, SEG 74th meeting, Colorado, USA

Heloise B. Lynn,Wallace Beckham E., 1999,P-Wave and S-wave azimuthal anisotropy at a naturally fractured gas reservoir, bluebell-Altamont Field, Utah, Geophysics Vol.64,No.4,Page1312-1328

Jenner, Edward, Marty Williams, and Tom Davis. "A new method for azimuthal velocity analysis and application to a 3D survey, Weyburn field, Saskatchewan, Canada." SEG Technical Program Expanded Abstracts 20.1, 2001, page102-05.

Kabbej A., Baina R., Duquet B. "Data driven automatic aperture optimization for Kirchhoff migration”, 75th Annual SEG International Meeting, Expanded Abstracts, 2005, page 1890-1893.

Khoshbalht, F, Memarian, H, Mohammadnia, M. "Comparison of Asmari, Pabdeh and Gurpi formation's fractures, derived from image log", Journal of Petrolum siecnce and engeneering, 2009

Liu K. , Bancrof J. C. , "The effect of dip-limited Kirchhoff migration and F-K migration", the university of Calgary, Calgary, Alberta,T2N 1N4, Canada

Liu Q. , Owusu J. , Alnemer J., "Fracture detection by P and C wave anisotropy from multiazimuth VSP", SEG, annule meeting, New Orleans, USA, 2006,

Lambaré, Gilles, Philippe Hermann, Jean-Paul Touré, Laure Capar, Patrice Guillaume, Nicolas Bousquié, Damien Grenié and Serge Zimine, 2007 a. "From time to depth imaging: An accurate workflow." SEG Technical Program Expanded Abstracts 26.1, 2007, page 3014-18.

Lambaré, Gilles, Philippe Hermann, Patrice Guillaume, Serge Zimine, Simon Wolfarth, Olivier Hermant and Suhail Butt, 2007 b, "From time to depth imaging with 'Beyond Dix"' First Break, volume 25 September , pages 71-76

Levin, Franklyn K. "Reflection from a dipping plane---Transversely isotropic solid." Geophysics 55.7,1990, page 851-55.

Letouzey J., S. Sherkati , J.M. Mengus , H. Motiei , M. Ehsani , A. Ahmadnia , J.L. Rudkiewicz , 2002. Regional structural interpretation of the Zagros Mountain belt in the Northern Fars and high Zagros. GEO 2002 meeting, Bahrain, Abstract.

Mari, J.L. et al; "Seismic Well Surveying", 1991, Editions Technip, Paris.

Mari, J. L. Online course of geophysic of the Lausanne University - Well seismic

Miriam Spinner, 3D CRS-based limited aperture Kirchhoff time migration “, SEG Expanded Abstracts 25, 2569, 2006, page 2569-2573

Miriam Spinner and Jurgen Mann, «CRS-based minimum-aperture time migration - A 2D land-data case study “,SEG Expanded Abstracts 26, 2354, 2007, page 2354-2358 
Naville C., US Patent no. 6,076,045, June 13, 2000,

Naville C., US Patent no. 2003/0086335 A1; May 8, 2003,

Naville C., Serbutoviez S., Bruneau J., 2001, SEISDIP: the "VSP dipmeter" from oriented 3 components, AAPG, Expanded Abstracts

Naville C. ,Serbutoviez S, Bruneau J. , Throo A. ,Japiot H. ,Daures R. , Gaborit J. Y. , Dip and Azimuths from 3 component Vertical seismic profiles(3C-VSP),IFP and CGG internal publication, 2001

Naville, C. " Detection of Anisotropy Using Shear-Wave Spitting in VSP surveys; Requirements and Alpplications", SEG Expanded Abstracts, $56^{\text {th }}$ int. SEG meeting, 1986, Houston, S5.2, page391-394.

Nicoletis, L., Cliet, C. \& Lefeuvre, F., " Shear-wave Splitting measurements from multishot VSP data, Expanded Abstracts, $58^{\text {th }}$ int. SEG meeting, 1988, Anaheim, POS 6.1, page527-530

P. N. Armstrong, "Method of estimating relative bearing of a borehole receiver", July 26, 2005, US pat. 6,922,373 B2

Robein, E. "Velocities, Time-imaging and Depth-imaging in reflection Seismics, principles and Methods" EAEG publication, 2003,

NIOC, Field survey report, Zero-offset VSP deviated well A, 2005,

NIOC, Processing VSP report, Zero-offset VSP well A, 2005,

NIOC, Field survey report, Rig source deviated VSP (Air Gun), well B, 2006,

NIOC, Processing VSP report, Rig source deviated VSP (Air Gun), well B, 2006,

Sepehr, M, Cosgrove, J W, The role of major fault zones in controlling the geometry and spatial organization of structures within the Zagros Fold-Thrust Belt, Geological Society, London, Special Publications, 2007, Vol: 272, Pages 419 - 436

Serbutoviez S., Naville C.,Bruneau J. , Huguet F. , Reinier M. ,2002,Pam-3C VSP- Preserved and true amplitude 3 component VSP, EAGE 64th conference \& Exhibition, Italy

Sherkati, S. Letouzey, J. ,variation of structural style and basin evolution in the central Zagros (Izeh zone and Dezful Embayment),Iran, Marine and petroleum geology 21 , 2004, page 535-554

Sherkati,S. Letouzey,J. and Frizon de Lamotte, D. 2006, Central Zagros fold-thrust belt (Iran): New insights from seismic data, field observation, and sandbox modeling, Tectonics, Vol. 25

Siliqi, R., et al. "High-order RMO picking using uncorrelated parameters." SEG Technical Program Expanded Abstracts 26.1, 2007, page 2772-76. 
Siliqi, Risto and Nicolas Bousquié. "Anelliptic time processing based on a shifted hyperbola approach." SEG Technical Program Expanded Abstracts 19.1, 2000, page 2245-48.

Soubaras, Robert. "Signal-preserving random noise attenuation by the f-x projection." SEG Technical Program Expanded Abstracts 13.1, 1994, page 1576-79.

Stewart A. Greenlagh and Ian M. Mason, "Orientation of a downhole triaxial geophone", 1995, Geophysics, VOL.60, NO4, page1234-1237.

Technical design for 3D seismic survey in AMK project, provided by BGP/OEOC companies, May 2004, NIOC Exploration

Well Completion reports of all wells, Aghajari Field, NIOC internal reports,

Wolfarth S., L. Capar, G. Lambaré, P. Guillaume, S. Butt and R. Siliqi, 2007, "Deriving a depth velocity model using Time Migrated Data-Case study", EAEG, London, Page53

Tabti,H. Gelius, L., Hellmann T., [2004], Fresnel aperture prestack depth migration: First Break, 22

Tabti, H. and P. Rasolofosaon. "Velocity analysis using nonhyperbolic move-out in anisotropic media of arbitrary symmetry: Synthetic and field data studies." Revue de 1 Institut Francais du Petrole 53.5, 1998, page 555-70.

Thomsen, Leon. "Weak elastic anisotropy." Geophysics 51.10, 1986, page1954-66.

Vinje, V., , Roberts, G., Taylor, R., [2008], Controlled beam migration: a versatile structural imaging tool, First Break, 26 (9), page109-113.

Xiaomin, Z. , Ran, Z. , Yingping, L. , peter, J. , David, D. , 2005, "Shear wave from nearoffset VSP survey and application", SEG, annual meeting Houston, USA

Yingping, L. Xiaomin, Z. ,Zhao R. ,Dushman D. , 2005, 3C VSP Tomography inversion for subsurface P-and S-wave velocity distribution, SEG annual meeting ,Houston, USA

Zhou R. Miandro R., Chinigo C., Lou M. , Patval Z. , Dary N. , 2006, Fracture orientation estimation using circular VSP, SEG, annual meeting New Orleans, USA

X. Zeng, G. A., McMechan, « Two methods for determining geophone orientation from VSP data », Geophysics, Vol. 71, N. 4, page V87-V97, 2006.

Zhao, Xiaomin, ran Zhou, Yingping Li, Peter Janak and david Dushman, "Shear waves from near-offset VSP survey and applications." SEG Technical Program Expanded Abstracts 24.1, 2005, page 2629-32.

Yilmaz O., 1988, Seismic Data Processing, volume2, SEG publication 

Appendixes 

Appendix 1 


\title{
BREVET D'INVENTION
}

\author{
$\star * * * * * * * * *$
}

IFP

$\star \star \star * * * * * * *$

\section{MÉTHODE DE POINTÉ-TEMPS ET D'ORIENTATION DE SIGNAUX SISMIQUES DE PUITS À TROIS COMPOSANTES}

\author{
$* * * * * * *$ \\ Invention de Charles NAVILLE, Sylvain SERBUTOVIEZ, Jean-Claude LECOMTE.
}

$\star * * * * * * *$

Abrégé descriptif

- Méthode de pointé de temps d'arrivée d'ondes sismiques et utilisation pour orienter les composantes d'un capteur multi-composantes.

- Après acquisition de données sismiques par une méthode de type PSV, au moyen d'un capteur multi - composantes, on construit un signal module en calculant la racine carrée de la somme des carrés d'au moins deux composantes sismiques orthogonales. Puis, on pointe des temps d'arrivée d'une onde sismique directe sur un extremum d'amplitude de ce signal module. A partir de ce pointé, on peut alors orienter les composantes sismiques dans un repère unique quelque soit la profondeur du capteur. Pour ce faire, on définit une fenêtre temporelle de part et d'autre des temps d'arrivée pointés, puis on détermine la direction azimutale par maximisation de l'énergie des composantes horizontales au sein de cette fenêtre temporelle. Enfin, on oriente les trois composantes dans un repère unique, défini par rapport à cette direction azimutale qui est identique pour chaque profondeur.

- Application à l'exploration ou la production de gisement pétrolier par exemple.

- Figure 5 à publier. 
La présente invention concerne le domaine de la sismique de puits, et plus particulièrement le domaine des techniques de prétraitement de données sismiques acquises au moyen de capteurs multicomposantes, obtenues lors d'opérations de Profil Sismique Verticale (PSV ou VSP en anglais).

La présente invention concerne notamment une méthode d'estimation de l'orientation d'un capteur sismique de puits multi-composantes.

La technique de PSV est couramment utilisée pour une meilleure connaissance de la structure du gisement et de son voisinage en phase d'exploration, ou en phase d'exploitation. Cette technique est également utilisée pour définir les structures géologiques au voisinage du puits pour guider le forage, ou redéfinir une déviation de la trajectoire de forage si ce dernier n'a malencontreusement pas atteint son objectif.

La mise en oeuvre la plus classique du profil sismique vertical (PSV) se fait au moyen d'une émission sismique effectuée par une source répétitive en surface, et d'une réception faite dans le puits. Cette dernière est effectuée grâce à une sonde de puits particulière, que l'on fait stationner successivement à différentes profondeurs. La sonde de puits comprend un 25 capteur, généralement à trois composantes, un système d'ancrage et une unité de numérisation pour la plupart des outils modernes. Le système d'ancrage et la conception mécanique de la sonde réceptrice de puits sont tels que l'enregistrement en trois composantes, du déplacement en trois dimensions de la formation au passage des diverses ondes sismiques est fidèle au déplacement effectif de la formation. Cette notion de fidélité vectorielle caractérise l'isotropie de l'ancrage mécanique des capteurs de la sonde réceptrice à la paroi du puits, et l'isotropie recherchée de la réception sismique en trois composantes. L'acquisition peut être faite avec un capteur triaxial placé à une profondeur unique, ou par une antenne de capteurs triaxiaux situés à des niveaux de réception profondeur adjacents. 
La figure 1 schématise de façon générale la géométrie d'acquisition d'un PSV 100 conduite dans un puits 101 foré sensiblement vertical, et en général faiblement dévié sur certains intervalles de profondeur. La profondeur du puits peut atteindre typiquement plusieurs milliers de mètres. La sonde sismique de puits 105 contenant les capteurs sismiques triaxiaux est descendue au moyen d'un câble 102, qui la relie à une unité d'enregistrement de surface 103 à partir de laquelle l'ingénieur terrain assure toutes les commandes de la sonde de puits, la télécommande de la source sismique 104, ainsi que le contrôle qualité des mesures. Afin de mesurer avec une bonne fidélité vectorielle le signal sismique qui se propage dans la formation rocheuse, en provenance de la source sismique de surface 104, cette sonde est appuyée fortement contre la paroi du puits à l'aide d'un dispositif d'ancrage symbolisé par le bras 106, préalablement à l'enregistrement de chaque station profondeur de mesure. Dans la configuration commune du PSV de base, la source sismique de surface 104 dite "zéro-offset", est placée en pratique dans un rayon de 100m par rapport à la tête de puits; compte tenu de la profondeur du puits, la source sismique 104 est dite "de surface" même si elle est placée à quelques mètres de profondeur. La sonde sismique 105 schématisée sur la figure1 peut représenter en réalité une sonde réceptrice comportant plusieurs niveaux profondeur de réception sismique adjacents, séparés typiquement d'une distance de 15 ou 20 mètres, chacun des niveaux correspondant à une sonde sismique indépendante, comportant des capteurs sismiques triaxiaux et un système d'ancrage.

La figure 2a schématise la géométrie de propagation 200 des ondes sismiques directes émises par la source de surface 206, reçues par les capteurs 201 à 204 situés dans le puits 205 : on peut constater, sur ce schéma, l'étroitesse de l'angle solide $207(\beta)$ de propagation en arrivée directe. Cet angle englobe l'ensemble de la partie profonde du puits dans laquelle les mesures de PSV sont réalisées avec un intervalle régulier fin, couramment de $15 \mathrm{~m}$. De fait, l'étroitesse de l'angle solide d'émission, garantit pour la réception dans le puits l'hypothèse de constance de forme d'onde des modes propres de propagation sismique en milieu stratifié sensiblement homogène pour des niveaux de mesure profondeur adjacents. De plus, on peut constater sur le schéma de la figure 2a que la différence de direction de propagation est extrêmement réduite entre les rais directs, associés à des niveaux de mesure de puits adjacents, comme par exemple les rais directs a1 et a2 associés au couple de positions 201-202, ou les rais directs a3 et a4 associés au couple 203-204. En pratique, la proximité immédiate des chemins de propagation entre une source commune et des récepteurs sensiblement adjacents, voisins d'une dizaine à une centaine de mètres par exemple, est également vérifiée pour une onde descendante plus complexe qu'une onde directe, par exemple une onde de type $\mathrm{P}$ convertie $\mathrm{S}$ au droit d'une interface située à une 
profondeur intermédiaire, par exemple dans la moitié supérieure de la profondeur totale du puits.

Les figures $2 b$ et $2 c$ illustrent deux géométries de propagation pour lesquelles l'arrivée directe de pression $\mathrm{P}$ arrive orthogonalement à la direction de la composante $\mathrm{Z}$ de direction spatiale connue. Par conséquent toute l'énergie d'onde $P$ est enregistrée par les capteurs $X$ et $\mathrm{Y}$ de direction spatiale inconnue, et il n'est donc pas aisé de pointer un phase remarquable de l'onde directe sur les signaux bruts de façon cohérente sur les niveaux de mesure profondeur adjacents.

La figure $2 \mathrm{~b}$ schématise la géométrie de propagation 210 des ondes sismiques directes 212 émises par la source de surface 216, reçues par le capteur 211 situé dans le puits 215, et arrivant perpendiculairement au puits 215. La trajectoire de ce puit est sensiblement horizontale au niveau de la position du capteur $\mathbf{2 1 1}$ dont la composante Z 213 est axiale au puits.

La figure 2c schématise la géométrie de propagation 220 des ondes sismiques directes 222 émises par la source de surface 226, reçues par le capteur 221 situé dans le puits 225, et arrivant perpendiculairement à la composante sensiblement verticale Z 223 du capteur 221 à trois composantes. La trajectoire de ce puit 225 est sensiblement verticale au niveau de la position du capteur 221.

Le terme "multicomposante » qualifie une disposition particulière d'une pluralité de capteurs sismiques. Par exemple, un capteur à trois composantes comprend trois récepteurs sismiques unidirectionnels disposés selon des axes orthogonaux, tels que des géophones ou des accéléromètres. Les ondes sismiques se propageant en trois dimensions, on utilise un capteur à trois composantes pour caractériser l'ensemble des ondes sismiques.

On appelle " composante », le signal issu d'un capteur sismique unitaire. Un capteur à trois composantes génère trois signaux électriques enregistrés selon trois axes orthogonaux. En général, la sonde réceptrice de fond comporte une composante d'axe connu, disposée soit selon la verticale, soit selon l'axe de l'outil. Cet axe de l'outil coïncide sensiblement avec l'axe du puits, après ancrage de la sonde à la paroi du puits, et de deux composantes orthogonales, dont on ne connaît pas la direction exacte dans le plan orthogonal.

Il est usuel lors de l'exploitation des données sismiques, obtenues par une méthode de type PSV au moyen de capteur à trois composantes, de traiter une seule des composantes enregistrées, en général soit la composante verticale, soit la composante axiale au puits, soit la composante correspondant à la direction spatiale maximisant l'énergie de l'arrivée directe d'onde de pression. Des exemples de traitement monocomposante de données sismiques 
sont décrits dans de nombreuses publications et ouvrages publiés, par exemple dans des ouvrages spécialisés suivants :

Hardage,B.; "Vertical Seismic Profiling" : Principles, Third updated and revised edition; in: Handbook of Geophysical Exploration, seismic exploration,Vol.14, 2000, Pergamon, Elsevier Science;

A.H. Balch \& Myung W. Lee; Vertical Seismic Profiling : technique, Applications, and case Histories, 1984, D. Reidel publishing Company;

Mari, J.L. et al; "Seismic Well Surveying", 1991, Editions Technip, Paris.

II est usuel également de prendre en compte la polarisation des ondes directes en mode de pression pour l'orientation et le traitement des profils sismiques verticaux avec offset, pour lesquels le puits est sensiblement vertical et la position de la source est située à une distance supérieure à $10 \%$ de la profondeur totale du puits. On calcule la composante orthogonale qui maximise l'énergie de l'arrivée directe d'onde de Pression (onde P) dans le plan orthogonal, et dans une fenêtre temps définie par le pointé temps de cette arrivée directe P. Pour déterminer cette direction azimutale dans un plan, à partir de deux composantes, on utilise une technique de maximisation de l'énergie, par exemple celle décrite dans le document suivant :

DiSiena, J. P., J. E. Gaiser, and D. Corrigan, 1984, "Horizontal components and shear wave analysis of three-component PSV data", in M. N. Toksöz and R. R. Stewart, eds., Vertical seismic profiling, Part B : Advanced concepts : Geophysical Press, 177-235.

Cependant, la limitation classique du traitement à une seule ou à deux des composantes conduit à des indéterminations potentielles dans l'identification du mode d'onde de certaines arrivées reçues par le capteur, pression ou cisaillement, d'une part, et dans le positionnement d'événements géologiques d'autre part, qu'il n'est pas possible de lever. L'inversion des temps, ou les techniques de migration des événements sismiques réfléchis uniquement, conduisent à une pluralité de solutions puisque la direction azimutale de pendage des réflecteurs demeure inconnue. De plus, lorsque deux événements sismiques arrivent en même temps avec des vitesses apparentes voisines, et figurent sur quelques traces profondeur adjacentes (six à douze par exemple), on vérifie qu'il est quasiment impossible de les séparer par un traitement monocomposante classique.

II apparaît donc indispensable, pour améliorer la fiabilité de l'interprétation des données sismiques de PSV, de traiter les trois composantes. Toutefois, l'étape d'acquisition ne permet pas de fournir l'orientation réelle des géophones du câble, alors que cette 
information est indispensable pour permettre de traiter les données. En effet, les composantes horizontales issues de PSV à trois composantes ont une orientation inconnue et aléatoire, car les câbles portant les capteurs sismiques (géophones) ne peuvent pas contrôler l'orientation de ces éléments. Traiter les trois composantes peut être envisagé, dans la mesure ou la qualité d'isotropie de réception mécanique de la sonde de PSV (résumée sous l'appellation de "fidélité vectorielle" de la réception) est suffisamment bonne. Ceci est le cas avec la plupart des outils de PSV modernes, dans lesquels le rapport entre la force d'ancrage de la partie de la sonde (ou de la sonde entière) supportant les capteurs triaxiaux, et le poids dans l'air du support (ou de la sonde) est supérieur à cinq. Toutefois, bien qu'il existe des accessoires de mesure d'orientation complète d'un outil dans un puits, comme les magnétomètres-inclinomètres et les gyroscopes de puits, ces accessoires matériels sophistiqués sont souvent inutilisés, car ils représentent un surcoût substantiel. De plus, ils peuvent détériorer les qualités d'ancrage mécanique de la sonde PSV à laquelle ils sont combinés. On constate que l'étape d'acquisition des PSV ne permet que rarement de fournir systématiquement l'orientation réelle et complète des capteurs trois composantes, alors que cette information est indispensable pour permettre le traitement des trois composantes. De fait, les composantes orthogonales issues de PSV à trois composantes ont la plupart du temps une orientation inconnue et aléatoire. C'est en particulier le cas dans les intervalles de profondeur du puits faiblement inclinés, notamment au dessous de 10 degrés d'inclinaison verticale, intervalles dans lesquels les dispositifs d'orientation partiels du type montage des capteurs sur cardans, ou adjonction d'un dispositif pendulaire sensible à la gravité mesurant l'angle de « relative bearing » dans le plan orthogonal à l'axe de l'outil, sont rendus inopérants.

Ainsi, afin de traiter de façon complète, efficace et bénéfique tous les signaux issus de capteurs multicomposantes, constituant un jeu usuel de données de sismique de puits, il est nécessaire d'orienter les géophones des capteurs multicomposantes.

\section{État de la technique}

Pour déterminer une direction azimutale dans l'espace à partir des trois composantes, on peut utiliser la technique d'analyse de matrice de covariance décrite dans les documents suivants :

Benhama,A., Cliet, C., and Dubesset, M., 1988, Study and application of spatial directional filtering in three component recordings : geophysical propecting, 36, 591-613,

Cliet, C., and Dubesset, M., 1987 : La paramétrisation des trajectoires de particules, Institut Français du pétrole, Report N 35080. 
Cette technique fait l'hypothèse que la direction de polarisation ainsi calculée appartient au plan vertical contenant les positions de source et de récepteur. Ceci est réaliste dans un milieu sédimentaire stratifié, même avec des valeurs de pendage faible à moyen (jusqu'à environ $20^{\circ}$ et pour tout pendage, si la source et le capteur de puits sont situés dans le plan de pendage structural local au voisinage puits). Ce processus fait appel au pointé de l'onde directe de pression effectué sur la composante verticale ou axiale au puits, dont la forme de signal est cohérente sur les traces de profondeurs adjacentes. Ce processus devient inapplicable si l'énergie d'arrivée directe de pression est trop faible sur les composantes orthogonales.

Cette méthode d'estimation de l'orientation des capteurs trois composantes par maximisation d'énergie du signal d'onde-P directe dans une fenêtre temps définie par pointé de la composante vertical ou axiale au puits, s'applique bien aux géométries d'acquisition comme le PSV avec offset, qui comprend au moins une source sismique de surface positionnée de façon fixe à une certaine distance offset du puits (typiquement égale à 0.2 à 1.5 fois la profondeur verticale totale du puits). On oriente de même les capteurs trois composantes des configurations de walkaway 2D ou 3D, et de walkaround, qui consistent à enregistrer sur une antenne de capteurs fixes $3 \mathrm{C}$ dans le puits, le signal émis à partir d'une pluralité de positions de source de surface selon une disposition qui détermine le type d'étude sismique de puits. Ainsi un walkaway 2D correspond à une disposition en ligne des points sources, un walkaway $3 \mathrm{D}$ correspond à une grille de points sources, plus ou moins régulière, et un walkaround correspond à un cercle de points sources tir autour du puits.

Par exemple, une méthode d'orientation des capteurs orthogonaux à l'aide de la polarisation d'arrivée directe d'onde $\mathrm{P}$ est bien illustrée dans la configuration d'une pluralité de points source placés à des azimuts variés autour du puits dans le document suivant :

P. N. Armstrong, "Method of estimating relative bearing of a borehole receiver", juillet 26,2005 , US pat. $6,922,373$ B2

Dans tous ces cas de figure, la pluralité de positions de source de surface permet d'en trouver plusieurs pour lesquelles le processus par maximisation d'arrivée directe d'onde-P est bien adapté à l'estimation précise et redondante de l'orientation. II n'est pas nécessaire que l'outil de fond soit muni de dispositif d'orientation des composantes.

Avec la redondance de points source, l'adoption des hypothèses de propagation commune dans le plan vertical source-récepteur, et pour autant que la rectilinéarité de l'arrivée directe d'onde $\mathrm{P}$ soit correcte pour une proportion suffisante de points source, il n'est même pas nécessaire de connaître la trajectoire du puits, comme en témoigne le document suivant : 
Stewart A. Greenlagh and lan M. Mason, "Orientation of a downhole triaxial geophone", 1995, Geophysics, VOL.60, NO4, p 1234-1237.

Enfin, on connaît également deux méthodes permettant de déterminer l'orientation des deux composantes horizontales lorsque l'on dispose d'une pluralité de positions de sources en surface, par le document :

X. Zeng, G. A., McMechan, « Two methods for determining geophone orientation from PSV data », Geophysics, Vol. 71, N. 4, p. V87-V97, 2006.

Une première méthode, basée sur le plan de polarisation, permet de déterminer l'orientation des composantes horizontales de capteurs montés sur cardans, à partir de l'énergie de polarisation d'une fenêtre temporelle autour de l'arrivée directe $P$, avec une indétermination de $\pi$ sur l'angle trouvé. II est notoire de constater dans ce document que cette indétermination de $\pi$ est maintenue, alors qu'elle pourrait être levée aisément en pointant une phase remarquable du signal d'arrivée $P$ sur la composante verticale, en particulier un extremum d'amplitude, et en imposant une polarité systématiquement identique sur la composante horizontale sortie du processus de maximisation d'amplitude, comme cela est pratiqué industriellement. Naturellement, cette méthode classique de polarisation, nommée PPDI, ne donne des résultats satisfaisants que si l'énergie d'onde $P$ est substantielle dans le plan horizontal, et cette méthode fait usage de la pluralité massive des points source de surface pour améliorer la fiabilité de l'orientation et pour lever l'indétermination de $\pi$.

Dans la seconde méthode, appelée RADI, on calcule l'azimut relatif entre deux stations profondeur adjacentes de géophones, par une méthode de maximisation d'une corrélation entre vecteurs bicomposantes, calculée sur une période ou une fenêtre temporelle autour de l'onde $\mathrm{P}$ directe de forte énergie sur les composantes horizontales. En pratique, la méthode RADI ne donne pas de bons résultats sur les données réelles, c'est pourquoi elle est généralement appliquée postérieurement à la première méthode PPDI pour lever l'indétermination de $\pi$ mentionnée précédemment.

On peut également utiliser les propriétés de polarisation des ondes sismiques directes $\mathrm{P}$ pour réorienter les composantes situées dans le plan perpendiculaire à l'axe du puits, dans le cas d'une géométrie particulière : géométrie d'acquisition dans un puits dévié de trajectoire connue, avec une source sismique de surface unique placée en offset de la tête de puits, et avec enregistrement par des capteurs triaxiaux fixes par rapport au corps d'un outil non 
pourvu d'accessoire de mesure de l'angle de "relative bearing ». On applique alors une procédure de maximisation de l'arrivée $P$ sur les composantes orthogonales. On fait également l'hypothèse que le rayon direct d'onde $\mathrm{P}$ est compris dans le plan vertical contenant la source de surface et le capteur de fond. Naturellement cette réorientation n'est valable que pour la diagraphie en cours, et elle est aisée lorsque l'arrivée directe $P$ est de polarisation sensiblement linéaire, descendante ou montante réfractée, et non interférée. Cette technique bien connue de l'homme de l'art est décrite par exemple dans le document suivant :

M. Becquey et M. Dubesset., " Three component sonde orientation in a deviated well" 10 Geophysics, 1990, vol.55 №10, p. 1386-1388.

Cette méthode d'orientation fournit en général soit deux solutions, soit une solution double, soit aucune solution. Dans le cas échéant, on lève l'indétermination de double solution de rayon d'arrivée directe d'onde- $P$, en ne conservant que celle qui est la plus proche de la droite définie par la source et le récepteur. S'il n'y a pas de solution, on conserve la solution double comme approximation : $\left(\Phi=\Phi_{0}\right.$ dans l'équation 6 , page $1387 \mathrm{du}$ document précédemment cité). Cette technique de réorientation a été utilisée avec succès sur plusieurs cas réels, comme en témoigne par exemple les illustrations en page $420 \mathrm{du}$ document suivant :

C. Cliet, L. Brodov, A. Tikhonov, D. Marin and D. Michon, "Anisotropy survey for reservoir definition", Geophys. J. Internat., 1991, 107, 417-427.

Une limitation à tous les processus d'orientation précédemment cités prenant en compte l'arrivée directe d'onde $\mathrm{P}$, survient lorsque l'énergie de la projection de l'arrivée directe sur les deux composantes non axiales, ou non verticales, est très faible. Ceci est par exemple le cas si le puits est vertical ou faiblement dévié, notamment avec la géométrie d'acquisition très courante dite de PSV "zéro-offset", pour laquelle la source de surface située proche de la tête de puits est activée en une position unique, à terre ou en mer, avec une structure géologique ayant des pendages locaux quelconques et souvent inconnus. II est connu de pallier à cette limitation en activant une source sismique supplémentaire à la source zéro-offset, localisée à une distance suffisante du puits, et de préférence dans la direction azimutale générale de la structure géologique dans le voisinage du puits. Mais cette solution alternative est rarement utilisée car elle entraîne des dépenses supplémentaires pour l'opération de mesure ainsi qu'un allongement de la durée d'acquisition, et donc de l'immobilisation de l'appareil de forage sur le site. L'activation de cette source additionnelle est effectuée successivement ou simultanément à la source zéro offset, avec la même 
position profondeur d'ancrage et de mesure que l'outil PSV, dont on désire orienter les trois composantes. De plus, ces palliatifs aux méthodes ou configurations géométriques de tir antérieurs, présentent l'inconvénient de ne pas être toujours applicables, soit à cause de reliefs accidentés par exemple, soit à cause d'indisponibilité matérielle ou financière de sources sismiques supplémentaires, soit à cause d'interférence de l'arrivée directe d'onde-P par une arrivée réfractée ou diffractée secondaire. Lorsqu'il est absolument nécessaire de connaître l'orientation des capteurs triaxiaux, il est judicieux d'envisager la mise en oeuvre d'une source supplémentaire en offset du puits, et de confronter cette solution avec le choix alternatif d'un outil de puits combinable avec un accessoire d'orientation. Mais en pratique, ces deux modes de mesure de PSV sont rarement mis en oeuvre.

Par ailleurs, après orientation des composantes triaxiales, certains programmes de traitement de PSV multi composantes se limitent aux deux composantes contenues dans le plan vertical contenant le capteur et la source, comme par exemple la méthode décrite dans le document suivant :

C. Esmersoy, "Velocity estimation from offset VSPs using Direct P and converted SVwaves", POS6.4, SEG abstracts 1987, p538-541.

Cependant, comme expliqué précédemment, la limitation classique du traitement à une seule ou à deux des composantes conduit à des indéterminations potentielles dans l'identification du mode d'onde de certaines arrivées reçues par le capteur, pression ou cisaillement, d'une part, et dans le positionnement d'événements géologiques d'autre part, qu'il n'est pas possible de lever.

Ainsi, afin de traiter de façon complète, efficace et bénéfique tous les signaux issus de capteurs multicomposantes, constituant un jeu usuel de données de sismique de puits, il est nécessaire d'orienter les géophones des capteurs multi composantes.

L'objet de l'invention est une méthode alternative d'orientation spatiale des géophones d'un capteur multi-composantes permettant de s'affranchir des difficultés d'orientation des techniques antérieures. La méthode permet d'orienter les géophones dans un repère localement cohérent et sensiblement unique pour tous les niveaux de mesure, inconnu à une rotation constante près, puis d'orienter les trois composantes dans un repère de directions géographiques liées au globe terrestre, afin de permettre le traitement isotrope des trois composantes, pour le bénéfice de l'interprétation structurale et géologique. La méthode se base sur une technique originale de pointé-temps des temps d'arrivée d'une onde directe $(P$ ou S) sur l'une des phases les plus énergiques d'un signal module. 


\section{La méthode selon l'invention}

Ainsi, l'invention concerne une méthode de prétraitement de données sismiques acquises au moyen d'une méthode de prospection sismique de type profil sismique vertical. Cette méthode de prospection sismique de type profil sismique vertical comprend une émission d'ondes sismiques et une réception de ces ondes sismiques au moyen d'au moins un capteur multi - composantes positionné au sein d'un puits et que l'on fait stationner à au moins deux profondeurs. Ce capteur comprend au moins trois géophones orthogonaux enregistrant en fonction du temps une première composante sismique dans une direction vectorielle connue, et au moins deux autres composantes sismiques dans deux directions orthogonales à cette direction vectorielle connue. La méthode comprend les étapes suivantes :

a- on construit un nouveau signal en calculant la racine carrée de la somme des carrés d'au moins deux composantes sismiques orthogonales, appelé signal module ;

b- $\quad$ on pointe des temps d'arrivée d'une onde sismique directe sur un extremum d'amplitude de ce signal module.

Selon un mode de réalisation, on peut construire le signal module en calculant la racine carrée de la somme des carrés des deux composantes sismiques orthogonales à la direction vectorielle connue. On pointe alors les temps d'arrivée d'une onde de cisaillement descendante.

Selon un autre mode de réalisation, on peut également pointer l'arrivée d'une onde de pression. On peut alors calculer des vitesses des ondes de cisaillement et de pression à partir de ces temps d'arrivée. On peut en déduire des rapports de vitesse et/ou un coefficient de Poisson.

Selon un autre mode de réalisation, on peut construire le signal module en calculant la racine carrée de la somme des carrés des trois composantes sismiques, et l'on pointe les temps d'arrivée d'une onde de pression directe.

De façon préférentielle, préalablement à l'étape a), on préserve l'isotropie du signal en trois composantes, en respectant des rapports d'amplitude et des différences de phase entre les composantes sismiques.

Selon l'invention, on peut améliorer un rapport signal sur bruit des trois composantes du signal brut, préalablement au calcul du module, au moyen d'une déconvolution isotrope des trois composantes par un signal unique d'onde de pression descendante extrait de la composante de direction vectorielle connue. On peut également, filtrer le signal module de 
façon à supprimer des composantes basses fréquences, avant de pointer les temps d'arrivée de l'onde sismique directe.

Selon l'invention, on peut orienter les composantes sismiques dans un repère unique quelque soit la profondeur du capteur, au moyen des étapes suivantes répétées pour chaque profondeur :

- on définit une fenêtre temporelle de part et d'autre des temps d'arrivée ;

- on détermine une direction azimutale par maximisation d'une énergie des composantes sismiques orthogonales à ladite direction vectorielle connue au sein de la fenêtre temporelle ;

- on oriente à 360 degrés près les composantes sismiques orthogonales à la direction vectorielle connue dans un repère unique défini par rapport à la direction azimutale qui est identique pour chaque profondeur.

Selon un mode réalisation, on peut déterminer l'orientation géographique de ce repère unique.

On peut déterminer l'orientation géographique du repère unique en montant le capteur multi-composantes sur un système de double cardans, ce système permettant d'orienter par gravité les composantes sismiques lorsque l'inclinaison du puits atteint une valeur d'au moins environ 10 degrés.

On peut également descendre dans le puits un outil de mesure de puits comportant le capteur multi-composantes monté de façon fixe. On détermine alors l'orientation géographique du repère unique au moyen d'un système de mesure d'un angle de "relative bearing" monté sur l'outil de mesure, qui permet de retrouver l'orientation du capteur multicomposantes lorsque l'inclinaison du puits atteint une valeur d'au moins environ 10 degrés.

Selon un mode de réalisation, on descend dans le puits un outil de mesure de puits comprenant une pluralité de capteurs multi - composantes placés à des profondeurs de mesure adjacentes, et on détermine l'orientation géographique du repère unique en couplant au moins l'un des capteurs multi - composantes à un outil de mesure d'orientation géographique, tel qu'un magnétomètre-inclinomètre ou un gyroscope.

Selon un autre mode de réalisation, au moins une portion du puits est horizontale et le capteur multi-composantes est monté de façon fixe dans un outil de mesure de puits. On détermine alors l'orientation géographique du repère unique en assimilant une direction de maximisation d'une onde de pression directe à une droite reliant une position du capteur à une position d'une source émettant les ondes sismiques. 
Selon l'invention, on peut également déterminer, sur différentes portions du puits, des repères uniques ayant un axe commun, ces portions ayant des zones de chevauchement permettant de déterminer un angle de rotation à appliquer à ces repères uniques, de façon à obtenir un repère unique pour l'ensemble du puits.

Enfin, on peut utiliser l'orientation dans un repère unique de façon automatisée pour obtenir un contrôle de la qualité des composantes sismiques, immédiatement après l'acquisition de mesures sur le terrain.

D'autres caractéristiques et avantages de la méthode selon l'invention, apparaîtront à la lecture de la description ci-après d'exemples non limitatifs de réalisations, en se référant aux figures annexées et décrites ci-après.

\section{Présentation succincte des figures}

- La figure 1 schématise de façon générale la géométrie d'acquisition d'un PSV avec source de surface et sonde comportant un seul niveau de réception sismique, descendue dans un puits sensiblement vertical au moyen d'un câble.

- La figure 2a illustre l'étroitesse de l'angle solide de propagation en arrivée directe, qui garantit l'hypothèse de constance de forme d'onde des modes propres de propagation sismique en milieu stratifié, sensiblement homogène pour des niveaux de mesure profondeur adjacents.

- Les figures $2 b$ et 2c illustrent deux géométries de propagation pour lesquelles l'arrivée directe de pression $\mathrm{P}$ arrive orthogonalement à la direction de la composante $\mathrm{Z}$ de direction spatiale connue; cette situation peut arriver en puits horizontal (fig. 2b), en puits dévié, ou en puits vertical (fig. 2c), lorsque la source est éloignée du puits d'une distance adéquate.

- Les figures $3 a$ et $3 b$ indiquent la disposition des trois composantes d'un capteur triaxial monté sur double cardans de type "tourelle", et placé dans un puits dévié : plan vertical tangent au puits (fig. 3a); plan horizontal (fig.3b).

- Les figures $4 \mathrm{a}$ et $4 \mathrm{~b}$ indiquent la disposition des trois composantes d'un capteur triaxial monté de façon fixe dans l'outil, dans le plan orthogonal à l'axe du puits et de la sonde (fig.4a), qui permet d'illustrer l'angle de « relative bearing », et dans le plan vertical tangent au puits (fig. $4 \mathrm{~b}$ ). 
- La figure 5 illustre l'attitude spatiale des trièdres des capteurs sismiques dans les parties verticales et déviées d'un puits avant orientation (a): montage fixe, c): montage sur doubles cardans), et la configuration des trièdres après orientation (b).

- Les figures $6 a, 6 b, 6 c$ et $6 d$ illustrent le principe de propagation des modes propres d'onde sismique volumique de cisaillement (ondes $S$ ) dans un milieu sensiblement homogène, et la méthode d'orientation des deux composantes orthogonales non orientées dans un repère intermédiaire unique selon l'invention.

- La figure 7 est un organigramme de l'ensemble du processus d'orientation selon l'invention.

- La figure 8 illustre le mouvement de particule de l'onde descendante $S$ dans la fenêtre temps de calcul d'orientation, dans le repère de l'outil; l'angle AG calculé et correspondant à la flèche superposée à l'hodogramme indique l'azimut de maximisation.

- Les figures 9a, 9b et 9c, représentent les rejeux bruts isotropes des composantes respectives $Z, X, Y$ mesurées par l'outil de puits, versus la profondeur croissante de gauche à droite.

- Les figures $10 \mathrm{a}, 10 \mathrm{~b}$ et $10 \mathrm{c}$ représentent les rejeux bruts isotropes des composantes géographiques orientées respectives Z-down verticale, $\mathrm{H}-\mathrm{Nord}(\mathrm{HN})$ et $\mathrm{H}$-East $(\mathrm{HE})$ de l'outil, versus la profondeur croissante de gauche à droite, et la cohérence obtenue sur l'arrivée $S$ dans la fenêtre rectangulaire, illustre l'efficacité de la méthode d'orientation des deux composantes orthogonales selon l'invention.

\section{Description détaillée de la méthode}

L'invention concerne une méthode de prétraitement spécifique de données sismiques acquises lors de la mise en oeuvre d'une opération de prospection sismique de type profil sismique vertical. Selon un mode de réalisation cette opération comprend une seule position de surface pour l'émission d'ondes sismiques, située à proximité de l'appareil de forage, et une réception des ondes sismiques au moyen d'un capteur multi - composantes positionné au sein d'un puits vertical à faiblement dévié. Le capteur (monté dans la sonde sismique) comporte trois géophones orthogonaux. Et on fait stationner ce capteur à au moins deux profondeurs voisines de quelques mètres. Le signal enregistré selon la direction axiale de chaque capteur unitaire est appelé composante du signal. La sonde réceptrice enregistre en fonction du temps au moins une composante dans une direction vectorielle connue, verticale ou axiale au puits de mesure, et deux composantes sismiques dites "orthogonales", c'est à dire orthogonales à la composante de direction vectorielle connue. 
Les figures $6 a$ et $6 \mathrm{~b}$ illustrent le principe physique de base de la propagation d'une onde sismique de cisaillement, dite $S$ (shear). II existe deux types d'ondes de volume : les ondes de pression (ondes $\mathrm{P}$ ), dont la polarisation, ou direction de déplacement de la formation au passage de l'onde, est parallèle à la direction de propagation, non illustrées sur la figure $6 a$, et les ondes transversales (ondes $S$ ) polarisées sensiblement perpendiculairement à la direction de propagation, illustrées sur la figure $6 a$.

La figure 6a représente un schéma d'un plan vertical de propagation 600 : la surface 601 symbolise non seulement la surface du sol mais également les premières couches de terrain hétérogènes jusqu'à environ 200 mètres d'épaisseur, ou même la surface rugueuse du fond de la mer, qui sont le siège de conversions de modes d'onde $\mathrm{P}$ en mode $\mathrm{S}$. Tous les modes d'ondes se propageant en profondeur suivant l'axe vertical 602. Ainsi, à partir d'une faible profondeur de terrain, l'onde $S$ se propage verticalement dans un milieu sensiblement homogène à l'échelle des longueurs d'onde sismiques utilisées. Le milieu est isotrope ou avec anisotropie faible pour la direction de propagation verticale (entraînant une biréfringence ordinaire), et il comporte une stratification éventuelle de couches de caractéristiques variables, avec un pendage éventuel identique pour toutes les couches ou non. Le milieu est ainsi représentatif de formations usuelles sédimentaires ou de socle. Le schéma de la figure $6 a$ illustre un milieu biréfringent dans lequel un train d'onde de cisaillement complexe se propage verticalement en profondeur selon l'axe 602 et comprend: - une onde de cisaillement rapide S-f ayant une forme de vibration 603 quelconque polarisée linéairement selon la direction constante $\mathbf{6 1 1}$ orthogonale à la direction de propagation 602 . ; - une onde de cisaillement lente S-s ayant une forme de vibration 604 en général différente de 603 et polarisée linéairement selon la direction constante $\mathbf{6 1 2}$ orthogonale à la fois aux axes $\mathbf{6 0 2}$ de propagation et $\mathbf{6 1 1}$ de polarisation d'onde S rapide S-f.

25 Les vecteurs de polarisation 611 et 612 symbolisent les directions de vibration de chacun des modes propres d'onde $S$ propagées selon la verticale, mais aussi l'amplitude du signal. L'amplitude du signal de vibration caractérise indifféremment le déplacement, la vitesse ou l'accélération du mouvement sismique de chacun des modes propres d'onde S-f et S-S, dont la direction et la forme d'onde demeurent constantes tout au long de la propagation, conformément à la physique du phénomène de propagation. La figure 6a montre, pour des instants successifs de propagation ti $\mathbf{6 1 0}(\mathrm{i}=0,1,2, \mathrm{i})$ mesurés à partir de l'instant d'activation de la source sismique de surface, la profondeur $z_{f}$ i 613 atteinte par l'onde rapide $S-f$, et la profondeur $z_{s}$ i 614 atteinte par l'onde lente $S-s$, le pied des vecteurs de polarisation respectifs 611 et 612 indiquant la profondeur exacte atteinte par chacune des deux ondes $S$ au temps ti $\mathbf{6 1 0}$. De fait, la profondeur $\mathrm{Z}_{\mathrm{f}}$ i $\mathbf{6 1 3}$ de l'onde rapide $\mathrm{S}$-f est progressivement et 
faiblement supérieure de quelques pourcents à la profondeur $z_{s}$ i 614 au cours de la propagation.

La figure $6 \mathrm{~b}$ représente dans le plan horizontal 650 , orthogonal à la direction de propagation verticale, des diagrammes de projection des formes du mouvement de vibration théoriques du train d'onde $S$. Ils représentent chacun une succession continue des points $[X(t), Y(t)]$ des signaux sismiques, dans une fenêtre temps centrée, dans le cas présent, autour du temps ti de l'arrivée d'onde $S$ à illustrer : ces diagrammes sont également connus sous les dénominations de "mouvement de particules" si les signaux $X$ et $Y$ représentent un déplacement au passage de l'onde sismique, ou même "hodogrammes" si les signaux $X$ et $Y$ représentent une vitesse de déplacement, mesurée par exemple par un capteur de type géophone. Ils sont encore appelés "diagramme de polarisation" ou simplement "polarisation" si les signaux $X$ et $Y$ représentent indifféremment un déplacement, une vitesse ou une accélération.

La figure $6 \mathrm{~b}$ montre sur la colonne de droite 653 au dessous de l'indication "IN", et pour des profondeurs verticales fixes successives zi $(i=0,1,2, i) \mathbf{6 6 0}$, atteintes par le train d'onde $S$ dans une fenêtre temps autour des instants de propagation ti $610(i=0,1,2, i)$ définis sur la figure $6 a$, les diagrammes de polarisations théoriques 651 d'orientation totalement aléatoire dans le plan horizontal pour chaque station profondeur zi, que l'on observe dans le repère d'une sonde sismique de puits utilisée pour l'enregistrement des PSV et qui s'ancre à la paroi de puits selon un azimut aléatoire. L'aléa de rotation du câble de diagraphie au bout duquel la sonde de puits est fixée est bien connu de l'homme du métier, cet aléa étant transmis aux capteurs sismiques à montage fixe dans une sonde. Par ailleurs, si ladite sonde réceptrice comporte des cardans de type tourelle sur lesquels sont montés les capteurs sismiques, la rotation des cardans autour de l'axe de la sonde est libre lorsque la sonde est dans un puits vertical, ce qui entraîne également une orientation aléatoire des capteurs horizontaux, même si l'on connaissait l'orientation azimutale de la sonde.

La figure $6 b$ indique symboliquement le processus P1 (666) suivant l'invention, via une flèche entre chaque diagramme de polarisation 651 des données entrées en colonne de droite "IN" 653, et le diagramme de polarisation orienté correspondant en colonne de gauche "OUT" 663; ce processus consiste à déterminer la direction d'amplitude maximale 652 sur le diagramme 651, de façon indépendante pour chaque profondeur de mesure sismique de puits, par une technique connue de régression linéaire du groupe des points $X(t), Y(t)$ dans une fenêtre -temps limitée appropriée, si possible plus courte que la fenêtre-temps correspondant au diagrammes 651 ou 661, puis à appliquer une rotation d'axe vertical aux signaux sismiques horizontaux de façon à faire coïncider la direction d'amplitude maximale 651 à une direction azimutale constante arbitraire 662 sur le diagramme 661, dans un 
premier temps. Ce processus est bien adapté au cas des PSV dits à zéro offset en puits vertical, où la source d'onde $P$ usuellement employée génère involontairement et très souvent en pratique des ondes de cisaillement énergiques au passage de la zone altérée et hétérogène de surface ou de la surface rugueuse du fond de la mer.

La figure $6 \mathrm{~b}$ montre sur la colonne de gauche 663 au dessous de l'indication "OUT", et pour des profondeurs verticales fixes successives $\mathrm{zi}(\mathrm{i}=0,1,2, \mathrm{i}) \mathbf{6 6 0}$, atteintes par le train d'onde $S$ dans une fenêtre temps autour des instants de propagation ti 610 ( $\mathrm{i}=0,1,2, \mathrm{i})$ définis sur la figure $6 a$, les diagrammes de polarisations théoriques 661 que l'on s'attend à observer dans un repère fixe du plan horizontal, par exemple un repère géographique (Nord, Est). On prend par exemple $z i=\left(z_{f} i+z_{s} i\right) / 2$, en référence à la figure 6a. On remarque qu'en cas d'isotropie du milieu de propagation pour la direction verticale, $z i=z_{f} i=z_{s} i$ représente la profondeur atteinte par deux ondes $S$ de polarisation orthogonale quelconque. Les diagrammes de polarisation 661 de la figure $6 \mathrm{~b}$ représentent les mouvements de particules du train d'onde $S$ illustré par la figure $6 a$, pour la même succession d'instants de propagation ti : on peut noter que les diagrammes successifs sur la colonne de gauche 663 sont de forme très similaires, avec une direction maximale d'amplitude 662 identique. Les différences de rotondité observées traduisent typiquement l'anisotropie azimutale de biréfringence, résultant du faible écart de vitesse entre onde S rapide S-f et onde S lente S-s. Compte tenu de la longueur temps des ondelettes sismiques 603 et 604 (figure 6a) associées aux deux modes propres S-f et S-s émis quasi simultanément, la forme des diagrammes de la figure $6 \mathrm{~b}$ est elliptique et témoigne de l'interférence des deux modes propres d'onde $S$ qui ne peuvent hélas pas être distingués visuellement en pratique dans le cas général sur ce genre de diagramme. On a constaté par expérience qu'une arrivée sismique énergétique individuelle en onde $\mathrm{P}$ de polarisation linéaire ou en onde $\mathrm{S}$ de polarisation elliptique quelconque, ondes

$25 \mathrm{P}$ ou $\mathrm{S}$ dont la forme de polarisation reste sensiblement constante au cours de la propagation, montre une cohérence du temps des extrema d'amplitudes d'une profondeur à la suivante, et en particulier, que la forme du signal module reste sensiblement constante pour une onde descendante de forte énergie. On peut trouver facilement des publications montrant des hodogrammes de trains d'onde $S$ directe émis par une source $S$ placée à faible distance du puits, et réorientés dans un repère fixe géographique à l'aide de source d'onde $P$ placée en offset et activée dans la même passe de mesure, et indiquant que la forme d'hodogramme est sensiblement stable tout au long de la propagation le long du puits vertical et dans un milieu à stratigraphie horizontale comportant une anisotropie azimutale notable en onde $\mathrm{S}$, par exemple dans les documents suivants : 
Charles Naville, " Detection of Anisotropy Using Shear-Wave Spitting in VSP surveys; Requirements and Alpplications", SEG Expanded Abstracts, 56 ${ }^{\text {th }}$ int. SEG meeting, 1986, Houston, S5.2, pp. 391-394.

Ian Bush and Stuart Crampin, "Paris Basin VSPs: case history establishing combinations of fine layer (or lithologic) anisotropy and crack anisotropy from modelling shear wavefields near point singulatities" Geophys. Journal Int., 1991, №107, pp. 433-437.

Nicoletis, L., Cliet, C. \& Lefeuvre, F., " Shear-wave Splitting measurements from multishot VSP data, Expanded Abstracts, $58^{\text {th }}$ int. SEG meeting, 1988, Anaheim, POS 6.1, pp.527-530;

Dans les trois documents précités, l'hypothèse de la fixité de polarisation des modes propres (figure 6a) pour une direction de propagation donnée est admise de façon explicite ou implicite, et l'on peut vérifier visuellement que pour une propagation verticale à faiblement déviée de la verticale, la forme du diagramme de polarisation demeure similaire avec la propagation, ainsi que la direction azimutale d'amplitude maximale. Ainsi, même en présence d'anisotropie de biréfringence sans atténuation différentielle d'amplitude drastique entre les deux modes propres d'onde $S$, on peut aisément vérifier mathématiquement que la direction d'amplitude maximale d'un signal à deux composantes d'un train d'onde $S$ direct non interféré, demeure sensiblement fixe le long d'une direction de propagation donnée. Ceci est schématisé sur la colonne de gauche de la figure $6 \mathrm{~b}$. Ainsi, en déterminant la direction azimutale pour chaque profondeur à laquelle on dispose d'un enregistrement des composantes horizontales, on définit un repère unique. Cette méthode est d'autant plus précise que (1) le couplage mécanique de la sonde réceptrice mono niveau ou de chacune des sondes réceptrices d'un outil de puits multi niveaux assure une bonne fidélité vectorielle du signal mesuré, et que (2) la source de surface reste en position fixe et émette toujours la même forme de signal pendant toute l'opération de PSV.

La méthode comporte une orientation des composantes sismiques dans un repère localement cohérent quelque soit la profondeur de la sonde. La figure 7 est un organigramme $\mathbf{7 0 0}$ d'un mode de réalisation de la méthode d'orientation selon l'invention. II s'agit d'orienter les deux composantes orthogonales à une composante verticale, ou à une composante axiale au puits. La méthode repose sur l'analyse des ondes $S$ descendantes pour définir une fenêtre temporelle nécessaire à la détermination d'un angle de rotation. En effet, les ondes $P$ enregistrées ayant habituellement une énergie trop faible sur les composantes horizontales des PSV, elles ne permettent pas de réorienter ces composantes. Ceci est notamment le cas pour les PSV sans déport, effectués avec des sources d'onde de 
pression dans des puits dont la trajectoire est proche de la verticale. En revanche, l'énergie des ondes $S$ directes ou d'ondes converties de $\mathrm{P}$ en $\mathrm{S}$ au cours de la propagation descendante est très souvent suffisante pour appliquer la méthode.

Ainsi, selon un mode de réalisation, la méthode comporte les étapes suivantes pour chaque station profondeur de mesure :

- dans le bloc 701, on déconvolue de façon isotrope les deux composantes orthogonales à orienter, c'est à dire que l'on applique un même opérateur, au même temps sur les deux composantes. Cette opération n'est envisagée que si le résultat des opérations 702 à 704 n'est pas satisfaisant.

10 - dans le bloc 702, on calcule le module, noté $M(t)$, des deux composantes brutes à orienter, ou une puissance exponentielle $(n)$ de ce dernier ;

- dans le bloc 703, on filtre le module $M(t)$, de façon à supprimer ses composantes basses fréquences et éventuellement des hautes fréquences ne contenant que du bruit, de façon à faciliter l'opération suivante.

- dans le bloc 704, on pointe le temps Tp d'une phase remarquable du signal du module filtré obtenu précédent. Par exemple on pointe un pic ou un creux d'amplitude, éventuellement affiné par application d'un algorithme industriel de pointé par corrélation ou par semblance.

- Les opérations 702 à 704 peuvent être automatiques, exécutables en cascade et en une seule passe, mais peuvent conduire à un pointé peu fiable ou peu précis. Dans un tel cas, on peut soit modifier le filtre du bloc $\mathbf{7 0 3}$, soit envisager d'appliquer au préalable des opérations 702 à 704 les opérations décrites au bloc 701, destinées à augmenter le rapport signal sur bruit de l'onde $S$ que l'on désire pointer, toutes les arrivées interférant avec l'arrivée $S$ désirée étant considérées comme du bruit.

25 - dans le bloc 705, on définit ensuite une fenêtre temporelle autour du temps pointé d'arrivée $S$, d'une longueur constante pour tous les niveaux profondeur de mesure et au moins égale à une demi période du signal module filtré, voire d'une longueur plus grande en cas d'onde $S$ interférée ou de faible rapport signal sur bruit

- dans le bloc 705, on détermine alors la direction azimutale, par maximisation de l'énergie des composantes d'entrée $X(t), Y(t)$, brutes ou filtrées pour ne retenir que les fréquences de rapport signal sur bruit le plus fort, selon un procédé industriel connu. On effectue ensuite une rotation des composantes d'entrée $X(t), Y(t)$ brutes dans le plan orthogonal à la composante brute $Z$, dans un repère unique intermédiaire défini par la direction azimutale calculée précédemment. On s'assure que l'amplitude du signal de 
sortie dans la direction de maximisation demeure de signe identique, par exemple positif, au temps pointé Tp issu de l'opération 704, pour tous les niveaux de mesure du PSV.

- dans le bloc 707, on calibre les composantes dans un repère intermédiaire unique, défini par rapport à un repère géographique, ou à un repère lié à la trajectoire connue du puits si l'on dispose d'information complémentaire pour ce faire.

- dans le bloc 708, on applique les rotations nécessaires au repère intermédiaire unique pour restituer les trois composantes du PSV dans un repère géographique lié au globe terrestre, selon une procédure connue.

Chacune des étapes précédentes est décrite ci-après :

\section{A. Pointé}

- bloc 701 : déconvolution préalable éventuelle :

dans certains cas, le signal du train d'arrivée directe d'onde $S$ est apparent sur un grand intervalle de temps d'enregistrement, il est potentiellement interféré par d'autres ondes d'énergie relative plus faible, mais non négligeable, ce qui a pour effet de rendre le pointé du module filtré plus imprécis. II peut être utile dans de tels cas d'appliquer une opération de déconvolution isotrope multicanale, identique pour les deux composantes " horizontales » (composantes orthogonales à la direction sensiblement verticale) de chacun des niveaux

20 profondeur de mesure, et identique sur plusieurs niveaux profondeurs. Ceci permet de réduire la longueur du train d'onde $S$ sur lequel on désire pointer le temps d'arrivée: la déconvolution peut être effectuée par extraction du signal d'onde $\mathrm{P}$ sur la composante verticale, afin de déconvoluer une onde convertie P-S sur les deux composantes horizontales, par exemple selon la méthode décrite dans les brevets de Nigel Anstey GB 1,569 581 du 27-09-1977 ou CA 1,106,957 du 9-12-1977 intitulés "Seismic delineation of oil and gas reservoirs using borehole geophones". La déconvolution peut également être effectuée simplement avec les algorithmes industriels isotropes et multicanaux existants pour la sismique de surface ou pour la sismique de puits, de type Wiener ou également d'équilibrage du spectre de fréquences, tous deux basés sur le spectre d'amplitude de la somme des signaux d'autocorrélation de chacune des composantes horizontales orthogonales à déconvoluer, compte tenu de l'invariance de cette somme de signaux d'autocorrélation vis à vis de l'orientation des deux composantes horizontales d'entrée.

- bloc 702 : Calcul du signal module $M(t)$ d'un signal à deux composantes, et invariance. 
Après s'être assuré que les opérations de prétraitement de base des enregistrements unitaires du PSV, telles que l'édition, la sommation verticale, la normalisation d'amplitude de source éventuelle préalable à la sommation..., ont été effectuées de façon isotrope, le signal module, noté $M(t)$, qui représente l'une des coordonnées polaires déduite des deux signaux bruts en coordonnées cartésiennes $X(t)$ et $Y(t, d)$ est calculé comme suit :

$$
M^{2}(t)=X^{2}(t)+Y^{2}(t), \text { pour tout temps } t
$$

Si l'outil de PSV, dont le couplage à la paroi est mécaniquement isotrope, est ancré à une profondeur donnée avec une direction azimutale différente d'un angle az inconnu autour de l'axe du puits vertical, l'outil enregistre les composantes horizontales $X 1(t)$ et $Y 1(t)$ qui s'expriment comme suit en fonction de $X(t, d)$ et $Y(t, d)$ :

$$
\begin{aligned}
& X 1(t)=X(t) \cdot \cos (a z)+Y(t) \cdot \sin (a z) \\
& Y 1(t)=-X(t) \cdot \sin (a z)+Y(t) \cdot \cos (a z)
\end{aligned}
$$

Il est aisé de constater que, pour toute valeur de l'angle az :

$$
X 1^{2}(t)+Y 1^{2}(t)=X^{2}(t)+Y^{2}(t)=M^{2}(t)
$$

Le module demeure identique quelle que soit l'orientation des capteurs associés aux composantes $X(t)$ et $Y(t)$, il est donc invariant eu égard à la rotation, à valeur toujours positive. Le module d'un signal à deux composantes est appelé également "M2" dans la suite de la description. Le module d'un signal à trois composantes, dit "M3", défini par :

$$
M^{2}(t)=X^{2}(t)+Y^{2}(t)+Z^{2}(t) \text {, est aussi un invariant eu égard à toute rotation spatiale. }
$$

II est intéressant de calculer le module M3 lorsque l'on cherche à pointer une onde de pression $(P)$ directe, dont la direction d'arrivée est orthogonale au puits dans certaines configurations de la géométrie d'acquisition. Par exemple, sur certains intervalles du puits de mesure proche de l'horizontale, lorsque la source est située proche de l'aplomb du capteur sismique $3 \mathrm{C}$, comme illustré sur la figure $2 \mathrm{~b}$, ou encore dans les configurations d'acquisition de type Offset-VSP et walkaway, sur certains intervalles du puits proches de la verticale, lorsque la source sismique de surface est suffisamment éloignée du puits, de sorte que le rayon sismique arrive au puits avec une incidence horizontale, comme illustré sur la figure 2c. Comme cette procédure de pointé de l'arrivée $P$ est valide quelle que soit l'incidence du rayon, on automatise le pointé de l'onde $\mathrm{P}$ directe en pointant le module $\mathrm{M} 3$, en particulier pour les études de type walkaway $2 D$, et walkaway $3 D$, cette dernière configuration également connue sous le nom de 3D-VSP. 
On peut aussi choisir de travailler sur une puissance exponentielle du signal $M(t)$, afin d'amplifier les variations d'amplitude de ce signal.

L'avantage de la propriété d'invariance du module est de permettre des opérations de pointé précis du temps d'une phase remarquable, lié à un instant particulier du train d'arrivée d'onde S, par exemple un extremum local bien individualisé, sans connaître l'orientation préalable des signaux constitutifs dudit module.

- $\quad$ bloc 703 : Filtrage du module $M(t)$

Selon un mode préférentiel de réalisation, la méthode comporte un filtrage du module $M(t)$, de façon à supprimer sa composante basse fréquence et à rendre ce signal plus lisible. On peut par exemple utiliser typiquement un filtre passe bande $5-60 \mathrm{~Hz}$ pour les PSV.

- $\quad$ bloc 704 : Pointé temps d'un extremum particulier du signal du module filtré.

Le pointé du temps Tp d'un extremum d'amplitude du module, de préférence filtré est décrit en relation avec les figures $6 c$ et $6 \mathrm{~d}$.

La figure 6c montre une projection horizontale $\mathbf{6 7 0}$ d'un diagramme de polarisation 671 d'un train d'arrivée directe $S$ choisi dans une large fenêtre temps appropriée.

Le signal module $M(t) 672$ est représenté graphiquement comme l'une des coordonnées polaires dérivée des deux signaux enregistrés orthogonaux bruts $X(t)$ et $Y(t)$ en coordonnées cartésiennes et d'orientation arbitraire.

Par définition, quel que soit le temps $t: M^{2}(t)=X^{2}(t)+Y^{2}(t)$.

20 Le signal $M(t) 672$ possède la caractéristique mathématique d'être invariant vis à vis du repère cartésien des signaux bruts $X(t)$ et $Y(t)$ mesurés, et dans lequel le module est calculé. De même, la forme du diagramme de polarisation 671 dans la fenêtre temps considérée est indépendante du repère, à une rotation près. Les vecteurs signaux bruts $X(t)$ et $Y(t)$, ainsi que le vecteur module $M(t)$, ont la même origine 673 (zéro des amplitudes), l'amplitude du vecteur $M(t)$ étant toujours positive ou nulle.

Compte tenu que le diagramme de polarisation de l'onde $S$ directe considérée est également quasi constant au cours de la propagation sismique en profondeur, le procédé selon l'invention permet de déterminer avec précision le pointé temps d'une phase remarquable du signal d'onde $S$ non orienté, de façon indépendante du repère cartésien des signaux bruts $\mathrm{X}(\mathrm{t})$ et $\mathrm{Y}(\mathrm{t})$, par exemple le temps de l'un des maxima locaux $674 \mathrm{du}$ signal module $M(t) 672$.

La figure $6 \mathrm{~d}$ montre un exemple schématique, en fonction du temps $\mathrm{t}$, du signal module d'un train d'onde $S$ dont l'énergie est supérieure à celle de toutes les autres ondes reçues au 
même temps par le capteur sismique. On constate, par expérience, que ce signal module reste sensiblement identique en fonction de la profondeur d'enregistrement, avec un décalage temps correspondant à la propagation des ondes S. En pratique, afin d'amplifier la reconnaissance des extrema locaux du signal module, on élimine les composantes basses fréquences par un filtre coupe bas, dont le résultat est le signal filtré Mf 693, qui représente également la différence entre le signal brut 691 et le signal lissé associé 692. On peut aussi élever les amplitudes du signal filtré Mf à une puissance exponentielle, afin d'en faciliter encore le pointé par méthode visuelle, ou par calcul de semblance ou de corrélation entre stations profondeur de mesure PSV. Le temps Tp du pic d'amplitude 695 est en pratique plus facile à pointer, sans ambiguïté, sur le signal filtré Mf 693 que le pic 694 sur le signal brut M 691, tant pour l'oeil que pour la plupart des algorithmes informatiques industriels de pointé du temps..

La méthode selon l'invention, permettant d'obtenir un pointé précis d'une phase remarquable d'un signal d'onde de cisaillement à deux composantes orthogonales dans le plan de polarisation sans orientation préalable, conduit à des applications immédiates : en effet, ce genre de pointé permet d'accéder à la connaissance d'un temps d'arrivée d'onde $S$ à une constante près identique pour toutes les stations profondeur de mesure du PSV, et par conséquent à la connaissance des vitesses d'intervalle en mode $S$. En combinant le temps $S$ avec la mesure du temps en onde $P$ généralement effectuée sur la composante verticale ou axiale au puits, on accède par exemple au rapport des vitesses d'intervalle $V s / V p$ et au coefficient de Poisson. On accède également au module d'Young si la densité de formation est connue par ailleurs. Cette invention permet également de pointer une onde $S$ sur des données en provenance d'outils de puits de mesure de diagraphie ultrasonique d'onde-S de type dipolaire ou quadrupolaire comportant des sources et des récepteurs d'ondes flexurale, sans nécessité de connaître l'orientation de l'outil. Ceci peut conduire à des simplifications de conception et à une diminution du coût opérationnel puisque les éléments matériels de mesure de l'orientation ne sont plus nécessaires.

\section{B. Orientation dans un repère unique et cohérent}

- bloc 705 : Détermination de la direction azimutale de maximisation de l'énergie des deux composantes brutes à orienter et rotation des signaux d'entrée dans un repère intermédiaire cohérent.

La définition d'une fenêtre temporelle de calcul est définie par un écart de temps constant de l'ordre de $10 \mathrm{~ms}$ à $20 \mathrm{~ms}$ de part et d'autre du temps pointé Tp sur le module filtré, de sorte que la fenêtre temporelle englobe au moins une demi période de la période 
dominante l'arrivée pointée. L'invariance du signal module eu égard à l'orientation des capteurs entraîne la cohérence du temps pointé de l'arrivée $S$, et garantit par conséquence sa validité pour des utilisations ultérieures ou pour connaître les vitesses d'onde $S$ en fonction de la profondeur.

On reprend les signaux bruts $X(t)$ et $Y(t)$ pour chacune des profondeurs de mesure du PSV, que l'on filtre éventuellement en coupant les hautes fréquences bruitées. Puis, on recherche la direction azimutale qui maximise l'énergie sismique dans le plan des deux composantes entrées et dans la fenêtre temporelle précédemment définie, en utilisant une technique courante de maximisation de l'énergie, telle que celle décrite dans les documents précités suivants :

DiSiena, J. P., J. E. Gaiser, and D. Corrigan, 1984, "Horizontal components and shear wave analysis of three-component PSV data", in M. N. Toksöz and R. R. Stewart, eds., Vertical seismic profiling, Part B : Advanced concepts :Geophysical Press, 177-235.

Benhama, A., Cliet, C., and Dubesset, M., 1988, Study and application of spatial directional filtering in three component recordings : geophysical prospecting, 36, 591-613,

La composante correspondante à cette direction de maximisation est notée $H \max (\mathrm{t})$, l'angle calculé entre $H \max (\mathrm{t})$ et la première composante $\mathrm{X}(\mathrm{t})$ est noté amax180 et n'est connu qu'à $180^{\circ}$ près. Cette indétermination est levée en choisissant, par exemple, que l'amplitude de la composante de sortie Hmax soit impérativement rendue de signe positif à l'instant du temps pointé Tp pour toute profondeur de mesure, en procédant comme suit :

si Hmax $(T p)>0$, alors on définit un angle amax360 = amax180, exprimé en degré

si $\operatorname{Hmax}(\mathrm{Tp})<0$, alors on définit un angle $\operatorname{amax360}=180+\operatorname{amax} 180$

La figure 8 montre des diagrammes de polarisation $\mathbf{8 0 0}$ de type hodogramme dessinés dans le repère $\mathbf{8 0 1}$ des composantes horizontales brutes $X$ et $Y$ d'un PSV réel, enregistré dans un puits vertical avec une source à très faible déport, et un outil de puits comportant trois capteurs fixes sans accessoire d'orientation et dont le couplage mécanique assure une bonne fidélité vectorielle. A gauche de chacun des hodogrammes, figure une légende indiquant la profondeur $\mathbf{8 0 2}$ de mesure PSV, les temps en milliseconde du début $\mathbf{8 0 3}$ et de la fin 804 de la fenêtre de calcul de maximisation de 55 ms qui suit le pointé temps effectué au préalable sur le module filtré, la valeur maximale $\mathbf{8 0 5}$ de l'amplitude du vecteur signal $\mathbf{8 0 7}$ calculé dans la direction angulaire de maximisation AG 806 exprimée en grade (GR) à partir de la composante de référence $X \mathbf{8 0 1}$ de l'outil de puits et dans le sens contraire des aiguilles d'une montre. L'angle 806 noté $A G$ sur la figure 8 correspond à l'angle amax360 défini plus haut modulo 360 degrés ou 400 grades. 
La rotation subséquente des composantes brutes $X(t)$ et $Y(t)$ d'angle amax360 qui est appliquée sur toute la longueur du signal enregistré, permet d'obtenir des signaux de sortie dans un repère unique qui les rend chacun cohérent en fonction de la profondeur. L'angle amax 360 peut être additionné d'une constante éventuelle.

Les figures 9 et 10 montrent les signaux trois composantes du PSV avant et après orientation. Les figures $9 a, 9 b$ et $9 c$, représentent les rejeux bruts isotropes 900 des composantes respectives $Z$ verticale et $X, Y$ horizontales de l'outil de puits, en fonction de la profondeur de mesure (« Measured Depth ») MD 901 croissante de gauche à droite. Le rejeu est dit "isotrope normalisé 3C", indiquant qu'un gain constant a été appliqué de façon identique aux amplitudes des trois composantes, mais variable en fonction de la profondeur, de telle sorte que l'amplitude d'arrivée directe d'onde $\mathrm{P}$ sur la composante verticale $Z$ soit identique à toute profondeur. L'arrivée directe d'onde $P 902$ est quasi invisible sur les composantes horizontales $\mathrm{X}, \mathrm{Y}$ sur lesquelles le temps d'onde $\mathrm{P}$ a été figuré par un trait $\mathbf{9 0 3}$. Une arrivée directe d'onde S 904 est clairement identifiée sur les composantes horizontales

$15 X, Y$ par sa pente plus importante que celle de l'onde P 902-903, et l'on observe des défauts de cohérence de la forme d'onde $S$ dans le rectangle 905 . Ces défauts sont associés à l'orientation aléatoire des capteurs horizontaux et de la direction de maximisation de l'arrivée directe $\mathrm{S}$ illustrée sur la figure 8.

Les figures 10 a, $10 \mathrm{~b}$ et $10 \mathrm{c}$ représentent les rejeux 1000 isotropes normalisés $3 \mathrm{C}$ des composantes orientées respectives $Z$ verticale, $\mathrm{HN}$ et $\mathrm{HE}$ horizontales orientées dans les directions géographiques respectives Nord et Est, en fonction de la profondeur MD 1001 croissante de gauche à droite, avec les mêmes échelles de temps et de profondeur que celles des figures 9a, 9b et 9c. Les quatre niveaux de mesures les moins profonds sont manquants. Le temps de l'arrivée directe d'onde P 1002 a été figuré par le trait 1003 sur les composantes horizontales HN et HE. La cohérence de l'arrivée directe d'onde S 1004 sur les composantes horizontales $\mathrm{HN}$ et $\mathrm{HE}$, dans le rectangle 1005 est bien meilleure que dans le rectangle 905 correspondant des figures $9 a, 9 b$ et $9 \mathrm{C}$, ce qui confirme la bonne orientation obtenue.

A ce stade, les trois composantes sont orientées à 360 degrés près dans un repère 30 sensiblement unique. Ce repère est donc cohérent pour chaque profondeur. Ce prétraitement permet de réaliser un traitement des trois composantes isotropes, même si ce repère est de direction azimutale inconnue.

C. Orientation dans un repère géographique 
- bloc 706 : Calibration du repère cohérent intermédiaire par rapport au repère géographique :

II est souhaitable, quand c'est possible, d'orienter de façon additionnelle les composantes horizontales dans un repère unique d'orientation géographique connue. Pour ce faire, il est nécessaire de déterminer l'orientation géographique du repère unique intermédiaire obtenu en sortie des opérations du bloc 705 de la figure 7. Cette opération de calibrage azimutal du repère unique permet l'interprétation géologique des résultats de traitement du PSV à trois composantes subséquent, tel que celui indiqué dans le brevet US 6076 045, focalisé sur la détermination de pendage et azimut de réflecteurs sismiques.

Plusieurs procédés de calibrage du repère unique peuvent être utilisés :

a) On peut, par exemple, utiliser l'énergie résiduelle de l'arrivée d'onde $P$ qui est parfois plus importante sur les composantes horizontales des niveaux de mesure les moins profonds du PSV, en faisant l'hypothèse classique que la polarisation de l'onde P directe est dans la direction azimutale du segment qui relie la position de la source et celle du capteur.

15 C'est ce qui a été accompli pour obtenir la figure 10, en effectuant une rotation supplémentaire d'angle constant par rapport à la direction azimutale de maximisation de l'arrivée directe $S$, de façon à orienter les composantes horizontales dans le repère géographique de représentation de la figure 10. En effet, l'arrivée d'onde directe P 1003 a une incidence verticale de l'ordre de 10 degrés sur les niveaux les moins profonds situés entre $1000 \mathrm{~m}$ et $1100 \mathrm{~m}$ pour les données du PSV représenté sur les figures 9a-9c (ref. profondeur mesurée 901) et sur les figures 10a-10c (signaux situés sur le côté droit des figures)

b) On peut, de façon alternative, utiliser des mesures directionnelles des trois composantes par divers instruments ou accessoires de mesures d'orientation complète ou partielle, si ces derniers ont été descendus de façon couplée à l'outil de PSV mono niveau.

L'orientation est dite complète, lorsque tous les paramètres permettant l'orientation (angles de "Relative Bearing ", de Déviation verticale du puits et d'Azimut du puits dévié) sont mesurés sur tous les niveaux profondeur de mesure du PSV. Ceci est possible avec un outil de type gyroscope couplé à l'outil PSV mono niveau par exemple.

L'orientation est dite partielle, si l'outil de mesure d'orientation est couplé à au moins un des satellites de mesure de l'outil sismique de PSV, si ce dernier comporte plusieurs niveaux profondeur mesurés simultanément. L'orientation est également dite partielle, si la mesure d'orientation est limitée à un intervalle profondeur donné (comme par exemple la limitation à l'intervalle de puits en trou ouvert, non chemisé avec des tubes métalliques pour un outil détectant le direction du Nord magnétique), ou à une plage d'angle de déviation du puits 
(comme par exemple les dispositifs de type inclinomètres, pendule de mesure du « Relative Bearing " et cardans, sensibles à la gravité, et rendus inopérants pour les faibles déviations verticales du puits).

- bloc 707 : Rotations des signaux sismiques entre le repère cohérent intermédiaire et le repère géographique, lorsque l'orientation de l'outil est partielle.

Afin de faciliter la compréhension de l'invention et de son objet, on expose ci dessous un panorama succinct des moyens matériels connus d'orientation des outils de puits sismiques et non sismiques :

- Moyens d'orientation exhaustive et précise d'une sonde de puits à coût opérationnel élevé : L'industrie de la diagraphie dispose de moyens magnétiques d'orientation repérant la direction du champ magnétique terrestre, si l'on opère en trou ouvert, souvent combinée à des inclinomètres précis rendus peu sensibles aux vibrations et capables d'effectuer des mesures en continu pendant la remontée des outils de mesure diagraphique également en fonctionnement continu. Les inclinomètres précis permettent de connaître le relative bearing d'un outil dans un puits tubé à partir de quelques degrés d'inclinaison verticale du puits, la trajectoire et les angles d'inclinaison et d'azimut du puits étant connue par ailleurs. Les gyroscopes de puits sont également d'un usage courant pour mesurer avec précision la trajectoire du puits; leur usage en combinaison avec d'autres outils de mesure diagraphique est sporadique, mais pas rare. A l'aide des moyens précédemment décrits, l'orientation des composantes est alors parfaitement mesurée en trou ouvert ou tubé.

- Moyens d'orientation partielle et peu précise, mais peu onéreuse, d'une sonde de puits :

a) pour les puits déviés de trajectoire connue, il est usuel de monter les capteurs triaxiaux sur des doubles cardans avec architecture dite "tourelle", comprenant un axe de rotation parallèle à l'axe puits, et d'un axe horizontal perpendiculaire au plan vertical tangent localement au puits. Les figures $3 a, b$ indiquent la disposition d'un capteur sismique triaxial $\mathbf{3 1 1}$ monté sur de tels cardans, et placé dans un puits dévié 310 : la figure 3a représente une projection $\mathbf{3 0 0}$ dans le plan vertical tangent au puits 310, qui comprend la composante verticale Z-down 301, orientée vers le bas, et la composante horizontale XH 302 orientée dans l'azimut des profondeurs mesurées croissantes du puits; l'autre composante horizontale YH 303 est orthogonale au plan vertical tangent représenté. L'angle d'inclinaison verticale du puits 304, ou déviation, est couramment référé par DEV dans l'industrie. La figure $3 \mathrm{~b}$ représente une projection $\mathbf{3 5 0}$ dans le plan horizontal en vue de dessus : la trajectoire du puits dévié 
310 apparaît comme une ligne quelconque, dans le repère géographique 320 , la composante horizontale $\mathrm{XH} 302$ est tangente au puits au niveau de la position du capteur 311, la composante horizontale $\mathrm{YH} 303$ est disposée à $+90^{\circ}$ par rapport à $\mathrm{XH} 302$, en vue de dessus. On recalcule les composantes sismiques $\mathrm{HE}$ et $\mathrm{HN}$ orientées dans un repère géographique 320 à partir des composantes $\mathrm{XH} 302$ et $\mathrm{YH}$ 303 par rotation d'angle HAZI 305 autour de la verticale, HAZI 305 correspondant à l'azimut du puits localement à la position du capteur 311. Les angles DEV 304 et HAZI 305 sont en général connus et mesurés indépendamment de l'opération de PSV par les mesures très précises de la trajectoire de puits effectuées à l'aide de moyens de type gyroscope ou magnétomètre - inclinomètre mentionnés plus haut. Le montage des capteurs triaxiaux sur double cardans de type tourelle permet d'orienter les capteurs sismiques trois composantes par gravité dans les puits suffisamment inclinés par rapport à la verticale, typiquement à partir d'une valeur seuil de l'ordre de 10 degrés de l'inclinaison verticale du puits, ce seuil pouvant varier d'une marque d'outil à une autre; en pratique, compte tenu des forces de frottements inhérentes à ce genre de dispositif mécanique, l'orientation devient plus précise lorsque l'inclinaison du puits dévié augmente. Pour les faibles valeurs de déviation du puits au dessous de la valeur seuil d'environ 10 degrés, l'orientation des composantes orthogonales n'est pas connue. Certes, il y a une incertitude du même ordre sur l'orientation réelle de la composante Z-down par rapport à la direction verticale réelle, mais cela n'altère pas significativement les résultats de traitement ni des conclusions interprétatives subséquentes.

b) De façon alternative et courante, il est connu de monter des capteurs sismiques à trois composantes de façon fixe dans un outil de PSV, comprenant en outre un dispositif de mesure de l'angle de Relative Bearing dans le plan orthogonal à l'axe de l'outil PSV : Naturellement ce genre de dispositif appelé communément "capteur de Relative Bearing" est inopérant en puits strictement vertical et restitue une mesure du Relative Bearing qui n'est significative qu'au delà d'une faible valeur de l'inclinaison verticale du puits, de l'ordre de 10 degrés; la mesure du Relative Bearing devient de plus en plus précise lorsque l'inclinaison du puits dévié augmente. Les figures $4 a, b$ indiquent la disposition d'un capteur sismique triaxial monté de façon fixe dans l'outil : la figure $4 a$ illustre la définition de l'angle de Relative Bearing par l'angle entre la génératrice haute du puits cylindrique et une direction de référence de l'outil PSV dans le plan orthogonal à l'axe de l'outil, avec une convention de signe positif dans le sens des aiguilles d'une montre lorsqu'on regarde le plan orthogonal dans la direction des profondeurs curvilignes croissantes du puits. La figure $4 a$ représente une 
projection 400 dans le plan orthogonal à l'axe $Z$ du puits 410 , au niveau du capteur 411, en vue de dessus, la flèche $\mathbf{4 1 2}$ indiquant la direction des profondeurs mesurées croissantes du puits; l'angle de relative bearing RB 430 est défini par l'angle entre la direction XV 422 orthogonale à l'axe du puits 410, contenue dans le plan vertical tangent au puits et pointant vers le haut, avec la direction $X \mathbf{4 1 9}$ de référence de la sonde contenant le capteur $\mathbf{4 1 1}$, correspondant au capteur sismique orthogonal $X$ 419 mesuré; l'angle RB 430 est mesuré positivement 431 dans le sens des aiguilles d'une montre lorsqu'on regarde dans la direction de la flèche $\mathbf{4 1 2}$.

La figure $4 \mathrm{~b}$ représente une projection $\mathbf{4 5 0}$ dans le plan vertical tangent au puits $\mathbf{4 1 0}$ localement à la position du capteur 411, qui comprend la composante $Z \mathbf{4 2 1}$ mesurée par l'outil, axiale au puits et pointant vers le bas et la composante XV422 précédemment calculée dans la direction de l'axe origine de l'angle de relative bearing $(\mathrm{RB}=0)$; la composante horizontale $\mathrm{YH} 403$ est orthogonale au plan vertical tangent représenté. L'angle d'inclinaison verticale du puits DEV 404 est indiqué entre les directions Z 421 axiale au puits et Z-down 401, composante sismique verticale orientée vers le bas; la composante horizontale XH 402 orientée dans l'azimut des profondeurs mesurées croissantes du puits et la composante sismique Z-down 401 sont obtenues à partir des composantes XV422 et Z 421 par rotation d'angle DEV 404 autour de l'axe YH 403.

Trois configurations de mesure partielle d'orientation sont considérées ci dessous :

C1: l'outil de puits mono niveau contient des capteurs triaxiaux montés sur des doubles cardans avec architecture dite "tourelle", comme illustré sur les figures $3 a$ et $3 b$, et dans un intervalle profondeur restreint contenant au moins une station de mesure PSV, la déviation du puits est suffisamment importante (au moins 10 degrés environ) pour permettre la rotation des cardans sous l'action de la gravité : la composante $\mathbf{3 0 1}$ Z-down est alors naturellement orientée selon la verticale (fig.3a). Les composantes horizontales géographiques $320 \mathrm{HN}$, HE (fig. 3b) sont obtenues par rotation des composantes XH 302 et YH 303 mesurées, autour de la verticale, de l'angle HAZI 305 connu à 360 degrés, correspondant à l'azimut du 30 plan vertical tangent au puits à la position du capteur.

$$
[H E, H N]=\operatorname{Rot}(H A Z I) .[X H, Y H]
$$

C2 : I'outil de puits mono niveau contient des capteurs triaxiaux montés de façon fixe dans l'outil de puits, comme illustré sur les figures $4 a$ et $4 b$, et dans un intervalle profondeur restreint contenant au moins une station de mesure PSV, la déviation du puits est suffisamment importante pour permettre une mesure précise à quelques degrés de l'angle 
de «relative bearing " RB 430 illustré sur Fig.4a: on applique alors trois rotations successives dans cet ordre :

$[\mathrm{XV}, \mathrm{YH}]=\operatorname{Rot}(\mathrm{RB}) .[\mathrm{X}, \mathrm{Y}]$, rotation dans le plan orthogonal à l'axe du puits,

puis $[X H, Z V$-down $]=\operatorname{Rot}(D E V) .[X V, Z]$, rotation dans le plan vertical tangent au puits à la position de l'outil de puits, selon l'illustration fig. $4 \mathrm{~b}$,

puis $[\mathrm{HE}, \mathrm{HN}]=\operatorname{Rot}(\mathrm{HAZI}) .[\mathrm{XH}, \mathrm{YH}]$, rotation dans le plan horizontal, selon l'illustration fig. 3b.

C3 : l'outil de puits comporte une pluralité de sondes réceptrices placées à des profondeurs de mesure adjacentes qui contiennent chacune des capteurs triaxiaux montés de façon fixe dans l'outil de puits; en outre, l'une des sondes est combinée avec un outil de mesure complète de l'orientation. Dans cette configuration, après rotation d'un des composantes orthogonales à l'axe du puits dans un repère unique, on calcule, pour toutes les stations profondeur mesurées avec la sonde couplée à l'outil de mesure de l'orientation la différence entre l'angle de rotation précédente et l'angle de "relative bearing " mesuré, puis on interpole la valeur de cette différence pour les niveaux profondeurs adjacents qui ne bénéficient pas de mesure d'orientation; l'angle différence interpolé obtenu est l'angle de « relative bearing " $\mathrm{RBi}$ à utiliser pour la rotation des composantes orthogonales du repère intermédiaire. On applique alors les trois rotations décrites pour la configuration C2 ci dessus, en prenant l'angle de « relative bearing » RBi pour la première des trois rotations.

Les figures $5 \mathrm{a}, 5 \mathrm{~b}$ et $5 \mathrm{c}$ illustrent, dans le plan vertical 500 de projection, l'attitude spatiale des trièdres des capteurs sismiques 511 à 513 et 521 à 523 dans un puits 510 comprenant une partie verticale $\mathbf{5 0 1}$ et une partie $\mathbf{5 0 2}$ déviée dans ledit plan vertical $\mathbf{5 0 0}$ représenté en projection.

La partie dite verticale $\mathbf{5 0 1}$ du puits $\mathbf{5 1 0}$ symbolise un intervalle profondeur pour laquelle la valeur de l'inclinaison verticale est au dessous de la valeur de seuil de fonctionnement efficace d'un dispositif de double cardans, ou d'un système de mesure gravitaire-pendulaire de l'angle de "relative bearing ", et contient les trièdres 511 à 513. La partie dite déviée 502, correspond à un intervalle pour lequel l'angle de déviation verticale du puits est au dessus de ladite valeur de seuil, et contient les trièdres 521 à 523.

La figure 5a représente l'attitude des trièdres correspondant à un montage fixe des trois capteurs sismiques orthogonaux dans la sonde de puits, dont $\mathbf{5 1 1}$ et $\mathbf{5 2 1}$, où l'axe du capteur généralement appelé Z-outil est aligné avec l'axe du puits et pointe vers le haut : le trièdre 511 dans la partie verticale 501 et le trièdre $\mathbf{5 2 1}$ dans la partie déviée 502 illustrent ainsi que 
l'angle de "relative bearing " qui repère la direction des capteurs orthogonaux à l'axe du puits par rapport à l'azimut du plan vertical $\mathbf{5 0 0}$ est aléatoire d'une station profondeur de PSV à une autre. La valeur mesurée du "relative bearing" ne peut être exploitée pour l'orientation des composantes que dans la partie déviée 502 (voir figure 4a et explications associées).

La figure $5 c$ représente l'attitude des trièdres correspondant à un montage des trois capteurs sismiques orthogonaux sur doubles cardans de type tourelle dans la sonde de puits, dont 512 et 522, où l'axe de l'un des capteurs est aligné avec la verticale et pointe vers le haut: le trièdre $\mathbf{5 1 2}$ et les trièdres des cotes adjacentes dans la partie verticale $\mathbf{5 0 1}$ illustrent ainsi que la direction azimutale des capteurs orthogonaux à l'axe du puits est aléatoire d'une station profondeur de PSV à une autre. Par contraste, le trièdre $\mathbf{5 2 2}$ dans la partie déviée 502, illustre que l'orientation du trièdre est totalement connue, l'une des composantes horizontales étant dans le plan vertical $\mathbf{5 0 0}$ de la partie déviée de la trajectoire du puits, et l'autre composante horizontale étant normale au plan $\mathbf{5 0 0}$ (voir figures $3 \mathrm{a}$ et $\mathbf{3 b}$ et explications associées).

La figure $5 \mathrm{~b}$ représente l'orientation unique connue des trièdres obtenue après application des procédures d'orientation selon l'un des modes de l'invention, dont $\mathbf{5 1 3}$ et $\mathbf{5 2 3}$, où l'axe de l'un des capteurs est aligné avec la verticale et pointe vers le haut, l'une des composantes horizontales étant dans le plan vertical $\mathbf{5 0 0}$ de la partie déviée de la trajectoire du puits et pointe dans l'azimut des profondeurs croissantes (identique à la direction de déviation du puits dans le cas présent), et l'autre composante horizontale étant normale au plan $\mathbf{5 0 0}$ : les trièdres $\mathbf{5 1 1}, \mathbf{5 1 2}$ et les trièdres des cotes adjacentes d'orientation aléatoire dans la partie verticale 501 sont réorientés dans un repère commun du trièdre 513 ou 523 à l'aide de la procédure P1 551 selon l'invention. Par contraste, le trièdre $\mathbf{5 2 1}$ dans la partie

25 déviée 502 est réorienté dans les directions du trièdre 523 à l'aide de deux rotations successives selon la procédure connue P2 552 et décrite précédemment (commentaires des figures $4 a$ et $4 b$ ). Le trièdre $\mathbf{5 2 2}$ dans la partie déviée $\mathbf{5 0 2}$ est naturellement orienté de façon identique au trièdre $\mathbf{5 2 3}$ et ses composantes ne nécessitent aucune intervention.

En pratique, la procédure P1 551 selon l'un des modes de l'invention est également 30 appliquée aux trièdres de la partie déviée $\mathbf{5 0 2}$ immédiatement adjacente à la partie verticale 501, dans un court intervalle de recouvrement et d'embrayage, de façon à calibrer l'azimut des composantes horizontales des trièdres de la partie verticale, dont 511 et 512, sur l'azimut connu des trièdres de la partie déviée 502. Au final, si l'on désire orienter tous les trièdres 513 à 523 représentés sur la figure $5 b$ dans un repère géographique, on applique une rotation azimutale similaire à celle décrite précédemment (commentaires de la figure $3 b)$. 


\section{Applications de l'invention}

La méthode selon l'invention peut être appliquée dans le cadre de prospection sismique par méthode de PSV conventionnel à très faible déport de l'unique position de source, afin de positionner dans l'espace à trois dimensions des événements géologiques au voisinage de puits. Une telle méthode de prospection sismique comporte alors les étapes suivantes :

- la réception par des capteurs sismiques triaxiaux, disposés dans un puits et couplés avec les formations environnant le puits, afin de mesurer aussi fidèlement que possible le signal vectoriel en trois composantes des ondes directes et réfléchies dans les modes $\mathrm{P}, \mathrm{S}$ ainsi que les modes d'ondes converties.

- l'orientation dans l'espace des capteurs sismiques multiaxes de réception. On utilise pour ce faire la méthode d'orientation selon l'invention.

- l'imagerie sismique de puits à partir de trois composantes orientées, telle que celle décrite par exemple dans le brevet US 6076 045: cette méthode fait appel au traitement isotrope des trois composantes orientées, permettant la lecture de la polarisation des événements réfléchis observés, puis de l'imagerie et du positionnement dans l'espace des réflecteurs correspondants, restituant ainsi le pendage et l'azimut de pendage à 360 degrés de chacun des réflecteurs.

Une application importante de la méthode selon l'invention concerne également l'amélioration du contrôle qualité des trois composantes enregistrées sur le site d'enregistrement, à l'aide des moyens informatiques disponibles: en effet, d'une part les méthodes informatiques, permettant l'orientation des données trois composantes dans un repère unique, sont aisées à mettre en oeuvre, et d'autre part il est plus facile d'évaluer visuellement la qualité générale d'enregistrement et le bon fonctionnement global de la chaîne d'acquisition sur des rejeux orientés des trois composantes par rapport à des rejeux bruts non orientés, pour toute profondeur. Ainsi, on peut utiliser l'orientation dans un repère unique de façon automatisée, pour obtenir un contrôle de la qualité de la mesure sismique en trois composantes, immédiatement après l'acquisition des mesures sur le terrain.

La méthode permet d'orienter les trois composantes des PSV, dans les intervalles profondeur proches de la verticale, en particulier lorsqu'une seule position de source sismique de surface localisée à proximité de l'appareil de forage a été exploitée, et que l'outil de mesure PSV descendu dans le puits n'est pas couplé à un outil de mesure précis de tous 
les angles permettant l'orientation des trois composantes des signaux dans un repère géographique. Ceci correspond à la configuration usuelle des PSV dans les puits d'exploration ou de production. La méthode selon l'invention s'applique avec efficacité sur un train d'onde de cisaillement descendante, y compris en présence d'anisotropie de biréfringence en propagation : en effet, la direction azimutale ne varie pas en présence d'anisotropie de vitesse des deux modes propres d'onde $S$, dont l'effet est très faible sur des niveaux profondeurs adjacents, pour autant que l'atténuation différentielle entre les deux ondes demeure également faible, ce qui est en général vérifié par expérience.

La méthode permet également d'orienter les trois composantes des outils de PSV comprenant plusieurs niveaux profondeur de mesure sismique $3 \mathrm{C}$ simultanés, pour lequel un seul niveau (ou un nombre incomplet de niveaux) est couplé à un outil de mesure d'orientation complète ou partielle.

La simplicité de mise en œuvre de la méthode, au moyen des ordinateurs de plus en plus puissants embarqués dans les systèmes d'acquisition, permet une amélioration du contrôle qualité globale sur site des données trois composantes enregistrées, grâce à la production en temps légèrement différé, voire en temps réel, du pointé temps de l'onde $S$ descendante et d'un rejeu des trois composantes orientées dans un repère unique, permettant à l'ingénieur d'acquisition de détecter rapidement sur site et avec fiabilité accrue les disfonctionnements éventuels de la chaîne d'acquisition des trois composantes.

L'avantage de la méthode est de permettre subséquemment le traitement isotrope des signaux PSV trois composantes, y compris pour des retraitements de jeux de données PSV anciennes pour lesquelles l'outil de fond n'était pas couplé à un outil de mesure d'orientation complète ou partielle.

Un autre avantage de la méthode est de permettre à l'opérateur qui prévoit d'enregistrer un PSV, d'affiner le choix le type d'outil sismique de puits ainsi que de l'outil d'orientation désirable à combiner, avant d'engager l'acquisition effective sur site du PSV en trois composantes, en fonction de l'objectif géologique poursuivi, de la déviation de trajectoire du puits considéré, et du type de traitement (1C ou $3 \mathrm{C}$ ) souhaité à la suite de l'acquisition des données au terrain.

La méthode s'applique à plusieurs configurations géométriques d'acquisition de sismique de puits, mais spécifiquement au PSV en puits vertical à faiblement dévié, avec source placée à faible distance de la tête du puits, configuration pour laquelle il n'y a pas d'alternative connue à la méthode selon l'invention.

Ainsi, la méthode est applicable aux cas très courants où aucun outil de mesure d'orientation complet et précis n'est couplé à l'outil de mesure de PSV, par exemple lorsque 
l'outil PSV comprend trois composantes de capteurs sismiques directionnels orthogonaux uniquement, dans les configurations suivantes :

a) capteurs sismiques 3C montés de façon fixe dans l'outil PSV,

b) capteurs sismiques $3 \mathrm{C}$ montés de façon fixe dans un outil de PSV comprenant en

5 outre un dispositif de mesure de l'angle de "Relative Bearing » dans le plan orthogonal à l'axe de l'outil PSV :

c) capteurs sismiques 3C sur des doubles cardans avec architecture dite "tourelle", c'est-à-dire comprenant un axe de rotation libre parallèle à l'axe de l'outil, donc parallèle à l'axe du puits à la station d'ancrage de l'outil PSV. Chaque capteur est monté conjointement avec une masse décentrée par rapport à l'axe des cardans de façon à obtenir un dispositif pendulaire qui s'oriente par la gravité dans un repère connu lié à la trajectoire du puits, qui est supposée connue, par exemple à partir d'une diagraphie de mesure de la trajectoire du puits par gyroscope, effectuée séparément.

Naturellement, le type de dispositif appelé communément "capteur de Relative Bearing", ainsi que le montage des capteurs sismiques sur doubles cardans monté en "tourelle" sont inopérants en puits strictement vertical et restituent une orientation des composantes sismiques horizontales qui n'est significative qu'au delà d'une faible valeur de l'ordre de 10 degrés de l'inclinaison verticale du puits, et qui devient de plus en plus précise lorsque l'inclinaison du puits dévié augmente.

La méthode selon l'invention peut également s'appliquer avec bénéfice sur le train d'onde-P descendante interféré dans un puits vertical, et dont la forme du signal trois composantes varie progressivement en fonction de la profondeur, mais avec une direction azimutale d'énergie totale stable pour le signal interféré, et dans le cas où un outil ancien comportant trois composantes à montage fixe dans l'outil, sans dispositif de mesure d'orientation est placé dans un drain horizontal, et où l'arrivée P directe ne montre aucune énergie sur la composante axiale au puits.

La méthode selon l'invention peut être également appliquée pour les configurations PSV de type "walkabove", lorsque la source est située sensiblement à l'aplomb d'un drain horizontal (figure $2 b$ ), les capteurs étant montés de façon fixe dans un outil de puits qui ne comporte aucun dispositif d'orientation. Après maximisation de l'arrivée directe $P$, on peut en première approximation, faire l'hypothèse que cette arrivée est confondue avec la droite qui relie la source et le récepteur, dont on peut déduire l'angle de « relative bearing » à partir de la connaissance de la trajectoire de puits et de la position relative de la source par rapport au puits. 
La méthode selon l'invention peut être également appliquée avec bénéfice, afin d'automatiser le pointé de l'onde $P$ et la détermination de l'orientation des capteurs, dans le cadre de prospection sismique par méthode de type walkaway conventionnel. Selon ce type de méthode, le dispositif de réception de puits peut être fixe ou non, et la source de surface est activée successivement à des positions voisines, soit sur une ligne d'azimut fixe ( walkaway 2D), soit sur un cercle concentrique au puits ou à la position géographique moyenne des capteurs de puits (walkaround). Les deux configurations précédentes peuvent être combinées, soit sur une grille plus ou moins complète de positions dans le voisinage du puits (walkaway 3D ou 3D-VSP). En particulier, la méthode selon l'invention présente l'avantage de fournir un pointé précis et automatique de l'onde directe $P$ lorsque celle-ci arrive orthogonalement à la composante de direction vectorielle connue (sensiblement verticale dans ce cas), sans devoir orienter au préalable les composantes horizontales, dans la configuration illustrée par la figure 2c.

Une application particulière de la méthode selon l'invention, consiste à monter un outil de PSV à trois composantes en combinaison avec un autre outil de diagraphie, dont on désire connaître l'orientation, dans le cas extrême ou les outils d'orientation usuels du genre gyroscope ou magnétomètre/inclinomètres ne sont plus opératoires, par exemple lorsque la température du puits excède $220^{\circ} \mathrm{C}$.

Selon un mode particulier de réalisation, pour des raisons de facilité de calcul et de fiabilité du résultat, on détermine, sur différentes portions du puits, des repères uniques au moyen de la méthode selon l'invention,. Ces repères ont un axe commun mais peuvent être d'orientation différentes. Les portions du puits ont des zones de chevauchement, qui permettent de déterminer un angle de rotation à appliquer aux repères uniques de chaque portion, de façon à obtenir un repère unique pour l'ensemble du puits.

Enfin, la technique de pointé d'un signal module filtré calculé à partir des composantes brutes, mesurées par un outil de diagraphie de type sonic dipolaire, ou quadrupolaire en forme d'onde complète, dit "full waveform", peut s'avérer utile dans le cas où l'on ne désire connaître que la lenteur et l'atténuation d'une onde de cisaillement sans rechercher les caractéristiques d'anisotropie azimutale. Dans un tel cas, il n'est pas utile de mesurer l'orientation de l'outil sonic dans le puits, ce qui allège l'opération de mesure diagraphique. 


\section{REVENDICATIONS}

1. Méthode de prétraitement de données sismiques acquises au moyen d'une méthode de prospection sismique de type profil sismique vertical qui comprend une émission d'ondes sismiques et une réception desdites ondes sismiques au moyen d'au moins un capteur multi - composantes positionné au sein d'un puits et que l'on fait stationner à au moins deux profondeurs, ledit capteur comprenant au moins trois géophones orthogonaux enregistrant en fonction du temps une première composante sismique dans une direction vectorielle connue, et au moins deux autres composantes sismiques dans deux directions orthogonales à ladite direction vectorielle connue, caractérisée en ce que :

a- on construit un nouveau signal en calculant la racine carrée de la somme des carrés d'au moins deux composantes sismiques orthogonales, appelé signal module ;

b- on pointe des temps d'arrivée d'une onde sismique directe sur un extremum d'amplitude dudit signal module

2. Méthode selon la revendication 1, dans laquelle on construit ledit signal module en calculant la racine carrée de la somme des carrés desdites deux composantes sismiques orthogonales à ladite direction vectorielle connue, et l'on pointe les temps d'arrivée d'une onde de cisaillement descendante.

20 3. Méthode selon la revendication 2, dans laquelle on pointe également l'arrivée d'une onde de pression, et on calcule des vitesses desdites ondes de cisaillement et de pression à partir desdits temps d'arrivée, et on en déduit des rapports de vitesse et/ou un coefficient de Poisson.

4. Méthode selon la revendication 1, dans laquelle on construit ledit signal module en calculant la racine carrée de la somme des carrés des trois composantes sismiques, et l'on pointe les temps d'arrivée d'une onde de pression directe.

5. Méthode selon l'une des revendications précédentes, dans laquelle, préalablement à l'étape a), on préserve l'isotropie du signal en trois composantes, en respectant des rapports d'amplitude et des différences de phase entre les composantes sismiques.

30 6. Méthode selon l'une des revendications précédentes, dans laquelle on améliore un rapport signal sur bruit des trois composantes du signal brut, préalablement au calcul du module, au moyen d'une déconvolution isotrope des trois composantes par un signal unique d'onde de pression descendante extrait de la composante sismique de direction vectorielle connue. 
7. Méthode selon l'une des revendications précédentes, dans laquelle on filtre ledit signal module de façon à supprimer des composantes basses fréquences, avant de pointer les temps d'arrivée de l'onde sismique directe.

8. Méthode selon l'une des revendications précédentes, dans laquelle on oriente lesdites composantes sismiques dans un repère unique quelque soit la profondeur dudit capteur, au moyen des étapes suivantes répétées pour chaque profondeur :

- on définit une fenêtre temporelle de part et d'autre desdits temps d'arrivée ;

- on détermine une direction azimutale par maximisation d'une énergie desdites composantes sismiques orthogonales à ladite direction vectorielle connue au sein de ladite fenêtre temporelle ;

- on oriente à 360 degrés près lesdites composantes sismiques orthogonales à ladite direction vectorielle connue dans un repère unique défini par rapport à la direction azimutale qui est identique pour chaque profondeur.

9. Méthode selon la revendication 8 , dans laquelle on détermine l'orientation géographique dudit repère unique.

10. Méthode selon la revendication 9 , dans laquelle on détermine l'orientation géographique dudit repère unique en montant ledit capteur multi-composantes sur un système de double cardans, ledit système permettant d'orienter par gravité lesdites composantes sismiques lorsque l'inclinaison du puits atteint une valeur d'au moins environ 10 degrés.

11. Méthode selon la revendication 9 , dans laquelle on descend dans le puits un outil de mesure de puits comportant ledit capteur multi-composantes monté de façon fixe, et l'on détermine l'orientation géographique dudit repère unique au moyen d'un système de mesure d'un angle de "relative bearing" monté sur ledit outil de mesure, qui permet de retrouver l'orientation dudit capteur multi-composantes lorsque l'inclinaison du puits atteint une valeur d'au moins environ 10 degrés.

12. Méthode selon la revendication 9 , dans laquelle on descend dans le puits un outil de mesure de puits comprenant une pluralité de capteurs multi - composantes placés à des profondeurs de mesure adjacentes, et on détermine l'orientation géographique dudit repère unique en couplant au moins l'un des capteurs multi - composantes à un outil de mesure d'orientation géographique, tel qu'un magnétomètre-inclinomètre ou un gyroscope.

13. Méthode selon la revendication 9 , dans laquelle au moins une portion du puits est sensiblement horizontale et ledit capteur multi-composantes est monté de façon fixe dans un outil de mesure descendu dans le puits, on détermine l'orientation 
géographique dudit repère unique en assimilant une direction de maximisation d'une onde de pression directe à une droite reliant une position dudit capteur à une position d'une source émettant lesdites ondes sismiques.

14. Méthode selon la revendication 8 , dans laquelle on détermine, sur différentes portions du puits, des repères uniques ayant un axe commun, lesdites portions ayant des zones de chevauchement permettant de déterminer un angle de rotation à appliquer auxdits repères uniques, de façon à obtenir un repère unique pour l'ensemble du puits.

15. Méthode selon la revendication 8 , dans laquelle on utilise l'orientation dans un repère unique de façon automatisée pour obtenir un contrôle de la qualité desdites composantes sismiques, immédiatement après l'acquisition de mesures sur le terrain. 

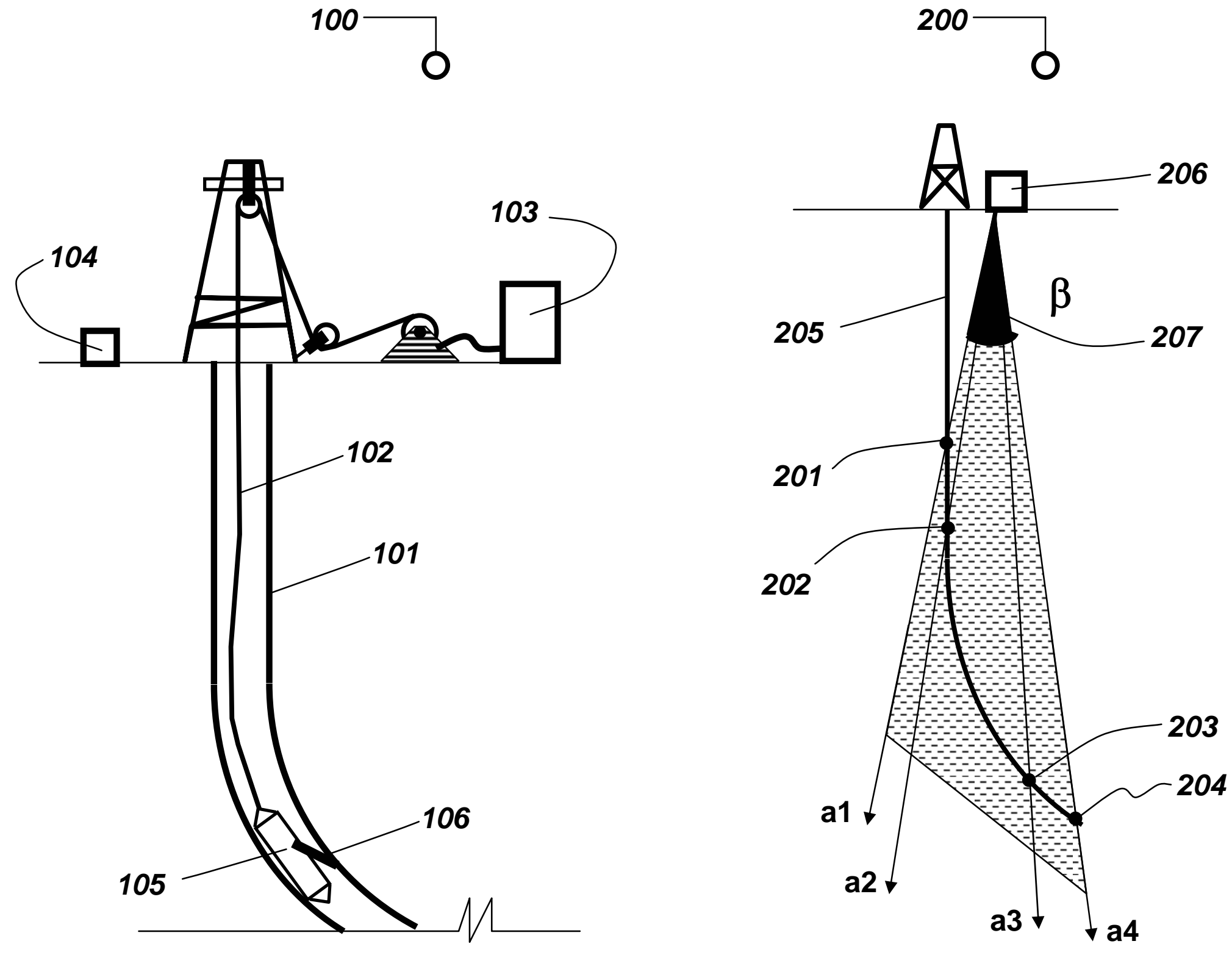

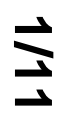

Fig. 1

Fig. 2a 

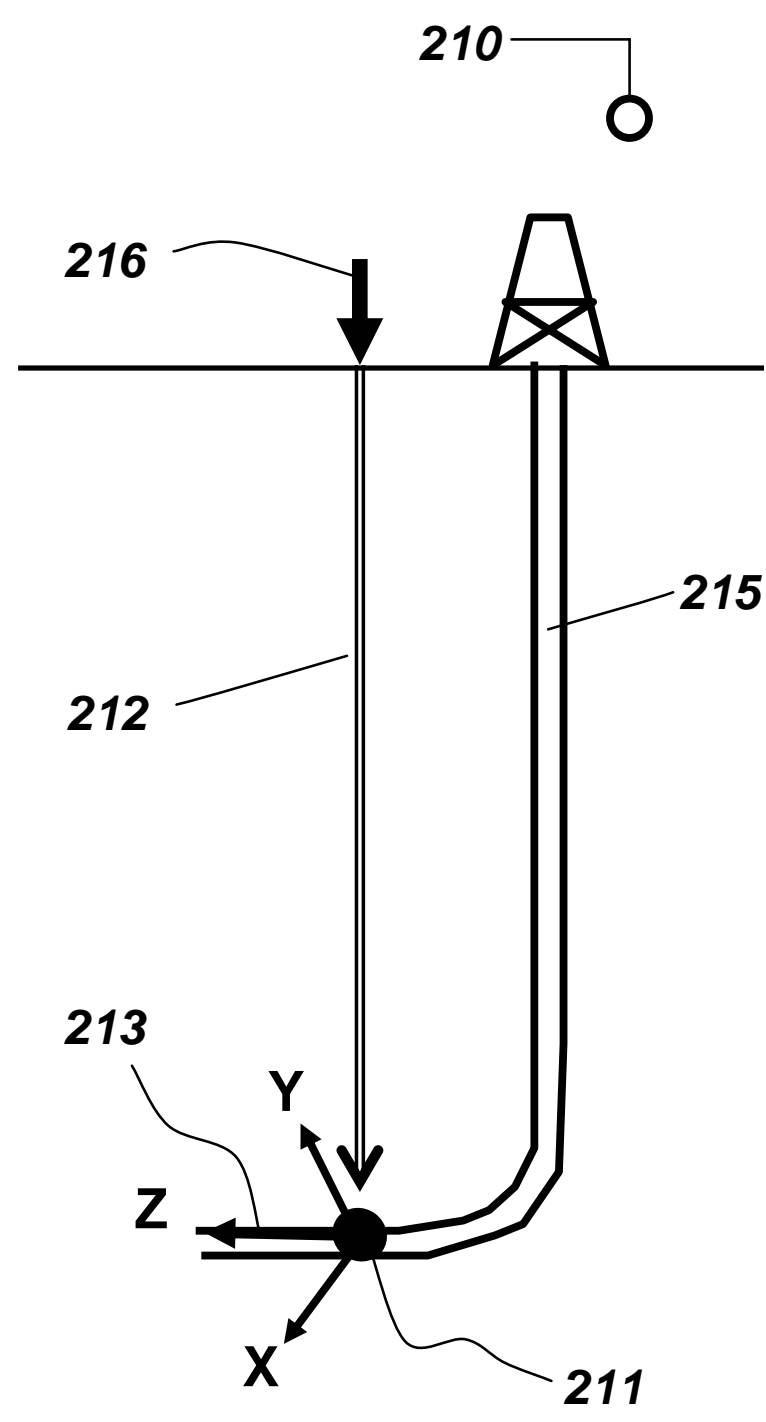

220

o

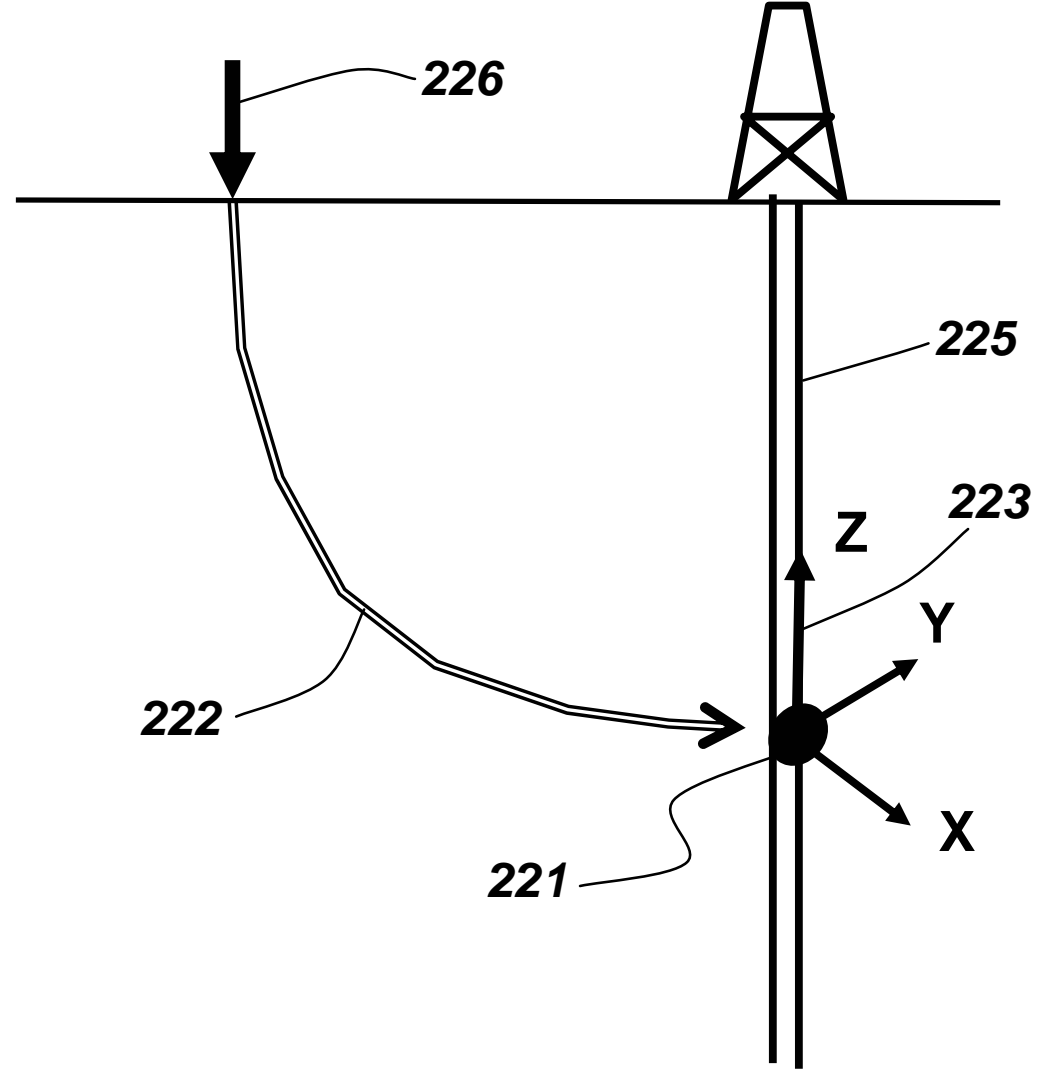

Fig. 2b

Fig. 2c 
300

0

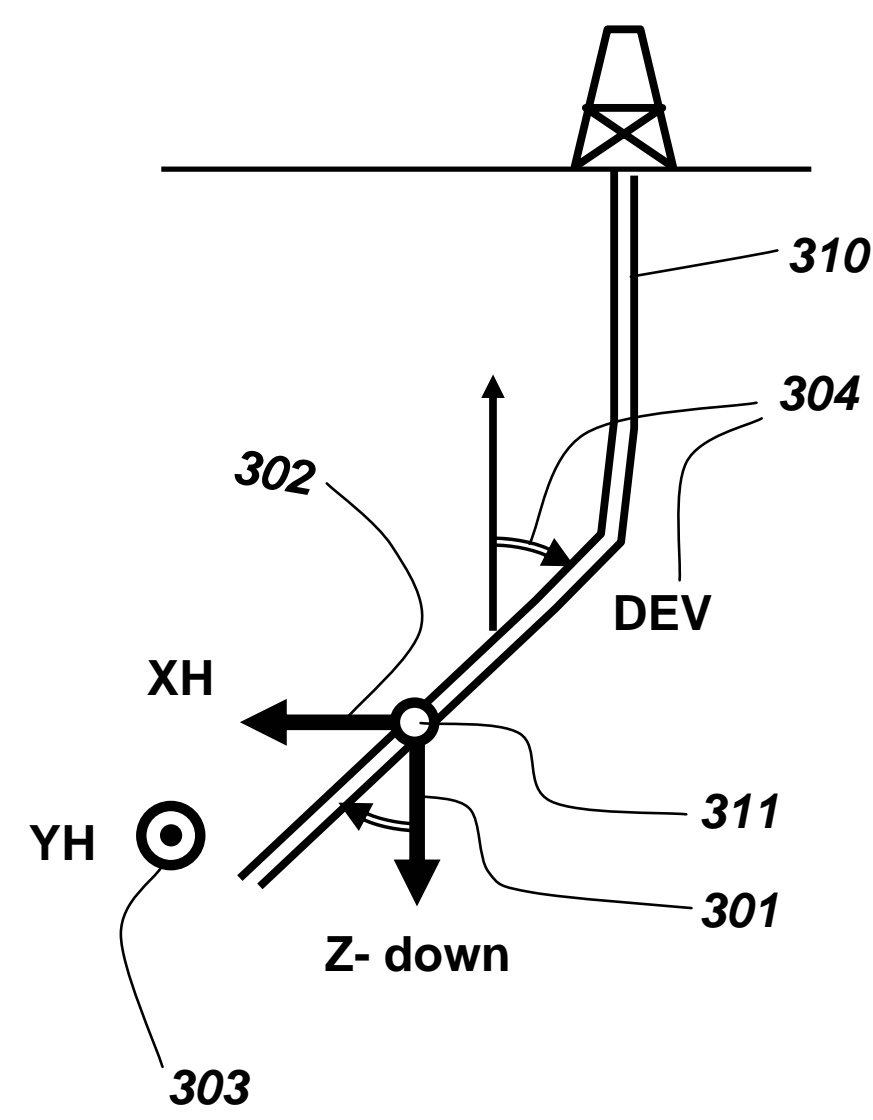

350

0

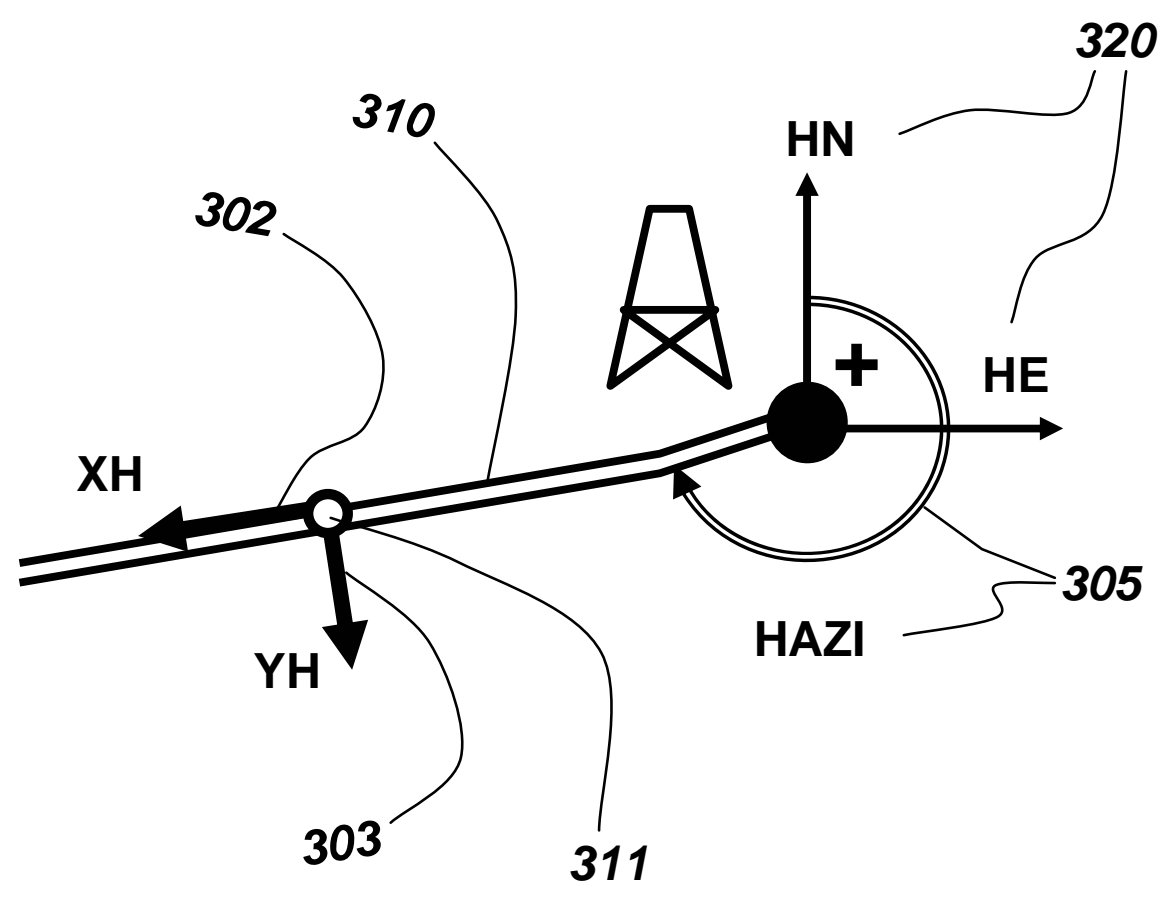


400

0

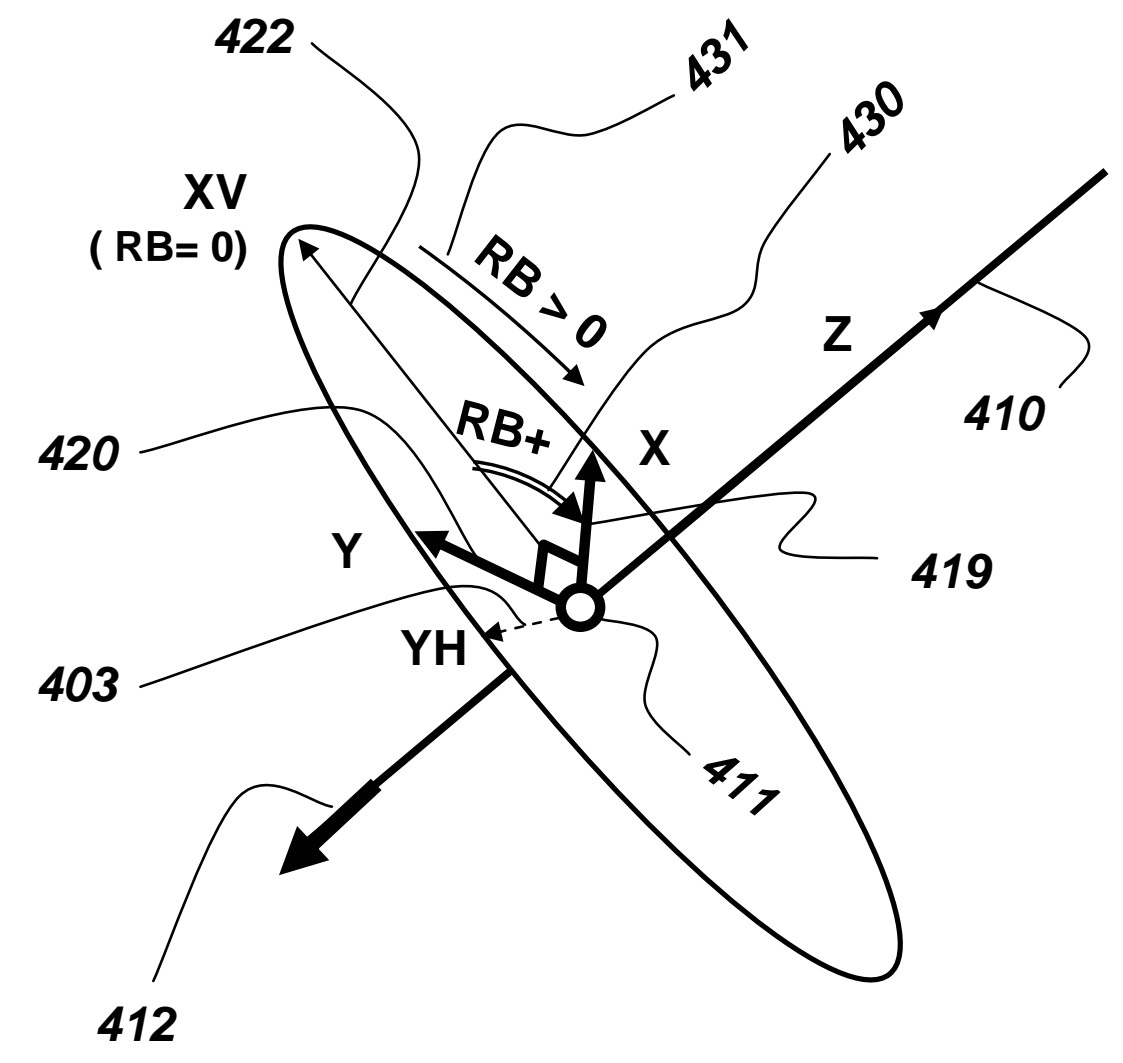

450 0

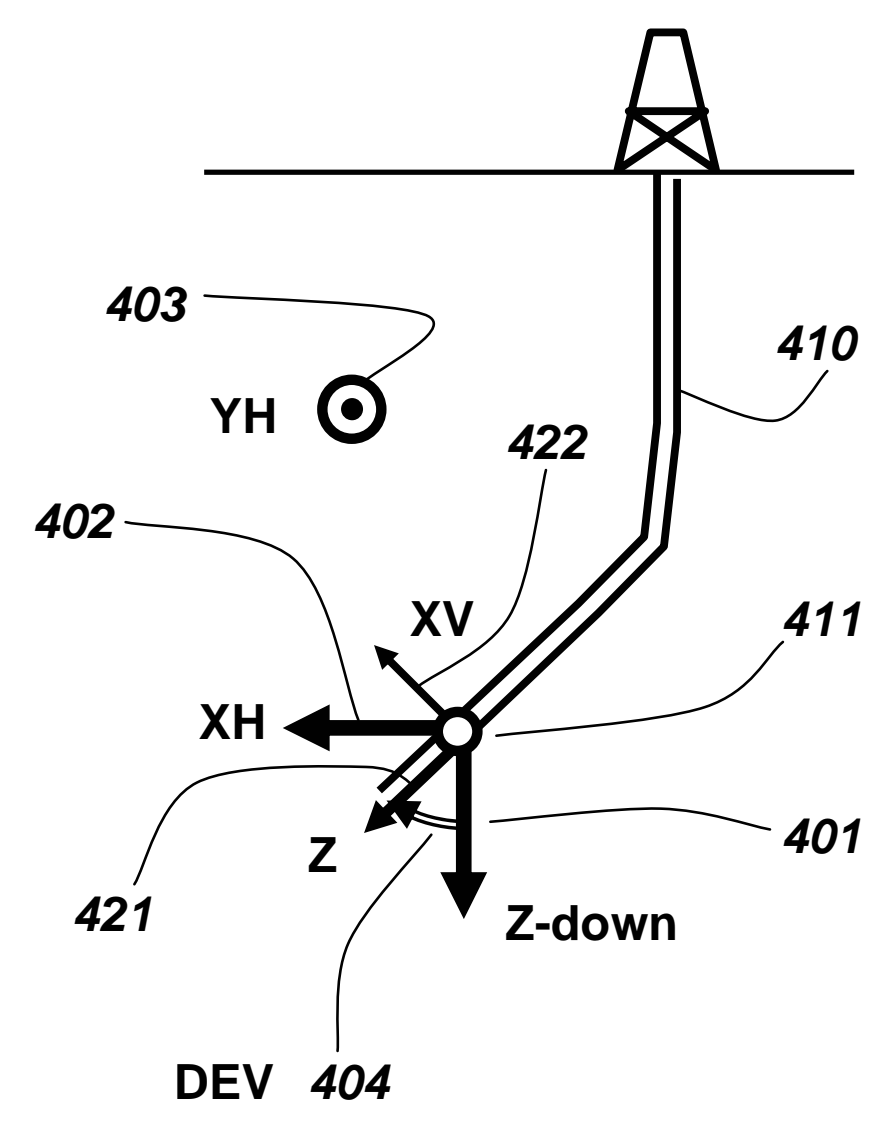

Fig. 4a

Fig. 4b 


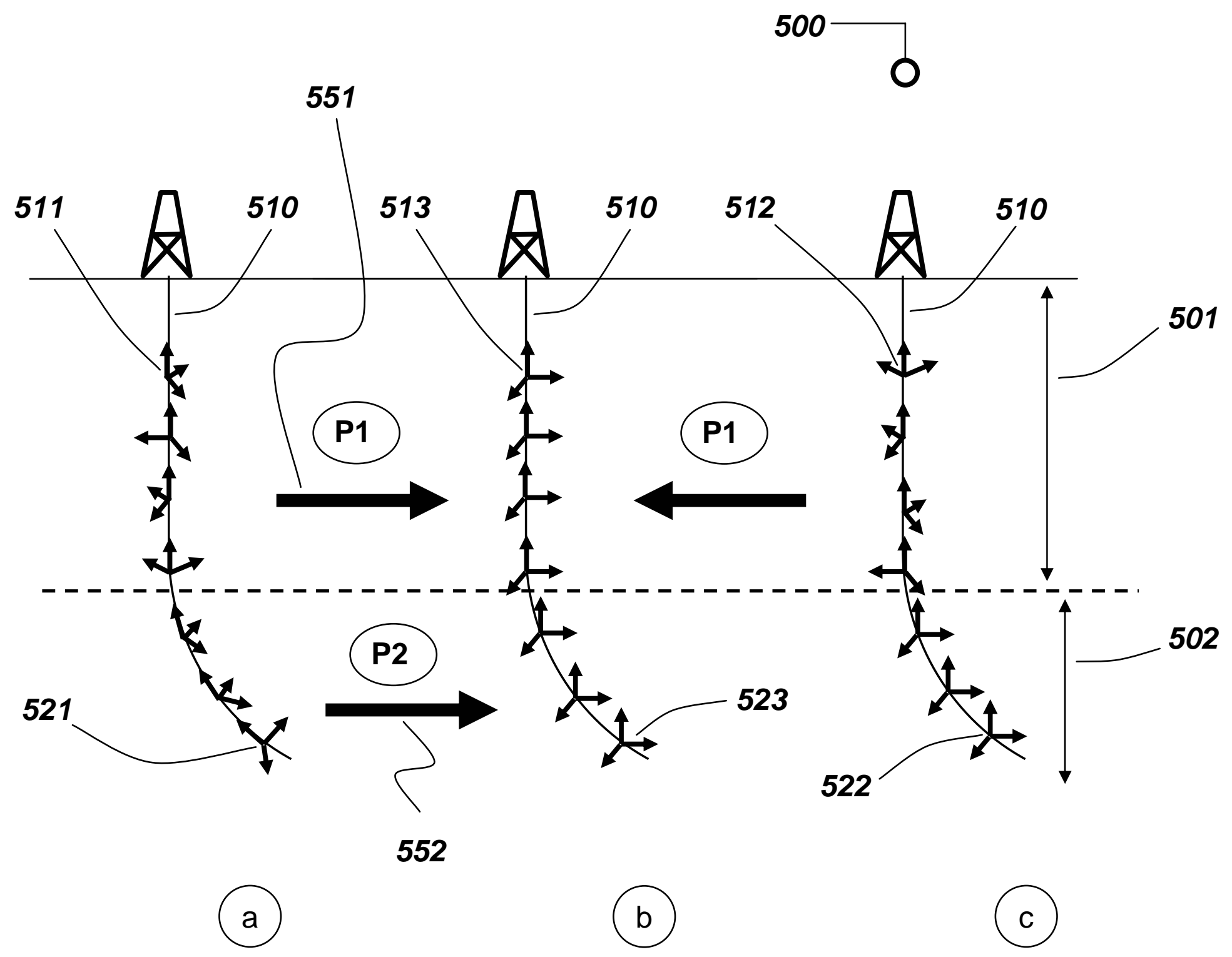

Fig. 5 
600

0

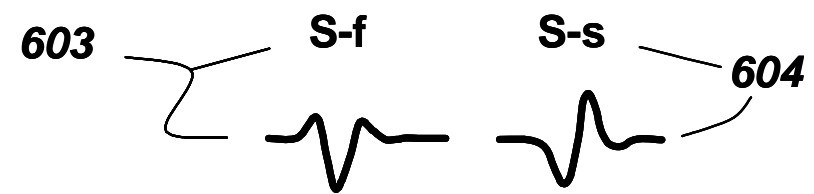

601

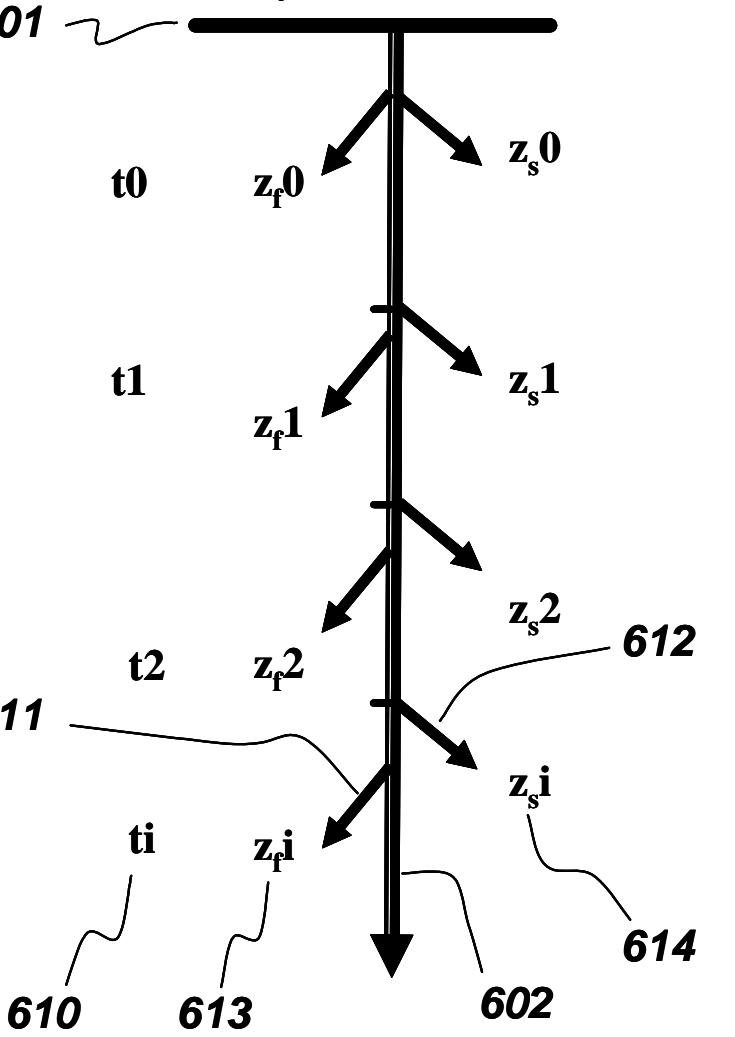

Fig. 6a
$650 \longrightarrow 0$<smiles>[Mg][Mg]CCC[SeH]</smiles><smiles>N#CC[AsH3]</smiles>
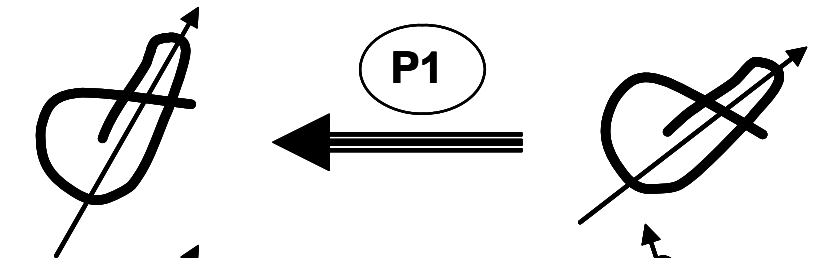

z0
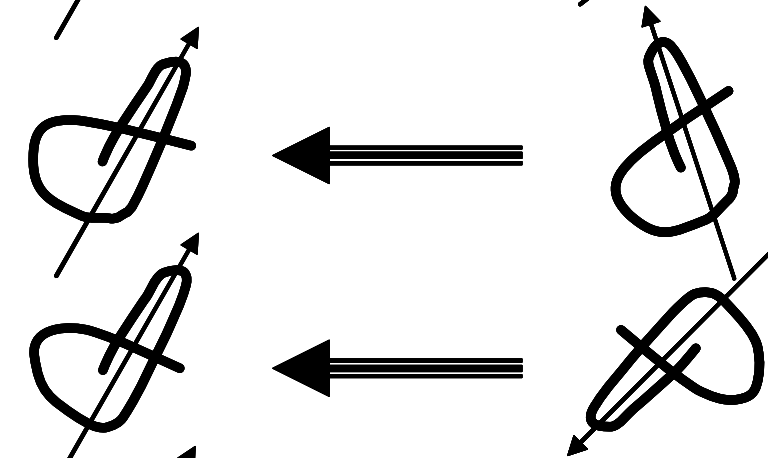

z1

$\stackrel{9}{!}$

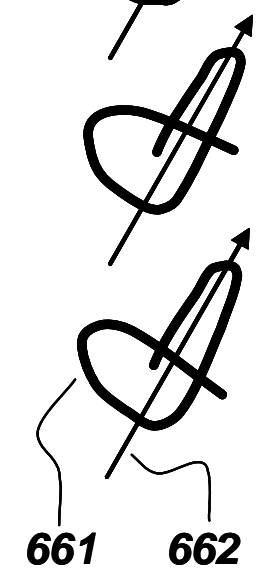

661662
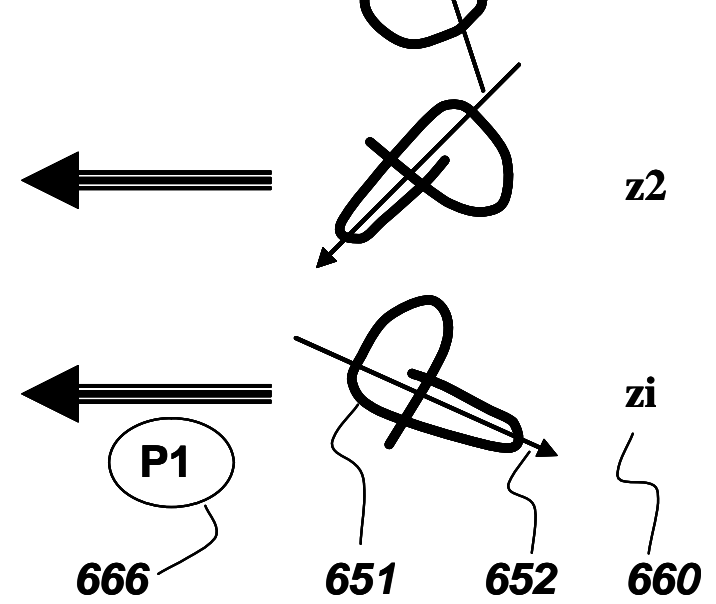

Fig. 6b 


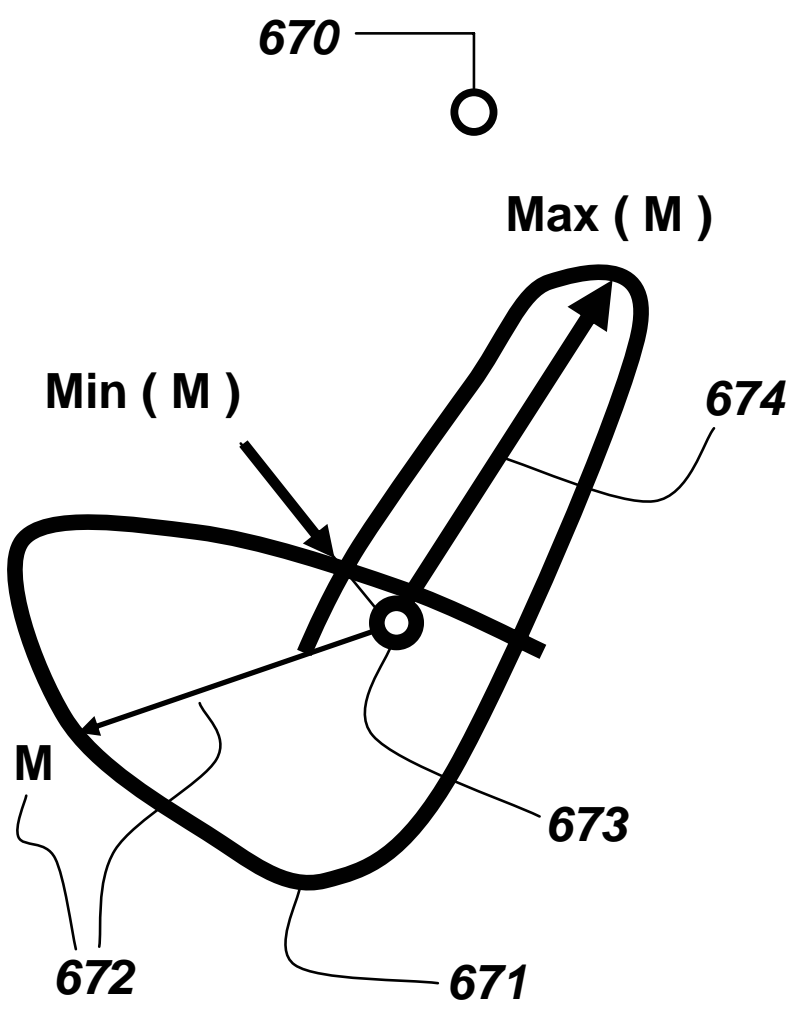

Fig. 6c

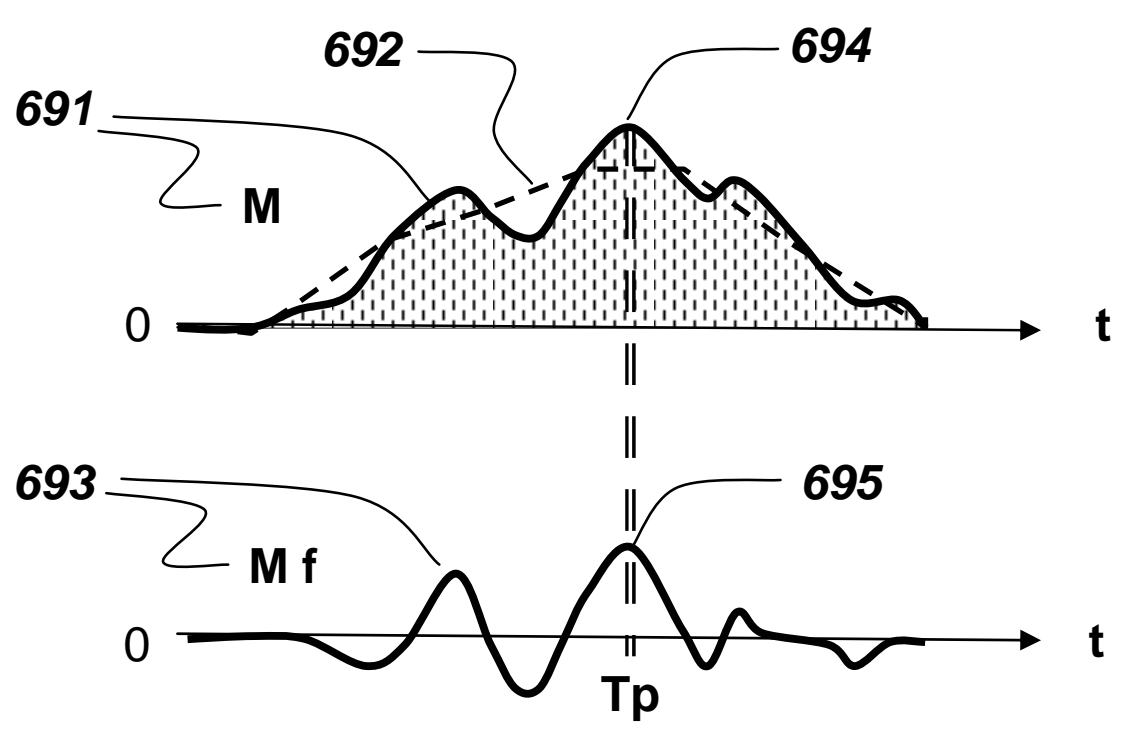

$\underline{v}$
Fig. 6d 


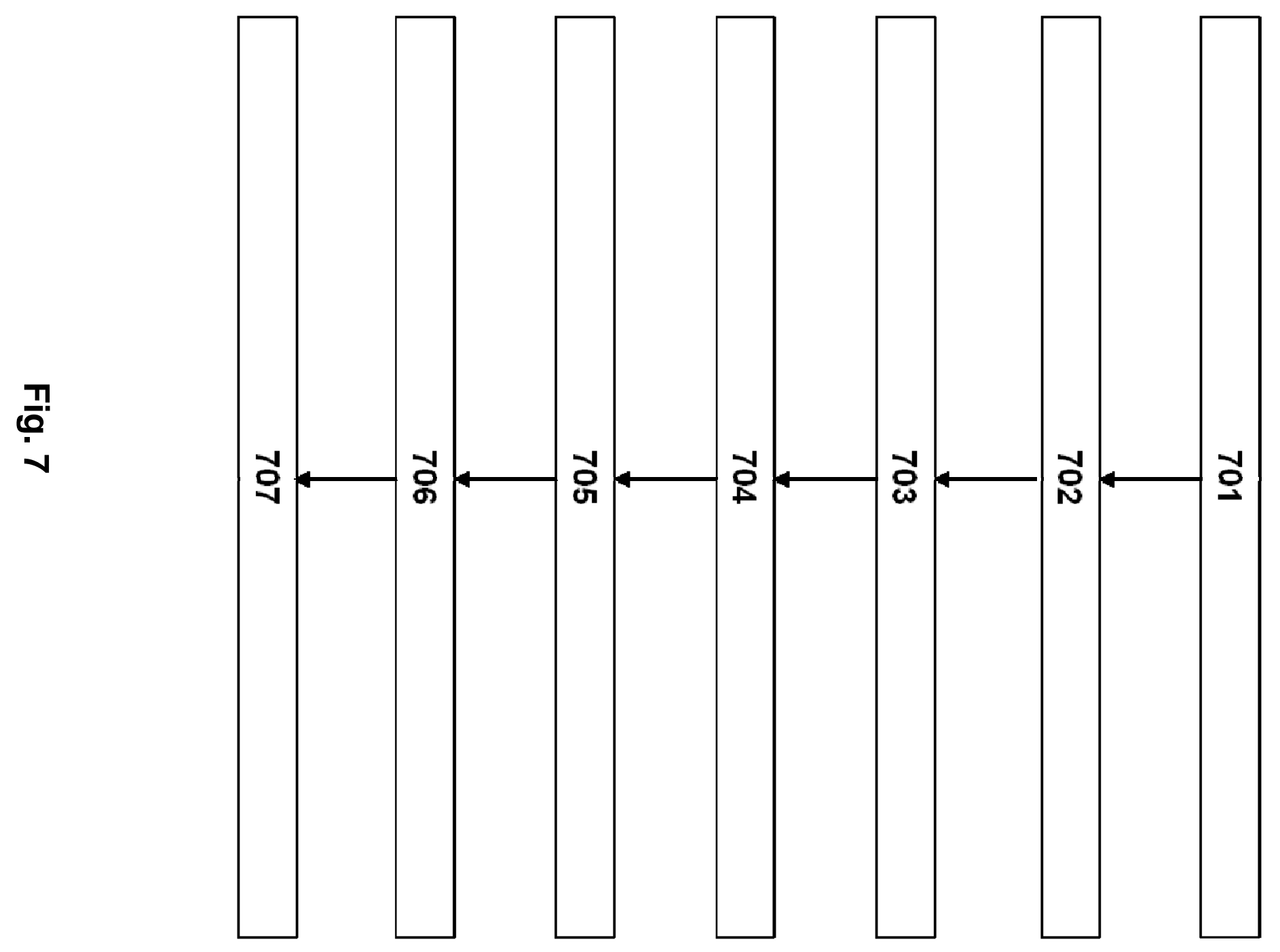

8

。

$\stackrel{\infty}{\mapsto}$ 


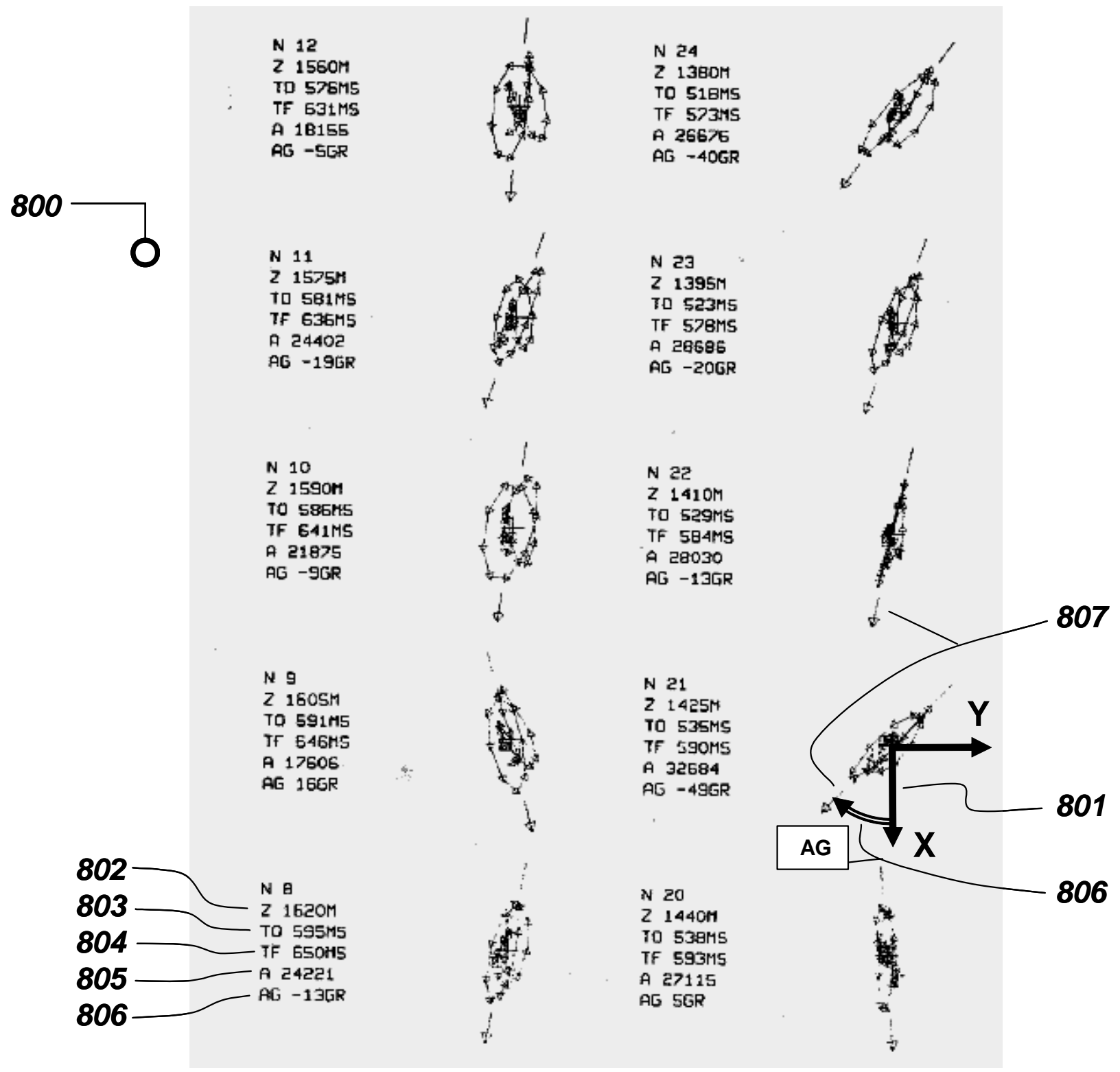

Fig. 8 


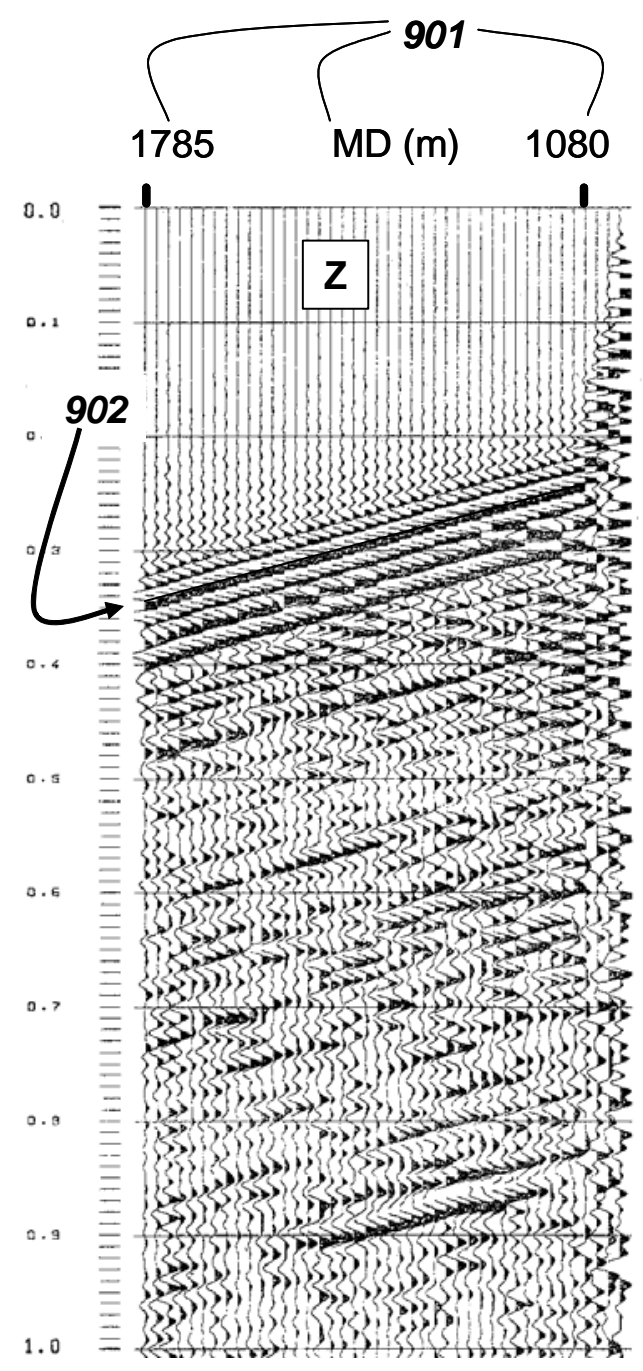

Fig. 9a

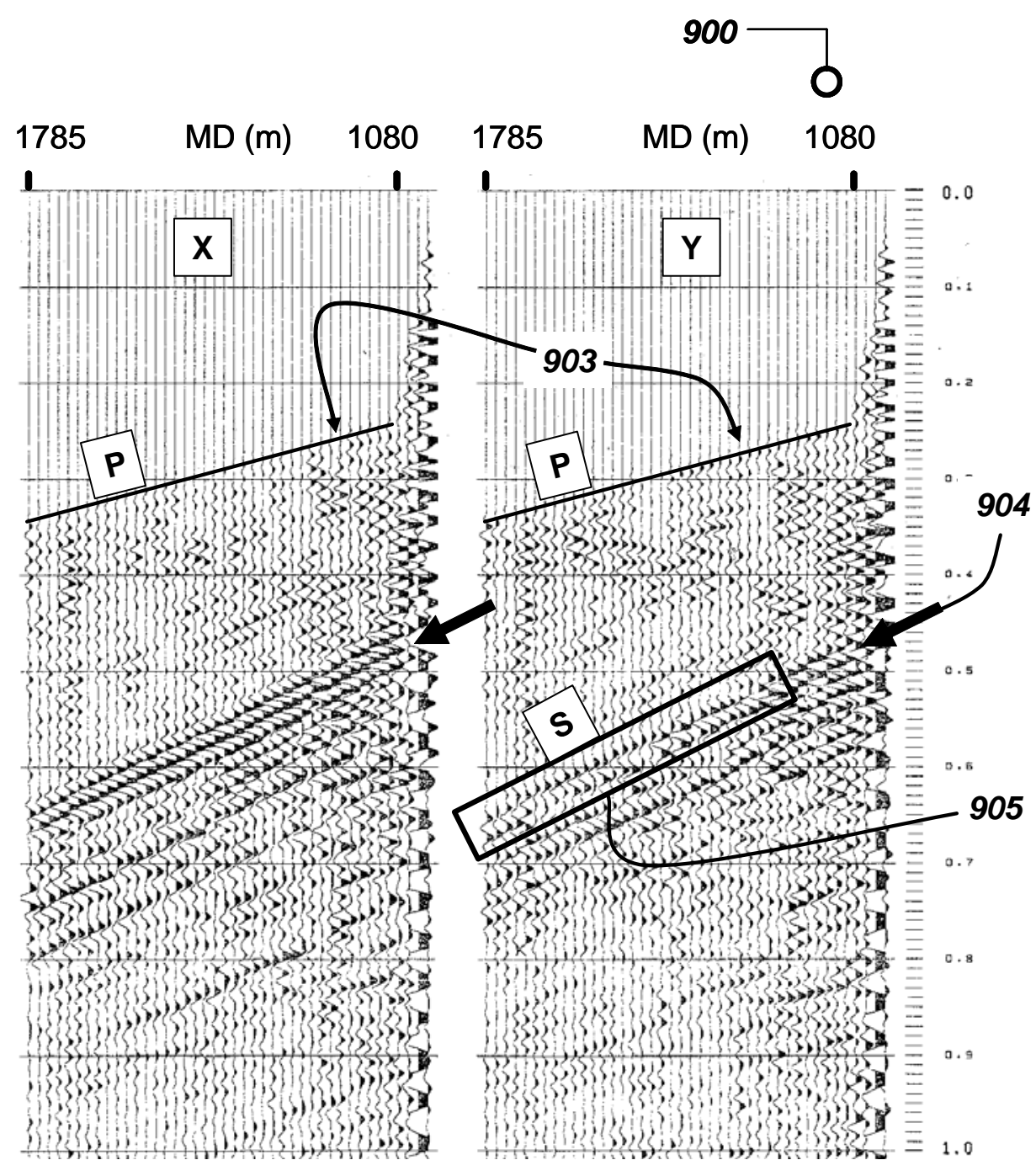

Fig. 9b
Fig. 9c 


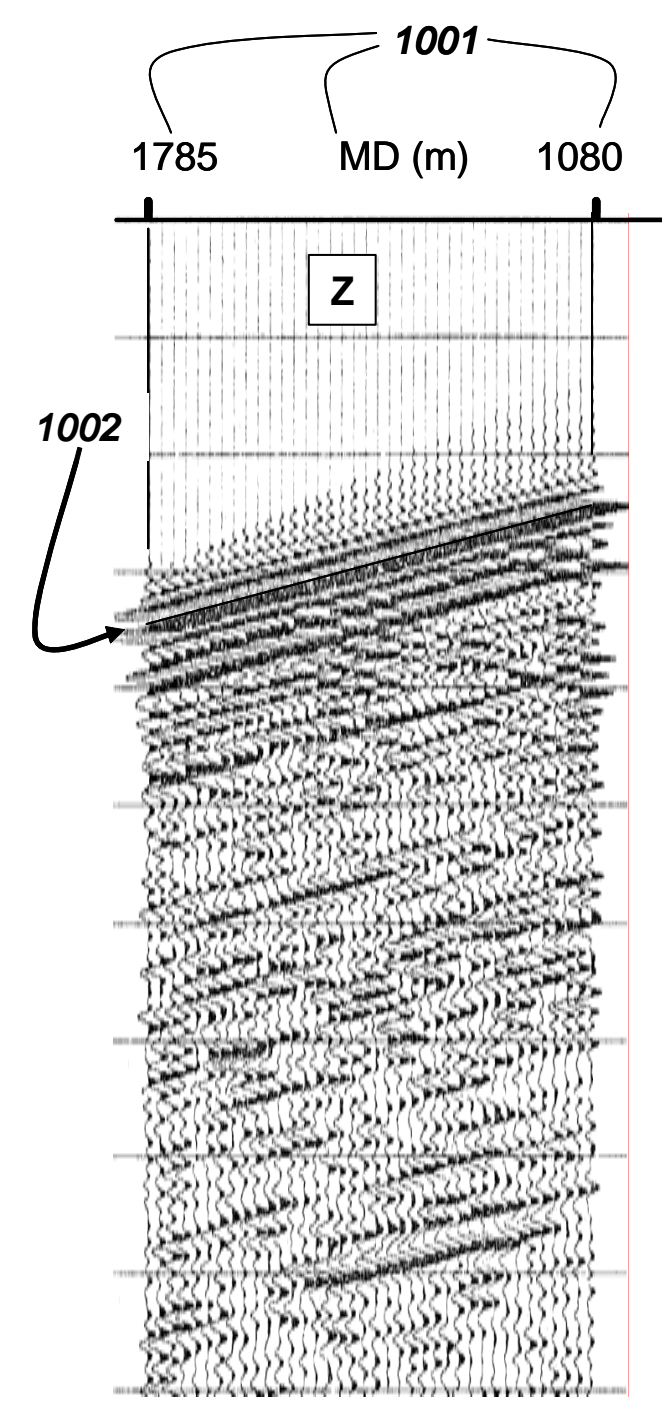

Fig. 10a

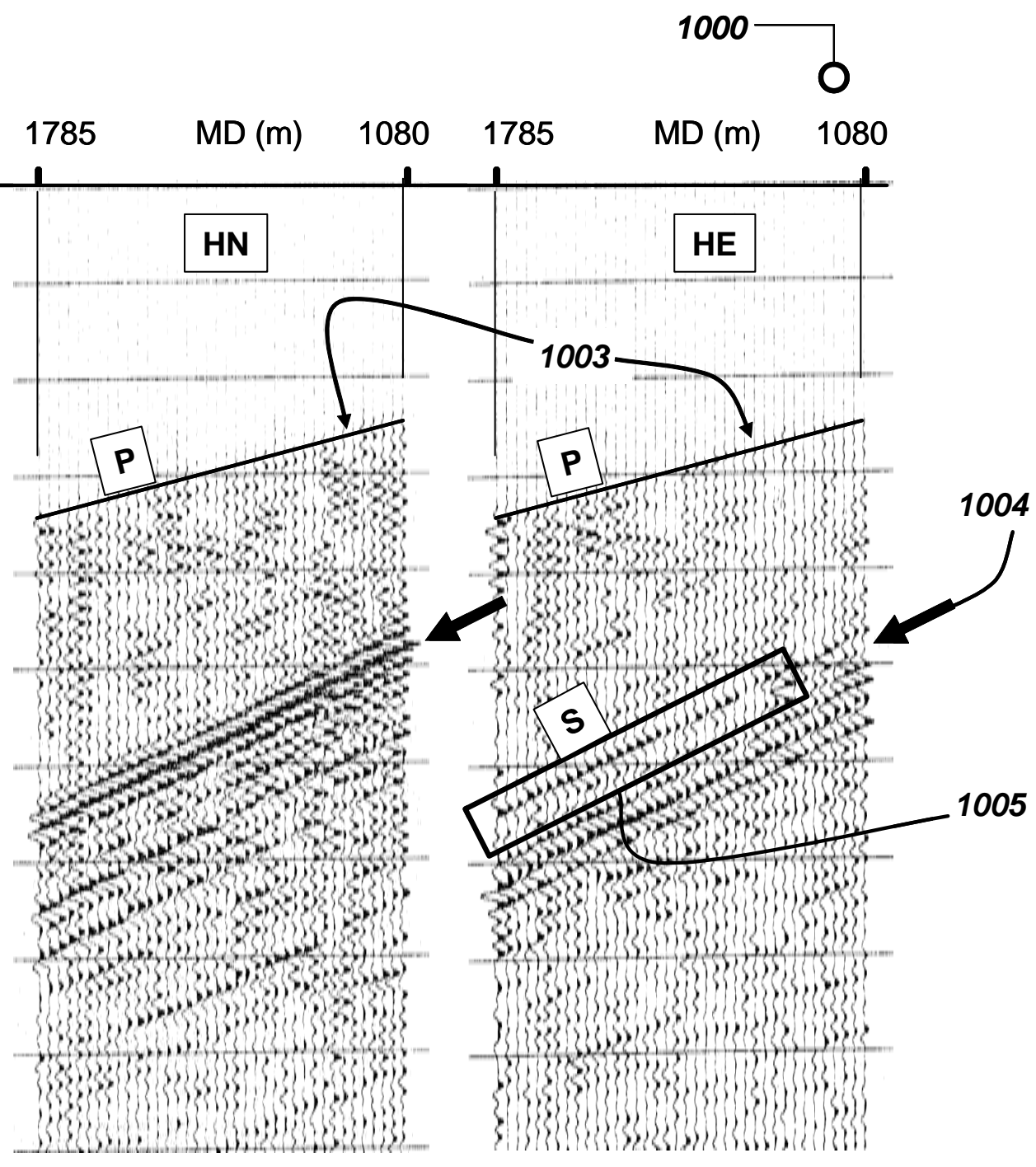

Fig. 10b
Fig. 10c 


\title{
BREVET D'INVENTION
}

\author{
$* * * * * * * * * *$
}

\section{IFP}

$\star \star \star * * * * * * *$

\section{MÉTHODE D'ORIENTATION DE SIGNAUX SISMIQUES DE PUITS À TROIS COMPOSANTES \\ $\star * * * * * *$}

\section{Invention de Charles NAVILLE, Sylvain SERBUTOVIEZ, Jean-Claude LECOMTE, Pascal FROIDEVAUX et Kazem KAZEMI.}

$* * * * * * * *$

\section{Abrégé descriptif}

- Méthode d'orientation des composantes d'un capteur multi-composantes dans un repère géographique connu.

- Après acquisition de données sismiques par une méthode de type PSV, au moyen d'un capteur multi - composantes, on construit le signal module des deux composantes sismiques horizontales. Puis, on pointe des temps d'arrivée d'une onde de cisaillement descendante sur ce signal module, à partir duquel on détermine une direction azimutale permettant d'orienter les composantes sismiques dans un repère unique quelque soit la profondeur du capteur. Enfin, on détermine l'orientation géographique de ce repère unique en lui appliquant une rotation jusqu'à ce qu'une direction azimutale, déterminée à partir de connaissances structurales et géologiques, telle que la direction de pendage d'une interface sédimentaire, coïncide avec une direction azimutale déterminée à partir des trois composantes: l'azimut de pendage d'un réflecteur sismique issu d'une analyse de polarisation du champ d'ondes réfléchies issu des composantes, ou la direction perpendiculaire à la direction d'anisotropie de biréfringence de l'onde de cisaillement.

- Application à l'exploration ou la production de gisement pétrolier par exemple.

- Figure 5 à publier. 
La présente invention concerne le domaine de la sismique de puits, et plus particulièrement le domaine des techniques de prétraitement de données sismiques acquises au moyen de capteurs multicomposantes, obtenues lors d'opérations de Profil Sismique Verticale (PSV ou VSP en anglais).

La présente invention concerne notamment une méthode d'estimation de l'orientation d'un capteur sismique de puits multi-composantes.

La technique de PSV est couramment utilisée pour une meilleure connaissance de la structure du gisement et de son voisinage en phase d'exploration, ou en phase d'exploitation. Cette technique est également utilisée pour définir les structures géologiques au voisinage du puits pour guider le forage, ou redéfinir une déviation de la trajectoire de forage si ce dernier n'a malencontreusement pas atteint son objectif.

La mise en oeuvre la plus classique du profil sismique vertical (PSV) se fait au moyen d'une émission sismique effectuée par une source répétitive en surface, et d'une réception faite dans le puits. Cette dernière est effectuée grâce à une sonde de puits particulière, que l'on fait stationner successivement à différentes profondeurs. La sonde de puits comprend un 25 capteur, généralement à trois composantes, un système d'ancrage et une unité de numérisation pour la plupart des outils modernes. Le système d'ancrage et la conception mécanique de la sonde réceptrice de puits sont tels que l'enregistrement en trois composantes, du déplacement en trois dimensions de la formation au passage des diverses ondes sismiques est fidèle au déplacement effectif de la formation. Cette notion de fidélité vectorielle caractérise l'isotropie de l'ancrage mécanique des capteurs de la sonde réceptrice à la paroi du puits, et l'isotropie recherchée de la réception sismique en trois composantes. L'acquisition peut être faite avec un capteur triaxial placé à une profondeur unique, ou par une antenne de capteurs triaxiaux situés à des niveaux de réception profondeur adjacents. 
La figure 1 schématise de façon générale la géométrie d'acquisition d'un PSV 100 conduite dans un puits 101 foré sensiblement vertical, et en général faiblement dévié sur certains intervalles de profondeur. La profondeur du puits peut atteindre typiquement plusieurs milliers de mètres. La sonde sismique de puits 105 contenant les capteurs sismiques triaxiaux est descendue au moyen d'un câble 102, qui la relie à une unité d'enregistrement de surface 103 à partir de laquelle l'ingénieur terrain assure toutes les commandes de la sonde de puits, la télécommande de la source sismique 104, ainsi que le contrôle qualité des mesures. Afin de mesurer avec une bonne fidélité vectorielle le signal sismique qui se propage dans la formation rocheuse, en provenance de la source sismique de surface 104, cette sonde est appuyée fortement contre la paroi du puits à l'aide d'un dispositif d'ancrage symbolisé par le bras 106, préalablement à l'enregistrement de chaque station profondeur de mesure. Dans la configuration commune du PSV de base, la source sismique de surface 104 dite "zéro-offset", est placée en pratique dans un rayon de 100m par rapport à la tête de puits; compte tenu de la profondeur du puits, la source sismique 104 est dite "de surface" même si elle est placée à quelques mètres de profondeur. La sonde sismique 105 schématisée sur la figure1 peut représenter en réalité une sonde réceptrice comportant plusieurs niveaux profondeur de réception sismique adjacents, séparés typiquement d'une distance de 15 ou 20 mètres, chacun des niveaux correspondant à une sonde sismique indépendante, comportant des capteurs sismiques triaxiaux et un système d'ancrage.

La figure 2 schématise la géométrie de propagation $\mathbf{2 0 0}$ des ondes sismiques directes émises par la source de surface 206, reçues par les capteurs 201 à 204 situés dans le puits 205 : on peut constater, sur ce schéma, l'étroitesse de l'angle solide $207(\beta)$ de propagation en arrivée directe. Cet angle englobe l'ensemble de la partie profonde du puits dans laquelle les mesures de PSV sont réalisées avec un intervalle régulier fin, couramment de $15 \mathrm{~m}$. De fait, l'étroitesse de l'angle solide d'émission, garantit pour la réception dans le puits I'hypothèse de constance de forme d'onde des modes propres de propagation sismique en milieu stratifié sensiblement homogène pour des niveaux de mesure profondeur adjacents. De plus, on peut constater sur le schéma de la figure 2a que la différence de direction de propagation est extrêmement réduite entre les rais directs, associés à des niveaux de mesure de puits adjacents, comme par exemple les rais directs a1 et a2 associés au couple de positions 201-202, ou les rais directs a3 et a4 associés au couple 203-204. En pratique, la proximité immédiate des chemins de propagation entre une source commune et des récepteurs sensiblement adjacents, voisins d'une dizaine à une centaine de mètres par exemple, est également vérifiée pour une onde descendante plus complexe qu'une onde directe, par exemple une onde de type $\mathrm{P}$ convertie $\mathrm{S}$ au droit d'une interface située à une 
profondeur intermédiaire, par exemple dans la moitié supérieure de la profondeur totale du puits.

Le terme "multicomposante " qualifie une disposition particulière d'une pluralité de capteurs sismiques. Par exemple, un capteur à trois composantes comprend trois récepteurs sismiques unidirectionnels disposés selon des axes orthogonaux, tels que des géophones ou des accéléromètres. Les ondes sismiques se propageant en trois dimensions, on utilise un capteur à trois composantes pour caractériser l'ensemble des ondes sismiques.

On appelle " composante », le signal issu d'un capteur sismique unitaire. Un capteur à trois composantes génère trois signaux électriques enregistrés selon trois axes orthogonaux. En général, la sonde réceptrice de fond comporte une composante d'axe connu, disposée soit selon la verticale, soit selon l'axe de l'outil. Cet axe de l'outil coïncide sensiblement avec l'axe du puits, après ancrage de la sonde à la paroi du puits, et de deux composantes orthogonales, dont on ne connaît pas la direction exacte dans le plan orthogonal.

II est usuel lors de l'exploitation des données sismiques, obtenues par une méthode de type PSV au moyen de capteur à trois composantes, de traiter une seule des composantes enregistrées, en général soit la composante verticale, soit la composante axiale au puits, soit la composante correspondant à la direction spatiale maximisant l'énergie de l'arrivée directe d'onde de pression. Des exemples de traitement monocomposante de données sismiques sont décrits dans de nombreuses publications et ouvrages publiés, par exemple dans des ouvrages spécialisés suivants :

Hardage,B.; "Vertical Seismic Profiling" : Principles, Third updated and revised edition; in: Handbook of Geophysical Exploration, seismic exploration,Vol.14, 2000, Pergamon, Elsevier Science;

A.H. Balch \& Myung W. Lee; Vertical Seismic Profiling : technique, Applications, and case Histories, 1984, D. Reidel publishing Company;

Mari, J.L. et al; "Seismic Well Surveying", 1991, Editions Technip, Paris.

Il est usuel également de prendre en compte la polarisation des ondes directes en mode de pression pour l'orientation et le traitement des profils sismiques verticaux avec offset, pour lesquels le puits est sensiblement vertical et la position de la source est située à une distance supérieure à $10 \%$ de la profondeur totale du puits. On calcule la composante orthogonale qui maximise l'énergie de l'arrivée directe d'onde de Pression (onde P) dans le plan orthogonal, et dans une fenêtre temps définie par le pointé temps de cette arrivée directe $\mathrm{P}$. Pour déterminer cette direction azimutale dans un plan, à partir de deux composantes, on utilise une technique de maximisation de l'énergie, par exemple celle décrite dans le document suivant : 
DiSiena, J. P., J. E. Gaiser, and D. Corrigan, 1984, "Horizontal components and shear wave analysis of three-component PSV data", in M. N. Toksöz and R. R. Stewart, eds., Vertical seismic profiling, Part B : Advanced concepts : Geophysical Press, 177-235.

Cependant, la limitation classique du traitement à une seule ou à deux des composantes conduit à des indéterminations potentielles dans l'identification du mode d'onde de certaines arrivées reçues par le capteur, pression ou cisaillement, d'une part, et dans le positionnement d'événements géologiques d'autre part, qu'il n'est pas possible de lever. L'inversion des temps, ou les techniques de migration des événements sismiques réfléchis uniquement, conduisent à une pluralité de solutions puisque la direction azimutale de pendage des réflecteurs demeure inconnue. De plus, lorsque deux événements sismiques arrivent en même temps avec des vitesses apparentes voisines, et figurent sur quelques traces profondeur adjacentes (six à douze par exemple), on vérifie qu'il est quasiment impossible de les séparer par un traitement monocomposante classique.

II apparaît donc indispensable, pour améliorer la fiabilité de l'interprétation des données sismiques de PSV, de traiter les trois composantes. Toutefois, l'étape d'acquisition ne permet pas de fournir l'orientation réelle des géophones du câble, alors que cette information est indispensable pour permettre de traiter les données. En effet, les composantes horizontales issues de PSV à trois composantes ont une orientation inconnue et aléatoire, car les câbles portant les capteurs sismiques (géophones) ne peuvent pas contrôler l'orientation de ces éléments. Traiter les trois composantes peut être envisagé, dans la mesure ou la qualité d'isotropie de réception mécanique de la sonde de PSV (résumée sous l'appellation de "fidélité vectorielle" de la réception) est suffisamment bonne. Ceci est le cas avec la plupart des outils de PSV modernes, dans lesquels le rapport entre la force d'ancrage de la partie de la sonde (ou de la sonde entière) supportant les capteurs triaxiaux, et le poids dans l'air du support (ou de la sonde) est supérieur à cinq. Toutefois, bien qu'il existe des accessoires de mesure d'orientation complète d'un outil dans un puits, comme les magnétomètres-inclinomètres et les gyroscopes de puits, ces accessoires matériels sophistiqués sont souvent inutilisés, car ils représentent un surcoût substantiel. De plus, ils peuvent détériorer les qualités d'ancrage mécanique de la sonde PSV à laquelle ils sont combinés. On constate que l'étape d'acquisition des PSV ne permet que rarement de fournir systématiquement l'orientation réelle et complète des capteurs trois composantes, alors que cette information est indispensable pour permettre le traitement des trois composantes. De fait, les composantes orthogonales issues de PSV à trois composantes ont la plupart du temps une orientation inconnue et aléatoire. C'est en particulier le cas dans les intervalles de profondeur du puits faiblement inclinés, notamment au dessous de 10 degrés 
d'inclinaison verticale, intervalles dans lesquels les dispositifs d'orientation partiels du type montage des capteurs sur cardans, ou adjonction d'un dispositif pendulaire sensible à la gravité mesurant l'angle de « relative bearing " dans le plan orthogonal à l'axe de l'outil, sont rendus inopérants.

Ainsi, afin de traiter de façon complète, efficace et bénéfique tous les signaux issus de capteurs multicomposantes, constituant un jeu usuel de données de sismique de puits, il est nécessaire d'orienter les géophones des capteurs multicomposantes.

\section{État de la technique}

Pour déterminer une direction azimutale dans l'espace à partir des trois composantes, on peut utiliser la technique d'analyse de matrice de covariance décrite dans les documents suivants :

Benhama,A., Cliet, C., and Dubesset, M., 1988, Study and application of spatial directional filtering in three component recordings : geophysical propecting, 36, 591-613,

Cliet, C., and Dubesset, M., 1987 : La paramétrisation des trajectoires de particules, Institut Français du pétrole, Report $N^{\circ} 35080$.

Cette technique fait l'hypothèse que la direction de polarisation ainsi calculée appartient au plan vertical contenant les positions de source et de récepteur. Ceci est réaliste dans un milieu sédimentaire stratifié, même avec des valeurs de pendage faible à moyen (jusqu'à environ $20^{\circ}$ et pour tout pendage, si la source et le capteur de puits sont situés dans le plan de pendage structural local au voisinage puits). Ce processus fait appel au pointé de l'onde directe de pression effectué sur la composante verticale ou axiale au puits, dont la forme de signal est cohérente sur les traces de profondeurs adjacentes. Ce processus devient inapplicable si l'énergie d'arrivée directe de pression est trop faible sur les composantes orthogonales.

Cette méthode d'estimation de l'orientation des capteurs trois composantes par maximisation d'énergie du signal d'onde-P directe dans une fenêtre temps définie par pointé de la composante vertical ou axiale au puits, s'applique bien aux géométries d'acquisition comme le PSV avec offset, qui comprend au moins une source sismique de surface positionnée de façon fixe à une certaine distance offset du puits (typiquement égale à 0.2 à 1.5 fois la profondeur verticale totale du puits). On oriente de même les capteurs trois composantes des configurations de walkaway $2 \mathrm{D}$ ou $3 \mathrm{D}$, et de walkaround, qui consistent à enregistrer sur une antenne de capteurs fixes $3 \mathrm{C}$ dans le puits, le signal émis à partir d'une pluralité de positions de source de surface selon une disposition qui détermine le type 
d'étude sismique de puits. Ainsi un walkaway 2D correspond à une disposition en ligne des points sources, un walkaway 3D correspond à une grille de points sources, plus ou moins régulière, et un walkaround correspond à un cercle de points sources tir autour du puits.

Par exemple, une méthode d'orientation des capteurs orthogonaux à l'aide de la polarisation d'arrivée directe d'onde $\mathrm{P}$ est bien illustrée dans la configuration d'une pluralité de points source placés à des azimuts variés autour du puits dans le document suivant :

P. N. Armstrong, "Method of estimating relative bearing of a borehole receiver", juillet 26,2005 , US pat. $6,922,373$ B2

Dans tous ces cas de figure, la pluralité de positions de source de surface permet d'en trouver plusieurs pour lesquelles le processus par maximisation d'arrivée directe d'onde-P est bien adapté à l'estimation précise et redondante de l'orientation. II n'est pas nécessaire que l'outil de fond soit muni de dispositif d'orientation des composantes.

Avec la redondance de points source, l'adoption des hypothèses de propagation commune dans le plan vertical source-récepteur, et pour autant que la rectilinéarité de l'arrivée directe d'onde $\mathrm{P}$ soit correcte pour une proportion suffisante de points source, il n'est même pas nécessaire de connaître la trajectoire du puits, comme en témoigne le document suivant :

Stewart A. Greenlagh and lan M. Mason, "Orientation of a downhole triaxial geophone", 1995, Geophysics, VOL.60, NO4, p 1234-1237.

Enfin, on connaît également deux méthodes permettant de déterminer l'orientation des deux composantes horizontales lorsque l'on dispose d'une pluralité de positions de sources en surface, par le document :

X. Zeng, G. A., McMechan, « Two methods for determining geophone orientation from PSV data », Geophysics, Vol. 71, №. 4, p. V87-V97, 2006.

Une première méthode, basée sur le plan de polarisation, permet de déterminer l'orientation des composantes horizontales de capteurs montés sur cardans, à partir de l'énergie de polarisation d'une fenêtre temporelle autour de l'arrivée directe $P$, avec une indétermination de $\pi$ sur l'angle trouvé. II est notoire de constater dans ce document que cette indétermination de $\pi$ est maintenue, alors qu'elle pourrait être levée aisément en pointant une phase remarquable du signal d'arrivée $\mathrm{P}$ sur la composante verticale, en particulier un extremum d'amplitude, et en imposant une polarité systématiquement identique sur la composante horizontale sortie du processus de maximisation d'amplitude, comme cela est pratiqué industriellement. Naturellement, cette méthode classique de polarisation, 
nommée PPDI, ne donne des résultats satisfaisants que si l'énergie d'onde $P$ est substantielle dans le plan horizontal, et cette méthode fait usage de la pluralité massive des points source de surface pour améliorer la fiabilité de l'orientation et pour lever l'indétermination de $\pi$.

Dans la seconde méthode, appelée RADI, on calcule l'azimut relatif entre deux stations profondeur adjacentes de géophones, par une méthode de maximisation d'une corrélation entre vecteurs bicomposantes, calculée sur une période ou une fenêtre temporelle autour de l'onde $\mathrm{P}$ directe de forte énergie sur les composantes horizontales. En pratique, la méthode RADI ne donne pas de bons résultats sur les données réelles, c'est pourquoi elle est généralement appliquée postérieurement à la première méthode PPDI pour lever l'indétermination de $\pi$ mentionnée précédemment.

On peut également utiliser les propriétés de polarisation des ondes sismiques directes $P$ pour réorienter les composantes situées dans le plan perpendiculaire à l'axe du puits, dans le cas d'une géométrie particulière : géométrie d'acquisition dans un puits dévié de trajectoire connue, avec une source sismique de surface unique placée en offset de la tête de puits, et avec enregistrement par des capteurs triaxiaux fixes par rapport au corps d'un outil non pourvu d'accessoire de mesure de l'angle de "relative bearing ". On applique alors une procédure de maximisation de l'arrivée $P$ sur les composantes orthogonales. On fait également l'hypothèse que le rayon direct d'onde $\mathrm{P}$ est compris dans le plan vertical contenant la source de surface et le capteur de fond. Naturellement cette réorientation n'est valable que pour la diagraphie en cours, et elle est aisée lorsque l'arrivée directe P est de polarisation sensiblement linéaire, descendante ou montante réfractée, et non interférée. Cette technique bien connue de l'homme de l'art est décrite par exemple dans le document suivant :

M. Becquey et M. Dubesset., " Three component sonde orientation in a deviated well" Geophysics, 1990, vol.55 №10, p. 1386-1388.

Cette méthode d'orientation fournit en général soit deux solutions, soit une solution double, soit aucune solution. Dans le cas échéant, on lève l'indétermination de double solution de rayon d'arrivée directe d'onde-P, en ne conservant que celle qui est la plus proche de la droite définie par la source et le récepteur. S'il n'y a pas de solution, on conserve la solution double comme approximation : $\left(\Phi=\Phi_{0}\right.$ dans l'équation 6 , page $1387 \mathrm{du}$ document précédemment cité). Cette technique de réorientation a été utilisée avec succès sur plusieurs cas réels, comme en témoigne par exemple les illustrations en page 420 du document suivant : 
C. Cliet, L. Brodov, A. Tikhonov, D. Marin and D. Michon, "Anisotropy survey for reservoir definition", Geophys. J. Internat., 1991, 107, 417-427.

Une limitation à tous les processus d'orientation précédemment cités prenant en compte l'arrivée directe d'onde $\mathrm{P}$, survient lorsque l'énergie de la projection de l'arrivée directe sur les deux composantes non axiales, ou non verticales, est très faible. Ceci est par exemple le cas si le puits est vertical ou faiblement dévié, notamment avec la géométrie d'acquisition très courante dite de PSV "zéro-offset", pour laquelle la source de surface située proche de la tête de puits est activée en une position unique, à terre ou en mer, avec une structure géologique ayant des pendages locaux quelconques et souvent inconnus. II est connu de pallier à cette limitation en activant une source sismique supplémentaire à la source zéro-offset, localisée à une distance suffisante du puits, et de préférence dans la direction azimutale générale de la structure géologique dans le voisinage du puits. Mais cette solution alternative est rarement utilisée car elle entraîne des dépenses supplémentaires pour l'opération de mesure ainsi qu'un allongement de la durée d'acquisition, et donc de l'immobilisation de l'appareil de forage sur le site. L'activation de cette source additionnelle est effectuée successivement ou simultanément à la source zéro offset, avec la même position profondeur d'ancrage et de mesure que l'outil PSV, dont on désire orienter les trois composantes. De plus, ces palliatifs aux méthodes ou configurations géométriques de tir antérieurs, présentent l'inconvénient de ne pas être toujours applicables, soit à cause de reliefs accidentés par exemple, soit à cause d'indisponibilité matérielle ou financière de sources sismiques supplémentaires, soit à cause d'interférence de l'arrivée directe d'onde-P par une arrivée réfractée ou diffractée secondaire. Lorsqu'il est absolument nécessaire de connaître l'orientation des capteurs triaxiaux, il est judicieux d'envisager la mise en oeuvre

25 d'une source supplémentaire en offset du puits, et de confronter cette solution avec le choix alternatif d'un outil de puits combinable avec un accessoire d'orientation. Mais en pratique, ces deux modes de mesure de PSV sont rarement mis en oeuvre.

Par ailleurs, après orientation des composantes triaxiales, certains programmes de traitement de PSV multi composantes se limitent aux deux composantes contenues dans le plan vertical contenant le capteur et la source, comme par exemple la méthode décrite dans le document suivant :

C. Esmersoy, "Velocity estimation from offset VSPs using Direct P and converted SVwaves", POS6.4, SEG abstracts 1987, p538-541.

Cependant, comme expliqué précédemment, la limitation classique du traitement à une seule ou à deux des composantes conduit à des indéterminations potentielles dans 
l'identification du mode d'onde de certaines arrivées reçues par le capteur, pression ou cisaillement, d'une part, et dans le positionnement d'événements géologiques d'autre part, qu'il n'est pas possible de lever.

Ainsi, afin de traiter de façon complète, efficace et bénéfique tous les signaux issus de capteurs multicomposantes, constituant un jeu usuel de données de sismique de puits, il est nécessaire d'orienter les géophones des capteurs multi composantes.

L'objet de l'invention est une méthode alternative d'orientation spatiale des géophones d'un capteur multi-composantes permettant de s'affranchir des difficultés d'orientation des techniques antérieures. La méthode permet d'orienter les géophones dans un repère géographique connu, afin de permettre le traitement isotrope des trois composantes, pour le bénéfice de l'interprétation structurale et géologique.

\section{La méthode selon l'invention}

Ainsi, l'invention concerne une méthode de prétraitement de données sismiques acquises au moyen d'une méthode de prospection sismique de type profil sismique vertical. Cette méthode de prospection sismique de type profil sismique vertical comprend une émission d'ondes sismiques et une réception de ces ondes sismiques au moyen d'au moins un capteur multi - composantes positionné au sein d'un puits et que l'on fait stationner à au moins deux profondeurs. Ce capteur comprend au moins trois géophones orthogonaux enregistrant en fonction du temps une première composante sismique dans une direction vectorielle connue, et au moins deux autres composantes sismiques dans deux directions orthogonales à cette direction vectorielle connue. La méthode comprend les étapes suivantes :

25 a- on construit un nouveau signal en calculant la racine carrée de la somme des carrés desdites deux composantes sismiques orthogonales à ladite direction vectorielle connue, appelé signal module, et l'on pointe des temps d'arrivée d'une onde de cisaillement descendante sur un extremum d'amplitude dudit signal module ;

b- on oriente lesdites composantes sismiques dans un repère unique quelque soit la profondeur dudit capteur, au moyen des étapes suivantes répétées pour chaque profondeur :

- on définit une fenêtre temporelle de part et d'autre desdits temps d'arrivée ;

- on détermine une direction azimutale par maximisation d'une énergie desdites composantes sismiques orthogonales à ladite direction vectorielle connue au sein de ladite fenêtre temporelle ; 
- on oriente à 360 degrés près lesdites composantes sismiques orthogonales à ladite direction vectorielle connue dans un repère unique défini par rapport à la direction azimutale qui est identique pour chaque profondeur.

c- on détermine l'orientation géographique dudit repère unique en déterminant une direction azimutale de pendage d'au moins un réflecteur sismique ou une direction principale d'anisotropie de biréfringence d'onde de cisaillement, par traitement des trois composantes dans le repère unique, et en appliquant au repère unique une rotation jusqu'à ce que la direction ainsi déterminée coïncide avec une direction azimutale connue à partir de connaissances structurales et géologiques.

Selon un premier mode de réalisation, on peut réaliser l'étape c) en déterminant l'orientation géographique du repère unique au moyen des étapes suivantes :

- on extrait un champ d'ondes réfléchies au moyen d'un traitement isotrope des trois composantes dans le repère unique ;

- on détermine dans le repère unique un azimut de pendage d'au moins un réflecteur sismique au moyen d'une analyse de polarisation dudit champ d'ondes réfléchies ; et

- on détermine l'orientation géographique dudit repère unique en alignant ledit azimut de pendage sur une direction de pendage dudit réflecteur connue par ailleurs, à une même profondeur au droit du puits.

Selon un second mode de réalisation, on peut réaliser l'étape c) en déterminant l'orientation géographique du repère unique au moyen des étapes suivantes :

- on détecte, pour au moins un intervalle de profondeur, une anisotropie de biréfringence de modes rapide et lent d'onde de cisaillement par une analyse de signaux de l'onde de cisaillement dans le repère unique ;

- on détermine une direction principale de l'anisotropie de biréfringence ainsi détectée dans le repère unique déterminé à l'étape $b$;

- on détermine l'orientation géographique dudit repère unique en alignant la direction principale de mode lent d'anisotropie de biréfringence d'onde de cisaillement sur une direction de pendage stratigraphique déterminée par des connaissances structurales et géologiques.

Selon un troisième mode de réalisation, on peut réaliser l'étape $\mathrm{c}$ ) en déterminant l'orientation géographique du repère unique au moyen des étapes suivantes : 
- on détecte, pour au moins un intervalle de profondeur, une anisotropie de biréfringence de modes rapide et lent d'onde de cisaillement par une analyse de signaux de l'onde de cisaillement dans le repère unique ;

- on détermine une direction principale de l'anisotropie de biréfringence ainsi détectée dans le repère unique déterminé à l'étape $b$;

- on détermine l'orientation géographique dudit repère unique en alignant la direction principale de mode lent d'anisotropie de biréfringence d'onde de cisaillement sur une direction de contrainte horizontale maximale déterminée par des connaissances structurales et géologiques.

De façon préférentielle, préalablement à l'étape a), on préserve l'isotropie du signal en trois composantes, en respectant des rapports d'amplitude et des différences de phase entre les composantes sismiques.

Selon l'invention, on peut améliorer le rapport signal sur bruit des trois composantes du signal brut, préalablement au calcul du module, au moyen d'une déconvolution isotrope des trois composantes par un signal unique d'onde de pression descendante extrait de la composante de direction vectorielle connue. On peut également filtrer le signal module de façon à supprimer des composantes basses fréquences, avant de pointer les temps d'arrivée de l'onde sismique directe.

Selon au autre mode de réalisation de l'invention, on détermine l'orientation géographique du repère unique pour au moins deux profondeurs, et on effectue une rotation graduelle du repère unique entre ces profondeurs, pour compenser une différence d'orientation géographique entre ces profondeurs. On peut effectuer la rotation graduelle en appliquant un gradient d'angle de rotation linéaire avec la profondeur et limité à environ quinze degrés pour cent mètres de profondeur. On peut alors déterminer l'orientation géographique du repère unique sur au moins une profondeur, en montant le capteur multicomposantes sur un système de double cardans, le système permettant d'orienter par gravité les composantes sismiques lorsque l'inclinaison du puits atteint une valeur d'au moins environ 10 degrés. Alternativement, on peut descendre dans le puits un outil de mesure de puits comportant le capteur multi-composantes monté de façon fixe, et déterminer l'orientation géographique du repère unique sur au moins une profondeur au moyen d'un système de mesure d'un angle de "relative bearing" monté sur l'outil de mesure, qui permet de retrouver l'orientation du capteur multi-composantes lorsque l'inclinaison du puits atteint une valeur d'au moins environ 10 degrés. 
D'autres caractéristiques et avantages de la méthode selon l'invention, apparaîtront à la lecture de la description ci-après d'exemples non limitatifs de réalisations, en se référant aux figures annexées et décrites ci-après.

- La figure 1 schématise de façon générale la géométrie d'acquisition d'un PSV avec source de surface et sonde comportant un seul niveau de réception sismique, descendue dans un puits sensiblement vertical au moyen d'un câble.

- La figure 2 illustre l'étroitesse de l'angle solide de propagation en arrivée directe, qui

- La figure 5 illustre l'attitude spatiale des trièdres des capteurs sismiques dans les parties verticales et déviées d'un puits avant orientation (a): montage fixe, c): montage sur doubles cardans), et la configuration des trièdres après orientation (b).

- Les figures $6 a, 6 b, 6 c$ et $6 d$ illustrent le principe de propagation des modes propres d'onde sismique volumique de cisaillement (ondes S) dans un milieu sensiblement homogène, et la méthode d'orientation des deux composantes orthogonales non orientées dans un repère intermédiaire unique selon l'invention.

- La figure 7 est un organigramme de l'ensemble du processus d'orientation selon l'invention.

- La figure 8a illustre le mouvement de particule de l'onde descendante $S$ dans la fenêtre la flèche superposée à l'hodogramme indique l'azimut de maximisation. La figure 8b illustre le mouvement de particule de l'onde descendante $S$ dans le repère cohérent intermédiaire. 
- Les figures 9a, 9b, 9c représentent les rejeux bruts isotropes des composantes respectives $Z, X, Y$ de l'outil, versus profondeur croissante de gauche à droite, et la figure $9 \mathrm{~d}$ montre le signal module $M 2$ filtré calculé à partir de $X$ et $Y$ uniquement.

- Les figures 10a, 10b, 10c représentent les rejeux bruts isotropes des composantes géographiques respectives ZV-up verticale, $\mathrm{H}$-Nord et $\mathrm{H}$-East, versus profondeur croissante de gauche à droite, et la figure $10 \mathrm{~d}$ montre le signal module $\mathrm{M} 2$ filtré calculé à partir de $\mathrm{H}$ Nord et H-East. uniquement.

- Les figures 11a,b,c montrent des rejeux de signaux d'onde $S$ descendante en fonction de la profondeur mesurée croissante (MD) de gauche à droite, alignés sur un pointé du temps effectué sur une phase remarquable du signal module filtré, respectivement:

figure11a: le signal du module M2 filtré utilisé pour le pointé précis, puis aligné au temps 0.10 s pour toute profondeur;

figure11b: le signal de l'onde-S rapide, résultant du calcul de maximisation d'énergie, figure11c: le signal de l'onde-S lente, orthogonale à la composante d'onde-S rapide.

- La figure 12 illustre les polarisations linéaires d'une onde $S$ descendante incidente qui se dédouble suite à la propagation verticale dans un milieu stratifié incliné et biréfringent.

- La figure 13 illustre la direction azimutale vers la direction d'aval pendage, naturellement prise par une réflexion de type P-P issue d'une onde incidente verticale réfléchie sur un interface incliné.

20 - Les figure 14a, 14b, 14c illustrent les trois composantes du champ d'onde réfléchi déconvolué et isotrope issu des composantes brutes orientées respectives des figures 10a, 10b, 10c d'un PSV. On distingue clairement une forte réflexion P-P et P-S sur un réflecteur incliné recoupant le puits vers $2100 \mathrm{~m}$ de profondeur.

- La figure 15 montre une estimation de l'incertitude du processus d'orientation selon l'invention, effectuée à partir d'un PSV réel enregistré dans un puits vertical avec une source à très faible déport, au moyen de deux vibrateurs orthogonaux placés en surface et activés successivement à chaque position de capteur de fond: les deux courbes du haut représentent en abscisse la profondeur croissante de droite à gauche, et en ordonnée, l'angle de rotation obtenu indépendamment pour chacune des deux sources; la courbe du bas représente la différence des deux angles, illustrant l'incertitude d'orientation. 


\section{Description détaillée de la méthode}

L'invention concerne une méthode de prétraitement spécifique de données sismiques acquises lors de la mise en oeuvre d'une opération de prospection sismique de type profil sismique vertical. Selon un mode de réalisation cette opération comprend une seule position de surface pour l'émission d'ondes sismiques, située à proximité de l'appareil de forage, et une réception des ondes sismiques au moyen d'un capteur multi - composantes positionné au sein d'un puits vertical à faiblement dévié. Le capteur (monté dans la sonde sismique) comporte trois géophones orthogonaux. Et on fait stationner ce capteur à au moins deux profondeurs voisines de quelques mètres. Le signal enregistré selon la direction axiale de chaque capteur unitaire est appelé composante du signal. La sonde réceptrice enregistre en fonction du temps au moins une composante dans une direction vectorielle connue, verticale ou axiale au puits de mesure, et deux composantes sismiques dites "orthogonales", c'est à dire orthogonales à la composante de direction vectorielle connue.

Les figures $6 a$ et $6 b$ illustrent le principe physique de base de la propagation d'une onde sismique de cisaillement, dite $S$ (shear). II existe deux types d'ondes de volume : les ondes de pression (ondes $\mathrm{P}$ ), dont la polarisation, ou direction de déplacement de la formation au passage de l'onde, est parallèle à la direction de propagation, non illustrées sur la figure $6 \mathrm{a}$, et les ondes transversales (ondes $\mathrm{S}$ ) polarisées sensiblement perpendiculairement à la direction de propagation, illustrées sur la figure $6 a$.

La figure 6a représente un schéma d'un plan vertical de propagation 600 : la surface 601 symbolise non seulement la surface du sol mais également les premières couches de terrain hétérogènes jusqu'à environ 200 mètres d'épaisseur, ou même la surface rugueuse du fond de la mer, qui sont le siège de conversions de modes d'onde $\mathrm{P}$ en mode $\mathrm{S}$. Tous les modes d'ondes se propageant en profondeur suivant l'axe vertical 602. Ainsi, à partir d'une faible profondeur de terrain, l'onde $S$ se propage verticalement dans un milieu sensiblement homogène à l'échelle des longueurs d'onde sismiques utilisées. Le milieu est isotrope ou avec anisotropie faible pour la direction de propagation verticale (entraînant une biréfringence ordinaire), et il comporte une stratification éventuelle de couches de caractéristiques variables, avec un pendage éventuel identique pour toutes les couches ou non. Le milieu est ainsi représentatif de formations usuelles sédimentaires ou de socle. Le schéma de la figure $6 a$ illustre un milieu biréfringent dans lequel un train d'onde de cisaillement complexe se propage verticalement en profondeur selon l'axe 602 et comprend : - une onde de cisaillement rapide S-f ayant une forme de vibration 603 quelconque polarisée linéairement selon la direction constante 611 orthogonale à la direction de propagation 602 . ; 
- une onde de cisaillement lente S-s ayant une forme de vibration 604 en général différente de 603 et polarisée linéairement selon la direction constante $\mathbf{6 1 2}$ orthogonale à la fois aux axes $\mathbf{6 0 2}$ de propagation et $\mathbf{6 1 1}$ de polarisation d'onde S rapide S-f.

Les vecteurs de polarisation $\mathbf{6 1 1}$ et $\mathbf{6 1 2}$ symbolisent les directions de vibration de chacun des modes propres d'onde $S$ propagées selon la verticale, mais aussi l'amplitude du signal. L'amplitude du signal de vibration caractérise indifféremment le déplacement, la vitesse ou l'accélération du mouvement sismique de chacun des modes propres d'onde S-f et S-s, dont la direction et la forme d'onde demeurent constantes tout au long de la propagation, conformément à la physique du phénomène de propagation. La figure 6a montre, pour des instants successifs de propagation ti 610 ( $\mathrm{i}=0,1,2, \mathrm{i})$ mesurés à partir de l'instant d'activation de la source sismique de surface, la profondeur $z_{f}$ i 613 atteinte par l'onde rapide S-f, et la profondeur $z_{s}$ i 614 atteinte par l'onde lente $S-s$, le pied des vecteurs de polarisation respectifs 611 et 612 indiquant la profondeur exacte atteinte par chacune des deux ondes $S$ au temps ti $\mathbf{6 1 0}$. De fait, la profondeur $z_{f} i \mathbf{6 1 3}$ de l'onde rapide $S-f$ est progressivement et faiblement supérieure de quelques pourcents à la profondeur $z_{s}$ i 614 au cours de la propagation.

La figure $6 \mathrm{~b}$ représente dans le plan horizontal 650 , orthogonal à la direction de propagation verticale, des diagrammes de projection des formes du mouvement de vibration théoriques du train d'onde $S$. Ils représentent chacun une succession continue des points $[X(t), Y(t)]$ des signaux sismiques, dans une fenêtre temps centrée, dans le cas présent, autour du temps ti de l'arrivée d'onde $S$ à illustrer : ces diagrammes sont également connus sous les dénominations de "mouvement de particules" si les signaux $X$ et $Y$ représentent un déplacement au passage de l'onde sismique, ou même "hodogrammes" si les signaux $X$ et $Y$ représentent une vitesse de déplacement, mesurée par exemple par un capteur de type géophone. Ils sont encore appelés "diagramme de polarisation" ou simplement "polarisation" si les signaux $X$ et $Y$ représentent indifféremment un déplacement, une vitesse ou une accélération.

La figure $6 \mathrm{~b}$ montre sur la colonne de droite 653 au dessous de l'indication "IN", et pour des profondeurs verticales fixes successives zi $(i=0,1,2, i) \mathbf{6 6 0}$, atteintes par le train d'onde $30 S$ dans une fenêtre temps autour des instants de propagation ti 610 ( $i=0,1,2, i)$ définis sur la figure $6 a$, les diagrammes de polarisations théoriques 651 d'orientation totalement aléatoire dans le plan horizontal pour chaque station profondeur zi, que l'on observe dans le repère d'une sonde sismique de puits utilisée pour l'enregistrement des PSV et qui s'ancre à la paroi de puits selon un azimut aléatoire. L'aléa de rotation du câble de diagraphie au bout duquel la sonde de puits est fixée est bien connu de l'homme du métier, cet aléa étant transmis aux capteurs sismiques à montage fixe dans une sonde. Par ailleurs, si ladite sonde réceptrice 
comporte des cardans de type tourelle sur lesquels sont montés les capteurs sismiques, la rotation des cardans autour de l'axe de la sonde est libre lorsque la sonde est dans un puits vertical, ce qui entraîne également une orientation aléatoire des capteurs horizontaux, même si l'on connaissait l'orientation azimutale de la sonde.

La figure $6 \mathrm{~b}$ indique symboliquement le processus P1 (666) suivant l'invention, via une flèche entre chaque diagramme de polarisation 651 des données entrées en colonne de droite "IN" 653, et le diagramme de polarisation orienté correspondant en colonne de gauche "OUT" 663; ce processus consiste à déterminer la direction d'amplitude maximale 652 sur le diagramme 651, de façon indépendante pour chaque profondeur de mesure sismique de puits, par une technique connue de régression linéaire du groupe des points $X(t), Y(t)$ dans une fenêtre -temps limitée appropriée, si possible plus courte que la fenêtre-temps correspondant au diagrammes 651 ou 661, puis à appliquer une rotation d'axe vertical aux signaux sismiques horizontaux de façon à faire coïncider la direction d'amplitude maximale 651 à une direction azimutale constante arbitraire 662 sur le diagramme 661, dans un premier temps. Ce processus est bien adapté au cas des PSV dits à zéro offset en puits vertical, où la source d'onde $P$ usuellement employée génère involontairement et très souvent en pratique des ondes de cisaillement énergiques au passage de la zone altérée et hétérogène de surface ou de la surface rugueuse du fond de la mer.

La figure $6 \mathrm{~b}$ montre sur la colonne de gauche 663 au dessous de l'indication "OUT", et pour des profondeurs verticales fixes successives zi $(i=0,1,2, i) 660$, atteintes par le train d'onde $S$ dans une fenêtre temps autour des instants de propagation ti 610 ( $i=0,1,2, i)$ définis sur la figure $6 \mathrm{a}$, les diagrammes de polarisations théoriques 661 que l'on s'attend à observer dans un repère fixe du plan horizontal, par exemple un repère géographique (Nord, Est). On prend par exemple $z i=\left(z_{f} i+z_{s} i\right) / 2$, en référence à la figure 6a. On remarque qu'en cas d'isotropie du milieu de propagation pour la direction verticale, $z i=z_{f} i=z_{s}$ i représente la profondeur atteinte par deux ondes $S$ de polarisation orthogonale quelconque. Les diagrammes de polarisation 661 de la figure $6 \mathrm{~b}$ représentent les mouvements de particules du train d'onde $S$ illustré par la figure $6 a$, pour la même succession d'instants de propagation ti : on peut noter que les diagrammes successifs sur la colonne de gauche $\mathbf{6 6 3}$ sont de forme très similaires, avec une direction maximale d'amplitude 662 identique. Les différences de rotondité observées traduisent typiquement l'anisotropie azimutale de biréfringence, résultant du faible écart de vitesse entre onde S rapide S-f et onde S lente S-s. Compte tenu de la longueur temps des ondelettes sismiques 603 et 604 (figure 6a) associées aux deux modes propres S-f et S-s émis quasi simultanément, la forme des diagrammes de la figure $6 \mathrm{~b}$ est elliptique et témoigne de l'interférence des deux modes propres d'onde $S$ qui ne peuvent hélas pas être distingués visuellement en pratique dans le cas général sur ce genre de 
diagramme. On a constaté par expérience qu'une arrivée sismique énergétique individuelle en onde $\mathrm{P}$ de polarisation linéaire ou en onde $\mathrm{S}$ de polarisation elliptique quelconque, ondes $\mathrm{P}$ ou $\mathrm{S}$ dont la forme de polarisation reste sensiblement constante au cours de la propagation, montre une cohérence du temps des extrema d'amplitudes d'une profondeur à la suivante, et en particulier, que la forme du signal module reste sensiblement constante pour une onde descendante de forte énergie. On peut trouver facilement des publications montrant des hodogrammes de trains d'onde $S$ directe émis par une source $S$ placée à faible distance du puits, et réorientés dans un repère fixe géographique à l'aide de source d'onde $P$ placée en offset et activée dans la même passe de mesure, et indiquant que la forme d'hodogramme est sensiblement stable tout au long de la propagation le long du puits vertical et dans un milieu à stratigraphie horizontale comportant une anisotropie azimutale notable en onde $\mathrm{S}$, par exemple dans les documents suivants :

Charles Naville, " Detection of Anisotropy Using Shear-Wave Spitting in VSP surveys; Requirements and Alpplications", SEG Expanded Abstracts, 56 ${ }^{\text {th }}$ int. SEG meeting, 1986, Houston, S5.2, pp. 391-394.

Ian Bush and Stuart Crampin, "Paris Basin VSPs: case history establishing combinations of fine layer (or lithologic) anisotropy and crack anisotropy from modelling shear wavefields near point singulatities" Geophys. Journal Int., 1991, №107, pp. 433-437.

Nicoletis, L., Cliet, C. \& Lefeuvre, F., " Shear-wave Splitting measurements from multishot VSP data, Expanded Abstracts, $58^{\text {th }}$ int. SEG meeting, 1988, Anaheim, POS 6.1, pp.527-530;

Dans les trois documents précités, l'hypothèse de la fixité de polarisation des modes propres (figure $6 \mathrm{a}$ ) pour une direction de propagation donnée est admise de façon explicite ou implicite, et l'on peut vérifier visuellement que pour une propagation verticale à faiblement déviée de la verticale, la forme du diagramme de polarisation demeure similaire avec la propagation, ainsi que la direction azimutale d'amplitude maximale. Ainsi, même en présence d'anisotropie de biréfringence sans atténuation différentielle d'amplitude drastique entre les deux modes propres d'onde $S$, on peut aisément vérifier mathématiquement que la direction d'amplitude maximale d'un signal à deux composantes d'un train d'onde $S$ direct non interféré, demeure sensiblement fixe le long d'une direction de propagation donnée. Ceci est schématisé sur la colonne de gauche de la figure $6 \mathrm{~b}$. Ainsi, en déterminant la direction azimutale pour chaque profondeur à laquelle on dispose d'un enregistrement des composantes horizontales, on définit un repère unique. Cette méthode est d'autant plus précise que (1) le couplage mécanique de la sonde réceptrice mono niveau ou de chacune des sondes réceptrices d'un outil de puits multi niveaux assure une bonne fidélité vectorielle 
du signal mesuré, et que (2) la source de surface reste en position fixe et émette toujours la même forme de signal pendant toute l'opération de PSV.

La méthode comporte une orientation des composantes sismiques dans un repère géographique connu quelque soit la profondeur de la sonde. La figure 7 est un organigramme $\mathbf{7 0 0}$ d'un mode de réalisation de la méthode d'orientation selon l'invention. II s'agit d'orienter les deux composantes orthogonales à une composante verticale, ou à une composante axiale au puits. La méthode repose sur l'analyse des ondes $\mathbf{S}$ descendantes pour définir une fenêtre temporelle nécessaire à la détermination d'un angle de rotation. En effet, les ondes $P$ enregistrées ayant habituellement une énergie trop faible sur les composantes horizontales des PSV, elles ne permettent pas de réorienter ces composantes. Ceci est notamment le cas pour les PSV sans déport, effectués avec des sources d'onde de pression dans des puits dont la trajectoire est proche de la verticale. En revanche, l'énergie des ondes $\mathrm{S}$ directes ou d'ondes converties de $\mathrm{P}$ en $\mathrm{S}$ au cours de la propagation descendante est très souvent suffisante pour appliquer la méthode.

Ainsi, selon un mode de réalisation, la méthode comporte les étapes suivantes pour chaque station profondeur de mesure :

- dans le bloc 701, on déconvolue de façon isotrope les deux composantes orthogonales à orienter, c'est à dire que l'on applique un même opérateur, au même temps sur les deux composantes. Cette opération n'est envisagée que si le résultat des opérations 702 à 704 n'est pas satisfaisant.

- dans le bloc 702, on calcule le module, noté $M(t)$, des deux composantes brutes à orienter, ou une puissance exponentielle $(n)$ de ce dernier ;

- dans le bloc 703, on filtre le module $M(t)$, de façon à supprimer ses composantes basses fréquences et éventuellement des hautes fréquences ne contenant que du bruit, de façon à faciliter l'opération suivante.

- dans le bloc 704, on pointe le temps Tp d'une phase remarquable du signal du module filtré obtenu précédent. Par exemple on pointe un pic ou un creux d'amplitude, éventuellement affiné par application d'un algorithme industriel de pointé par corrélation ou par semblance.

- Les opérations 702 à 704 peuvent être automatiques, exécutables en cascade et en une seule passe, mais peuvent conduire à un pointé peu fiable ou peu précis. Dans un tel cas, on peut soit modifier le filtre du bloc $\mathbf{7 0 3}$, soit envisager d'appliquer au préalable des opérations 702 à 704 les opérations décrites au bloc 701, destinées à augmenter le 
rapport signal sur bruit de l'onde $S$ que l'on désire pointer, toutes les arrivées interférant avec l'arrivée $S$ désirée étant considérées comme du bruit.

- dans le bloc 705, on définit ensuite une fenêtre temporelle autour du temps pointé d'arrivée $S$, d'une longueur constante pour tous les niveaux profondeur de mesure et au moins égale à une demi période du signal module filtré, voire d'une longueur plus grande en cas d'onde $S$ interférée ou de faible rapport signal sur bruit

- dans le bloc 705, on détermine alors la direction azimutale, par maximisation de l'énergie des composantes d'entrée $X(t), Y(t)$, brutes ou filtrées pour ne retenir que les fréquences de rapport signal sur bruit le plus fort, selon un procédé industriel connu. On effectue ensuite une rotation des composantes d'entrée $X(t), Y(t)$ brutes dans le plan orthogonal à la composante brute $Z$, dans un repère unique intermédiaire défini par la direction azimutale calculée précédemment. On s'assure que l'amplitude du signal de sortie dans la direction de maximisation demeure de signe identique, par exemple positif, au temps pointé Tp issu de l'opération 704, pour tous les niveaux de mesure du PSV.

- dans le bloc 707, on calibre les composantes dans un repère intermédiaire unique, défini par rapport à un repère géographique, ou à un repère lié à la trajectoire connue du puits si l'on dispose d'information complémentaire pour ce faire.

- dans le bloc 708, on applique les rotations nécessaires au repère intermédiaire unique pour restituer les trois composantes du PSV dans un repère géographique lié au globe terrestre, selon une procédure connue.

Chacune des étapes précédentes est décrite ci-après :

A. Pointé

- $\quad$ bloc 701 : déconvolution préalable éventuelle :

dans certains cas, le signal du train d'arrivée directe d'onde $S$ est apparent sur un grand intervalle de temps d'enregistrement, il est potentiellement interféré par d'autres ondes d'énergie relative plus faible, mais non négligeable, ce qui a pour effet de rendre le pointé du module filtré plus imprécis. II peut être utile dans de tels cas d'appliquer une opération de déconvolution isotrope multicanale, identique pour les deux composantes "horizontales » (composantes orthogonales à la direction sensiblement verticale) de chacun des niveaux profondeur de mesure, et identique sur plusieurs niveaux profondeurs. Ceci permet de réduire la longueur du train d'onde $S$ sur lequel on désire pointer le temps d'arrivée: la déconvolution peut être effectuée par extraction du signal d'onde $\mathrm{P}$ sur la composante 
verticale, afin de déconvoluer une onde convertie P-S sur les deux composantes horizontales, par exemple selon la méthode décrite dans les brevets de Nigel Anstey GB 1,569 581 du 27-09-1977 ou CA 1,106,957 du 9-12-1977 intitulés "Seismic delineation of oil and gas reservoirs using borehole geophones". La déconvolution peut également être effectuée simplement avec les algorithmes industriels isotropes et multicanaux existants pour la sismique de surface ou pour la sismique de puits, de type Wiener ou également d'équilibrage du spectre de fréquences, tous deux basés sur le spectre d'amplitude de la somme des signaux d'autocorrélation de chacune des composantes horizontales orthogonales à déconvoluer, compte tenu de l'invariance de cette somme de signaux d'autocorrélation vis à vis de l'orientation des deux composantes horizontales d'entrée.

- bloc 702: Calcul du signal module $M(t)$ d'un signal à deux composantes, et invariance.

Après s'être assuré que les opérations de prétraitement de base des enregistrements unitaires du PSV, telles que l'édition, la sommation verticale, la normalisation d'amplitude de source éventuelle préalable à la sommation..., ont été effectuées de façon isotrope, le signal module, noté $M(t)$, qui représente l'une des coordonnées polaires déduite des deux signaux bruts en coordonnées cartésiennes $X(t)$ et $Y(t, d)$ est calculé comme suit :

$$
M^{2}(t)=X^{2}(t)+Y^{2}(t), \text { pour tout temps } t
$$

Si l'outil de PSV, dont le couplage à la paroi est mécaniquement isotrope, est ancré à une profondeur donnée avec une direction azimutale différente d'un angle az inconnu autour de l'axe du puits vertical, l'outil enregistre les composantes horizontales $X 1(t)$ et $Y 1(t)$ qui s'expriment comme suit en fonction de $X(t, d)$ et $Y(t, d)$ :

$$
\begin{aligned}
& X 1(t)=X(t) \cdot \cos (a z)+Y(t) \cdot \sin (a z) \\
& Y 1(t)=-X(t) \cdot \sin (a z)+Y(t) \cdot \cos (a z)
\end{aligned}
$$

Il est aisé de constater que, pour toute valeur de l'angle az:

$$
X 1^{2}(t)+Y 1^{2}(t)=X^{2}(t)+Y^{2}(t)=M^{2}(t)
$$

Le module demeure identique quelle que soit l'orientation des capteurs associés aux composantes $X(t)$ et $Y(t)$, il est donc invariant eu égard à la rotation, à valeur toujours positive. Le module d'un signal à deux composantes est appelé également "M2" dans la suite de la description. Le module d'un signal à trois composantes, dit "M3", défini par :

$$
M^{2}(t)=X^{2}(t)+Y^{2}(t)+Z^{2}(t) \text {, est aussi un invariant eu égard à toute rotation spatiale. }
$$


II est intéressant de calculer le module M3 lorsque l'on cherche à pointer une onde de pression $(P)$ directe, dont la direction d'arrivée est orthogonale au puits dans certaines configurations de la géométrie d'acquisition. Par exemple, sur certains intervalles du puits de mesure proche de l'horizontale, lorsque la source est située proche de l'aplomb du capteur sismique $3 \mathrm{C}$, comme illustré sur la figure $2 \mathrm{~b}$, ou encore dans les configurations d'acquisition de type Offset-VSP et walkaway, sur certains intervalles du puits proches de la verticale, lorsque la source sismique de surface est suffisamment éloignée du puits, de sorte que le rayon sismique arrive au puits avec une incidence horizontale, comme illustré sur la figure 2c. Comme cette procédure de pointé de l'arrivée $P$ est valide quelle que soit l'incidence du rayon, on automatise le pointé de l'onde $P$ directe en pointant le module $M 3$, en particulier pour les études de type walkaway $2 \mathrm{D}$, et walkaway $3 \mathrm{D}$, cette dernière configuration également connue sous le nom de 3D-VSP.

On peut aussi choisir de travailler sur une puissance exponentielle du signal $M(t)$, afin d'amplifier les variations d'amplitude de ce signal.

L'avantage de la propriété d'invariance du module est de permettre des opérations de pointé précis du temps d'une phase remarquable, lié à un instant particulier du train d'arrivée d'onde $S$, par exemple un extremum local bien individualisé, sans connaître l'orientation préalable des signaux constitutifs dudit module.

- bloc 703 : Filtrage du module $M(t)$

Selon un mode préférentiel de réalisation, la méthode comporte un filtrage du module $M(t)$, de façon à supprimer sa composante basse fréquence et à rendre ce signal plus lisible. On peut par exemple utiliser typiquement un filtre passe bande $5-60 \mathrm{~Hz}$ pour les $\mathrm{PSV}$.

- $\quad$ bloc 704 : Pointé temps d'un extremum particulier du signal du module filtré.

Le pointé du temps Tp d'un extremum d'amplitude du module, de préférence filtré est décrit en relation avec les figures $6 c$ et $6 \mathrm{~d}$.

La figure 6c montre une projection horizontale $\mathbf{6 7 0}$ d'un diagramme de polarisation 671 d'un train d'arrivée directe $S$ choisi dans une large fenêtre temps appropriée.

Le signal module $M(t) 672$ est représenté graphiquement comme l'une des coordonnées polaires dérivée des deux signaux enregistrés orthogonaux bruts $X(t)$ et $Y(t)$ en coordonnées cartésiennes et d'orientation arbitraire.

Par définition, quel que soit le temps $t: M^{2}(t)=X^{2}(t)+Y^{2}(t)$.

Le signal $M(t) 672$ possède la caractéristique mathématique d'être invariant vis à vis du repère cartésien des signaux bruts $X(t)$ et $Y(t)$ mesurés, et dans lequel le module est calculé. 
De même, la forme du diagramme de polarisation 671 dans la fenêtre temps considérée est indépendante du repère, à une rotation près. Les vecteurs signaux bruts $X(t)$ et $Y(t)$, ainsi que le vecteur module $M(t)$, ont la même origine 673 (zéro des amplitudes), l'amplitude du vecteur $M(t)$ étant toujours positive ou nulle.

Compte tenu que le diagramme de polarisation de l'onde $S$ directe considérée est également quasi constant au cours de la propagation sismique en profondeur, le procédé selon l'invention permet de déterminer avec précision le pointé temps d'une phase remarquable du signal d'onde $S$ non orienté, de façon indépendante du repère cartésien des signaux bruts $\mathrm{X}(\mathrm{t})$ et $\mathrm{Y}(\mathrm{t})$, par exemple le temps de l'un des maxima locaux 674 du signal module $M(t) 672$.

La figure $6 \mathrm{~d}$ montre un exemple schématique, en fonction du temps $\mathrm{t}$, du signal module d'un train d'onde $S$ dont l'énergie est supérieure à celle de toutes les autres ondes reçues au même temps par le capteur sismique. On constate, par expérience, que ce signal module reste sensiblement identique en fonction de la profondeur d'enregistrement, avec un décalage temps correspondant à la propagation des ondes S. En pratique, afin d'amplifier la reconnaissance des extrema locaux du signal module, on élimine les composantes basses fréquences par un filtre coupe bas, dont le résultat est le signal filtré Mf 693, qui représente également la différence entre le signal brut 691 et le signal lissé associé 692 . On peut aussi élever les amplitudes du signal filtré Mf à une puissance exponentielle, afin d'en faciliter encore le pointé par méthode visuelle, ou par calcul de semblance ou de corrélation entre stations profondeur de mesure PSV. Le temps Tp du pic d'amplitude 695 est en pratique plus facile à pointer, sans ambiguïté, sur le signal filtré Mf 693 que le pic 694 sur le signal brut M 691, tant pour l'oeil que pour la plupart des algorithmes informatiques industriels de pointé du temps..

Le pointé précis d'une phase remarquable d'un signal d'onde de cisaillement à deux composantes orthogonales dans le plan de polarisation sans orientation préalable, conduit à des applications immédiates: en effet, ce genre de pointé permet d'accéder à la connaissance d'un temps d'arrivée d'onde $S$ à une constante près identique pour toutes les stations profondeur de mesure du PSV, et par conséquent à la connaissance des vitesses d'intervalle en mode $S$. En combinant le temps $S$ avec la mesure du temps en onde $P$ généralement effectuée sur la composante verticale ou axiale au puits, on accède par exemple au rapport des vitesses d'intervalle $\mathrm{Vs} / \mathrm{Vp}$ et au coefficient de Poisson. On accède également au module d'Young si la densité de formation est connue par ailleurs. Cette méthode permet également de pointer une onde $S$ sur des données en provenance d'outils de puits de mesure de diagraphie ultrasonique d'onde-S de type dipolaire ou quadrupolaire comportant des sources et des récepteurs d'ondes flexurale, sans nécessité de connaître 
l'orientation de l'outil. Ceci peut conduire à des simplifications de conception et à une diminution du coût opérationnel puisque les éléments matériels de mesure de l'orientation ne sont plus nécessaires.

5 B. Orientation dans un repère unique et cohérent

- bloc 705 : Détermination de la direction azimutale de maximisation de l'énergie des deux composantes brutes à orienter et rotation des signaux d'entrée dans un repère intermédiaire cohérent.

La définition d'une fenêtre temporelle de calcul est définie par un écart de temps constant de l'ordre de $10 \mathrm{~ms}$ à $20 \mathrm{~ms}$ de part et d'autre du temps pointé Tp sur le module filtré, de sorte que la fenêtre temporelle englobe au moins une demi période de la période dominante l'arrivée pointée. L'invariance du signal module eu égard à l'orientation des capteurs entraîne la cohérence du temps pointé de l'arrivée $S$, et garantit par conséquence sa validité pour des utilisations ultérieures ou pour connaître les vitesses d'onde $S$ en fonction de la profondeur.

On reprend les signaux bruts $X(t)$ et $Y(t)$ pour chacune des profondeurs de mesure du PSV, que l'on filtre éventuellement en coupant les hautes fréquences bruitées. Puis, on recherche la direction azimutale qui maximise l'énergie sismique dans le plan des deux composantes entrées et dans la fenêtre temporelle précédemment définie, en utilisant une technique courante de maximisation de l'énergie, telle que celle décrite dans les documents précités suivants :

DiSiena, J. P., J. E. Gaiser, and D. Corrigan, 1984, "Horizontal components and shear wave analysis of three-component PSV data", in M. N. Toksöz and R. R. Stewart, eds., Vertical seismic profiling, Part B : Advanced concepts :Geophysical Press, 177-235.

Benhama, A., Cliet, C., and Dubesset, M., 1988, Study and application of spatial directional filtering in three component recordings : geophysical prospecting, 36, 591-613,

La composante correspondante à cette direction de maximisation est notée Hmax(t), l'angle calculé entre $H \max (\mathrm{t})$ et la première composante $\mathrm{X}(\mathrm{t})$ est noté amax180 et n'est connu qu'à $180^{\circ}$ près. Cette indétermination est levée en choisissant, par exemple, que l'amplitude de la composante de sortie Hmax soit impérativement rendue de signe positif à l'instant du temps pointé Tp pour toute profondeur de mesure, en procédant comme suit :

si Hmax $(T p)>0$, alors on définit un angle amax360 = amax180, exprimé en degré si $\mathrm{Hmax}(\mathrm{Tp})<0$, alors on définit un angle $\operatorname{amax360}=180+\operatorname{amax} 180$ 
La figure 8a montre des diagrammes de polarisation $\mathbf{8 0 0}$ de l'onde descendante $\mathbf{S}$, de type hodogramme, dessinés dans le repère $\mathbf{8 0 1}$ des composantes horizontales brutes $X$ et $Y$ d'un PSV réel, enregistré dans un puits vertical avec une source à très faible déport, et un outil de puits comportant trois capteurs fixes avec un dispositif de mesure de l'angle de «relative bearing». Le couplage mécanique de l'outil assure une excellente fidélité vectorielle dans le cas illustré. A gauche de chacun des hodogrammes figure une légende indiquant :

- la profondeur $\mathbf{8 0 2}$ de mesure PSV ;

- les temps en milliseconde du début 803 et de la fin 804 de la fenêtre de calcul de maximisation de $30 \mathrm{~ms}$ (la fenêtre est définie à partir du pointé temps effectué au préalable sur le module filtré) ;

- le vecteur signal 807 calculé dans la direction angulaire de maximisation AG 806. Cette direction est exprimée en degrés (DEG) à partir de la composante de référence $X \mathbf{8 0 1}$ de l'outil de puits, et dans le sens contraire des aiguilles d'une montre. L'angle 806 noté AG sur les figures $8 \mathrm{a}, 8 \mathrm{~b}$ correspond à l'angle amax360 défini précédemment modulo 360 degrés

La figure $8 \mathrm{~b}$ montre les hodogrammes 850 dessinés dans le repère 851 des composantes orthogonales XV et $\mathrm{YH}$ liées à la trajectoire connue du puits (figures $4 a, 4 b$ ), obtenus par une rotation subséquente des composantes brutes $X(t)$ et $Y(t)$ d'angle amax360 diminué de l'angle d'azimut du puits et d'une constante de calage avec les stations de mesure PSV dans une partie suffisamment déviée du puits pour laquelle l'angle de « relative bearing " mesuré est représentatif. Pour construire la figure $8 a$, on considère que l'angle azimutal 856 de la trajectoire du puits localement à la profondeur curviligne 852 du capteur est sensiblement identique en projection sur le plan orthogonal au puits, pour les faibles déviations du puits, inférieures à $12^{\circ}$. A gauche de chacun des hodogrammes, figure une légende indiquant la profondeur $\mathbf{8 5 2}$ de mesure PSV, les temps en milliseconde du début $\mathbf{8 5 3}$ et de la fin $\mathbf{8 5 4}$ des mêmes fenêtres de calcul de maximisation de 30 ms illustrées sur la figure $8 \mathrm{a}$. On constate sur la figure $8 \mathrm{~b}$ la bonne linéarité des hodogrammes dans la direction de maximisation indiquée par la flèche de régression 857, alignée avec l'azimut du puits sensiblement constant, et la bonne cohérence de forme des portions d'hodogramme considérées, correspondant à un intervalle temps très restreint autour du temps pointé

Les figures 9 et 10 montrent les signaux trois composantes du PSV avant et après orientation complète dans le repère géographique, ainsi que le signal module, invariant eu égard au repère de calcul. 
Les figures $9 a, 9 b, 9 c$, représentent les rejeux bruts isotropes 900 normalisés des trois composantes respectives $Z$ axiale et $X, Y$ orthogonales de l'outil de puits, en fonction de la profondeur de mesure (" Measured Depth" ) MD 901 croissante de gauche à droite. La figure $9 \mathrm{~d}$ montre le signal module M2 filtré calculé à partir des composantes $X$ et $Y$ mesurées dans le plan orthogonal à l'axe de puits. Le rejeu est dit "isotrope normalisé $3 C$ ", indiquant qu'un gain constant a été appliqué de façon identique aux amplitudes des trois composantes, mais variable en fonction de la profondeur, de telle sorte que l'amplitude d'arrivée directe d'onde P sur la composante verticale Z (fig.9a) soit identique à toute profondeur. L'arrivée directe d'onde P 902 est quasi invisible sur les composantes horizontales X,Y sur lesquelles le temps d'onde $\mathrm{P}$ a été figuré par un trait 903; une arrivée directe d'onde S 904 est clairement identifiée sur les composantes horizontales $X, Y$ et sur le signal du module filtré ( Fig.9d) par sa pente plus importante que celle de l'onde $P$ 902-903. Oon observe des défauts de cohérence de la forme d'onde $S$ sur les composantes orthogonales $X$ et $Y$ ( resp. figs.9b et $9 c)$, ces défauts étant associés à l'orientation aléatoire des capteurs horizontaux et de la direction de maximisation de l'arrivée directe $S$ illustrée sur la figure 8a.

Les figures $10 \mathrm{a}, 10 \mathrm{~b}$ et $10 \mathrm{c}$ représentent les rejeux 1000 isotropes normalisés $3 \mathrm{C}$ des composantes géographiques respectives $\mathrm{ZV}$ verticale, $\mathrm{HN}$ et $\mathrm{HE}$ horizontales orientées dans les directions géographiques respectives Nord et Est, en fonction de la profondeur MD 1001 croissante de gauche à droite, avec les mêmes échelles de temps et de profondeur que celles des figures $9 \mathrm{a}, 9 \mathrm{~b}$ et $9 \mathrm{c}$. La figure $10 \mathrm{~d}$ montre le signal module M2 filtré calculé à partir de $\mathrm{HN}$ et $\mathrm{HE}$, qui est sensiblement identique au module calculé à partir de $X$ et $Y$, figure $9 \mathrm{~d}$, illustrant ainsi l'invariance du module eu égard au repère de calcul. Le temps de l'arrivée directe d'onde P 1002 a été figuré par le trait 1003 sur les composantes horizontales HN et HE. La cohérence de forme du signal d'arrivée directe d'onde S 1004 sur les composantes

25 horizontales $\mathrm{HN}$ et $\mathrm{HE}$ est excellente, et contraste drastiquement avec l'incohérence observée sur les composantes $X$ et $Y$ correspondantes des figures $9 b$ et $9 c$, ce qui confirme la bonne orientation obtenue.

A ce stade, les trois composantes sont orientées à 360 degrés près dans un repère sensiblement unique. Ce repère est donc cohérent pour chaque profondeur. $\mathrm{Ce}$ prétraitement permet de réaliser un traitement des trois composantes isotropes, même si ce repère est de direction azimutale inconnue.

\section{Orientation dans un repère géographique}


- bloc 706 : Calibration du repère cohérent intermédiaire par rapport au repère géographique :

Selon l'invention, il est ensuite nécessaire d'orienter de façon additionnelle les composantes horizontales dans un repère unique d'orientation géographique connue. Pour ce faire, il est nécessaire de déterminer l'orientation géographique du repère unique intermédiaire obtenu en sortie des opérations du bloc 705 de la figure 7. Cette opération de calibrage azimutal du repère unique permet l'interprétation géologique des résultats de traitement du PSV à trois composantes subséquent, tel que celui indiqué dans le brevet US 6076 045, focalisé sur la détermination de pendage et azimut de réflecteurs sismiques.

Plusieurs procédés de calibrage du repère unique peuvent être utilisés.

Par exemple, selon les exemples a) et b) ci-après décrits, on peut déterminer l'orientation géographique du repère unique en déterminant, par traitement des trois composantes dans le repère unique, la direction azimutale de pendage d'un réflecteur sismique ou la direction principale d'anisotropie de biréfringence d'onde de cisaillement, et en appliquant au repère unique une rotation jusqu'à ce que cette direction ainsi déterminée coïncide avec une direction azimutale connue à partir de connaissances structurales et géologiques.

\section{a) Utilisation de la biréfringence des ondes de cisaillement}

Selon une alternative au problème de l'orientation des composantes dans un repère géographique connu, on détermine cette orientation géographique au moyen des étapes suivantes :

- on détecte, pour au moins un intervalle de profondeur, une anisotropie de biréfringence des modes rapide et lent d'une onde de cisaillement par une analyse des signaux des mode rapide et lent de l'onde de cisaillement ;

- on détermine la direction principale de l'anisotropie de biréfringence à partir de la direction azimutale déterminée pour construire le repère unique (issue de la maximisation de l'énergie de l'étape 705);

- on détermine l'orientation géographique dudit repère unique en alignant la direction principale de l'anisotropie de biréfringence sur une direction azimutale connue par ailleurs à la même profondeur au droit du puits, telle que la direction orthogonale à un pendage stratigraphique connue, ou la direction de contrainte horizontale maximale connue. 
L'analyse des signaux des modes rapide et lent de l'onde de cisaillement peut être réalisée par une analyse visuelle. On représente, sous forme d'image, le signal du mode rapide de l'onde de cisaillement dans le repère unique. On peut, pour faciliter l'interprétation visuelle, effectuer une translation temporelle égale temps pointé sur le module filtré. Puis, on représente, sous forme d'image, le signal du mode lent de l'onde de cisaillement qui est orthogonale à la composante d'onde-S rapide, après translation temporelle égale au temps pointé. Puis on comparant ces deux images, on détecte au moins un intervalle de profondeur pour lequel il existe une anisotropie de biréfringence.

Les figures $11 \mathrm{a}, 11 \mathrm{~b}$ et $11 \mathrm{c}$ représentent le résultat du processus d'orientation dans un repère unique selon l'invention, à partir des données brutes du PSV représentées sur les figures 9a, 9b et 9c. Ces figures $11 \mathrm{a}, 11 \mathrm{~b}$ et $11 \mathrm{c}$ montrent des rejeux $\mathbf{1 1 0 0}$ de signaux d'onde $S$ descendante en fonction de la profondeur MD 1101, croissante de gauche à droite. Sur ces figures, les signaux sont alignés sur le pointé temps effectué sur le premier maximum d'amplitude du signal module filtré, dans une fenêtre temps 1102 de $0.20 \mathrm{~s}$. Le rectangle 1104 contient la courte fenêtre temps de 30ms utilisée pour la maximisation des signaux bruts dans le plan orthogonal, le signal maximal étant représenté par l'onde rapide sur la figure 11b, sur lequel on a imposé un signe négatif de sortie au temps pointé.

- la figure 11a représente le signal du module M2 filtré utilisé pour le pointé précis, puis aligné au temps 0.10 s pour toute profondeur, par translation temporelle égale temps pointé ; cette opération permet un contrôle visuel de la précision du pointé.

- la figure $11 \mathrm{~b}$ représente le signal de l'onde-S rapide, résultant du calcul de maximisation d'énergie, après translation temporelle égale temps pointé sur le module filtré, et inversion du signe.

- la figure $11 \mathrm{c}$ représente le signal de l'onde-S lente, orthogonale à la composante d'onde-S rapide, après translation temporelle égale temps pointé. L'onde S-lente est également repérable par la phase noire $\mathbf{1 1 0 5}$ sur le signal module représenté sur la figure 11a. Cette onde S-lente accuse un retard de propagation bien visible dans l'intervalle profondeur 1106, entre $600 \mathrm{~m}$ et $1500 \mathrm{~m}$, et uniquement dans cet intervalle qui est ainsi anisotrope à la propagation des ondes $S$ ou biréfringent, les autres intervalles étant isotropes. Les indications chiffrées 1103 représentent l'inclinaison verticale de la trajectoire de puits en degrés. L'intervalle profondeur 1107 est utilisé pour calibrer l'orientation des signaux des ondes S-rapide et S-lente par rapport au Nord géographique, ainsi que l'orientation du repère unique, grâce aux indications d'angle de relative bearing mesurées par l'outil de puits. 
La biréfringence ainsi détectée, possède une direction principale qui correspond à la direction azimutale déterminée pour construire le repère unique (issue de la maximisation de l'énergie de l'étape 705).

Par ailleurs, il est connu que la biréfringence des ondes de cisaillement dans un milieu peut être due soit à la présence de contraintes horizontales dans le milieu, soit à la présence de couches sédimentaires de pendage non nul. La figure 12 représente un schéma 1200 illustrant les polarisations linéaires d'une onde S descendante incidente S-i 1201 qui se dédouble suite à la propagation verticale dans un milieu stratifié penté et biréfringent 1210, en une onde S-rapide S-f 1202, de polarisation linéaire perpendiculaire à la direction de pendage "Dip" 1211, et une onde S-lente S-s 1203, de polarisation linéaire sensiblement parallèle à la direction de pendage "Dip" 1211. Ainsi, la direction principale d'onde-S rapide de biréfringence correspond à la direction perpendiculaire à la direction du pendage stratigraphique.

Or il est classique de déterminer, localement au niveau d'un puits, les directions de pendage d'un milieu sédimentaire stratifié, au moyen de données externes, telles qu'une carte structurale dérivée d'une étude sismique 3D ou d'une pluralité de puits, ou d'outils diagraphique de pendagemétrie. On peut également déterminer, de manière connue des spécialistes, la direction de la contrainte horizontale maximale, qui correspond à la direction principale d'onde-S rapide de biréfringence.

Ainsi, par déduction, connaissant une direction azimutale par des connaissances structurales et géologiques externes, et connaissant localement au niveau du puits la direction principale d'anisotropie de biréfringence, on peut déterminer la direction géographique du repère unique. En effet, il suffit de faire "tourner » le repère unique de façon à ce que la direction principale d'anisotropie de biréfringence dans le repère unique corresponde à la direction azimutale connue par ailleurs: direction perpendiculaire à la direction azimutale d'un pendage stratigraphique ou direction de la contrainte horizontale maximale.

\section{b) Utilisation d'un champ d'ondes réfléchies}

Selon une alternative au problème de l'orientation des composantes dans un repère géographique connu, on détermine cette orientation géographique au moyen des étapes suivantes :

- on extrait un champ d'ondes réfléchies au moyen d'un traitement isotrope des trois composantes dans le repère unique ; 
- on détermine dans le repère unique un azimut de pendage d'au moins un réflecteur sismique au moyen d'une analyse de polarisation de ce champ d'ondes réfléchies ; et

- on détermine l'orientation géographique du repère unique en alignant cet azimut de pendage sur un azimut de pendage du même réflecteur connue par ailleurs, à une même profondeur au droit du puits.

Une méthode de traitement isotrope des trois composantes permettant d'extraire un champ d'ondes réfléchies est décrite par exemple dans le brevet US 6076 045. II s'agit de la méthode SEISDIP (marque déposée).

La figure 13 représente un schéma 1300 illustrant la propagation d'une onde sismique verticale incidente I 1301 qui se réfléchit sur un réflecteur sismique (interface) 1310 de pendage "Dip $\alpha$ " 1311 : la direction azimutale prise par l'onde réfléchie R 1302, de type P-P, est naturellement orientée vers la direction d'aval pendage, et l'angle d'incidence verticale 1303 du rai réfléchi $R$ est $2 \alpha$. De telles circonstances de propagation dans un milieu stratifié penté correspondent en effet à une réflexion notoire observée après traitement des données orientées du PSV illustrées par les figures $10 a, 10 b$ et 10c.

Par ailleurs, il est classique de déterminer, localement au niveau d'un puits, les directions de pendage d'un milieu sédimentaire stratifié, au moyen de données externes, telles qu'une carte structurale dérivée d'une étude sismique 3D ou d'une pluralité de puits, ou d'outils diagraphique de pendagemétrie.

Ainsi, par déduction, connaissant localement au niveau du puits la direction de pendage du réflecteur sismique $\mathbf{1 3 1 0}$, on peut déterminer la direction géographique du repère unique. En effet, il suffit de faire "tourner » le repère unique de façon à ce que l'azimut de pendage du réflecteur sismique dans le repère unique corresponde à la direction d'aval pendage connue par ailleurs.

Les résultats d'un tel traitement (méthode SEISDIP®) sont montrés par le champ d'onde réfléchi isotrope $\mathbf{1 4 0 0}$ représenté en trois composantes par les figures $14 \mathrm{a}, 14 \mathrm{~b}$ et $14 \mathrm{c}$, en temps double vertical 1401, échelle en seconde. On observe de façon évidente une forte réflexion en mode P-P 1421 accompagnée d'une forte réflexion en mode P-S 1422, toutes deux générées sur le même réflecteur $R$ 1423, situé à la profondeur $2100 \mathrm{~m}$ lue sur l'échelle profondeur MD 1402. Ces arrivées réfléchies apparaissent principalement sur la composante verticale Z 1410 (figure 14a) et sur la composante horizontale H-Ss 1411 (figure 14b). La composante horizontale H-Ss 1411 correspond à la direction azimutale de l'onde Slente représentée figure10c, et la seconde composante horizontale H-Sf 1412 (figure 14c), correspond à la direction d'onde-S rapide. Cette dernière ne montre aucune énergie pour les arrivées réfléchies P-P 1421 ou P-S 1422, confirmant ainsi son orthogonalité au plan de 
pendage du réflecteur et des couches sédimentaires dans l'intervalle profondeur où a lieu la réflexion. La réflexion P-P 1421 est de polarité identique sur la composante verticale Z 1410 orientée vers le haut, et sur la composante H-Ss 1411. Cette dernière est orientée vers la direction d'aval pendage $\mathrm{N} 55^{\circ} \mathrm{E}$, localement connu par les cartes structurales dérivées des

puits et de la sismique de surface 3D. De façon cohérente, la réflexion P-S apparaît en signe opposé sur les composantes verticale et horizontale H-Ss. Le procédé de calibration décrit ci-dessus s'avère utile pour vérifier éventuellement la bonne orientation géographique obtenue par d'autres voies, ainsi que la précision intrinsèque du processus d'orientation dans le repère unique.

\section{c) Autres alternatives}

- Selon une autre alternative, on peut utiliser l'énergie résiduelle de l'arrivée d'onde P qui est parfois plus importante sur les composantes horizontales des niveaux de mesure les moins profonds du PSV, en faisant l'hypothèse classique que la polarisation de l'onde P directe est dans la direction azimutale du segment qui relie la position de la source et celle du capteur.

15 - On peut également, de façon alternative, utiliser des mesures directionnelles des trois composantes par divers instruments ou accessoires de mesures d'orientation complète ou partielle, si ces derniers ont été descendus de façon couplée à l'outil de PSV mono niveau.

L'orientation est dite complète, lorsque tous les paramètres permettant l'orientation (angles de "Relative Bearing ", de Déviation verticale du puits et d'Azimut du puits dévié) sont mesurés sur tous les niveaux profondeur de mesure du PSV. Ceci est possible avec un outil de type gyroscope couplé à l'outil PSV mono niveau par exemple.

L'orientation est dite partielle, si l'outil de mesure d'orientation est couplé à au moins un des satellites de mesure de l'outil sismique de PSV, si ce dernier comporte plusieurs niveaux profondeur mesurés simultanément. L'orientation est également dite partielle, si la mesure d'orientation est limitée à un intervalle profondeur donné (comme par exemple la limitation à l'intervalle de puits en trou ouvert, non chemisé avec des tubes métalliques pour un outil détectant le direction du Nord magnétique), ou à une plage d'angle de déviation du puits (comme par exemple les dispositifs de type inclinomètres, pendule de mesure du «Relative Bearing » et cardans, sensibles à la gravité, et rendus inopérants pour les faibles déviations verticales du puits).

- bloc 707 : Rotations des signaux sismiques entre le repère cohérent intermédiaire et le repère géographique, lorsque l'orientation de l'outil est partielle. 
Afin de faciliter la compréhension de l'invention et de son objet, on expose ci dessous un panorama succinct des moyens matériels connus d'orientation des outils de puits sismiques et non sismiques :

- Moyens d'orientation exhaustive et précise d'une sonde de puits à coût opérationnel élevé : L'industrie de la diagraphie dispose de moyens magnétiques d'orientation repérant la direction du champ magnétique terrestre, si l'on opère en trou ouvert, souvent combinée à des inclinomètres précis rendus peu sensibles aux vibrations et capables d'effectuer des mesures en continu pendant la remontée des outils de mesure diagraphique également en fonctionnement continu. Les inclinomètres précis permettent de connaître le relative bearing d'un outil dans un puits tubé à partir de quelques degrés d'inclinaison verticale du puits, la trajectoire et les angles d'inclinaison et d'azimut du puits étant connue par ailleurs. Les gyroscopes de puits sont également d'un usage courant pour mesurer avec précision la trajectoire du puits; leur usage en combinaison avec d'autres outils de mesure diagraphique est sporadique, mais pas rare. A l'aide des moyens précédemment décrits, l'orientation des composantes est alors parfaitement mesurée en trou ouvert ou tubé.

- Moyens d'orientation partielle et peu précise, mais peu onéreuse, d'une sonde de puits :

a) pour les puits déviés de trajectoire connue, il est usuel de monter les capteurs triaxiaux sur des doubles cardans avec architecture dite "tourelle", comprenant un axe de rotation parallèle à l'axe puits, et d'un axe horizontal perpendiculaire au plan vertical tangent localement au puits. Les figures $3 a, b$ indiquent la disposition d'un capteur sismique triaxial $\mathbf{3 1 1}$ monté sur de tels cardans, et placé dans un puits dévié 310 : la figure 3a représente une projection $\mathbf{3 0 0}$ dans le plan vertical tangent au puits 310, qui comprend la composante verticale Z-down 301, orientée vers le bas, et la composante horizontale XH 302 orientée dans l'azimut des profondeurs mesurées croissantes du puits; l'autre composante horizontale YH 303 est orthogonale au plan vertical tangent représenté. L'angle d'inclinaison verticale du puits 304, ou déviation, est couramment référé par DEV dans l'industrie. La figure $3 \mathrm{~b}$ représente une 30 projection $\mathbf{3 5 0}$ dans le plan horizontal en vue de dessus : la trajectoire du puits dévié 310 apparaît comme une ligne quelconque, dans le repère géographique 320 , la composante horizontale $\mathrm{XH} 302$ est tangente au puits au niveau de la position du capteur 311, la composante horizontale $\mathrm{YH} 303$ est disposée à $+90^{\circ}$ par rapport à $\mathrm{XH302}$, en vue de dessus. On recalcule les composantes sismiques $\mathrm{HE}$ et HN orientées dans un repère géographique 320 à partir des composantes $\mathrm{XH} 302$ et $\mathrm{YH}$ 303 par rotation d'angle HAZI 305 autour de la verticale, HAZI 305 correspondant à 
l'azimut du puits localement à la position du capteur 311. Les angles DEV 304 et HAZI 305 sont en général connus et mesurés indépendamment de l'opération de PSV par les mesures très précises de la trajectoire de puits effectuées à l'aide de moyens de type gyroscope ou magnétomètre - inclinomètre mentionnés plus haut. Le montage des capteurs triaxiaux sur double cardans de type tourelle permet d'orienter les capteurs sismiques trois composantes par gravité dans les puits suffisamment inclinés par rapport à la verticale, typiquement à partir d'une valeur seuil de l'ordre de 10 degrés de l'inclinaison verticale du puits, ce seuil pouvant varier d'une marque d'outil à une autre; en pratique, compte tenu des forces de frottements inhérentes à ce genre de dispositif mécanique, l'orientation devient plus précise lorsque l'inclinaison du puits dévié augmente. Pour les faibles valeurs de déviation du puits au dessous de la valeur seuil d'environ 10 degrés, l'orientation des composantes orthogonales n'est pas connue. Certes, il y a une incertitude du même ordre sur l'orientation réelle de la composante Z-down par rapport à la direction verticale réelle, mais cela n'altère pas significativement les résultats de traitement ni des conclusions interprétatives subséquentes.

b) De façon alternative et courante, il est connu de monter des capteurs sismiques à trois composantes de façon fixe dans un outil de PSV, comprenant en outre un dispositif de mesure de l'angle de Relative Bearing dans le plan orthogonal à l'axe de l'outil PSV : Naturellement ce genre de dispositif appelé communément "capteur de Relative Bearing" est inopérant en puits strictement vertical et restitue une mesure du Relative Bearing qui n'est significative qu'au delà d'une faible valeur de l'inclinaison verticale du puits, de l'ordre de 10 degrés; la mesure du Relative Bearing devient de plus en plus précise lorsque l'inclinaison du puits dévié augmente. Les figures $4 a, b$ indiquent la disposition d'un capteur sismique triaxial monté de façon fixe dans l'outil : la figure 4a illustre la définition de l'angle de Relative Bearing par l'angle entre la génératrice haute du puits cylindrique et une direction de référence de l'outil PSV dans le plan orthogonal à l'axe de l'outil, avec une convention de signe positif dans le sens des aiguilles d'une montre lorsqu'on regarde le plan orthogonal dans la direction des profondeurs curvilignes croissantes du puits. La figure 4a représente une projection 400 dans le plan orthogonal à l'axe $Z$ du puits 410 , au niveau du capteur 411, en vue de dessus, la flèche 412 indiquant la direction des profondeurs mesurées croissantes du puits; l'angle de relative bearing RB 430 est défini par l'angle entre la direction XV 422 orthogonale à l'axe du puits 410 , contenue dans le plan vertical tangent au puits et pointant vers le haut, avec la direction $X \mathbf{4 1 9}$ de référence de la sonde contenant le capteur $\mathbf{4 1 1}$, correspondant au capteur sismique orthogonal $X$ 
419 mesuré; l'angle RB 430 est mesuré positivement 431 dans le sens des aiguilles d'une montre lorsqu'on regarde dans la direction de la flèche $\mathbf{4 1 2}$.

La figure $4 \mathrm{~b}$ représente une projection $\mathbf{4 5 0}$ dans le plan vertical tangent au puits $\mathbf{4 1 0}$ localement à la position du capteur 411, qui comprend la composante Z 421 mesurée par l'outil, axiale au puits et pointant vers le bas et la composante XV422 précédemment calculée dans la direction de l'axe origine de l'angle de relative bearing $(\mathrm{RB}=0)$; la composante horizontale $\mathrm{YH} 403$ est orthogonale au plan vertical tangent représenté. L'angle d'inclinaison verticale du puits DEV 404 est indiqué entre les directions Z 421 axiale au puits et Z-down 401, composante sismique verticale orientée vers le bas; la composante horizontale XH 402 orientée dans l'azimut des profondeurs mesurées croissantes du puits et la composante sismique Z-down 401 sont obtenues à partir des composantes XV422 et Z 421 par rotation d'angle DEV 404 autour de l'axe YH 403.

Par exemple, l'orientation géographique obtenue sur les figures $10 \mathrm{a}, 10 \mathrm{~b}$ et $10 \mathrm{c}$ a été obtenue par calibration de l'azimut du repère unique grâce à des mesures du «Relative Bearing», par un dispositif pendulaire dans un intervalle profondeur restreint, où l'inclinaison verticale du puits dépasse 13 degrés d'angle.

Trois configurations de mesure partielle d'orientation sont considérées ci dessous :

20 C1: l'outil de puits mono niveau contient des capteurs triaxiaux montés sur des doubles cardans avec architecture dite "tourelle", comme illustré sur les figures $3 a$ et $3 b$, et dans un intervalle profondeur restreint contenant au moins une station de mesure PSV, la déviation du puits est suffisamment importante (au moins 10 degrés environ) pour permettre la rotation des cardans sous l'action de la gravité : la composante 301 Z-down est alors naturellement orientée selon la verticale (fig.3a). Les composantes horizontales géographiques $320 \mathrm{HN}$, HE (fig. 3b) sont obtenues par rotation des composantes XH 302 et YH 303 mesurées, autour de la verticale, de l'angle HAZI 305 connu à 360 degrés, correspondant à l'azimut du plan vertical tangent au puits à la position du capteur.

$$
[\mathrm{HE}, \mathrm{HN}]=\operatorname{Rot}(\mathrm{HAZI}) \cdot[\mathrm{XH}, \mathrm{YH}]
$$

30 C2 : l'outil de puits mono niveau contient des capteurs triaxiaux montés de façon fixe dans l'outil de puits, comme illustré sur les figures $4 a$ et $4 b$, et dans un intervalle profondeur restreint contenant au moins une station de mesure PSV, la déviation du puits est suffisamment importante pour permettre une mesure précise à quelques degrés de l'angle de «relative bearing» RB 430 illustré sur Fig.4a: on applique alors trois rotations successives dans cet ordre : 
$[X V, Y H]=\operatorname{Rot}(R B) .[X, Y]$, rotation dans le plan orthogonal à l'axe du puits,

puis $[X H, Z V-$ down $]=\operatorname{Rot}(D E V)$. $[X V, Z]$, rotation dans le plan vertical tangent au puits à la position de l'outil de puits, selon l'illustration fig. $4 \mathrm{~b}$,

puis $[H E, H N]=\operatorname{Rot}(H A Z I) .[X H, Y H]$, rotation dans le plan horizontal, selon l'illustration 5 fig. $3 b$.

C3 : l'outil de puits comporte une pluralité de sondes réceptrices placées à des profondeurs de mesure adjacentes qui contiennent chacune des capteurs triaxiaux montés de façon fixe dans l'outil de puits; en outre, l'une des sondes est combinée avec un outil de mesure complète de l'orientation. Dans cette configuration, après rotation d'un des composantes orthogonales à l'axe du puits dans un repère unique, on calcule, pour toutes les stations profondeur mesurées avec la sonde couplée à l'outil de mesure de l'orientation la différence entre l'angle de rotation précédente et l'angle de "relative bearing " mesuré, puis on interpole la valeur de cette différence pour les niveaux profondeurs adjacents qui ne bénéficient pas de mesure d'orientation; l'angle différence interpolé obtenu est l'angle de « relative bearing » $\mathrm{RBi}$ à utiliser pour la rotation des composantes orthogonales du repère intermédiaire. On applique alors les trois rotations décrites pour la configuration C2 ci dessus, en prenant l'angle de « relative bearing » RBi pour la première des trois rotations.

Les figures $5 a, 5 b$ et $5 c$ illustrent, dans le plan vertical $\mathbf{5 0 0}$ de projection, l'attitude spatiale des trièdres des capteurs sismiques 511 à 513 et 521 à 523 dans un puits 510 comprenant une partie verticale $\mathbf{5 0 1}$ et une partie $\mathbf{5 0 2}$ déviée dans ledit plan vertical $\mathbf{5 0 0}$ représenté en projection.

La partie dite verticale $\mathbf{5 0 1}$ du puits $\mathbf{5 1 0}$ symbolise un intervalle profondeur pour laquelle la valeur de l'inclinaison verticale est au dessous de la valeur de seuil de fonctionnement efficace d'un dispositif de double cardans, ou d'un système de mesure gravitaire-pendulaire de l'angle de "relative bearing ", et contient les trièdres 511 à 513. La partie dite déviée 502, correspond à un intervalle pour lequel l'angle de déviation verticale du puits est au dessus de ladite valeur de seuil, et contient les trièdres 521 à 523.

La figure 5a représente l'attitude des trièdres correspondant à un montage fixe des trois capteurs sismiques orthogonaux dans la sonde de puits, dont 511 et $\mathbf{5 2 1}$, où l'axe du capteur généralement appelé Z-outil est aligné avec l'axe du puits et pointe vers le haut : le trièdre $\mathbf{5 1 1}$ dans la partie verticale $\mathbf{5 0 1}$ et le trièdre $\mathbf{5 2 1}$ dans la partie déviée $\mathbf{5 0 2}$ illustrent ainsi que l'angle de « relative bearing " qui repère la direction des capteurs orthogonaux à l'axe du puits par rapport à l'azimut du plan vertical $\mathbf{5 0 0}$ est aléatoire d'une station profondeur de PSV à une autre. La valeur mesurée du "relative bearing» ne peut être exploitée pour 
l'orientation des composantes que dans la partie déviée 502 (voir figure 4a et explications associées).

La figure $5 \mathrm{c}$ représente l'attitude des trièdres correspondant à un montage des trois capteurs sismiques orthogonaux sur doubles cardans de type tourelle dans la sonde de puits, dont 512 et 522, où l'axe de l'un des capteurs est aligné avec la verticale et pointe vers le haut: le trièdre $\mathbf{5 1 2}$ et les trièdres des cotes adjacentes dans la partie verticale 501 illustrent ainsi que la direction azimutale des capteurs orthogonaux à l'axe du puits est aléatoire d'une station profondeur de PSV à une autre. Par contraste, le trièdre $\mathbf{5 2 2}$ dans la partie déviée 502, illustre que l'orientation du trièdre est totalement connue, l'une des composantes horizontales étant dans le plan vertical $\mathbf{5 0 0}$ de la partie déviée de la trajectoire du puits, et l'autre composante horizontale étant normale au plan $\mathbf{5 0 0}$ (voir figures 3a et 3b et explications associées).

La figure $5 \mathrm{~b}$ représente l'orientation unique connue des trièdres obtenue après application des procédures d'orientation selon l'un des modes de l'invention, dont $\mathbf{5 1 3}$ et $\mathbf{5 2 3}$, où l'axe de l'un des capteurs est aligné avec la verticale et pointe vers le haut, l'une des composantes horizontales étant dans le plan vertical $\mathbf{5 0 0}$ de la partie déviée de la trajectoire du puits et pointe dans l'azimut des profondeurs croissantes (identique à la direction de déviation du puits dans le cas présent), et l'autre composante horizontale étant normale au plan $\mathbf{5 0 0}$ : les trièdres $\mathbf{5 1 1}, \mathbf{5 1 2}$ et les trièdres des cotes adjacentes d'orientation aléatoire dans la partie verticale 501 sont réorientés dans un repère commun du trièdre 513 ou 523 à l'aide de la procédure P1 551 selon l'invention. Par contraste, le trièdre $\mathbf{5 2 1}$ dans la partie déviée 502 est réorienté dans les directions du trièdre 523 à l'aide de deux rotations successives selon la procédure connue P2 552 et décrite précédemment (commentaires des figures $4 a$ et $4 b$ ). Le trièdre $\mathbf{5 2 2}$ dans la partie déviée $\mathbf{5 0 2}$ est naturellement orienté de façon identique au trièdre $\mathbf{5 2 3}$ et ses composantes ne nécessitent aucune intervention.

En pratique, la procédure P1 551 selon l'un des modes de l'invention est également appliquée aux trièdres de la partie déviée $\mathbf{5 0 2}$ immédiatement adjacente à la partie verticale 501, dans un court intervalle de recouvrement et d'embrayage, de façon à calibrer l'azimut des composantes horizontales des trièdres de la partie verticale, dont 511 et $\mathbf{5 1 2}$, sur

30 l'azimut connu des trièdres de la partie déviée 502. Au final, si l'on désire orienter tous les trièdres 513 à 523 représentés sur la figure $5 b$ dans un repère géographique, on applique une rotation azimutale similaire à celle décrite précédemment (commentaires de la figure $3 b)$. 
La figure 15 montre sous forme d'un graphique de synthèse 1500 les résultats d'une double orientation selon l'invention, obtenue de façon indépendante, sur l'arrivée directe $S$, à partir des deux composantes horizontales d'un PSV réel enregistré dans un puits vertical, au moyen de deux vibrateurs horizontaux placés orthogonalement en surface à très faible déport du puits, et activés successivement à chaque position de capteur de fond, en une seule passe de l'outil de puits (appelée "run"). Au préalable, les composantes horizontales sont réorientées en utilisant l'arrivée d'onde de pression d'un PSV avec déport tiré avec un vibrateur vertical, dans la même passe d'outil PSV (le même "run"). Cependant, le déport du vibrateur vertical étant trop faible, les cotes profondeur des mesures les plus profondes présentent des orientations aberrantes justifiant une reprise du processus d'orientation.

Les courbes sont représentées en fonction de l'abscisse profondeur 1501 croissante de droite à gauche, dans l'intervalle $2700 \mathrm{~m}$ à $3480 \mathrm{~m}$. En ordonnée, les courbes 1502 et 1503 représentent l'angle d'orientation obtenu indépendamment pour chacune des deux sources. Les échelles d'angles respectives 1512 et 1513 sont exprimées en degré d'azimut à partir de la direction du Nord géographique, sur le côté droit du graphique.

La courbe 1504 représente la différence entre les deux angles représentés par les courbes 1502 et 1503. L'échelle d'angle 1514 en degrés de la courbe 1504 apparaît sur le côté droit du graphique. La droite 1505 correspond à la régression linéaire de la courbe 1504.

De façon intéressante, la droite 1505 illustre l'incertitude d'orientation selon l'invention en ce qu'elle ne correspond pas à une constante, et présente une variation de l'ordre de 11 degrés d'angle, sur l'intervalle global de mesure, de $2700 \mathrm{~m}$ à $3480 \mathrm{~m}$ : de $67^{\circ}$ pour l'abscisse profondeur $2700 \mathrm{~m}$ à l'extrémité droite du graphique, à $56^{\circ}$ pour l'abscisse profondeur $3480 \mathrm{~m}$ à l'extrémité gauche du graphique. Cela signifie que le processus d'orientation selon l'invention peut être entaché d'une dérive angulaire de l'ordre de 15 degrés par intervalle de 1000 mètres de profondeur. II est par conséquent préférable d'effectuer plusieurs calages angulaires géographiques du repère fixe, même si le fait de disposer d'un seul calage demeure le strict minimum.

Au final, on observe que la différence d'angle aléatoire entre la courbe 1504 et sa 30 régression 1505, appelé "jitter", est de l'ordre de 5 degrés d'écart moyen dans l'intervalle $2700 \mathrm{~m}$ à $3340 \mathrm{~m}$. Par ailleurs, on observe une différence angulaire brutale d'environ $50^{\circ}$ sur les courbes d'angles 1502 et 1503 entre les cotes de mesures situées au dessus de $3340 \mathrm{~m}$ et celles situées au dessous, dans la partie profonde : cette différence est sensiblement compensée par le processus de réorientation, car la courbe de différence 1504 ne montre plus cette variation brutale, malgré un "jitter" plus important entre les cotes $3340 \mathrm{~m}$ et $3420 \mathrm{~m}$, 
témoignant d'une dégradation locale du rapport signal sur bruit sur les données sismiques initiales.

Ainsi, selon un mode particulier de réalisation, une étape supplémentaire d'ajustement est réalisée. Elle comprend les étapes suivantes :

- on réalise au moins deux calages angulaires géographiques du repère unique à différentes profondeurs, par des méthodes similaires ou différentes ; et

- si ces calages sont suffisamment précis et restituent des valeurs d'angles différentes, on effectue une rotation graduelle du repère unique entre les profondeurs de calage, de préférence en appliquant un gradient d'angle de rotation linéaire avec la profondeur et limité à environ quinze degrés pour cent mètres de profondeur.

\section{Applications de l'invention}

La méthode selon l'invention peut être appliquée dans le cadre de prospection sismique par méthode de PSV conventionnel à très faible déport de l'unique position de source, afin de positionner dans l'espace à trois dimensions des événements géologiques au voisinage de puits. Une telle méthode de prospection sismique comporte alors les étapes suivantes :

- la réception par des capteurs sismiques triaxiaux, disposés dans un puits et couplés avec les formations environnant le puits, afin de mesurer aussi fidèlement que possible le signal vectoriel en trois composantes des ondes directes et réfléchies dans les modes $\mathrm{P}, \mathrm{S}$ ainsi que les modes d'ondes converties.

- l'orientation dans l'espace des capteurs sismiques multiaxes de réception. On utilise pour ce faire la méthode d'orientation selon l'invention.

- l'imagerie sismique de puits à partir de trois composantes orientées, telle que celle décrite par exemple dans le brevet US 6076 045: cette méthode fait appel au traitement isotrope des trois composantes orientées, permettant la lecture de la polarisation des événements réfléchis observés, puis de l'imagerie et du positionnement dans l'espace des réflecteurs correspondants, restituant ainsi le pendage et l'azimut de pendage à 360 degrés de chacun des réflecteurs.

Une application importante de la méthode selon l'invention concerne également l'amélioration du contrôle qualité des trois composantes enregistrées sur le site d'enregistrement, à l'aide des moyens informatiques disponibles : en effet, d'une part les méthodes informatiques, permettant l'orientation des données trois composantes dans un repère unique, sont aisées à mettre en oeuvre, et d'autre part il est plus facile d'évaluer 
visuellement la qualité générale d'enregistrement et le bon fonctionnement global de la chaîne d'acquisition sur des rejeux orientés des trois composantes par rapport à des rejeux bruts non orientés, pour toute profondeur. Ainsi, on peut utiliser l'orientation dans un repère unique de façon automatisée, pour obtenir un contrôle de la qualité de la mesure sismique en trois composantes, immédiatement après l'acquisition des mesures sur le terrain.

La méthode permet d'orienter les trois composantes des PSV, dans les intervalles profondeur proches de la verticale, en particulier lorsqu'une seule position de source sismique de surface localisée à proximité de l'appareil de forage a été exploitée, et que l'outil de mesure PSV descendu dans le puits n'est pas couplé à un outil de mesure précis de tous les angles permettant l'orientation des trois composantes des signaux dans un repère géographique. Ceci correspond à la configuration usuelle des PSV dans les puits d'exploration ou de production. La méthode selon l'invention s'applique avec efficacité sur un train d'onde de cisaillement descendante, y compris en présence d'anisotropie de biréfringence en propagation : en effet, la direction azimutale ne varie pas en présence d'anisotropie de vitesse des deux modes propres d'onde $S$, dont l'effet est très faible sur des niveaux profondeurs adjacents, pour autant que l'atténuation différentielle entre les deux ondes demeure également faible, ce qui est en général vérifié par expérience.

La méthode permet également d'orienter les trois composantes des outils de PSV comprenant plusieurs niveaux profondeur de mesure sismique $3 \mathrm{C}$ simultanés, pour lequel un seul niveau (ou un nombre incomplet de niveaux) est couplé à un outil de mesure d'orientation complète ou partielle.

La simplicité de mise en œuvre de la méthode, au moyen des ordinateurs de plus en plus puissants embarqués dans les systèmes d'acquisition, permet une amélioration du contrôle qualité globale sur site des données trois composantes enregistrées, grâce à la production en temps légèrement différé, voire en temps réel, du pointé temps de l'onde $S$ descendante et d'un rejeu des trois composantes orientées dans un repère unique, permettant à l'ingénieur d'acquisition de détecter rapidement sur site et avec fiabilité accrue les dysfonctionnements éventuels de la chaîne d'acquisition des trois composantes.

L'avantage de la méthode est de permettre subséquemment le traitement isotrope des signaux PSV trois composantes, y compris pour des retraitements de jeux de données PSV anciennes pour lesquelles l'outil de fond n'était pas couplé à un outil de mesure d'orientation complète ou partielle.

Un autre avantage de la méthode est de permettre à l'opérateur qui prévoit d'enregistrer un PSV, d'affiner le choix le type d'outil sismique de puits ainsi que de l'outil 
d'orientation désirable à combiner, avant d'engager l'acquisition effective sur site du PSV en trois composantes, en fonction de l'objectif géologique poursuivi, de la déviation de trajectoire du puits considéré, et du type de traitement (1C ou $3 \mathrm{C}$ ) souhaité à la suite de l'acquisition des données au terrain.

La méthode s'applique à plusieurs configurations géométriques d'acquisition de sismique de puits, mais spécifiquement au PSV en puits vertical à faiblement dévié, avec source placée à faible distance de la tête du puits, configuration pour laquelle il n'y a pas d'alternative connue à la méthode selon l'invention.

Ainsi, la méthode est applicable aux cas très courants où aucun outil de mesure d'orientation complet et précis n'est couplé à l'outil de mesure de PSV, par exemple lorsque l'outil PSV comprend trois composantes de capteurs sismiques directionnels orthogonaux uniquement, dans les configurations suivantes :

a) capteurs sismiques $3 \mathrm{C}$ montés de façon fixe dans l'outil PSV,

b) capteurs sismiques $3 \mathrm{C}$ montés de façon fixe dans un outil de PSV comprenant en outre un dispositif de mesure de l'angle de «Relative Bearing " dans le plan orthogonal à l'axe de l'outil PSV :

c) capteurs sismiques $3 \mathrm{C}$ sur des doubles cardans avec architecture dite "tourelle", c'est-à-dire comprenant un axe de rotation libre parallèle à l'axe de l'outil, donc parallèle à l'axe du puits à la station d'ancrage de l'outil PSV. Chaque capteur est monté conjointement avec une masse décentrée par rapport à l'axe des cardans de façon à obtenir un dispositif pendulaire qui s'oriente par la gravité dans un repère connu lié à la trajectoire du puits, qui est supposée connue, par exemple à partir d'une diagraphie de mesure de la trajectoire du puits par gyroscope, effectuée séparément.

Naturellement, le type de dispositif appelé communément "capteur de Relative Bearing", ainsi que le montage des capteurs sismiques sur doubles cardans monté en "tourelle" sont inopérants en puits strictement vertical et restituent une orientation des composantes sismiques horizontales qui n'est significative qu'au delà d'une faible valeur de l'ordre de 10 degrés de l'inclinaison verticale du puits, et qui devient de plus en plus précise lorsque l'inclinaison du puits dévié augmente.

Une application particulière de la méthode selon l'invention, consiste à monter un outil de PSV à trois composantes en combinaison avec un autre outil de diagraphie, dont on désire connaître l'orientation, dans le cas extrême ou les outils d'orientation usuels du genre 
gyroscope ou magnétomètre/inclinomètres ne sont plus opératoires, par exemple lorsque la température du puits excède $220^{\circ} \mathrm{C}$. 


\section{REVENDICATIONS}

1. Méthode de prétraitement de données sismiques acquises au moyen d'une méthode de prospection sismique de type profil sismique vertical qui comprend une émission d'ondes sismiques et une réception desdites ondes sismiques au moyen d'au moins un capteur multicomposantes positionné au sein d'un puits et que l'on fait stationner à au moins deux profondeurs, ledit capteur comprenant au moins trois géophones orthogonaux enregistrant en fonction du temps une première composante sismique dans une direction vectorielle connue, et au moins deux autres composantes sismiques dans deux directions orthogonales à ladite direction vectorielle connue, caractérisée en ce que :

a- on construit un nouveau signal en calculant la racine carrée de la somme des carrés desdites deux composantes sismiques orthogonales à ladite direction vectorielle connue, appelé signal module, et l'on pointe des temps d'arrivée d'une onde de cisaillement descendante sur un extremum d'amplitude dudit signal module ;

b- on oriente lesdites composantes sismiques dans un repère unique quelque soit la profondeur dudit capteur, au moyen des étapes suivantes répétées pour chaque profondeur:

- on définit une fenêtre temporelle de part et d'autre desdits temps d'arrivée ;

- on détermine une direction azimutale par maximisation d'une énergie desdites composantes sismiques orthogonales à ladite direction vectorielle connue au sein de ladite fenêtre temporelle ;

- on oriente à 360 degrés près lesdites composantes sismiques orthogonales à ladite direction vectorielle connue dans un repère unique défini par rapport à la direction azimutale qui est identique pour chaque profondeur.

c- on détermine l'orientation géographique dudit repère unique en déterminant une direction azimutale de pendage d'au moins un réflecteur sismique ou une direction principale d'anisotropie de biréfringence d'onde de cisaillement, par traitement des trois composantes dans le repère unique, et en appliquant au repère unique une rotation jusqu'à ce que la direction ainsi déterminée coïncide avec une direction azimutale connue à partir de connaissances structurales et géologiques. 
2. Méthode selon la revendication 1 , dans laquelle on réalise l'étape $\mathrm{c}$ ), en déterminant l'orientation géographique dudit repère unique au moyen des étapes suivantes :

- on extrait un champ d'ondes réfléchies au moyen d'un traitement isotrope des trois composantes dans le repère unique ;

- on détermine dans le repère unique un azimut de pendage d'au moins un réflecteur sismique au moyen d'une analyse de polarisation dudit champ d'ondes réfléchies ; et

- on détermine l'orientation géographique dudit repère unique en alignant ledit azimut de pendage sur une direction de pendage dudit réflecteur connue par ailleurs, à une même profondeur au droit du puits.

3. Méthode selon la revendication 1, dans laquelle on réalise l'étape c), en déterminant l'orientation géographique dudit repère unique au moyen des étapes suivantes :

- on détecte, pour au moins un intervalle de profondeur, une anisotropie de biréfringence de modes rapide et lent d'onde de cisaillement par une analyse de signaux de l'onde de cisaillement dans le repère unique ;

- on détermine une direction principale de l'anisotropie de biréfringence ainsi détectée dans le repère unique déterminé à l'étape $b$;

- on détermine l'orientation géographique dudit repère unique en alignant la direction principale de mode lent d'anisotropie de biréfringence d'onde de cisaillement sur une direction de pendage stratigraphique déterminée par des connaissances structurales et géologiques.

4. Méthode selon la revendication 1 , dans laquelle on réalise l'étape $\mathrm{c}$ ), en déterminant l'orientation géographique dudit repère unique au moyen des étapes suivantes :

- on détecte, pour au moins un intervalle de profondeur, une anisotropie de biréfringence de modes rapide et lent d'onde de cisaillement par une analyse de signaux de l'onde de cisaillement dans le repère unique ;

- on détermine une direction principale de l'anisotropie de biréfringence ainsi détectée dans le repère unique déterminé à l'étape $b$;

- on détermine l'orientation géographique dudit repère unique en alignant la direction principale de mode lent d'anisotropie de biréfringence d'onde de cisaillement sur une direction de contrainte horizontale maximale déterminée par des connaissances structurales et géologiques. 
5. Méthode selon l'une des revendications précédentes, dans laquelle, préalablement à l'étape a), on préserve l'isotropie du signal en trois composantes, en respectant des rapports d'amplitude et des différences de phase entre les composantes sismiques.

6. Méthode selon l'une des revendications précédentes, dans laquelle on améliore un rapport signal sur bruit des trois composantes du signal brut, préalablement au calcul du module, au moyen d'une déconvolution isotrope des trois composantes par un signal unique d'onde de pression descendante extrait de la composante sismique de direction vectorielle connue.

7. Méthode selon l'une des revendications précédentes, dans laquelle on filtre ledit signal module de façon à supprimer des composantes basses fréquences, avant de pointer les temps d'arrivée de l'onde sismique directe.

8. Méthode selon l'une des revendications précédentes, dans laquelle on détermine l'orientation géographique dudit repère unique pour au moins deux profondeurs, et on effectue une rotation graduelle du repère unique entre lesdites profondeurs, pour compenser une différence d'orientation géographique entre lesdites profondeurs.

9. Méthode selon la revendication 8, dans laquelle on effectue la rotation graduelle en appliquant un gradient d'angle de rotation linéaire avec la profondeur et limité à environ quinze degrés pour cent mètres de profondeur.

10. Méthode selon l'une des revendications 8 et 9 , dans laquelle on détermine l'orientation géographique dudit repère unique sur au moins une profondeur en montant ledit capteur multi-composantes sur un système de double cardans, ledit système permettant d'orienter par gravité lesdites composantes sismiques lorsque l'inclinaison du puits atteint une valeur d'au moins environ 10 degrés.

11. Méthode selon l'une des revendications 8 et 9 , dans laquelle on descend dans le puits un outil de mesure de puits comportant ledit capteur multi-composantes monté de façon fixe, et l'on détermine l'orientation géographique dudit repère unique sur au moins une profondeur au moyen d'un système de mesure d'un angle de "relative bearing" monté sur ledit outil de mesure, qui permet de retrouver l'orientation dudit capteur multi-composantes lorsque l'inclinaison du puits atteint une valeur d'au moins environ 10 degrés. 
$100 \longrightarrow$

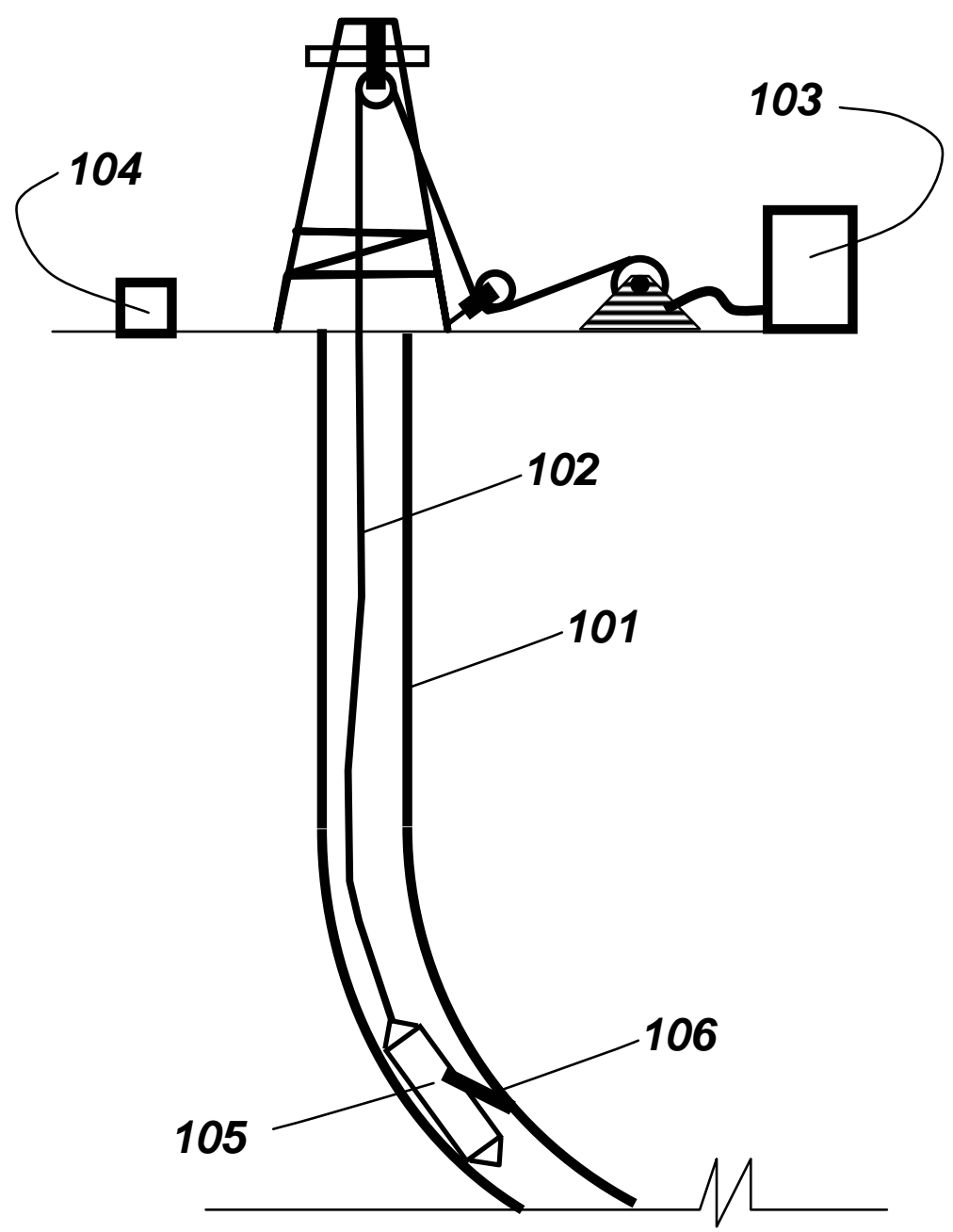

卢

Fig. 1 
0

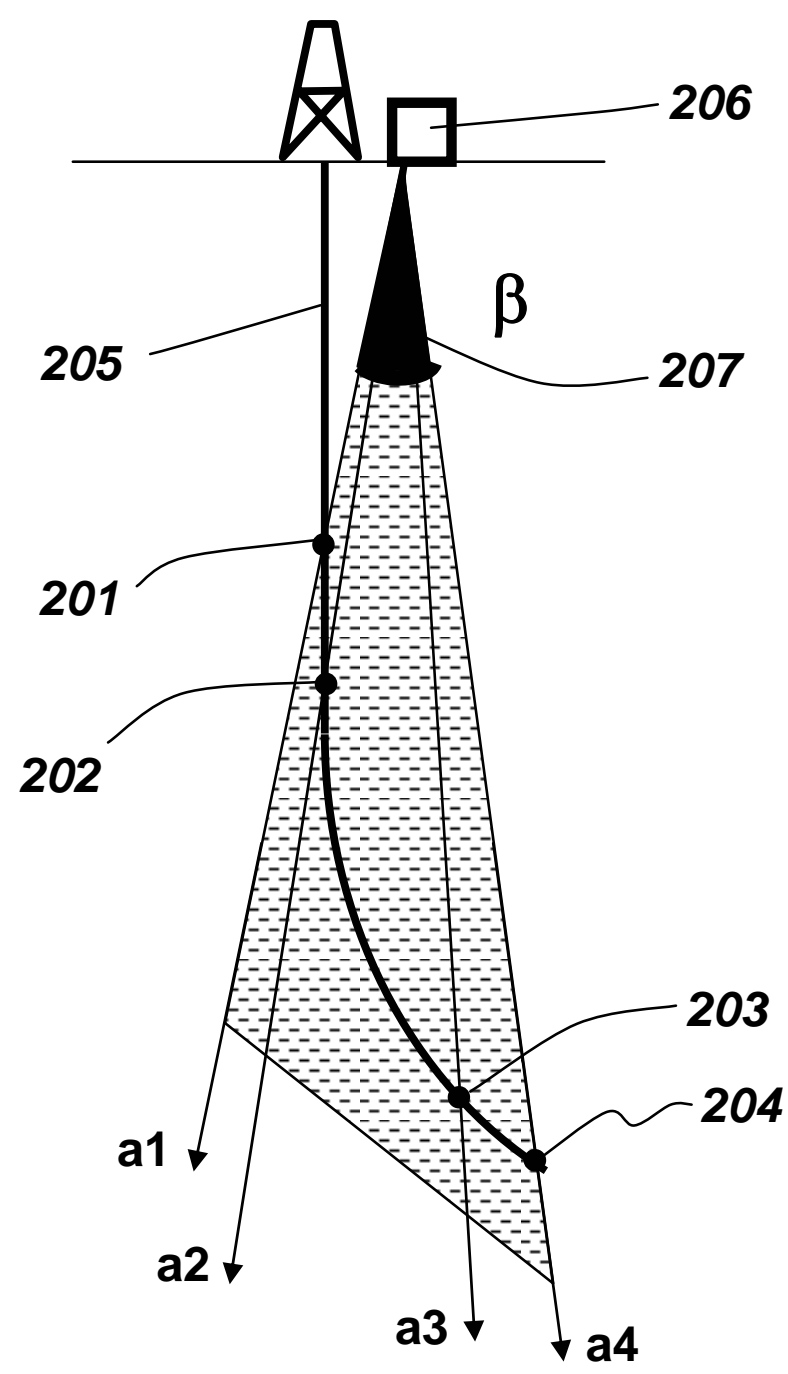

$\stackrel{N}{\mathrm{v}}$

Fig. 2 
300

0

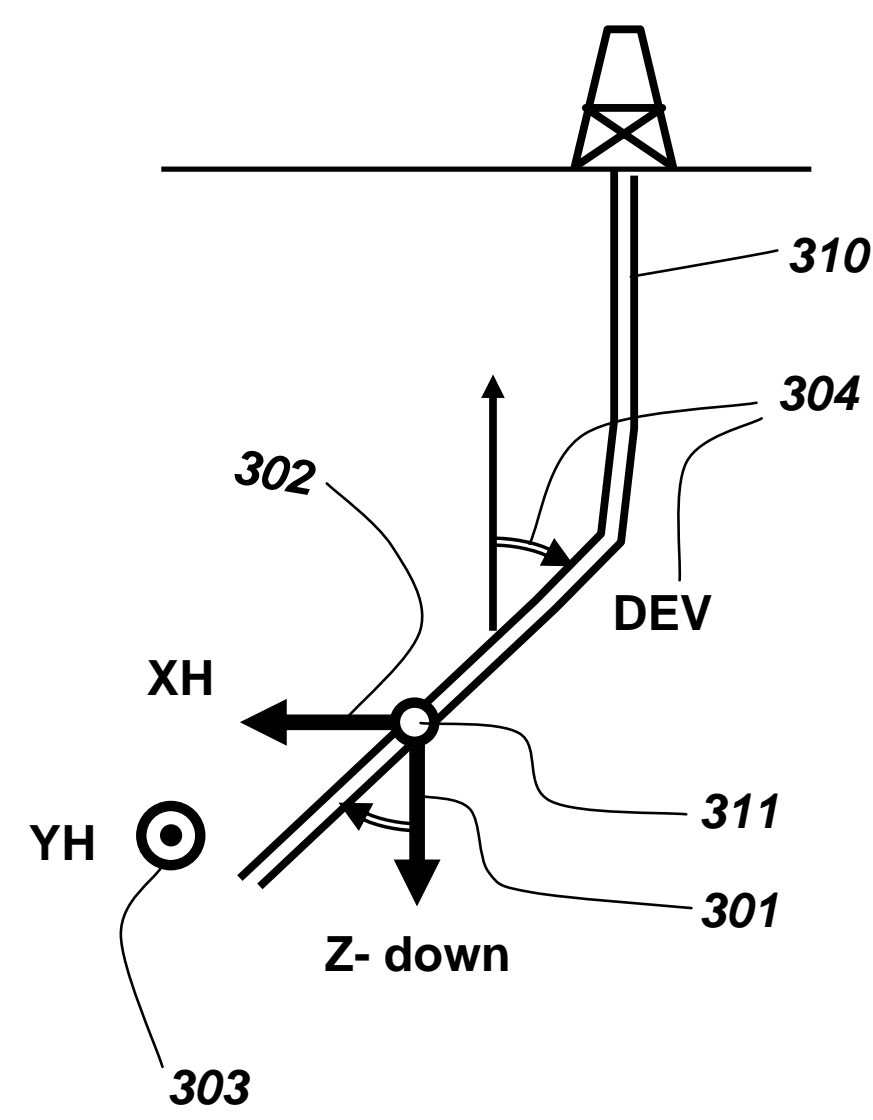

350

0

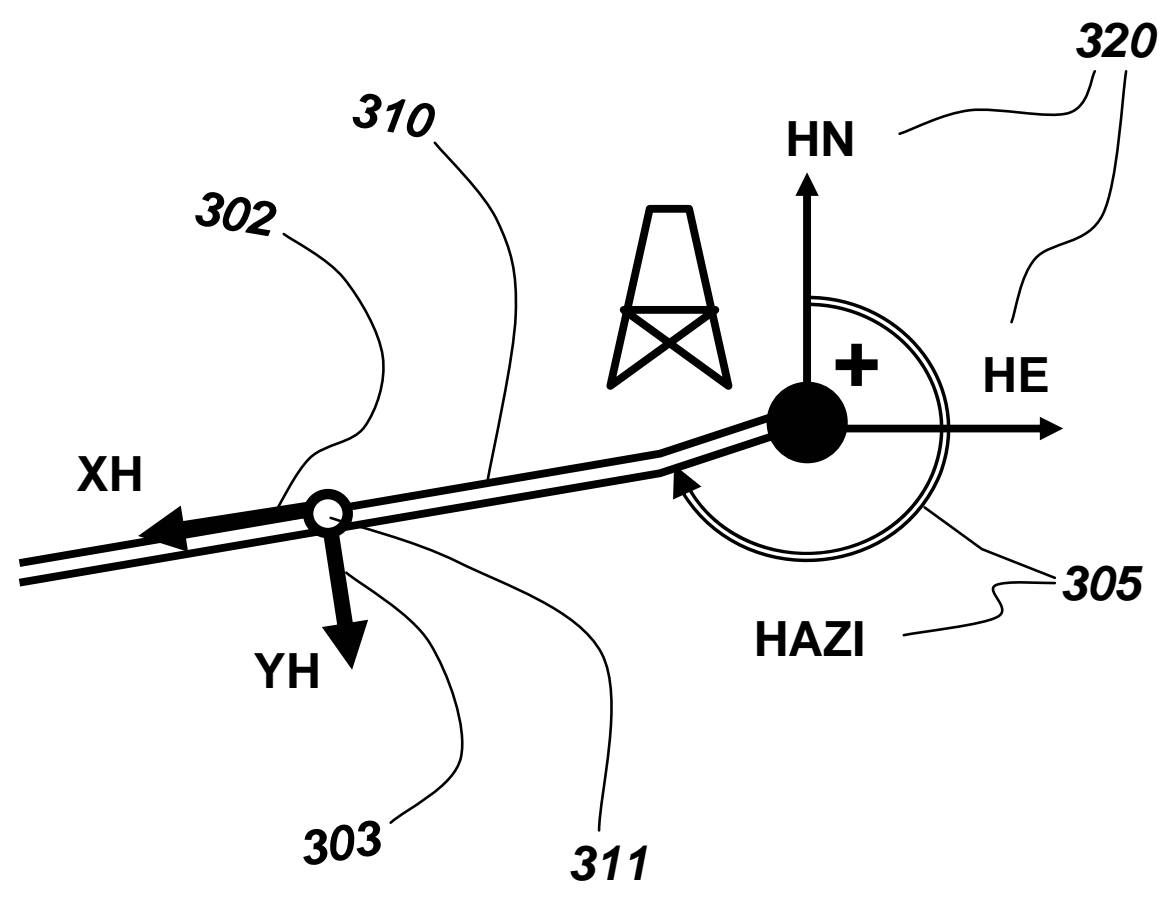


400

0

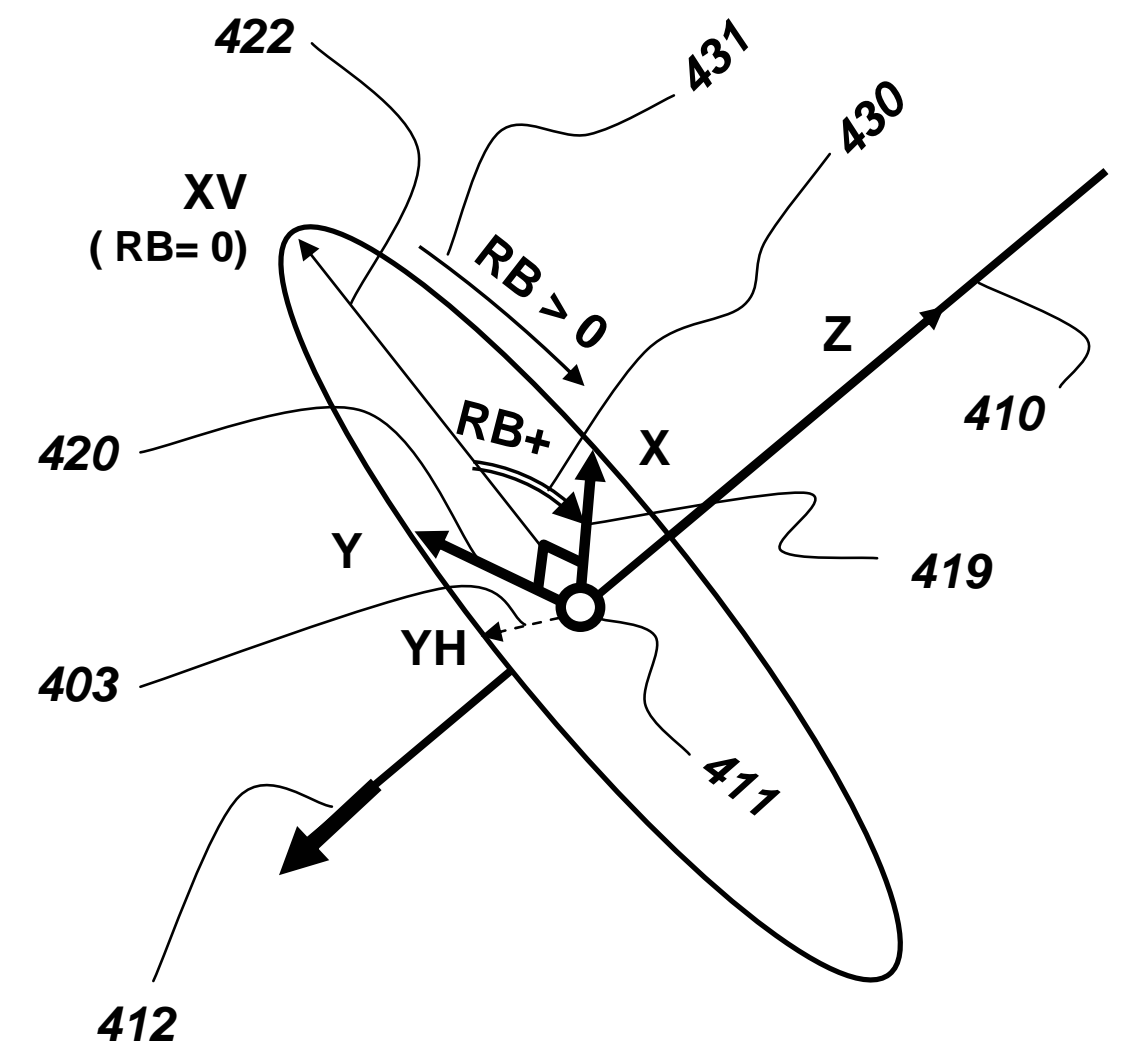

450 0

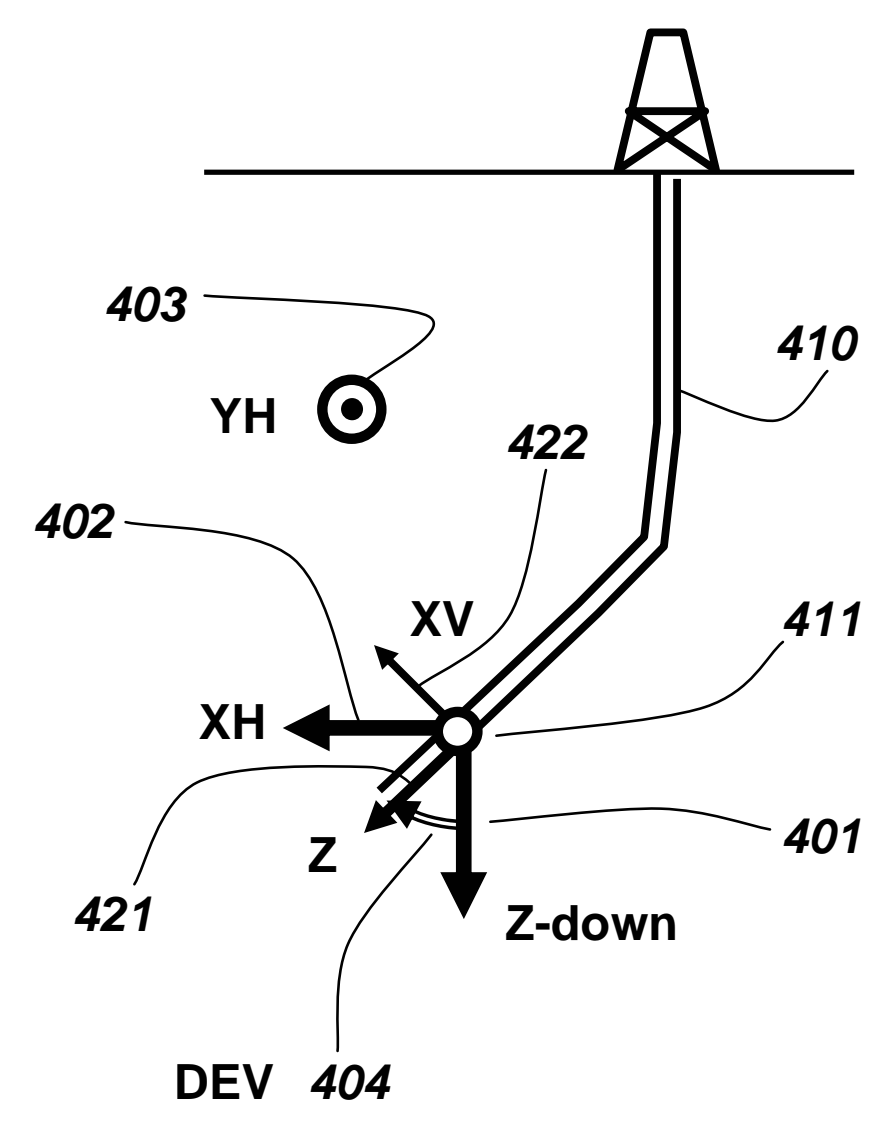

Fig. 4a

Fig. 4b 


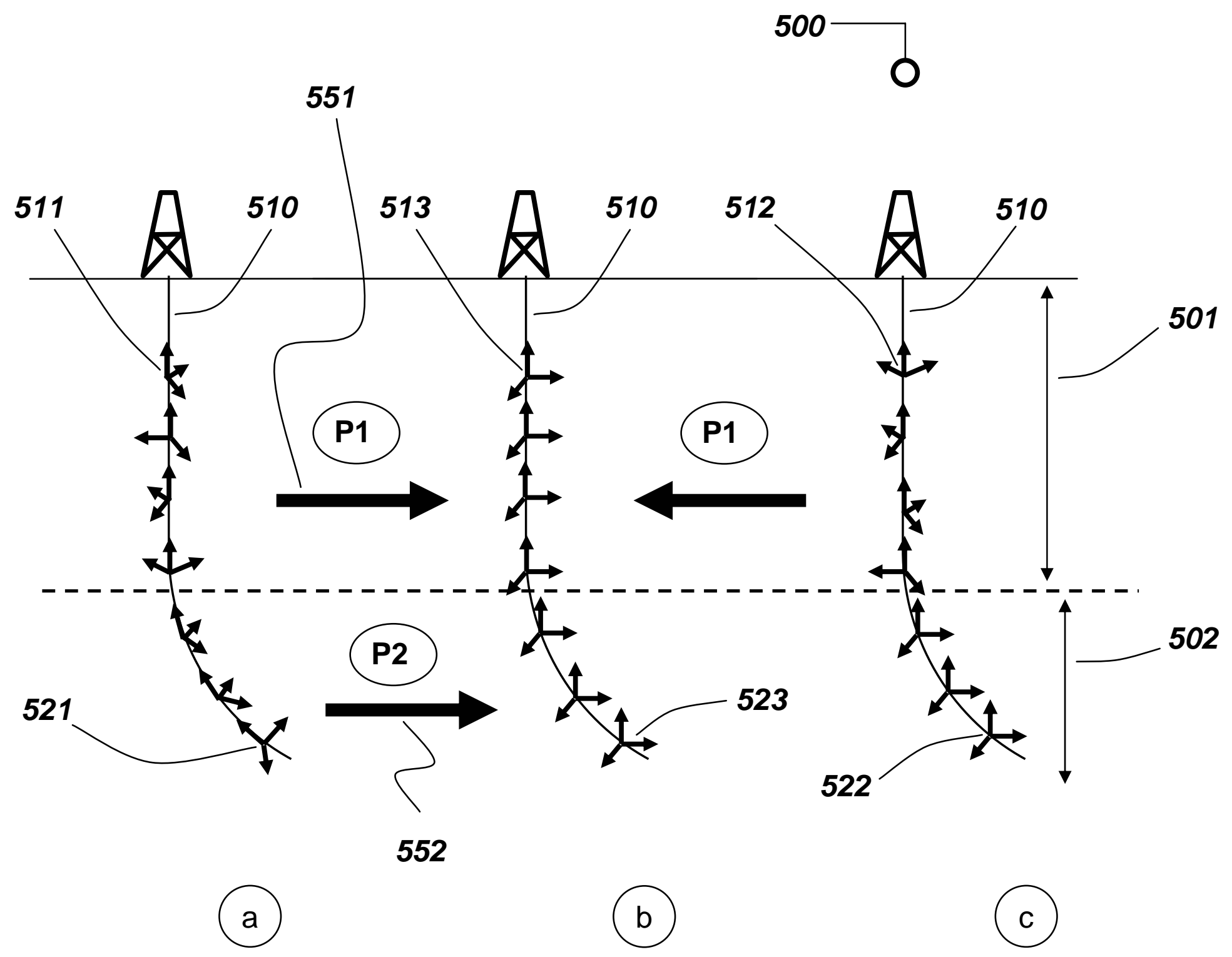

Fig. 5 
600

0

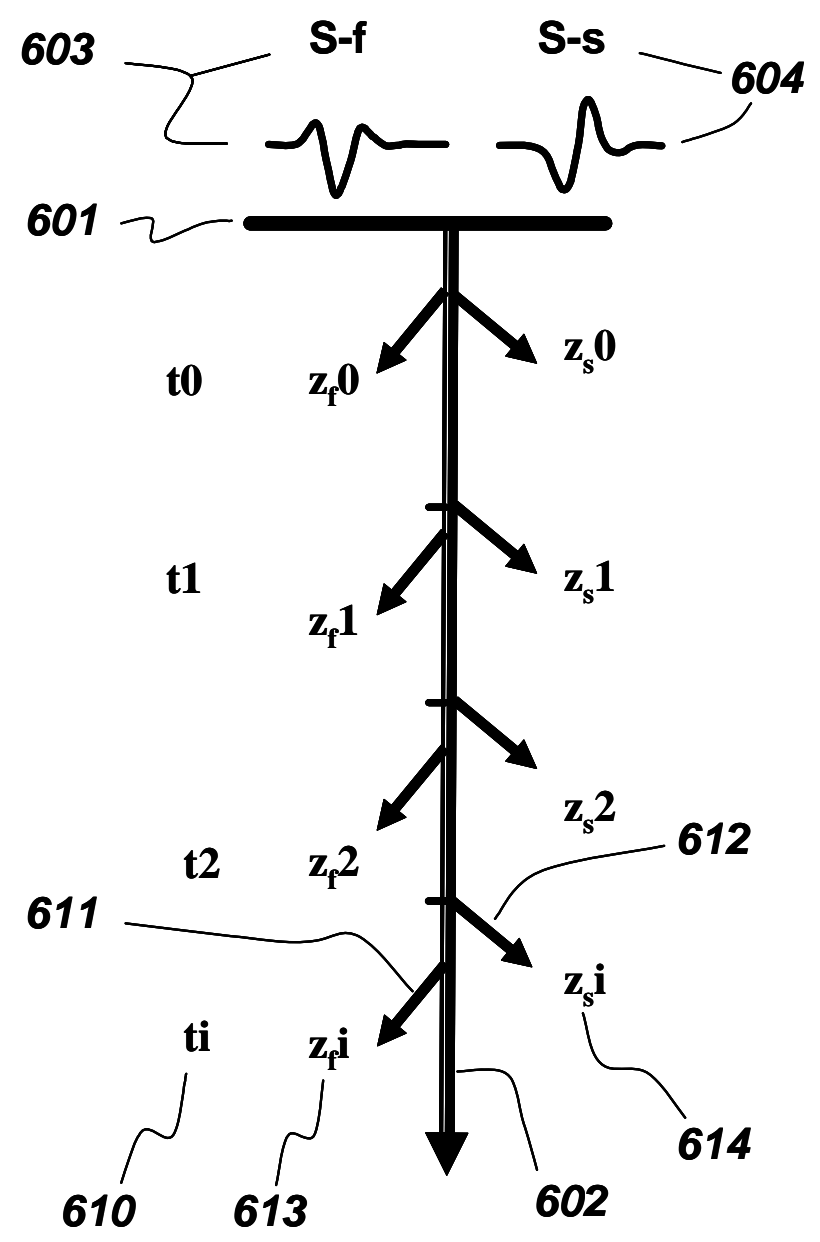

601

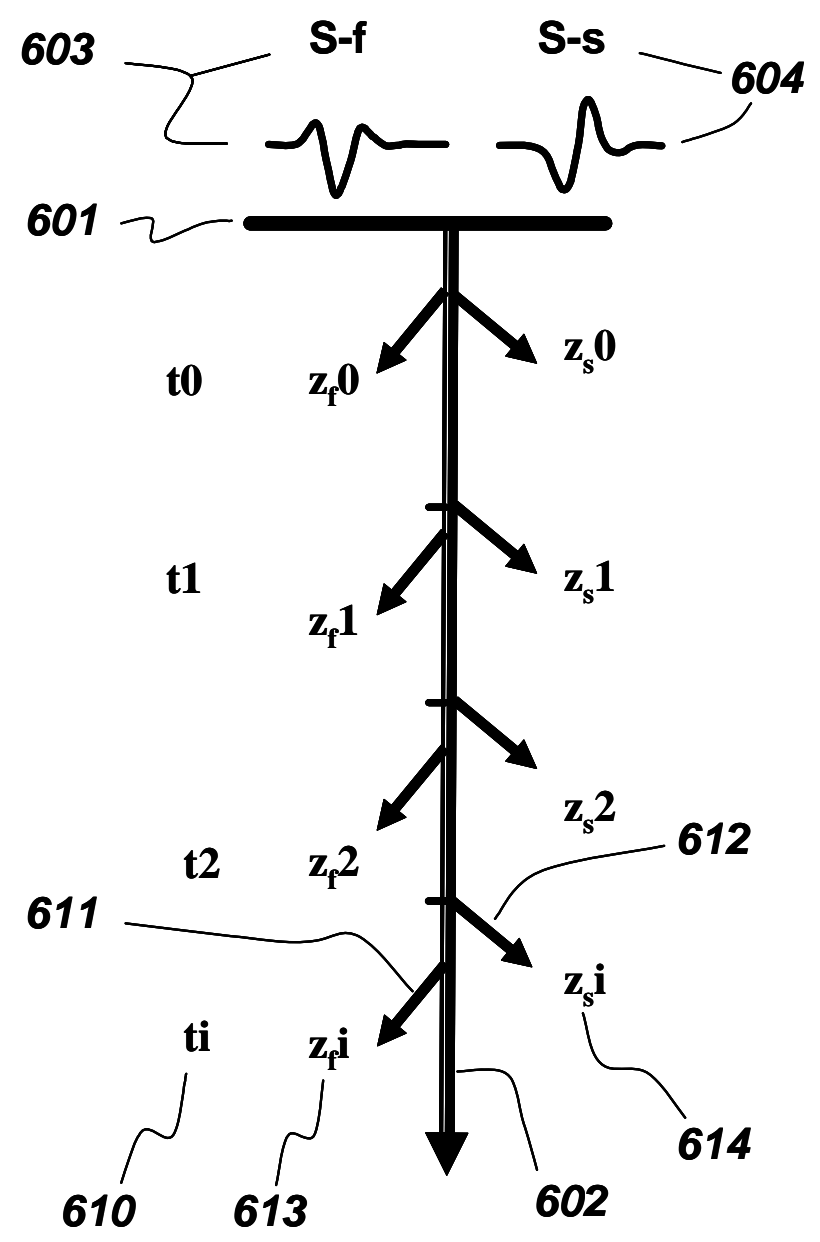

Fig. 6a
650

0

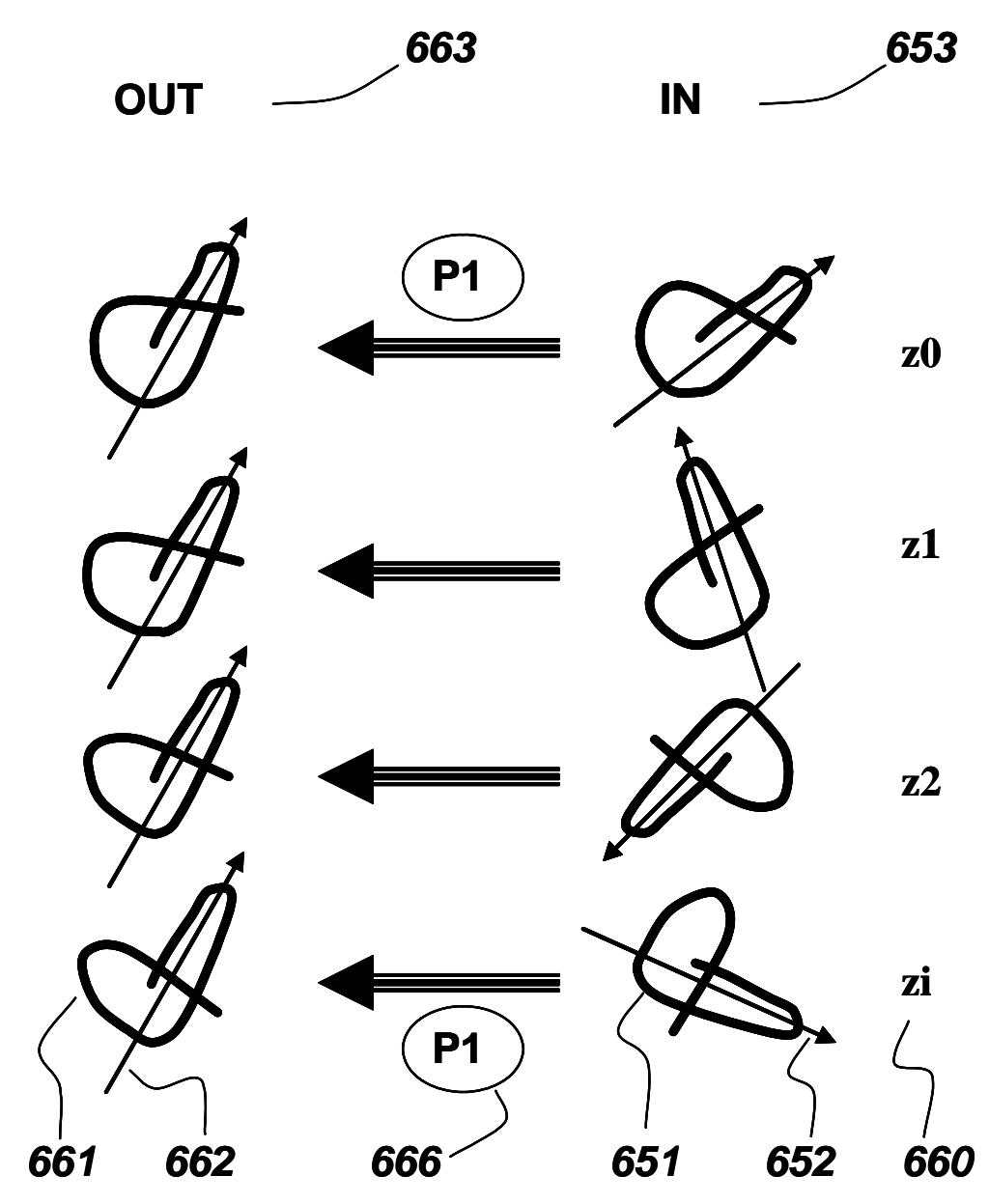

Fig. 6b 


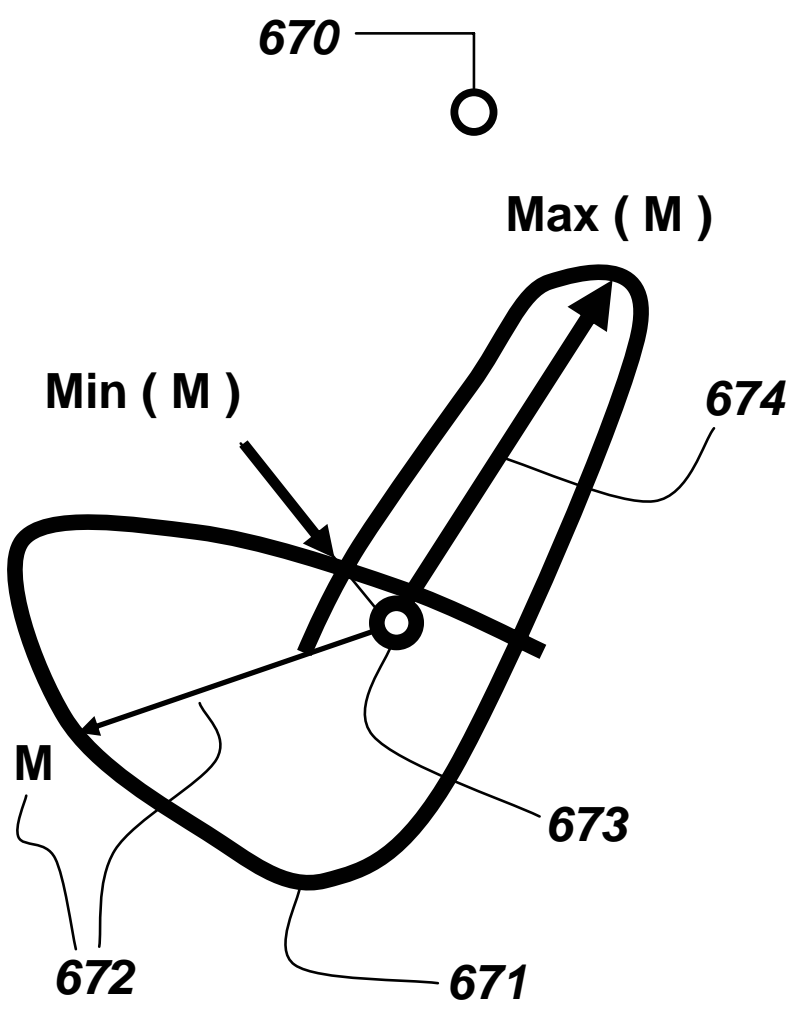

Fig. 6c

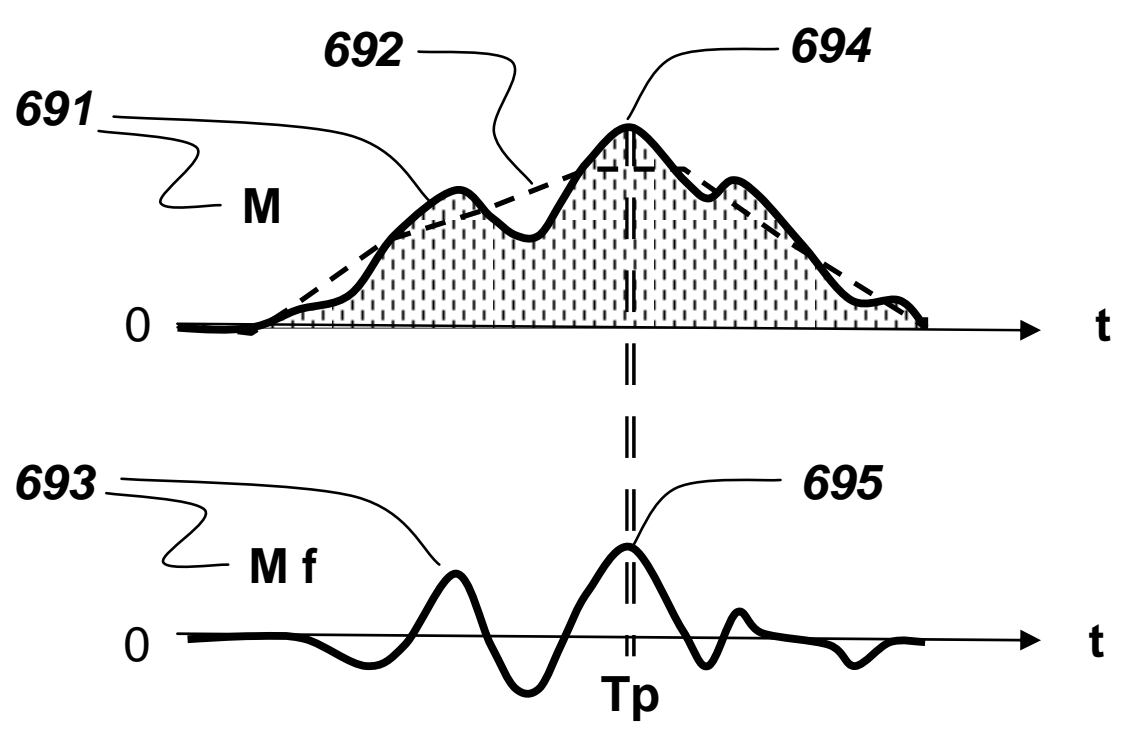

v
Fig. 6d 


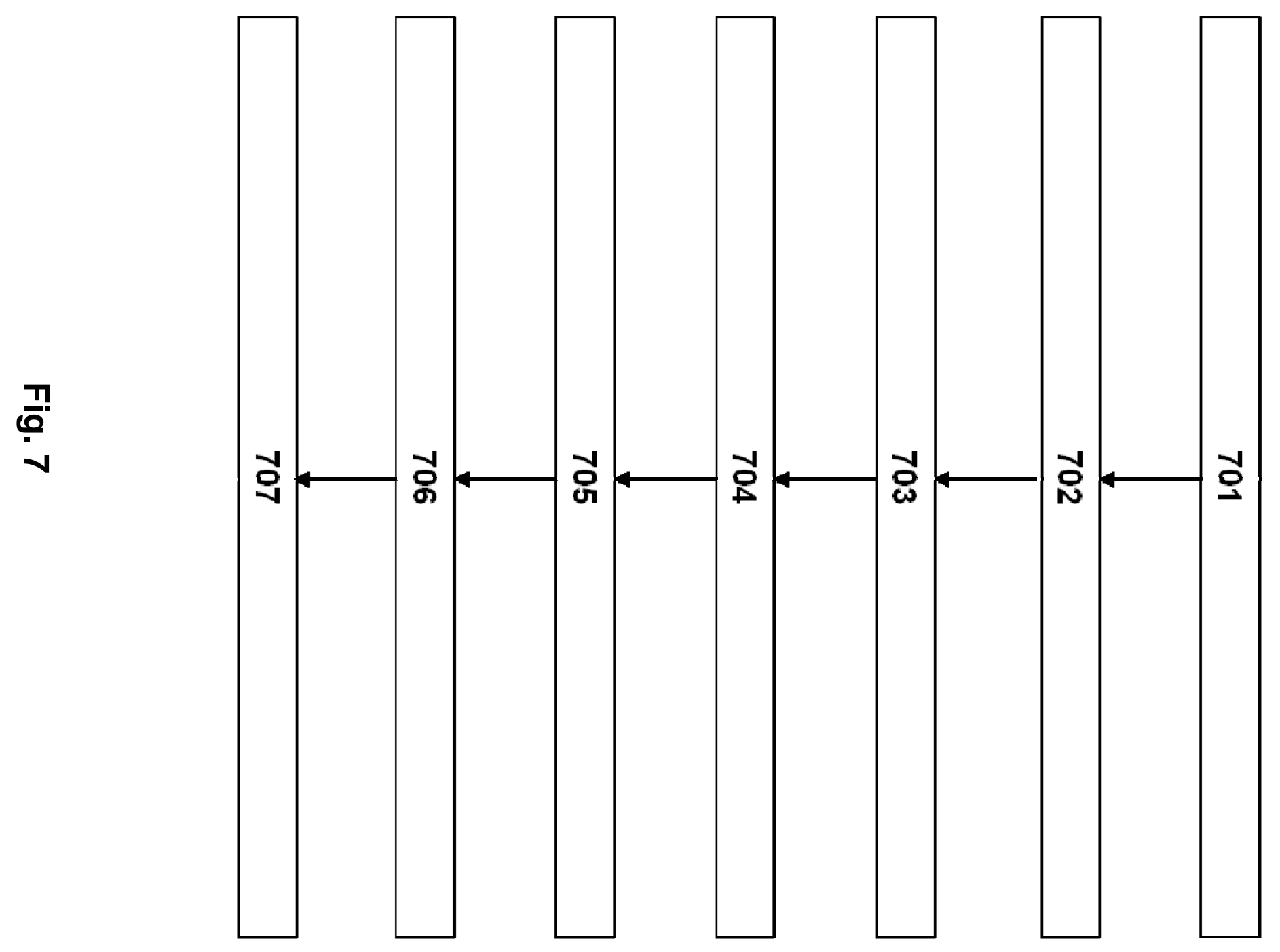

8

。

$\stackrel{\infty}{\vec{v}}$ 


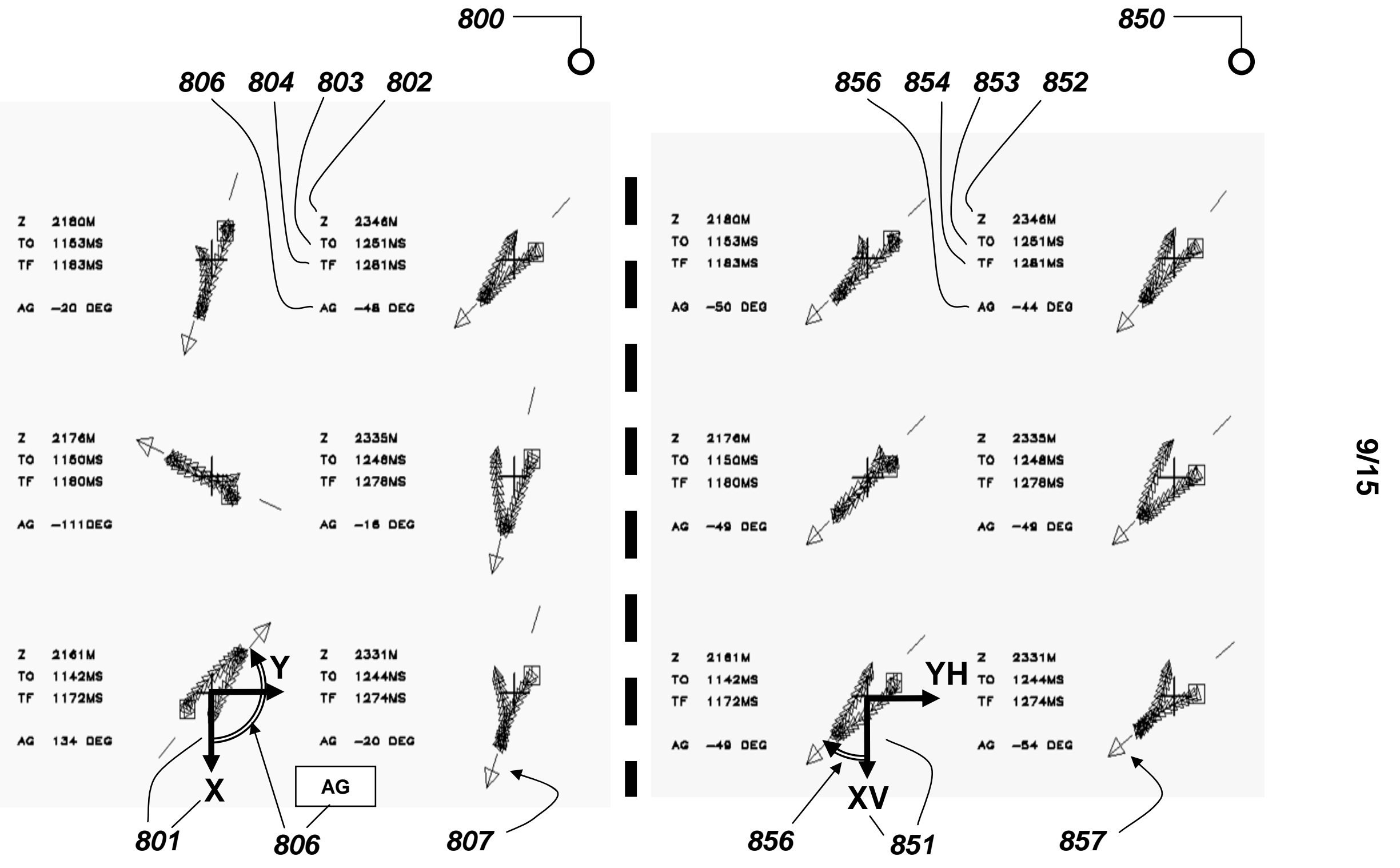

Fig.8a

Fig.8b 

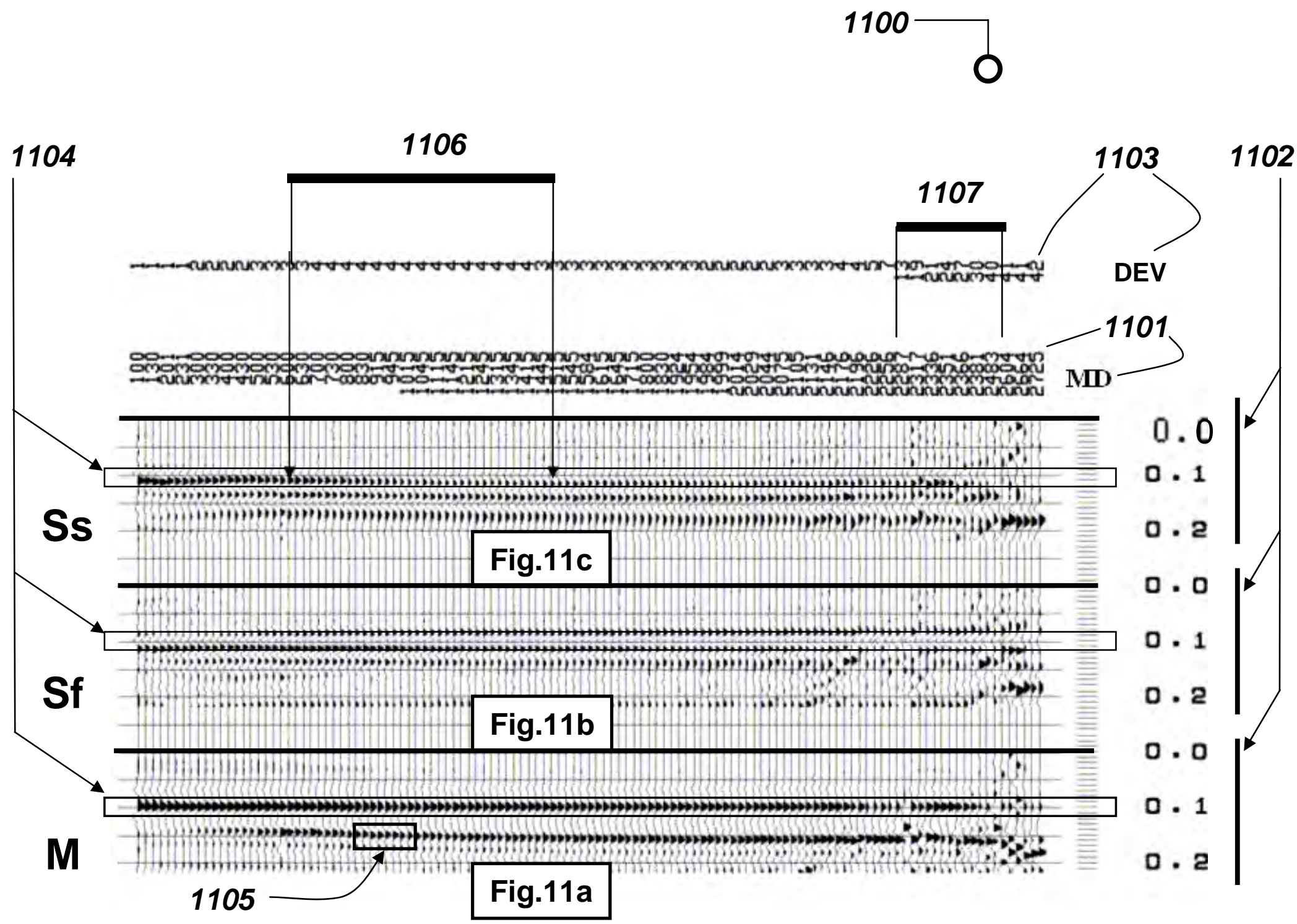
1200

0

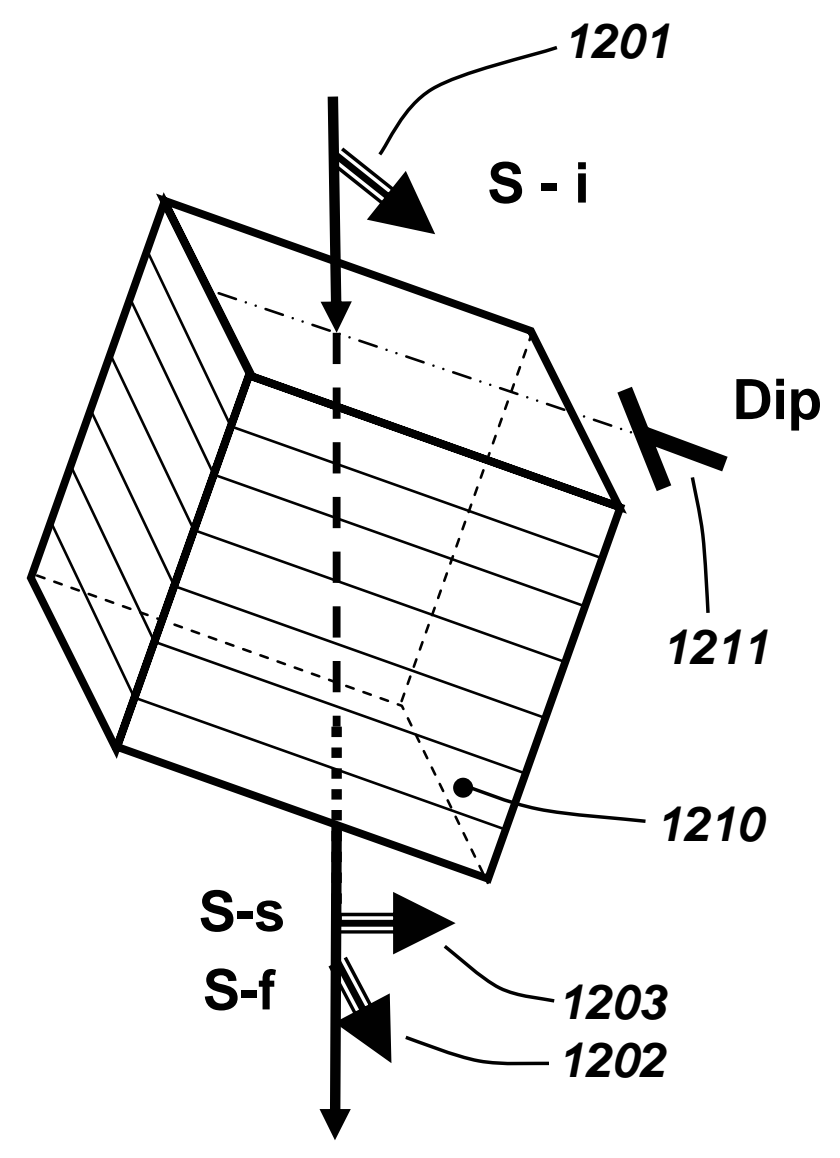

1300

0

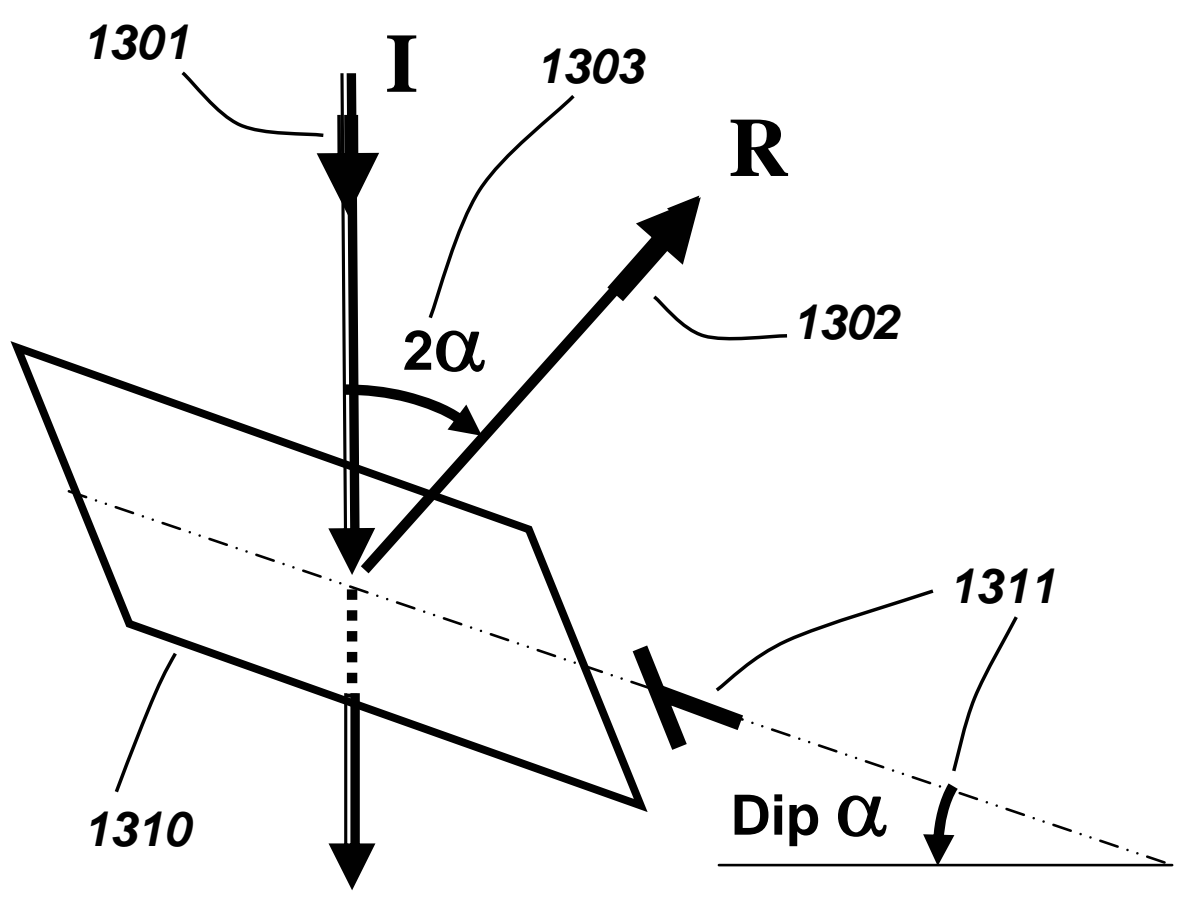




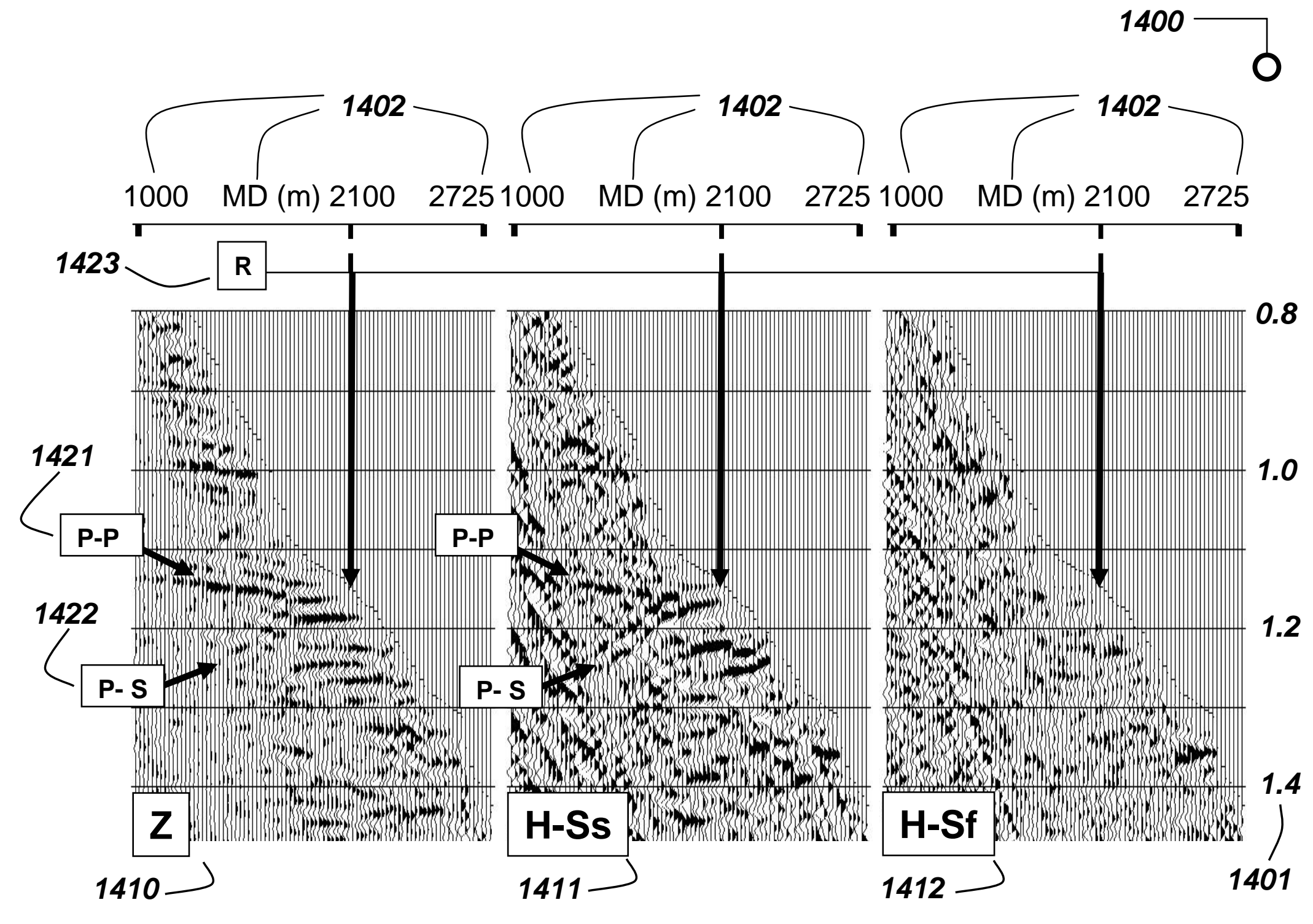

Fig.14a

Fig.14b

Fig.14c 


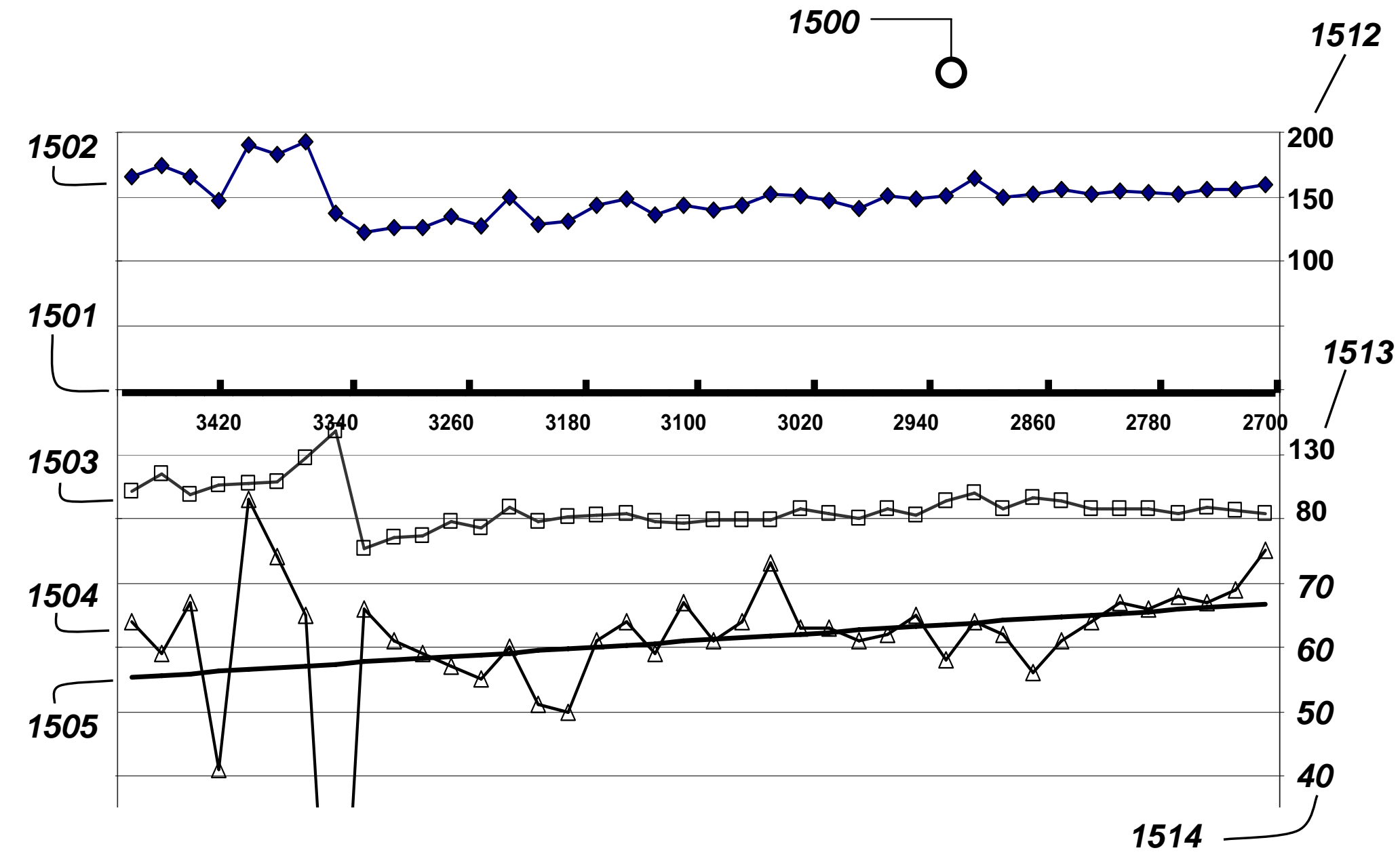

$\underset{v}{\vec{v}}$

Fig.15 

Appendix 2 



\title{
Seismic imaging on the Aghajari field: pitfalls and solutions
}

Kazem Kazemi* , Pascal Froidevaux¹, Laurence Nicoletis,

\begin{abstract}
Aghajari field is located in the Dezful Embayment, one of major structural zone in Zagros foldthrust belt containing most of Iranian oil fields. Asmari formation is the main reservoir unit and consists of dolomite and limestone, highly fractured in the more producing area. In the area of interest, the Asmari is strongly folded and recovered by evaporitic sequence with large thickness variations and heterogeneous content. The recent and first 3D seismic acquisition in the area has produced poor image of the reservoir after a standard processing and PSTM workflow. As PSTM kinematic attributes are key data to initiate velocity model inversion, this paper analyses the parameters of classic PSTM and proposes a better PSTM approach for this dataset.
\end{abstract}




\section{Amsterdam|'o9}

\section{Introduction}

The Aghajari oil field is located in the Dezful Embayment, one of major structural zone in Zagros fold-thrust belt containing most of Iranian oil fields. The Aghajari oil field consists of an anticline with the axis oriented approximately northwest to southeast. Southern flank is usually the steeper with dips of up to $70^{\circ}$ but averaging about $40^{\circ}$, while the average dip on the northeast flank is about $30^{\circ}$, with values up to $50^{\circ}$ locally developed. There is a thrust fault in the southwest flank of Aghajari field and the separation with the Marun field located southwest of the fault 2200 meters below MSL is a big doubt. One of the objectives of the first 3D seismic survey in the area is to bring information on the structural link between these two fields.

The Asmari and deeper formations are mainly limestones and dolomites with high velocities (5500 $\mathrm{m} / \mathrm{s}$ and above) while the overburden formations have velocities between $3000 \mathrm{~m} / \mathrm{s}$ and $4000 \mathrm{~m} / \mathrm{s}$. The reservoir formation is overlaid by the the evaporitic (salt and anhydrites) that presents important thickness variations and syntectonic deformation (Sherkati and Letouzey, 2005). As the reservoir porosity and production is mainly controlled by fractures, a second objective for the 3D seismic survey is the identification of faults system and possibly main fractures in the reservoir area. For both objectives, the first step is to obtain a good structural image.

PSTM imaging is appropriate as long as lateral velocity variations are mild to moderate. When the lateral velocity gradients are significant, as it is the case in the south West flank of the Aghagari structure, we need to use depth migration that requires to build a depth velocity model by tomographic inversion. The final aim of this work is to use the "Beyond Dix" method to build the initial velocity field from pre-stack time migrated gathers. It is based on a dense volumetric dip and residual moveout (RMO) picking in pre-stack time migrated domain, and a tomographic inversion of the kinematic invariants computed in the un-migrated time domain (Lambare et al, 2007). This method already showed significant improvements in imaging faults and steeply dipping structure in the Golf of Mexico, and other complex geological setting, using marine data. Here, it is the first application of the "Beyond Dix" method to standard orthogonal land dataset in a compressive and folded environment.

In the first part of the paper, we present the results obtained by this method, starting with standard aperture parameters for the initial PSTM. As the results were not satisfactory, we analysed in a second step, the different imaging parameters and we optimized migration apertures in the azimutal domain in order to recover dipping part of the reservoir.

\section{Acquisition and Processing}

This survey was shot by dynamite, using $300 \mathrm{~m}$ spacing between source and receivers lines and $50 \mathrm{~m}$ spacing between sources and groups. The maximum offset is $1800 \mathrm{~m}$ in cross line (strike) direction and $6000 \mathrm{~m}$ in the receiver line (dip) direction. The final bin is $25 \mathrm{~m}$ by $25 \mathrm{~m}$ and the maximum fold of 80 and 48 when considering a "standard" $2500 \mathrm{~m}$ offset. The preprocessing included regional and residual statics, noise elimination and a first path of PSTM leading to initial PSTM velocity laws.

\section{PSTM and attributes picking in the time migrated domain}

Based on acquisition parameters and geological data (in particular steep dips up to $60^{\circ}$ ), aperture widths (horizontal vector offset between image points and midpoints) were chosen at maximum values and local dips up to $65^{\circ}$. We manually picked PSTM velocities and obtained an "optimum" field shown on Figure 1. In order to get residual move-out and to improve the quality of final stack the following steps were performed on prestack gathers:

-Prediction and subtraction of multiples in the prestack migrated gathers, -High density automatic bispectral picking of non-hyperbolic residual move-out. 
At the end we obtained the stack shown on the figure 2, where the unconformity (strong marker just above top reservoir) is picked in blue. In this figure, we see the large fault that outcrops on the surface and the heterogeneous evaporitic formation with important thickness variation, just above the unconformity. However, the unconformity does not show up in the dipping part (reservoir area). In consequence, the "migrated facets" and RMO attributes cannot be properly picked in the reservoir area. Finally, the velocity model obtained by inversion of corresponding time invariants was not constrained in the complex area and application of Kirchhoff PSDM did not bring a better illumination of the steep parts of the structure.

At this stage, the question is to know if reflections on dipping flanks do exist in the recorded dataset and if so, how to recover them?

\section{Improving PSTM}

We reviewed all parameters of the PSTM processing in order to retrieve, if possible, the dipping features on the flanks of the reservoir. We found a solution that involves:

- not to apply any migration anti-alias operator that progressively kills dipping reflectors above $40^{\circ}$, possibly because the bin size driving the anti-alias was too large $(50 \mathrm{~m})$, with regards to the nominal bin size,

- to apply an azimuthal aperture selection in migration offset vector (differences between $\mathrm{X}$ and $\mathrm{Y}$ coordinates of the image and midpoint): the best results were obtained for a $42^{\circ}$ in-line sector that filters out traces corresponding to strike dips and attenuate somehow horizontal reflectors compared to dipping reflectors ,

- to stack the gathers only in the 0 to $2500 \mathrm{~m}$ (acquisition) offset range .

\section{Discussion and conclusions}

The main problem to be solved, prior to the picking of kinematics attributes is to recover dipping parts of reflectors that correspond to reservoir area. This problem is a recurrent difficulty of all surveys in the area. One of the problems may be the anti-alias protection that attenuates the dipping reflectors because of the bin size. Another problem is the high level of migration noise present in the data when the migration aperture is large. Partial solution has been found by selecting carefully and manually the migration aperture. A better and more practical solution would be to automatically and densely select the best (specular) dip (Kabbej et al, 2005, Tabti et al, 2004). Another solution could be to use CRS attributes measured on pre-migrated data space in order to automatically limit migration aperture (Spinner, 2006) or beam migration (Vetle et al, 2008). Such methods have not yet been applied and the results obtained so far make us confident that some solution could be found using such new methods 


\section{Amsterdam|'o9}

\section{Acknowledgments}

We acknowledge National Iranian Oil Company, Exploration Directorate for providing the data, CGGVeritas and IFP for financial support. We gratefully acknowledge Gilles Lambare and Serge Zimine (CGGVeritas) for his comments and insightful discussions, P. Mitouard , P. Charrier and R. Oukhedouma (CGGVeritas) for their support and supervision and J. Letouzey for his useful geological comments .

\section{References}

Kabbej, A., Baina, R., Duquet, B. [2005] Data driven automatic aperture optimization for Kirchhoff migration, 75th Annual SEG International Meeting, Expanded Abstracts, 1890-1893.

Lambare, G., Herrmann, P., Guillaume, P., Zimine, S., Wolfarth, S., Hermant, O., Butt, S., [2007] From time to depth imaging with 'Beyond Dix', First Break, 25 (9) , 71-76.

Sherkati, S. Letouzey, J. Lamotte D., [2006] The centeral Zagros fold-thrust belt (Iran): New insights from seismic data, feild observation and sandbox modelling, Tectonics, 25

Spinner, M., [2006] 3D CRS-based limited aperture Kirchhoff time migration, 76 th Annual SEG International Meeting, Expanded Abstracts, 2569-2573

Tabti,H. Gelius, L., Hellmann T., [2004], Fresnel aperture prestack depth migration, First Break, 22 (3), 39-46

Vetle, V., V., Roberts, G., Taylor, R., [2008], Controlled beam migration: a versatile structural imaging tool, First Break, 26 (9), 109-113.

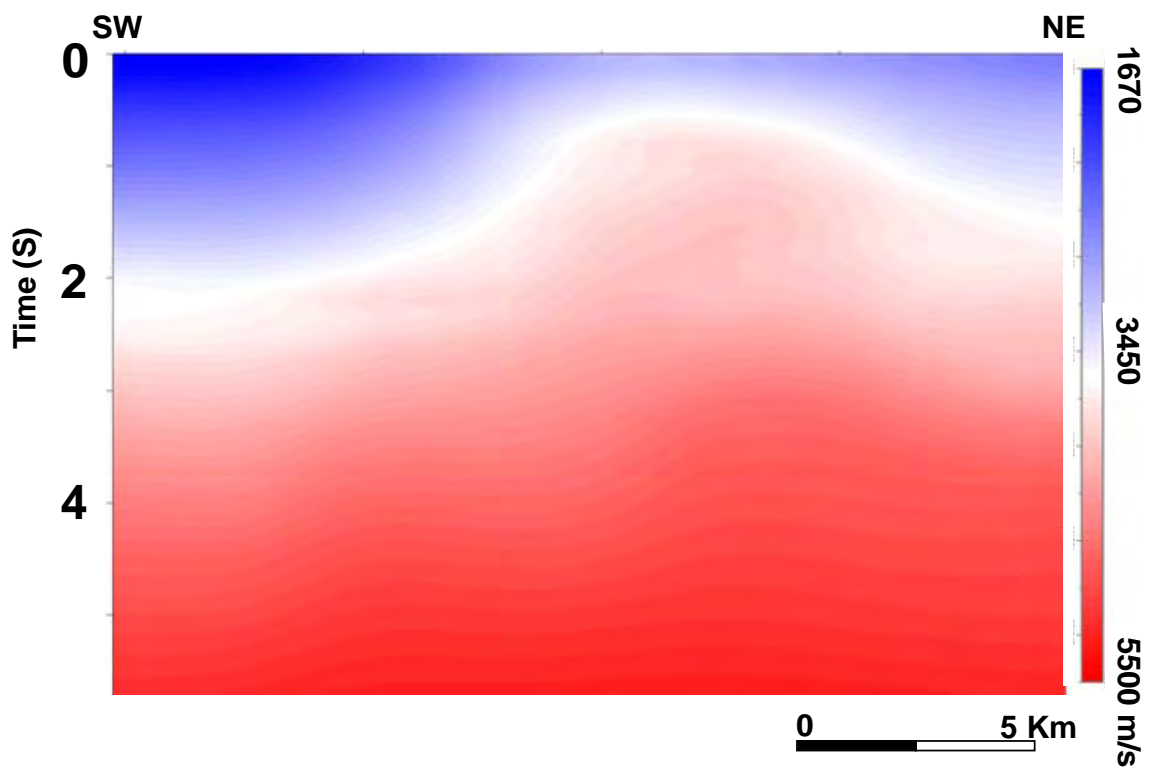

Figure 1: PSTM velocity profile 


\section{0 \\ Amsterdaml'og}

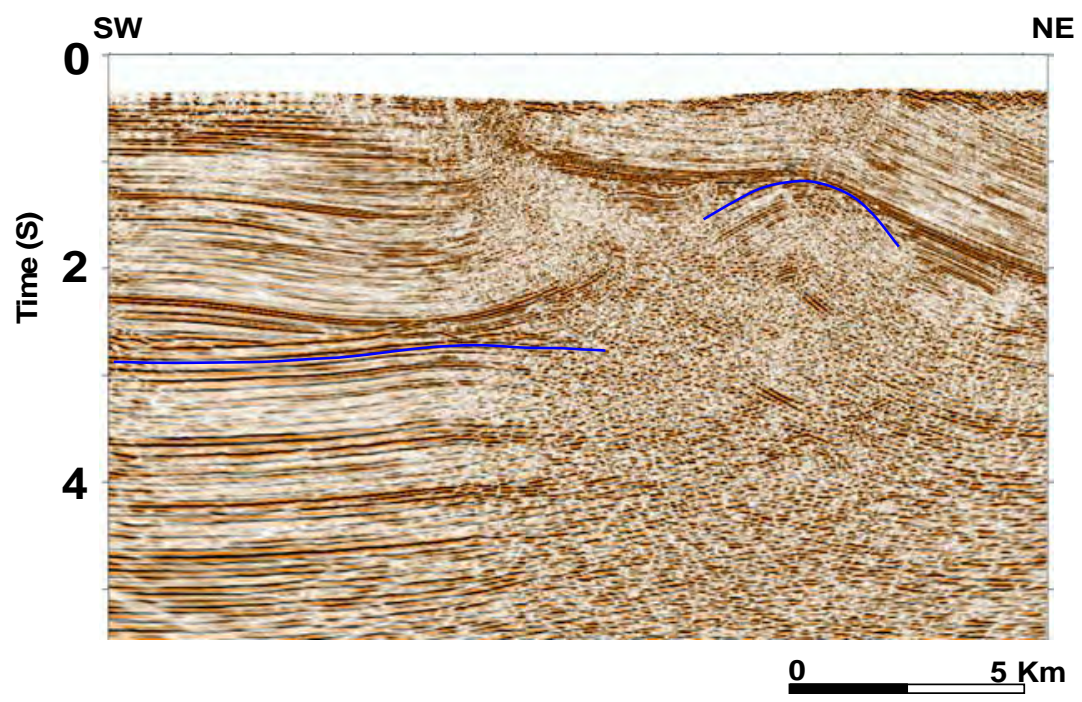

Figure 2: stack after first PSTM

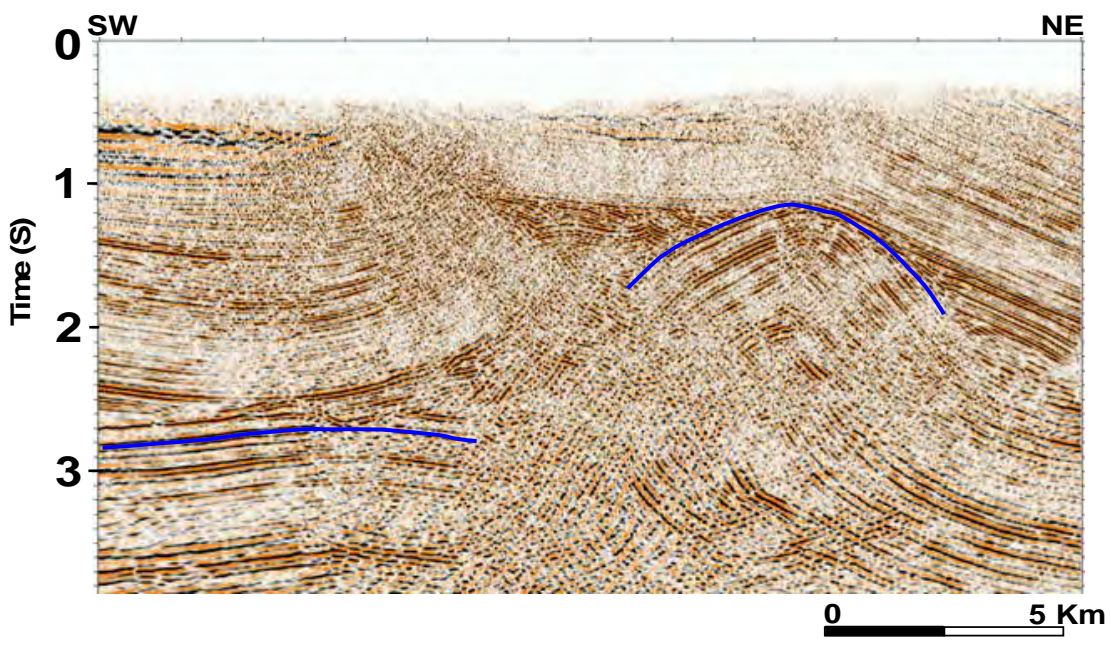

Figure 3: Stack after final PSTM 